Cochrane Database of Systematic Reviews

\title{
Intensive case management for severe mental illness (Review)
}

Dieterich M, Irving CB, Bergman H, Khokhar MA, Park B, Marshall M

Dieterich M, Irving CB, Bergman H, Khokhar MA, Park B, Marshall M.

Intensive case management for severe mental illness.

Cochrane Database of Systematic Reviews 2017, Issue 1. Art. No.: CD007906.

DOI: 10.1002/14651858.CD007906.pub3. 
TABLE OF CONTENTS

ABSTRACT 1

PLAIN LANGUAGE SUMMARY

SUMMARY OF FINDINGS

BACKGROUND

OBJECTIVES

METHODS

Figure 1.

RESULTS

Figure 2.

Figure 3.

Figure 4.

Figure 5.

Figure 6.

Figure 7.

Figure 8.

Figure 9.

Figure 10.

Figure 11.

DISCUSSION

AUTHORS' CONCLUSIONS

ACKNOWLEDGEMENTS

REFERENCES

CHARACTERISTICS OF STUDIES

DATA AND ANALYSES

Analysis 1.1. Comparison 1 Intensive Case Management versus standard care, Outcome 1 Service use: 1 . Average number of days in hospital per month - by about 24 months.

Analysis 1.2. Comparison 1 Intensive Case Management versus standard care, Outcome 2 Service use: 1a. Number of days in hospital - by follow -up (skewed data, sample size $\geqq 200$ ).

Analysis 1.3. Comparison 1 Intensive Case Management versus standard care, Outcome 3 Service use: 2 . Not remaining in contact with psychiatric services.

Analysis 1.4. Comparison 1 Intensive Case Management versus standard care, Outcome 4 Service use: 3a. Admitted to hospital.

Analysis 1.5. Comparison 1 Intensive Case Management versus standard care, Outcome 5 Service use: 3b. Average number of admissions per month (skewed data).

Analysis 1.6. Comparison 1 Intensive Case Management versus standard care, Outcome 6 Service use: 4a. Admitted to ER - by long term.

Analysis 1.7. Comparison 1 Intensive Case Management versus standard care, Outcome 7 Service use: 4b. Average number of admissions to ER (skewed data) - by medium term.

Analysis 1.8. Comparison 1 Intensive Case Management versus standard care, Outcome 8 Service use: $5 a$. Received day hospital care - by short term FUP.

Analysis 1.9. Comparison 1 Intensive Case Management versus standard care, Outcome 9 Service use: 5b. Outpatient visits by short term FUP (6 months).

Analysis 1.10. Comparison 1 Intensive Case Management versus standard care, Outcome 10 Service use: $5 c$. Outpatient visits - by medium term (skewed data).

Analysis 1.11. Comparison 1 Intensive Case Management versus standard care, Outcome 11 Service use: 5d. Received home visits - by short term FUP.

Analysis 1.12. Comparison 1 Intensive Case Management versus standard care, Outcome 12 Adverse event: 1a. Death - any cause.

Analysis 1.13. Comparison 1 Intensive Case Management versus standard care, Outcome 13 Adverse event: 1 b. Death - suicide.

Analysis 1.14. Comparison 1 Intensive Case Management versus standard care, Outcome 14 Global state: 1. Leaving the study early.

Analysis 1.15. Comparison 1 Intensive Case Management versus standard care, Outcome 15 Global state: 2. Average endpoint score $(\mathrm{GAF}$, high $=$ good $)$. 
Analysis 1.16. Comparison 1 Intensive Case Management versus standard care, Outcome 16 Global state: 3. Not compliant with medication - by long term.

Analysis 1.17. Comparison 1 Intensive Case Management versus standard care, Outcome 17 Social functioning: 1a. Contact with legal system (various measurements).

Analysis 1.18. Comparison 1 Intensive Case Management versus standard care, Outcome 18 Social functioning: $1 \mathrm{~b}$. Mean contacts with legal system (skewed data) - by medium term.

Analysis 1.19. Comparison 1 Intensive Case Management versus standard care, Outcome 19 Social functioning: 2. Employment status (various measurements).

Analysis 1.20. Comparison 1 Intensive Case Management versus standard care, Outcome 20 Social functioning: 3a. Accommodation status (various measurements).

Analysis 1.21. Comparison 1 Intensive Case Management versus standard care, Outcome 21 Social functioning: 3b. Accomodation status: mean number of days in supported house (skewed data, sample size $\geqq 200$ ).

Analysis 1.22. Comparison 1 Intensive Case Management versus standard care, Outcome 22 Social functioning: 3c. Accommodation status (various measurements, skewed data).

Analysis 1.23. Comparison 1 Intensive Case Management versus standard care, Outcome 23 Social functioning: 4a. Substance abuse.

Analysis 1.24. Comparison 1 Intensive Case Management versus standard care, Outcome 24 Social functioning: 4b. Substance abuse (DALI, skewness not detectable) - by medium term.

Analysis 1.25. Comparison 1 Intensive Case Management versus standard care, Outcome 25 Social functioning: 4c. Substance abuse - days used per month (skewed data).

Analysis 1.26. Comparison 1 Intensive Case Management versus standard care, Outcome 26 Social functioning: 5a. Average endpoint score (various scales).

Analysis 1.27. Comparison 1 Intensive Case Management versus standard care, Outcome 27 Social functioning: 5b. Average endpoint score (various scales, skewed data).

Analysis 1.28. Comparison 1 Intensive Case Management versus standard care, Outcome 28 Mental state: 1a. General symptoms - average endpoint score (various scales).

Analysis 1.29. Comparison 1 Intensive Case Management versus standard care, Outcome 29 Mental state: 1 b. General symptoms - mean change from baseline (CSI, low = poor ) - by long term.

Analysis 1.30. Comparison 1 Intensive Case Management versus standard care, Outcome 30 Mental state: 1c. General symptoms - average endpoint score (various scales, skewed data).

Analysis 1.31. Comparison 1 Intensive Case Management versus standard care, Outcome 31 Mental state: 2 a. Specific symptoms - depression at follow up interview.

Analysis 1.32. Comparison 1 Intensive Case Management versus standard care, Outcome 32 Mental state: $2 \mathrm{~b}$. Specific symptoms - average endpoint score (various scales, skewed data, sample size $\geqq 200$ ).

Analysis 1.33. Comparison 1 Intensive Case Management versus standard care, Outcome 33 Mental state: 2 c. Specific symptoms - average endpoint score (various scales, skewed data).

Analysis 1.34. Comparison 1 Intensive Case Management versus standard care, Outcome 34 Behaviour: 1. Specific behaviour - self-harm.

Analysis 1.35. Comparison 1 Intensive Case Management versus standard care, Outcome 35 Behaviour: 2. Social behaviour average endpoint score (SBS, high = poor).

Analysis 1.36. Comparison 1 Intensive Case Management versus standard care, Outcome 36 Quality of life: 1a. Average endpoint score (various scales).

Analysis 1.37. Comparison 1 Intensive Case Management versus standard care, Outcome 37 Quality of life: $1 \mathrm{~b}$. Mean change from baseline (QOLI, high = better, skewed data) - by long term.

Analysis 1.38. Comparison 1 Intensive Case Management versus standard care, Outcome 38 Participant satisfaction: 1a. Average endpoint score (CSQ, high = better).

Analysis 1.39. Comparison 1 Intensive Case Management versus standard care, Outcome 39 Participants satisfaction: $1 \mathrm{~b}$. Average endpoint score (CSQ, high = better, skewed data) - by short term.

Analysis 1.40. Comparison 1 Intensive Case Management versus standard care, Outcome 40 Participants need: 1 . Average endpoint score (various scales, skewed data).

Analysis 1.41. Comparison 1 Intensive Case Management versus standard care, Outcome 41 Costs: 1a. Direct costs of psychiatric hospital care - by medium term (Unit cost = USD, fiscal year 1990).

Analysis 1.42. Comparison 1 Intensive Case Management versus standard care, Outcome 42 Costs: $1 \mathrm{~b}$. Direct costs of psychiatric hospital care - skewed data.

Analysis 1.43. Comparison 1 Intensive Case Management versus standard care, Outcome 43 Costs: 2a. Direct healthcare costs - by long term (Unit cost = USD, fiscal year 1988). 
Analysis 1.44. Comparison 1 Intensive Case Management versus standard care, Outcome 44 Costs: $2 \mathrm{~b}$. Direct healthcare costs - skewed data.

Analysis 1.45. Comparison 1 Intensive Case Management versus standard care, Outcome 45 Costs: 3 . Direct costs - other data - skewed data.

Analysis 2.1. Comparison 2 Intensive Case Management versus non-Intensive Case Management, Outcome 1 Service use: 1. Average number of days in hospital per month - by about 24 months.

Analysis 2.2. Comparison 2 Intensive Case Management versus non-Intensive Case Management, Outcome 2 Service use: 1 a. Average number of days in hospital per month - by medium/long term follow up (skewed data, sample size $\geqq 200$ ).

Analysis 2.3. Comparison 2 Intensive Case Management versus non-Intensive Case Management, Outcome 3 Service use: 2 . Not remaining in contact with psychiatric services.

Analysis 2.4. Comparison 2 Intensive Case Management versus non-Intensive Case Management, Outcome 4 Service use: $3 a$. Admitted to hospital - by long term.

Analysis 2.5. Comparison 2 Intensive Case Management versus non-Intensive Case Management, Outcome 5 Service use: $3 \mathrm{~b}$. Average number of admissions (skewed data -sample size $\geqq 200$ ).

Analysis 2.6. Comparison 2 Intensive Case Management versus non-Intensive Case Management, Outcome 6 Service use: $3 c$. Average number of admissions (skewed data) - by medium term.

Analysis 2.7. Comparison 2 Intensive Case Management versus non-Intensive Case Management, Outcome 7 Adverse event: 1a. Death - any cause.

Analysis 2.8. Comparison 2 Intensive Case Management versus non-Intensive Case Management, Outcome 8 Adverse event: 1b. Death - suicide.

Analysis 2.9. Comparison 2 Intensive Case Management versus non-Intensive Case Management, Outcome 9 Global state: 1. Leaving the study early.

Analysis 2.10. Comparison 2 Intensive Case Management versus non-Intensive Case Management, Outcome 10 Global state: 2a. Average endpoint score (HoNOS, high = poor) - by long term.

Analysis 2.11. Comparison 2 Intensive Case Management versus non-Intensive Case Management, Outcome 11 Global state: 2b. Average endpoint score (HoNOS, high = poor) - skewed data.

Analysis 2.12. Comparison 2 Intensive Case Management versus non-Intensive Case Management, Outcome 12 Global state: 3a. Not compliant with medication - by medium term.

Analysis 2.13. Comparison 2 Intensive Case Management versus non-Intensive Case Management, Outcome 13 Global state: 3b. Compliance with medication - average endpoint sub-scale score (ROMI) - by long term.

Analysis 2.14. Comparison 2 Intensive Case Management versus non-Intensive Case Management, Outcome 14 Global state: $3 c$. Compliance with medication - average endpoint sub-scale score (ROMI, score 1-3, skewed data).

Analysis 2.15. Comparison 2 Intensive Case Management versus non-Intensive Case Management, Outcome 15 Social functioning: 1 . Contact with legal system (various measurements).

Analysis 2.16. Comparison 2 Intensive Case Management versus non-Intensive Case Management, Outcome 16 Social functioning 2. Employment status (various measurements).

Analysis 2.17. Comparison 2 Intensive Case Management versus non-Intensive Case Management, Outcome 17 Social functioning: 3a. Accommodation status (various measurements).

Analysis 2.18. Comparison 2 Intensive Case Management versus non-Intensive Case Management, Outcome 18 Social functioning: 3b. Accommodation status - average days per month in stable accommodation.

Analysis 2.19. Comparison 2 Intensive Case Management versus non-Intensive Case Management, Outcome 19 Social functioning: 4a. Substance abuse - by long term.

Analysis 2.20. Comparison 2 Intensive Case Management versus non-Intensive Case Management, Outcome 20 Social functioning: 4b. Substance abuse - average endpoint score (SATS, low = poor).

Analysis 2.21. Comparison 2 Intensive Case Management versus non-Intensive Case Management, Outcome 21 Social functioning: 4c. Alcohol - abuse (various measurements, skewed data).

Analysis 2.22. Comparison 2 Intensive Case Management versus non-Intensive Case Management, Outcome 22 Social functioning: 5a. Average endpoint score (LSP, high = poor) - by long term.

Analysis 2.23. Comparison 2 Intensive Case Management versus non-Intensive Case Management, Outcome 23 Social functioning: 5b. Average endpoint score (SFQ, high = poor) - skewed data.

Analysis 2.24. Comparison 2 Intensive Case Management versus non-Intensive Case Management, Outcome 24 Mental state: 1a. General symptoms - average endpoint score (various scales).

Analysis 2.25. Comparison 2 Intensive Case Management versus non-Intensive Case Management, Outcome 25 Mental state: 1b. General symptoms - average endpoint scores (various scales, skewed data).

Analysis 2.26. Comparison 2 Intensive Case Management versus non-Intensive Case Management, Outcome 26 Mental state: 2a. Specific symptoms: negative symptoms - average endpoint score (SANS, high = poor) - by long term. 
Analysis 2.27. Comparison 2 Intensive Case Management versus non-Intensive Case Management, Outcome 27 Mental state: 2b. Specific symptoms - average endpoint scores (various scales, skewed data).

Analysis 2.28. Comparison 2 Intensive Case Management versus non-Intensive Case Management, Outcome 28 Behaviour: 1. Specific behaviour (various measurements).

Analysis 2.29. Comparison 2 Intensive Case Management versus non-Intensive Case Management, Outcome 29 Quality of life: 1. Average endpoint score (various scales).

Analysis 2.30. Comparison 2 Intensive Case Management versus non-Intensive Case Management, Outcome 30 Participant satisfaction/need: 1 . Average endpoint scores (various scale) - by long term.

Analysis 2.31. Comparison 2 Intensive Case Management versus non-Intensive Case Management, Outcome 31 Participants need: 1 . Average endpoint scores (various scales, skewed data).

Analysis 2.32. Comparison 2 Intensive Case Management versus non-Intensive Case Management, Outcome 32 Participant satisfaction: 1. Average endpoint scores (CSQ-modified, high = better, skewed data) - by long term.

Analysis 2.33. Comparison 2 Intensive Case Management versus non-Intensive Case Management, Outcome 33 Costs: 1 . Direct costs of psychiatric hospital care (skewed data).

Analysis 2.34. Comparison 2 Intensive Case Management versus non-Intensive Case Management, Outcome 34 Costs: 2a. Direct costs of all care - by long term (2 years). Unit cost GBP, fiscal year 1997/98.

Analysis 2.35. Comparison 2 Intensive Case Management versus non-Intensive Case Management, Outcome 35 Costs: 2 b. Direct costs of all care - by medium term (skewed data).

Analysis 2.36. Comparison 2 Intensive Case Management versus non-Intensive Case Management, Outcome 36 Costs: 3 . Total costs of care per patient - Unit cost GBP.

ADDITIONAL TABLES

APPENDICES

WHAT'S NEW

HISTORY

CONTRIBUTIONS OF AUTHORS

DECLARATIONS OF INTEREST

SOURCES OF SUPPORT

DIFFERENCES BETWEEN PROTOCOL AND REVIEW 
[Intervention Review]

\section{Intensive case management for severe mental illness}

Marina Dieterich ${ }^{1}$, Claire B Irving 2 , Hanna Bergman³, Mariam A Khokhar ${ }^{4}$, Bert Park ${ }^{5}$, Max Marshall6

1Department of Psychiatry, Azienda USL Toscana Nord Ovest, Livorno, Italy. ${ }^{2}$ Cochrane Schizophrenia Group, The University of Nottingham, Nottingham, UK. ${ }^{3}$ Enhance Reviews Ltd, Wantage, UK. ${ }^{4}$ Oral Health and Development, University of Sheffield, Nottingham, UK. ${ }^{5}$ Nottinghamshire Healthcare NHS Trust, Nottingham, UK. ${ }^{6}$ University of Manchester, The Lantern Centre, Preston, UK

Contact: Marina Dieterich, Department of Psychiatry, Azienda USL Toscana Nord Ovest, Livorno, Italy. marinadieterich.md@gmail.com.

Editorial group: Cochrane Schizophrenia Group.

Publication status and date: New search for studies and content updated (no change to conclusions), published in Issue 1, 2017.

Citation: Dieterich M, Irving CB, Bergman H, Khokhar MA, Park B, Marshall M. Intensive case management for severe mental illness. Cochrane Database of Systematic Reviews 2017, Issue 1. Art. No.: CD007906. DOI: 10.1002/14651858.CD007906.pub3.

Copyright ( 2017 The Cochrane Collaboration. Published by John Wiley \& Sons, Ltd.

\section{A B S T R A C T}

\section{Background}

Intensive Case Management (ICM) is a community-based package of care aiming to provide long-term care for severely mentally ill people who do not require immediate admission. Intensive Case Management evolved from two original community models of care, Assertive Community Treatment (ACT) and Case Management (CM), where ICM emphasises the importance of small caseload (fewer than 20) and high-intensity input.

\section{Objectives}

To assess the effects of ICM as a means of caring for severely mentally ill people in the community in comparison with non-ICM (caseload greater than 20) and with standard community care. We did not distinguish between models of ICM. In addition, to assess whether the effect of ICM on hospitalisation (mean number of days per month in hospital) is influenced by the intervention's fidelity to the ACT model and by the rate of hospital use in the setting where the trial was conducted (baseline level of hospital use).

\section{Search methods}

We searched the Cochrane Schizophrenia Group's Trials Register (last update search 10 April 2015).

\section{Selection criteria}

All relevant randomised clinical trials focusing on people with severe mental illness, aged 18 to 65 years and treated in the community care setting, where ICM is compared to non-ICM or standard care.

\section{Data collection and analysis}

At least two review authors independently selected trials, assessed quality, and extracted data. For binary outcomes, we calculated risk ratio (RR) and its $95 \%$ confidence interval (CI), on an intention-to-treat basis. For continuous data, we estimated mean difference (MD) between groups and its $95 \% \mathrm{Cl}$. We employed a random-effects model for analyses.

We performed a random-effects meta-regression analysis to examine the association of the intervention's fidelity to the ACT model and the rate of hospital use in the setting where the trial was conducted with the treatment effect. We assessed overall quality for clinically important outcomes using the GRADE approach and investigated possible risk of bias within included trials.

\section{Main results}

The 2016 update included two more studies $(n=196)$ and more publications with additional data for four already included studies. The updated review therefore includes 7524 participants from 40 randomised controlled trials (RCTs). We found data relevant to two 
comparisons: ICM versus standard care, and ICM versus non-ICM. The majority of studies had a high risk of selective reporting. No studies provided data for relapse or important improvement in mental state.

\section{ICM versus standard care}

When ICM was compared with standard care for the outcome service use, ICM slightly reduced the number of days in hospital per month ( $n=3595,24$ RCTs, MD $-0.86,95 \% \mathrm{Cl}-1.37$ to -0.34 ,low-quality evidence). Similarly, for the outcome global state, ICM reduced the number of people leaving the trial early $(n=1798,13 \mathrm{RCTs}$, RR $0.68,95 \% \mathrm{Cl} 0.58$ to 0.79 , low-quality evidence). For the outcome adverse events, the evidence showed that ICM may make little or no difference in reducing death by suicide ( $\mathrm{n}=1456,9 \mathrm{RCTs}, \mathrm{RR} 0.68,95 \% \mathrm{Cl} 0.31$ to 1.51 , lowquality evidence). In addition, for the outcome social functioning, there was uncertainty about the effect of ICM on unemployment due to very low-quality evidence $(n=1129,4 \mathrm{RCTs}$, RR $0.70,95 \% \mathrm{Cl} 0.49$ to 1.0 , very low-quality evidence).

\section{ICM versus non-ICM}

When ICM was compared with non-ICM for the outcome service use, there was moderate-quality evidence that ICM probably makes little or no difference in the average number of days in hospital per month $(n=2220,21 \mathrm{RCTs}, \mathrm{MD}-0.08,95 \% \mathrm{Cl}-0.37$ to 0.21 , moderate-quality evidence) or in the average number of admissions ( $n=678,1 \mathrm{RCT}, \mathrm{MD}-0.18,95 \% \mathrm{Cl}-0.41$ to 0.05 , moderate-quality evidence) compared to non-ICM. Similarly, the results showed that ICM may reduce the number of participants leaving the intervention early $(n=1970,7$ RCTs, RR $0.70,95 \% \mathrm{Cl} 0.52$ to 0.95 , low-quality evidence) and that ICM may make little or no difference in reducing death by suicide ( $\mathrm{n}=1152,3 \mathrm{RCTs}$, $\mathrm{RR} 0.88,95 \% \mathrm{Cl} 0.27$ to 2.84 , low-quality evidence). Finally, for the outcome social functioning, there was uncertainty about the effect of ICM on unemployment as compared to non-ICM ( $n=73,1$ RCT, RR $1.46,95 \% \mathrm{Cl} 0.45$ to 4.74 , very low-quality evidence).

\section{Fidelity to $\mathrm{ACT}$}

Within the meta-regression we found that i.) the more ICM is adherent to the ACT model, the better it is at decreasing time in hospital ('organisation fidelity' variable coefficient $-0.36,95 \% \mathrm{Cl}-0.66$ to -0.07 ); and ii.) the higher the baseline hospital use in the population, the better ICM is at decreasing time in hospital ('baseline hospital use' variable coefficient $-0.20,95 \% \mathrm{Cl}-0.32$ to -0.10 ). Combining both these variables within the model, 'organisation fidelity' is no longer significant, but the 'baseline hospital use' result still significantly influences time in hospital (regression coefficient $-0.18,95 \% \mathrm{Cl}-0.29$ to $-0.07, \mathrm{P}=0.0027$ ).

\section{Authors' conclusions}

Based on very low- to moderate-quality evidence, ICM is effective in ameliorating many outcomes relevant to people with severe mental illness. Compared to standard care, ICM may reduce hospitalisation and increase retention in care. It also globally improved social functioning, although ICM's effect on mental state and quality of life remains unclear. Intensive Case Management is at least valuable to people with severe mental illnesses in the subgroup of those with a high level of hospitalisation (about four days per month in past two years). Intensive Case Management models with high fidelity to the original team organisation of ACT model were more effective at reducing time in hospital.

However, it is unclear what overall gain ICM provides on top of a less formal non-ICM approach.

We do not think that more trials comparing current ICM with standard care or non-ICM are justified, however we currently know of no review comparing non-ICM with standard care, and this should be undertaken.

\section{PLAIN LANGUAGE SUMMARY}

\section{Intensive case management for people with severe mental illness}

\section{Background}

Severe mental illnesses are defined by diagnosis, degree of disability and the presence of some abnormal behaviour. Including schizophrenia and psychosis, severe mood problems, and personality disorder, severe mental illness can cause considerable distress over a long period of time to both the person affected and his or her family and friends.

Until the 1970s, it was common for those suffering from these disorders to remain in an institution for most of their lives, but in most of the countries of the world, they are now managed in the community with one of several different types of intervention. Intensive Case Management (ICM) is one such intervention. It consists of management of the mental health problem and the rehabilitation and social support needs of the person concerned, over an indefinite period of time, by a team of people who have a fairly small group of clients (fewer than 20). Twenty-four-hour help is offered and clients are seen in a non-clinical setting.

\section{Aims of the review}

To find and present good-quality evidence concerning the effectiveness of ICM compared with non-ICM (where people receive the same package of care, but the professionals have caseloads of more than 20 people) and standard care (where people are seen as outpatients, but their support needs are less clearly defined) for people with severe mental illness. 


\section{Searching for evidence}

We carried out electronic searches for randomised controlled trials comparing ICM with non-ICM or standard care in 2009, 2012 , and 2015.

\section{Results}

We included 40 trials involving 7524 people. The trials took place in Australia, Canada, China, Europe, and the USA. When ICM was compared to standard care, those in the ICM group were more likely to stay with the service, have improved general functioning, get a job, not be homeless, and have shorter stays in hospital (especially when they had had very long stays in hospital previously). When ICM was compared to non-ICM, the only clear difference was that those in the ICM group were more likely to be kept in care.

\section{Conclusions}

None of the evidence for the main outcomes of interest was high quality; at best the evidence was of moderate quality. In addition, the healthcare and social support systems of the countries where the studies took place were quite different, so it was difficult to make valid overall conclusions. Furthermore, we were unable to use much of the data on quality of life and patient and carer satisfaction because the trials used many different scales to measure these outcomes, some of which were not validated. The development of an overall scale and its validation would be very beneficial in producing services that people favour.

(Plain language summary initially prepared for this review by Janey Antoniou of RETHINK, UK (rethink.org)) 


\begin{tabular}{|c|c|c|c|c|c|c|}
\hline $\begin{array}{l}\text { S U M M A R Y O F F I N D I N G S } \\
\text { Summary of findings for the main cor }\end{array}$ & aparison. Inte & sive Case Management vers & standard care $f$ & severe ment & llness & \\
\hline \multicolumn{7}{|c|}{ Intensive Case Management versus standard care for severe mental illness } \\
\hline \multicolumn{7}{|c|}{$\begin{array}{l}\text { Patient or population: people with severe mental illness } \\
\text { Settings: community } \\
\text { Intervention: Intensive Case Management versus standard care }\end{array}$} \\
\hline \multirow[t]{3}{*}{ Outcomes } & \multicolumn{2}{|c|}{ Illustrative comparative risks* $(95 \% \mathrm{Cl})$} & \multirow{3}{*}{$\begin{array}{l}\text { Relative effect } \\
(95 \% \mathrm{CI})\end{array}$} & \multirow{3}{*}{$\begin{array}{l}\text { No of Partici- } \\
\text { pants } \\
\text { (studies) }\end{array}$} & \multirow{3}{*}{$\begin{array}{l}\text { Quality of the } \\
\text { evidence } \\
\text { (GRADE) }\end{array}$} & \multirow[t]{3}{*}{ Comments } \\
\hline & Assumed risk & Corresponding risk & & & & \\
\hline & Control & $\begin{array}{l}\text { Intensive Case Manage- } \\
\text { mentversus standard care }\end{array}$ & & & & \\
\hline $\begin{array}{l}\text { Service use: } 1 \text {. Average number of } \\
\text { days in hospital per month - by about } \\
24 \text { months }\end{array}$ & - & $\begin{array}{l}\text { The mean service use: } 1 . \text { av- } \\
\text { erage number of days in hos- } \\
\text { pital per month - by about } 24 \\
\text { months in the intervention } \\
\text { groups was } \\
\mathbf{0 . 8 6} \text { lower } \\
\text { (1.37 lower to } 0.34 \text { lower) }\end{array}$ & - & $\begin{array}{l}3595 \\
\text { (24 studies) }\end{array}$ & $\begin{array}{l}\oplus \oplus \oplus \ominus \\
\text { low } 1,2\end{array}$ & \\
\hline $\begin{array}{l}\text { Adverse event: } 1 \text { b. Death - suicide - by } \\
\text { long term }\end{array}$ & 20 per 1000 & $\begin{array}{l}13 \text { per } 1000 \\
(6 \text { to } 30)\end{array}$ & $\begin{array}{l}\text { RR } \mathbf{0 . 6 8} \\
\text { (0.31 to } 1.51)\end{array}$ & $\begin{array}{l}1456 \\
\text { (9 studies) }\end{array}$ & $\begin{array}{l}\oplus \oplus \odot \ominus \\
\text { low } 1,4\end{array}$ & \\
\hline Global state: 1. Relapse - by long term & - & - & - & - & - & $\begin{array}{l}\text { No data avail- } \\
\text { able }\end{array}$ \\
\hline $\begin{array}{l}\text { Global state: } 1 \text {. Leaving the study ear- } \\
\text { ly - by long term }\end{array}$ & 331 per 1000 & $\begin{array}{l}\mathbf{2 2 5} \text { per } \mathbf{1 0 0 0} \\
\text { (192 to } 262)\end{array}$ & $\begin{array}{l}\text { RR } 0.68 \\
(0.58 \text { to } 0.79)\end{array}$ & $\begin{array}{l}1798 \\
\text { (13 studies) }\end{array}$ & $\begin{array}{l}\oplus \oplus \ominus \ominus \\
\text { low } 1,3\end{array}$ & \\
\hline $\begin{array}{l}\text { Social functioning: } 2 \text {. Employment } \\
\text { status (various measurements) - by } \\
\text { long term - not employed at the end of } \\
\text { the trial }\end{array}$ & 766 per 1000 & $\begin{array}{l}\mathbf{5 3 6} \text { per } \mathbf{1 0 0 0} \\
(375 \text { to } 766)\end{array}$ & $\begin{array}{l}\text { RR } 0.7 \\
(0.49 \text { to } 1)\end{array}$ & $\begin{array}{l}1129 \\
\text { (4 studies) }\end{array}$ & $\begin{array}{l}\oplus \odot \odot \ominus \\
\text { very low } 1,5\end{array}$ & \\
\hline $\begin{array}{l}\text { Mental state: not improved to an im- } \\
\text { portant extent - by long term }\end{array}$ & - & - & - & - & - & $\begin{array}{l}\text { No data avail- } \\
\text { able }\end{array}$ \\
\hline
\end{tabular}

${ }^{*}$ The basis for the assumed risk (e.g. the median control group risk across studies) is provided in footnotes. The corresponding risk (and its $95 \%$ confidence interval) is 
GRADE Working Group grades of evidence

High quality: Further research is very unlikely to change our confidence in the estimate of effect.

Moderate quality: Further research is likely to have an important impact on our confidence in the estimate of effect and may change the estimate.

Low quality: Further research is very likely to have an important impact on our confidence in the estimate of effect and is likely to change the estimate.

Very low quality: We are very uncertain about the estimate.

1Downgraded one step for risk of bias: randomisation not well described; problematic to blind.

2Downgraded one step for inconsistency: substantial heterogeneity $\left(I^{2}=74 \%\right)$.

3Downgraded one step for selective reporting bias: only 13 studies reported fully on the flow of participants through the study.

${ }^{4}$ Downgraded one step for imprecision: the $95 \% \mathrm{Cl}$ includes both appreciable benefit and appreciable harm.

5 Downgraded two steps for inconsistency: considerable heterogeneity $(12=94 \%)$

\section{Summary of findings 2. Intensive Case Management versus non-Intensive Case Management for severe mental illness}

Intensive Case Management versus non-Intensive Case Management for severe mental illness

Patient or population: people with severe mental illness

Settings: community

Intervention: Intensive Case Management versus non-Intensive Case Management

\begin{tabular}{|c|c|c|c|c|c|c|}
\hline \multirow[t]{3}{*}{ Outcomes } & \multicolumn{2}{|c|}{ Illustrative comparative risks* $(95 \% \mathrm{CI})$} & \multirow{3}{*}{$\begin{array}{l}\text { Relative effect } \\
(95 \% \mathrm{CI})\end{array}$} & \multirow{3}{*}{$\begin{array}{l}\text { No of Partici- } \\
\text { pants } \\
\text { (studies) }\end{array}$} & \multirow{3}{*}{$\begin{array}{l}\text { Quality of the } \\
\text { evidence } \\
\text { (GRADE) }\end{array}$} & \multirow[t]{3}{*}{ Comments } \\
\hline & Assumed risk & Corresponding risk & & & & \\
\hline & Control & $\begin{array}{l}\text { Intensive Case Management versus } \\
\text { non-Intensive Case Management }\end{array}$ & & & & \\
\hline $\begin{array}{l}\text { Service use: } 1 \text {. Average number } \\
\text { of days in hospital per month - by } \\
\text { about } 24 \text { months }\end{array}$ & - & $\begin{array}{l}\text { The mean service use: } 1 \text {. average } \\
\text { number of days in hospital per } \\
\text { month - by about } 24 \text { months in the } \\
\text { intervention groups was } \\
\mathbf{0 . 0 8} \text { lower } \\
\text { ( } 0.37 \text { lower to } 0.21 \text { higher) }\end{array}$ & - & $\begin{array}{l}2220 \\
\text { (21 studies) }\end{array}$ & $\begin{array}{l}\oplus \oplus \oplus \ominus \\
\text { moderate } 1\end{array}$ & \\
\hline $\begin{array}{l}\text { Service use: } 3 \text { b. Average num- } \\
\text { ber of admissions (skewed data - } \\
\text { sample size } \geqq 200 \text { ) - by long term }\end{array}$ & - & $\begin{array}{l}\text { The mean service use: } 3 \mathrm{~b} \text {. average } \\
\text { number of admissions (skewed data } \\
\text { - sample size } \geqq 200 \text { ) - by long term in } \\
\text { the intervention groups was } \\
\mathbf{0 . 1 8} \text { lower } \\
\text { ( } 0.41 \text { lower to } 0.05 \text { higher) }\end{array}$ & - & $\begin{array}{l}678 \\
\text { (1 studies) }\end{array}$ & $\begin{array}{l}\oplus \oplus \oplus \odot \\
\text { moderate } 1\end{array}$ & \\
\hline
\end{tabular}




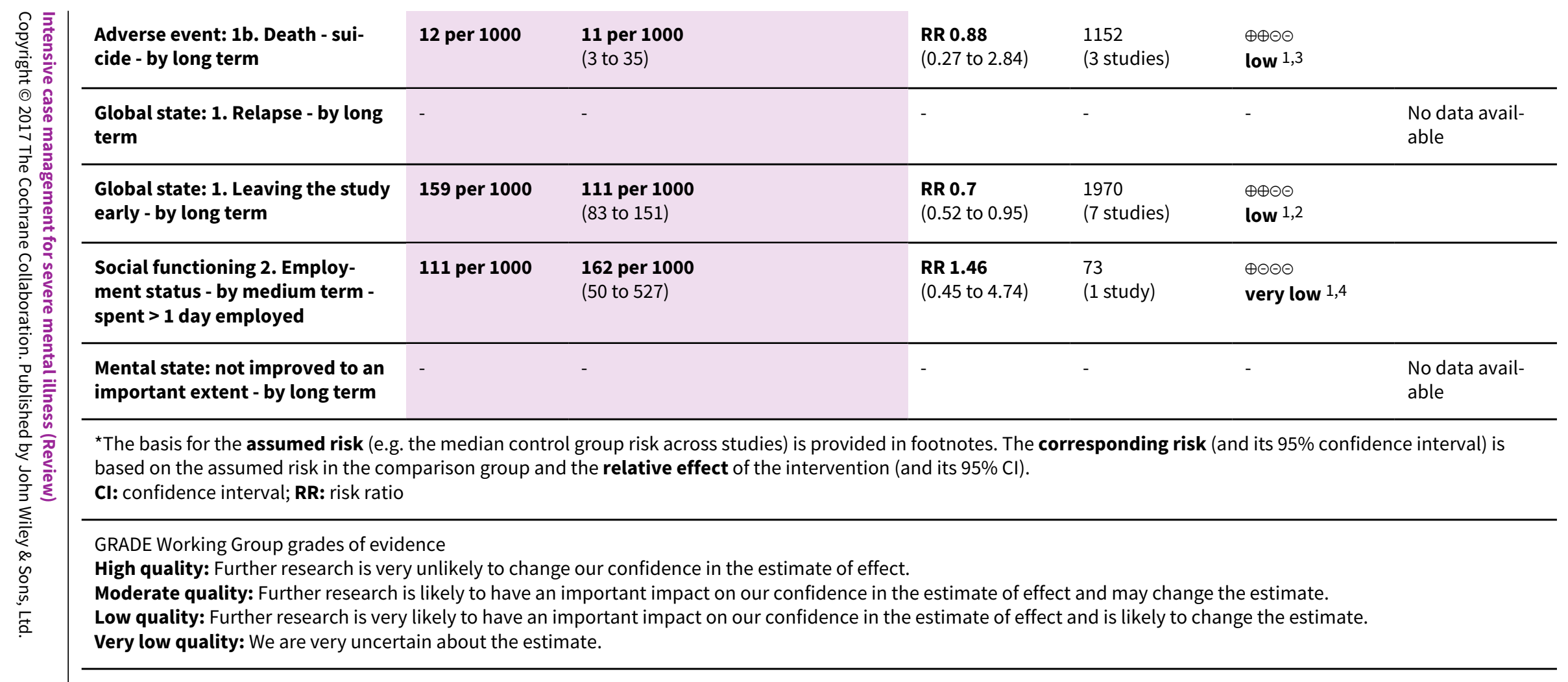

1Downgraded one step for risk of bias: randomisation not well described; problematic to blind.

2Downgraded one step for selective reporting bias: only 7 studies reported fully on the flow of participants through the study.

3 Downgraded one step for imprecision: the $95 \% \mathrm{Cl}$ includes both appreciable benefit and appreciable harm.

4 Downgraded two steps for imprecision: the $95 \% \mathrm{Cl}$ includes both appreciable benefit and appreciable harm, and only 73 participants were included. 


\section{B A C K G R O U N D}

\section{Description of the condition}

Worldwide, more than $25 \%$ of people develop one or more mental or behavioural disorders during their lifetime (WHO 2001). Schizophrenia is one illness that heavily contributes to the numbers of people considered severely mentally ill. The lifetime prevalence of schizophrenia is $0.58 \%$ in the adult population (Warner 1995). It is currently 26th on the list of diseases ranked according to contribution to overall burden in term of disability-adjusted life years (DALYs). Its ranking is projected to rise to 20th by the year 2020, with more than 17 million DALYs lost (accounting for $1.25 \%$ of overall burden) (Murray 1996). However, other psychiatric/ psychological conditions can also profoundly affect a person's functioning. Many people with other types of non-organic psychotic illness, or even non-psychotic disorders such as personality disorder, can be considered to be severely mentally ill.

There has been lack of consensus over the definition of 'severe mental illness', but the most common dimensions used to identify this group are i.) diagnosis, ii.) disability, iii.) duration, and iv.) abnormal behaviour. However, there is little consistency between dimensions and thresholds used in different settings (Slade 1997). The definition of severe mental illness with the widest consensus is that of the National Institute of Mental Health (NIMH) (Schinnar 1990). Their definition is based on three criteria: i.) diagnosis of non-organic psychosis or personality disorder; ii.) duration characterised as involving 'prolonged illness' and 'long term treatment' and operationalised as a two-year or longer history of mental illness or treatment; and iii.) disability, which includes dangerous or disturbing social behaviour, moderate impairment in work and non-work activities, and mild impairment in basic needs (National Institute of Mental Health 1987).

A survey conducted in Europe to calculate prevalence rates of severe mental illness according to the NIMH definition put the total population-based annual prevalence at approximately 2 per 1000 (Ruggeri 2000).

\section{Description of the intervention}

Since the 1960s, there has been an almost worldwide trend towards the closure of institutions for the mentally ill. Coupled with these closures, many government policies have focused on reducing the number of hospital beds for people with severe mental illness in favour of providing care in a variety of non-hospital settings - outpatient clinics, day centres, or community mental health centres. These changes were consistent with the increasing shift from hospital-based care in favour of a more community-focused approach (Malone 2007).

Assertive Community Treatment and Case Management (Table 1) are community-based packages of care developed in the early 1970s. They were initially conceived to co-ordinate the care of severely mentally ill people discharged from closing mental hospitals. However, they were soon more widely applied as a means of caring for severely mentally ill people who did not require immediate admission (Thompson 1990).

Core features of Assertive Community Treatment (ACT) were clearly stated since the first paradigm-shifting study of Stein and Test (Stein 1980), and successively critical ingredients of ACT have operationally defined by developing fidelity scale (McGrew 1994; McGrew 1995).

Case Management was not likewise defined; brokerage case management was rapidly abandoned in favour of Clinical Case Management (Holloway 1995), and more sophisticated, but poorly defined models were developed. In these models case managers have clinical training, provide at least some clinical services, and operate with low caseloads (Rubin 1992; Solomon 1992).

Assertive Community Treatment and Case Management do share common goals such as maintaining contact, reducing hospitalisation (and hence cost), and improving outcome. However, there are, at least in theory and with respect to the original models, important structural distinctions between them. Nonetheless, across time through clinical practice the two interventions have evolved and tended to converge into a package of care known as Intensive Case Management, which contains elements from the two original models (Burns 2008; Scott 1995). In both clinical trials and clinical practice, what is currently called 'Case Management' is thus likely to contain some elements of ACT practice. These models can be called 'Clinical Case Management', 'Intensive Case Management', and 'Strengths Case Management' (Solomon 1992). However, 'Intensive Case Management' is a broader term often used interchangeably with Assertive Community Treatment but distinguished from it on the grounds that it often lacks one or more ACT programme elements (Burns 2001). Intensive Case Management (ICM) emphasises the importance of small caseload (usually considerably fewer than 20) and high-intensity input. Intensive case managers are usually clinicians who act as therapist in addition to their case management duties (Marshall 2008).

Until a few years ago, the approaches to care within community mental health teams differed. These approaches (evolved over the last 30 years) fell into two main categories: i.) services with welldelimited aims, such as crisis resolution and home treatments teams, vocational rehabilitation, and early intervention service; and ii.) services aimed at meeting a wide range of patient needs, such as ACT and Case Management (CM) (merging in the Intensive Case Management model) (Ruggeri 2008).

In the last decade such a distinction has no longer been so relevant. Intensive Case Management partly lost its purity and closeness to the original models (ACT and CM), where many services are offering a less intensive but more flexible and responsive form of assertive outreach (Drukker 2008), investing on the 'critical ingredients' of ICM research helped to identify (Burns 2007) (Killaspy 2012). Many emerging practices are developed within ICM framework, where their aim is to address specific target populations and outcome domains (Bond 2015). Specifically, many specialised models of intervention within community mental health teams are based on adaptation of key principles of ICM, where they are addressing specific population subgroups (difficult to engage in traditional settings, high-risk and revolving door, with comorbidity) (Brewer 2015). Among these, there are packages of care for homeless populations with severe mental illness (Coldwell 2007); populations with severe mental illness and substance abuse (Pettersen 2014), substance abuse, Kirk 2013 or alcohol dependence only (Gilburt 2012), and early intervention in first-episode psychosis (Brewer 2015). The recent proliferation of models inspired by ICM that focus on a special issue was permitted by the structure and flexibility of the original ACT model, but 
exploring this emerging area goes beyond the objectives of this review.

\section{How the intervention might work}

The theory behind care in the community is that it enables people to live as independently as possible within their own homes or 'homely settings' out of hospital, because unnecessary hospital care is wasteful, untherapeutic, and stigmatising. It was hoped that living in the community would increase opportunities for people with severe mental illness to achieve their full potential as autonomous members of society (Department of Health 1990). Community care policies are also aimed at promoting choice and independence for people experiencing mental health difficulties.

Intensive Case Management is an intervention at the level of local service organisation. It is a way of organising teams, rather than a specific treatment model (Johnson 2008). Intensive Case Management should provide a mental health service that is a reliable, systematic, flexible, and co-ordinated care method, addressed to answer the unique combination of health and social care needs of people with severe mental illness. It represents a long-term intensive approach to the patient in the community (Killaspy 2008), providing a comprehensive range of treatment, rehabilitation, and support services (Scott 1995); in the last decade ICM has absorbed the recovery principle of promoting emancipation, through policies encouraging graduation (Finnerty 2015). Intensive Case Management aims to help people with severe mental illness acquire material resources (such as food, shelter, clothing, and medical care) and to improve their psychosocial functioning; to provide sufficient support to keep the patient involved in community life and to encourage growth towards greater autonomy; to develop coping skills to meet the demands of community life; and to ensure continuity of care among treatment agencies (Stein 1980). Key purposes of ICM are to improve outcome, reduce hospitalisation, and prevent loss of contact with services.

A cornerstone in the research field was a study by Burns and colleagues exploring the mechanism for ICM to be effective (Burns 2007). It suggested that the success of ICM depends on its fidelity to the ACT model (i.e. if a team approach is properly implemented) and on the setting (i.e. it would work better where there is a high baseline level of bed use).

\section{Why it is important to do this review}

With the evolution of the original intervention models, there was a need to update and merge two previous relevant Cochrane reviews (Marshall 2000a; Marshall 2000b), and to take into account the findings of work by the same authoring team (Burns 2007). During the last 15 years, not only have intervention models been modified, merged, and become more difficult to distinguish in practice, but also research has been more widespread, with new studies evaluating these approaches outside of the USA.

Since early 2000 , ICM has been a very implemented and widespread intervention in the community care setting, with many nations in Europe, North America, and Australia, investing great efforts and resources in its promotion and dissemination (in England Care Programme Approach promoting ACT team (Department of Health 1999)).

Since then, research providing long-term follow-up outcomes and data on the impact of ACT teams on inpatient service use in specific national settings has been published with emerging data casting doubt on the opportunity of such an initial enthusiastic approach, especially in England, one of the nations where there had been stronger investments in it (Glover 2006). This topic is therefore still under an international debate (Burns 2009; Burns 2010; Burns 2012; Killaspy 2012; Rosen 2013). Almost in the same years (since the mid-2000s), ICM landed in Asia, where the idea of comprehensive community programmes is gradually catching on, and wide implementation of both programs has inspired programmes highly faithful to ICM (Low 2013; Nishio 2014).

The effects of the currently implemented packages of care in different settings should be fully understood across a range of outcomes.

\section{O B JECT IVES}

To assess the effects of ICM as a means of caring for severely mentally ill people in the community in comparison with non-ICM (caseload greater than 20) and with standard community care. We did not distinguish between models of ICM. In addition, to assess whether the effect of ICM on hospitalisation (mean number of days per month in hospital) is influenced by the intervention's fidelity to the ACT model and by the rate of hospital use in the setting where the trial was conducted (baseline level of hospital use).

\section{METHODS}

\section{Criteria for considering studies for this review}

\section{Types of studies}

We considered all relevant randomised controlled trials, and economic evaluations conducted alongside included randomised controlled trials. We excluded quasi-randomised studies, such as those allocating by alternate days of the week. Where trials were described in some way as to suggest or imply that the study was randomised and where the demographic details of each group's participants were similar, we included these trials and undertook a Sensitivity analysis.

\section{Types of participants}

We required the majority of participants to be:

1. within the age range of 18 to 65 years;

2. suffering from severe mental illness, preferably as defined by National Institute of Mental Health 1987, or in the absence of this, from illness such as schizophrenia, schizophrenia-like disorders, bipolar disorder, depression with psychotic features or/and personality disorder; and

3. not acutely ill.

We did not consider substance abuse to be a severe mental disorder in its own right, however studies were eligible if they dealt with people with both diagnoses, that is those with severe mental illness plus substance abuse. Dementia and mental retardation are not considered to be severe mental disorders, hence we excluded studies focusing on these populations. We considered only participants treated in the community care setting.

\section{Types of interventions}

We considered only interventions and management packages not focused primarily on alternatives to acute hospital admission. 


\section{Intensive Case Management}

We defined Intensive Case Management as where the majority of people received:

a. a package of care shaped on the:

- Assertive Community Treatment model, being based on the Treatment in Community Living, Assertive Community Treatment (Stein 1980);

- Assertive Outreach model (Witheridge 1982; Witheridge 1991) (i.e. multidisciplinary team-based approach, practising 'assertive outreach', offering 24-hour emergency cover, providing care themselves) (McGrew 1995); or

- Case Management model (Intagliata 1982), however described as such in the trial report.

b. with a caseload up to and including 20 people.

\section{Non-Intensive Case Management}

We defined non-Intensive Case Management as where the majority of people received:

a. a package of care shaped on the:

- Assertive Community Treatment model, being based on the Treatment in Community Living, Assertive Community Treatment (Stein 1980);

- Assertive Outreach model (Witheridge 1982; Witheridge 1991) (i.e. multidisciplinary team-based approach, practising 'assertive outreach', offering 24-hour emergency cover, providing care themselves) (McGrew 1995); or

- Case Management model (Intagliata 1982), however described as such in the trial report.

b. with a caseload over 20 people.

\section{Standard care}

We defined standard care as where the majority of people received a community or outpatient model of care not specifically shaped on either the model of Assertive Community Treatment and Case Management, and not working within a designated named package or approach to care. If data were available on the standard care caseload, we undertook a final sensitivity analysis testing how prone the primary outcomes were to change when trials comparing Intensive Case Management with standard community care only (caseload greater than 20) were included.

\section{Types of outcome measures}

We grouped outcomes by time into short term (up to and including 6 months), medium term (7 months to up to and including 12 months), and long term (over 12 months). Where available, 24 months was the preferred follow-up point for calculating mean days per months in hospital. If more than one follow-up point within the same period was available, we reported the latest one. During this period, participants remained allocated in their trial arm.

We grouped outcomes assessed after active intervention was stopped or after participants could choose to which arm they were transferred, by time into short-term follow-up (up to and including one year), medium-term follow-up (from one to five years), and long-term follow-up (over five years). We calculated follow-up length as time since intervention stopped.

To simplify distinguishing between outcomes assessed during and after the active intervention, we entered ones explicitly reporting follow-up (FUP) length.

\section{Primary outcomes}

\section{Service use}

1.1 Hospitalisation: mean number of days per month in hospital 1.2 Not remaining in contact with psychiatric services

\section{Secondary outcomes}

\section{Service use}

1.1 Admitted to hospital

1.2 Hospital admission rate

1.3 Use of services outside of mental health provision (i.e. emergency services)

\section{Adverse effects}

2.1 Death - all causes and suicide

\section{Global state}

3.1 Leaving the study early (lost to follow-up)

3.2 Relapse (as defined in trial)

3.3 Not improved to a clinically meaningful extent (as defined in trial)

3.4 Not improved

3.5 Average endpoint score

3.6 Average change score

3.7 Compliance with medication

3.8 Average endpoint score

3.9 Average change score

\section{Social functioning}

4.1 Contact with legal system (i.e. police contacts, arrests, imprisonments)

4.2 Employment status (number unemployed at end of study)

4.3 Accommodation status (number homeless or not living independently during or at the end of the study, mean days homeless and mean days in stable accommodation per month in study)

4.4 Alcohol use

4.5 Illicit drug use

4.6 Average endpoint score

4.7 Average change score

\section{Mental state}

5.1 General symptoms

5.1.1 Not improved to a clinically meaningful extent (as defined in trial)

5.1.2 Not improved

5.1.3 Average endpoint score

5.1.4 Average change score

5.2 Specific symptoms

5.2.1 Positive symptoms (delusions, hallucinations, disordered thinking)

5.2.1.1 Not improved to a clinically meaningful extent (as defined in trial) 
5.2.1.2 Not improved

5.2.1.3 Average endpoint score

5.2.1.4 Average change score

5.2.2 Negative symptoms (poor volition, poor self care, blunted affect)

5.2.2.1 Not improved to a clinically meaningful extent (as defined in trial)

5.2.2.2 Not improved

5.2.2.3 Average endpoint score

5.2.2.4 Average change score

5.2.3 Mood depression

5.2.3.1 Not improved to a clinically meaningful extent (as defined in trial)

5.2.3.2 Not improved

5.2.3.3 Average endpoint score

5.2.3.4 Average change score

\section{Behaviour}

6.1 General behaviour

6.2 Not improved to a clinically meaningful extent (as defined in trial)

6.3 Not improved

6.4 Average endpoint score

6.5 Average change score

6.6 Specific behaviours (i.e. self harm; injury to others or property)

\section{Quality of life}

7.1 Not improved to a clinically meaningful extent (as defined in trial)

7.2 Not improved

7.3 Average endpoint score

7.4 Average change score

\section{Satisfaction}

\subsection{Participant satisfaction}

8.1.1 Not improved to a clinically meaningful extent (as defined in trial)

8.1.2 Not improved

8.1.3 Average endpoint score

8.1.4 Average change score

\subsection{Carer satisfaction}

8.2.1 Not improved to a clinically meaningful extent (as defined in trial)

8.2.2 Not improved

8.2.3 Average endpoint score

8.2.4 Average change score

\section{Costs}

9.1 Direct costs of psychiatric hospital care

9.2 Direct healthcare costs (including all medical and psychiatric care and the costs of case management, but excluding accommodation other than hospital care)

9.3 Direct costs of all care (including costs of accommodation and subtracting benefits, such as earnings, where these are known)

\section{Summary of findings}

We used the GRADE approach to interpret findings, Schünemann 2008, and GRADEpro, GRADEpro, to import data from Review
Manager 5, Review Manager, to create 'Summary of findings' tables. These tables provide outcome-specific information concerning the overall quality of evidence from each included study in the comparison, the magnitude of effect of the interventions examined, and the sum of available data on all outcomes we rated as important to patient care and decision making. We selected the following main outcomes for inclusion in the 'Summary of findings' table.

1. Service use: average number of days in hospital per month by about 24 months

2. Service use: average number of admissions (skewed data sample size $\geq 200$ ) by long term (> 12 months)

3. Adverse event: death - suicide by long term (>12 months)

4. Global state: relapse

5. Global state: leaving the study early by long term ( $>12$ months)

6. Social functioning: employment status - spent less than 1 day employed - by medium term ( 6 to 12 months)

7. Mental state: not improved to an important extent

\section{Search methods for identification of studies}

\section{Electronic searches}

\section{Cochrane Schizophrenia Group's Trials Register}

On 10 April 2015, the Information Specialist searched the Register using the following search strategy:

( ${ }^{\star} \mathrm{ca}$ ?e manag* OR * ${ }^{*} \mathrm{cpa}^{\star} \mathrm{OR}$ *community treat ${ }^{\star} \mathrm{OR}$ *community team* OR * community cent ${ }^{\star}$ OR * community care* OR *madison model $^{\star} \mathrm{OR}^{*}$ outreach ${ }^{\star} \mathrm{OR}{ }^{\star}$ hostel ${ }^{\star} \mathrm{OR}$ * aftercare $^{\star} \mathrm{OR}^{*}$ residential ${ }^{\star}$ OR *housing* OR *transitional* OR *post?hospital* OR *partial hospitali?ation* OR *foster* OR *guardianship* OR *daily living program ${ }^{\star} O R^{\star}$ crisis interven ${ }^{\star} O R^{*}$ early interven ${ }^{\star} O R^{*}$ ambulatory ${ }^{\star}$ OR * community liv* OR *social support* OR * patient care team* OR ${ }^{*}$ community mental health* $\mathrm{OR}^{*}$ patient participation ${ }^{\star} \mathrm{OR}$ *drop? in $^{\star}$ OR *day hospital ${ }^{\star}$ OR *day care* OR *day treat* OR *day cent* $O R$ *day unit* $O R{ }^{*}$ intensive care* $O R$ *intensive interven* $O R$ ${ }^{*}$ intensive treat* ${ }^{\star} R^{*}$ intensive therap ${ }^{\star} O R{ }^{*}$ intensive manag* $O R$ *intensive model ${ }^{\star} \mathrm{OR}$ *intensive program* $\mathrm{OR}$ *intensive team OR *intensive service ${ }^{\star}$ OR * mobile care* $O{ }^{*}{ }^{*}$ mobile interven* $\mathrm{OR}{ }^{*}$ mobile treat ${ }^{\star} \mathrm{OR}{ }^{*}$ mobile therap* $\mathrm{OR}^{*}$ mobile manag* OR *mobile model ${ }^{*} \mathrm{OR}{ }^{*}$ mobile program* OR *mobile team* $\mathrm{OR}{ }^{*}$ mobile service ${ }^{\star} \mathrm{OR}{ }^{*}$ community interven* $\mathrm{OR}{ }^{*}$ community therap* OR *community manag* OR *community model ${ }^{*}$ OR ${ }^{*}$ community program* OR * community service* OR *community base* OR *home care* OR *home interven" OR *home treat ${ }^{\star}$ OR *home therap* OR *home manag* OR *home model* OR *home program* OR *home team* OR *home service* OR *home base* OR *broker* OR *care program*) in Title, Abstract, and Index Terms of REFERENCE OR (*ca?e manag* OR *community ${ }^{*}$ OR *outreach* OR *hostel* OR *aftercare* OR * ${ }^{\star}$ residential* OR *hous* $\mathrm{OR}^{\star}$ transitional ${ }^{\star} \mathrm{OR}$ * ${ }^{*}$ foster ${ }^{\star} \mathrm{OR}^{*}$ crisis interven ${ }^{\star} \mathrm{OR}$ * early interven* OR *ambul ${ }^{\star}$ OR * social support* OR *drop-in* OR *day * $\mathrm{OR}^{\star}$ (intensive $)^{\star} \mathrm{OR}^{\star}(\text { home })^{\star} \mathrm{OR}^{\star}$ care program $\left.{ }^{\star}\right)$ in Intervention of STUDY

The Cochrane Schizophrenia Group's Register of Trials is compiled by systematic searches of major resources (including MEDLINE, Embase, AMED, BIOSIS, CINAHL, PsycINFO, PubMed, and registries of clinical trials) and their monthly updates, handsearches, grey literature, and conference proceedings (see Group Module). There 
is no language, date, document type, or publication status limitations for inclusion of records into the register.

For search methods of previous versions of this review, please see Appendix 1.

\section{Searching other resources}

\section{References}

Should an included or excluded study suggest that another study was relevant, we identified the reference and acquired the full text.

\section{Personal contact}

We contacted authors of trials for additional data where required. We did not systematically contact all authors for additional papers.

\section{Data collection and analysis}

Methods used for this version are presented below; previous methods are presented in Appendix 2.

\section{Selection of studies}

Two review authors (HB, MK) inspected results of the update search and identified potentially relevant reports. Disagreements were resolved by discussion, or where there was still doubt, we aquired the full article for further inspection. We obtained the full articles of relevant reports for reassessment and inspected them carefully to decide on inclusion or exclusion (see Criteria for considering studies for this review). Review authors were not blinded to the names of the authors, institutions, or journal of publication. Where difficulties or disputes arose, we discussed; if we were unable decide, we added these studies to those awaiting assessment and contacted the authors of the papers for clarification.

\section{Data extraction and management}

\section{Extraction}

\subsection{Data extraction for criteria and outcomes}

Three review authors (MD, HB, MK) independently extracted data from the included studies and compared results of the data extraction. We would have discussed any disagreements, documented our decisions, and contacted the authors of studies for clarification where necessary. Whenever possible, we would have extracted data presented only in graphs and figures and included the data if two review authors independently reached the same result. In order to obtain any missing information or for clarification, we attempted to contact authors through an open-ended request. Where possible, we would have extracted data relevant to each component centre of multicentre studies separately.

\subsection{Additional data extraction}

\subsubsection{Fidelity}

We rated fidelity of the ICM intervention to ACT on the 'team membership' and 'team structure and organisation' subscales of the Index of Fidelity to Assertive Community Treatment (IFACT) (McGrew 1994). This index was derived from a survey of 20 clinical experts in ACT and validated in a survey of 18 programmes.

a. The 'team membership' subscale comprises four items:
- total size of team;

- extent of psychiatric input;

- extent of nursing input to the team.

b. The 'structure and organisation' subscale comprises seven items, whether the team is:

- the primary source of care for its patients;

- situated away from the hospital;

- meets daily;

- shares responsibility for caseloads;

- is available 24 hours a day;

- has a team leader who is also a case manager;

- offers unlimited time for its services.

We chose IFACT because the subscales are brief and can be completed from published or unpublished text. For each item on the index, a score of 1 indicates high fidelity to the model. Score ranges from 0 to 11 , where the maximum score available on the 'team membership' subscale is 4 and on the 'structure and organisation' subscale is 7 , with higher scores indicating higher fidelity to the model.

We obtained fidelity data from published and unpublished trial reports, direct contact with trialists, and data previously obtained directly from trialists reported by previous reviews (Burns 2001; Burns 2007; Catty 2002). Two raters (MD and CBI) independently combined these data into a single fidelity score. Multicentre trials of ICM often struggle to implement a uniform approach, with centres operating at differing degrees of fidelity. Where possible, we rated each component centre separately.

\subsubsection{Baseline hospital use}

We extracted data relating to the average number of days per month in hospital for all participants in the two years before the study began. We obtained this data from published and unpublished trial reports and from direct contact with trialists.

\subsubsection{Service use: hospitalisation}

We obtained the primary outcome mean number of days per month in hospital for the included studies from published and unpublished trial reports, direct contact with trialists, and data previously obtained directly from trialists reported by a previous review (Burns 2007).

\section{Management}

\subsection{Forms}

Two review authors (HB, MK) extracted data onto simple, standard forms.

\subsection{Data from multicentre trials}

For the original version, where possible review authors $\mathrm{MD}$ and $\mathrm{CBI}$ verified independently calculated centre data against original trial reports.

\subsection{Scale-derived data}

We included continuous data from rating scales only if: a. the psychometric properties of the measuring instrument had been described in a peer-reviewed journal (Marshall 2000);

- ratio of patients to staff; 
b. the measuring instrument was not written or modified by one of the trialists for that particular trial; and

c. the measuring instrument was either i.) a self report or ii.) completed by an independent rater or relative.

\subsection{Endpoint versus change data}

Both endpoint and change data have advantages. Change data can remove a component of between-person variability from the analysis. On the other hand, calculation of change needs two assessments (baseline and endpoint), which can be difficult in unstable and difficult-to-measure conditions such as schizophrenia. We decided to primarily use endpoint data, and only use change data if the former were not available. When relevant, we combined endpoint and change data in the analysis, as we aimed to use mean differences rather than standardised mean differences throughout (Higgins 2011).

\subsection{Skewed data}

Continuous data on clinical and social outcomes are often not normally distributed. To avoid the pitfall of applying parametric tests to non-parametric data, we applied the following standards to relevant continuous data before inclusion.

We entered all relevant data from studies of more than 200 participants in the analysis irrespective of the following rules, because skewed data pose less of a problem in large studies. We also entered all relevant change data, as when continuous data are presented on a scale that includes a possibility of negative values (such as change data), it is difficult to tell whether data are skewed or not.

For endpoint data from studies of fewer than 200 participants, we used the following methods.

a. If a scale started from the finite number zero, we subtracted the lowest possible value from the mean, and divided this by the standard deviation (SD). If this value is lower than 1 , it strongly suggests a skew, and we excluded these data. If this ratio is higher than 1 but below 2, there is suggestion of skew. We entered these data to test whether their inclusion or exclusion changed the results substantially. Finally, if the ratio was larger than 2, we included these data, because skew is less likely (Altman 1996; Higgins 2011).

b. If a scale starts from a positive value (such as the Positive and Negative Syndrome Scale (PANSS), which can have values from 30 to 210) (Kay 1986), we modified the calculation described above to take the scale starting point into account. In these cases skew is present if $2 \mathrm{SD}>(\mathrm{S}-\mathrm{S} \mathrm{min})$, where $\mathrm{S}$ is the mean score and ' $\mathrm{S}$ min' is the minimum score.

\section{Exception to above rules - mean number of days in hospital}

We implemented one exception to the above rules in order to present more data, recognising that this is a post hoc decision, but also that the rules with regards to management of skewed data and how robust skewed data are within meta-analysis are unclear (Higgins 2011). Where mean number of days in hospital data were skewed, and they were provided by studies of fewer than 200 participants, we entered those data into a subgroup of the overall analysis. We also presented the overall effect from all data pooled.

\subsection{Common measure}

To facilitate comparison between trials, we converted variables that can be reported in different metrics, such as days in hospital (mean days per year, per week, or per month) to a common metric (e.g. mean days per month).

\subsection{Conversion of continuous to binary}

Where possible, we attempted to convert outcome measures to dichotomous data. This can be done by identifying cutoff points on rating scales and dividing participants accordingly into 'clinically improved' or 'not clinically improved'. It was generally assumed that if there had been a $50 \%$ reduction in a scale-derived score such as the Brief Psychiatric Rating Scale (BPRS), in Overall 1962, or the PANSS (Kay 1986), this could be considered to be a clinically significant response (Leucht 2005a; Leucht 2005b). If data based on these thresholds were not available, we used the primary cutoff presented by the original authors.

\subsection{Direction of graphs}

Where possible, we entered data in such a way that the area to the left of the line of no effect indicated a favourable outcome for ICM.

\section{Assessment of risk of bias in included studies}

For this version of the review, two review authors (HB, MK) assessed risk of bias of all new included studies using the tool described in the Cochrane Handbook for Systematic Reviews of Interventions (Higgins 2011).

This set of criteria is based on evidence of associations between overestimate of effect and high risk of bias of the article such as sequence generation, allocation concealment, blinding, incomplete outcome data, and selective reporting.

If the raters had disagreed, we planned to make the final rating by consensus. Where inadequate details of randomisation and other characteristics of trials were provided, we contacted the authors of the studies to obtain further information. We would have reported non-concurrence in quality assessment, and if disputes had arisen as to which category a trial was to be allocated, again, we would have resolved this by discussion.

We noted the level of risk of bias in Risk of bias in included studies, Summary of findings for the main comparison, Summary of findings 2, and Figure 1. 
Figure 1. Methodological quality summary: review authors' judgements about each methodological quality item for each included study.

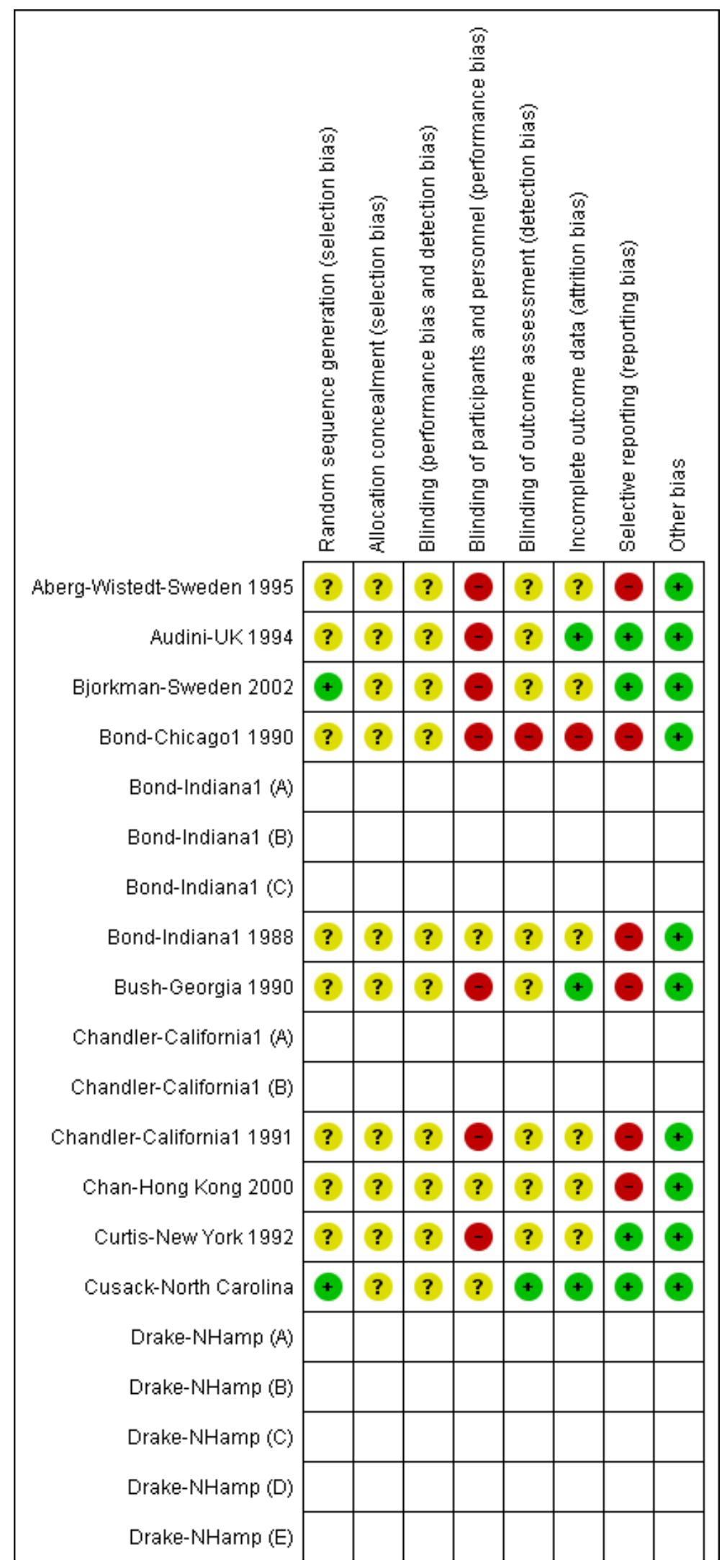


Figure 1. (Continued)

\begin{tabular}{|c|c|c|c|c|c|c|c|c|}
\hline \multicolumn{9}{|l|}{ Drake-NHamp (E) } \\
\hline \multicolumn{9}{|l|}{ Drake-NHamp $(\mathrm{F})$} \\
\hline \multicolumn{9}{|l|}{ Drake-NHamp (G) } \\
\hline Drake-NHamp 1998 & $?$ & $?$ & $?$ & $?$ & $?$ & $?$ & $\oplus$ & + \\
\hline Essock-Connecticut1 1995 & $?$ & $?$ & $?$ & $?$ & $?$ & + & $\odot$ & + \\
\hline Essock-Connecticut2 2006 & $\odot$ & $?$ & $?$ & $?$ & $?$ & $?$ & - & + \\
\hline Ford-UK 1995 & $\odot$ & $?$ & $?$ & - & $?$ & $\odot$ & + & $\oplus$ \\
\hline \multicolumn{9}{|l|}{ Hampton-Illinois (A) } \\
\hline \multicolumn{9}{|l|}{ Hampton-Illinois (B) } \\
\hline Hampton-Illinois 1992 & ? & $?$ & $?$ & ? & $?$ & $?$ & - & ? \\
\hline Harrison-Read-UK 2000 & $\odot$ & $?$ & $?$ & $?$ & $\odot$ & $?$ & $\odot$ & + \\
\hline Herinck-Oregon 1996 & ? & $?$ & $?$ & - & $?$ & $?$ & - & + \\
\hline Holloway-UK 1996 & $?$ & $?$ & $?$ & - & $?$ & + & + & + \\
\hline Jerrell-SCarolina1 1991 & ? & ? & $?$ & ? & $?$ & $?$ & - & + \\
\hline Johnston-Australia 1998 & $?$ & $?$ & $?$ & $?$ & $?$ & $\odot$ & $\odot$ & $\odot$ \\
\hline Lehman-Maryland1 1994 & ? & $?$ & $?$ & - & $?$ & $?$ & - & $\odot$ \\
\hline Macias-Utah 1994 & $?$ & $?$ & $?$ & ? & $?$ & $?$ & ? & + \\
\hline Marshall-UK 1995 & + & $?$ & $?$ & - & $?$ & $?$ & + & + \\
\hline \multicolumn{9}{|l|}{ McDonel-Indiana (A) } \\
\hline \multicolumn{9}{|l|}{ McDonel-Indiana (B) } \\
\hline McDonel-Indiana 1997 & ? & $?$ & $?$ & ? & $?$ & $?$ & - & 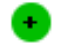 \\
\hline Morse-Missouri1 1992 & ? & $?$ & $?$ & - & $?$ & $?$ & - & + \\
\hline Morse-Missouri3 2005 & $?$ & $?$ & $?$ & - & $?$ & $?$ & + & + \\
\hline Muijen-UK2 1994 & ? & $?$ & $?$ & - & $?$ & ? & $\Theta$ & + \\
\hline Muller-Clemm-Canada 1996 & $?$ & $?$ & $?$ & $\Theta$ & $?$ & $?$ & $\odot$ & + \\
\hline Okpaku-Tennessee 1997 & $?$ & $?$ & $?$ & ? & $\odot$ & $?$ & $\odot$ & + \\
\hline OPUS-Denmark 1999 & + & + & $?$ & - & $?$ & $\odot$ & $\odot$ & + \\
\hline Pique-California 1999 & + & $?$ & $?$ & - & $?$ & $?$ & - & + \\
\hline Quinlivan-California 1995 & ? & $?$ & $?$ & ? & + & $?$ & + & + \\
\hline REACT-UK 2002 & $\odot$ & + & $?$ & $?$ & - & $\odot$ & + & + \\
\hline Rosenheck-USA 1993 & + & ? & $?$ & 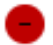 & $?$ & $?$ & - & + \\
\hline
\end{tabular}


Figure 1. (Continued)

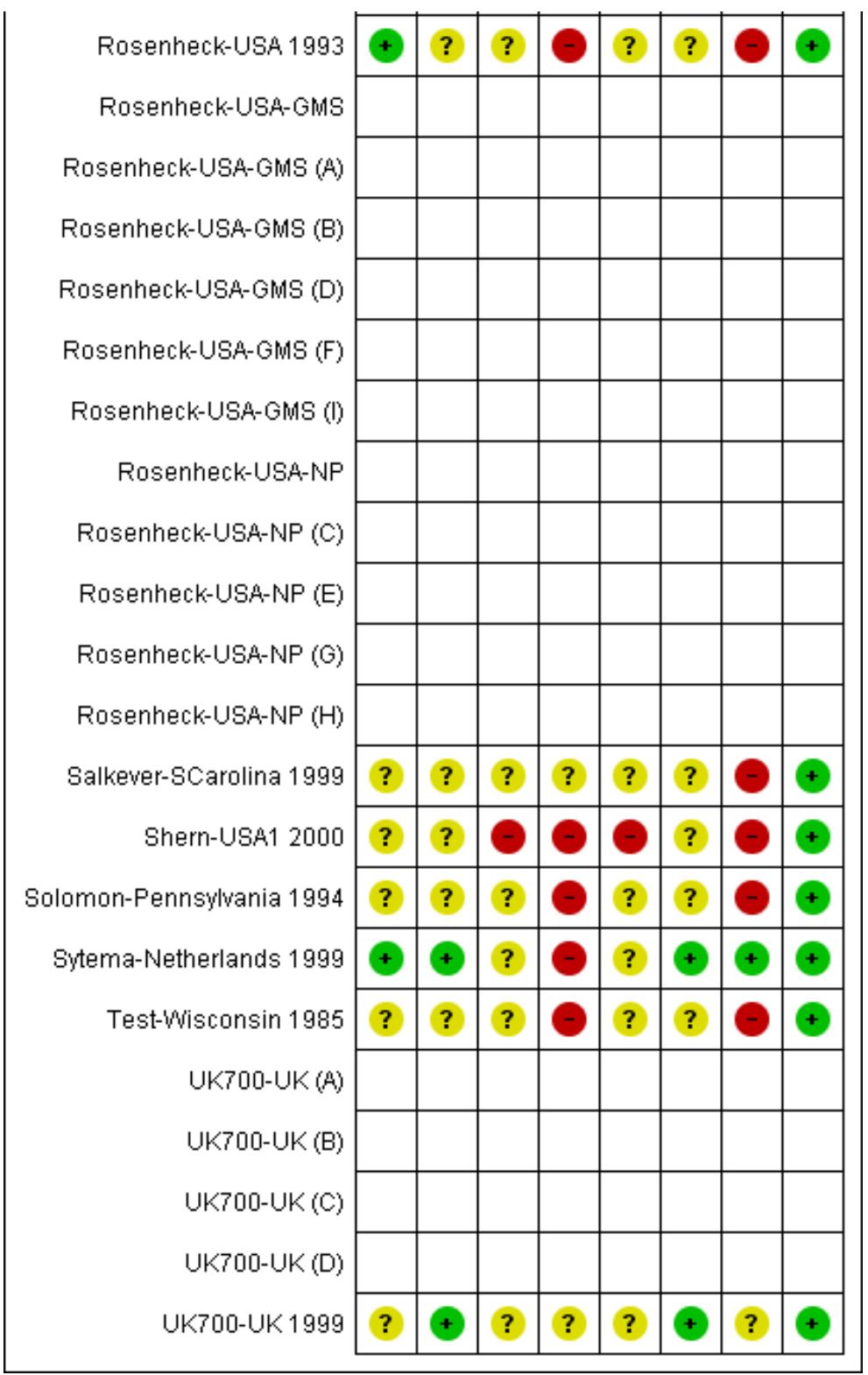

\section{Measures of treatment effect}

\section{Binary data}

For binary outcomes, we calculated a standard estimation of the random-effects risk ratio (RR) and its 95\% confidence interval. It has been shown that $\mathrm{RR}$ is more intuitive than odds ratios (OR), and that clinicians tend to interpret ORs as RR (Boissel 1999; Deeks 2000). Within the 'Summary of findings' table, we aimed to calculate the lowest control risk applied to all data. We assumed the same for the highest-risk groups. We used the 'Summary of findings' table to calculate absolute risk reduction for primary outcomes.

\section{Continuous data}

For continuous outcomes, we estimated the mean difference between groups. We preferred not to calculate effect size measures (standardised mean difference). However, if in future versions of this review scales of very considerable similarity are used, we will presume there is a small difference in measurement and will calculate effect size and transform the effect back to the units of one or more of the specific instruments.

\section{Unit of analysis issues}

\section{Cluster trials}

Studies increasingly employ 'cluster randomisation' (such as randomisation by clinician or practice), but analysis and pooling of clustered data pose problems. Firstly, authors often fail to account for intraclass correlation in clustered studies, leading to a 'unit of analysis' error (Divine 1992), whereby $P$ values are spuriously low, confidence intervals unduly narrow, and statistical significance overestimated. This causes type I errors (Bland 1997; Gulliford 1999). 
Where clustering is not accounted for in primary studies, we would present data in a table, with a $\left(^{*}\right)$ symbol to indicate the presence of a probable unit of analysis error. If we find such studies in subsequent versions of this review, we will attempt to contact first authors of studies to obtain intraclass correlation coefficients for their clustered data and to adjust for this by using accepted methods (Gulliford 1999). Where clustering has been incorporated into the analysis of primary studies, we would present these data as if from a non-cluster randomised study, but adjust for the clustering effect.

We have sought statistical advice and have been advised that the binary data as presented in a report should be divided by a 'design effect'. This is calculated using the mean number of participants per cluster $(\mathrm{m})$ and the intraclass correlation coefficient (ICC) [Design effect $=1+(m-1)^{\star} I C C$ ] (Donner 2002). If the ICC is not reported, we would assume it to be 0.1 (Ukoumunne 1999).

If cluster studies have been appropriately analysed taking into account intraclass correlation coefficients, and relevant data documented in the report, synthesis with other studies would be possible using the generic inverse variance technique.

\section{Cross-over trials}

A major concern of cross-over trials is the carry-over effect, which occurs if an effect (e.g. pharmacological, physiological, or psychological) of the treatment in the first phase is carried over to the second phase. As a consequence, on entry to the second phase the participants can differ systematically from their initial state despite a wash-out phase. For the same reason, cross-over trials are not appropriate if the condition of interest is unstable (Elbourne 2002). As both effects are very likely in severe mental illness, we only used data from the first phase of cross-over studies.

\section{Studies with multiple treatment groups}

Where a study involved more than two treatment arms, we presented the additional treatment arms in comparisons if relevant. Where the additional treatment arms were not relevant, we did not reproduce these data.

\section{Dealing with missing data}

\section{Overall loss of credibility}

At some degree of loss of follow-up data loses credibility (Xia 2009). For any particular outcome, should more than $50 \%$ of data be unaccounted for, we did not reproduce these data or use them within analyses. If, however, more than $50 \%$ of participants in one arm of a study were lost, but the total loss was less than $50 \%$, we marked such data with $\left({ }^{*}\right)$ to indicate that such a result may well be prone to bias.

\section{Binary}

Where attrition for a binary outcome was between 0 and $50 \%$, and where these data were not clearly described, we presented data on a 'once-randomised-always-analyse' basis (an intention-to-treat analysis). We assumed all those leaving the study early to have the same rates of negative outcome as those who completed, with the exception of the outcome of death. We undertook a Sensitivity analysis testing how prone the primary outcomes were to change when we compared data from only those who completed the study with intention-to-treat data using the assumption outlined above.
Where number of deaths was more than $10 \%$ of the sample overall, we applied the above statement but did not impute attrition due to death.

\section{Continuous}

\subsection{Attrition}

Where attrition for a continuous outcome was between 0 and $50 \%$, and data from only those who completed the study were reported, we reproduced these.

\subsection{Standard deviations}

\subsubsection{General}

Where there were missing measures of variance for continuous data, but exact standard errors or confidence intervals for group means, or either ' $P$ ' or ' $t$ ' values for differences in means, we calculated standard deviation value according to the method described in Section 7.7.3 of the Cochrane Handbook for Systematic Reviews of Interventions (Higgins 2011). If standard deviations were not reported and could not be calculated from the available data, we asked authors to supply the data. In the absence of data from authors, we used the mean standard deviation from other studies.

\subsubsection{Standard deviation mean number of days per month in hospital}

For the primary outcome mean number of days per month in hospital, if standard deviations were not reported and could not be calculated from available data, we asked the authors for additional information. In the absence of data from authors, we imputed the missing standard deviations using a regression analysis of SD against mean from those trials that provided both. We documented in what studies we imputed SDs according to the above technique in Table 2.

\subsection{Last observation carried forward}

We anticipated that in some studies the method of last observation carried forward (LOCF) would be employed within the study report. As with all methods of imputation to deal with missing data, LOCF introduces uncertainty about the reliability of the results. Therefore, where LOCF data had been used in the trial, if less than $50 \%$ of the data had been assumed, we reproduced these data and indicated that they were the product of LOCF assumptions.

\subsection{Incomplete data for meta-regression}

We anticipated that in some cases not all IFACT score variables would be available. If we could not calculate IFACT score from the available data, we imputed it by multiple imputation using the Multiple Imputation with Diagnostics (mi) library in R (R 2008). As explained above, we only made these assumptions if we were able to directly rate over $50 \%$ of the data. We documented in what studies we calculated IFACT score according to the above technique in Table 3.

We anticipated that in some cases not all baseline hospital use data would be available. We imputed missing data as for the IFACT scores. As explained above, we only made these assumptions if we were able to directly rate over $50 \%$ of the data. We documented for which studies we calculated baseline hospital use data according to the above technique (Table 3 ).

We undertook a Sensitivity analysis testing how prone the results from meta-regression were to change when we compared data 
from only those who completed the studies with the imputed data using the assumption outlined above.

\section{Assessment of heterogeneity}

\section{Clinical heterogeneity}

We considered all included studies initially, without seeing comparison data, to judge clinical heterogeneity. We simply inspected all studies for clearly outlying situations or people that we had not predicted would arise. When such situations or participant groups arose, we discussed these fully.

In addition, we specified two potential sources of heterogeneity a priori (fidelity and baseline level of hospital use) (Data extraction and management). We extracted these data as described above.

\section{Methodological heterogeneity}

We considered all included studies initially, without seeing comparison data, to judge methodological heterogeneity. We simply inspected all studies for clearly outlying methods that we had not predicted would arise. If such methodological outliers arose, we discussed these fully.

\section{Statistical heterogeneity}

\subsection{Visual inspection}

We visually inspected graphs to investigate the possibility of statistical heterogeneity.

\subsection{Employing the $I^{2}$ statistic}

We investigated heterogeneity between studies by considering the $\mathrm{I}^{2}$ statistic alongside the $\mathrm{Chi}^{2} \mathrm{P}$ value. The $\mathrm{I}^{2}$ provides an estimate of the percentage of inconsistency thought to be due to chance (Higgins 2003). The importance of the observed value of 12 depends on i.) magnitude and direction of effects, and ii.) strength of evidence for heterogeneity (e.g. $\mathrm{P}$ value from $\mathrm{Chi}^{2}$ test, or a confidence interval for $\mathrm{1}^{2}$ ). We interpreted an $\mathrm{I}^{2}$ estimate greater than or equal to $50 \%$ accompanied by a statistically significant $\mathrm{Chi}^{2}$ statistic, as evidence of substantial levels of heterogeneity (Section 9.5.2; Higgins 2011). When we found substantial levels of heterogeneity in the primary outcome, we explored reasons for the heterogeneity (Subgroup analysis and investigation of heterogeneity).

\section{Assessment of reporting biases}

Reporting biases arise when the dissemination of research findings is influenced by the nature and direction of results (Egger 1997). These are described in Chapter 10 of the Cochrane Handbook for Systematic Reviews of Interventions (Higgins 2011). We are aware that funnel plots may be useful in investigating reporting biases but are of limited power to detect small-study effects. We did not use funnel plots for outcomes where there were 10 or fewer studies, or where all studies were of similar size. In other cases, where funnel plots were possible, we sought statistical advice in their interpretation.

\section{Data synthesis}

Where possible, we employed a random-effects model for analyses. We understand that there is no closed argument for preference for use of fixed-effect or random-effects models. The randomeffects method incorporates an assumption that different studies are estimating different, yet related, intervention effects. According to our hypothesis of an existing variation across studies, to be explored further in the meta-regression analysis, despite being cautious that random-effects methods do put added weight onto the smaller of the studies, we favoured using random-effects model.

\section{Subgroup analysis and investigation of heterogeneity}

\section{Subgroup analyses}

We anticipated conducting two subgroup analyses. For the first version of the protocol for this review, we did not anticipate any subgroup analyses. On further consideration, we now realise that analysis at separate time periods could be thought of as subgroups. The second subgroup is within the primary outcome and relates to skewed and non-skewed data. We introduced this late into the protocol, and it could be considered post hoc. However, we are also aware that our original rule for management of these data could be considered overly cautious and result in some important data not being presented (Higgins 2011).

\section{Investigation of heterogeneity}

\subsection{Anticipated heterogeneity - outcome of mean days per month in} hospital

Investigation of heterogeneity formed part of the secondary objectives of the review. We hypothesised that the effect of ICM on one of our primary outcomes (mean number of days per month in hospital) differs according to fidelity of intervention to the ACT model and the baseline level of hospital use.

We examined the association of the IFACT score and the baseline number of days in hospital with the treatment effect by performing random-effects meta-regression analysis in $R$ ( $R$ 2008). The script we used to perform meta-regression analyses is reported in Appendix 3. We also carried out meta-regression using both variables within the same model. In addition, we examined the relationship between the treatment effect and the two variables using a thin plate spline. If possible, we aimed to enter data from multicentre studies in the meta-regression disaggregated into the component centre with outcome and fidelity data for each.

Meta-regression was performed only if at least 10 studies per comparison were available (Higgins 2011). rWe also tested comparison type as an additional regressor in the model.

\subsection{Unanticipated heterogeneity - other outcomes}

\subsubsection{For outcomes other than the second primary outcome (not remaining in contact with psychiatric services)}

We reported if inconsistency was high and undertook no exploration.

\subsubsection{For outcome 'not remaining in contact with psychiatric services'}

We reported if inconsistency was high. First we investigated whether data had been entered correctly. Second, if data was correct, we visually inspected the graph and successively removed studies outside of the company of the rest to see if homogeneity was restored. Should this occur with no more than $10 \%$ of the data being excluded, we presented the data. If not, these data were not pooled. 
Should unanticipated clinical or methodological heterogeneity have been obvious, we simply stated hypotheses regarding these for future reviews or versions of this review. We did not anticipate undertaking analyses relating to these.

\section{Sensitivity analysis}

\section{Implication of randomisation}

We aimed to include trials in a sensitivity analysis if they were described in some way as to imply randomisation. For the primary outcomes, we included these studies, and if there was no substantive difference when the implied randomised studies were added to those with a better description of randomisation, then we employed all data from these studies.

\section{Standard-care caseload}

If data were available, we undertook a sensitivity analysis testing how prone the primary outcomes were to change when trials comparing ICM to standard community care caseload less than or equal to 20 were compared with trials comparing ICM to standard community care caseload greater than 20 . If there was a substantial difference, we reported the results and discussed them but continued to pool the data.

\section{Assumptions for lost binary data}

Where we needed to make assumptions regarding participants lost to follow-up (see Dealing with missing data), we compared the findings of the primary outcomes when we used our assumption compared with completer data only. If there was a substantial difference, we reported the results and discussed them but continued to employ our assumption.

\section{Assumptions for incomplete data for meta-regression}

Where we needed to make assumptions regarding missing SDs data in studies entering meta-regression (see Dealing with missing data), we compared the findings of the meta-regression on our primary outcome when we used our assumption compared with data taken from only those who completed the studies. We tested how prone results from meta-regression were to change when we compared data from those who completed with imputed data using the assumption outlined above. If there was a substantial difference, we reported results and discussed them but continued to employ our assumption.

\section{RE S U L T S}

\section{Description of studies}

\section{Results of the search}

We have presented the results of the latest update search below; for previous results, please see Appendix 4.

The April 2015 update search of Cochrane Schizophrenia Group's Register of Trials yielded 299 references. We selected 87 for further inspection. One hundred and twenty-seven references (corresponding to 96 studies with 31 companion papers) were available from the 'awaiting classification' section of the previous version of the review and were all selected for further inspection. We excluded a total of 85 studies from the review. Only two trials met the inclusion criteria and were included (Chan-Hong Kong 2000; Cusack-North Carolina). There were 26 new companion papers to previously included studies such as Morse-Missouri3 2005, OPUS-Denmark 1999, REACT-UK 2002, and UK700-UK 1999.

We have entered 20 trials in the 'awaiting classification' section and have sought further information. We added five new studies to the ongoing studies.

\section{Problematic trials}

There were two problematic trials worth special mention.

Jerrell-SCarolina1 1991 was a three-arm trial, with two of the arms qualifying as Intensive Case Management (Programme Assertive Community Treatment and Intensive Broker Case Management) and one a control (standard care). As results were reported separately for each arm, it was not possible to present continuous data from two ICM arms pooled together. One option was to treat each arm as a separate 'site', effectively treating the study as two trials, but with the same control group. A second option was to include only one of the experimental arms. Although aware of excluding potentially useful data on an arbitrary basis, we decided to include only one of the arms compared to standard care, per the second option. The main reason for this was to avoid a unit of analysis error, which would have occurred in the first option. We undertook a sensitivity analysis testing how prone results were to change when this trial was not included in meta-analysis.

Curtis-New York 1992 was a trial comparing ICM with standard care presenting two main difficulties. The first was regarding ICM caseload size. The study reported caseload ratio as 1:35 (above the 1:20 ratio defining an ICM intervention). As we derived estimation of caseload size by dividing the number of intervention participants by the number of whole-time equivalent clinical staff in the team (not just those formally classified as 'case managers'), we found that the actual staff:participant ratio was about 1:17. We therefore found this trial eligible for inclusion in the review. The second issue was regarding the peculiar way this trial provided the ICM intervention. Both experimental and control interventions were community-oriented and fit fully into the review's inclusion criteria, but the ICM team was located in hospital. While undesirable, the team office being based in hospital is not unusual. In any case, the case management was provided in the community. We therefore confirmed inclusion of the study, not wanting to penalise it because it reported details that were not available for all trials. However, we found a discrepancy in the data the study provided on service use outcomes (average number of days in hospital per month, admitted to hospital). Curtis-New York 1992 was an outlier, being the only study clearly favouring standard care over ICM. We undertook a sensitivity analysis testing how prone results were to change when this trial was not included in the meta-analysis. Neither results for the primary outcome 'average number of days per month in hospital' nor for the outcome 'admitted to hospital' changed significantly when this study was dropped, but it did significantly affect the level of heterogeneity. We could just advance the hypothesis that the reason for heterogeneity could be the unusual way the intervention was provided in this trial (Table 4).

For a summary of the trial selection from the 2015 search, please see Figure 2. 
Figure 2. Study flow diagram 2015 update

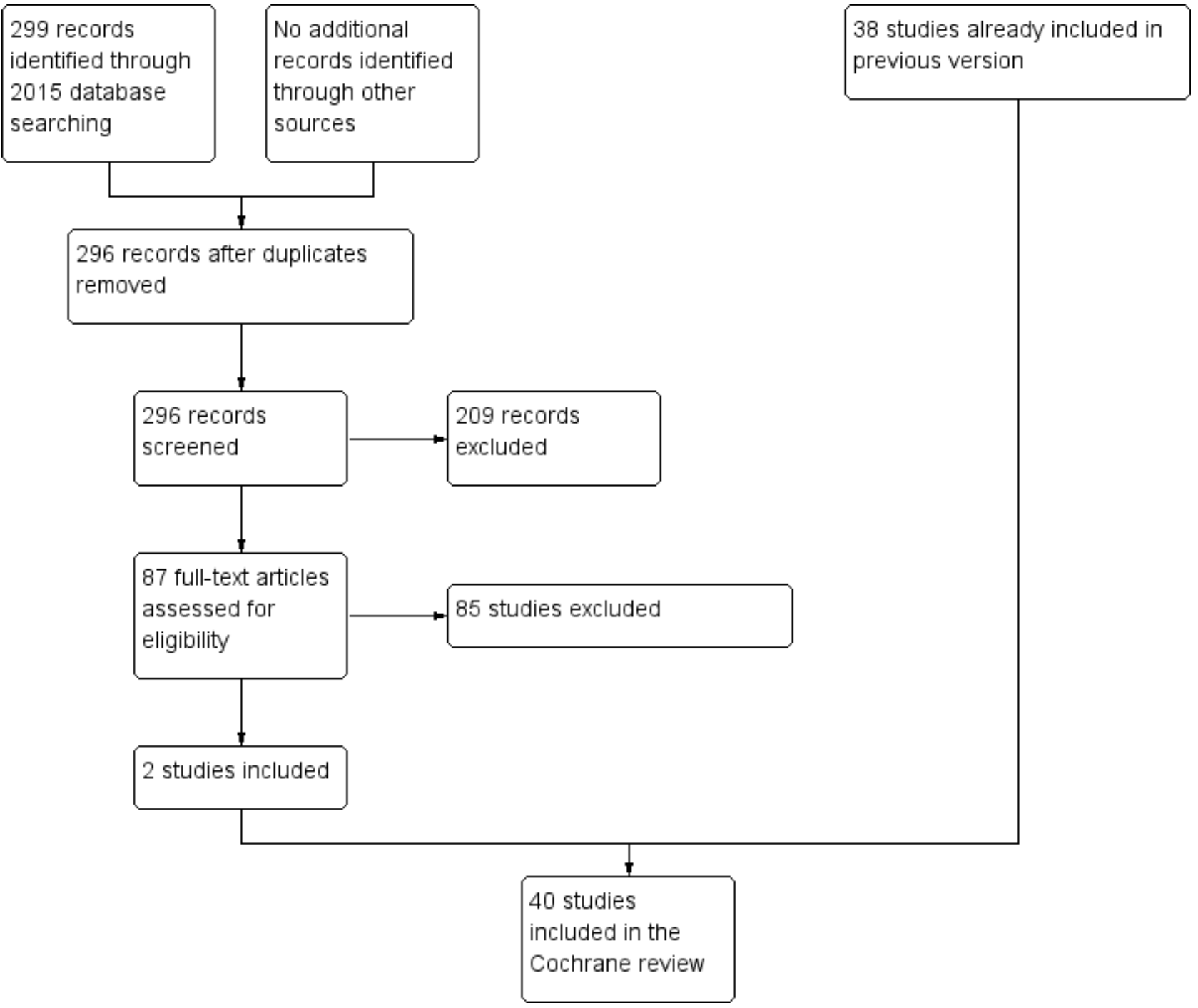

\section{Included studies}

See: Characteristics of included studies.

The previous updates of two reviews included 176 reports describing 38 studies. This review now includes these 38 separate trials with an additional two studies including data on 196 participants (Chan-Hong Kong 2000; Cusack-North Carolina). These 40 trials provide data on 7524 randomised people. Twenty-four of these studies had already been included in two original reviews (as included or awaiting assessment), with 14 more derived from the 2008 search and two more derived from the 2015 search. Twenty-eight trials provided data for the ICM versus standard care comparison, 11 for the ICM versus non-ICM comparison, and only one for both comparisons. Both of the two newly included studies provided data for the ICM versus standard care comparison. Please note that it was possible to report data for several separate centres of seven multicentre trials (Bond-Indiana1 1988 (3); ChandlerCalifornia1 1991 (2); Drake-NHamp 1998 (7); Hampton-Illinois 1992 (2); McDonel-Indiana 1997 (2); Rosenheck-USA 1993 (9); and UK700UK 1999 (4)). Several of these centres are reported separately in the Characteristics of included studies table.

\section{Study length}

Only one study fell into the short-term category, with a maximum length of six months (Bond-Indiana1 1988); nine studies reported medium-term data, with only one study reporting data by seven months, Okpaku-Tennessee 1997, and the remaining eight studies reporting data by 12 months (Bond-Chicago1 1990; Bush-Georgia 1990; Hampton-Illinois 1992; Johnston-Australia 1998; LehmanMaryland1 1994; Morse-Missouri1 1992; Solomon-Pennsylvania 1994; Sytema-Netherlands 1999). The remaining 29 studies all fell into the long-term category, with a maximum length of four years (Test-Wisconsin 1985), and an average length of 23.5 months.

One study reported data only on short-term follow-up (five months of active intervention followed by six months' follow-up) (Chan-Hong Kong 2000), not reporting data assessed during the intervention. Newly retrieved companion papers provided data from medium- and long-term follow-up for two studies already included in the previous version of this review. Those two studies were OPUS-Denmark 1999, now reporting data at three and eight years after active intervention was discontinued (i.e. 5 and 10 years after randomisation), and REACT-UK 2002, now reporting data at 18 
months and 8.5 years after participants could choose to which arm they were allocated (i.e. 3 and 10 years after randomisation). During the follow-up period, all participants in OPUS-Denmark 1999 received the control intervention, where participants in REACT-UK 2002 could remain in the originally allocated intervention or be transferred to the control one.

\section{Design}

All included studies were randomised with a parallel longitudinal design. Twelve were multicentre trials, but only seven of these provided data for single centres (see above).

\section{Participants}

We included a total of 7524 participants from 40 trials. Most trials included from the previous review were conducted in Australia, Canada, and the USA. Specifically, most of the trials included from the previous two reviews were conducted in the USA ( 16 trials; 3474 participants), and only five were European (345 participants). In the first update of 2010, we added 1964 participants from seven trials conducted in Europe, and 1545 participants from 10 studies conducted in Australia, Canada, and the USA. In the current version of the review, we added 62 participants from one trial conducted in China (Chan-Hong Kong 2000), and 134 participants from one trial conducted in the USA (Cusack-North Carolina).

The review now provides data on 27 trials, including 5153 participants, conducted in Australia, Canada, and the USA; 12 trials, including 2309 participants, conducted in Europe; and one trial, including 62 participants, conducted in China.

Twenty studies included participants with severe mental illness. None of these studies provided operationalised definitions addressing dimensions of diagnosis, duration, and disability. However, 14 provided criteria for either the diagnosis, impairment, or level of service use. The remaining six studies provided no criteria for defining serious mental illness. Diagnoses of 'severe mental illness' varied across studies, from schizophrenic disorder alone to wider diagnostic groups including schizophrenic, affective, and personality disorder.

Of the remaining 20 studies, 18 involved participants with various diagnoses, but the great majority had some psychotic disorder, and most trials reported criteria for service use or impairment, or both. Two studies included participants with a high level of impairment or service use due to psychiatric illness, but provided no diagnostic criteria for inclusion (Harrison-Read-UK 2000; Okpaku-Tennessee 1997).

Most trials (23) involved participants that had been diagnosed using operationalised criteria (DSM, ICD, OPCRIT, RDC, SADS, see Characteristics of included studies footnotes), whilst 17 (10 in the group including participants with serious mental illness and six in the group including participants with various diagnoses) did not report using any diagnostic tool, but only stated type of illness or level of impairment. Only OPUS-Denmark 1999 included participants with a first episode of psychotic illness.

Four studies included a total of 742 dually diagnosed participants (Drake-NHamp 1998; Essock-Connecticut2 2006; Morse-Missouri3 2005; Muller-Clemm-Canada 1996), and eight studies included a total of 1337 homeless participants.
Information on mean age was available from 32 trials (6473 participants). The average age was about 38 years old. Only MaciasUtah 1994 did not report information on participant age.

\section{Settings}

As stated in the inclusion criteria, all of the included studies took place in a community setting, provided both by private and public mental health services. No study was carried out in a low-income country, as the included studies were conducted in Australia, Canada, the USA, Europe, and China.

\section{Interventions}

Twenty-nine trials were included in the comparison ICM versus standard care, and 12 in the ICM versus non-ICM comparison. Quinlivan-California 1995 was a three-arm trial (ICM, non-ICM, and standard care) and provided data for both comparisons. We considered REACT-UK 2002 to be an ICM versus non-ICM comparison due to our assumption that standard care could be considered to be Care Programme Approach, even if not clearly reported by trial authors. The Care Programme Approach was introduced in England in the mid-1990s and become standard care thereafter; it is a combination of non-Intensive Case Management and care from a Community Mental Health Team (Department of Health 1999), hence to be considered as non-ICM according to the definitions in this review.

\subsection{Intensive Case Management}

On average, the ICM included in this review was well defined. The majority of experimental interventions were explicitly modelled on the ACT model, being based on the Treatment in Community Living model of Stein 1980. Only a few studies based ICM on the Case Management model. The experimental intervention was provided either by an already existing team or ICM services newly established for the trial. Cusack-North Carolina was the only trial that included ICM as forensic adaptation of ACT compared with standard care.

\subsection{Non-Intensive Case Management}

There were no discernable differences between the practice of non-ICM and ICM except for the intensity of contact. The degree of training and skill of the staff was similar in the ICM and nonICM teams. In some studies, non-ICM was itself an experimental intervention, but it mostly represented the average standard care, as what in this review we call 'standard care' has increasingly shifted towards non-ICM across decades. Mental health systems increasingly included elements from ICM, melding them with community mental health service. English mental health policy is one example, where the Care Programme Approach was introduced in the UK in the mid-1990s and become standard care thereafter. It is a combination of non-ICM and care from a Community Mental Health Team, hence to be considered as non-ICM, according to the definitions in this review. Therefore the REACT-UK 2002 study was considered in the ICM versus non-ICM comparison due to our assumption that standard care could be considered as Care Programme Approach, even if not clearly reported by trial authors.

\subsection{Standard care}

On average, the definition of standard care was blurred, as this intervention was modelled on a generalist model. Its core was being provided by a community mental health service, but its features were variable across trials run in different countries at 
different time periods. Presence of further specialised services, such as rehabilitation or psychotherapist services, were variable within standard care services. In a few studies, both ICM and standard care incorporated services for substance abuse treatment and homelessness care.

\section{Outcome measures}

\subsection{Overall}

The outcomes for which we could obtain usable data were: service use, adverse effects, global state, social functioning, mental state - general and specific symptoms, behaviour, quality of life, satisfaction, and costs.

Many trials used different scales in assessing treatment effects in various outcomes (global state, social functioning, mental state - general and specific symptoms, behaviour, quality of life, and satisfaction). As most of the scales were used by only one study in each comparison group, it was not possible to enter these data in a unique analysis. Even where studies used the same scale, they often applied different rating scores. Therefore, again, data could not be entered together in the analysis. Some studies failed to clearly report the rating score they used for a pre-stated scale. We noted this in the 'Risk of bias' tables (see 'Outcomes' in Characteristics of included studies table). No studies assessed improvement by measuring it on scales. We did not calculate effect size measures (standardised mean difference, see Measures of treatment effect).

\subsection{Outcome scales}

Only details of the scales that provided usable data are shown below. Reasons for exclusions of data are provided under 'Outcomes' in Characteristics of included studies table.

\subsubsection{Global Assessment Scale (GAS) (Endicott 1976): in Audini-UK} 1994, Muijen-UK2 1994, Rosenheck-USA 1993

This is an observer-rated scale for evaluating the overall functioning of a person during a specified time period on a continuum from psychological or psychiatric sickness to health. The score ranges from 0 to 100, where a higher score indicates a better outcome. A modified version of the GAS was included in the Diagnostic and Statistical Manual of Mental Disorders (DSM-III) as the Global Assessment of Functioning Scale (GAF) (APA 1987): in BjorkmanSweden 2002. Outcomes from the two scales are reported together as GAF, as these two scales are very similar, and they report results on the same score range.

6.2.2 Health of the Nation Outcome Scale (HoNOS) (Stein 1999; Wing 1998): in Harrison-Read-UK 2000, REACT-UK 2002

This scale provides a systematic summary of behaviours and functioning, measuring mental health and social/behavioural functioning. It consists of four areas (behaviour, impairment, symptoms, and social), each assessed through 12 items on a fivepoint scale (0 to 4). Ratings are from 0 to 48; high score means severe dysfunction.

\subsubsection{Rating of Medication Influence (ROMI) (Weiden 1994): in Harrison-Read-UK 2000, REACT-UK 2002}

This 20-item scale measures the influence of factor on medication adherence. Each item is rated according to the degree of influence on medication-taking behaviour: none (1), mild (2), and strong (3).
It has two subscales: patient-reported compliance (items 1 to 7 ) and patient-reported non-compliance (items 8 to 20). A high score on the compliance subscale indicates high compliance; a high score on the non-compliance subscale indicates high non-compliance. Results from the two studies are presented on a different range score (Harrison-Read-UK 2000 range score of 1 to 3; REACT-UK 2002 range score not clearly reported).

6.2.4 Disability Assessment Schedule (DAS) (WHO 2001a): in Holloway-UK 1996

The World Health Organization's Psychiatric Disability Assessment Schedule (DAS) is a measure of functioning and disability. It contains 36 items with six domains of functioning, including: understanding and communicating, getting around, self care, getting along with others, household and work activities, and participation in society. Higher scores indicate a worse outcome.

6.2.5 Interview Schedule for Social Interaction - abbreviated version (ISSI) (Henderson 1980; Unden 1989): in Bjorkman-Sweden 2002

The ISSI scale is a self report scale consisting of 30 items that measures social integration and attachment. The maximum score is 30 points; higher scores indicate better social integration and attachment.

6.2.6 Life Skills Profile (LSP) (Parker 1992; Rosen 1989): in REACT-UK 2002

The Life Skills Profile is a clinician-rated questionnaire developed in Australia primarily for use with people with psychotic illnesses. Thirty-nine items and five subscales assess the general domain of disability over the last three months. The five subscales measure self care, non-turbulence, social contact, communication, and responsibility. Each of the 39 items on the scale range from 'not at all disabled' to 'extremely disabled'.

\subsubsection{REHAB Scale (REHAB) (Baker 1983): in Marshall-UK 1995}

The REHAB Scale is an observer-rated measure of social functioning, covering social activity, self care, speech disturbance, and community skills. It rates the frequency of items of embarrassing or disruptive behaviour, such as violence, self harm, shouting and swearing, and sexual offensiveness (deviant behaviour-REHAB DB); and lack of general skills (general behaviour - REHAB GB). The scale ranges from 0 to 144, with higher scores indicating poorer functioning.

6.2.8 Role Functioning Scale (RFS) (Green 1987): in Jerrell-SCarolina2 1994

This is a self report scale whereby the total of four subscales measures global role functioning. Higher scores indicate better functioning.

6.2.9 Social Adjustment Scale (SAS) (Weissman 1971; Weissman 1974): in Audini-UK 1994, Muijen-UK2 1994

Measures social functioning in a number of life domains (work, social, extended family, marital, parental, family unit, and economic adequacy). Score ranges from 1 to 7 , with higher score indicating poorer outcome. 
6.2.10 Social Adjustment Scale-II (SAS-II) (Schooler 1979): in JerrellSCarolina1 1991

Revised version of the Social Adjustment Scale (see above), used to assess social adjustment. Self reported scale similar to SAS, but adapted for schizophrenia; it comprises 24 items covering seven areas including social, family, and work functioning. The scoring system of the two versions appears to differ, perhaps because this was an adapted version. Higher score indicates better outcome.

6.2.11 Social Functioning Questionnaire (SFQ) (Tyrer 1990; Tyrer 2005): in Harrison-Read-UK 2000

An eight-item self report scale (score range is 0 to 24). It provides a quick assessment of perceived social function. Higher score indicates poorer social functioning.

6.2.12 Strauss-Carpenter Outcome Scale (Strauss 1972; Strauss 1974): in Bjorkman-Sweden 2002

The Strauss-Carpenter Outcome Scale assesses a 21 items exploring frequency of social contacts, employment duration, symptomatology, and duration of rehospitalisation. The scaling of each item extends from 0 (maximal negative) to 4 (maximal positive). The scoring range of the scale extends from 0 (maximal negative) to 84 (maximal positive).

6.2.13 Alcohol Use Scale (AUS) (Drake 1996; Mueser 1995): in DrakeNHamp 1998

A five-point scale based on clinicians' ratings of the severity of the disorder, ranging from 1 (abstinence) to 5 (severe dependence).

6.2.14 Dartmouth Assessment of Lifestyle Interview (DALI) (Rosenberg 1998): in Sytema-Netherlands 1999

An 18-item, interviewer-administered scale addressing the detection of substance use disorder in people with severe mental illness. DALI focuses on alcohol, cannabis, and cocaine use disorders. DALI-alcohol: scores range from -4 to +6 , higher scores indicate higher risk of alcohol abuse. DALI-drugs: scores range from -4 to +4 , higher scores indicate higher risk of drug abuse. As scale ranges from negative to positive value, skew is difficult to detect. We therefore entered data from this scale in Additional tables rather than into an analysis.

6.2.15 Substance Abuse Treatment Scale (SATS) (Drake 1996; McHugo 1995): in Drake-NHamp 1998

An eight-point scale indicating progression toward recovery, ranging from 1 (early stages of engagement) to 8 (relapse prevention). Higher scores indicate greater progression.

6.2.16 Timeline Followback (TLFB) (Sobell 1980): in Drake-NHamp 1998

Scale administered by an interviewer to assess days of alcohol and drug use over the previous six months. Outcome reported as binary data.

6.2.17 Brief Psychiatric Rating Scale (BPRS) (Overall 1962): in AudiniUK 1994, Drake-NHamp 1998, Muijen-UK2 1994, REACT-UK 2002, Sytema-Netherlands 1999 (BPRS 24-item - Velligan 2005); in ChanHong Kong 2000, Ford-UK 1995, Rosenheck-USA 1993 (BPRS 18item)
The BPRS measures positive symptoms, general psychopathology, and affective symptoms. The original scale has 16 items, but a revised 18 -item scale is commonly used. Symptoms are reported in several ways (i.e. on a scale of 0 to 6 or 1 to 7 ), but it is most common for each item to be rated on a seven-point scale $(1=$ not present to 7 = extremely severe). The 18-item scale can range from 18 to 126 or from 0 to 108 (as in Ford-UK 1995 and Rosenheck-USA 1993).A further version is a 24-item scale ranging from 24 to 168 . For all of the scales, higher scores indicate more severe symptoms.

6.2.18 Brief Symptom Inventory (BSI) (Derogatis 1983): in RosenheckUSA 1993

A brief rating scale used by an independent rater to assess the severity of psychiatric symptoms. Scores range from 0 to 4 , with higher scores indicating more symptoms.

6.2.19 Comprehensive Psychopathological Rating Scale (CPRS) (Asberg 1978): in Holloway-UK 1996, UK700-UK 1999

This is an interview rating scale covering a wide range of psychiatric symptoms; it can be used in total or as subscales. CPRS consists of 65 items that cover the range of psychopathology over the preceding week ( 40 symptom items are rated by the participant, and 25 observed items are rated by the rater during the interview). Each item is rated on a 0 to 3 scale ranging from 'not present' to 'extremely severe', with higher scores indicating more severe symptoms.

6.2.20 Colorado Symptom Index (CSI) (Shern 1994): in LehmanMaryland1 1994, Shern-USA1 2000

A brief rating scale used by an independent rater to assess the severity of a range of psychiatric symptoms. A lower score indicates more symptoms.

6.2.21 Krawiecka Scale (KS) alias Manchester Scale (Krawiecka 1977): in Harrison-Read-UK 2000

This scale rates severity of psychiatric symptoms. It consists of eight categories of symptoms assessed on a five-point scale, which are depression, anxiety, hallucinations, delusions, flattened and incongruous effect, psychomotor retardation, incoherence and irrelevance of speech, and poverty of speech. A score of 0 or 1 denotes an absence of pathology, while ratings of 2, 3, or 4 denote the presence of the target symptoms in increasing severity. Rating is from 0 to 36 . Higher scores indicate a worse outcome.

6.2.22 Present State Examination (PSE) (Wing 1974): in Audini-UK 1994, Muijen-UK2 1994

This is a clinician-rated scale measuring mental status. The scale rates and combines 140 symptom items to give various syndrome and subsyndrome scores. A short version covering the first 40 'neurotic' symptoms has been used in several population surveys. Score ranges from 1 to 120 . Higher scores indicate greater clinical impairment.

6.2.23 Symptom Checklist-90 (SCL-90) (Hopkins Symptoms Checklist) (Derogatis 1974): in Bjorkman-Sweden 2002

This is a self report clinical rating scale of psychiatric symptomatology comprised of 90 symptom-related questions. Out of 90 items, 83 items represent nine subscales: somatisation (12 
items), obsessive-compulsive (10 items), interpersonal sensitivity (9 items), depression (3 items), anxiety (10 items), anger-hostility (6 items), phobic anxiety ( 7 items), paranoid ideation (6 items), and psychoticism (10 items). Seven additional items include disturbances in appetite and sleep. The SCL-90 also utilises three global distress indices: Global Severity Index (GSI), Positive Symptom Distress Index (PSDI), and Positive Symptom Total (PST). The participant assesses the degree of severity of each symptom. Items are rated on a five-point Likert scale, ranging from 'not at all distressing' (0) to 'extremely distressing' (4), with higher scores indicating greater symptomatology.

\subsubsection{Beck Depression Inventory (BDI) (Beck 1979): in Holloway-UK 1996}

A 21-item self rating scale for depression. Each item comprises 4 statements (rated from 0 to 4 ) describing increasing severity of the abnormality concerned. The person completing the scale is required to read each group of statements and identify the one that best describes the way they have felt over the preceding week. Score ranges from 0 to 84 , with higher score indicating more severe symptoms.

6.2.25 Hospital Anxiety and Depression Scale (HADS) (Zigmond 1983): Harrison-Read-UK 2000

This scale is a questionnaire composed of statements relevant to either generalised anxiety or depression referring to the past week. Seven items in the questionnaire reflect anxiety, and seven reflect depression. The participant answers each item on a four-point $(0$ to 3 ) response category; the possible scores range from 0 to 21 for anxiety and 0 to 21 for depression. Higher score indicates a worse outcome.

6.2.26 Scale for the Assessment of Negative Symptoms (SANS) (Andreasen 1982; Andreasen 1989): in Holloway-UK 1996, UK700UK 1999

This scale assesses five symptom complexes to obtain clinical ratings of negative symptoms in people with schizophrenia over the preceding week. They are: affective blunting, alogia (impoverished speech), avolition/apathy, anhedonia/asociality, and disturbance of attention. The final symptom complex seems to have less obvious relevance to negative symptoms than the other four complexes. Assessments are conducted on a six-point scale (from 0 indicating 'not at all' to 5 indicating 'severe'). Higher scores indicate a worse outcome.

6.2.27 Scale for the Assessment of Positive Symptoms (SAPS) (Andreasen 1984): in OPUS-Denmark 1999

The SAPS measures positive symptoms in schizophrenia. It is intended to serve as a complementary instrument to the Scale for the Assessment of Negative Symptoms (SANS). SAPS is split into four domains: hallucinations, delusions, bizarre behavior, and positive formal thought disorder. Within each domain, separate symptoms are rated from 0 (absent) to 5 (severe). Higher scores indicate a worse outcome.

6.2.28 Social Behaviour Schedule (SBS) (Wykes 1986): in HollowayUK 1996

The SBS is a 21-item scale designed to assess a range of areas of functioning in people with long-term mental illness. The scale covers areas such as social behaviour and communication, self care, and inappropriate behaviour. The respondent's behaviour on each item during the previous month is scored by a person familiar with him or her. Each item is rated on a five-point Likert scale (from 0 to 4), with higher scores indicating greater deficits.

6.2.29 Lancashire Quality of Life Profile (LQoLP) (Oliver 1996; Oliver 1997): in Bjorkman-Sweden 2002, Holloway-UK 1996, UK700-UK 1999

A structured self report interview with 105 items, combining objective and subjective measures in the following nine life domains (range of values 1 to 7): living situation, social relationships, work and education, legal status and safety, religion, family relations, leisure activities, finances, and health. The LQoLP also measures the following additional areas: positive and negative affect (with the Bradburn Affect-Balance Scale), self esteem, global well-being (Cantril's Ladder and Happiness Scale), perceived quality of life, and the quality of life of the patient independent of the patient's own opinion (with the Quality of Life Uniscale). The measures from LQoLP used in Bjorkman-Sweden 2002 were: overall quality of life (which is the mean of subjective quality of life in nine life domains) and global well-being. Higher score indicates better subjective quality of life/satisfaction.

6.2.30 Manchester Short Assessment of Quality of Life (MANSA) (Priebe 1999): REACT-UK 2002, Sytema-Netherlands 1999

A 16-item scale composed of 4 objective and 12 subjective questions. The 12 subjective items are rated on a 7-point scale (from 'couldn't be worse' to 'couldn't be better', scored from 1 to 7 , range 12 to 84 ) assessing satisfaction with life 'in general', and in a range of domains such as vocational, financial, friendships, leisure, personal safety, physical health, and mental health. Four objective items, answered yes or no, assess the existence of a close friend, contacts with friends per week, accusation of a crime, and victimisation of physical violence. Higher score indicates better quality of life. In REACT-UK 2002 and Sytema-Netherlands 1999, score is reported as a mean ranging from 1 to 7 .

6.2.31 Lehman's Quality of Life Interview (QOLI) (Lehman 1988): in Drake-NHamp 1998, Lehman-Maryland1 1994, Shern-USA1 2000; (Lehman 1993) in Ford-UK 1995; (Lehman 1983) in Marshall-UK 1995

The QOLI contains 153 items that measure global life satisfaction as well as objective and subjective quality of life, in eight life domains (living situation, daily activities and functioning, family relations, social relations, finances, work and school, legal and safety issues, and health). The QOLI is rated on a seven-point scale, with higher scores indicating better quality of life. Subjective assessment of general life satisfaction ranges from 1 to 7 (terrible to delighted). Ford-UK 1995 reported objective quality of life, and Marshall-UK 1995 reported subjective quality of life.

6.2.32 Camberwell Assessment of Need interview (CAN) (Phelan 1993): in Bjorkman-Sweden 2002, Harrison-Read-UK 2000, UK700UK 1999

The Camberwell Assessment of Need assesses the health and social needs of people with mental health problems. It measures 22 areas to yield numbers of met and unmet needs as rated by the participant. Possible scores range from 0 to 22, with a higher score indicating poorer level of met needs. 
6.2.33 Camberwell Assessment of Need Short Appraisal Schedule (CANSAS) (Phelan 1995; Slade 1999): in REACT-UK 2002, SytemaNetherlands 1999

This is an abbreviated form of the above CAN.

6.2.34 Client Satisfaction Questionnaire (CSQ) (Larsen 1979): in Audini-UK 1994, Muijen-UK2 1994, OPUS-Denmark 1999, SytemaNetherlands 1999

The CQS is a self report instrument designed to measure patient's global satisfaction with services. Items are concerned with quality of services received, how well services met the client's needs, and general satisfaction. The CSQ is substantially correlated with treatment attrition, number of therapy sessions attended, and change in client-reported symptoms. It consists of eight items that are scored on four-point Likert scales (1 to 4). Total score ranges from 8 to 32. Higher scores indicate greater satisfaction.

6.2.35 Client Satisfaction Questionnaire (CSQ) - modified version (Gerber 1999; Larsen 1979): in REACT-UK 2002

This survey has 35 questions covering the location of services, services clients expect, delays in obtaining services, client's input into treatment, information received about drug treatment, satisfaction with treatment, access to clinical files, satisfaction with the therapist, family involvement in treatment, the treatment process, and overall satisfaction. Possible responses to most items range from 1 (most negative) to 7 (most positive). A rating of zero on certain items enables the respondent to indicate that the question was not relevant to his or her situation. Six items within the questionnaire, also on a seven-point scale, form a general satisfaction questionnaire. Higher score indicates greater satisfaction.

\subsubsection{Patient's satisfaction with health services (Tyrer 1979): in UK700-UK 1999}

A self reporting questionnaire that rates nine components of satisfaction with services, each on a four-point scale (1 to 4). Scores can range from 9 to 36 , with higher values indicating less satisfaction.

\subsubsection{Patient Satisfaction Instrument (PSI) (Risser 1975): in Chan- Hong Kong 2000}

The PSI assesses patient and client satisfaction. It is a 26item questionnaire designed to measure clients' satisfaction with nursing care in the community setting.

6.2.38 Specific Level of Functioning Scale (SLOF) (Schneider 1983): in Chan-Hong Kong 2000

The SLOF assesses clients' behavioural functioning and daily skills. It is a 43-item behavioural rating scale designed for use in clients with chronic mental illness in the community.

\subsection{Missing outcomes}

No trial reported or rated relapse, mental state: not improved, or carer satisfaction.

\section{Excluded studies}

See: Characteristics of excluded studies.
Eighty-one studies were excluded from the previous versions of this review, while a total of 85 studies were excluded from this version.

The earlier update excluded four trials included in the original reviews as the trials did not match the new inclusion criteria. (De Cangas-Canada 1994; Franklin-Texas 1987; Lafave-Canada 1996; Marx-Wisconsin 1973). Morse-Missouri2 1997 was also excluded, as it did not report the number of people randomised to each treatment group.

One further trial (Tyrer-UK 1995), originally included in the Case Management (CM) review, was now excluded because of a methodological issue. Tyrer-UK 1995 is a trial comparing ICM to standard care. The first issue was to clarify the ICM caseload. Thanks to further information provided from the author, we clarified that there were 25 key workers in the service looking after 400 patients on the register, therefore each key worker had a caseload of 1 to 16 , or 'high-intensity' case management by this review's definition. The second issue that arose was that the case managers in the treatment group were also workers in the control group. The problem was therefore one of contamination, as requiring someone to carry out close monitoring of one participant in the treatment group could affect their care of a similar participant in the control group in an unpredictable way. We decided to exclude this study on the grounds that we could not be sure that high-intensity case management was really being compared with standard care.

We also excluded three trials that had been awaiting assessment in the original Case Management and Assertive Case Management reviews (see Other published versions of this review) for varying reasons (Godley-Illinois 1994; Jerrell-California 1989; MulderMissouri 1985) (for more details see Characteristics of excluded studies).

Jerrell-California 1989 was a partially published trial previously classified as 'awaiting further assessment' because we needed information on number of people excluded after randomisation (participants were excluded if they refused to participate after randomisation or if they had not been discharged from hospital within six months of entering the study). As these data have not become available, we have now excluded the trial.

Godley-Illinois 1994 was an unpublished, two-centre trial initially classified as 'awaiting further assessment' because we could not determine if the intervention was ACT or CM. During the current update we classified the intervention as ICM versus standard care. We had to exclude this study because it contained no usable data due to incomplete data reporting, and no further information became available (i.e. there was an apparent error in the reporting of numbers admitted to hospital: in one table admission rates were reported as 31/52 experimental group and 33/45 control group, while in another table admission rates were reported as $31 / 52$ experimental and $25 / 45$ control).

Mulder-Missouri 1985 was a report of data from randomised and non-randomised participants. We excluded this study because these data were not reported separately and, in addition, the intervention did not fit our inclusion criteria (ICM was compared to acute hospital admission).

Overall, we excluded 42 studies because they were not randomised or because randomisation was compromised (Jerrell-California 1989). We had to exclude five studies because participants 
required immediate hospital admission (Fenton-Canada 1978; Hoult-Australia 1981; Muijen-UK1 1992; Mulder-Missouri 1985; Stein-Wisconsin), one study because participants were dually diagnosed with intellectual disability and mental illness (MartinUK 2005), and one additional study because the majority of participants were simply homeless and not clearly ill (ToroNew York 1997). Most trials had to be excluded because of the intervention: 54 because the experimental intervention was not ICM. We had to exclude Modcrin-Kansas 1988 as caseload was not reported in either the experimental or the control group. We excluded 48 trials as the comparison intervention was neither standard care nor non-ICM. We excluded 10 trials as the intervention administered to the experimental group was not only ICM (Chandler-California2 2006; COAST-UK 2004; Cosden-California 2005; Gold-SCarolina 2006; Grawe-Norway 2005; Lehman-Maryland2 1993; LEO-UK 1994; McHugo-Washington DC 2004; Shern-USA2; Shern-USA3). We excluded 10 trials as they did not present with relevant comparisons. Finally, we excluded two trials, Godley-Illinois 1994 and Morse-Missouri2 1997, because we could extract no usable data from the study report (as previously explained).

\section{Awaiting classification}

See: Characteristics of studies awaiting classification.

Five trials described as 'awaiting classification' in the previous review have now been excluded as more data have become available (Agius-Croatia 2007; Kane-Virginia 2004; Klotz-California 2001; Li-China 2004; NCT00781079); two more have now been excluded based only on the abstract, and so they were not included in the excluded studies section (Huang-China and Johnson-UK).

Twenty trials, of which three are in the Chinese language, are awaiting classification, and their authors have been contacted for further clarification.

\section{Ongoing studies}

See: Characteristics of ongoing studies.

We are aware of six currently ongoing trials, five more than the ongoing studies in the previous version of this review (WalshConnecticut).

\section{Risk of bias in included studies}

For multicentre trials that provided data for individual single centres, we did not assess the risk of bias for each centre. Our judgements regarding the overall risk of bias in individual studies are illustrated in Figure 1.

\section{Allocation}

All 40 studies were stated to be randomised, but only 11 provided descriptions of the methods used to generate the sequence. We therefore classified these studies as at low risk of selection bias. The most common method of randomisation was random allocation according to a sequence of random numbers generated by a computer program in one of two sites (Bjorkman-Sweden 2002; Essock-Connecticut2 2006; Ford-UK 1995; Harrison-Read-UK 2000; OPUS-Denmark 1999), while Cusack-North Carolina employed randomisation using a random number table, assigned in blocks of two. Three trials used permuted block (Marshall-UK 1995; REACTUK 2002; Sytema-Netherlands 1999), one used a table of random permutation (Pique-California 1999), and one used coin tossing (Rosenheck-USA 1993). In one of the two sites of the OPUSDenmark 1999 trial (Aahrus site), allocation was performed by drawing lots - from among five red and five white lots from a black box. Overall, however, most studies, including Chan-Hong Kong 2000 , were classified as at unclear risk of selection bias with an overestimate of positive effect, as no description of the methods used to generate the sequence was provided.

Regarding the allocation concealment, we rated only four studies as low risk of bias as they provided descriptions of the methods used to conceal random allocation (OPUS-Denmark 1999; REACT-UK 2002; Sytema-Netherlands 1999; UK700-UK 1999). All four studies used centralised allocation carried on by telephone, fax, or mail. We classified the remaining 33 studies as at unclear risk of selection bias with an overestimate of positive effect.

\section{Blinding}

We classified blinding with respect to only primary outcomes. Due to intervention characteristics, that is being a model of service organisation, we assumed participants and clinicians implicitly not being blind to treatment assignment. We also assumed that primary outcomes were likely to be influenced by participant and clinician lack of blinding, as the knowledge of treatment allocation could determine both performance and attrition bias at a level that is difficult to predict/quantify. Whereas we did not consider the primary outcomes as interviewer mediated, we assumed that lack of interviewer blinding would produce less detection bias. We therefore classified all studies providing primary outcome data as at unclear risk of performance and attrition bias. This creates further potential for overestimate of positive effects and underestimate of negative ones.

We have reported blinding to secondary outcomes in the 'Risk of bias' table, but we did not account for it in the global rating of the study blinding risk of bias. Again, if the secondary outcome was clinician/participant mediated, we rated it as unclear. If it was interviewer rated, we assessed it according to information provided in the study. We rated only Shern-USA1 2000 as at high risk of bias, as it provided only secondary outcome data and was only interviewer mediated, and it was therefore possible to assess risk of bias for this study with greater confidence. We rated Chan-Hong Kong 2000 as at unclear risk of performance and detection bias, as it did not clearly report any information on blinding.

\section{Incomplete outcome data}

Where information was available, we assessed incomplete outcome data separately for primary and secondary outcomes and presented both assessments in Figure 1. However, we only rated risk of bias with respect to primary outcome. Only three trials provided separate information for incomplete primary and secondary outcome data, and so we could assess the risk of bias separately (Holloway-UK 1996; Johnston-Australia 1998; REACT-UK 2002). We judged nine trials as adequately addressing incomplete outcome data and rated them as at low risk of attrition bias. We so rated four of these studies because there were no missing outcome data (Bush-Georgia 1990; Holloway-UK 1996; REACT-UK 2002; Sytema-Netherlands 1999); three because they made the number and reason for missing data explicit, and the missing data were balanced across groups (Audini-UK 1994; Essock-Connecticut1 1995; Johnston-Australia 1998); and two because they undertook 
intention-to-treat (ITT) analysis (OPUS-Denmark 1999; UK700-UK 1999).

In Cusack-North Carolina, all analyses were intention to treat and outcomes were observed regardless of active or continued participation. Although Chan-Hong Kong 2000 reported the analyses as ITT, it was not clearly reported whether any participants left early.

We judged Bond-Chicago1 1990 and Ford-UK 1995 as at high risk of attrition bias because, although clearly reporting number and reasons for missing data, the reasons for missing outcome data were likely to be related to true outcome, with imbalance either in numbers or reasons for missing data across intervention group. However, our protocol compensated for this somewhat (see Dealing with missing data), and despite the high rating, information from these studies remains included.

We rated the remaining trials as at unclear risk of attrition bias. Either they did not address this issue or presented insufficient information of attrition/exclusions to permit judgement (i.e. no reasons for missing data provided or number randomised not stated. Jerrell-SCarolina1 1991 reported only number of randomised participants completing the study period).

Some specific examples may serve to illustrate the difficulty in rating this issue. Essock-Connecticut2 2006 was not an ITT analysis (seven participants were excluded from the study immediately after randomisation because they were lost to follow-up), but the authors failed to provide information on to what intervention those participants had been allocated and reason for leaving the study early. As this study provided only continuous outcome data, we reproduced completer-only data that were reported. No action was undertaken to deal with other missing data.

Macias-Utah 1994 had three problems. First, the study was not an ITT analysis: seven participants were excluded from the study because they were lost to follow-up. The authors of this study broke with standard practice by failing to provide any data on participants lost to follow-up, in particular data on admissions to hospital. Second, one participant (presumably randomised to the treatment group) was excluded after randomisation, having refused to participate (again, it was unclear whether this person had been admitted to hospital). Third, five further participants were added (randomly) to the treatment and control groups part way through the study, some as late as "late 1990" (final assessments took place in February 1991). No further information has become available since first review publication, which potentially could substantially affect findings.

In Morse-Missouri1 1992, 28 further participants were added (randomly) to the initial randomised sample, to replace participants leaving the study within the first month after entering. As replacement was carried out through randomised assignment, we did not raise any questions on the replacement issue and included the study. We presented data from the final sample, obtained after randomised replacement had occurred.

In Muller-Clemm-Canada 1996, the number randomised was not clearly reported; authors declared that "Clients who withdrew from the study within the first 6 months were replaced by other clients".
Finally, Sytema-Netherlands 1999 randomised 119 participants, but one was excluded because he or she moved to another area directly after randomisation. (We performed ITT analysis on the remaining 118 participants.)

\section{Selective reporting}

We rated most of the trials (24) as at high risk of reporting bias, as their data was presented in such a way that we could not consider it to be free of the suggestion of selective outcome reporting (i.e. prespecified outcomes were not reported, or they were reported incompletely so that they could not be entered in the analysis, or outcomes were reported that were not prespecified). We rated 16 studies as at low risk of reporting bias. We assessed two studies as at unclear risk of reporting bias.

\section{Other potential sources of bias}

Only Hampton-Illinois 1992 was rated as at unclear risk of other potential sources of bias, as it was unclear whether the study was interrupted early in one of the two centres.

We rated all of the remaining trials as at low risk of other potential sources of bias, as we found no evidence of other bias. Most of these studies were publicly funded. No declaration of interest was made by authors, and we assume there was none to be made. However, many study authors were active pioneers in the development and implementation of the experimental intervention model across the scientific community and the clinical world. This raises the issue of how researcher beliefs could affect the entire process of evaluating an intervention in a randomised clinical trial. Although conscious of this issue, we decided not to make any attempt in rating it as it is very difficult to judge, and erroneous quantification could drive bias into our conclusions.

\section{Effects of interventions}

See: Summary of findings for the main comparison Intensive Case Management versus standard care for severe mental illness; Summary of findings 2 Intensive Case Management versus nonIntensive Case Management for severe mental illness

We categorised studies into two comparisons: Intensive Case Management versus standard care, and Intensive Case Management versus non-Intensive Case Management. The nine main indices of outcome were:
1. service use;
2. adverse effects;
3. global state;
4. social functioning;
5. mental state;
6. behaviour;
7. quality of life;
8. satisfaction; and
9. direct costs.

We considered each index in turn for each of the two comparisons. We were able to extract numerical data from 40 randomised trials, among which seven multicentre trials provided data for individual single centre. 


\section{COMPARISON 1: INTENSIVE CASE MANAGEMENT versus STANDARD CARE}

(Summary of findings for the main comparison). There were 45 outcomes in this comparison.

\subsection{Service use: 1. Average number of days in hospital per month - by about 24 months}

Data were available from five studies presenting skewed data from a sample size greater than or equal to 200 participants and from 19 trials reporting skewed data from sample sizes less than 200 . We entered these data in separate subgroups, but we also presented the overall data.

\subsubsection{Skewed data (sample size $\geqq 200$ )}

In the first subgroup analysis (i.e. skewed data from studies with sample size greater than or equal to 200 participants), we found no significant difference in length of hospitalisation per month ( $\mathrm{n}=1812,5$ randomised controlled trials (RCTs), mean difference (MD) -0.46 , confidence interval $(\mathrm{Cl})-0.95$ to 0.03 ), although data suggested a trend favouring ICM $(P=0.06)$. This subgroup had moderate levels of heterogeneity $\left(\mathrm{Chi}^{2}=6.36 ; \mathrm{df}=4.0 ; \mathrm{P}=0.17 ; \mathrm{I}^{2}\right.$ $=37 \%$; Analysis 1.1 .

\subsubsection{Skewed data (sample size $<200$ )}

In the second subgroup analysis (i.e. skewed data from study sample size less than 200 participants), there was a significant difference between groups, favouring the ICM group in reducing length of hospitalisation ( $\mathrm{n}=1783,19 \mathrm{RCTs}$, MD $-1.01,95 \% \mathrm{Cl}-1.74$ to -0.28$)$, but these data were highly heterogeneous $\left(\mathrm{Chi}^{2}=79.27\right.$; $\mathrm{df}=18.0 ; \mathrm{P}=0.0 ; \mathrm{I}^{2}=77 \%$; Analysis 1.1 ).

1.1.3 Overall data (skewed data: sample size $\geqq 200$ and sample size < 200)

When synthesising data from the two subgroups, we found that length of hospitalisation was significantly reduced in the ICM group ( $n=3595,24$ RCTs, MD $-0.86,95 \% \mathrm{Cl}-1.37$ to -0.34 ), but the level of heterogeneity was high $\left(\mathrm{Chi}^{2}=89.43 ; \mathrm{df}=23.0 ; \mathrm{P}=0.0 ; \mathrm{I}^{2}=\right.$ $74 \%$; Figure 3). We investigated the heterogeneity by checking again for correctness of data and removing one outlier study from the analysis (Curtis-New York 1992), as it was the only study favouring standard care. After excluding Curtis-New York 1992, the level of heterogeneity was still high $\left(I^{2}=59 \%\right.$; $\left.P<0.0002\right)$. We therefore removed the second-most outlier study from the analysis (BondIndiana1 (A), one of three centres from a multicentre study), as this was the most extreme result (favouring ICM). By excluding Bond-Indiana1 (A), data remained significant, favouring ICM ( $\mathrm{n}=$ $3245,22 \mathrm{RCTs}, \mathrm{MD}-0.79,95 \% \mathrm{Cl}-1.22$ to -0.36 ). The heterogeneity was reduced to just within our cutoff point $\left(I^{2}=49 \%\right.$; $\left.P=0.005\right)$. Removing two further outliers (Bond-Indiana1 (C) and QuinlivanCalifornia 1995) reduced heterogeneity still further $\left(I^{2}=36 \%\right.$; $P$ $=0.05)$ as well as the overall estimate, but ICM still seemed to significantly decrease time in hospital $(n=3143,20$ RCTs, MD -0.62 , $95 \% \mathrm{Cl}-1.00$ to -0.23 , Figure 4).

Figure 3. Forest plot of comparison: 1 Intensive Case Management versus standard care, outcome: 1.1 Service use: 1. Average number of days in hospital per month - at about 24 months.

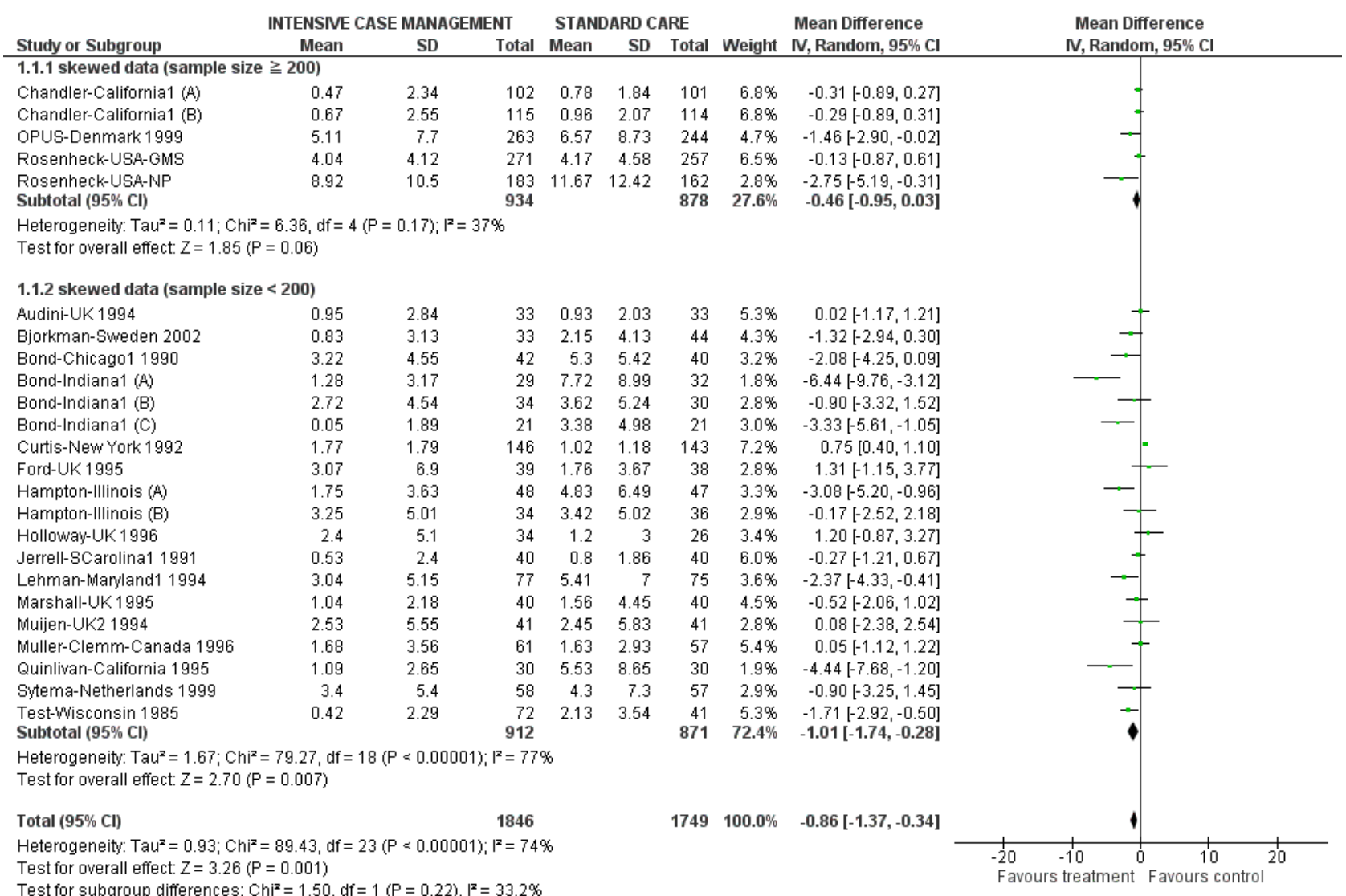


Figure 4. Service use: 1. Average number of days in hospital per month - at about 24 months - restoring homogeneity - 4 studies removed from analysis.

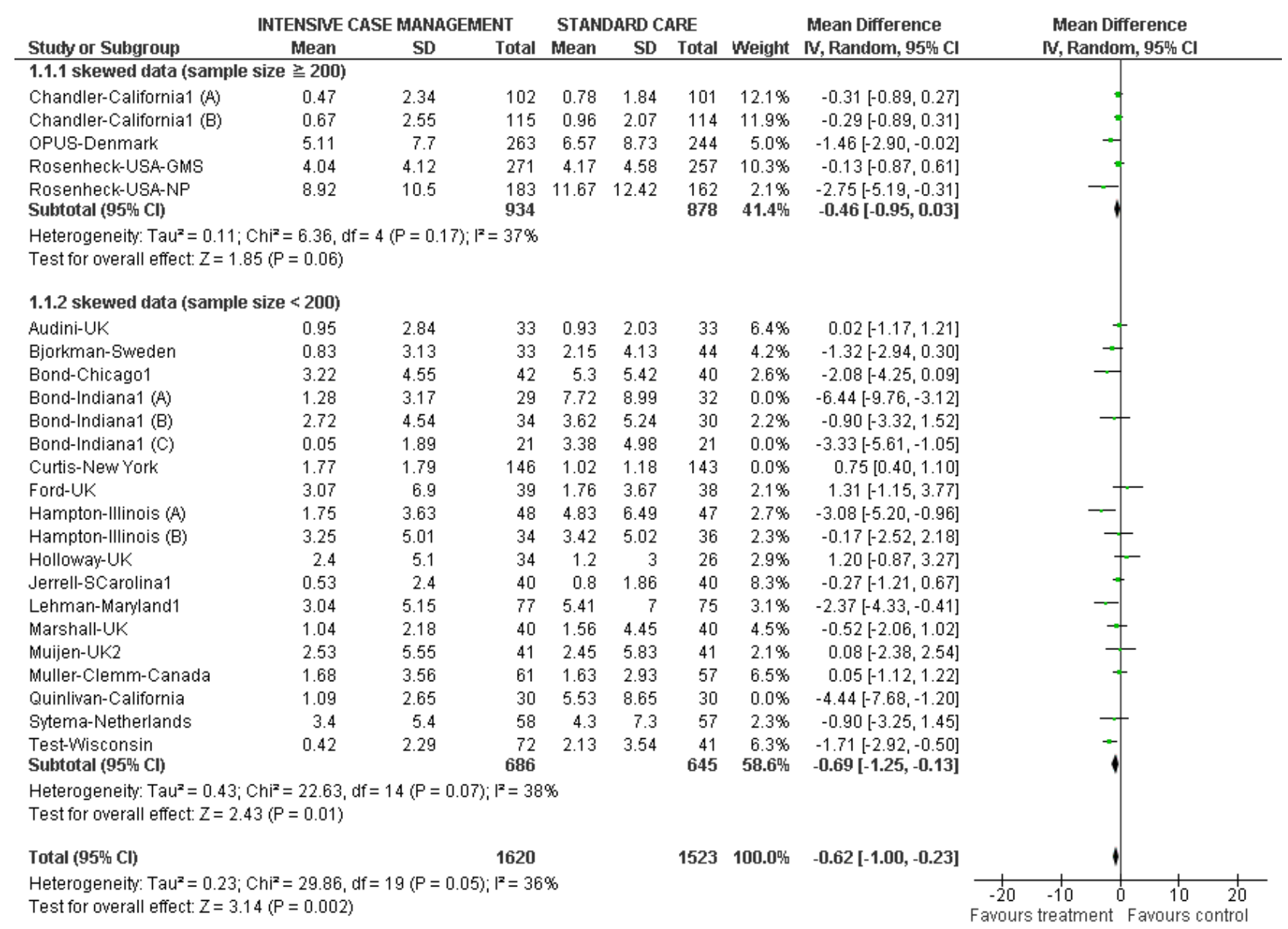

No substantial reporting biases were highlighted when investigated through visual inspection of funnel plot (Figure 5). Two studies -
Bond-Indiana1 (A) and Quinlivan-California 1995 - seemed most heterogeneous (see above). 
Figure 5. Funnel plot of comparison: 1 Intensive Case Management versus standard care, outcome: 1.1 Service use: 1. Average number of days in hospital per month - by about 24 months.

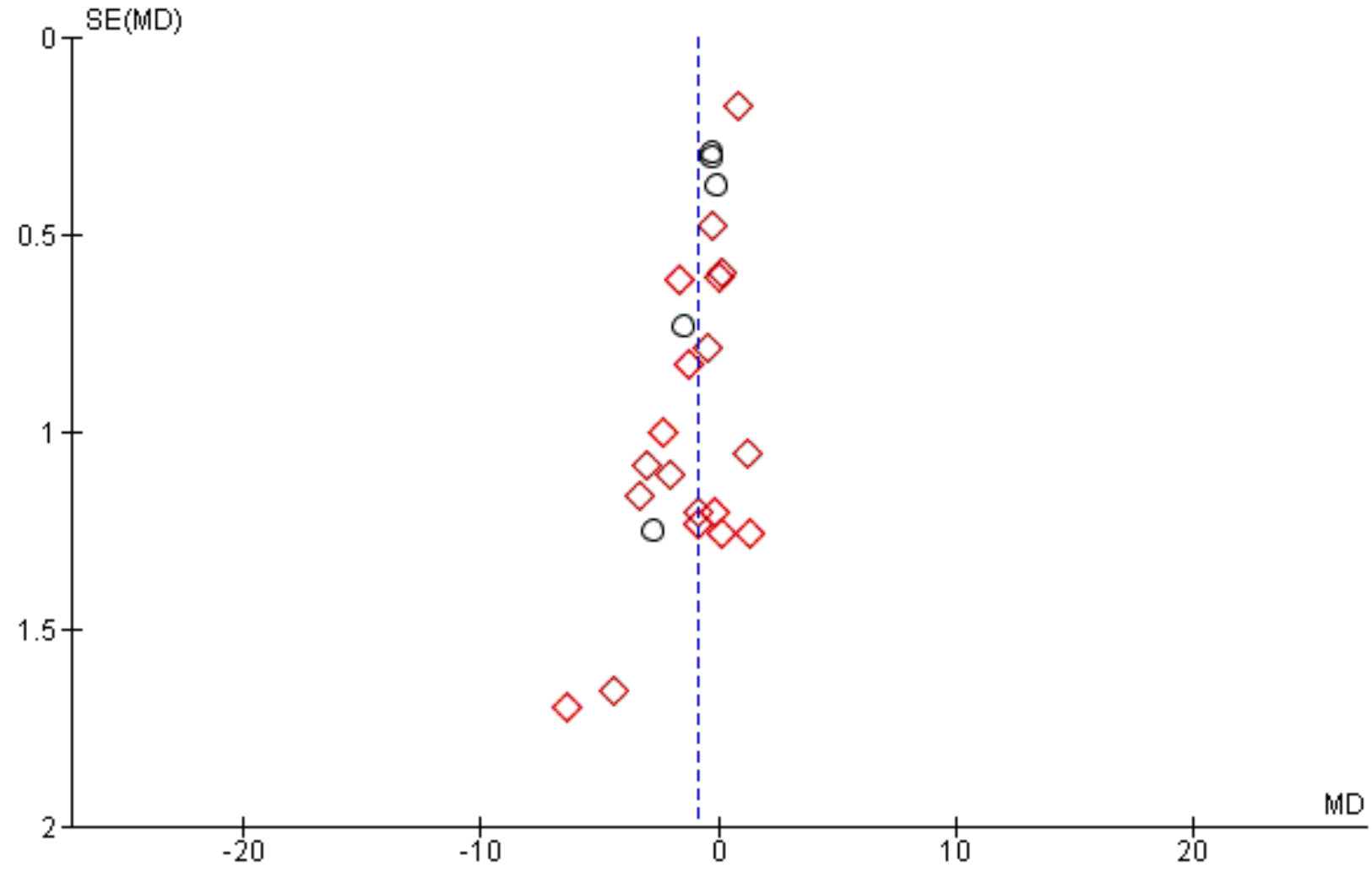

Subgroups

skewed data (sample size $\geqq 200$ )

skewed data (sample size $<200$ )

We ran meta-regression on trials providing data for the primary outcome 'average number of days in hospital per month - at about 24 months' (combining data from all ICM studies within Comparison 1 and 2). Within the meta-regression we found that i.) the more ICM is adherent to the organisation model, the better it is at decreasing time in hospital ('organisation fidelity' variable coefficient $-0.36,95 \% \mathrm{Cl}-0.66$ to -0.07 , Figure 6); and ii.) the higher the baseline hospital use in the population, the better ICM is at decreasing time in hospital ('baseline hospital use' variable coefficient $-0.20,95 \% \mathrm{Cl}-0.32$ to -0.10 , Figure 7 ). Combining both these variables within the model, 'organisation fidelity' is no longer significant (regression coefficient $-0.24,95 \% \mathrm{Cl}-0.52$ to $0.04, \mathrm{P}=$ 0.089 ), but 'baseline hospital use' resultstill significantly influences time in hospital, although it seems to lose some of its potency (regression coefficient $-0.18,95 \% \mathrm{Cl}-0.29$ to $-0.07, \mathrm{P}=0.0027$ ) (Figure 8). Figure 8 shows the interaction of the two variables on study outcome graphically through the use of thin plate spline modelling. The plot provides a locally weighted two-dimensional representation of the collinearity between the variables used in the regression. 
Figure 6. Meta-regression: Scatterplot of IFACT organisation subscore versus mean days per month in hospital.

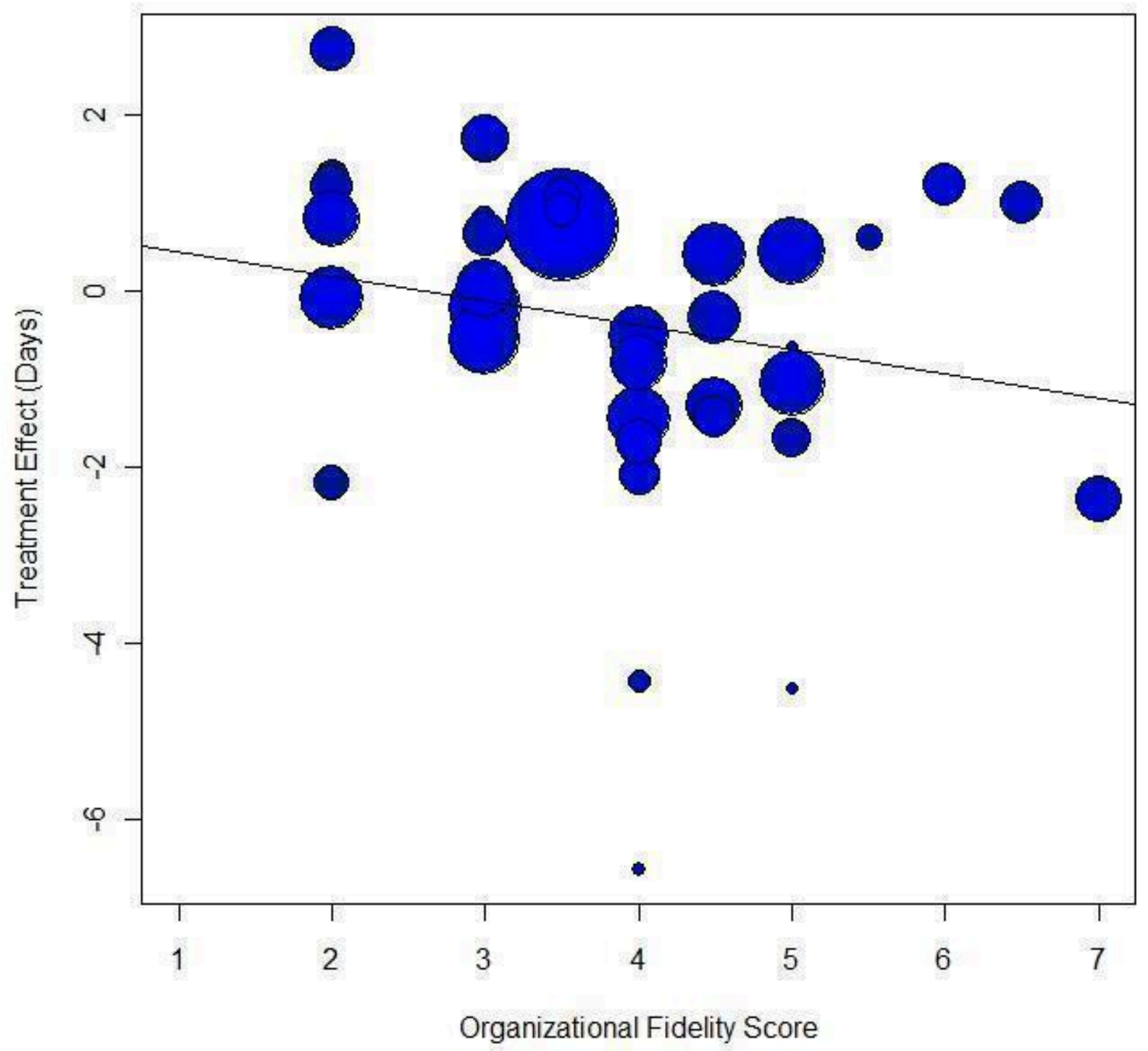


Figure 7. Meta-regression: Scatterplot of mean baseline days in hospital versus mean days per month in hospital.

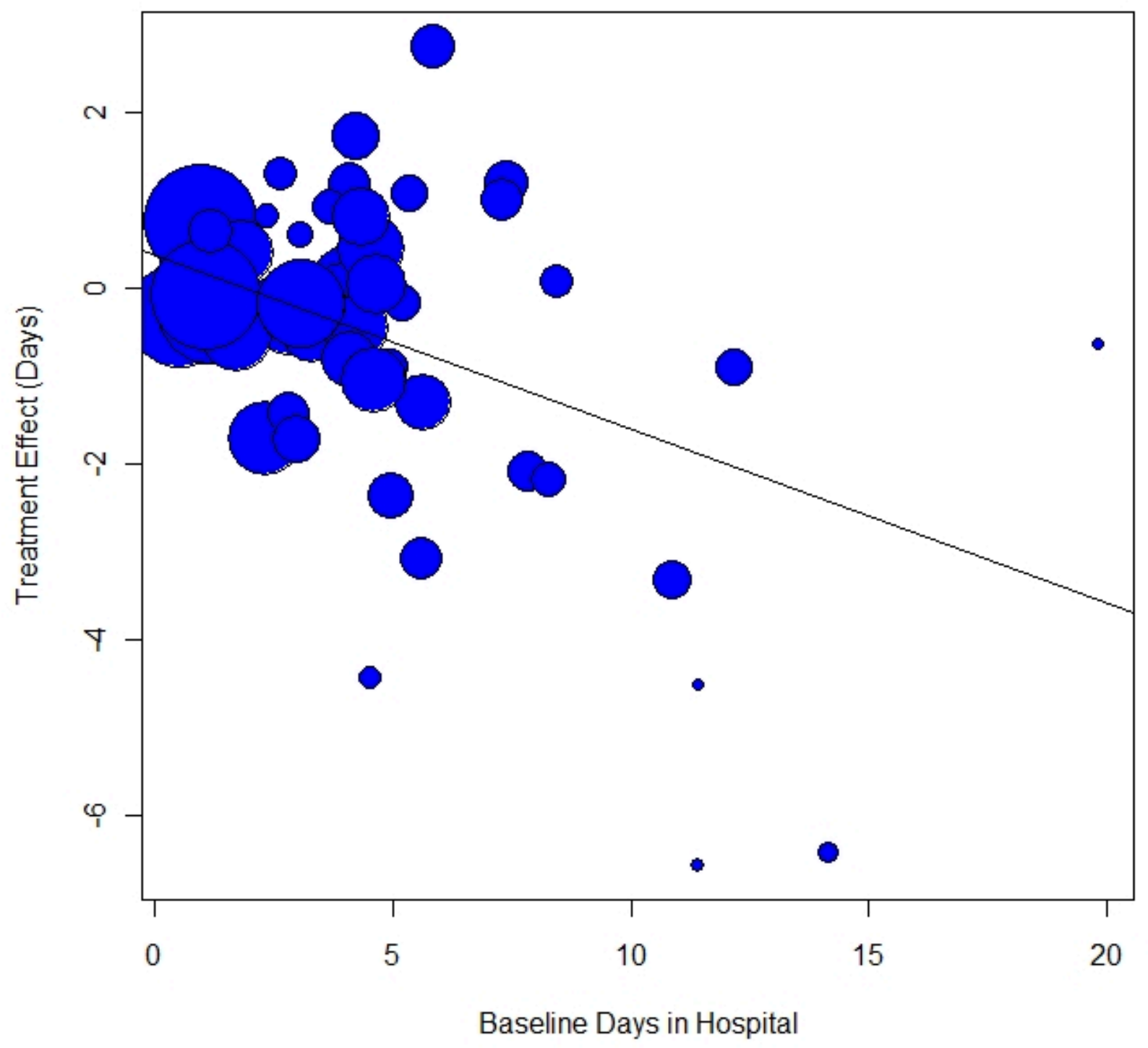


Figure 8. Weighted thin plate spline regression showing combined effect of baseline days in hospital and organisational fidelity score on treatment effect.

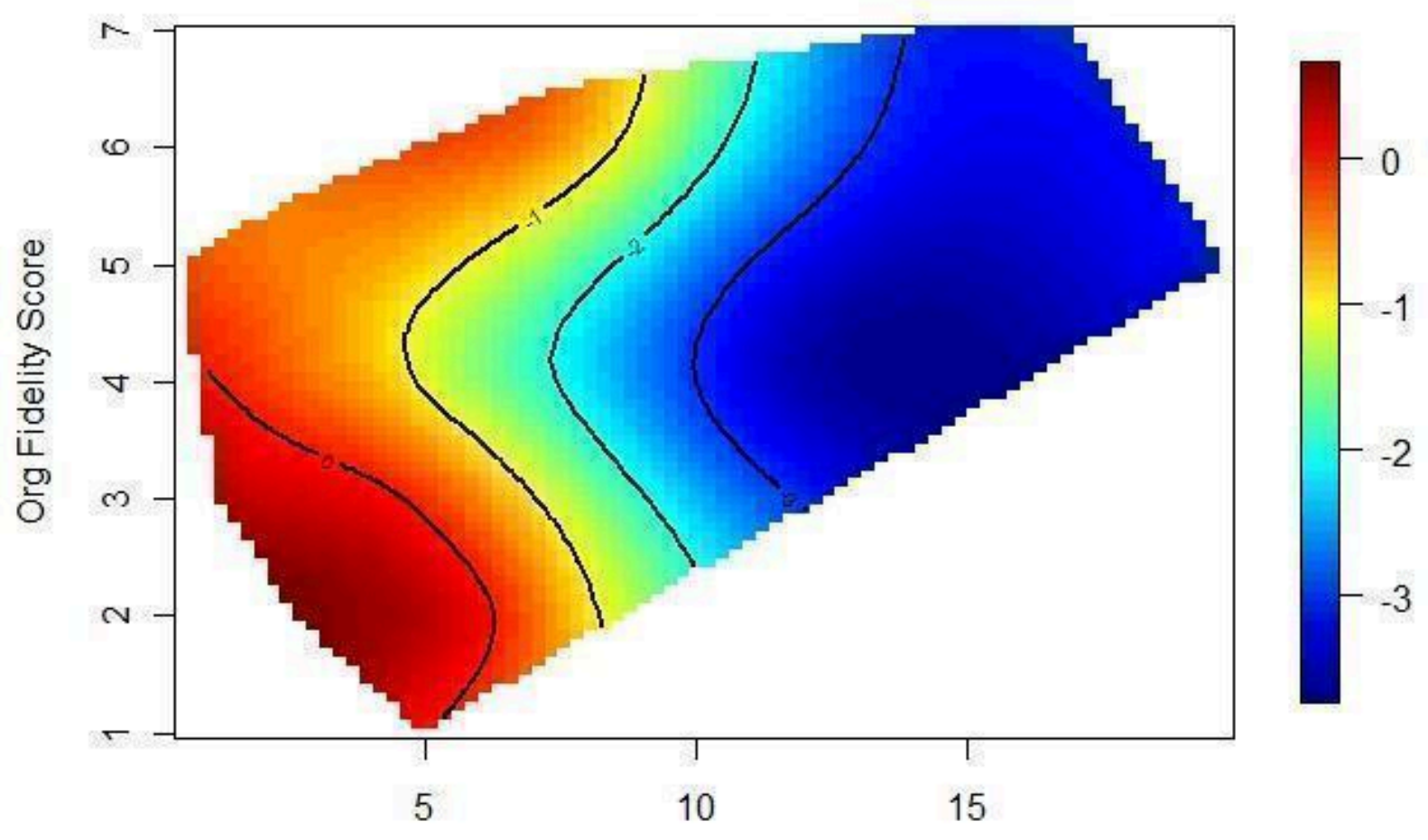

Baseline Days in Hospital

1.2 Service use: 1 Number of days in hospital - by follow-up (skewed data, sample size \# 200)

We identified one study relevant to this outcome, providing data by medium- and long-term follow-up (FUP) and measuring outcome during the previous year.

\subsubsection{By medium-term FUP ( 3 years) (previous year)}

We found one trial to be relevant to this subgroup, with a total of 547 participants. For this subgroup, we did not find evidence of a clear difference between the two treatments (MD 0.1,95\% Cl-10.26 to 10.46; Analysis 1.2).

\subsubsection{By long-term FUP (8 years) (previous year)}

There was a single trial in this subgroup, with a total of 547 participants. There was no clear difference between ICM and standard care within this subgroup (MD 4.3, 95\% Cl -4.63 to 13.23; Analysis 1.2).

\subsection{Service use: 2. Not remaining in contact with psychiatric services}

We found nine relevant studies (total $n=1633$ ) for this outcome, providing data on different follow-up length. Overall, when pooling studies from different time subgroups, we found a significant advantage to the ICM group, where people were less likely to be lost 
to psychiatric services than people in the standard care group (n $=1633,9 \mathrm{RCTs}$, risk ratio (RR) $0.43,95 \% \mathrm{Cl} 0.30$ to 0.61 , Figure 9).
Heterogeneity was moderately high for this outcome $\left(\mathrm{Chi}^{2}=15.57\right.$; $d f=8.0 ; P=0.05 ;\left.\right|^{2}=48 \%$ ).

Figure 9. Forest plot of comparison: 1 Intensive Case Management versus standard care, outcome: 1.2 Service use: 2. Not remaining in contact with psychiatric services by short, medium, long term and overall.

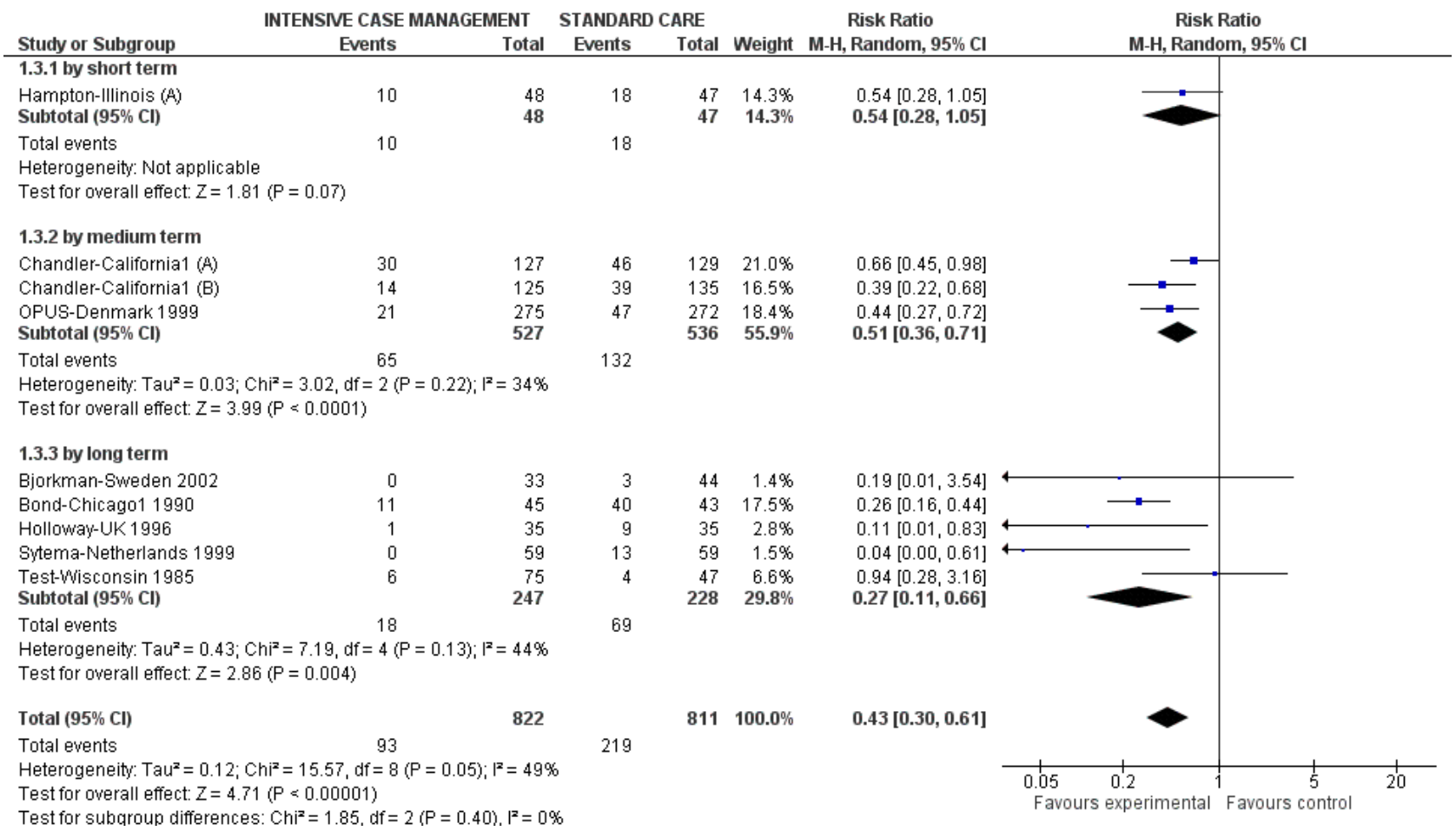

As there were fewer than 10 studies for this outcome, we did not use a funnel plot (see Assessment of reporting biases).

\subsubsection{By short term}

We included only one short-term study and found no significant difference between treatment groups ( $\mathrm{n}=95,1$ RCT, RR 0.54, 95\% $\mathrm{Cl} 0.28$ to 1.05 ; Analysis 1.3).

\subsubsection{By medium term}

Medium-term data available from three studies showed a significant difference between treatment groups, favouring the ICM group, where participants had a lower risk of not remaining in contact with psychiatric services compared with participants in the standard care group ( $\mathrm{n}=1063,3$ RCTs, RR $0.51,95 \% \mathrm{Cl} 0.36$ to 0.71 ). Heterogeneity was moderately high for this subgroup $\left(\mathrm{Chi}^{2}=3.02\right.$; $\mathrm{df}=2.0 ; \mathrm{P}=0.22 ; 1^{2}=33 \%$; Analysis 1.3).

\subsubsection{By long term}

Six long-term studies data confirmed the trend favouring ICM, showing a significant advantage for the ICM group ( $n=653,6$ RCTs, $\mathrm{RR} 0.35,95 \% \mathrm{Cl} 0.18$ to 0.68$)$, but data were heterogeneous $\left(\mathrm{I}^{2}=\right.$ $63 \% ; P=0.02$ ). Herinckx-Oregon 1996 seemed to be the sole cause of this, and on further consideration post hoc, we think we were in error in including the outcome from this study because it was defined so differently from the other trials. Herinckx-Oregon 1996 did not include refusing to re-interview, moving out, and death as all the other studies had done - and it was impossible to amend this at this stage. We therefore feel justified in removing this study altogether from this part of the review outcomes. Once HerinckxOregon 1996 was removed, the five remaining trials confirmed the significant advantage for the ICM group ( $n=475,5$ RCTs, RR 0.27 , $95 \% \mathrm{Cl} 0.11$ to 0.66 ), and heterogeneity was restored to a moderate level $\left(\mathrm{Chi}^{2}=7.19 ; \mathrm{df}=4.0 ; \mathrm{P}=0.13 ; \mathrm{I}^{2}=44 \%\right.$; Analysis 1.3$)$.

\subsection{Service use: 3a. Admitted to hospital}

We identified 16 studies relevant to this outcome and categorised data into five subgroups (in keeping with our protocol).

\subsubsection{By short term}

Data were available from two short-term studies and showed no significant differences between treatment groups ( $n=244,2$ RCTs, RR $0.61,95 \% \mathrm{Cl} 0.22$ to 1.69 ), but these data were heterogeneous $\left(\mathrm{Chi}^{2}=5.36 ; \mathrm{df}=1.0 ; \mathrm{P}=0.02 ; \mathrm{I}^{2}=81 \% ;\right.$ Analysis 1.4$)$.

\subsubsection{By medium term}

Five studies reported medium-term data, and these favoured the ICM group, which had less admission to hospital across time compared with standard care ( $\mathrm{n}=1303,5 \mathrm{RCTs}$, RR $0.85,95 \% \mathrm{Cl} 0.77$ to 0.93 ; Analysis 1.4).

\subsubsection{By long term}

Eleven studies provided long-term data. As with the short-term data, they showed no significant differences between treatment groups ( $\mathrm{n}=1516,11$ RCTs, RR $0.96,95 \% \mathrm{Cl} 0.74$ to 1.23 ), but data were heterogeneous $\left(\mathrm{Chi}^{2}=32.88 ; \mathrm{df}=10.0 ; \mathrm{P}=0.0 ; \mathrm{I}^{2}=69 \%\right.$; Analysis 1.4). 


\subsubsection{By long term- during previous 12 months}

Only one study reported data by long term, but referring to the number of admissions across the previous year. We therefore could not enter this data in the long-term data subgroup analysis. This data showed a significant effect favouring the ICM group $(n=547$, $1 \mathrm{RCT}, \mathrm{RR} 0.67,95 \% \mathrm{Cl} 0.52$ to 0.86 ), therefore not consistent with long-term data shown above. As these findings were based on data from one study only, we consider them less robust than the findings from the 11 long-term studies Analysis 1.4.

\subsubsection{By short term FUP - unplanned admission through emergency department (ED)}

We found one trial to be relevant to this subgroup (total $n=62$ ). For this subgroup, we did not find evidence of a clear difference between the two treatments ( $\mathrm{RR} 1.0,95 \% \mathrm{Cl} 0.07$ to 15.28 ; Analysis 1.4).

\subsection{Service use: 3b. Average number of admissions per month (skewed data)}

Data describing the average number of admissions per month were available from one medium-term and three long-term studies. All of these data were skewed and did not enter the analysis. Data from the medium-term study suggested a trend favouring the ICM group. Data from the long-term studies showed no trend favouring one group over the other. Audini-UK 1994 and MullerClemm-Canada 1996 did not report variance measurements. We assumed consistency between studies and used the fully reported variance for Sytema-Netherlands 1999, and employed these data for Audini-UK 1994 and Muller-Clemm-Canada 1996 as well.

\subsection{Service use: 4a. Admitted to ER - by long term}

The only study identified describing 'number admitted to ER' showed a non-significant difference between the two groups ( $\mathrm{n}=$ $178,1$ RCT, RR $1.13,95 \% \mathrm{Cl} 0.72$ to 1.76$)$.

\subsection{Service use: $4 b$. Average number of admissions to ER (skewed data) - by medium term}

The two studies describing the average number of admissions to ER reported skewed data. Skewed data were not consistent, as one study did not show any trend in the direction of effect, and the other study showed a trend favouring the ICM group. As in one study the variance measurement was not reported (Jerrell-SCarolina1 1991), we carried the standard deviation over from the other available study (Lehman-Maryland1 1994).

\subsection{Service use: 5a. Received day hospital care - by short-term FUP}

We identified only one study relevant to this outcome (total $n=62$ ). There was not a significant difference between ICM and standard care (RR 2.0, 95\% Cl 0.19 to 20.93; Analysis 1.8).

\subsection{Service use: 5 b. Outpatient visits - by short-term FUP (6 months)}

We identified only one study relevant to this outcome. There was not a significant difference between ICM and standard care $(n=62$, $1 \mathrm{RCT}, \mathrm{MD} 0.29,95 \% \mathrm{Cl}-0.14$ to 0.72 ; Analysis 1.9 ).
1.10 Service use: 5c. Outpatient visits - by medium term (skewed data)

One more small study provided data for this outcome $(n=134)$, but as data were skewed and the total sample less than 200 , we entered them as 'Other' data. Data showed a trend favouring ICM, where the ICM group received more outpatient visits than the standard care group by medium term (Analysis 1.10).

\subsection{Service use: $5 d$. Received home visits - by short-term FUP}

We found a single study showing a significant difference between ICM and standard care in the mean number of home visits received, favouring the ICM group ( $\mathrm{n}=62,1 \mathrm{RCT}$, MD $4.32,95 \% \mathrm{Cl} 3.42$ to 5.22). Note that for this outcome the right graph label favours ICM (experimental group).

\subsection{Adverse event: 1a. Death - any cause}

We found 14 relevant studies for this outcome, the data from which we divided into five subgroups according to different time to followup. We found similar results in mortality across different subgroups, none of which showed a significant difference between ICM and standard carefor overall mortality.

\subsubsection{By short term}

We found two trials relevant to this subgroup, with a total of 161 participants. For this subgroup, two deaths occurred in the 81 people treated with ICM compared with two deaths in the 80 people treated with standard care ( $\mathrm{n}=161,2 \mathrm{RCTs}$, RR $1.04,95 \% \mathrm{Cl} 0.16$ to 6.91; Analysis 1.12).

\subsubsection{By medium term}

There were six relevant trials in this subgroup (total $n=901$ ). For this subgroup, five deaths occurred in 453 people treated with ICM compared with six deaths in 448 people treated with standard care ( $\mathrm{n}=901,6 \mathrm{RCTs}$, RR $0.78,95 \% \mathrm{Cl} 0.23$ to 2.62 ; Analysis 1.12 ).

\subsubsection{By long term}

We found nine trials relevant to this subgroup, with a total of 1456 participants. For this subgroup, 24 deaths occurred in 741 people treated with ICM compared with 27 deaths in 715 people treated with standard care $(\mathrm{n}=1456,9 \mathrm{RCTs}$, RR $0.84,95 \% \mathrm{Cl} 0.48$ to 1.47 ; Analysis 1.12).

\subsubsection{By medium-term FUP ( 3 years)}

There was a single trial in this subgroup, in which six deaths occurred in 275 people treated with ICM compared with 10 deaths in 272 people treated with standard care, showing no significant difference between ICM and standard care $(n=547,1$ RCT, RR 0.59, $95 \% \mathrm{Cl} 0.22$ to 1.61 ; Analysis 1.12).

\subsubsection{By long-term FUP (8 years)}

There was a single trial in this subgroup, in which 14 deaths occurred in 275 people treated with ICM compared with 15 deaths in 272 people treated with standard care, showing no significant difference between ICM and standard care $(n=547,1$ RCT, RR 0.92, $95 \% \mathrm{Cl} 0.45$ to 1.88; Analysis 1.12).

\subsection{Adverse event: $1 b$. Death - suicide}

We found 12 relevant studies for this outcome and categorised data into four subgroups. Our results for mortality due to suicide were 
similar to those found for mortality due to all causes, that is no significant difference in suicide rate between the two intervention groups.

\subsubsection{By short term}

Data by short term were available from two studies, where no suicides occurred in 62 people treated with ICM compared with two suicides in 65 people treated with standard care $(n=127,2$ RCTs, RR $0.35,95 \% \mathrm{Cl} 0.04$ to 3.27 ; Analysis 1.13).

\subsubsection{By medium term}

Data by medium term were available from four studies, where two suicides occurred in 412 people treated with ICM compared with two suicides in 407 people treated with standard care $(n=819,4$ RCTs, RR 0.98, 95\% Cl 0.17 to 5.60; Analysis 1.13).

\subsubsection{By long term}

Data by long term were available from nine studies, where 10 suicides occurred in 741 people treated with ICM compared with 14 suicides in 715 people treated with standard care $(n=1456,9$ RCTs, RR $0.68,95 \% \mathrm{Cl} 0.31$ to 1.51 ; Analysis 1.13 ).

\subsubsection{By medium-term FUP ( 3 years)}

Data by medium-term follow-up (3 years) were available from one study, where three suicides occurred in 275 people treated with ICM compared with four suicides in 272 people treated with standard care ( $n=547,1$ RCT, RR $0.74,95 \% \mathrm{Cl} 0.17$ to 3.28 ; Analysis 1.13 ).

\subsection{Global state: 1. Leaving the study early}

We identified 21 studies relevant to this outcome and categorised data into five subgroups (in keeping with our protocol).

\subsubsection{By short term}

We included five short-term studies and found no significant differences between treatment groups for number of participants leaving the study early ( $\mathrm{n}=598,5 \mathrm{RCTs}$, RR $0.79,95 \% \mathrm{Cl} 0.44$ to 1.41 ), but data were heterogeneous $\left(\mathrm{Chi}^{2}=80.24 ; \mathrm{df}=4.0 ; \mathrm{P}=0.0 ; 12=95 \%\right.$; Analysis 1.14).

\subsubsection{By medium term}

We included eight medium-term studies and found the risk of leaving the study early was lower for participants in the ICM group ( $n=1699$, 8 RCTs, RR 0.60, 95\% Cl 0.51 to 0.70 ; Analysis 1.14).

\subsubsection{By long term}

Data from 13 long-term studies confirmed data from medium-term studies, showing a significant advantage for ICM ( $n=1798,13$ RCTs, RR 0.68, 95\% Cl 0.58 to 0.79; Analysis 1.14).

\subsubsection{By medium-term FUP ( 3 years)}

There was a single trial in this subgroup, showing no significant difference between ICM and standard care ( $n=547,1$ RCT, RR 1.01, $95 \% \mathrm{Cl} 0.84$ to 1.21 ; Analysis 1.14).

\subsubsection{By long-term FUP (8 years)}

We found one trial relevant to this subgroup, showing no significant difference between ICM and standard care $(n=547,1$ RCT, RR 0.88, $95 \% \mathrm{Cl} 0.7$ to 1.09; Analysis 1.14).

\subsection{Global state: 2. Average endpoint score (Global Assessment} of Functioning Scale, high $=$ good)

We identified five studies relevant to this outcome and categorised data into three subgroups. Note that for this outcome the right graph label favours ICM (experimental group).

\subsubsection{By short term}

There were four relevant trials in this subgroup (total $n=797$ ). For this subgroup, the Global Assessment of Functioning Scale (GAF) score favoured ICM (MD 2.07, 95\% $\mathrm{Cl} 0.28$ to 3.86; Analysis 1.15).

\subsubsection{By medium term}

Medium-term GAF data from three studies ( $\mathrm{n}=722$, 3 RCTs, MD $0.09,95 \% \mathrm{Cl}-3.11$ to 3.28 ) showed no significant difference between groups. This subgroup had important levels of heterogeneity (Chi2 $=4.42 ; \mathrm{df}=2.0 ; \mathrm{P}=0.11 ; \mathrm{I}^{2}=54 \%$; Analysis 1.15 ).

\subsubsection{By long term}

We found five trials relevant to this subgroup, with a total of 818 participants. For this subgroup, the GAF score favoured the ICM group (MD 3.41, 95\% Cl 1.66 to 5.16; Analysis 1.15).

\subsection{Global state: 3. Not compliant with medication - by long term}

We only found data from one long-term study, which favoured the ICM group ( $\mathrm{n}=71,1 \mathrm{RCT}$, RR $0.35,95 \% \mathrm{CI} 0.15$ to 0.81 ).

\subsection{Social functioning: 1a. Contact with legal system (various measurements)}

We found 11 relevant studies for this outcome and categorised data into six subgroups, according to different time to follow-up and different outcomes described.

\subsubsection{By short term - contact with the police}

We only found data from one study for short-term outcomes, describing the outcome 'contact with the police'. This study did not reveal any significant difference in the rate of contact with the police between treatment groups $(n=61,1$ RCT, RR $2.57,95 \% \mathrm{Cl} 0.73$ to 9.04; Analysis 1.17).

\subsubsection{By medium term - arrested}

We found three studies describing the outcome 'number of arrested'. These studies failed to show a significant difference between the two intervention groups ( $n=604,3$ RCTs, RR 1.08, 95\% $\mathrm{Cl} 0.61$ to 1.90 ; Analysis 1.17).

\subsubsection{By medium term - contact with the police}

Only one medium-term study was available providing data on 'contact with the police'. These data favoured the ICM group in reducing the number of contacts with the police $(n=88,1 R C T, R R$ $0.23,95 \% \mathrm{Cl} 0.09$ to 0.55 ; Analysis 1.17 ).

\subsubsection{By medium term - imprisoned}

We found four medium-term studies describing 'number of imprisoned'. These studies showed no significant advantage for the ICM group ( $\mathrm{n}=804,4 \mathrm{RCTs}$, RR $0.80,95 \% \mathrm{Cl} 0.39$ to 1.64 ). This subgroup had important levels of heterogeneity $\left(\mathrm{Chi}^{2}=6.21 ; \mathrm{df}=\right.$ 3.0; $P=0.1 ;\left.\right|^{2}=51 \%$; Analysis 1.17). 


\subsubsection{By long term - arrested}

We found data from one study for long-term outcomes, describing the outcome 'number of arrested', and it showed no significant advantage for ICM ( $\mathrm{n}=178,1 \mathrm{RCT}$, RR $0.66,95 \% \mathrm{Cl} 0.32$ to 1.37 ; Analysis 1.17).

\subsubsection{By long term - imprisoned}

We found also five long-term studies reporting data on 'number of imprisoned', again not showing any significant advantage for ICM in reducing the number of participants imprisoned by long term $(\mathrm{n}=$ 908, 5 RCTs, RR 0.86, 95\% Cl 0.45 to 1.65; Analysis 1.17).

\subsection{Social functioning: 1 b. Mean contacts with legal system (skewed data) - by medium term}

Data were available from one medium-term trial, describing three different outcomes: bookings, jail days, and convictions. All these data were skewed and did not enter the analysis, therefore we have presented them in Analysis 1.18. Data on booking and jail days suggested a trend favouring the ICM group, reducing contacts with legal system. Data on convictions did not show a trend favouring one group over the other.

\subsection{Social functioning: 2. Employment status (various measurements)}

We found six relevant studies for this outcome and categorised data into six subgroups, according to different time to follow-up and various outcomes described.

\subsubsection{By medium term - not competitively employed at the end of the trial}

One study reported data on 'not competitively employed at the end of the trial', and these data did not show a significant advantage for ICM in improving the number of competitively employed people ( $\mathrm{n}$ $=88,1 \mathrm{RCT}, \mathrm{RR} 1.00,95 \% \mathrm{Cl} 0.91$ to 1.10 ; Analysis 1.19).

\subsubsection{By medium term - not employed at the end of the trial}

We found four trials relevant to this subgroup; these also failed to show a significant difference between groups, although data did suggest a trend favouring ICM ( $\mathrm{n}=1136$, 4 RCTs, RR $0.89,95 \% \mathrm{Cl} 0.79$ to 1.0$)$. However, heterogeneity was present $\left(\mathrm{Chi}^{2}=11.86 ; \mathrm{df}=3.0\right.$; $\mathrm{P}=0.01 ; \mathrm{I}^{2}=74 \%$; Analysis 1.19 ).

\subsubsection{By long term - not employed at the end of the trial}

We found four trials relevant to this subgroup. As in the mediumterm comparison, data failed to show a significant difference, although they suggested a trend favouring the ICM group $(n=1129$, 4 RCTs, RR $0.70,95 \% \mathrm{Cl} 0.49$ to 1.00$)$. However, again, there was considerable heterogeneity $\left(\mathrm{Chi}^{2}=46.48 ; \mathrm{df}=3.0 ; \mathrm{P}=0.0 ; \mathrm{I}^{2}=93 \%\right.$; Analysis 1.19).

\subsubsection{By long term - not working/studying in the previous year}

We found one trial relevant to this subgroup, with a total of 547 participants. Data showed a significant difference between groups, favouring ICM in reducing the risk of being 'not working or not studying' compared to standard care ( $n=547,1$ RCT, RR 0.86, 95\% $\mathrm{Cl} 0.74$ to 0.99$)$. Heterogeneity for this outcome was high $\left(\mathrm{Chi}^{2}=0.0\right.$; $\mathrm{df}=0.0 ; \mathrm{P}=0.0 ; \mathrm{I}^{2}=100 \%$; Analysis 1.19).
1.19.5 By medium-term FUP (3 years) - not working/studying in the previous year

There was a single trial in this subgroup. Data failed to show a signiifcant difference between groups ( $n=547,1$ RCT, RR 1.02, 95\% $\mathrm{Cl} 0.9$ to 1.16 ; Analysis 1.19).

\subsubsection{By long-term FUP (8 years) - not working/studying in the previous year}

We found one trial relevant to this subgroup. Again, we did not find evidence of a clear difference between the two treatments $(n=547$, 1 RCT, RR 0.99, 95\% Cl 0.88 to 1.11; Analysis 1.19).

\subsection{Social functioning: 3a. Accommodation status (various measurements)}

We found 10 relevant studies for this outcome, the data from which we divided into six subgroups according to different time to followup and various outcomes described.

\subsubsection{By short term - homelessness}

We found data on the outcome 'homelessness' from one short-term study. This small study revealed a significant reduction in the rate of homelessness in the ICM group ( $n=95,1$ RCT, RR $0.04,95 \% \mathrm{Cl} 0.00$ to 0.70 ; Analysis 1.20).

\subsubsection{By medium term - homelessness}

Medium-term data on the outcome 'homelessness' were available from one small study. Data did not reveal any significant difference between groups in the rate of homelessness by the medium term ( $\mathrm{n}$ $=88,1 \mathrm{RCT}$, RR $0.32,95 \% \mathrm{Cl} 0.03$ to 2.95 ; Analysis 1.20 ).

\subsubsection{By medium term - not living independently}

We found five trials relevant to this subgroup. These data showed a significant advantage for ICM in reducing the number of participants not living independently ( $n=1303,5$ RCTs, RR 0.80, 95\% $\mathrm{Cl} 0.66$ to 0.97 ). Heterogeneity for this subgroup was moderately high $\left(\mathrm{Chi}^{2}=5.81 ; \mathrm{df}=4.0 ; \mathrm{P}=0.21 ; \mathrm{I}^{2}=31 \%\right.$; Analysis 1.20$)$.

\subsubsection{By long term - homelessness}

We found 'homelessness' data in three long-term studies, which did not reveal any significant difference between intervention groups ( $n=418,3$ RCTs, RR $0.78,95 \% \mathrm{Cl} 0.34$ to 1.82 ). This subgroup had moderate levels of heterogeneity $\left(\mathrm{Chi}^{2}=3.27 ; \mathrm{df}=2.0 ; \mathrm{P}=0.19 ; \mathrm{I}^{2}=\right.$ $38 \%$; Analysis 1.20).

\subsubsection{By long term - not living independently}

There were four relevant trials in this subgroup. Data for this subgroup favoured the ICM group, where the incidence of not living independently was lower compared with the standard care group ( $n=1185,4$ RCTs, RR $0.65,95 \% \mathrm{Cl} 0.49$ to 0.88 ). This subgroup had moderate levels of heterogeneity $\left(\mathrm{Chi}^{2}=5.39 ; \mathrm{df}=3.0 ; \mathrm{P}=0.15 ; \mathrm{I}^{2}=\right.$ 44\%; Analysis 1.20).

\subsubsection{By long term - not living in stable accommodation}

The outcome 'not living in stable accommodation' was only available from one study. We found that data favoured the ICM group in reducing the number of participants not living in stable accommodation ( $\mathrm{n}=168,1 \mathrm{RCT}, \mathrm{RR} 0.80,95 \% \mathrm{Cl} 0.65$ to 0.98 ; Analysis 1.20). 
1.21 Social functioning: $3 b$. Accomodation status: mean number of days in supported house (skewed data, sample size \# 200)

We identified only one study relevant to this outcome, providing data at different times, but always referring to the previous year of follow-up. Note that for this outcome the right graph label favours ICM (experimental group).

\subsubsection{By long term (previous year)}

There was no significant difference between ICM and standard care within this subgroup ( $n=547,1$ RCT, MD $0.3,95 \% \mathrm{Cl}-13.98$ to 14.58 ; Analysis 1.21).

\subsubsection{By medium-term FUP ( 3 years) (previous year)}

We found evidence of a significant difference between ICM and standard care within this subgroup, with data favouring standard care over ICM ( $\mathrm{n}=547,1 \mathrm{RCT}, \mathrm{MD}-22.2,95 \% \mathrm{Cl}-38.47$ to -5.93 ; Analysis 1.21).

\subsubsection{By long-term FUP (8 years) (previous year)}

We did not find evidence of a significant difference between the two treatments for this subgroup ( $\mathrm{n}=547,1 \mathrm{RCT}, \mathrm{MD}-6.7,95 \% \mathrm{Cl}-19.35$ to 5.95; Analysis 1.21).

\subsection{Social functioning: 3c. Accommodation status (various measurements, skewed data)}

Data on this outcome were available from three studies; as all of these data were skewed and did not enter the analysis, we have presented them in Analysis 1.22.

Two studies provided medium-term data on 'average days in stable accommodation', which showed a trend favouring the ICM group, consistent with results previously described for 'not living in stable accommodation' by long term.

Two studies provided long-term data on 'average days per month in sheltered homes'. These data were equivocal, as data from one study favoured ICM, whilst data from the second study favoured the standard care group.

\subsection{Social functioning: $4 a$. Substance abuse}

We identified only one study relevant to this outcome, providing various measures at different time of follow-up.

\subsubsection{Alcohol abuse - by long term}

We found one trial relevant to this subgroup (total $n=547$ ). There was no significant difference between ICM and standard care within this subgroup ( $\mathrm{n}=547,1 \mathrm{RCT}, \mathrm{RR} 0.55,95 \% \mathrm{Cl} 0.26$ to 1.17 ; Analysis 1.23).

\subsubsection{Illicit drug abuse - by long term}

As for alcohol abuse, data failed to show a significant difference between groups for this subgroup ( $\mathrm{n}=547,1 \mathrm{RCT}$, RR $0.96,95 \% \mathrm{Cl}$ 0.63 to 1.47 ; Analysis 1.23).

\subsubsection{Substance abuse - by medium-term FUP ( 3 years)}

We found one trial relevant to this subgroup (total $n=547$ ). As for the two previous outcomes, data failed to show a significant difference between groups ( $\mathrm{RR} 0.91,95 \% \mathrm{Cl} 0.67$ to 1.24 ; Analysis 1.23).

\subsection{Social functioning: $4 b$. Substance abuse (Dartmouth Assessment of Lifestyle Interview (DALI), skewness not detectable) - by medium term}

We were unable to enter two types of data into the analysis: medium-term data assessing alcohol and drug abuse on DALI scale. As the DALI scale averages values from positive to negative, skew is very difficult to detect, we did not enter these data in the analysis. These data tended to favour the standard care group for both the alcohol and drug abuse outcomes; we have presented them in Analysis 1.25.

\subsection{Social functioning: $4 c$. Substance abuse - days used per month (skewed data)}

One study provided medium- and long-term data from the outcome 'days of substance use per month', which were equivocal, but skewed; we have therefore presented them in Analysis 1.25.

\subsection{Social functioning: $5 a$. Average endpoint score (various scales)}

Three studies provided data from five different scales (Disability Assessment Scale (DAS), Interview Schedule for Social Interaction (ISSI), Role Functioning Scale (RFS), Social Adjustment Scale (SASadapted version), Strauss-Carpenter Outcome Scale) assessing social functioning by short, medium, and long term. As no more than one study used the same scale per time period, we did not enter more than one study per subgroup. Data from each available time period failed to show any significant difference between treatment groups, with the exception of two outcomes by long term (1.26.7 on ISSI and 1.26.8 on RFS), the first favouring the standard care group, and the second favouring the ICM group.

\subsubsection{By short term (RFS, low = poor)}

One trial providing data, no significant difference between groups ( $\mathrm{n}=80,1 \mathrm{RCT}, \mathrm{MD}-0.62,95 \% \mathrm{Cl}-2.23$ to 0.99 ; Analysis 1.26 ).

\subsubsection{By short term (SAS-adapted version, low = poor)}

One trial providing data, no significant difference between groups ( $\mathrm{n}=80,1 \mathrm{RCT}, \mathrm{MD}-3.34,95 \% \mathrm{Cl}-7.55$ to 0.87 ; Analysis 1.26 ).

1.26.3 By medium term - social role performance (DAS, high = poor)

One trial providing data, no significant difference between groups ( $n=55,1 \mathrm{RCT}, \mathrm{MD} 0.1,95 \% \mathrm{Cl}-0.4$ to 0.6 ; Analysis 1.26 ).

\subsubsection{By medium term (RFS, low = poor)}

One trial providing data, no significant difference between groups ( $n=80,1$ RCT, MD -0.86, 95\% Cl-2.72 to 1.0; Analysis 1.26).

\subsubsection{By medium term (SAS-adapted version, low = poor)}

One trial providing data, no significant difference between groups ( $\mathrm{n}=80,1 \mathrm{RCT}, \mathrm{MD}-3.3,95 \% \mathrm{Cl}-7.83$ to 1.23 ; Analysis 1.26 ).

\subsubsection{By long term - social role performance (DAS, high = poor)}

One trial providing data, no significant difference between groups ( $\mathrm{n}=58,1 \mathrm{RCT}, \mathrm{MD}-0.2,95 \% \mathrm{Cl}-0.67$ to 0.27 ; Analysis 1.26 ).

\subsubsection{By long term (ISSI, low = poor)}

One trial providing data, showing a significant difference between groups favouring standard careover ICM ( $n=62,1$ RCT, MD 3.2, 95\% Cl 0.11 to 6.29; Analysis 1.26). 


\subsubsection{By long term (RFS, low = poor)}

One trial providing data, showing a significant difference between groups favouring ICM over standard care ( $\mathrm{n}=80,1 \mathrm{RCT}, \mathrm{MD}-2.35$, $95 \% \mathrm{Cl}-4.05$ to -0.65 ; Analysis 1.26$)$.

\subsubsection{By long term (SAS-adapted version, low = poor)}

One trial providing data, no significant difference between groups ( $n=80,1$ RCT, MD -2.75, 95\% Cl -7.13 to 1.63; Analysis 1.26).

\subsubsection{By long term (Strauss-Carpenter Outcome Scale, low = poor)}

One trial providing data, no significant difference between groups ( $n=60,1$ RCT, MD 0.1, 95\% Cl -1.17 to 1.37 ; Analysis 1.26 ).

\subsection{Social functioning: 5 b. Average endpoint score (various scales, skewed data)}

Skewed data on SAS score were available by short, medium, and long term from two studies. These data were equivocal and not consistent between the two studies. One study provided long-term data on REHAB Scale score, which tended to favour the ICM group, but were also skewed. We have presented them in Analysis 1.27.

\subsection{Mental state: 1a. General symptoms - average endpoint score (various scales)}

Two sets of data were available: i.) non-skewed data or skewed data from a sample size greater than or equal to 200 participants per study: entering analysis together; and ii.) skewed data: not entering analysis.

We identified four studies relevant to this outcome entering analysis, providing data from three different scales (Brief Psychiatric Rating Scale (BPRS-18 items), Brief Symptom Inventory (BSI), Colorado Symptom Index (CSI)) per different time period.

\subsubsection{By short term (BPRS-18 items, high = poor)}

We found two trials relevant to this subgroup. There was no significant difference between ICM and standard care within this subgroup ( $\mathrm{n}=668,2 \mathrm{RCTs}, \mathrm{MD}-1.56,95 \% \mathrm{Cl}-6.85$ to 3.73 ), but data were heterogeneous $\left(\mathrm{Chi}^{2}=12.58 ; \mathrm{df}=1.0 ; \mathrm{P}=0.0 ; \mathrm{I}^{2}=92 \%\right.$; Analysis 1.28).

\subsubsection{By short term (BSI, high = poor)}

Short-term mental state scores assessed with the BSI were available from the same two studies providing BPRS short-term data. Again, data were not significantly different $(n=668,2$ RCTs, MD $-0.06,95 \%$ $\mathrm{Cl}-0.19$ to 0.06 ; Analysis 1.28); however, different from the BPRS results, these data were homogeneous.

\subsubsection{By short term (CSI, low = poor)}

One further trial was available providing short-term data on mental state assessed with the CSI. These data showed a significant difference between groups favouring the ICM group $(n=125,1$ RCT, MD - $0.56,95 \% \mathrm{Cl}-0.84$ to -0.28 ; Analysis 1.28 ).

\subsubsection{By medium term (BPRS-18 items, high = poor)}

We found two trials relevant to this subgroup, with a total of 662 people. We did not find evidence of a significant difference between ICM and standard care within this subgroup (MD - $0.96,95 \% \mathrm{Cl}-2.42$ to 0.51 ; Analysis 1.28 ).

\subsubsection{By medium term (BSI, high = poor)}

We found two trials relevant to this subgroup (total $n=662$ ). We did not find evidence of a significant difference between ICM and standard care within this subgroup (MD $-0.02,95 \% \mathrm{Cl}-0.15$ to 0.1 ; Analysis 1.28).

\subsubsection{By medium term (CSI, low = poor)}

There was a single trial in this subgroup. We found evidence favouring the ICM group over the standard care group ( $\mathrm{n}=125,1$ RCT, MD $-0.35,95 \% \mathrm{Cl}-0.65$ to -0.05 ; Analysis 1.28 ).

\subsubsection{By long term (BPRS-18 items, high $=$ poor)}

We found three trials relevant to this subgroup (total $n=777$ ). There was no significant difference between ICM and standard care within this subgroup ( $\mathrm{n}=777,3 \mathrm{RCTs}$, MD $-1.48,95 \% \mathrm{Cl}-3.69$ to 0.74 ), but data were heterogeneous $\left(\mathrm{Chi}^{2}=13.13 ; \mathrm{df}=2.0 ; \mathrm{P}=0.0 ; \mathrm{I}^{2}=84 \%\right.$; Analysis 1.28).

\subsubsection{By long term $(\mathrm{BSI}, \mathrm{high}=$ poor $)$}

We found two trials relevant to this subgroup, with data favouring the ICM group, in which participants reached a better mental state score by long term compared to the standard care group $(n=647,2$ RCTs, MD - $0.18,95 \% \mathrm{Cl}-0.31$ to -0.06 ; Analysis 1.28 ).

\subsection{Mental state: 1 b. General symptoms - mean change from baseline (CSI, low = poor) - by long term}

Finally, one study assessed the mean change from baseline on the CSI, with data showing no difference between groups ( $n=168,1$ $\mathrm{RCT}, \mathrm{MD}-0.32,95 \% \mathrm{Cl}-0.53$ to -0.11$)$.

\subsection{Mental state: 1c. General symptoms - average endpoint score (various scales, skewed data)}

Skewed data were available from six studies assessing mental state by different time periods and with different scales (BPRS-18 items, BPRS-24 items, CPRS, PSE, SCL-90). Data failed to show a significant trend favouring one group over the other, this report being consistent across different studies and different rating scales. We considered these data to not be robust as they were skewed, but they were in accordance with short-, medium-, and long-term data. We have presented them in Analysis 1.30.

\subsection{Mental state: 2a. Specific symptoms - depression at follow- up interview}

We found data on specific symptoms from only one study, which provided data on depression incidence per different time period. There was no significant difference between groups by medium term, long term, and medium-term follow-up (three years).

\subsubsection{By medium term}

One trial providing data, no significant difference between groups ( $\mathrm{n}=547,1 \mathrm{RCT}, \mathrm{RR} 0.77,95 \% \mathrm{Cl} 0.56$ to 1.04 ; Analysis 1.31 ).

\subsubsection{By long term}

One trial providing data, no significant difference between groups ( $\mathrm{n}=547,1$ RCT, RR $0.83,95 \%$ Cl 0.57 to 1.21 ; Analysis 1.31 ).

\subsubsection{By medium term FUP ( 3 years)}

One trial providing data, no significant difference between groups ( $n=547,1$ RCT, RR 1.25, 95\% Cl 0.91 to 1.72; Analysis 1.31). 
1.32 Mental state: 2 b. Specific symptoms - average endpoint score (various scales, skewed data, sample size \# 200)

We identified only one study $(n=547)$ relevant to this outcome, providing data on two different dimensions (positive and negative symptoms) assessed at different times. Although skewed, data entered the analyses, as the sample size was greater than or equal to 200 participants per study. Only one comparison showed a significant advantage for the ICM group, in reducing risk of negative symptoms by long term.

1.32.1 By long term - positive symptoms (Scale for the Assessment of Positive Symptoms (SAPS), high = poor)

One trial providing data, showing no significant difference between groups, although tending to favour the ICM group $(n=547,1 \mathrm{RCT}$, MD - $0.22,95 \% \mathrm{Cl}-0.45$ to 0.01 ; Analysis 1.32 ).

1.32.2 By long term - negative symptoms (Scale for the Assessment of Negative Symptoms (SANS), high = poor)

One trial providing data, showing evidence of a significant difference between groups favouring ICM over standard care ( $\mathrm{n}=$ 547, 1 RCT, MD -0.42, 95\% Cl -0.62 to -0.22; Analysis 1.32 ).

1.32.3 By medium-term FUP (3 years) - positive symptoms (SAPS, high $=$ poor)

One trial providing data, no significant difference between groups ( $n=547,1$ RCT, MD 0.12, 95\% Cl -0.15 to 0.39; Analysis 1.32).

1.32.4 By medium-term FUP (3 years) - negative symptoms (SANS, high = poor)

One trial providing data, no significant difference between groups ( $n=547,1$ RCT, MD -0.1, 95\% Cl -0.33 to 0.13; Analysis 1.32).

\author{
1.32.5 By long-term FUP (8 years) - positive symptoms (SAPS, high = \\ poor)
}

One trial providing data, no significant difference between groups ( $n=547,1$ RCT, MD 0.03, 95\% Cl -0.21 to 0.27 ; Analysis 1.32 ).

\subsubsection{By long-term FUP (8 years) - negative symptoms (SANS, high = poor)}

One trial providing data, no significant difference between groups ( $n=547,1$ RCT, MD 0.06, 95\% Cl -0.13 to 0.25 ; Analysis 1.32 ).

\subsection{Mental state: 2c. Specific symptoms - average endpoint score (various scales, skewed data)}

We found a second small study $(\mathrm{n}=70$ ) providing skewed data on depression symptoms assessed with the Beck Depression Inventory, and negative symptoms assessed with SANS. Neither data set was entered into the analysis. Skewed depression scores favoured the ICM group at medium and long term, whilst skewed negative symptoms scores by medium and long term were equivocal. We have reported these data in Analysis 1.33.

\subsection{Behaviour: 1. Specific behaviour - self harm}

We found three relevant studies for this outcome providing data per different time period. We detected no significant difference between ICM and standard care in either reducing the risk for self harm or reducing the risk for attempting suicide.

\subsubsection{By medium term}

There were two relevant trials in this subgroup, in which 30 events occurred in 312 people treated with ICM compared with 30 events in 308 people treated with standard care. There was no significant difference in number of participants who committed self harm between the groups ( $n=620,2$ RCTs, RR $0.99,95 \% \mathrm{Cl} 0.61$ to 1.59 ; Analysis 1.34).

\subsubsection{By long term}

There is a single relevant trial in this subgroup, in which 2 events occurred in 63 people treated with ICM compared with 2 events in 60 people treated with standard care. There was no significant difference in number of participants who committed self harm between the groups ( $n=123,1$ RCT, RR $0.95,95 \% \mathrm{Cl} 0.14$ to 6.55 ; Analysis 1.34).

\subsubsection{Attempted suicide - by long term (during last 12 months)}

Long term data on self harm during the previous 12 months available from a single study confirmed medium- and long-term data ( $\mathrm{n}=547,1 \mathrm{RCT}$, RR $0.81,95 \% \mathrm{Cl} 0.47$ to 1.38 ; Analysis 1.34), that is failing to show any significant difference between the two groups.

\subsubsection{Attempted suicide - by medium-term FUP (during last 3 years)}

The above data were again confirmed, from medium-term followup data on suicide attempts during the previous three years available from a single study. There was no significant difference between ICM and standard care ( $\mathrm{n}=547,1$ RCT, RR 0.95, 95\% CI 0.56 to 1.62; Analysis 1.34).

\subsection{Behaviour: 2. Social behaviour - average endpoint score (Social Behaviour Scale, high = poor)}

Skewed data were available from one small study $(n=70)$ assessing behaviour with the Social Behaviour Scale by medium and long term. These data tended to favour the ICM group.

\subsection{Quality of life: 1a. Average endpoint score (various scales)}

We found seven studies assessing quality of life with various scales by different time periods, and categorised data into five subgroups. Note that for this outcome the right graph label favours ICM (experimental group).

1.36.1 By short term - general well-being (Lehman's Quality of Life Interview (QOLI), high = better)

The only significant result we found was by short term: data were available from a single study and showed a significantly higher quality of life in the ICM group as assessed on the QOLI general wellbeing subscale ( $\mathrm{n}=125,1$ RCT, MD $0.53,95 \% \mathrm{Cl} 0.09$ to 0.97 ; Analysis 1.36).

\subsubsection{By medium term (Lancashire Quality of Life Profile (LQoLP), high $=$ better)}

Medium-term data assessing quality of life with LQoLP (one study) did not show a significant difference between groups ( $n=52,1$ RCT, MD $0.09,95 \% \mathrm{Cl}-0.60$ to 0.78 ; Analysis 1.36 ).

\subsubsection{By medium term (Manchester Short Assessment of Quality of Life (MANSA) - range 1 to 7 , high = better)}

Medium-term data assessing quality of life with MANSA (one study) did not show a significant difference between groups ( $\mathrm{n}=81,1 \mathrm{RCT}$, MD 0.20, $95 \% \mathrm{Cl}-0.29$ to 0.69 ; Analysis 1.36 ). 


\subsubsection{By long term (LQoLP, high = better)}

As with the medium-term data, long-term data assessing quality of life with LQoLP (three studies) did not show a significant difference between groups $(\mathrm{n}=274,3$ RCTs, MD $-0.13,95 \% \mathrm{Cl}-0.38$ to 0.12 ; Analysis 1.36).

\subsubsection{By long term (QOLI, high = better)}

Again, as with the medium-term data, long-term data assessing quality of life with QOLI (two studies) did not show a significant difference between groups ( $\mathrm{n}=132,2$ RCTs, MD 0.09, 95\% Cl -0.24 to 0.42 ; Analysis 1.36).

\subsection{Quality of life: 1 b. Mean change from baseline $(Q O L I$, high $=$ better, skewed data) - by long term}

We found one further study providing data by long term for this comparison, but as data were skewed, measuring mean change from baseline on the QOLI, the study was not entered into the analysis. These data tended to favour the ICM group. We have reported these data in Analysis 1.37.

\subsection{Participant satisfaction: 1a. Average endpoint score (Client Satisfaction Questionnaire (CSQ), high = better)}

We found three relevant studies for this outcome, providing data per different time period. We found that participant satisfaction assessed with the CSQ was significantly greater in the ICM group compared with the standard care group in all three time period assessments. Note that for this outcome the right graph label favours ICM (experimental group).

\subsubsection{By short term}

Short-term data were available from only one small study and showed a significant difference between groups, favouring the ICM intervention ( $\mathrm{n}=61,1 \mathrm{RCT}, \mathrm{MD} 6.2,95 \% \mathrm{Cl} 2.6$ to 9.8; Analysis 1.38).

\subsubsection{By medium term}

Medium-term data from two studies confirmed the above results (n $=500,2$ RCTs, MD 1.93, 95\% Cl 0.86 to 3.01; Analysis 1.38).

\subsubsection{By long term}

Long-term data also favoured the ICM group ( $\mathrm{n}=423,2$ RCTs, MD $3.23,95 \% \mathrm{Cl} 2.31$ to 4.14 ; Analysis 1.38 ).

\subsection{Participant satisfaction: 1 b. Average endpoint score (CSQ, high $=$ better, skewed data) - by short term}

One further small trial provided short-term data, but the data were skewed: attrition in the standard care arm was higher than $50 \%$. Participant satisfaction was assessed with the CSQ, and it tended to favour the ICM group. We have reported these data in Analysis 1.39.

\subsection{Participant need: 1. Average endpoint score (various scales, skewed data)}

We found more skewed data from two studies assessing participant need on two other scales (Camberwell Assessment of Need Interview (CAN), Camberwell Assessment of Need Short Appraisal Schedule (CANSAS)). Medium-term data from one study assessed on CANSAS failed to show any difference between groups. Longterm data assessed in one study with the CAN tended to favour the ICM group. We have reported these data in Analysis 1.40.

\subsection{Costs: 1a. Direct costs of psychiatric hospital care - by} medium term (unit cost = USD, fiscal year 1990)

Direct medium-term costs of psychiatric hospital care were available from two studies reporting skewed data, but with a sample size greater than 200 (Chandler-California1 (A); ChandlerCalifornia1 (B)). Data favoured ICM ( $n=426,2$ RCTs, MD USD -143.74, $95 \% \mathrm{Cl}-272.40$ to -15.08 ; Analysis 1.41).

\subsection{Costs: 1 b. Direct costs of psychiatric hospital care - skewed data}

Five additional studies did describe 'direct costs of psychiatric hospital care', but data were markedly skewed. Some of these data showed a trend favouring ICM, while some favoured standard care, therefore we could not highlight any trend confirming the findings from meta-analysis. We have presented these data in Analysis 1.42

\subsection{Costs: $2 a$. Direct healthcare costs - by long term (unit cost = USD, fiscal year 1988)}

Long-term data for direct healthcare cost were available from two studies; again studies reported skewed data, but with a sample size greater than 200 , and so these data could be entered into a meta-analysis. These data were inconclusive ( $n=873,2$ RCTs, MD USD -529.24, $95 \% \mathrm{Cl}-2143.59$ to 1085.1 ; Analysis 1.43$)$, as they were highly heterogeneous $\left(\mathrm{Chi}^{2}=17.83 ; \mathrm{df}=1.0 ; \mathrm{P}=0.0 ; \mathrm{I}^{2}=94 \%\right)$ with inconsistency in direction of effect.

\subsection{Costs: $2 b$. Direct healthcare costs - skewed data}

Other skewed data from two studies with a sample size of less than 200 could not be entered into the meta-analysis. These studies assessed direct healthcare costs by medium term (one study) and by short-term follow-up (the other study). Medium-term data did not show any significant difference between interventions, whilst short-term FUP data seemed to favour standard care in reducing direct healthcare costs. We have reported these data in Analysis 1.44 .

\subsection{Costs: 3. Direct costs - other data - skewed data}

Five studies described direct costs for "all care" by short, medium, and long term, and one more study described direct costs for "specific" outcome (outpatient care and prison) by medium term. As these data were skewed and from studies with a sample size of less than 200 , they could not be entered into the meta-analysis. We have presented these data in Analysis 1.45.

Costs for all care by short term seemed to favour the ICM group, where costs were reduced (one study); by medium term one study favoured standard care (where costs were reduced), whilst the other study failed to show any difference between the two groups; and five studies provided data by long term: some of these data showed a trend favouring ICM, and some favoured standard care; these data were therefore inconclusive, and we could not highlight any trend.

Medium-term data from one study on cost for outpatient care showed costs were higher in the ICM group compared to standard care. Data from the same study on cost for prison showed costs were higher for the standard care group compared to ICM. 
2. COMPARISON 2: INTENSIVE CASE MANAGEMENT versUS NONINTENSIVE CASE MANAGEMENT

Summary of findings 2 . This comparison has 36 outcomes.

\subsection{Service use: 1. Average number of days in hospital per month} - by about 24 months

We found 21 relevant studies for this outcome and categorised data into two subgroups (skewed data but with a sample size greater than or equal to 200 , and skewed data with a sample size of less than 200). Overall, combining the two pools of studies, we found no clear difference between ICM and non-ICM ( $n=2220,21$ RCTs, MD $-0.08,95 \% \mathrm{Cl}-0.37$ to 0.21 , Figure 10 ). A funnel plot did not show any significant reporting bias (Figure 11).

Figure 10. Forest plot of comparison: 2 Intensive Case Management versus non-Intensive Case Management, outcome: 2.1 Service use: 1. Average number of days in hospital per month - at about 24 months.

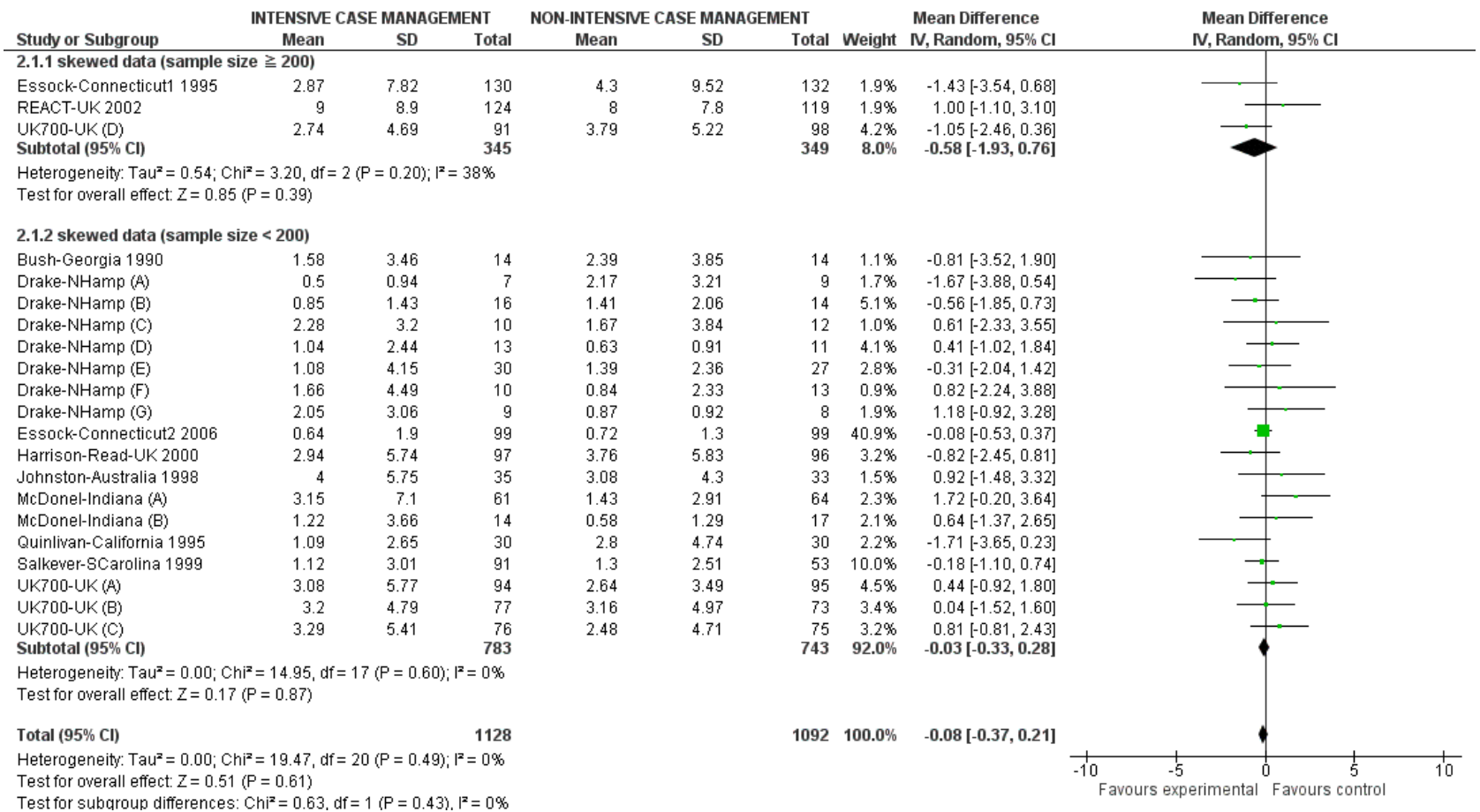


Figure 11. Funnel plot of comparison: 2 Intensive Case Management versus non-Intensive Case Management, outcome: 2.1 Service use: 1. Average number of days in hospital per month - by about 24 months.

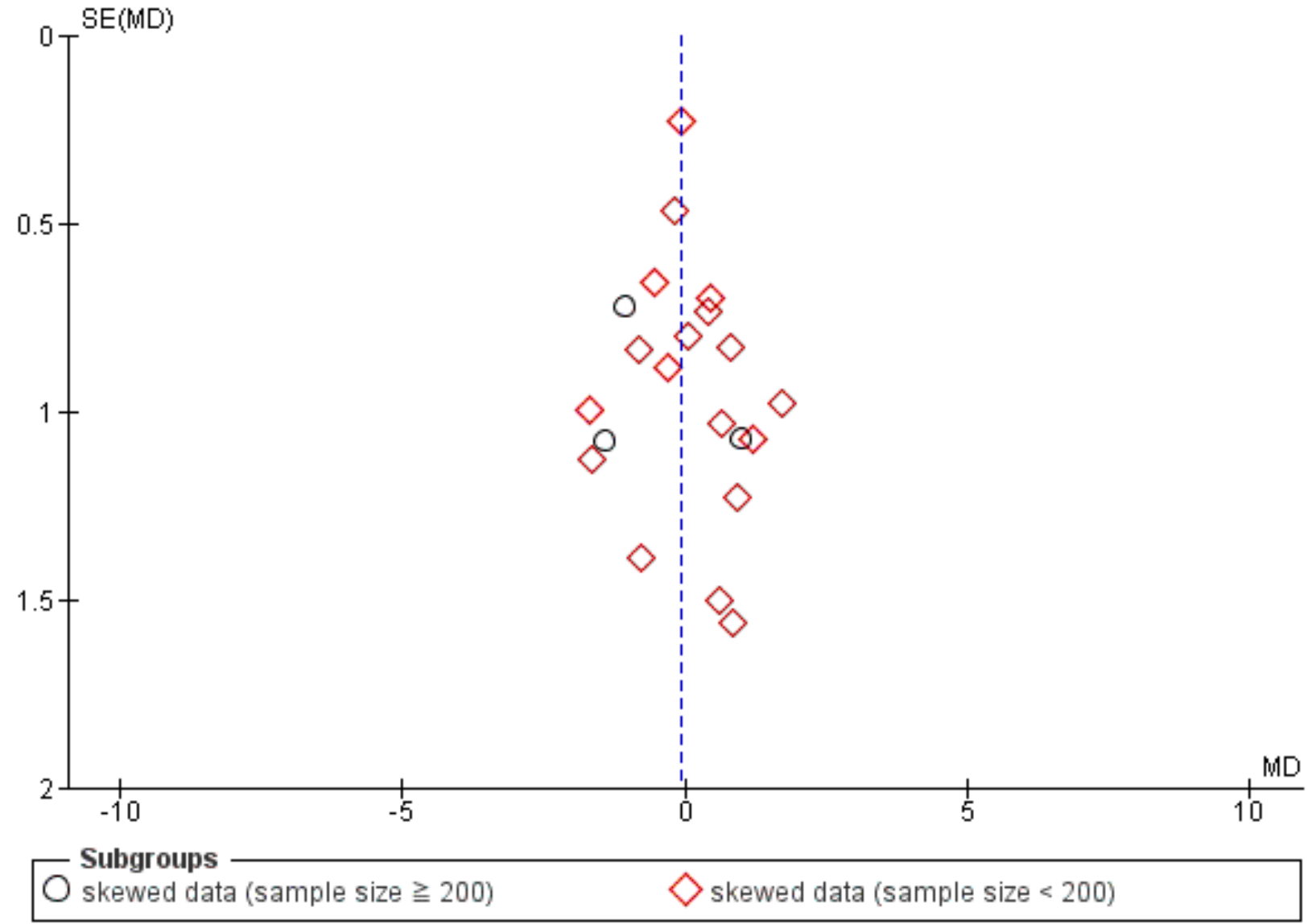

\subsubsection{Skewed data (sample size $\geqq 200$ )}

We found three studies reporting skewed data but with a sample size greater than or equal to 200 . There was no significant difference between groups for reducing length of hospitalisation ( $n=694,3$ RCTs, MD $-0.58,95 \% \mathrm{Cl}-1.93$ to 0.76 ; Analysis 2.1 ). These findings were in accordance with the second subgroup analysis, skewed data from studies with a sample size of less than 200 participants.

\subsubsection{Skewed data (sample size $<200$ )}

There were 18 relevant trials in this subgroup, with a total of 1526 people. Again, these data did not show a significant difference between groups ( $n=1526,18$ RCTs, MD $-0.03,95 \% \mathrm{Cl}-0.33$ to 0.28 ; Analysis 2.1).

2.2 Service use: 1a. Average number of days in hospital per month - by medium/long-term follow-up (skewed data, sample size \# 200)

One study provided data by medium- and long-term follow-up, and as the sample size was greater than 200 , skewed data entered the analysis.

\subsubsection{By medium-term FUP (18 months)}

One study provided data on medium-term follow-up (18 months), confirming the results described above by 24 months. There was no clear difference between ICM and non-ICM within this subgroup ( $\mathrm{n}$ $=237,1$ RCTs, MD 0.6, 95\% Cl-1.25 to 2.45; Analysis 2.2).

\subsubsection{By long-term FUP (8.5 years)}

The same study provided data on long-term follow-up (8.5 years), again showing no significant differences between interventions in reducing number of days in hospital $(n=203,1$ RCTs, MD 0.8, 95\% $\mathrm{Cl}-1.47$ to 3.07; Analysis 2.2).

\subsection{Service use: 2 . Not remaining in contact with psychiatric services}

We found four relevant studies for this outcome and categorised data into three subgroups, by different time period. Short-term data were not available. When we pooled studies from medium and long term, data did not show significant differences between interventions, but heterogeneity was substantial ( $n=1255,4$ RCTs, RR $0.63,95 \% \mathrm{Cl} 0.27$ to $1.49, \mathrm{I}^{2}=81 \%, \mathrm{P}=0.001$ ). We addressed this finding by checking again for correctness of data and explored heterogeneity by dropping each study out of the analysis. Only by removing both Drake-NHamp 1998 and UK700-UK 1999 was homogeneity restored. As we could not ascertain any clear reason for the heterogeneity, we have therefore chosen not to pool these data, as it could be misleading to quote an average value for the intervention effect - particularly in this case, when there is inconsistency in direction of effect. We did not use a funnel plot 
for this outcome because there were fewer than 10 studies (see Assessment of reporting biases).

\subsubsection{By medium term}

One small study provided medium-term data showing a significant difference favouring the ICM group $(\mathrm{n}=73,1 \mathrm{RCT}, \mathrm{RR} 0.27,95 \% \mathrm{Cl}$ 0.08 to 0.87 ; Analysis 2.3 ).

\subsubsection{By long term}

Long-term data were available from three studies. Pooled data were not statistically significant $(n=1182,3$ RCTs, RR $0.82,95 \% \mathrm{Cl}$ 0.34 to 1.98$)$, but data were heterogeneous $\left(\mathrm{Chi}^{2}=10.86 ; \mathrm{df}=2.0 ; \mathrm{P}=\right.$ $0.0 ; 1^{2}=81 \%$ ) with inconsistency in direction of effect (Analysis 2.3).

\subsubsection{By medium-term FUP (18 months)}

One single trial provided data for this outcome, and we found no evidence of a clear difference between the two treatments ( $n=251$, 1 RCT, RR 0.42, 95\% Cl 0.17 to 1.05 ; Analysis 2.3).

\subsection{Service use: 3a. Admitted to hospital - by long term}

Binary data describing this outcome were available from three long-term studies, reporting 'number of admitted to hospital'. These data showed a non-significant difference in number of participants admitted to hospital between groups $(n=1132,3$ RCTs, RR $0.91,95 \% \mathrm{Cl} 0.75$ to 1.12 ; Analysis 2.4 ). Heterogeneity was high for this outcome $\left(\mathrm{Chi}^{2}=5.26 ; \mathrm{df}=2.0 ; \mathrm{P}=0.07 ; \mathrm{I}^{2}=62 \%\right)$.

\subsection{Service use: $3 b$. Average number of admissions (skewed data - sample size \# 200)}

We identified two studies relevant to this outcome, one providing data by long term, the other by medium- and long-term follow-up. Data were skewed, but as the trial sample size were greater than or equal to 200 , we entered these data in the analysis. Data from different time periods failed to show any significant differences between ICM and non-ICM in average number of admissions.

\subsubsection{By long term ( 24 months)}

Data from one long-term study failed to show any significant differences between groups $(\mathrm{n}=678,1 \mathrm{RCT}$, MD $-0.18,95 \% \mathrm{Cl}-0.41$ to 0.05 ; Analysis 2.5).

\subsubsection{By medium term FUP (18 months)}

One single study provided data by medium-term follow-up (18 months). Data failed to show any significant differences between groups ( $n=237,1$ RCT, MD $-0.1,95 \% \mathrm{Cl}-0.6$ to 0.4 ; Analysis 2.5 ).

\subsubsection{By long-term FUP (8.5 years)}

One single study provided data by medium-term follow-up ( 8.5 years). Data failed to show any significant differences between groups ( $n=203,1 \mathrm{RCT}, \mathrm{MD} 1.0,95 \% \mathrm{Cl}-0.25$ to 2.25 ; Analysis 2.5 ).

\subsection{Service use: 3c. Average number of admissions (skewed data) - by medium term}

A trial that included fewer than 200 participants presented skewed data that could not be entered into the meta-analysis. Data were reported for the outcome 'average number of admissions' by medium term. As with previous findings, they failed to show any trend in effect between groups.

\subsection{Adverse event: 1a. Death - any cause}

We found seven relevant studies for this outcome and categorised data into five subgroups, by different time period.

\subsubsection{By short term}

Short-term data on mortality were available from one study $(\mathrm{n}=$ 193) reporting no deaths, therefore a measure of effect was not estimable (Analysis 2.7).

\subsubsection{By medium term}

Medium-term data were available from three studies, where 1 death occurred in 148 people treated with ICM, compared with no deaths in 146 people treated with non-ICM. There were no significant differences in mortality between groups ( $n=294,3$ RCTs, RR 2.92, $95 \% \mathrm{Cl} 0.12$ to 69.43 ; Analysis 2.7).

\subsubsection{By long term}

We found long-term data in five studies, reporting 16 deaths occurring in 816 people treated with ICM, compared with 18 deaths in 821 people treated with non-ICM. These data confirmed mediumterm findings showing no differences in mortality between groups ( $n=1637,5$ RCTs, RR 0.90, 95\% Cl 0.46 to 1.75 ; Analysis 2.7 ).

\subsubsection{By medium-term FUP (18 months)}

Medium-term follow-up data were available from one study, where 6 deaths occurred in 127 people treated with ICM, compared with 6 deaths in 124 people treated with non-ICM. These data confirmed the above results, showing no differences in mortality between groups ( $n=251,1$ RCT, RR 0.98, 95\% Cl 0.32 to 2.95; Analysis 2.7).

\subsubsection{By long-term FUP (8.5 years)}

Long-term follow-up data were available from one study, where 20 deaths occurred in 127 people treated with ICM, compared with 17 deaths in 124 people treated with non-ICM. These data confirmed the above results, showing no differences in mortality between groups ( $n=251,1$ RCT, RR 1.15, 95\% Cl 0.63 to 2.09; Analysis 2.7).

\subsection{Adverse event: 1 b. Death - suicide}

We found eight relevant studies for this outcome and categorised data into four subgroups, by different time period.

\subsubsection{By short term}

Short-term data on suicide mortality were available from one study ( $n=193$ ), again reporting no deaths, and therefore a measure of effect was not estimable.

\subsubsection{By medium term}

Medium-term data were available from six studies, where 5 suicides occurred in 464 people treated with ICM, compared with 3 suicides in 465 people treated with non-ICM. There were no significant differences in the suicide rate between groups ( $n=929,6$ RCTs, RR $1.61,95 \% \mathrm{Cl} 0.26$ to 9.85 ; Analysis 2.8).

\subsubsection{By long term}

Long-term data were available from three studies, reporting 6 suicides occurring in 577 people treated with ICM, compared with 7 suicides in 575 people treated with non-ICM. These data confirmed medium-term data on overall mortality and on suicide, with no 
significant differences in the suicide rate between groups $(\mathrm{n}=1152$, 3 RCTs, RR 0.88, $95 \%$ Cl 0.27 to 2.84; Analysis 2.8).

\subsubsection{By medium-term FUP (18 months)}

Medium-term follow-up data on suicide mortality were available from one study, reporting 1 suicide occurring in 127 people treated with ICM, compared with 3 suicides in 124 people treated with nonICM. These data confirmed also in the follow-up period, mediumand long-term data on overall mortality and on suicide, with no significant differences in the suicide rate between groups $(n=251$, 1 RCT, RR $0.33,95 \%$ Cl 0.03 to 3.09; Analysis 2.8).

\subsection{Global state: 1. Leaving the study early}

We found nine studies providing medium- and long-term data, but not short-term data. When pooling data from sub-groups for two time periods, we found data still significant, showing an advantage for ICM in reducing the number of participants lost to follow-up ( $n=$ 2195, 9 RCTs, RR $0.72,95 \% \mathrm{Cl} 0.52$ to 0.99 ). Although heterogeneity was reduced in comparison to the medium-term subgroup data, heterogeneity was still substantial $\left(\mathrm{Chi}^{2}=19.58 ; \mathrm{df}=8.0 ; \mathrm{P}=0.01\right.$; $\left.\mathrm{I}^{2}=59 \%\right)$ (Analysis 2.9).

\subsubsection{By medium term}

Medium-term data from two trials showed no treatment effect in reducing number of participants lost to follow-up ( $n=225,2$ RCTs, $\mathrm{RR} 0.64,95 \% \mathrm{Cl} 0.13$ to 3.07 ), but these data presented a substantial level of heterogeneity $\left(\mathrm{Chi}^{2}=6.38 ; \mathrm{df}=1.0 ; \mathrm{P}=0.01 ; \mathrm{I}^{2}=84 \%\right)$. In addition, there was inconsistency in the direction of effect between the two studies (Analysis 2.9).

\subsubsection{By long term}

Long-term data were available from seven studies, and we found a significant advantage for ICM in reducing the number of participants lost to follow-up ( $\mathrm{n}=1970,7 \mathrm{RCTs}$, RR $0.70,95 \% \mathrm{Cl} 0.52$ to 0.95$)$. Different from the medium-term data, long-term subgroup analyses did not show substantial heterogeneity $\left(\mathrm{Chi}^{2}=9.88 ; \mathrm{df}=\right.$ $6.0 ; P=0.13 ; 1^{2}=39 \%$ ) (Analysis 2.9).

\subsection{Global state: 2a. Average endpoint score (Health of the Nation Outcome Scale (HoNOS), high = poor) - by long term}

One study reported long-term data on global state assessed with HoNOS. These data were skewed, but as the study sample size was greater than or equal to 200 participants they entered the analysis. Data failed to show a significant difference between interventions ( $n=239,1$ RCT, MD $-0.40,95 \% \mathrm{Cl}-1.77$ to 0.97 ; Analysis 2.10 ).

\subsection{Global state: 2 b. Average endpoint score (HoNOS, high = poor) - skewed data}

We found skewed data describing global state with HoNOS by medium and long term from one trial. These data tended to favour the standard care group (Analysis 2.11).

\subsection{Global state: 3a. Not compliant with medication - by medium term}

One study reported medium-term data for the binary outcome 'number of participants not compliant with medication'. There was no significant difference between groups ( $n=73,1$ RCT, RR 1.14, $95 \% \mathrm{Cl} 0.42$ to 3.05 ; Analysis 2.12).
2.13 Global state: $3 b$. Compliance with medication - average endpoint subscale score (Rating of Medication Influences (ROMI)) - by long term

Long-term compliance scores assessed with the ROMI compliance and non-compliance subscales were not significantly different (compliance subscale: $\mathrm{n}=239,1 \mathrm{RCT}$, MD $0.60,95 \% \mathrm{Cl}-0.05$ to 1.25 ; non-compliance subscale: $\mathrm{n}=239,1 \mathrm{RCT}, \mathrm{MD}-0.60,95 \% \mathrm{Cl}-1.63$ to 0.43 ), although both subscale scores tended to favour ICM. Note that for the compliance subscale (high = good), the right side of the graph favours experimental (ICM).

\subsubsection{Compliance subscale $($ high $=$ good $)$}

There was a single trial in this subgroup, with a total of 239 people. There was no clear difference between ICM and non-ICM within this subgroup (MD 0.6, 95\% Cl -0.05 to 1.25; Analysis 2.13).

\subsubsection{Non-compliance subscale (high = poor)}

There was a single trial in this subgroup, with a total of 239 people. There was no clear difference between ICM and non-ICM within this subgroup (MD $-0.6,95 \% \mathrm{Cl}-1.63$ to 0.43 ). This subgroup had important levels of heterogeneity $\left(\mathrm{Chi}^{2}=0.0 ; \mathrm{df}=0.0 ; \mathrm{P}=0.0 ; \mathrm{I}^{2}=\right.$ 100\%) (Analysis 2.13).

\subsection{Global state: 3c. Compliance with medication - average endpoint subscale score (ROMI, score 1 to 3, skewed data)}

Medium- and long-term skewed data were available from one study assessing compliance scores with the ROMI compliance and noncompliance subscales. These data tended to favour standard care, where participants had a higher level of compliance (Analysis 2.14).

\subsection{Social functioning: 1. Contact with legal system (various measurements)}

We identified three studies relevant to this outcome, providing data on different time periods and different measures.

\subsubsection{By medium term - contact with the police}

Medium-term data were available from one study reporting 'contact with the police'. We found no significant difference between groups ( $n=73,1$ RCT, RR 0.32, 95\% Cl 0.04 to 2.97; Analysis 2.15).

\subsubsection{By long term - imprisoned}

Long-term data were available on both binary outcomes of 'imprisoned' and 'arrested'. We found two studies reporting the outcome 'imprisoned', and data failed to show any difference in the number of people imprisoned ( $n=959,2$ RCTs, RR 1.15, 95\% Cl 0.64 to 2.08; Analysis 2.15).

\subsubsection{By long term - arrested}

Only one study provided data on the outcome 'arrested'. Again, there was no clear difference between groups ( $n=251,1$ RCT, RR $0.87,95 \% \mathrm{Cl} 0.53$ to 1.42 ; Analysis 2.15 ).

\subsubsection{By medium-term FUP ( 18 months) - imprisoned}

We found one trial relevant to this subgroup, with a total of 251 participants. There was no clear difference between ICM and nonICM within this subgroup ( $\mathrm{n}=251,1 \mathrm{RCT}$, RR $1.07,95 \% \mathrm{Cl} 0.47$ to 2.44; Analysis 2.15). 


\subsubsection{By long-term FUP (8.5 years) - imprisoned}

There was a single trial in this subgroup. There was no clear difference between ICM and non-ICM within this subgroup ( $n=214$, 1 RCT, RR $0.70,95 \%$ Cl 0.43 to 1.14 ; Analysis 2.15).

\subsection{Social functioning 2. Employment status (various measurements)}

We identified two studies relevant to this outcome: one mediumterm study, reporting 'participant who spent more than one day employed' and 'participants on paid employment', and one long-term follow-up study, reporting 'unemployed'. Both mediumterm outcomes showed no significant difference between groups. The long-term follow-up outcome confirmed medium-term data, showing no significant difference between ICM and non-ICM in employment status.

\subsubsection{Spent $>1$ day employed - by medium term}

There was no significant difference between ICM and non-ICM in increasing number of days of employment ( $\mathrm{n}=73,1 \mathrm{RCT}, \mathrm{RR} 1.46$, $95 \% \mathrm{Cl} 0.45$ to 4.74 ; Analysis 2.16).

\subsubsection{On paid employment - by medium term}

There was no significant difference between ICM and non-ICM in increasing the chance of being on paid employment by medium term ( $\mathrm{n}=73,1 \mathrm{RCT}$, RR 0.97, 95\% Cl 0.14 to 6.54; Analysis 2.16).

\subsubsection{Unemployed - by long-term FUP (8.5 years)}

There was no significant difference between ICM and non-ICM in decreasing the risk of unemployment $(n=214,1$ RCT, RR 1.10, 95\% $\mathrm{Cl} 0.91$ to 1.34 ; Analysis 2.16).

\subsection{Social functioning: 3a. Accommodation status (various measurements)}

We found two relevant studies for this outcome, one providing data only for the outcome 'living in supported accomodation' by medium term. The second study provided data for all of the other outcomes, using different measures to assess accomodation status. The data failed to show significant differences between groups at any time period.

\subsubsection{By medium term - living in supported accommodation}

The outcome 'living in supported accommodation' was only available for the medium term from one study. Data failed to show a significant difference between groups ( $n=73,1$ RCT, RR 2.59, 95\% $\mathrm{Cl} 0.75$ to 9.01 ; Analysis 2.17).

\subsubsection{By long term - homelessness}

The outcome 'homelessness' was available for the long term from one study. There were no significant differences between treatment groups in the number of people who were homeless ( $n=251,1$ RCT, RR 0.69, 95\% Cl 0.34 to 1.38; Analysis 2.17).

\subsubsection{By medium-term FUP (18 months) - living independently}

One study provided data and failed to show a significant difference between groups ( $\mathrm{n}=251,1$ RCT, RR $0.98,95 \% \mathrm{Cl} 0.84$ to 1.13 ; Analysis 2.17).
2.17.4 By medium-term FUP (18 months) - living in supported accomodation

One study provided data and failed to show a significant difference between groups ( $\mathrm{n}=251,1 \mathrm{RCT}$, RR $0.83,95 \% \mathrm{Cl} 0.38$ to 1.77 ). This subgroup had important levels of heterogeneity $\left(C^{2} i^{2}=0.0 ; \mathrm{df}=0.0\right.$; $P=0.0 ; I^{2}=100 \%$; Analysis 2.17).

\subsubsection{By medium-term FUP (18 months) - homelessness}

One study provided data and failed to show a significant difference between groups ( $\mathrm{n}=251,1 \mathrm{RCT}$, RR $0.84,95 \% \mathrm{Cl} 0.47$ to 1.49 ). Heterogeneity was high for this outcome $\left(\mathrm{Chi}^{2}=0.0 ; \mathrm{df}=0.0 ; \mathrm{P}=0.0\right.$; $\mathrm{I}^{2}=100 \%$; Analysis 2.17).

\subsubsection{By long-term FUP (8.5 years) - living in supported accomodation}

One study provided data and failed to show a significant difference between groups ( $\mathrm{n}=214,1$ RCT, RR $1.05,95 \% \mathrm{Cl} 0.75$ to 1.48 ; Analysis 2.17).

\subsubsection{By long-term FUP (8.5 years) - homelessness}

One study provided data and failed to show a significant difference between groups ( $\mathrm{n}=214,1$ RCT, RR 0.92, 95\% Cl 0.55 to 1.53; Analysis 2.17).

\subsection{Social functioning: 3 b. Accommodation status - average days per month in stable accommodation}

The continuous outcome 'average days per month in stable accommodation' was available for short, medium, and long term (2 RCTs). Data did not show significant differences between groups at any time period.

\subsubsection{By short term}

One trial was relevant to this subgroup. We did not find evidence of a clear difference between the two treatments ( $n=203,1$ RCT, MD $-0.2,95 \% \mathrm{Cl}-2.48$ to 2.08 ; Analysis 2.18 ).

\subsubsection{By medium term}

One trial was relevant to this subgroup. We did not find evidence of a clear difference between the two treatments ( $n=203,1$ RCT, MD $0.1,95 \% \mathrm{Cl}-2.15$ to 2.35$)$. This subgroup had important levels of heterogeneity $\left(\mathrm{Chi}^{2}=0.0 ; \mathrm{df}=0.0 ; \mathrm{P}=0.0 ; \mathrm{I}^{2}=100 \%\right.$; Analysis 2.18).

\subsubsection{By long term}

We found two trials relevant to this subgroup (total $n=901$ ). We did not find evidence of a clear difference between ICM and non-ICM within this subgroup ( $\mathrm{n}=901,2 \mathrm{RCTs}$, MD $-0.19,95 \% \mathrm{Cl}-1.37$ to 1.0 ; Analysis 2.18).

\subsection{Social functioning: $4 a$. Substance abuse - by long term}

We found two relevant studies for this outcome. Long-term binary data on 'substance abuse' differentiated between 'alcohol abuse' and 'illicit drug abuse'. Data did not show significant differences between groups with any measures.

\subsubsection{Alcohol abuse}

There was no difference between ICM and non-ICM in the number of people abusing alcohol $(n=251,1$ RCT, RR 1.10, 95\% Cl 0.67 to 1.83; Analysis 2.19). 


\subsubsection{Illicit drug abuse}

There was no difference between ICM and non-ICM in the number of people abusing illicit drugs ( $n=251,1$ RCT, RR 1.08, 95\% Cl 0.69 to 1.71; Analysis 2.19).

2.19.3 Alcohol - remission from alcohol use disorder (Alcohol Use Scale (AUS) score <3)

Binary data on 'remission from alcohol use disorder' (defined AUS score $<3$ ) also did not show any significant difference between groups by long term ( $\mathrm{n}=223,1 \mathrm{RCT}$, RR $0.86,95 \% \mathrm{Cl} 0.65$ to 1.14 ; Analysis 2.19).

2.20 Social functioning: $4 b$. Substance abuse - average endpoint score (Substance Abuse Treatment Scale (SATS), low = poor)

Short-, medium-, and long-term continuous data were available from one study, assessing substance abuse with the SATS. These data failed to show a significant difference between groups at any time period assessment.

\subsubsection{By short term}

We did not find evidence of a clear difference between ICM and nonICM within this subgroup $(\mathrm{n}=203,1 \mathrm{RCT}, \mathrm{MD} 0.07,95 \% \mathrm{Cl}-0.28$ to 0.42; Analysis 2.20).

\subsubsection{By medium term}

We did not find evidence of a clear difference between ICM and nonICM within this subgroup $(\mathrm{n}=203,1 \mathrm{RCT}, \mathrm{MD}-0.11,95 \% \mathrm{Cl}-0.55$ to 0.33; Analysis 2.20).

\subsubsection{By long term}

We did not find evidence of a clear difference between ICM and nonICM within this subgroup $(\mathrm{n}=203,1 \mathrm{RCT}, \mathrm{MD} 0.11,95 \% \mathrm{Cl}-0.41$ to $0.63)$. This subgroup had important levels of heterogeneity $\left(\mathrm{Chi}^{2}=\right.$ $0.0 ; \mathrm{df}=0.0 ; \mathrm{P}=0.0 ; \mathrm{I}^{2}=100 \%$; Analysis 2.20).

\subsection{Social functioning: $4 c$. Alcohol - abuse (various measurements, skewed data)}

Skewed data were available from one study assessing 'days using alcohol' and 'AUS score' by short, medium, and long term. Data on 'days using alcohol' showed a trend towards a higher alcohol consumption in the ICM group in each assessed time period. This trend was confirmed by the short- and medium-term findings on the AUS, where the ICM group rating had a worse outcome than the non-ICM group; however, long-term data showed a worse outcome in the non-ICM group (Analysis 2.21).

\subsection{Social functioning: 5a. Average endpoint score (Life Skill Profile (LSP), high = poor) - by long term}

We found the LSP social functioning score did not favour one group over the other by long term ( $n=239,1$ RCT, MD $4.0,95 \% \mathrm{Cl}-0.61$ to 8.61).

\subsection{Social functioning: 5 b. Average endpoint score (Social Functioning Questionnaire (SFQ), high = poor) - skewed data}

Skewed data on SFQ social functioning scores showed equivocal results by medium term, and a worse outcome in the ICM group by long term. We entered these data as 'other' data (Analysis 2.23).

\subsection{Mental state: 1a. General symptoms - average endpoint score (various scales)}

We identified two studies relevant to this outcome, assessing 'mental state: general symptoms' on two different scales (BPRS-24 items and (PRS) at different time periods. We found BPRS mental state scores favoured neither group during the short-, medium-, and long-term analysis. These data were confirmed by long-term findings on the CPRS, where there was no significant difference between ICM and non-ICM.

\subsubsection{By short term (BPRS-24 items, high = poor)}

The single trial in this subgroup showed no difference between the two treatments $(n=203,1$ RCT, MD $-0.65,95 \% \mathrm{Cl}-3.99$ to 2.69; Analysis 2.24).

\subsubsection{By medium term (BPRS-24 items, high = poor)}

The single trial in this subgroup showed no difference between the two treatments $(n=203,1$ RCT, MD $-1.62,95 \% \mathrm{Cl}-4.76$ to 1.52 ; Analysis 2.24).

\subsubsection{By long term (BPRS-24 items, high = poor)}

The single trial in this subgroup showed no difference between the two treatments $(n=203,1$ RCT, MD $-0.22,95 \% \mathrm{Cl}-3.32$ to 2.88 ; Analysis 2.24).

\subsubsection{By long term (CPRS, high = poor)}

The single trial in this subgroup showed no difference between the two treatments ( $\mathrm{n}=595,1 \mathrm{RCT}, \mathrm{MD} 0.40,95 \% \mathrm{Cl}-1.83$ to 2.63; Analysis 2.24).

\subsection{Mental state: 1 b. General symptoms - average endpoint scores (various scales, skewed data)}

Medium- and long-term skewed data from the Krawiecka Scale and long-term skewed data from the BPRS scale could not be entered into meta-analysis. These data, provided from two different trials, consistently suggested a better mental state outcome in the nonICM group.

\subsection{Mental state: $2 a$. Specific symptoms: negative symptoms - average endpoint score (SANS, high = poor) - by long term}

Long-term continuous data on negative symptoms from one longterm trial did not favour either group ( $n=593,1$ RCT, MD 0.20, 95\% $\mathrm{Cl}-2.32$ to 2.72 ).

\subsection{Mental state: 2 b. Specific symptoms - average endpoint scores (various scales, skewed data)}

Skewed data were available on anxiety and depression symptoms assessed with the Hospital Anxiety and Depression Scale (HADS) anxiety and depression subscale by medium and long term. These data, provided by the same study, were not consistent between medium and long term, as they showed a better outcome in the ICM group by medium term and a worse outcome in the ICM group by long term, compared with the non-ICM group (Analysis 2.27).

\subsection{Behaviour: 1. Specific behaviour (various measurements)}

We found three relevant studies for this outcome, assessing the outcome at different time periods and using different measures. Data failed to show a significant difference between groups at any time period for any outcomes. 


\subsubsection{By medium term - harm to self or others}

Medium-term data reporting 'harm to self or others' did not show a significant difference between groups ( $\mathrm{n}=73,1$ RCT, RR 0.88, 95\% $\mathrm{Cl} 0.4$ to 1.9; Analysis 2.28).

\subsubsection{By long term - self-harm}

We found two trials relevant to this subgroup. There was no clear difference between ICM and non-ICM within this subgroup ( $\mathrm{n}=959$, 2 RCTs, RR 1.00, 95\% Cl 0.69 to 1.46 ; Analysis 2.28).

\subsubsection{By long term - injury/assault to others}

We found two trials relevant to this subgroup. There was no clear difference between ICM and non-ICM within this subgroup ( $\mathrm{n}=959$, 2 RCTs, RR $1.09,95 \% \mathrm{Cl} 0.85$ to 1.4 ; Analysis 2.28).

\subsubsection{By medium-term FUP (18 months) - self harm}

There was a single trial in this subgroup. We did not find evidence of a clear difference between the two treatments ( $n=251$, RR 0.85, $95 \% \mathrm{Cl} 0.44$ to 1.67 ; Analysis 2.28).

\subsubsection{By medium-term FUP (18 months) - injury/assault to others}

There was a single trial in this subgroup. We did not find evidence of a clear difference between the two treatments ( $n=251,1$ RCT, RR $1.35,95 \% \mathrm{Cl} 0.87$ to 2.1 ; Analysis 2.28 ).

\subsubsection{By long-term FUP (8.5 years) - self harm}

There was a single trial in this subgroup. We did not find evidence of a clear difference between the two treatments ( $n=214,1$ RCT, RR $0.81,95 \% \mathrm{Cl} 0.51$ to 1.27 ; Analysis 2.28).

\subsubsection{By long-term FUP (8.5 years) - injury/assault to others}

There was a single trial in this subgroup. We did not find evidence of a clear difference between the two treatments ( $n=214,1$ RCT, RR 0.95, 95\% Cl 0.83 to 1.09; Analysis 2.28).

\subsection{Quality of life: 1. Average endpoint score (various scales)}

We found three studies assessing quality of life with three different scales (LQoLP, MANSA, QOLI) at different time periods. There were no significant differences between ICM and non-ICM in any of these measures at any time period. Data on QOLI scores were available by short and medium term from one study. Long-term data were available from three different studies measuring quality of life with three different scales, therefore not entering the analysis together. No results showed any significant difference between groups. Note that for this outcome the right graph label favours ICM (experimental group).

\subsubsection{By short term - overall life satisfaction (QOLI, high = better)}

One study provided data, showing no difference between groups ( $\mathrm{n}$ $=203,1 \mathrm{RCT}, \mathrm{MD}-0.02,95 \% \mathrm{Cl}-0.43$ to 0.39 ; Analysis 2.29 ).

\subsubsection{By medium term - overall life satisfaction (QOLI, high = better)}

One study provided data, showing no difference between groups ( $\mathrm{n}$ $=203,1$ RCT, MD $-0.04,95 \% \mathrm{Cl}-0.43$ to 0.35 ; Analysis 2.29 ).

\subsubsection{By long term $(L Q \circ L P$, high $=$ better $)$}

One study provided data, showing no difference between groups ( $\mathrm{n}$ $=526,1 \mathrm{RCT}, \mathrm{MD} 0.03,95 \% \mathrm{Cl}-0.10$ to 0.16 ; Analysis 2.29 ).

\subsubsection{By long term (MANSA, range 1 to 7 , high = better)}

One study provided data, showing no difference between groups ( $\mathrm{n}$ $=166,1$ RCT, MD 0.10, 95\% Cl -0.19 to 0.39).

\subsubsection{By long term - overall life satisfaction (QOLI, high = better)}

One study provided data, showing no difference between groups (n = 203, 1 RCT, MD 0.1, 95\% Cl -0.25 to 0.45; Analysis 2.29).

\subsection{Participant satisfaction/need: 1. Average endpoint scores (various scales) - by long term}

Long-term data were available from one study, assessing participant need with CAN, and participant satisfaction with health service scale. There were no significant differences between groups as assessed with the two scales.

\subsubsection{Patient need: CAN (high = poor)}

One study provided data, showing no difference between groups ( $\mathrm{n}$ $=585,1 \mathrm{RCT}, \mathrm{MD}-0.29,95 \% \mathrm{Cl}-0.69$ to 0.11 ; Analysis 2.30$)$.

\subsubsection{Patient Satisfaction With Health Services (high = poor)}

One study provided data, showing no difference between groups ( $\mathrm{n}$ $=490,1 \mathrm{RCT}, \mathrm{MD}-0.4,95 \% \mathrm{Cl}-1.25$ to 0.45 ; Analysis 2.30 ).

\subsection{Participant need: 1. Average endpoint scores (various scales, skewed data)}

One study provided data suggesting a difference between interventions in participant need as assessed with CAN scores, showing a worse outcome in the ICM group in the medium and long term, but these data were skewed. A second study provided data on participant need, assessed with CANSAS by long term. These data failed to show any difference between groups. We have presented these data as 'other' data (Analysis 2.31).

\subsection{Participant satisfaction: 1. Average endpoint scores (CSQ- modified, high = better, skewed data) - by long term}

Skewed data from a different study suggested long-term better satisfaction with treatment for the ICM group (data assessed with CSQ). We have reported these data as 'Other' data, (Analysis 2.32).

\subsection{Costs: 1. Direct costs of psychiatric hospital care (skewed data)}

Costs were assessed measuring 'direct costs of psychiatric hospital care' and 'direct cost of all care'. No data were available on 'direct healthcare costs'.

Skewed data on direct costs of psychiatric hospital care were available from two studies by medium and long term. Mediumand long-term data from one study found no significant difference between groups, whilst the second long-term study data found costs were significantly lower for the ICM group. The latter study was small (Quinlivan-California 1995, $\mathrm{n}=60$ ), where the former one was larger (Harrison-Read-UK 2000, $n=193$ ). We have presented these data as 'Other' data, (Analysis 2.33).

\subsection{Costs: 2a. Direct costs of all care - by long term ( 2 years) - unit cost GBP, fiscal year 1997/98}

Direct costs of all care were available from one study reporting skewed data (UK700-UK 1999), but with a sample size greater than 200. There was no significant difference between groups for 
reducing direct costs of all care $(n=667,1 \mathrm{RCT}, \mathrm{MD} 77.00,95 \% \mathrm{Cl}$ -66.63 to 220.63), but findings showed a trend suggesting greater cost in the ICM group. This trend suggested a cost increase of GBP 77 per person per month by long term, referred to as fiscal year 1997/98.

\subsection{Costs: 2b. Direct costs of all care (skewed data) - by medium term}

One study $(n=58)$ reported skewed data on the same outcome (direct costs of all care) (Johnston-Australia 1998). This data set showed no significant differences between groups by medium term, substantially confirming data by long term. We have reported these data as 'Other' data, (Analysis 2.35).

\subsection{Costs: 3. Total costs of care per patient - unit cost GBP)}

Total costs of care were available from two studies reporting skewed data (REACT-UK 2002; UK700-UK 1999), but with a sample size greater than 200. Total costs of care included direct and indirect costs (i.e. informal care, prison, court, probation officer, police custody, etc.).

\subsubsection{By 24 months, fiscal year 1997/1998}

There was no significant difference between groups for reducing total costs of care ( $n=667,1$ RCT, MD $1849.00,95 \% \mathrm{Cl}-1598.23$ to 5296.23), but findings showed a trend suggesting greater cost in the ICM group (Analysis 2.36).

\subsubsection{By 18 months, fiscal year 2003/2004 (GBP 1 = USD 1.58)}

There was no significant difference between groups for reducing total costs of care ( $n=243,1$ RCT, MD $4031.00,95 \% \mathrm{Cl}-2724.13$ to $10,786.13)$, but findings showed a trend suggesting greater cost in the ICM group (Analysis 2.36).

\section{SENSITIVITY ANALYSES}

As anticipated in the Methods section (see Methods, Sensitivity analysis), we performed the following sensitivity analyses.

\subsection{Implication of randomisation}

\subsubsection{COMPARISON 1: INTENSIVE CASE MANAGEMENT versus STANDARD CARE}

All of the included studies were described as randomised, and none were described in a way as to imply randomisation, therefore we did not include any trials in a sensitivity analysis.

\subsubsection{COMPARISON 2: INTENSIVE CASE MANAGEMENT versus NON- INTENSIVE CASE MANAGEMENT}

All of the included studies were described as randomised, and none were described in a way as to imply randomisation, therefore we did not include any trials in a sensitivity analysis

\subsection{Standard care caseload is over 20}

\subsubsection{COMPARISON 1: INTENSIVE CASE MANAGEMENT versus STANDARD CARE}

Among studies reporting on our primary outcomes, only six studies reported the ratio of staff to participants, and for each study it was greater than 1:20 (Bond-Chicago1 1990; Bond-Indiana1 1988; Herinckx-Oregon 1996; Jerrell-SCarolina1 1991; OPUS-Denmark 1999; Sytema-Netherlands 1999).
When we entered these studies (where standard care group caseload was greater than 20) in the analysis, the first primary outcome 'average days per month in hospital' showed a significantly favourable effect in the ICM group in reducing the length of hospitalisation ( $\mathrm{n}=951,7$ RCTs/study centres, MD -2.01, $95 \% \mathrm{Cl}-3.36$ to -0.67$)$, but heterogeneity was substantial $(12=70 \%$, $P=0.003)$. These findings confirm those obtained when all studies were entered in the analysis, regardless of caseload in the standard care comparison group.

The second primary outcome 'not remaining in contact with psychiatric services' showed that participants in the ICM group were significantly less likely to lose contact with psychiatric services than participants in the standard care group by medium term, long term, and overall $(\mathrm{n}=931,4 \mathrm{RCTs}$, MD $0.38,95 \% \mathrm{Cl}$ 0.23 to 0.63$)$, but heterogeneity was substantial $\left(I^{2}=67 \%, P=\right.$ $0.03)$. These findings confirm those obtained when all studies were entered in the analysis, regardless of caseload in the standard care comparison group, however data in the sensitivity analysis showed a substantial level of heterogeneity not present when all studies were entered in the analysis.

\subsection{Assumptions for lost binary data}

\subsubsection{COMPARISON 1: INTENSIVE CASE MANAGEMENT versuS STANDARD CARE}

No assumptions needed to be made regarding people lost to followup for the primary binary outcome 'not remaining in contact with psychiatric services' for this comparison, therefore we did not include any trials in a sensitivity analysis.

\subsubsection{COMPARISON 2: INTENSIVE CASE MANAGEMENT versUS NON- INTENSIVE CASE MANAGEMENT}

For the second comparison, we needed to make assumptions regarding people lost to follow-up for the primary binary outcome 'not remaining in contact with psychiatric services' for one trial (REACT-UK 2002). When we compared the findings of the outcome when we used our assumption to when we used completer data only, results did not differ substantially.

\subsection{Assumptions for lost continuous data (for meta-regression)}

We undertook sensitivity analysis, removing 13 out of 52 trials originally entering meta-regression. These were trials where primary outcome standard deviation was imputed (see Table 3). Meta-regression was therefore run on the remaining pool of 39 non-imputed studies. Results no longer reached significance for both variables 'organisation fidelity subscore' and 'baseline hospital use' as $\mathrm{P}$ is at trend 0.07 ('organisation fidelity subscore': regression coefficient $-0.2,95 \% \mathrm{Cl}-0.48$ to $0.02, \mathrm{P}=0.067$; 'baseline hospital use': regression coefficient $-0.18,95 \% \mathrm{Cl}-0.39$ to $0.02, \mathrm{P}=$ 0.077). Removing a high-influence outlier (Rosenheck-USA-NP (C)), significance returns for the effect of 'baseline hospital use' $(-0.26$, $95 \% \mathrm{Cl}-0.51$ to $-0.01, \mathrm{P}=0.046)$, but not for 'organisation fidelity'. When combining the two variables within the model ('organisation fidelity subscore' and 'baseline hospital use'), the pattern of results did not change for the non-imputed studies. 


\section{DISCUSSION}

\section{Summary of main results}

\section{COMPARISON 1: INTENSIVE CASE MANAGEMENT versus STANDARD CARE}

\subsection{Service use}

\subsubsection{Service use: Average number of days in hospital per month - at about 24 months}

ICM does seem to reduce length of hospitalisation when compared with standard care (by 0.86 day per month over 24 months). However, these data were low quality and from a heterogeneous analysis; when certain studies are removed from the analysis, the now-homogeneous result suggests that the saving is in the region of 0.6 days per month. We were unsure which of the two figures was the most reliable, but in any event trial findings suggest a saving of time in hospital per person of between about 7 and 10 days in hospital per year.

What is important, however, is that there was a high duration of hospital stay for these people in the two previous years (averaging 6 days per month). This was higher than for those in the ICM versus non-ICM comparison (averaging 3.4 days per month), which found no difference between groups (see Figure 10). The impressive saving of time in hospital for ICM was greater than that of standard care, but the saving in standard care was also considerable (absolute decline for ICM was about 3 days, standard care was 1.8 days). Well-organised standard care, for people with what would seem to be a history of considerable periods spent in hospital over the last 24 months, does seem to reduce the time in hospital for the ensuing two years. However, ICM adds more than an extra day to that gain.

The meta-regression is a tool of limited power, employed on weak data. However, the results were in keeping with the results of one other review (Burns 2007), which suggests that the gain of ICM was not so much linked with the total fidelity score, the staff subscale, or the comparison group. It did suggest a link with the organisational subscore of the Index of Fidelity to Assertive Community Treatment (IFACT) scale ( 0.36 day per month out of hospital gained by each point increase of this subscore). It also suggested that ICM effect is linked to length of hospitalisation during the previous two years ( 0.2 day per month out of hospital gained by each day increase in the day in hospital per month during previous two years).

In other words, this means that:

- if the ICM team ratio staff:client is less than 1:20 (calculated dividing the number of active clients on the caseload by the number of full-time equivalents of direct service staff on the team), then it makes no difference how much the caseload is lower than 1:20;

- the ICM team size does not matter (calculated as the number of full-time clinical staff equivalents, as defined earlier);

- the availability of a psychiatrist on the team is not pivotal; and/or

- the availability of a nurse on the team is not pivotal.

It also suggested that gains in IFACT scores can reduce average hospital stay. McGrew 1994 and McGrew 1995 suggest that gain can be mediated by:
- ensuring that the ICM team performed the role of primary therapist for the client (the primary therapist role designates the person within the local mental health system with primary clinical and record keeping (e.g. treatment plans) responsibility for the client);

- the ICM team's offices being located in a separate building from the parent agency's main offices (i.e. usually away from the hospital site);

- the ICM team sharing caseloads (rated as the degree to which all staff members on the team had contact with all clients on a regular basis, e.g. through rotation), in contrast to individual caseloads in which specific staff workers are responsible for specific clients;

- the ICM team meeting as a group each weekday to discuss their entire caseload;

- the ICM team's supervisor devoting at least half time to client contacts, either cojointly during supervision of team members, or individually as part of his or her duties as a member of the team;

- the ICM team providing 24-hour direct access to the team (if access to the ICM team was triaged through the community mental health centre emergency 24-hour on-call service, intermediate score of 0.5 is obtained, therefore the advantage on decreasing days in hospital per month would be halved $(-0.2$ days in hospital per month)); and/or

- the ICM team serving clients without any expectation of transferring them to another programme.

\subsubsection{Service use: Average number of days in hospital per month - by follow-up}

When the same outcome 'average number of days in hospital per month' was assessed on medium- and long-term follow up, data did not confirm the effect of ICM in reducing length of hospitalisation assessed by 24 months. These data were provided by one large study $(n=547)$, where during the follow-up period all participants received the control intervention (standard care). These data are suggestive of a loss of effect on reducing number of days in hospital over time, if ICM intervention is discontinued.

\subsubsection{Service use: Not remaining in contact with psychiatric services}

Overall, we found ICM to be better than standard care for retaining people in psychiatric services $(n=1633,9$ RCTs, RR $0.43,95 \% \mathrm{Cl}$ 0.30 to 0.61 ). However, this effect was not seen for the short-term analysis ( $\mathrm{n}=95,1 \mathrm{RCT}$, RR $0.54,95 \% \mathrm{Cl} 0.28$ to 1.05 ), although confidence intervals were compatible with an effect favouring ICM $(P=0.07)$. Medium-term findings did suggest an overall effect of ICM being better in reducing loss to follow-up to psychiatric service contact ( $\mathrm{n}=1063,3$ RCTs, RR $0.51,95 \% \mathrm{Cl} 0.36$ to 0.71 ). In the longer term, this same effect was heterogeneous, due to an outlying finding of a single study (Herinckx-Oregon). This trial had a peculiar definition for this outcome, which was different from the other studies. In Herinckx-Oregon 'not remaining in contact with psychiatric services' did not include refusing re-interview, moving out, and death, whereas for the other trials it did. If we exclude Herinckx-Oregon, and only five long-term trials are retained in the analysis, then ICM appears to be effective in preventing loss to follow-up ( $n=475,5$ RCTs, RR $0.27,95 \% \mathrm{Cl} 0.11$ to 0.66 ).

The result of a better retention in care for the ICM group strengthens the relevance of the result for the first primary outcome (i.e. ICM decreasing time in hospital). ICM decreases days of hospitalisation 
in a severe mentally ill population where patients are kept in close contact with services, therefore the ICM effect on reducing days in hospital might not be dissipated by a higher rate of loss to followup.

\subsubsection{Service use: Use of hospital}

We found that ICM reduced the number of people admitted to hospital more than standard care, at least in the medium term ( $\mathrm{n}=1303,5 \mathrm{RCTs}$, RR $0.85,95 \% \mathrm{Cl} 0.77$ to 0.93 ). Skewed data for 'average number of admissions in the medium term' were in accordance with these first findings, whilst skewed data for 'average number of admissions in the long term' failed to show any trend in the effect of intervention. Synthesis of short- and-long term studies in this outcome produced heterogeneous findings. The short-term data were from only two studies, and when the most positive one is removed (Bond-Indiana1), the finding becomes clearly null. The long-term data was from 11 trials; removing the three clearly outlying studies, Curtis-New York, Macias-Utah, and Test-Wisconsin, also restores homogeneity and moves the finding squarely towards the null. One small study provided inconclusive data on the unplanned admission to the emergency department, an outcome describing only one option of psychiatric admission; for this reason, and because these data come from a single small study, we considered this finding weak.

Overall, the effect of ICM on admission to hospital is not strong. Whereas the time spent in hospital does seem to be less if allocated ICM - at least for those whose baseline use of hospital was high the number of admissions is not greatly changed. Admissions are shorter, but not by much, if any less frequent.

\subsubsection{Service use: Use of services outside of mental health provision}

More outcomes were available describing 'use of services outside of mental health provision'. Studies did not report a convincing difference in 'use of emergency room', 'rate of use of emergency room', 'use of day hospital care', or 'rate of outpatient visits'. Only 'rate of home visits' was higher for the ICM group. However, we did not consider these data very robust, as they were based on single studies providing data per each outcome or on skewed data that were very difficult to interpret.

\subsection{Adverse event}

\subsubsection{Death - all cause and suicide}

This review did not find any difference in mortality either due to all causes or to suicide in the short, medium, and long term and in medium- and long-term follow-up. Although death is a rare adverse event, the duration of the longer studies ( $\sim 24$ months) means that some deaths would have been expected to occur and did $3.2 \%$ ICM versus $3.8 \%$ standard care). This difference was not statistically significant, but is homogeneous, and suggests a trend in terms of risk reduction (RR $0.84,95 \% \mathrm{Cl} 0.48$ to 1.47 ), partly confirmed in one large study on medium-term follow-up (RR $0.59,95 \% \mathrm{Cl}$ 0.22 to 1.61). The same trend was confirmed in the ICM effect on mortality due to suicide by long term (suicide rate $1.3 \%$ ICM versus $2 \%$ standard care). Again, this difference was not statistically significant, but is homogeneous, and stronger compared to death due to all causes. If the suicide risk is in actuality so much reduced (RR $0.68,95 \% \mathrm{Cl} 0.31$ to 1.51 ), this would be a very important finding, although the quality of the evidence is low (Summary of findings table 1 ).
Few things have changed the outcome of death for people with schizophrenia. If, for this group of people, set in the context of a standard care with a high baseline risk of admission, ICM could decrease death, and by so much, this would be a strong argument in favour of ICM. These data on suicide are the findings of nine studies with only a total number of participants of 1456 and lowquality evidence. A single larger trial might be able to confirm this important suggestion.

\subsection{Global state}

\subsubsection{Global state: Relapse}

No data were provided for the important outcome of relapse.

\subsubsection{Global state: Leaving the study early}

ICM data regarding number of participants lost to follow-up for the short term were heterogeneous. However, we found ICM to be more advantageous than standard care in reducing rate of lost to followup both in the medium term ( $\mathrm{n}=1699,8$ RCTs, RR $0.60,95 \% \mathrm{Cl}$ 0.51 to 0.70$)$ and long term ( $n=1798,13$ RCTs, RR $0.68,95 \% \mathrm{Cl}$ 0.58 to 0.79 , low-quality evidence). The impression remains that $\mathrm{ICM}$, and again with the proviso that these findings may apply most specifically to a group with high baseline admission, holds on to people more tightly across time. This may not significantly lower admission rate, but loss to follow-up and length of admission may decrease. Overall, these data were not of high quality (Summary of findings table 1), but there is a belief that ICM is advantageous over standard care for the higher-risk groups. For example, in groups with only $10 \%$ loss to follow-up across the long term, only 3 more people are not lost for every 100 given ICM. However, with more realistic figures of $50 \%$ loss to follow-up, this figure rises to 15 more people out of every 100 who are kept in care compared with those allocated standard care.

\subsubsection{Global state: Global Assessment of Functioning Scale (GAF)}

Short-term studies indicated a better improvement in GAF endpoint score for participants in the ICM group compared with those allocated standard care (short term: $\mathrm{n}=797,4 \mathrm{RCTs}$, MD 2.07, 95\% $\mathrm{Cl} 0.28$ to 3.86). This effect was confirmed by the long-term data (n $=818,5 \mathrm{RCTs}, \mathrm{MD} 3.41,95 \% \mathrm{Cl} 1.66$ to 5.16 ). Whilst this is favourable for those allocated ICM, we are unsure of the clinical meaning of these data, as changes of two or three points on a scale that runs to 100 does not seem to be much. We have found no reference to the clinical meaning of such small changes.

\subsubsection{Global state: Not compliant with medication}

Only one long-term study provided data on compliance with medication, and these indicated a higher compliance level in those allocated to ICM compared with those in the standard care group ( $\mathrm{n}$ $=71,1 \mathrm{RCT}$, RR $0.35,95 \% \mathrm{Cl} 0.15$ to 0.81 ). Again, this is an important finding and should be replicated. We are surprised that such easily recorded data are not reported in more studies.

\subsection{Social functioning}

\subsubsection{Social functioning: Contact with legal system}

Studies measuring contact with legal system used different definitions over varying time periods, which makes interpretation difficult. There was no real suggestion that ICM either increases or decreases the measures of this outcome. The 'arrested' and 'imprisoned' outcomes were the only ones with some consistency. They showed no significant difference in the intervention effect 
between groups. The 'contact with the police' outcome findings were from only one study and were not significant in the short term, but became significantly different in the medium term (favouring the ICM group). Overall, the legal outcomes were not convincing, and there is a need for more consistency in approach to this area of research.

\subsubsection{Social functioning: Employment status}

This review did not reveal any significant difference between ICM and standard care in employment status, as measured by different outcomes. Medium-term findings on 'number of people not competitively employed' were based on only one trial. Both medium- and long-term data reporting 'not employed' tended to favour ICM, although data were heterogeneous, and overall the quality of the evidence was very low (Summary of findings table 1). Data from one large trial on medium- and long-term follow-up failed to show any difference between interventions. Again, this is an important outcome for which more data are needed before firm conclusions can be drawn.

\subsubsection{Social functioning: Accommodation status}

Data on the outcome 'homelessness' were not convincing by short, medium, or long term, but were mostly derived from just a few trials.

The outcome 'not living in stable accommodation' was scarcely reported, available from only one long-term study.

Regarding the 'not living independently' outcome, we found that people allocated to ICM were more likely to live independently compared with those allocated standard care - in the medium term, and even more so in the long term. This is another important finding of this review. If the risk of not living independently is in actuality substantially reduced by this ICM package (18\% ICM versus $26 \%$ standard care, long term), at least for people with high baseline risk of admission, and if this is a desired outcome for this particular client group, then, combined with the other moderate but cumulative advantages, this finding further highlights the advantage of ICM over standard care.

Only one large study reported the outcome 'days in supported house', assessing it by long term and by medium- and long-term follow-up. The advantage for standard care reported on mediumterm follow-up was not confirmed on long term or on long-term follow-up, failing to show a significant trend of effect.

\subsubsection{Social functioning: Substance abuse}

Only one study $(n=547)$ reported usable binary data for alcohol and drug abuse. A long-term single study failed to show any advantage for participants treated with ICM compared to standard care. Skewed data were supplied by Sytema-Netherlands $(n=81)$, with Dartmouth Assessment of Lifestyle Interviewscores tending to favour the ICM group for both drug use and alcohol consumption. However, we are unsure of the clinical meaning of these scores. Morse-Missouri3 $(n=103)$ reported skewed data on days substances were used per month. There was no indication of any difference between groups. There is currently no compelling evidence that ICM affects abuse of substances or alcohol.

\subsubsection{Social functioning: Various scales}

Few studies reported usable data for social functioning scale, and outcome data were complicated by the use of different scales within single studies, making meta-analysis impossible. In this confusion of evidence, we see no advantage for participants treated with ICM compared to those treated with standard care in terms of social functioning measures. This does not seem to concur with other findings on independent living. It could be that the fine-grain measures of functioning are picking up subtle parameters of social function not effected by the package. It could also be that the measures are not sensitive enough to broad and important issues of social function.

\subsection{Mental state}

Rating of mental state in these trials illustrates the confusion of how such symptoms are recorded in randomised studies. Timings of use of the scales differ, and the findings are so problematic to interpret from the clinical perspective that we are left to make safe but bland conclusions.

No data were provided for the important outcome 'mental state: not improved to an important extent', and there does not seem to be any compelling evidence that, in this group of people, set where the baseline risk of admission is high, ICM in actuality substantially affects a person's mental state.

\subsection{Behaviour: self harm}

Based on findings from the larger of the studies, self harm was not convincingly reduced by use of the ICM model. The mortality finding discussed above, however, does seem to suggest that ICM reduced the risk of death. These findings are a little at odds with each other, although not entirely, providing all the more reason to continue to research into this area for these, the simplest of outcomes.

\subsection{Quality of life}

Few studies reported relevant outcomes. Short- and medium-term outcome data were complicated by the use of different scales within single studies, making meta-analysis impossible. We found that one short-term study $(n=125)$ showed a better quality of life in the ICM group on the Lehman's Quality of Life Interview scale, but more medium- and long-term data failed to show any advantage for participants treated in the ICM group compared with standard care. The few skewed data seem to concur with the impression that for quality of life measures used in trials, ICM confers no advantage over standard care.

\subsection{Participant satisfaction/need}

Participants administered ICM were more satisfied with their treatment compared with those administered standard care in these trials. These findings were based on data that were quite strong (short term, $\mathrm{n}=61$; medium term, $\mathrm{n}=500$; long term, $n=423$ ). More satisfaction with care could enhance medication compliance, the will to keep in services, housing status, and a host of other variables. We are left doubting the size and meaning of the overall finding. We are unsure how encouraged we should be that these packages of care deliver an average of a two- to three-point improvement in the Client Satisfaction Questionnaire.

Several of the smaller trials did measure need and unmet need. These skewed data were difficult to interpret, but did not seem to convincingly favour either of the groups. 


\subsection{Costs}

With respect to cost of inpatient psychiatric care, ICM was consistently superior to standard care for the outcome 'direct costs of psychiatric hospital care', suggesting a saving of money per person of about USD 144 per month (fiscal year 1990). Two studies taking part in the same multicentre trial provided these data. Skewed data were contradictory, neither showing a trend confirming nor disputing these data.

We found no difference between groups with respect to direct healthcare costs (where skewed data were contradictory and provided by only two small studies). Results on 'direct costs of all care' were inconcludent, as data were skewed and different trials reported contradictory effects.

\section{COMPARISON 2: INTENSIVE CASE MANAGEMENT versus NON- INTENSIVE CASE MANAGEMENT}

\subsection{Service use}

\subsubsection{Service use: Average number of days in hospital per month - at} about 24 months

Moderate quality evidence from this review showed no significant advantage of ICM in reducing the average length of hospitalisation when compared with non-ICM. This could be an important finding, and we see no good reason not to trust this result. The implications from this finding could be that if services are already providing non-ICM, there is no point in investing in further intensiveness. We currently know of no review comparing non-ICM with standard care and reporting relevant outcomes. This should be undertaken. It is possible that there are other features of ICM that may improve outcome, but we are not stipulating that we should specifically investigate for these. What was different between the two sets of comparisons was the baseline risk of admission in the previous two years (about 6 days per month for Comparison 1 versus about 3.4 days per month for Comparison 2). This was highlighted by the meta-regression process. This generates further hypotheses. Baseline hospital risk is linked to service provision, service culture, severity of illness, and other issues. We do not have the sophistication of data to investigate these. What we are left with is the possibility that in a situation where people with severe mental illness have a duration in hospital of less than 4 days per month in the two years preceding the ICM package of care, the increased intensity of approach may not be justified.

\subsubsection{Service use: Average number of days in hospital per month - at follow-up}

Data on medium- and long-term follow-up from one study ( $\mathrm{n}=$ 237) failed to show a significant advantage of ICM in reducing the average length of hospitalisation when compared with nonICM. During the follow-up period participants could remain in the originally allocated intervention or be transferred to the control one. These data on follow-up confirmed the data at 24 months discussed above.

\subsubsection{Service use: Not remaining in contact with psychiatric service}

We found ICM to be more effective in increasing the number of people retained in contact with psychiatric service in the medium term, but we did not consider these findings robust as they were based only on one small trial $(n=73)$. We found no difference between interventions in the long term, but data were heterogeneous. Overall, when pooling medium- and long-term data, we found no advantage for participants treated with ICM compared to non-ICM for better retention in psychiatric service but, again, these data were heterogeneous, and we found no obvious explanation for the heterogeneity ( $n=1255,4$ RCTs). Medium-term (18 months) follow-up data showed a trend favouring ICM in increasing the number of people retained in contact with psychiatric service.

\subsubsection{Service use: Admissions}

We found no difference between groups in the risk of being admitted to hospital in the long term ( $\mathrm{n}=1132$, 3 RCTs, RR 0.91, $95 \% \mathrm{Cl} 0.75$ to 1.12 ). These findings were confirmed by data from one long-term study $(n=678)$ and one medium-term study $(n=68)$ on the average number of admissions, where no advantage was shown between treatments in reducing number of admissions in the long term (moderate-quality evidence, Summary of findings 2) or in the medium- and long-term follow-up. Data on frequency of admission and on length of hospitalisation therefore consistently show no effect of ICM for both outcomes.

\subsection{Adverse events}

\subsubsection{Death due to all causes and to suicide}

This review did not find provide strong evidence on mortality rate either due to all causes or to suicide in the short, medium, and long term, or in the medium- and long-term follow-up. These data are quite informative, especially those from long-term studies, where the study length might balance the rarity of the event in detecting any difference between intervention effects. Some deaths occurred in the long-term studies ( $2.0 \%$ ICM versus $2.2 \%$ non-ICM) $(n=1634$, $5 \mathrm{RCTs}$, RR $0.90,95 \% \mathrm{Cl} 0.46$ to 1.75 ). This impression was confirmed for studies reporting suicide only, as shown in Summary of findings 2, where low-quality evidence also showed no difference between groups.

\subsection{Global state}

\subsubsection{Global state: Relapse}

No data were provided for this important outcome.

\subsubsection{Global state: Leaving the study early}

No studies were available for the short-term outcome.

Data showed no difference between interventions by the medium term, but these data were not strong as they came from a small sample of two studies $(n=225)$ and were heterogeneous with inconsistency of effect.

We found ICM to be more advantageous than non-ICM in reducing rate of lost to follow-up by the long term.

Overall, pooling studies from subgroups for two time points, we found heterogeneous data, but substantially confirming homogeneous data obtained by long term. ICM was confirmed to be more advantageous than non-ICM in reducing rate of lost to follow-up ( $\mathrm{n}=2195,9 \mathrm{RCTs}$, RR $0.72,95 \% \mathrm{Cl} 0.52$ to 0.99 ). If we consider this outcome as proxy of a better retention in care, it might overcome the inconsistency of data on 'number of people remaining in contact with psychiatric service' (see Discussion - 2.1.3 Service use: Not remaining in contact with psychiatric service). ICM therefore seems to positively reduce number of lost to follow-up, but does not affect length and frequency of admission. These data 
were of low quality (Summary of findings 2), but it appears that ICM has an advantage over non-ICM for the higher-risk groups. For example, in groups with only $10 \%$ loss to follow-up across the long term, only three more people are not lost for every 100 given ICM. However, with a more realistic figure of $50 \%$ loss to follow-up, this rises to 14 more people out of every 100 are kept in care compared with those allocated standard care.

\subsubsection{Global state: Health of the Nation Outcome Scale (HoNOS)}

Not enough studies were available to run a meta-analysis on data derived from the HoNOS or from any other scales assessing global state. Considering the comprehensiveness of the scale assessment of other outcomes, it appears that global state as an outcome is under-recorded by trialists, despite being informative and relevant from a clinical point of view.

\subsubsection{Global state: Compliance with medication}

As for the previous outcome, not enough studies were available to run a meta-analysis. Again, a very meaningful outcome from a clinical point of view is neglected.

\subsection{Social functioning}

\subsubsection{Social functioning: Contact with legal system}

As for studies included in Comparison 1, studies measuring contact with legal system used different definitions over a variety of time periods. This makes interpretation difficult. In addition, only a few studies addressed this outcome (three trials overall). There was no real suggestion that ICM either increases or decreases the measures of this outcome. The 'imprisoned' outcome was the only one with some consistency, showing no significant difference in the intervention effect between groups. The 'contact with the police' and 'arrested' outcome findings were from only one study each and were not significant in the short and long term, respectively. Overall, the legal outcomes were not convincing, and there is a need for more consistency in approach to this area of research.

\subsubsection{Social functioning: Employment status}

Data available for this outcome were substantially inconclusive, reported by only one small trial $(n=73)$. This trial measured employment status according to two different definitions: 'spent more than one day employed' and 'on paid employment'. Both findings were not significant in the medium term, and overall quality of evidence was low (Summary of findings 2). One larger study $(n=214)$ provided data on long-term follow-up ( 8.5 years after the randomised allocation was broken), showing no difference between the two groups. These data did not add much to the understanding of the impact of ICM on employment status compared to non-ICM. Again, this is an important outcome underestimated in the current studies, and at this stage more data are needed before firm conclusions can be drawn.

\subsubsection{Social functioning: Accommodation status}

Available data on this outcome were surprisingly scarce, as this outcome was reported in just four studies. Two studies described this outcome with binary data: one measuring just 'living in supported accommodation' by medium term, the second measuring 'living in supported accommodation', 'homelessness', and 'living independently' by long term and on follow-up. All data on different measures at different time periods were based on only one trial and they were not significant.
Two more studies described this outcome with continuous data on 'average days per months in stable accomodation', again failing to show any difference between the two groups. Findings seemed to point at a non-significant difference in effects of intervention, although these findings were inconclusive due to the scarcity of available data, despite being easily recorded and very relevant from the perspective of a community-based service.

\subsubsection{Social functioning: Substance abuse}

Only two studies measured substance abuse, and they described this outcome as binary and continuous measures, and not consistently across studies. This makes interpretation difficult and findings unconvincing, as a single study entered each measurement, and we therefore could not carry out any metaanalysis. As far as we could assess, there was no indication of any long-term difference between groups in 'number of people abusing alcohol', 'number of people abusing illicit drug', or 'remission from alcohol use disorder' (defined as Alcohol Use Scale score less than 3). These findings were confirmed by those continuous data, assessing the substance abuse with Substance Abuse Treatment Scale. These data failed to show a significant difference between groups at any time period assessment. As for the first comparison, currently there is no compelling evidence that ICM affects abuse of substances or alcohol.

\subsubsection{Social functioning: Scale data}

Findings were equivocal on scale data measuring social functioning, as provided by only one study. We do not think that future studies should address this outcome by use of scale measurement. Scales are not sensitive measures of social functioning. More effort should be placed on consistent and wide measurements of the main issues of social functioning (such as accommodation status, employment status, contact with legal system, rate of permanent social benefits).

\subsection{Mental state: General symptoms and specific symptoms}

Again, outcomes measured on scales were substantially inconclusive, as the data were spread across single studies on different scales and at different time periods, making meta-analysis impossible. According to the low-quality data available, there does not seem to be any compelling evidence that ICM substantially affects mental state. No data were available for the significant outcome of 'important improvement in mental health'.

\subsection{Behaviour: Self harm and injury to others}

This review did not reveal any long-term significant difference between ICM and non-ICM in risk of committing self harm or injury to others. These data were based on findings from two studies ( $\mathrm{n}$ = 959), one of which is the largest study (UK700-UK 1999), and the other the only study providing data on medium- and long-term follow-up (REACT-UK 2002). These findings are consistent with the mortality findings discussed above, where no significant difference was shown in death rate between groups, either for suicide and for all causes. Although these data are suggestive of no difference of effects between interventions, they are still quite weak due to limited sample size. More trials should address this outcome, one of the simplest ones to collect. 


\subsection{Quality of life}

Quality of life rating in these trials illustrates the confusion of how such symptoms are recorded in randomised studies. The scales differ, and the timings of use of the scales also differ. There was such an inconsistency in approach to this area of research to make meta-analysis impossible. None of the available findings showed any significant difference between interventions. There does not seem to be any compelling evidence that ICM substantially affects the quality of life of a person with severe mental illness.

\subsection{Participant satisfaction/need}

Findings tended to favour the ICM group in being better satisfied with health services and in reducing need. However, this difference was not significant, and both findings were based on data from the same trial, the largest one (UK700-UK 1999, $n=585$ ). We therefore cannot draw any conclusions, but highlight a possible favourable effect in the ICM group, which needs to be confirmed.

\subsection{Costs}

Studies assessed 'direct costs of psychiatric hospital care', 'direct cost of all care', and 'total cost of care'. Regarding the first outcome, findings were based on skewed data, provided by one small trial (Quinlivan-California 1995, $\mathrm{n}=60$ ) and one larger one (HarrisonRead-UK 2000, $n=193$ ). There did not appear to be any compelling evidence that ICM substantially affects 'direct costs of psychiatric hospital care', either by medium or long term. Also, findings on long-term 'direct cost of all care' did not show any difference between interventions. Again, findings on 'total cost of care' failed to show any difference between ICM and non-ICM.

\section{Overall completeness and applicability of evidence}

\section{Completeness}

\subsection{Duration of follow-up}

The majority of studies presented long-term data, that is over one year of follow-up. This is a reasonable length of time to sensibly assess any difference in the intervention effects.

Two studies, one in the first comparison (ICM versus standard care) and one in the second comparison (ICM versus non-ICM) presented long-term follow-up (from 18 months to 8 years), assessing outcomes after the active intervention was discontinued or after participants could chose to which arm they were allocated.

\subsection{Coverage of outcomes}

As the experimental intervention is a service organisation model, its realisation involves health policy and research should account for efficacy and cost evaluation. The outcomes reported were mainly service use and social functioning oriented. No studies reported data on relapse (see Summary of findings for the main comparison, Summary of findings 2), carer satisfaction and family burden. Participant satisfaction was scarcely reported and in a fragmented way, therefore available data are only partially informative on the effects of these approaches. Few studies provided cost data.

\section{Applicability}

\subsection{Origin}

The origin of the data has changed in the last decade since the two original reviews (Marshall 2000a; Marshall 2000b). There are now more included trials from Europe, whereas in the past the data source was largely North America, with a few trials from Australia. Thirty per cent of the total sample included in the current review comprises randomised people from Europe. These studies add power to the result for the primary outcome, narrowing the confidence intervals, but otherwise not substantially changing the findings. As only one study was from China, and all the remaining included studies were from Europe, North America, and Australia, the findings of this review still lack applicability to low-income countries and, more generally, to countries where mental health systems are not community based.

\subsection{People}

Studies included people presenting a variability that we feel is likely to reflect the heterogeneous population a clinician faces in daily practice when treating people affected by severe mental illness. This variability was in terms of diagnosis (where participants were affected by a wide diagnostic group including schizophrenic, affective, and personality disorder); comorbidity (where four studies included dually diagnosed participants) (Drake-NHamp 1998; Essock-Connecticut2 2006; Morse-Missouri3 2005; MullerClemm-Canada 1996); and social characteristics (where eight trials included homeless participants). On average, studies included people with a long history of illness; only OPUS-Denmark 1999 included participants with a first episode of psychotic illness. This fits with the concept of severe mental illness, where this label includes certain criteria relating to length of illness.

\subsection{Interventions}

Some studies showed a greater applicability because the experimental intervention was provided by pre-existing team, therefore closer to the real world and less contaminated by the experimental setting.

The majority of new included trials from the 2010 update compared ICM with non-ICM (8 out of 14 trials), and they are all from Europe, Australia, and North America. This confirms the trend of psychiatric services in those particular areas to increasingly include some elements of the original model, but also to dilute and contaminate them with the current organisation. What we call 'standard care' is therefore converging toward non-ICM. The two studies newly included from the 2015 update compare ICM with standard care: one is from the USA and assesses ICM adapted to the forensic setting, and the other is from China, where only recently community care is catching on. For those of us who practice in Europe, the second of the two comparisons in this review may well be more applicable to everyday care. Importantly, this comparison did not illustrate a substantial difference between ICM and non-ICM.

\section{Quality of the evidence}

As illustrated in Figure 1, it appears there is an overall unclear risk of bias in these trials. This would therefore mean there is a moderate risk of overestimate of positive effect. Also, making difficult judgements about quality has been greatly helped by a discernable improvement in reporting of methodology.

\section{Potential biases in the review process}

There were several potential biases. We have worked mainly with published reports, and only in few cases with unpublished material. By doing this we may be perpetuating a reporting and 
publishing bias. It would have been better to have much more original individual participant data. This review follows from two past Cochrane reviews (Marshall 2000a; Marshall 2000b), as well as much work already published in paper format (Burns 2007). The conduct of these reports has influenced this document, and it is possible that we have failed to identify systematic biases in the way we have conducted the reviews across time.

An author of this review is an active pioneer in the development and implementation of the experimental intervention model across the scientific community and clinical world (MM), and one included study is his (Marshall-UK 1995). As a team, we tried to ensure that decisions were made by rational consensus, and not to have an expert in the team would have been an inadvisable omission.

In some cases, protocol rules were unclear, and need for subsequent clarification arose and post hoc decisions had to be taken (see Differences between protocol and review). This could have affected the review process in various cases. This has probably lowered the quality of the data included in the review, but to not include so much, for example, skewed data, would have omitted much information. Also, by breaking down studies into their centres, many fell below the 200-participant cutoff point. We have included these data in the 'less than 200' category, whereas in previous versions of the review they would have been in the 'greater than 200' category. Due to the overall effect of the changes in protocol, it appears that we have a more inclusive review, with data that are more heterogeneous and also more favourable for the experimental interventions than otherwise would have been the case should we have used a more limited data set. Nevertheless, we did feel it important to present all of these data for the reader to consider.

We prespecified what characteristics of studies could be associated with heterogeneity, and therefore we stated in the protocol what variables were to be explored in the meta-regression before inspecting the results of the studies. Despite this prespecification, we were not blind to what variables were probably more related to heterogeneity, as we were familiar with some study results previously published. The undertaken exploration of heterogeneity might therefore at best lead to generation of hypothesis, but it cannot provide reliable conclusions.

\section{Agreements and disagreements with other studies or reviews}

This review merges two older Cochrane reviews, fully bringing up to date how these data should be considered. This version does not disagree with the older reviews; it simply replaces them with a more current viewpoint of the data. A major improvement in this version is data on duration of admission, which were previously lacking from past reviews. Other research in this area do not provide a full summary of available evidence on ICM effects across various outcomes (Burns 2007). This Cochrane review does not disagree with the paper version; it is just much more comprehensive. Regarding the meta-regression, this review substantially confirms the hypothesis stated elsewhere that baseline hospital use and fidelity to the model affects outcome (Burns 2007).

\section{AUTHORS' CONCLUSIONS}

\section{Implications for practice}

\section{For people with severe mental illnesses}

We found ICM to be effective in ameliorating many outcomes relevant to people with severe mental illness. Compared to standard care, ICM may reduce hospitalisation and increase retention in care. In fact, ICM was shown to reduce hospitalisation, in terms of less frequent and shorter admission to hospital; increase retention in care; probably reduce the risk of death and suicide; and globally improve social functioning in terms of a better accomodation status, employment status, and showing a trend in reducing contact with legal system. Although its effect on mental state and quality of life remains unclear, ICM seems to significantly help global state compared with standard care. However, it is unclear what gain ICM provides on top of a less formal non-ICM approach. The latter may better suit some people with severe mental illness than the more intensive full-ICM model. Data on satisfaction with care for ICM versus non-ICM were very few and difficult to interpret.

\section{For clinicians}

ICM formalises a holistic approach to care of people with severe mental illness in the era of limiting hospital admissions and subsequent hospital closure. This review suggests that this formalising is helpful across several outcomes over and above standard care, the latter largely based in outpatient departments, and that this seems acceptable to people with severe mental illness. However, when the fully formal holistic approach (ICM) is compared with the less formal, but also holistic non-ICM, the differences are not so clear. This could be seen as encouraging, as for various reasons many clinicians are unlikely to rigidly apply full ICM. This does not abrogate the need to know and apply key components of the model of care within ICM.

\section{For policymakers}

We know at this juncture that ICM is of value at least to people with severe mental illness in the subgroup with a high level of hospitalisation (about 4 days per month in past 2 years). The intervention should be performed close to the original model, therefore training should be planned for relevant mental health workers. Data on costs are still scarce, and we could not draw conclusions on cost-effectiveness. Where ICM features are already available in the community psychiatric service (the non-ICM intervention), it is unclear if additional full development to the rigid model of ICM is of value. The results of this review could guide policies on the introduction of such an ICM service in those countries where a community psychiatric service is already set up but ICM is not in use, and in countries where a shift from hospitalbased care in favour of a more community-focused approach has still to be developed. Particular consideration should be given to the setting where ICM is to be developed, as its value was shown where the level of hospitalisation is high. It is unclear whether the introduction of some but not all of the ICM features (the non-ICM intervention) is of value compared with community-based standard care, as more research is needed to clarify the effects of non-ICM versus those of standard care. 


\section{Implications for research}

\section{General}

First, as we have said previously, reporting of research does seem to have improved across time, and as a result the details of the practice of modern studies are much easier to understand.

However, this review illustrates how scale measurements are much more widespread than simple clinical questions for assessing clinical outcomes. We suggest that binary data are less ambiguous than continuous. There were many scales for the same outcome, further complicating matters: we found many studies assessing the same outcome on different scales and therefore did not feel justified to run a meta-analysis. For example, there was need for consistency in approach regarding social functioning outcomes. Heterogeneous measurements were used to describe the same outcome. This is not very informative - but this review illustrates opportunities lost by researchers. As it is possible that the time for more studies has past, by not having consistency, we will always be left in doubt about important effects of care. Finally, we presume that the use of scales may discourage any worker committed to patient care from taking part in an experimental study.

More attention should be placed on patient and family perspectives, in terms of detecting patient and carer satisfaction, quality of life, and family burden.

\section{Specific}

\subsection{More reviews}

We currently know of no review comparing non-ICM with standard care and reporting relevant outcomes. This should be undertaken. In addition, we excluded several good studies from this review as they evaluated mixed models of care, or models plus other interventions such as cognitive behavioural therapy. These studies do merit further attention from reviewers and could help clarify further ways by which either the ICM model develops or new and yet more holistic approaches evolve.

\subsection{Developing this review}

A full data set with all individual participant data would help in avoiding some biases and allow re-analysis using unified definitions of outcome. Any relevant studies in this area should make a provision for prospectively providing data compatible with this review.

\subsection{More trials}

We do not think that more trials comparing current ICM with standard care or non-ICM are justified. We do think that the features of ICM that may improve outcome should be researched, as it may be that the model of intervention is effective only because of some of its features. This work may involve more observational studies in order to evolve the ICM model to new and better packages of care.

\section{ACKN OWLEDGEMENTS}

We acknowledge the additional authors of the two previous Cochrane Reviews, Marshall 2000a and Marshall 2000b, and the authors of a further study, Burns 2007, that were the forerunners of this review: Tom Burns, Jocelyn Catty, Michael Dash, Alastair Gray, Rex Green, Austin Lockwood, and Chris Roberts.

We would like to thank Miranda Mugford and lan Shemilt for economic advice and Julian Higgins for statistical advice.

We acknowledge the following authors who provided us with further information on their trials through personal communication: Bjorkman-Sweden 2002, Essock-Connecticut1 1995, Essock-Connecticut2 2006, Hargreaves-California, HollowayUK 1996, LEO-UK 1994, Malm-Sweden 2003, REACT-UK 2002, RutterUK, Shern-USA2, Tyrer-UK 1995.

We are grateful for the substantial contribution of Clive Adams and the staff at the Cochrane Schizophrenia Group in Nottingham, UK, for all their practical help and spiritual support during our review process.

Parts of this review were generated using RevMan HAL v 4.2. You can find more information about RevMan HAL here.

We would also like to acknowledge the The National Institute for Health Research (NIHR), the largest single funder of the Cochrane Schizophrenia Group. NIHR provided an incentive grant to help authors incorporate results from the 2015 update search (see Sources of support).

Disclaimer: The views and opinions expressed therein are those of the authors and do not necessarily reflect those of the NIHR, NHS or the Department of Health. 


\section{R E F E R E N C E S}

\section{References to studies included in this review}

\section{Aberg-Wistedt-Sweden 1995 \{published data only\}}

Aberg-Wistedt A, Cressell T, Lidberg Y, Liljenberg B, Osby U. Two-year outcome of team-based Intensive Case Management for patients with schizophrenia. Psychiatric Services 1995;46:1263-6.

\section{Audini-UK 1994 \{published data only\}}

* Audini B, Marks IM, Lawrence RE, Connolly J, Watts V. Homebased versus out-patient-in-patient care for people with serious mental illness. Phase II of a controlled study. British Journal of Psychiatry 1994;165(August):204-10.

Knapp MRJ, Marks IM, Wolstenholme J, Beecham JK, Astin J, Audini B, et al. Home-based versus hospital-based care for serious mental illness: controlled cost-effectiveness study over four years. British Journal of Psychiatry 1998;172(6):506-12.

Bjorkman-Sweden 2002 \{published and unpublished data\} Bjorkman T. Further information on case management study [personal communication]. Email to: M Dieterich 22 April 2009.

Bjorkman T, Hansson L, Sandlund M. Outcome of case management based on the strengths model compared to standard care. A randomised controlled trial. Social Psychiatry and Psychiatric Epidemiology 2002;37(4):147-52.

\section{Bond-Chicago1 1990 \{published data only\}}

Bond GR, Witheridge TF, Dincin J, Wasmer D, Webb J, De GraafKaser R. Assertive community treatment for frequent users of psychiatric hospitals in a large city: a controlled study. American Journal of Community Psychology 1990;18(6):865-91.

\section{Bond-Indiana1 (A) \{published data only\}}

Bond GR, Miller LD, Krumwied RD, Ward RS. Assertive case management in three CMHCs: a controlled study. Hospital and Community Psychiatry 1988;39(4):411-8. [PUBMED: 2836295]

Bond-Indiana1 (B) \{published data only\}

See above Bond-Indiana1.

Bond-Indiana1 (C) \{published data only\} See above Bond-Indiana1.

\section{Bond-Indiana1 1988 \{published data only\}}

Bond GR, Miller LD, Krumwied RD, Ward RS. Assertive case management in three CMHCs: a controlled study. Hospital and Community Psychiatry 1988;39(4):411-8. [PUBMED: 2836295]

\section{Bush-Georgia 1990 \{published data only\}}

Bush CT, Langford MW, Rosen P, Gott W. Operation outreach: Intensive Case Management for severely psychiatrically disabled adults. Hospital and Community Psychiatry 1990;41(6):647-9. [PUBMED: 2361668]

Chandler-California1 (A) \{published data only\} See Chandler-California 1991.
Chandler-California1 (B) \{published data only\} See Chandler-California 1991.

Chandler-California1 1991 \{published data only\}

Chandler D, Hu WT, Meisel J, McGowen M, Madison K. Mental health costs, other public costs, and family burden among mental health clients in capitated integrated service agencies. Journal of Mental Health Administration 1997;24(2):178-88.

Chandler D, Meisel J, Hu T, McGowen M, Madison K. A capitated model for a cross-section of severely mentally ill clients: employment outcomes. Community Mental Health Journal 1997;33(6):501-16. [PUBMED: 9435997]

Chandler D, Meisel J, Hu TW, McGowen M, Madison K. Administrative update. A capitated model for a cross-section of severely mentally ill clients: hospitalization. Community Mental Health Journal 1998;34(1):13-26. [PUBMED: 9559237]

* Chandler D, Meisel J, Hu TW, McGowen M, Madison K. Client outcomes in a three year controlled study of an integrated service agency model. Psychiatric Services 1996;47(12):1337-43.

Chandler D, Meisel J, McGowen M, Mintz J, Madison K. Client outcomes in two model capitated integrated service agencies. Psychiatric Services 1996;47(2):175-80. [PUBMED: 8825255]

Hargreaves WA. A capitation model for providing mental health services in California. Hospital and Community Psychiatry 1992;43:275-7.

Hu T, Jerrell J. Cost-effectiveness of alternative approaches in treating severely mentally ill in California. Schizophrenia Bulletin 1991;17(3):461-8. [PUBMED: 1947870]

Meisel J, Chandler D. The Integrated Service Agency Model. California: Lewin-VHI, 1995.

\section{Chan-Hong Kong 2000 \{published data only\}}

Chan S, Mackenzie A, Jacobs P. Cost-effectiveness analysis of case management versus a routine community care organization for patients with chronic schizophrenia. Archives of Psychiatric Nursing 2000;14(2):98-104. [MEDLINE: 10783528]

Curtis-New York 1992 \{published data only\}

Curtis JL, Millman EJ, Struening E, D'Ercole A. Deaths among former psychiatric inpatients in an outreach case management programme. Psychiatric Services 1996;47(4):398-402.

Curtis JL, Millman EJ, Struening E, D'Ercole A. Effect of case management on rehospitalization and utilization of ambulatory care services. Hospital and Community Psychiatry 1992;43(9):895-9.

Curtis JL, Millman EJ, Struening EL, D'Ercole A. Does outreach case management improve patients' quality of life?. Psychiatric Services 1998;49(3):351-4.

D'Ercole A, Struening E, Curtis JL, Millman EJ, Morris A. Effects of diagnosis, demographic characteristics, and case management on rehospitalization. Psychiatric Services 1997;48(5):682-8. 


\section{Cusack-North Carolina \{published data only\}}

Cusack KJ, Morrissey JP, Cuddeback GS, Prins A, Williams DM. Criminal justice involvement, behavioral health service use, and costs of forensic assertive community treatment: a randomized trial. Community Mental Health Journal 2010;46(4):356-63. [MEDLINE: 20217230]

Drake-NHamp (A) \{published data only\}

See above Drake-NHamp.

Drake-NHamp (B) \{published data only\}

See above Drake-NHamp.

Drake-NHamp (C) \{published data only\}

See above Drake-NHamp.

Drake-NHamp (D) \{published data only\}

See above Drake-NHamp.

Drake-NHamp (E) \{published data only\}

See above Drake-NHamp.

Drake-NHamp (F) \{published data only\}

See above Drake-NHamp.

Drake-NHamp (G) \{published data only\}

See above Drake-NHamp.

Drake-NHamp 1998 \{published and unpublished data\}

Clark RE, Teague GB, Ricketts SK, Bush PW, Xie H, McGuire TG, et al. Cost-effectiveness of assertive community treatment versus standard case management for persons with co-occurring severe mental illness and substance use disorders. Health Services Research 1998;33(5):1285-308.

Cleary M, Hunt G, Matheson S, Siegfried N, Walter G. Psychosocial interventions for people with both severe mental illness and substance misuse. Cochrane Database of Systematic Reviews 2008, Issue 1. [DOI: 10.1002/14651858.CD001088.pub2]

* Drake RE, McHugo GJ, Clark RE, Teague GB, Xie H, Miles K, et al. Assertive community treatment for patients with co-occurring severe mental illness and substance use disorder: a clinical trial. American Journal of Orthopsychiatry 1998;68(2):201-15.

McHugo GJ, Drake RE, Teague GB, Xie H. Fidelity to assertive community treatment and client outcomes in the New Hampshire dual disorders study. Psychiatric Services 1999;50(6):818-24.

Xie H, McHugo GJ, Helmstetter BS, Drake RE. Three-year recovery outcomes for long term patients with co-occurring schizophrenic and substance use disorders. Schizophrenia Research 2005;75(2-3):337-48. [PUBMED: 15885525]

Essock-Connecticut1 1995 \{published and unpublished data\} Essock S. Further information about a trial [personal communication]. Email to: M Dieterich 13 May 2009.
Essock SM. Act versus standard case management clients use different services. 153rd Annual Meeting of the American Psychiatric Association; 2000 May 13-18, Chicago (IL). 2000.

Essock SM, Frisman LK, Kontos NJ. Cost-effectiveness of assertive community treatment teams. American Journal of Orthopsychiatry 1998;68(2):179-90.

* Essock SM, Kontos N. Implementing assertive community treatment teams. Psychiatric Services 1995;46(7):679-83.

Essock-Connecticut2 2006 \{published and unpublished data\} Essock S. Further information about a trial [personal communication]. Email to: M Dieterich 13 May 2009.

Essock SM. Assertive community treatment for the dually diagnosed. CRISP Database (www-commons.cit.nih.gov/crisp/ index.html) (accessed 19 February 2001).

* Essock SM, Mueser KT, Drake RE, Covell NH, McHugo GJ, Frisman LK, et al. Comparison of ACT and standard case management for delivering integrated treatment for cooccurring disorders. Psychiatric Services 2006;57(2):185-96. [PUBMED: 16452695]

Manuel JI, Covell NH, Jackson CT, Essock SM. Does assertive community treatment increase medication adherence for people with co-occurring psychotic and substance use disorders?. Journal of the American Psychiatric Nurses Association 2011;17(1):51-6.

\section{Ford-UK 1995 \{published data only\}}

Ford R, Barnes A, Davies R, Chalmers C, Hardy P, Muijen M. Maintaining contact with people with severe mental illness: 5year follow-up of assertive outreach. Psychiatric Epidemiology 2001;36(9):444-7.

* Ford R, Beadsmoore A, Ryan P, Repper J, Craig T, Muijen M. Providing the safety net: case management for people with a serious mental illness. Journal of Mental Health 1995;4(1):91-7.

Ford R, Raftery J, Ryan P, Beadsmoore A, Craig T, Muijen M. Intensive case management for people with serious mental illness - Site 2: cost-effectiveness. Journal of Mental Health 1997;6(2):191-9.

* Ford R, Ryan P, Beadsmore A, Craig T, Muijen M. Intensive care management for people with serious mental illness site 2: clinical and social outcome. Journal of Mental Health 1997;6(2):181-90.

Ryan P, Ford R, Beadsmoore A, Muijen M. The enduring relevance of case management. British Journal of Social Work 1999;29(1):97-125.

Hampton-Illinois (A) \{published data only\} See above Hampton-Illinois.

Hampton-Illinois (B) \{published data only\} See above Hampton-Illinois. 
Hampton-Illinois 1992 \{published data only\}

* Hampton B, Korr W, Bond G, Mayes J, Havis P. Integration services system approach to avert homelessness; CSP homeless prevention project for $\mathrm{HMI}$ adults. Final report to State of Illinois NIMH Demonstration Grant Project 1992.

Korr WS, Joseph A. Housing the homeless mentally ill: findings from Chicago. Journal of Social Service Research 1996;21(1):53-68.

\section{Harrison-Read-UK 2000 \{published data only\}}

Harrison-Read P. A randomised controlled trial of short term intensive community case management of severe mental illness: an assessment of its benefit to 'heavy users' of hospital services and cost effectiveness. National Research Register 2000.

Harrison-Read P. A randomised controlled trial of short term intensive community case management of severe mental illness: an assessment of its benefit to 'heavy users' of hospital services and cost effectiveness. www.isrctn.com/ ISRCTN56473864 (accessed: 31 January 2004). [ISRCTN: 56473864]

* Harrison-Read P, Lucas B, Tyrer P, Ray J, Shipley K, Simmonds S, et al. Heavy users of acute psychiatric beds: randomized controlled trial of enhanced community management in an outer London borough. Psychological Medicine 2002;32(3):403-16.

\section{Herinckx-Oregon 1996 \{published data only\}}

* Clarke GN, Herinckx HA, Kinney RF, Paulson RI, Cutler DL, Lewis K, et al. Psychiatric hospitalizations, arrests, emergency room visits, and homelessness of clients with serious and persistent mental illness: findings from a randomized trial of two ACT programmes vs. usual care. Mental Health Services Research 2000;2(3):155-64. [PUBMED: 11256724]

* Herinckx HA, Kinney RF, Clarke GN, Paulson RI. Assertive community treatment versus usual care in engaging and retaining clients with severe mental illness. Psychiatric Services 1997;48(10):1297-306. [PUBMED: 9323749]

Paulson R, Herinckx H, Demmler J, Clarke G, Cutler D, Birecree E. Comparing practice patterns of consumer and nonconsumer mental health service providers. Community Mental Health Journal 1999;35(3):251-69.

Paulson RI, Clarke G, Herinckx H, Kinney R, Lewis K, Oxman E. First year outcomes from a randomised trial comparing consumer and non-consumer assertive community treatment teams with usual care. Fourth International Conference on Schizophrenia - 1996: Breaking Down the Barriers; 1996 Oct 6-9; Vancouver, Canada. 1996.

\section{Holloway-UK 1996 \{published and unpublished data\}}

Cullen D, Waite A, Oliver N, Carson J, Holloway F. Case management for the mentally ill: a comparative evaluation of client satisfaction. Health and Social Care in the Community 1997;5:106-15.

Holloway F. Further information on ICM study [personal communication]. Email to: M Dieterich 15 April 2009.
* Holloway F, Carson J. Intensive case management for the severely mentally ill. Controlled trial. British Journal of Psychiatry 1998;172:19-22. [PUBMED: 9534826]

Holloway F, Carson J. Intensive case management: does it work?. Eighth Congress of the Association of European Psychiatrists; 1996 Jul 7-12; London, UK. 1996.

Holloway F, Carson J. Subjective quality of life, psychopathology, satisfaction with care and insight: an exploratory study. International Journal of Social Psychiatry 1999;45(4):259-67. [PUBMED: 10689609]

Holloway F, Murray M, Squire C, Carson J. Intensive case management: putting it into practice. Psychiatric Bulletin 1996;20:395-7.

\section{Jerrell-SCarolina1 1991 \{published data only\}}

$\mathrm{Hu}$ T, Jerrell J. Cost-effectiveness of alternative approaches in treating severely mentally ill in California. Schizophrenia Bulletin 1991;17(3):461-8. [PUBMED: 1947870]

Hu TW, Jerrell JM. Estimating the cost impact of three case management programmes for treating people with severe mental illness. British Journal of Psychiatry 1998;173(36):26-32. [PUBMED: 9829008]

* Jerrell JM. Toward managed care for persons with severe mental illness: implications from a cost-effectiveness study. Health Affairs 1995;14:197-207. [PUBMED: 7498892]

Schmidt-Posner J, Jerrell JM. Qualitative analysis of three case management programmes. Community Mental Health Journal 1998;34(4):381-92.

\section{Johnston-Australia 1998 \{published data only\}}

* Issakidis C, Sanderson K, Teesson M, Johnston S, Buhrich N. Intensive case management in Australia: a randomized controlled trial. Acta Psychiatrica Scandinavica 1999;99:360-7. [PUBMED: 10353452]

* Johnston S, Salkeld G, Sanderson K, Issakidis C, Teesson M, Buhrich N. Intensive case management: a cost effectiveness analysis. Australian and New Zealand Journal of Psychiatry 1998;32(4):551-9. [PUBMED: 9711370]

\section{Lehman-Maryland1 1994 \{published and unpublished data\}} Dixon L, Bruce D, Eimer K, Anthony F. Outcomes of homeless persons with mental illness and substance use disorders. 149th Annual Meeting of the American Psychiatric Association; 1996 May 4-9, New York (NY). 1996.

Dixon L, Kernan E, Krauss N, Lehman A, Deforge BR. Assertive community treatment for homeless adults with severe mental illness in Baltimore. In: Breakey WR, Thompson JW editor(s). Mentally Ill and Homeless: Special Programs for Special Needs. Chronic Mental Illness. Harwood Academic, 1997.

Dixon L, Krauss N, Myers P, Lehman A. Clinical and treatment correlates of access to section 8 certificates for homeless mentally ill persons. Hospital and Community Psychiatry 1994;45(12):1196-200. 
Dixon L, Weiden P, Torres M, Lehman A. Assertive community treatment and medication compliance in the homeless mentally ill. American Journal of Psychiatry 1997;154(9):1302-4.

Hackman AL. Assertive community treatment with homeless individuals. 156th Annual Meeting of the American Psychiatric Association; 2003 May 17-22; San Francisco. 2003.

Hackman AL, Dixon LB, Postrado LT, Delahanty JC. Somatic issues in persons with severe psychiatric illness and homelessness. 151st Annual Meeting of the American Psychiatric Association; 1998 May 30 - Jun 4; Toronto. 1998.

Lehman AF, Dixon L, Hoch JS, DeForge B, Kernan E, Frank R. Cost-effectiveness of assertive community treatment for homeless persons with severe mental illness. British Journal of Psychiatry 1999;174:346-52. [PUBMED: 10533554]

Lehman AF, Dixon LB, DeForge BR, Kernan E. Assertive community treatment for homeless persons with schizophrenia and other chronic mental disorders. Schizophrenia Research 1995;15(1, 2):128.

* Lehman AF, Dixon LB, Kernan E, DeForge BR, Postrado LT. A randomized trial of assertive community treatment for homeless persons with severe mental illness. Archives of General Psychiatry 1997;54(11):1038-43. [PUBMED: 9366661]

Lehman AF, Dixon LB, Kernan E, Deforge B. Assertive treatment for the homeless mentally ill. 148th Annual Meeting of the American Psychiatric Association; 1995 May 20-25; Miami. 1995.

Marshall M, Lockwood A. Assertive community treatment for people with severe mental disorders. Cochrane Database of Systematic Reviews 2000, Issue 2. [DOI: 10.1002/14651858.CD001089]

Macias-Utah 1994 \{published data only\}

Macias C, Kinney R, Farley OW, Jackson R, Vos B. The role of case management within a community support system: partnership with psychosocial rehabilitation. Community Mental Health Journal 1994;30(4):323-39. [PUBMED: 7956109]

Marshall-UK 1995 \{published and unpublished data\}

Gray AM, Marshall M, Lockwood A, Morris J. Problems in conducting economic evaluations alongside clinical trials. Lessons from a study of case management for people with mental disorders. British Journal of Psychiatry 1997;170:47-52. [PUBMED: 9068775]

Marshall M, Gray A, Lockwood A, Green R. Case management for people with severe mental disorders . Cochrane Database of Systematic Reviews 2000, Issue 2. [DOI: 10.1002/14651858.CD000050]

Marshall M, Lockwood A, Gath D. Social services casemanagement for long term mental disorders: a randomised controlled trial. Lancet 1995;345(8947):409-12. [PUBMED: 7853949]

McDonel-Indiana (A) \{published data only\} See above McDonel-Indiana.
McDonel-Indiana (B) \{published data only\}

See above McDonel-Indiana.

McDonel-Indiana 1997 \{published data only\}

* Fekete DM, Bond GR, McDonel EC, Salyers M, Chen A, Miller L. Rural assertive community treatment: a field experiment. Psychiatric Rehabilitation Journal 1998;21(4):371-9.

McDonel EC, Bond GR, Salyers M, Fekete D, Chen A, McGrew JH, et al. Implementing assertive community treatment programmes in rural settings. Administration and Policy in Mental Health 1997;25(2):153-73.

Morse-Missouri1 1992 \{published data only\}

Morse GA, Calsyn RJ, Allen G, Tempelhoff B, Smith R. Experimental comparison of the effects of three treatment programmes for homeless mentally ill people. Hospital and Community Psychiatry 1992;43(10):1005-9.

\section{Morse-Missouri3 2005 \{published data only\}}

Calsyn RJ, Yonker RD, Lemming MR, Morse GA, Klinkenberg WD. Impact of assertive community treatment and client characteristics on criminal justice outcomes in dual disorder homeless individuals. Criminal Behaviour and Mental Health 2005;15(4):236-48. [PUBMED: 16575844]

Fletcher TD, Cunningham JL, Calsyn RJ, Morse GA, Klinkenberg WD. Evaluation of treatment programmes for dual disorder individuals: modelling longitudinal and mediation effects. Administration and Policy in Mental Health 2008;35(4):319-36. [PUBMED: 18506618]

* Morse GA, Calsyn RJ, Dean Klinkenberg W, Helminiak TW, Wolff N, Drake RE, et al. Treating homeless clients with severe mental illness and substance use disorders: costs and outcomes. Community Mental Health Journal 2006;42(4):377-404. [PUBMED: 16897413]

Morse GA, Calsyn RJ, Klinkenberg WD, Cunningham J, Lemming MR. Integrated treatment for homeless clients with dual disorders: a quasi-experimental evaluation. Journal of Dual Diagnosis 2008;4(3):219-37.

Muijen-UK2 1994 \{published data only\}

* McCrone P, Beecham J, Knapp M. Community psychiatric nurse teams: cost-effectiveness of intensive support versus generic care. British Journal of Psychiatry 1994;164(2):218-21.

Muijen M, Cooney M, Strathdee G, Bell R, Hudson A. Community psychiatric nurse teams: intensive support versus generic care. British Journal of Psychiatry 1994;165:211-7.

Muller-Clemm-Canada 1996 \{published data only\}

Muller-Clemm WJ. Halting the 'Revolving Door' of Serious Mental Illness: Evaluating an assertive case management programme (deinstitutionalization, community mental health). University of Victoria, Canada, 1996.

Okpaku-Tennessee 1997 \{published data only\}

Okpaku SO, Anderson KH, Sibulkin AE, Butler JS, Bickman L. The effectiveness of a multidisciplinary case management 
intervention on the employment of SSDI applicants and beneficiaries. Psychiatric Rehabilitation Journal 1997;20:34-41.

\section{OPUS-Denmark 1999 \{published data only\}}

Austin S, Seche R, Hagen R, Mors O, Nordentoft M. Remission, metacognitive processes and quality of life-outcomes from opus trial. A 10 year follow-up of a randomized multi-center trial of intensive early intervention vs. standard treatment for patients with first episode schizophrenia spectrum disorder. Schizophrenia Bulletin 2011;1:258.

Bertelsen M. Randomized controlled trial of two-years integrated treatment versus standard treatment of patients with first-episode of schizophrenia or psychosis, five years follow-up. The opus trial. Schizophrenia Bulletin 2005;31:519-20.

Bertelsen M, Jeppesen P, Petersen L, Thorup A, Ohlenschlaeger J, Le Quach P, et al. Course of illness in a sample of 265 patients with first-episode psychosis - five-year follow-up of the Danish OPUS trial. Schizophrenia Research 2009;107(2-3):173-8. [MEDLINE: 18945593]

Bertelsen M, Jeppesen P, Petersen L, Thorup A, Ohlenschlaeger J, Le Quach P, et al. First episode of psychosis intensive early intervention programme versus standard treatment - secondary publication. Ugeskrift for Laeger 2009;171(41):2992-5. [MEDLINE: 19824225]

* Bertelsen M, Jeppesen P, Petersen L, Thorup A, Ohlenschlaeger J, Le Quach P, et al. Five-year follow-up of a randomized multi-centre trial of intensive early intervention vs standard treatment for patients with a first episode of psychotic illness: the OPUS trial. Archives of General Psychiatry 2008;65(7):762-71. [PUBMED: 18606949]

Bertelsen M, Jeppesen P, Petersen L, Thorup A, Ohlenschlaeger J, Le Quach P, et al. The OPUS-trial; a randomised multicentre single-blinded trial of integrated versus standard treatment for patients with a first episode of psychotic illness - five-years follow-up. Early Intervention in Psychiatry 2008;2(Suppl 1):A6.

Bertelsen M, Le Quach P, Jeppesen P, Petersen L, Thorup A, Ohlenschlaeger J, et al. The OPUS trial: a randomised multicentre trial of intensive early-intervention programme versus standard treatment for 547 first-episode psychotic patients - a five-year follow up. The International Congress on Schizophrenia Research; 2009 Mar 28 - Apr 1; San Diego (CA). 2009.

Bertelsen M, Nordentoft M, Jeppesen P, Petersen L, Thorup A, Le Quach P. The opus trial: results from the five-years follow-up. Schizophrenia Bulletin 2007;33(2):421.

Bertelsen M, Thorup A, Petersen L, Jeppesen P, Oehlenschlager J, Joergensen $P$, et al. The OPUS trial: results from the five-year follow-up. Schizophrenia Research 2006;86(Suppl 1):S43.

Bertelsen MB. RCT of integrated treatment versus standard treatment of patients with first-episode of schizophrenia - 5 years follow up. Schizophrenia Research 2004;70(1):32.
Hastrup LH, Kronborg C, Bertelsen M, Jeppesen P, Jorgensen P, Petersen L, et al. Cost-effectiveness of early intervention in first-episode psychosis: Economic evaluation of a randomised controlled trial (the opus study). British Journal of Psychiatry 2013;202(1):35-41.

Hastrup LH, Kronborg C, Bertelsen M, Jeppesen P, Jorgensen $\mathrm{P}$, Petersen $L$, et al. Cost-effectiveness of early intervention in first-episode psychosis: economic evaluation of a randomised controlled trial (the OPUS study). British Journal of Psychiatry 2013;202(1):35-41. [DOI: 10.1192/bjp.bp.112.112300; PUBMED: 23174515]

Hastrup LH, Kronborg C, Nordentoft M, Simonsen E. Costeffectiveness of a randomized multicenter trial in first-episode psychosis (OPUS) in Denmark. Journal of Mental Health Policy and Economics 2011:10.

Jeppesen P, Abel MB, Krarup G, Jorgensen P, Nordentoft M. Family burden and expressed emotion in first episode psychosis. The OPUS-trial. Third International Conference on Early Psychosis; 2002 Sep 25-28; Copenhagen. 2002.

Jeppesen P, Hemmingsen R, Jírgensen P, Reisby N, Abel MB, Nordentoft M. Opus project: impact of mental disorder on caregivers. 11th World Congress of Psychiatry; 1999 Aug 6-11; Hamburg. 1999; Vol. 2.

Jeppesen P, Hemmingsen R, Reisby N, Jørgensen P, Nordentoft M, Abel MB. The impact of mental disorder on caregivers. 11th World Congress of Psychiatry; 1999 Aug 6-11; Hamburg. 1999; Vol. 2.

Jeppesen P, Nordentoft M, Abel M, Hemmingsen RP, Joergensen, Kassow P. Opus-project: a RCT of integrated psychiatric treatment for recent onset psychotic patients. Schizophrenia Research 2001;49(1, 2):262.

Jeppesen P, Nordentoft M, Jorgensen P, Abel MB, Reisby N, Hemmingsen R, et al. Opus-project: better compliance? A randomised-controlled trial of integrated care of first-episode psychotic patients. Conference abstract. Schizophrenia Research 1999;36(1-3):327.

Jeppesen P, Petersen L, Thorup A, Abel MB, Oehlenschlaeger J, Christensen TO, et al. Integrated treatment of first-episode psychosis: effect of treatment on family burden: OPUS trial. British Journal of Psychiatry 2005;48(Suppl):s85-90. [PUBMED: 16055815]

Jørgensen P, Jeppesen P, Abel MB, Kassow P, Krarup G, Hemmingsen R, et al. Early intervention in schizophrenia. Nordic Journal of Psychiatry 2002;56(2):8.

* Jørgensen P, Nordentoft M, Abel MB, Gouliaev G, Jeppesen P, Kassow $P$. Early detection and assertive community treatment of young psychotics: the Opus study rationale and design of the trial. Social Psychiatry and Psychiatric Epidemiology 2000;35(7):283-7. [PUBMED: 11016522]

Melau M. Treatment response, working alliance and patients' perspective: results from opus i and opus ii. Early Intervention in Psychiatry 2010;4(Suppl 1):152. 
Melau M, Bertelsen M, Jeppesen P, Krarup G, Nordentoft M, Thorup A, et al. A randomised clinical trial of the effect of fiveyears versus two-years specialised assertive intervention for first episode psychosis - the opus-II trial. Schizophrenia Research 2010;117(2-3):526.

Melau M, Jeppesen P, Thorup A, Bertelsen M, Petersen L, Gluud C, et al. The effect of five years versus two years of specialised assertive intervention for first episode psychosis opus ii: study protocol for a randomized controlled trial. Trials 2011;12:72.

Melau M, Thorup A, Bertelsen M, Jeppesen P, Krarup G, Nordentoft M. Does extended specialized intervention for patients with first episode psychosis improve outcome in the critical period? The opus ii trial. Schizophrenia Bulletin 2011;1:315.

NCT00914238. Extended specialized assertive intervention for first episode psychosis. clinicaltrials.gov/ct2/show/ NCT00914238 (first received 28 May 2009).

Nordentoft M. Randomised clinical trial of integrated treatment versus standard treatment in first episode psychosis. www.clinicaltrials.gov (first received 2005).

Nordentoft M, Bertelsen M, Jeppesen P, Thorup A, Petersen L, Ohlenschlaeger J, et al. OPUS trial: a randomised multicentre trial of integrated versus standard treatment for patients with a first episode of psychotic illness. Nordic Journal of Psychiatry 2007;61(6):488.

Nordentoft M, Bertelsen M, Thorup A, Jeppesen P, Petersen L. The opus-trial; a randomised multi-centre trial of integrated versus standard treatment for patients with a first episode of psychotic illness - five-years follow-up. 12th International Congress on Schizophrenia Research; 2009 Mar 28 - Apr 1; San Diego (CA). 2009:370.

Nordentoft M, Bertelsen M, Thorup A, Petersen L. Two versus 5 years of early intensive intervention in first episode psychosis. Early Intervention in Psychiatry 2008;2(Suppl 1):A7.

Nordentoft M, Jeppesen P, Abel M, Kassow P, Petersen L, Thorup A, et al. OPUS study: suicidal behaviour, suicidal ideation and hopelessness among patients with first-episode psychosis. One-year follow-up of a randomised controlled trial. British Journal of Psychiatry 2002;181(Suppl 43):S98-106. [PUBMED: 12271808]

Nordentoft M, Jeppesen P, Abel M, Petersen L, Thorup A, Christensen T, et al. Opus-project: a randomised controlled trial of integrated psychiatric treatment in first-episode psychosis clinical outcome improved. Third International Conference on Early Psychosis; 2002 Sep 25-28; Copenhagen. 2002.

Nordentoft M, Jeppesen P, Jorgensen P, Abel MB, Kassow P, Reisby N, et al. OPUS-project: a randomised controlled trial of first episode psychotic patients: better compliance. Nordic Journal of Psychiatry 2000;54:16.

Nordentoft M, Jeppesen P, Jørgensen P, Abel MB, Kassow P, Reisby N, et al. Opus - project: a randomised controlled trial of first episode psychotic patients: better compliance. Second
International Conference on Early Psychosis; 2000 Mar 31 - Apr 2, New York (NY). 2000.

Nordentoft M, Jeppesen P, Kassow P, Abel M, Petersen L, Thorup A, et al. Opus-project: a randomised controlled trial of integrated psychiatric treatment in first-episode psychosis clinical outcome improved. Schizophrenia Research 2002;53(3 Suppl 1):51.

Nordentoft M, Jeppesen P, Petersen L, Thorup A, Abel M. OPUS project: a randomised controlled trial of integrated psychiatric treatment in first episode psychosis. Ninth International Congress on Schizophrenia Research; 2003 Mar 29 - Apr 2, Colorado Springs (CO). 2003.

Nordentoft M, Jeppesen P, Petersen L, Thorup A, Abel M, Ohlenschlaeger JK, et al. OPUS project: a randomised controlled trial of integrated psychiatric treatment in first episode psychosis. Schizophrenia Research 2003;60(1):297.

Nordentoft M, Jeppesen P, Petersen L, Thorup A, Jorgensen P. Duration of untreated psychosis predicts psychotic symptoms but not negative symptoms. Schizophrenia Bulletin 2005;31:234.

Nordentoft M, Jeppesen P, Petersen L, Thorup A, Krarup G, Abel M, et al. The Danish opus-trial: a randomised controlled trial of integrated treatment among 547 first-episode psychotic patients. One and two years follow-up. Schizophrenia Research 2004;67(1):35-6.

Nordentoft M, Jeppesen P, Petersen L, Thorup A, Ohlenschlaeger J, Christensen T, et al. The OPUS trial: a randomised multi-centre trial of integrated versus standard treatment for 547 first-episode psychotic patients. 12th Biennial Winter Workshop on Schizophrenia; 2004 Feb 7-13, Davos, Switzerland. 2004.

Nordentoft M, Jeppesen P, Petersen L, Thorup A, Ohlenschlaeger J, Christensen T, et al. Transition rates from schizotypal disorder to psychotic disorder for first-contact patients included in the opus trial. A randomized clinical trial of integrated treatment and standard treatment. European Psychiatry 2007;22:S129.

Nordentoft M, Jeppesen P, Ventegodt AT, Joergensen P, Abel M, Petersen L, et al. Opus-project: a randomised controlled trial of first episode psychotic patients: patient satisfaction, depression and suicidal behaviour. Schizophrenia Research 2001;49(1, 2):265.

Nordentoft M, Jorgensen P, Jeppesen P, Kassow P, Abel MB, Resiby N, et al. Opus-project: differences in clinical and social outcome of a randomized controlled trial of integrated care of first-episode psychotic patients. Schizophrenia Research 1999;36(1-3):330.

Nordentoft M, Reisby N, Jeppesen P, Abel M-B, Kassow P, Jírgensen P. Opus-project: differences in treatment outcome of a randomised controlled trial of integrated psychiatric treatment of first-episode psychotic patients. 11th World Congress of Psychiatry; 1999 Aug 6-11; Hamburg. 1999.

Nordentoft M, Thorup A, Petersen L, Jeppesen P, Krarup G, Christensen T, et al. The opus trial: A randomised multi-centre 
trial of integrated versus standard treatment for 547 firstepisode psychotic patients. 13th Biennial Winter Workshop on Schizophrenia Research; 2006 Feb 4-10; Davos, Switzerland. 2006.

Nordentoft M, Thorup A, Petersen L, Ohlenschlaeger J, Melau M, Christensen TO, et al. Transition rates from schizotypal disorder to psychotic disorder for first-contact patients included in the OPUS trial. A randomized clinical trial of integrated treatment and standard treatment. Schizophrenia Research 2006;83(1):29-40. [PUBMED: 16504481]

Nordentoft M, Thorup A, Petersen L, Ohlenschlaeger J, Melau M, Christensen TO, et al. Transition rates from schizotypal disorder to psychotic disorder for first-contact patients included in the opus trial. A randomized clinical trial of integrated treatment and standard treatment. Schizophrenia Research 2006;86(Suppl 1):S44.

Ohlenschlaeger J, Nordentoft M, Thorup A, Jeppesen P, Petersen L, Christensen TO, et al. Effect of integrated treatment on the use of coercive measures in first-episode schizophreniaspectrum disorder. A randomized clinical trial. International Journal of Law and Psychiatry 2008;31(1):72-6. [PUBMED: 18191455]

Ohlenschlaeger J, Thorup A, Petersen L, Jeppesen P, Koster A, Munkner R, et al. Intensive treatment models and coercion. Nordic Journal of Psychiatry 2007;61(5):369-78. [PUBMED: 17990199]

* Petersen L, Jeppesen P, Thorup A, Abel MB, Ohlenschlaeger J, Christensen $\mathrm{TO}$, et al. A randomised multi-centre trial of integrated versus standard treatment for patients with a first episode of psychotic illness. BMJ 2005;331(7517):602-8. [PUBMED: 16141449]

Petersen L, Jeppesen P, Thorup A, Ohlenschlaeger J, Christensen T, Krarup G, et al. Substance abuse in first-episode schizophrenia-spectrum disorders. Schizophrenia Research 2006;86(Suppl 1):S44.

Petersen L, Jeppesen P, Thorup A, Ohlenschlaeger J, Krarup G, Østergård T, et al. Substance abuse and first-episode schizophrenia-spectrum disorders. The Danish OPUS trial. Early Intervention in Psychiatry 2007;1(1):88-96.

Petersen L, Jeppesen P, Ventegodt AT, Abel M, Nordentoft M, Kassow $P$, et al. Opus-project: a randomised controlled trial of first episode psychotic patients: predictors of outcome. Schizophrenia Research 2001;49(1, 2):266.

Petersen L, Nordentoft M, Jeppesen P, Ohlenschaeger J, Thorup A, Christensen TO, et al. Improving 1-year outcome in first-episode psychosis: OPUS trial. British Journal of Psychiatry 2005;48(Suppl):s98-103. [PUBMED: 16055817]

Petersen L, Nordentoft M, Thorup A, Oehlenschlaeger J, Jeppesen P, Christensen T, et al. The OPUS trial: a randomised multi-centre trial of integrated versus standard treatment for 547 first-episode psychotic patients. Schizophrenia Bulletin 2005;31:531.
Secher RG, Hjorthoj CR, Austin SF, Thorup A, Jeppesen P, Mors O, et al. Ten-year follow-up of the OPUS Specialized Early Intervention Trial for Patients With a First Episode of Psychosis. Schizophrenia Bulletin 2015;41(3):617-26.

* Secher RG, Hjorthoj CR, Austin SF, Thorup A, Jeppesen P, Mors O, et al. Ten-year follow-up of the OPUS Specialized Early Intervention Trial for Patients With a First Episode of Psychosis. Schizophrenia Bulletin 2015;41:617-26.

Secher RG, Nordentoft M, Austin S, Mors O. The opus trial: intensive, early, psychosocial intervention versus treatment as usual for people with a first episode within the schizophrenic spectrum. Results from the 10-year follow-up. Early Intervention in Psychiatry 2012;6:21.

Secher RG, Nordentoft M, Austin S, Mors O. The opus trial: intensive, early, psychosocial intervention versus treatment as usual for people with a first episode within the schizophrenic spectrum. Results from the 10-year follow-up. Early Intervention in Psychiatry 2012;6:21.

Stevens H, Agerbo E, Dean K, Mortensen PB, Nordentoft M. Reduction of crime in first-onset psychosis: a secondary analysis of the OPUS randomized trial. Journal of Clinical Psychiatry 2013;74(5):e439-44.

Thorup A, Nordentoft M, Petersen L, Oehlensschlaeger J, Abel M, Jeppesen $P$, et al. The Danish OPUS-project: psychopathology and gender differences in first episode psychotic patients. Third International Conference on Early Psychosis; 2002 Sep 25-28; Copenhagen. 2002.

Thorup A, Petersen L, Jeppesen P, Nordentoft M. The quality of life among first-episode psychotic patients in the opus trial. Schizophrenia Research 2010;116(1):27-34.

Thorup A, Petersen L, Jeppesen P, Ohlenschlaeger J, Christensen T, Krarup G, et al. Integrated treatment ameliorates negative symptoms in first episode psychosis - results from the Danish OPUS trial. Schizophrenia Research 2005;79(1):95-105.

Thorup A, Petersen L, Jeppesen P, Ohlenschlaeger J, Christensen T, Krarup G, et al. Social network among young adults with first-episode schizophrenia spectrum disorders: results from the Danish OPUS trial. Social Psychiatry and Psychiatric Epidemiology 2006;41(10):761-70. [PUBMED: 16900304]

Ventegodt AT, Jeppesen P, Petersen L, Abel M, Nordentoft M, Kassow $P$, et al. Opus-project: a randomised controlled trial of first episode psychotic patients: gender differences, social network and negative symptoms. Schizophrenia Research 2001;49(1, 2):267.

\section{Pique-California 1999 \{published data only\}}

Pique T. Cost-Effectiveness of an African-American Focus Assertive Community Treatment Programme. Alameda: California School of Professional Psychology, 1999.

\section{Quinlivan-California 1995 \{published data only\}}

Quinlivan R, Hough R, Crowell A, Beach C, Hofstetter R, Kenworthy K. Service utilization and costs of care for 
severely mentally ill clients in an Intensive Case Management programme. Psychiatric Services 1995;46:365-71.

REACT-UK 2002 \{published and unpublished data\}

Killaspy $\mathrm{H}$. Further information on REACT study for Cochrane Systematic Review [personal communication]. Email to: M Dieterich 8 June 2009.

* Killaspy H, Bebbington P, Blizard R, Johnson S, Nolan F, Pilling $S$, et al. The REACT study: randomised evaluation of assertive community treatment in north London. BMJ 2006;332(7545):815-8

Killaspy H, Kingett S, Bebbington P, Blizard R, Johnson S, Nolan F, et al. Randomised evaluation of assertive community treatment: 3-year outcomes. British Journal of Psychiatry 2009;195(1):81-2.

Killaspy H, Mas-Exposito L, Marston L, King M. Ten year outcomes of participants in the REACT (Randomised Evaluation of Assertive Community Treatment in North London) study. BMC Psychiatry 2014;14:296. [PUBMED: 25342641]

King M. Effectiveness of assertive outreach - a randomised controlled trial. National Research Register 2002; Vol. 1.

McCrone P, Killaspy H, Bebbington P, Johnson S, Nolan F, Pilling S, et al. The REACT study: cost-effectiveness analysis of assertive community treatment in north London. Psychiatric Services 2009;60(7):908-13.

\section{Rosenheck-USA 1993 \{published data only\}}

Rosenheck R. Intersite variation in the impact of intensive psychiatric community care on hospital use: a research network to evaluate assertive community treatment. American Journal of Orthopsychiatry 1998;68(2):191-200.

Rosenheck R, Neale M, Gallup P. Community-oriented mental health care: assessing diversity in clinical practice. Psychosocial Rehabilitation Journal 1993;16:39-50.

Rosenheck R, Neale M, Leaf P, Milstein R, Frisman L. Multisite experimental cost study of intensive psychiatric community care. Schizophrenia Bulletin 1995;21:129-40.

Rosenheck RA, Neale MS. Cost-effectiveness of intensive psychiatric community care for high users of in-patient services. Archives of General Psychiatry 1998;55(5):459-66.

Rosenheck-USA-GMS \{published data only\}

See above Rosenheck-USA

Rosenheck-USA-GMS (A) \{published data only\}

See above Rosenheck-USA.

Rosenheck-USA-GMS (B) \{published data only\}

See above Rosenheck-USA

Rosenheck-USA-GMS (D) \{published data only\}

See above Rosenheck-USA.

Rosenheck-USA-GMS (F) \{published data only\}

See above Rosenheck-USA.
Rosenheck-USA-GMS (I) \{published data only\} See above Rosenheck-USA.

Rosenheck-USA-NP \{published data only\} See above Rosenheck-USA.

Rosenheck-USA-NP (C) \{published data only\} See above Rosenheck-USA.

Rosenheck-USA-NP (E) \{published data only\} See above Rosenheck-USA.

Rosenheck-USA-NP (G) \{published data only\} See above Rosenheck-USA.

Rosenheck-USA-NP (H) \{published data only\} See above Rosenheck-USA.

Salkever-SCarolina 1999 \{published data only\}

Salkever D, Domino ME, Burns BJ, Santos AB, Deci PA, Dias J, et al. Assertive community treatment for people with severe mental illness: the effect on hospital use and costs. Health Services Research 1999;34(2):577-601. [PUBMED: 10357291]

Shern-USA1 2000 \{published data only\}

* Shern DL, Tsemberis S, Anthony W, Lovell AM, Richmond L, Felton CJ, et al. Serving street-dwelling individuals with psychiatric disabilities: Outcomes of a psychiatric rehabilitation clinical trial. American Journal of Public Health 2000;90(12):1873-8. [PUBMED: 11111259]

Tsemberis SJ, Moran L, Shinn M, Asmussen SM, Shern DL. Consumer preference programmes for individuals who are homeless and have psychiatric disabilities: a drop-in center and a supported housing programme. American Journal of Community Psychology 2003;32(3-4):305-17. [PUBMED: 14703266]

\section{Solomon-Pennsylvania 1994 \{published data only\}}

Draine JN. Case Management for Homeless Mentally Ill Adult Offenders: a Re-Examination of Random Field Trial Data. Philadelphia: University of Pennsylvania, 1995.

Solomon P, Draine J. Issues in serving the forensic client. Social Work 1995;40(1):25-33.

Solomon P, Draine J. Jail recidivism in a forensic case management programme. Health and Social Work 1995;20(3):167-73.

Solomon P, Draine J. One-year outcomes of a randomized trial of case management with seriously mentally ill clients leaving jail. Evaluation Review 1995;19(3):256-73.

Solomon P, Draine J, Meyerson A. Jail recidivism and receipt of community mental health services. Hospital and Community Psychiatry 1994;45(8):793-7. [PUBMED: 7982695]

Sytema-Netherlands 1999 \{published data only\}

Burns T. The UK700 study: impact of case load size in case management. Current Opinion in Psychiatry 1999;12:302. 
Byford S, Fiander M, Torgerson DJ, Burns T, Van Horn E, Gilvarry $C$, et al. Cost-effectiveness of intensive v. standard case management for severe psychotic illness - UK700 case management trial. British Journal of Psychiatry 2000;176:537-43.

ISRCTN11281756. Effectiveness of assertive community treatment versus care-as-usual for patients with severe and persistent psychiatric disorders. www.isrctn.com/ ISRCTN11281756 (first received 20 December 2005).

Samele C, Gilvarry C, Walsh E, Manley C, Van Os J, Murray R. Patients' perceptions of intensive case management. Psychiatric Services 2002;53(11):1432-7.

Samele C, Walsh E, Gilvarry C, Manley C, Tattan T, Fahy T, et al. Non-alcohol substance misuse, outcome and intensive case management. European Psychiatry 2002;17(Suppl 1):216s.

Sytema S, Wunderink L, Bloemers W, Roorda L, Wiersma D. Assertive community treatment in the Netherlands: a randomized controlled trial. Acta Psychiatrica Scandinavica 2007;116(2):105-12. [PUBMED: 17650271]

\section{Test-Wisconsin 1985 \{published data only\}}

Angell B, Test MA. The relationship of clinical factors and environmental opportunities to social functioning in young adults with schizophrenia. Schizophrenia Bulletin 2002;28(2):259-71.

Cohen LJ, Test MA, Brown RL. Suicide and schizophrenia: data from a prospective community treatment study. American Journal of Psychiatry 1990;147:602-7. [PUBMED: 2327487]

Test MA, Knoedler W, Allness D, Burke S, Brown R, Wallisch L. Community care of schizophrenia: two year findings. 142nd Annual Meeting of the American Psychiatric Association; 1989 May 6-11; San Francisco. 1989.

Test MA, Knoedler WH, Allness DJ, Burke SS. Characteristics of young adults with schizophrenic disorders treated in the community. Hospital and Community Psychiatry 1985;36(8):853-8. [PUBMED: 4029910]

Test MA, Knoedler WH, Allness DJ, Burke SS, Brown RL, Wallisch LS. Long-term community care through an assertive continuous treatment team. Advances in Neuropsychiatry and Psychopharmacology 1991;1:239-46.

\section{UK700-UK (A) \{published data only\}}

See above UK700-UK.

UK700-UK (B) \{published data only\}

See below UK700-UK.

UK700-UK (C) \{published data only\}

See below UK700-UK.

UK700-UK (D) \{published data only\}

See below UK700-UK.
UK700-UK 1999 \{published data only\}

Austwick C. The design and analysis of randomised trials in health services research, with particular reference to the UK case management trial of psychotic patients. National Research Register 2003.

Burns T. Erratum: Intensive versus standard case management for severe psychotic illness: a randomised trial. Lancet 1999;354(9184):1128.

Burns T. Predictors of outcome at 4 years of psychotic patients treated in two forms of case management. National Research Register 2000.

Burns T. Random controlled trial of Intensive Case Management for people with severe mental illness. National Research Register 2001; Vol. 1.

Burns T, Creed F, Fahy T, Thompson S, Tyrer P, White I. Intensive versus standard case management for severe psychotic illness: a randomised trial. UK 700 Group. Lancet 1999;353(9171):2185-9. [PUBMED: 10392982]

Burns T, Fiander M, Kent A, Ukoumunne OC, Byford S, Fahy T, et al. Effects of caseload size on the process of care of patients with severe psychotic illness. Report from the UK700 trial. British Journal of Psychiatry 2000; Vol. 177:427-33. [PUBMED: 11059996]

Burns T, White I, Byford S, Fiander M, Creed F, Fahy T. Exposure to case management: relationships to patient characteristics and outcome. Report from the UK700 trial. British Journal of Psychiatry 2002;181:236-41. [PUBMED: 12204929]

Byford S, Barber JA, Fiander M, Marshall S, Green J. Factors that influence the cost of caring for patients with severe psychotic illness: report from the UK 700 trial. British Journal of Psychiatry 2001;178:441-7. [MEDLINE: 11331560]

Creed F. Comparison of intensive and standard case management programmes for psychotic patients. www.isrctn.com/ISRCTN15904286 (first received 23 January 2004).

Creed F, Burns T, Butler T, Byford S, Murray R, Thompson S, et al. Comparison of intensive and standard case management for patients with psychosis: rationale of the trial. British Journal of Psychiatry 1999;174:74-8. [PUBMED: 10211155]

Creed F, Butler T, Fraser J, Gater R, Huxley P, Tarrier N, et al. Comparison of intensive and standard case management programmes for psychotic patients (UK 700 study). Data on file 1999.

Fahy TA, Burns T, Creed F, Tyrer P, White I. The outcome of intensive versus standard case management for severe psychotic illness: the UK 700-case management trial conference abstract. Schizophrenia Research 1999;36(1-3):324-5.

Fiander M, Burns T, Ukoumunne OC, Fahy T, Creed F, Tyrer P, et al. Do care patterns change over time in a newly established mental health service? A report from the UK700 trial. European Psychiatry 2006;21(5):300-6. [PUBMED: 16824736] 
Harvey K. The effect of Intensive Case Management on relatives' appraisal of caregiving and psychological distress. National Research Register 2001; Vol. 1.

Harvey K, Burns T, Fiander M, Huxley P, Manley C, Fahy T. The effect of Intensive Case Management on the relatives of patients with severe mental illness. Psychiatric Services 2002;53(12):1580-5. [PUBMED: 12461219]

Hassiotis A, Ukoumunne O, Tyrer P, Piachaud J, Gilvarry C, Harvey K, et al. Prevalence and characteristics of patients with severe mental illness and borderline intellectual functioning: report from the UK700 randomised controlled trial of case management. British Journal of Psychiatry 1999;175:135-40. [PUBMED: 10627795]

Hassiotis A, Ukoumunne OC, Byford S, Tyrer P, Harvey K, Piachaud J, et al. Intellectual functioning and outcome of patients with severe psychotic illness randomised to Intensive Case Management: report from the UK700 trial. British Journal of Psychiatry 2001;178:166-71. [PUBMED: 11157431]

Huxley P, Evans S, Burns T, Fahy T, Green J. Quality of life outcome in a randomized controlled trial of case management. Social Psychiatry and Psychiatric Epidemiology 2001;36(5):249-55. [PUBMED: 11515703]

Jabben N, van Os J, Burns T, Creed F, Tattan T, Green J, et al. Is processing speed predictive of functional outcome in psychosis?. Social Psychiatry and Psychiatric Epidemiology 2008;6:437-44. [DOI: 10.1007/s00127-008-0328-y]

McKenzie K, Samele C, van Horn E, Tattan T, van Os J, Murray R. Comparison of the outcome and treatment of psychosis in people of Caribbean origin living in the UK and British Whites. Report from the UK700 trial. British Journal of Psychiatry 2001;178:160-5. [PUBMED: 11157430]

Moran P, Walsh E, Tyrer P, Burns T, Creed F, Fahy T, et al. Impact of comorbid personality disorder on violence in psychosis. British Journal of Psychiatry 2003;182:129-34.

Theresa T. The effect of Intensive Case Management on compliance with medication and psychiatric services. National Research Register 2000.

Tyrer P, Hassiotis A, Ukoumunne O, Piachaud J, Harvey K. Intensive case management for psychotic patients with borderline intelligence. Lancet 1999;354(9183):999-1000. [MEDLINE: 10501366]

Tyrer P, Manley C, Van Horn E, Leddy D, Ukoumunne OC. Personality abnormality in severe mental illness and its influence on outcome of intensive and standard case management: a randomised controlled trial. European Psychiatry 2000;15(Suppl 1):7-10. [PUBMED: 11520467]

UK700 Group. Cost-effectiveness of intensive v. standard case management for severe psychotic illness: UK700 case management trial. British Journal of Psychiatry 2000;176:537-43. [PUBMED: 10974959]

Walsh E, Gilvarry C, Samele C, Harvey K, Manley C, Tyrer P, et al. Reducing violence in severe mental illness: randomised controlled trial of Intensive Case Management compared with standard care. BMJ 2001;323(7321):1093-6. [PUBMED: 11701572]

Walsh E, Harvey K, White I, Higgitt A, Fraser J, Murray R. Suicidal behaviour in psychosis: prevalence and predictors from a randomised controlled trial of case management: report from the UK700 trial. British Journal of Psychiatry 2001;178:255-60. [PUBMED: 11230037]

Walsh E, Harvey K, White I, Manley C, Fraser J, Stanbridge S, et al. Prevalence and predictors of parasuicide in chronic psychosis. UK700 group. Acta Psychiatrica Scandinavica 1999;100(5):375-82. [PUBMED: 10563455]

Walsh E, Leese M, Byford S, Gilvarry C, Samele C, Tyrer P, et al. Do violent patients benefit from Intensive Case Management? Schizophrenia Research 2002;53(3 Suppl 1):234.

Walsh E, Leese M, Byford S, Gilvarry K, Samele C, Tatten T, et al. The impact of Intensive Case Management on violent patients with psychosis. European Psychiatry 2002;17(Suppl 1):S142.

Walsh E, Moran P, Scott C, Burns T, Tyrer P, Creed F. Reducing violent victimization in severe mental illness. Schizophrenia Research 2004;67(1):11.

Walsh EM. Community risk in psychosis: predictors and management. 158th Annual Meeting of the American Psychiatric Association; 2005 May 21-26; Atlanta (GA). 2005.

Walsh EM, Gilvarry CM, Samele C, Creed F, Tyrer P, Fahy TJ, et al. Reducing violence in psychosis: the effect of Intensive Case Management. 154th Annual Meeting of the American Psychiatric Association; 2001 May 5-10; New Orleans. 2001.

Walsh EM, Gilvarry CM, Samele C, Creed F, Tyrer P, Fahy TJ, et al. Reducing violence in psychosis: the effect of Intensive Case Management. 155th Annual Meeting of the American Psychiatric Association; 2002 May 18-23; Philadelphia. 2002.

\section{References to studies excluded from this review}

Agius-Croatia 2007 \{published data only\}

Agius M, Shah S, Ramkisson R, Murphy S, Zaman R. Three year outcomes of an early intervention for psychosis service as compared with treatment as usual for first psychotic episodes in a standard community mental health team - final results. Psychiatria Danubina 2007;19(3):130-8. [PUBMED: 17914313]

Agius M, Shah S, Ramkisson R, Murphy S, Zaman R. Three year outcomes of an early intervention for psychosis service as compared with treatment as usual for first psychotic episodes in a standard community mental health team. Preliminary results [see comment]. Psychiatria Danubina 2007;19(1-2):10-9. [PUBMED: 17603411]

\section{An 2013 \{published data only\}}

An XD, Li SC 安晓东, 李守春. Effects of different interventions on quality of life and rehabilitation outcomes in patients with chronic schizophrenia [不同干预方式对慢性精神分裂症患者 生活质量和康复影响的研究] . Journal of Psychiatry [精神医 学杂志] 2013, issue 01:25-7. 


\section{An Qi 2013 \{published data only\}}

An Q, Wang HG, Meng XJ 安琦, 王宏刚, 孟祥军. The effects of family intervention on the rehabilitation of patients with newly diagnosed schizophrenia [家庭干预对首诊精神分裂症患者康 复的影响]. Heilongjiang Medicine and Pharmacy [黑龙江医药 科学] 2013, issue 03:59-60.

Bao 2012 \{published data only\}

Bao LP, Shen X, Zhang SF, Cui XQ, Cheng WL 鲍丽萍, 沈曦, 张 世芳, 崔晓青, 程万良. Comparative study of the effects of family intervention for patients with schizophrenia [精神分裂 症家庭干预效果的对照研究]. Sichuan Mental Health [四川精 神卫生] 2012, issue 01:50-1.

Bao-China 2005 \{published data only\}

Bao WQ, Sun XJ, Wang ML. Research on home intervention to community schizophreniform by applying PDCA. Journal of Practical Nursing 2005;21(3A):9-11.

\section{Barbic 2009 \{published data only\}}

Barbic S, Krupa T, Armstrong I. A randomized controlled trial of the effectiveness of a modified recovery workbook program: preliminary findings. Psychiatric Services 2009;60(4):491-7.

Barekatain-Iran 2014 \{published data only\}

Barekatain M, Maracy MR, Rajabi F, Baratian H. Aftercare services for patients with severe mental disorder: a randomized controlled trial. Journal of Research in Medical Sciences 2014;19:240-5.

\section{Bigelow-Oregon 1991 \{published data only\}}

Bigelow DA, Young DJ. Effectiveness of a case management programme. Community Mental Health Journal 1991;27:115-23.

\section{Bond-Chicago2 1989 \{published data only\}}

Bond GR, Witheridge TF, Wasmer D, Dincin J, McRae SA, Mayes J, et al. A comparison of two crisis housing alternatives to psychiatric hospitalization. Hospital and Community Psychiatry 1989;40(2):177-83. [PUBMED: 2914671]

\section{Bond-Indiana2 1991 \{published data only\}}

Bond GR, McDonel EC, Miller LD, Pensec M. Assertive community treatment and reference groups - an evaluation of their effectiveness for young adults with serious mental illness and substance abuse problems. Special Issue - Serving persons with dual disorders of mental illness and substance use. Psychosocial Rehabilitation Journal 1991;15(2):31-43.

\section{Borland-Washington 1989 \{published data only\}}

Borland A, McRae J, Lycan C. Outcomes of five years of continuous Intensive Case Management. Hospital and Community Psychiatry 1989;40:369-76.

\section{Borras 2009 \{published data only\}}

Borras L, Boucherie M, Mohr S, Lecomte T, Perroud N, Huguelet $P$. Increasing self-esteem: efficacy of a group intervention for individuals with severe mental disorders. European Psychiatry 2009;24(5):307-16. [MEDLINE: 19321313]
Botha 2014 \{published data only\}

Botha UA, Koen L, Galal U, Jordaan E, Niehaus DJ. The rise of assertive community interventions in South Africa: a randomized control trial assessing the impact of a modified assertive intervention on readmission rates; a three year followup. BMC Psychiatry 2014;14:56.

Botha UA, Koen L, Joska JA, Hering LM, Oosthuizen PP. Assessing the efficacy of a modified assertive community-based treatment programme in a developing country. BMC Psychiatry 2010;10:73. [MEDLINE: 20843301]

Burns 2013 \{published data only\}

Burns T, Rugkasa J, Molodynski A, Dawson K, Yeeles K, VazquezMontes $\mathrm{M}$, et al. Community treatment orders for patients with psychosis (OCTET): a randomised controlled trial. Lancet 2013;381:1627-33.

\section{Burns-UK 1993 \{published data only\}}

Burns T, Beadsmoore A, Bhat AV, Oliver A, Mathers C. A controlled trial of home-based acute psychiatric services. I: Clinical and social outcome. British Journal of Psychiatry 1993;163:49-54. [PUBMED: 8353699]

Burns T, Raftery J. Cost of schizophrenia in a randomized trial of home based treatment. Schizophrenia Bulletin 1991;17(3):407-10. [PUBMED: 1947865]

Burns T, Raftery J, Beadsmoore A, McGuigan S, Dickson M. A controlled trial of home-based acute psychiatric services. II: Treatment patterns and costs. British Journal of Psychiatry 1993;163:55-61. [PUBMED: 8353700]

\section{Champney-Ohio 1992 \{published data only\}}

Champney TF, Dzurec LC. Involvement in productive activities and satisfaction with living situation among severely mentally disabled adults. Hospital and Community Psychiatry 1992:43:899-903.

\section{Chandler 1999 \{published data only\}}

Chandler D, Spicer G, Wagner M, Hargreaves W. Costeffectiveness of a capitated assertive community treatment program. Psychiatric Rehabilitation Journal 1999;22:327-36.

\section{Chandler 2000 \{published data only\}}

Chandler D, Quinlivan R. Social skills training modules in an intensive community support program. Administration and Policy in Mental Health 2000;27(4):211-20.

\section{Chandler-California2 2006 \{published data only\}}

Chandler DW, Spicer G. Integrated treatment for jail recidivists with co-occurring psychiatric and substance use disorders. Community Mental Health Journal 2006;42(4):405-25. [PUBMED: 16933087]

\section{Chang 2013 \{published data only\}}

* Chang WC, Chan GH, Jim OT, Lau ES, Hui CL, Chan SK, et al. Optimal duration of an early intervention programme for firstepisode psychosis: randomised controlled trial. British Journal of Psychiatry 2015;206(6):492-500. 
Chang WC, Chan HK, Jim TT, Wong HY, Hui LM, Chan KW, et al. Randomized controlled trial evaluating 1-year extended case management for first-episode psychosis patients discharged from EASY program in Hong Kong. 14th International Congress on Schizophrenia Research (ICOSR); 2013 April 21-25; Orlando (FL). 2013:324-5.

Chen EYH, Chang WC, Chan SKW, Lam MML, Hung SF, Chung DWS, et al. Community case management for early psychosis: is two years an optimal duration? A randomized controlled study. Hong Kong Medical Journal 2015;21:23-6.

\section{Chatterjee 2014 \{published data only\}}

Chatterjee S, Naik S, John S, Dabholkar H, Balaji M, Koschorke M, et al. Effectiveness of a community-based intervention for people with schizophrenia and their caregivers in India (COPSI): a randomised controlled trial. Lancet 2014;383:1385-94.

\section{Chen-China 2007 \{published data only\}}

Chen LS, Li ZG, Chen YF, Xiao CL, Huang JJ, Bao GL, et al. Comparative study of relapse prevention in schizophrenia by network. Linchuang Jingshen Yixue Zazhi 2007;17(4):234-6.

\section{Chen XZ 2012 \{published data only\}}

Chen XZ, Huang XF, Deng XM, Hong HZ 陈秀珍, 黄秀芳, 邓晓 明, 洪华珍. The effect of home care intervention on long-term prognosis of patients with schizophrenia [居家护理干预对精神 分裂症患者长期预后的影响]. Sichuan Mental Health [四川精 神卫生] 2012, issue 02:112-4.

\section{COAST-UK 2004 \{published data only\}}

Kuipers E, Holloway F, Rabe-Hesketh S, Tennakoon L. An RCT of early intervention in psychosis: Croydon Outreach and Assertive Support Team (COAST). Social Psychiatry and Psychiatric Epidemiology 2004;39(5):358-63. [PUBMED: 15133591]

\section{COMO-UK 2003 \{published data only\}}

Hughes E, Wanigaratne S, Gournay K, Johnson S, Thornicroft G, Finch $\mathrm{E}$, et al. Training in dual diagnosis interventions (the COMO Study): randomised controlled trial. BMC Psychiatry 2008;8:12. [PUBMED: 18304310]

Johnson S, Thornicroft G, Afuwape S, Leese M, White IR, Hughes $E$, et al. Effects of training community staff in interventions for substance misuse in dual diagnosis patients with psychosis (COMO study): cluster randomised trial. British Journal of Psychiatry 2007;191:451-2. [PUBMED: 17978327]

Miles H, Johnson S, Amponsah-Afuwape S, Finch E, Leese M, Thornicroft G. Characteristics of sub-groups of individuals with psychotic illness and a comorbid substance use disorder. Psychiatric Services 2003;54(4):554-61. [PUBMED: 12663845]

Thornicroft G. Prevention of admission to psychiatric hospital. A randomised controlled trial of service use, health and social care outcomes of a community mental health team intervention specific to dual diagnosis (psychosis and substance misuse) patients. www.isrctn.com/ISRCTN98891022 (first received 14 March 2007).

\section{Cook 2013 \{published data only\}}

Cook JA, Jonikas JA, Hamilton MM, Goldrick V, Steigman PJ, Grey DD, et al. Impact of Wellness Recovery Action Planning on service utilization and need in a randomized controlled trial. Psychiatric Rehabilitation Journal 2013;36:250-7.

\section{CORE 2014 \{published data only\}}

University of Melbourne. The CORE Study: a stepped wedge cluster randomised controlled trial to test a co-design technique to optimise psychosocial recovery outcomes for people affected by mental illness in the community mental health setting. www.apps.who.int/trialsearch/Trial2.aspx? TrialID=ACTRN12614000457640 (first received 1 May 2014).

\section{Cosden-California 2005 \{published data only\}}

Cosden M, Ellens J, Schnell J, Yamini-Diouf Y. Efficacy of a mental health treatment court with assertive community treatment. Behavioral Sciences and the Law 2005;23(2):199-214. [PUBMED: 15818609]

\section{CRIMSON-UK 2008 \{published data only\}}

Thornicroft G. CRIMSON study: randomised controlled trial (RCT) of joint crisis plans to reduce compulsory treatment of people with psychosis. www.isrctn.com/ISRCTN11501328 (first received 13 March 2008).

\section{Cui 2012 \{published data only\}}

Cui H, Shao H, He XJ, Ding HQ 崔虹, 邵华, 何夏君, 丁寒琴. The effect of case management on social function in patients with schizophrenia in community [个案管理在改善社区精神分 裂症患者社会功能中的作用]. Modern Clinical Nursing [现代 临床护理] 2012, issue 11:38-40.

Dean-UK1 1990 \{published data only\}

Dean C, Gadd EM. Home treatment for acute psychiatric illness. BMJ 1990;301:1021-3.

\section{Dean-UK2 1993 \{published data only\}}

Dean C, Phillips J, Gadd EM, Joseph M, England S. Comparison of community based services with hospital based service for people with acute, severe psychiatric illness. BMJ 1993;307:473-6.

\section{De Cangas-Canada 1994 \{published data only\}}

De Cangas JPC. Assertive case management: a comprehensive evaluation of a hospital based case management programme [Le "case management" affirmatif: une évaluation complète d'un programme du genre en milieu hospitalier]. Santé Mentale au Québec 1994;19(1):75-91.

De Cangas JPC. Psychiatric nursing assertive case management: a comprehensive evaluation of the effectiveness and outcomes of hospital based treatment versus a nurse directed assertive case management programme. International Journal of Psychiatric Nursing Research 1995;1(3):72-81.

\section{Dekker-Netherlands 2002 \{published data only\}}

Dekker J, Wijdenes W, Koning YA, Gardien R, HermandesWillenborg L, Nusselder H. Assertive community treatment in Amsterdam. Community Mental Health Journal 2002;38(5):425-34. [PUBMED: 12236412] 
Deng-China 2006 \{published data only\}

Deng QY, Huang ZL, Xie ZY. Research the effect of early intervention of first episode schizophrenia. Medical Journal of Chinese Civil Administration 2006;18(9):794-6.

\section{Dharwadkar-Victoria 1994 \{published data only\}}

Dharwadkar N. Effectiveness of an assertive outreach community treatment programme. Australian and New Zealand Journal of Psychiatry 1994;28:244-9.

\section{Dinitz 1965 \{published data only\}}

Dinitz S, Scarpitti FR, Albini JL, Lefton M, Pasamanick B. An experimental study in the prevention of hospitalization of schizophrenics; thirty months of experience. American Journal of Orthopsychiatry 1965;35:1-9.

\section{Er 2013 \{published data only\}}

E ED 额尔敦. The role of regular family visit to prevent recurrence of patients with chronic schizophrenia [定期家访 对慢性精神分裂症预防复发的作用] . Inner Mongol Journal of Traditional Chinese Medicine [内蒙古中医药] 2013, issue 07:42-4.

\section{Fenton-Canada 1978 \{published data only\}}

Fenton FR, Tessier L, Contandriopoulos AP, Nguyen H, Struening EL. A comparative trial of home and hospital psychiatric treatment: financial costs. Canadian Journal of Psychiatry 1982;27(3):177-87. [PUBMED: 6807524]

Fenton FR, Tessier L, Struening EL. A comparative trial of home and hospital psychiatric care. One-year follow-up. Archives of General Psychiatry 1979;36(10):1073-9. [PUBMED: 475542]

Fenton FR, Tessier L, Struening EL, Smith FA, Benoit C, Contandriopoulos A, et al. A two-year follow-up of a comparative trial of the cost-effectiveness of home and hospital psychiatric treatment. Canadian Journal of Psychiatry 1984;29(3):205-11.

Smith FA, Fenton FR, Benoit C, Barzell E, Tessier L. Home care treatment of acutely ill psychiatric patients. A one year follow up. Canadian Psychiatric Association Journal 1978;23(2):73-6. [PUBMED: 647604]

\section{Franklin-Texas 1987 \{published data only\}}

Dozier M, Lee SW, Keir SS, Toprac M, Mason M. A case management programme in Texas revisited. Psychosocial Rehabilitation Journal 1993;17(2):183-9.

Franklin JL, Solovitz B, Mason M, Clemons JR, Miller GE. An evaluation of case management. American Journal of Public Health 1987;77(6):674-8. [PUBMED: 3578614$]$

\section{Gao 2013 \{published data only\}}

Gao HR, Liang JM, Wang Y, Bai CM, Guo XW, Su ZZ 高红锐, 梁 金梅, 王艳, 白翠梅, 郭雄伟, 苏焯焯. The effect of FamilyCommunity-Hospital integrated management for patients with schizophrenia [家庭-社区-医院一体化管理对精神分裂症治疗 效果的影响]. Medical Journal of Chinese People's Health [中 国民康医学] 2013, issue 12:15-16+87.

\section{Gaughran 2013 \{published data only\}}

Gaughran F, Stahl D, Ismail K, Atakan Z, Lally Gardner-Sood JP, Patel A, et al. Improving physical health and reducing substance use in psychosis - randomised control trial (IMPACT RCT): study protocol for a cluster randomised controlled trial. BMC Psychiatry 2013;13:263.

\section{Glick-New York 1986 \{published data only\}}

Glick ID, Fleming L, DeChillo N, Meyerkopf N, Jackson C, Muscara D, et al. A controlled study of transitional day care for non-chronically ill patients. American Journal of Psychiatry 1986;143:1551-6.

\section{Godley-Illinois 1994 \{published data only\}}

Godley SH. The Illinois MI/SA Project: A treatment system united for persons with mental illness and substance abuse. Bloomington (IL): Chestnut Health Systems, Inc., 1995.

Harrington-Godley S, Hoewing-Roberson R, Godley MD. Final MISA report. Technical report submitted to the Illinois Department of Mental Health and Developmental Disabilities and the Illinois Department of Alcoholism and Substance Abuse. Bloomington (IL): Chestnut Health Systems, Inc. 1994.

\section{Goering-Canada 1988 \{published data only\}}

Goering PN, Wasylenki DA, Farkas M, Lancee WJ, Ballantyne R. What difference does case management make?. Hospital and Community Psychiatry 1988;39:272-6.

Gold-SCarolina 2006 \{published data only\}

Gold PB, Meisler N, Santos AB, Carnemolla MA, Williams OH, Keleher J. Randomized trial of supported employment integrated with assertive community treatment for rural adults with severe mental illness. Schizophrenia Bulletin 2006;32(2):378-95. [PUBMED: 16177278]

\section{Gong 2014 \{published data only\}}

Gong W, Xu D, Zhou L, Shelton Brown H 3rd, Smith KL, Xiao S. Village doctor-assisted case management for patients with schizophrenia in rural communities: a randomized control study. Implementation Science 2014;9:13.

\section{Grawe-Norway 2005 \{published data only\}}

Gotestam KG. Continued early intervention for recent-onset schizophrenia. A randomized controlled study. clinicaltrials.gov/ ct2/show/NCT00184509 (first received 15 September 2005).

Grawe RW, Falloon IRH, Widen JH, Skogvoll E. Two years of continued early treatment for recent-onset schizophrenia: a randomised controlled study. Acta Psychiatrica Scandinavica 2006;114(5):328-36. [PUBMED: 17022792]

Morken G, Grawe RW, Widen JH. A randomized controlled trial in recent-onset schizophrenia. Effects on compliance of two years of continued intervention. European Neuropsychopharmacology 2005;15(Suppl 3):S521.

Morken G, Grawe RW, Widen JH. Effects of integrated treatment on antipsychotic medication adherence in a randomized trial in recent-onset schizophrenia. Journal of Clinical Psychiatry 2007;68(4):566-71. [PUBMED: 17474812] 
Gu-China 2010 \{published data only\}

Gu XH 顾秀华. Effects of program training on the social function in community patients with schizophrenia [程式训 练对社区慢性精神分裂症患者社会功能的影响]. Nursing Practice and Research [\#\#\#\#\#\#\#] 2010;7(1):10-2.

Han SH 2012 \{published data only\}

Han SH 韩树华. The effects of family intervention on the rehabilitation of patients with schizophrenia [家庭护理干预对 精神分裂症患者康复影响的研究]. Medical Journal of Chinese People's Health [中国民康医学] 2012, issue 07:867-71.

\section{Hargreaves-California \{published and unpublished data\}} Hargreaves WA. Further information on your study [personal communication]. Email to: M Dieterich 26 May 2009.

Hargreaves WA, Ja DY, Gee M. Assertive community treatment can be cost-effective for schizophrenia. 39th Annual Meeting of the American College of Neuropsychopharmacology; 2000 Dec 10-14; San Juan, Puerto Rico. 2000.

\section{Havassy-California 2000 \{published data only\}}

Havassy BE, Shopshire MS. Implications of substance dependence for severe and persistent mental illness. NIDA Research Monograph 2000;180:119.

Havassy BE, Shopshire MS, Quigley LA. Effects of substance dependence on outcomes of patients in a randomized trial of two case management models. Psychiatric Services 2000;51(5):639-44. [PUBMED: 10783183]

\section{He-China 2004 \{published data only\}}

He JY, Jiang YH, Liang CS. Effects of synthetical intervention on prognosis in rural schizophrenia. Chinese Journal of Psychiatry 2004;37(2):96-8.

\section{Hornstra-Kansas 1993 \{published data only\}}

Hornstra RK, Bruce-Wolfe V, Sagduyu K, Riffle DW. The effect of Intensive Case Management on hospitalization of patients with schizophrenia. Hospital and Community Psychiatry 1993;44:844-7.

\section{Hoult-Australia 1981 \{published data only\}}

Hoult J. Community care of the acutely mentally ill. British Journal of Psychiatry 1986;149:137-44. [PUBMED: 3779274$]$

Hoult J, Reynolds I. Schizophrenia. A comparative trial of community orientated and hospital orientated psychiatric care. Acta Psychiatrica Scandinavica 1984;69(5):359-72. [PUBMED: 6730991]

Hoult J, Reynolds I, Charbonneau Powis M, Coles P, Briggs J. A controlled study of psychiatric hospital versus community treatment - the effect on relatives. Australian and New Zealand Journal of Psychiatry 1981;15(4):323-8. [PUBMED: 6951573]

Hoult J, Reynolds I, Charbonneau Powis M, Weekes P, Briggs J. Psychiatric hospital versus community treatment: the results of a randomised trial. Australian and New Zealand Journal of Psychiatry 1983;17(2):160-7. [PUBMED: 6578788]
Hoult J, Rosen A, Reynolds I. Community orientated treatment compared to psychiatric hospital orientated treatment. Social Science and Medicine 1984;18(11):1005-10. [PUBMED: 6740335]

Reynolds I, Hoult JE. The relatives of the mentally ill. A comparative trial of community-oriented and hospital-oriented psychiatric care. Journal of Nervous and Mental Disease 1984;172(8):480-9. [PUBMED: 6086834]

Huang 2012 \{published data only\}

Huang LH 黄丽宏. The effect of family intervention on the rehabilitation of inpatients with schizophrenia [家庭护理模式 在住院精神分裂症患者康复期的应用]. Chinese Community Doctors [中国社区医师(医学专业)] 2012, issue 35:270-1.

\section{Huang 2013 \{published data only\}}

Huang CY 黄春艳. The clinical treatment effects of community nursing care for the convalescent schizophrenics: a comparative study [社区护理干预对康复期精神分裂症患者的临床治疗影 响的对照研究]. Today Nurse [当代护士(专科版)] 2013, issue 07:133-5.

\section{Hui 2014 \{published data only\}}

Chang WC, Hui CLM, Lai DC, Tam WWY, Tang JYM, Wong GHY, et al. Clinical and social determinants of duration of untreated psychosis: a 4-year randomized controlled trial of specialized early intervention service for adult-onset first-episode. Early Intervention in Psychiatry 2012;6:77.

Hui CL, Chang WC, Chan SK, Lee EH, Tam WW, Lai DC, et al. Early intervention and evaluation for adult-onset psychosis: the JCEP study rationale and design. Early Intervention in Psychiatry 2014;8:261-8.

NCT00919620. Stage-specific case management for early psychosis. clinicaltrials.gov/ct2/show/NCT00919620 (first received 10 June 2009).

\section{Hurlburt-California 1996 \{published data only\}}

Hurlburt MS, Hough RL, Wood PA. Effects of substance abuse on housing stability of homeless mentally ill persons in supported housing. Psychiatric Services 1996;47(7):731-6. [PUBMED: 8807687]

Wood PA, Hurlburt MS, Hough RL, Hofstetter CR. Longitudinal assessment of family support among homeless mentally ill participants in a supported housing programme. Journal of Community Psychology 1998;26(4):327-44.

ISRCTN73683215 \{published data only\}

ISRCTN73683215. Effectiveness of the psychiatric rehabilitation approach in the Netherlands. www.isrctn.com/ISRCTN73683215 (first received 20 December 2005).

\section{Jerrell-California 1989 \{published data only\}}

Jerrell J, Hu T. Cost-effectiveness of intensive clinical and case management compared with an existing system of care. Inquiry 1989;26:224-34. 
Jerrell-SCarolina2 1994 \{published data only\}

Jerrell JM. Cost-effective treatment for persons with dual disorders. New Directions for Mental Health Services 1996;70:79-91. [PUBMED: 8754232]

Jerrell JM. Toward cost-effective care for persons with dual diagnoses. Journal of Mental Health Administration 1996;23:329-37. [PUBMED: 10172689]

Jerrell JM, Hu T, Ridgely MS. Cost-effectiveness of substance disorder interventions for people with severe mental illness. Journal of Mental Health Administration 1994;21(3):283-97.

Jerrell JM, Ridgely MS. Comparative effectiveness of three approaches to serving people with severe mental illness and substance abuse disorders. Journal of Nervous and Mental Disease 1995;183(9):566-76. [PUBMED: 7561818]

Jerrell JM, Ridgely MS. Evaluating changes in symptoms and functioning of dually diagnosed clients in specialized treatment. Psychiatric Services 1995;46(3):233-8. [PUBMED: 7796208]

Jerrell JM, Ridgely MS. Impact of robustness of programme implementation on outcomes of clients in dual diagnosis programmes. Psychiatric Services 1999;50:109-12. [PUBMED: 9890592]

Jerrell JM, Wilson JL. The utility of dual diagnosis services for consumers from nonwhite ethnic groups. Psychiatric Services 1996;47(11):1256-8. [PUBMED: 8916247]

\section{Jiang 2012 \{published data only\}}

Jiang XF, Zhang C, Wu ZG, Xiong XY, Zhang J, Zhu XQ 江学锋, 张晨, 吴志国, 熊祥玉, 张洁, 朱学勤. A follow-up study of family intervention as adjuvant therapy for first-episode schizophrenica [家庭干预对首发精神分裂症患者辅助治疗的 随访研究]. Journal of Clinical Psychiatry [临床精神医学杂志] 2012, issue 01:1-4.

Jiang 2013 \{published data only\}

Jiang MX, Ji YW, Zhou LJ, Xu WW, Dai LQ, Tang WZ, et al 姜明 霞, 季一薇, 周路佳, 徐婉婉, 戴丽琴, 唐文忠, et al. Social support for patients with chronic schizophrenia [慢性精神分裂 症患者的社会支持]. China Journal of Health Psychology [中 国健康心理学杂志] 2013, issue 08:1139-41.

\section{Jorgensen 2012 \{published data only\}}

Jorgensen R, Munk-Jorgensen P, Hansson L, Zoffmann V. Meaningful change with the method guided self-determination - a randomised controlled study for outpatients diagnosed with schizophrenia. 14th International Congress on Schizophrenia Research; 2013 April 21-25; Grande Lakes (FL). 2013.

\section{Kane-Virginia 2004 \{published data only\}}

Kane CF, Blank MB. NPACT: enhancing programmes of assertive community treatment for the seriously mentally ill. Community Mental Health Journal 2004;40(6):549-59. [PUBMED: 15672693]

\section{Kilbourne 2014 \{published data only\}}

Kilbourne AM, Almirall D, Goodrich DE, Lai Z, Abraham KM, Nord KM, et al. Enhancing outreach for persons with serious mental illness: 12-month results from a cluster randomized trial of an adaptive implementation strategy. Implementation Science 2014;9:163.

\section{Klotz-California 2001 \{published data only\}}

Klotz TA, Summers BH, Richardson L, Gomberg DI, Maloney M. Community reintegration for severely mentally ill patients leaving jail. 154th Annual Meeting of the American Psychiatric Association; 2001 May 5-10; New Orleans. 2001.

Maloney M. MIOCRG Grant Application - FORward MOMentum study. Data on file 2001.

\section{Knight-California 1990 \{published data only\}}

Knight RG, Carter PM. Reduction of psychiatric inpatient stay for older adults by Intensive Case Management. Gerontologist 1990;30:510-5.

\section{Kuldau-California 1977 \{published data only\}}

Kuldau JM, Dirks SJ. Controlled evaluation of a hospitaloriginated community transitional system. Archives of General Psychiatry 1977;34:1331-40.

\section{Lafave-Canada 1996 \{published data only\}}

Lafave HG, De Souza HR, Gerber GJ. Assertive community treatment of severe mental illness - a Canadian experience. Psychiatric Services 1996;47(7):757-9.

\section{Langley 2009 \{published data only\}}

Langley J, Gehrs M, Wasylenki D, Dewa C, Rueda S, Rourke S. Suicidality in seriously mentally ill clients of two intensive community mental health programs. Canadian Journal of Community Mental Health 2009;28(1):151-64.

\section{Langsley-Colorado 1968 \{published data only\}}

Langsley DG, Machotka P, Flomenhaft K. Follow up evaluation of family crisis therapy. American Journal of Orthopsychiatry 1969;39:753-9.

Langsley DG, Machotka P, Flomenshaft K. Avoiding mental hospital admissions: a follow up study. American Journal of Psychiatry 1971;127:1391-4.

Langsley DG, Pittman FS, Machotka P, Flomenhaft K. Family crisis therapy - results and implications. Family Process 1968; 7:145-58.

\section{Lehman-Maryland2 1993 \{published data only\}}

Lehman AF, Herron JD, Schwartz RP, Myers CP. Rehabilitation for adults with severe mental illness and substance use disorders. A clinical trial. Journal of Nervous and Mental Disease 1993;181:86-90.

\section{LEO-UK 1994 \{published and unpublished data\}}

Craig T. Brixton early psychosis project. National Research Register 2001; Vol. 3.

Craig T. Brixton early psychosis project. www.isrctn.com/ ISRCTN73679874 (first received 23 January 2004).

Craig T. Further information on Brixton Early Psychosis Project [personal communication]. Email to: M Dieterich 22 May 2009. 
Craig TKJ. Brixton early psychosis project. National Research Register 2000.

Craig TKJ, Garety P, Power P, Rahaman N, Colbert S, FornellsAmbrojo M, et al. The Lambeth Early Onset (LEO) Team: randomised controlled trial of the effectiveness of specialised care for early psychosis. BMJ 2004;329(7474):1067-70. [PUBMED: 15485934]

Garety P. Randomised controlled trial of early psychosis in Lambeth. National Research Register 2000.

Garety P. Randomised controlled trial of early psychosis in Lambeth. National Research Register 2001; Vol. 1.

Garety PA, Craig TKJ, Dunn G, Fornells-Ambrojo M, Colbert S, Rahaman N, et al. Specialised care for early psychosis: symptoms, social functioning and patient satisfaction: randomised controlled trial. British Journal of Psychiatry 2006;188:37-45. [PUBMED: 16388068]

Knapp M. Brixton early psychosis study project \#432. National Research Register 2004; Vol. 1.

Power P, Craig T, Garety P, Rahaman N, Colbert S, FornellsAmbrojo M. Lambeth Early Onset (LEO) trial: a randomised controlled trial of assertive community follow-up in early psychosis: initial 6 month data. Data on file 1994.

Power P, Craig T, Garety P, Rahaman N, Fornells-Ambrojo M, Colbert $\mathrm{S}$. A randomised controlled trial of assertive community follow-up in early psychosis: preliminary results. Schizophrenia Research 2002;53(3 Suppl 1):42.

\section{Li 2011 \{published data only\}}

Li SC, Chen CY 李守春, 陈常云. Effects of comprehensive intervention on mental health status of first-degree relatives of inpatients with first-episode schizophrenia [综合干预对首发住 院精神分裂症患者一级亲属心理健康状况的影响]. Journal of Psychiatry [\#\#\#\#\#] 2011;24(03):218-20.

\section{Li 2012 \{published data only\}}

Li ZC 李振超. The effect of community comprehensive intervention on cognitive function of first-episode schizophrenia [社区防治综合措施对首发精神分裂症认知功能 的影响] . China Modern Medicine [中国当代医药] 2012, issue $33: 164-5$

\section{Li 2013 \{published data only\}}

Li SC, Lu ZS, An XD 李守春, 卢振胜, 安晓东. A follow-up study of the effects of family health education and individualized management on quality of life of patients with chronic schizophrenia [家庭健康教育和个体化管理对慢性精神分 裂症患者生活质量的随访研究]. Medical Journal of Chinese People's Health [中国民康医学] 2013, issue 13:91-2.

\section{Liang 2009 \{published data only\}}

Liang GY, Zhang YL, He YT, Fu LM 梁国英, 张艳莉, 何育涛, 傅兰 梅. The effect of community nursing care for the rehabilitation in patients with schizophrenia [社区护理对精神分裂症患 者复发的影响]. Chinese Journal of Misdiagnostics [\#\#\#\#\#\#] 2009;9(35):8591-2.

\section{Liang 2012 \{published data only\}}

Liang JM 梁金梅. The effect of integrated family intervention for schizophrenia patients in rehabilitation period [家庭干预综 合护理措施对康复期精神分裂症患者作用的研究]. Medical Journal of Chinese People's Health [中国民康医学] 2012, issue 07:863-4.

\section{Liao 2010 \{published data only\}}

Liao GS 廖更生. Effects of comprehensive intervention for rehabilitation of first-episode schizophrenia [综合干预对首发 精神分裂症康复的影响]. Chinese Journal of Health Psychology [\#\#\#\#\#\#\#] 2010;18(9):1038-40.

\section{Li-China 2004 \{published data only\}}

Li TY, Li YY, Wu TC, Yan WY, Chen YH, An Y, et al. Effect of early intervention on quality of life in patients with first episode schizophrenia. Chinese Journal of Clinical Rehabilitation 2004;8(18):3464-5.

\section{Lichtenberg-Israel 2008 \{published data only\}}

Lichtenberg P, Levinson D, Sharshevsky Y, Feldman D, Lachman M. Clinical case management of revolving door patients - a semi-randomized study. Acta Psychiatrica Scandinavica 2008;117(6):449-54. [PUBMED: 18331577]

\section{Li JX 2013 \{published data only\}}

Li JH, Wang YZ 李金惠, 王颖昭. The hospital and home rehabilitation training for patients with first-episode schizophrenia [医院-居家康复训练对首发分裂症患者康 复的影响]. Hainan Medical Journal [海南医学] 2013, issue 20:3108-10

\section{Li MD 2013 \{published data only\}}

Li MD, Zhao LQ, Zhang YS 李明德, 赵丽琼, 张跃坤. The effects of family intervention on social support in patients with schizophrenia in rural areas [家庭干预对农村精神分裂症患 者社会支持度影响的对照研究]. Medical Journal of Chinese People's Health [中国民康医学] 2013, issue 08:22-3.

Lin-China 1998 \{published data only\}

Lin Y, Zhang, Zhen S. Community based rehabilitation programme for schizophrenia in Shantou: a three year follow up. Journal of Clinical Psychological Medicine 1998;8(5):280-2.

Lin YQ, Zheng SX, Hong XH. An assessment of community based rehabilitation management for psychiatric patients in Shantou. Chinese Journal of Behavioral Medical Science 2002;11(02):149, 150-1.

\section{Li Ning 2013 \{published data only\}}

Li N, Han Y, Yan HL, Du JJ, Zheng H, Gao YQ 李宁, 韩嫣, 严宏 力, 杜建军, 郑宏, 高运庆. Comparative study on the effects of community-based comprehensive intervention on relapse frequency in patients with chronic schizophrenia [社区综合干 预对慢性精神分裂症患者复发次数影响的对照研究]. Journal of Xinxiang Medical College [新乡医学院学报] 2013, issue 09:749-51.

\section{Liu 2010 \{published data only\}}

Liu H, Qin W, Hu SY, Qian ZP 刘华, 秦伟, 胡姝颖, 钱志萍. Effect of systematic rehabilitation intervention on the treatment 
compliance in patients with schizophrenia [系统康复干预对 精神分裂症康复期患者治疗依从性的影响]. Nursing Practice and Research [\#\#\#\#\#\#\#] 2010;7(2):17-9.

\section{Li WX 2013 \{published data only\}}

Li WX 李文秀. The effect of community rehabilitation intervention versus long-term hospitalisation for patients with schizophrenia [精神分裂症社区康复与长期住院治疗模式对照 分析]. Hebei Medicine [河北医学] 2013, issue 08:1173-5.

\section{Lloyd 2000 \{published data only\}}

Lloyd KR, Cleary M, Pearson C, Brooks R. A controlled clinical trial of intensive home treatment for persons with severe mental illness. European Psychiatry 2000;15:291S-2S.

\section{Lu 2013 \{published data only\}}

Lu GF 鲁国芬. Effects of integrated hospital and community management for patients with schizophrenia [医院社区一体 化管理模式对精神分裂症患者的管理效果研究]. Chinese General Practice [中国全科医学] 2013, issue 05:453-5.

\section{Malm-Sweden 2003 \{published and unpublished data\}} Malm U. Further information about your trial [personal communication]. Email to: M Dieterich 25 May 2009.

Malm U, Ivarsson B, Allebeck P, Falloon IR. Integrated care in schizophrenia: a 2-year randomized controlled study of two community-based treatment programmes. Acta Psychiatrica Scandinavica 2003;107(6):415-23. [PUBMED: 12752017]

\section{Martin-Delaware 1993 \{published data only\}}

Martin SM, Scarpitti FR. An Intensive Case Management approach for paroled IV drug users. Journal of Drug Issues 1993;23:43-59

\section{Martin-UK 2005 \{published data only\}}

Martin G, Costello H, Leese M, Slade M, Bouras N, Higgins S, et al. An exploratory study of assertive community treatment for people with intellectual disability and psychiatric disorders: conceptual, clinical, and service issues. Journal of Intellectual Disability Research 2005;49(Pt 7):516-24. [PUBMED: 15966959]

\section{Marx-Wisconsin 1973 \{published data only\}}

Marx AJ, Test MA, Stein LI. Extrohospital management of severe mental illness. Feasibility and effects of social functioning. Archives of General Psychiatry 1973;29(4):505-11. [PUBMED 4748311]

\section{McDonell 2013 \{published data only\}}

McDonell MG, Srebnik D, Angelo F, McPherson S, Lowe JM, Sugar A, et al. Randomized controlled trial of contingency management for stimulant use in community mental health patients with serious mental illness. American Journal of Psychiatry 2013;170:94-101.

\section{McFarlane-New York 1992 \{published data only\}}

McFarlane WR, Dushay R, Lukens E, Stastny P, Deakins S, Link B. Work outcomes in family-aided assertive community treatment: vocational rehabilitation for persons with psychotic disorders. Epidemiologia e Psichiatria Sociale 1999;8(3):174-82.
McFarlane WR, Dushay RA, Deakins SM, Stastny P, Lukens EP, Toran J, et al. Employment outcomes in family-aided assertive community treatment. American Journal of Orthopsychiatry 1999;70(2):203-14.

McFarlane WR, Dushay RA, Stastny P, Deakins SM, Link B. A comparison of two levels of family-aided assertive community treatment. Psychiatric Services 1996;47(7):744-50.

* McFarlane WR, Stastny P, Deakins S. Family-aided assertive community treatment: a comprehensive rehabilitation and Intensive Case Management approach for persons with schizophrenic disorders. New Directions for Mental Health Services 1992;53:43-54.

\section{McGowan-California 1995 \{published data only\}}

McGowan M, Madison K, Meisel J, Chandler D. AB3777 Final Report: The Integrated Service Agencies. Report to California Department of Mental Health. Sacramento: Lewin-VHI, Inc. , 1995.

\section{McGrew-Indiana 1994 \{published data only\}}

McGrew JH, Bond GR, Dietzen L, Salyers M. Measuring the fidelity of implementation of a mental health programme model. Journal of Consulting and Clinical Psychology 1994;62:670-8.

\section{McHugo-Washington DC 2004 \{published data only\}}

McHugo GJ, Bebout RR, Harris M, Cleghorn S, Herring G, Xie H, et al. A randomized controlled trial of integrated versus parallel housing services for homeless adults with severe mental illness. Schizophrenia Bulletin 2004;30(4):969-82. [PUBMED: 15957201]

MECCA-Europe 2002 \{published data only\}

Mecca protocol. www.mecca.eu.org/index.htm (accessed 10 December 2008).

McCabe R. Towards more effective community care for people with severe psychosis - MECCA - a randomised controlled trial on outcome management in six European countries. National Research Register 2004; Vol. 3.

McCabe R, Priebe S, the MECCA group. Aims and methods of the MECCA study in six European countries. Fifth ENMESH (European Network for Mental Health Service Evaluation) International Conference; 2002 May 31 - June 2; Sofia. 2002.

Priebe S. Outcome management in community mental health care in six European countries: the MECCA study. The Mental Health Research in East London Second Annual Presentation; 2002 Oct 30; London. East London \& City Mental Health Trust, 2002.

Priebe S. Towards more effective European community care for patients with severe psychosis. www.isrctn.com/ ISRCTN75571732 (first received 3 September 2005).

Priebe S. Towards more effective European community care for patients with severe psychosis (MECCA). National Research Register 2003; Vol. 1.

Priebe S. Towards more effective community care for patients with severe psychosis - a randomised controlled trial on 
outcome management in six European countries. National Research Register 2001; Vol. 1.

Priebe S. Towards more effective community care for patients with severe psychosis - a randomised controlled trial on outcome management in six European countries. National Research Register 2003.

Priebe S, Bullenkamp J, Hansson L, McCabe R, Roessler W, Torres-Gonzales F, et al. Outcome management in community mental health care in six European countries: the MECCA study. 10th Annual Meeting of the European Public Health Association (EUPHA); 2002 Nov 28-30; Dresden. 2002.

Priebe S, Bullenkamp J, McCabe R, Hansson L, Roessler W, Torres-Gonzalez F, et al. Impact of regular outcome assessment on treatment - the MECCA Study. European Psychiatry 2002;17(Suppl 1):7s.

Priebe S, Bullenkamp J, McCabe R, Hansson L, Rossler W, Torres-Gonzales $\mathrm{F}$, et al. The impact of routine outcome measurement on treatment processes in community mental health care: approach and methods of the MECCA study. Epidemiologia e Psichiatria Sociale 2002;11(3):198-205.

Wright $D$, McCabe R, Priebe S, the MECCA group. MECCA: How to improve community mental health care. First London Mental Health Research and Development Virtual Institute (LoMHR\&D) Conference; 2003 Jan 28; London. 2003.

\section{Meneghelli-Italy 2000 \{published data only\}}

Cocchi A, Meneghelli A, Preti A. Programmema 2000: Celebrating 10 years of activity of an Italian pilot programme on early intervention in psychosis. Australian and New Zealand Journal of Psychiatry 2008;42(12):1003-12. [PUBMED: 19016088]

Meneghelli A. An early intervention programme in a community mental health center in Italy. Second International Conference on Early Psychosis; 2000 Mar 31 - Apr 2; New York (NY). 2000.

\section{Merson-UK 1992 \{published data only\}}

Merson S, Tyrer P, Carlen D, Johnson T. The cost of treatment of psychiatric emergencies: a comparison of hospital and community services. Psychological Medicine 1996;26(4):727-34. [PUBMED: 8817707]

Merson S, Tyrer P, Onyett S, Lack S, Birkett P, Lynch S, et al. Early intervention in psychiatric emergencies: a controlled clinical trial. Lancet 1992;339(8805):1311-4. [PUBMED: 1349990]

Tyrer P, Merson S, Onyett S, Johnson T. The effect of personality disorder on clinical outcome, social networks and adjustment: a controlled clinical trial of psychiatric emergencies. Psychological Medicine 1994;24(3):731-40. [PUBMED: 7991755]

\section{Modcrin-Kansas 1988 \{published data only\}}

Modcrin M, Rapp C, Poertner J. The evaluation of case management services with the chronically mentally ill. Evaluation and Programme Planning 1988;11:307-14.

\section{Morse-Missouri2 1997 \{published data only\}}

Burger GK, Calsyn RJ, Morse GA, Klinkenberg WD. Prototypical profiles of the Brief Psychiatric Rating Scale. Journal of Personality Assessment 2000;75(3):373-86. [PUBMED: 11117152]

Calsyn RJ. A modified ESID approach to studying mental illness and homelessness. American Journal of Community Psychology 2003;32(3-4):319-31. [PUBMED: 14703267]

Calsyn RJ, Morse GA, Klinkenberg WD, Trusty ML, Allen G. The impact of assertive community treatment on the social relationships of people who are homeless and mentally ill. Community Mental Health Journal 1998;34(6):579-93. [PUBMED: 9833199]

Calsyn RJ, Morse GA, Klinkenberg WD, Yonker RD, Trusty ML. Moderators and mediators of client satisfaction in case management programmes for clients with severe mental illness. Mental Health Services Research 2002;4(4):267-75. [PUBMED: 12558015]

Kenny DA, Calsyn RJ, Morse GA, Klinkenberg WD, Winter JP, Trusty ML. Evaluation of treatment programmes for persons with severe mental illness: moderator and mediator effects. Evaluation Review 2004;28(4):294-324.

Klinkenberg WD, Calsyn RJ, Morse GA. The helping alliance in case management for homeless persons with severe mental illness. Community Mental Health Journal 1998;34(6):569-78. [PUBMED: 9833198]

Morse GA, Calsyn RJ, Klinkenber W, Trusty ML, Gerber F, Smith R, et al. An experimental comparison of three types of case management for homeless mentally ill persons. Psychiatric Services 1997;48(4):497-503. [PUBMED: 9090733]

Wolff N, Helminiak TW, Morse GA, Calsyn RJ, Klinkenberg WD, Trusty ML. Cost-effectiveness evaluation of three approaches to case management for homeless mentally ill clients. American Journal of Psychiatry 1997;154(3):341-8. [PUBMED: 9054781]

\section{Morthorst 2012 \{published data only\}}

Morthorst B, Krogh J, Erlangsen A, Alberdi F, Nordentoft M. Effect of assertive outreach after suicide attempt in the AID (assertive intervention for deliberate self harm) trial: randomised controlled trial. BMJ 2012;345:e4972. [DOI: 10.1136/bmj.e4972]

\section{Mosher-California 1975 \{published data only\}}

Bola JR, Mosher LR. Treatment of acute psychosis without neuroleptics: two-year outcomes from the Soteria project. Journal of Nervous and Mental Disease 2003;191(4):219-29. [PUBMED: 12695732]

Matthews SM, Roper MT, Mosher LR, Menn AZ. A non neuroleptic treatment for schizophrenia: analysis of the two year postdischarge risk of relapse. Schizophrenia Bulletin 1979;5(2):322-33. [PUBMED: 37598]

Mosher LR, Menn A, Matthew SM. Soteria: evaluation of a home-based treatment for schizophrenia. American Journal of Orthopsychiatry 1975;45(3):455-67. [PUBMED: 238399] 
Mosher LR, Menn AZ. Community residential treatment for schizophrenia: two year follow up. Hospital and Community Psychiatry 1978;29(11):715-23. [PUBMED: 700610]

\section{Muijen-UK1 1992 \{published data only\}}

Knapp M, Beecham J, Koutsogeorgopoulou V, Hallam A Fenyo A, Marks IM, et al. Service use and costs of homebased versus hospital-based care for people with serious mental illness. British Journal of Psychiatry 1994;165:195-203. [PUBMED: 1571681]

Marks IM, Connolly J, Muijen M, Audini B, McNamee G, Lawrence RE. Home-based versus hospital-based care for people with serious mental illness. British Journal of Psychiatry 1994;165:179-94.

Muijen M, Marks I, Connolly J, Audini B. Home based care and standard hospital care for patients with severe mental illness: a randomised controlled trial. BMJ 1992;304(6829):749-54. [PUBMED: 1571681]

Muijen M, Marks IM, Connolly J, Audini B, McNamee G. The daily living programme. Preliminary comparison of community versus hospital-based treatment for the seriously mentally ill facing emergency admission. British Journal of Psychiatry 1992;160:379-84. [PUBMED: 1562865]

Simpson CJ, Seager CP, Robertson JA. Home-based care and standard hospital care for patients with severe mental illness: a randomised controlled trial. British Journal of Psychiatry 1993;162:239-43. [PUBMED: 8435695]

\section{Mulder-Missouri 1985 \{published data only\}}

Mowbray CT, Collins ME, Plum TB, Masterton T, Mulder R. Harbinger. I: The development and evaluation of the first PACT replication. Administration and Policy in Mental Health 1997;25(2):105-23. [PUBMED: 9727211]

Mulder R. Evaluation of the Harbringer Programme. Data on file 1985.

\section{NCT00781079 \{published data only\}}

NCT00781079. Do consumer providers enhance recovery? (PEER). clinicaltrials.gov/ct2/show/NCT00781079 (first received 27 October 2008).

\section{NCT01597141 \{published data only\}}

NCT01597141. Psychosis: Early detection, intervention and prevention. clinicaltrials.gov/show/NCT01597141 (first received 9 May 2012).

\section{Nieves 2002 \{published data only\}}

Nieves EJ. The effectiveness of the assertive community treatment model. Administration and Policy in Mental Health 2002;29(6):461-80.

\section{Odom 2005 \{published data only\}}

Odom AE. A Randomized Study of Integrated Outpatient Treatment and Assertive Community Treatment for Patients With Comorbid Mental Illness and Substance Use Disorders: Comparing Treatment Outcome for Domiciled and Homeless Patients [PhD dissertation]. New School University, 2005.

\section{Pai-India 1982 \{published data only\}}

Pai S, Kapur RL. Evaluation of home care treatment for schizophrenic patients. Acta Psychiatrica Scandinavica 1983;67(2):80-8. [PUBMED: 6846041]

Pai S, Kapur RL. Impact of treatment intervention on the relationship between dimensions of clinical psychopathology, social dysfunction and burden on the family of psychiatric patients. Psychological Medicine 1982;12(3):651-8. [PUBMED: 7134321]

Pai S, Nagarajaiah. Treatment of schizophrenic patients in their homes through a visiting nurse - some issues in the nurse's training. International Journal of Nursing Studies 1982;19(3):167-72. [PUBMED: 6293993]

Pai S, Roberts EJ. Follow-up study of schizophrenic patients initially treated with home care. British Journal of Psychiatry 1983;143:447-50. [PUBMED: 6640212]

\section{Pioli 2006 \{published data only\}}

Pioli R, Vittorielli M, Gigantesco A, Rossi G, Basso L, Caprioli C, et al. Outcome assessment of the VADO approach in psychiatric rehabilitation: a partially randomised multicentric trial. Clinical Practice and Epidemiology in Mental Health 2006;2(5):1-8.

Polak-Colorado 1976 \{published data only\}

Polak PR, Kirkby MW. A model to replace psychiatric hospitals. Journal of Nervous and Mental Disease 1976;162:13-22.

\section{PRiSM-UK 1998 \{published data only\}}

Becker T, Holloway F, McCrone P, Thornicroft G. Evolving service interventions in Nunhead and Norwood. PRiSM Psychosis Study 2. British Journal of Psychiatry 1998;173:371-5. [PUBMED: 9926052]

Johnson S, Leese M, Brooks L, Clarkson P, Guite H, Thornicroft G, et al. Frequency and predictors of adverse events. PRiSM Psychosis Study 3. British Journal of Psychiatry 1998;173:376-84. [PUBMED: 9926053]

Leese M, Johnson S, Slade M, Parkman S, Kelly F, Phelan M, et al. User perspective on needs and satisfaction with mental health services. PRiSM psychosis study 8 . British Journal of Psychiatry 1998;173:409-15. [PUBMED: 9926058]

McCrone P, Thornicroft G, Phelan M, Holloway F, Wykes T, Johnson S. Utilisation and costs of community mental health services. PRiSM Psychosis Study 5. British Journal of Psychiatry 1998;173:391-8. [PUBMED: 9926055]

Szmukler GI, Wykes T, Parkman S. Care giving and the impact on carers of a community mental health service. PRiSM Psychosis Study 6. British Journal of Psychiatry 1998;173:399-403. [PUBMED: 9926056]

Taylor RE, Leese M, Clarkson P, Holloway F, Thornicroft G. Quality of life outcomes for intensive versus standard community mental health services. PRiSM Psychosis Study 9. British Journal of Psychiatry 1998;173:416-22. [PUBMED: 9926059] 
Thornicroft G, Strathdee G, Phelan M, Holloway F, Wykes T, Dunn G, et al. Rationale and design. PRiSM Psychosis Study I. British Journal of Psychiatry 1998;173:363-70. [PUBMED: 9926051]

Thornicroft G, Wykes T, Holloway F, Johnson S, Szmukler G. From efficacy to effectiveness in community mental health services. PRiSM Psychosis Study 10. British Journal of Psychiatry 1998;173:423-7. [PUBMED: 9926060]

Wykes T, Leese M, Taylor R, Phelan M. Effects of community services on disability and symptoms. PRiSM Psychosis Study 4. British Journal of Psychiatry 1998;173:385-90. [PUBMED: 9926054]

\section{Ren-China 2004 \{published data only\}}

Ren E, Liang X, Yan H. The administration of openly comprehensive rehabilitation impacts on the self-efficacy of chronic schizophrenics. Medical Journal of Chinese Peoples Health 2004;16(5):308-9.

\section{Roldan-Merino 2012 \{published data only\}}

Roldan-Merino J, Garcia IC, Ramos-Pichardo JD, Foix-Sanjuan A, Quilez-Jover J, Montserrat-Martinez M. Impact of personalized in-home nursing care plans on dependence in ADLs/IADLs and on family burden among adults diagnosed with schizophrenia: a randomized controlled study. Perspectives in Psychiatric Care 2012;49:171-8.

\section{Rossler-Germany1 1992 \{published data only\}}

Rossler W, Loffler W, Fatkenheuer B, Reicher-Rossler A. Does case management reduce the rehospitalization rate?. Acta Psychiatrica Scandinavica 1992;86(6):445-9.

\section{Rossler-Germany2 1995 \{published data only\}}

Rossler W, Loffler B, Fatkenheuer A, Riecher-Rossler A. Case management for schizophrenic patients at risk of rehospitalization - a case control study. European Archives of Psychiatry and Clinical Neuroscience 1995;246(1):29-36.

\section{Rutter-UK \{published and unpublished data\}}

Rutter D, Tyrer P, Emmanuel J, Weaver T, Byford S, Hallam A, et al. Internal vs. external care management in severe mental illness: randomized controlled trial and qualitative study. Journal of Mental Health 2004;13(5):453-66.

Tyrer P. Further information about your trial [personal communication]. Email to: M Dieterich 19 May 2009.

Tyrer P. Integrated care management in the care of severe psychotic illness. National Research Register 2001; Vol. 1.

Tyrer P. Integrated care management in the care of severe psychotic illness. (In the process of application). National Research Register 2000.

Tyrer P. Randomised controlled trial (RCT) on qualitative analysis of internal and external care management in severe mental illness. www.isrctn.com/ISRCTN01712888 (first received 23 January 2004).
Tyrer P. Randomised controlled trial on qualitative analysis of internal and external care management in severe mental illness. National Research Register 2001.

\section{Salyers 2010 \{published data only\}}

Salyers MP, McGuire AB, Rollins AL, Bond GR, Mueser KT, Macy VR. Integrating assertive community treatment and illness management and recovery for consumers with severe mental illness. Community Mental Health Journal 2010;46(4):319-29.

Salyers 2014 \{published data only\}

Salyers MP, McGuire AB, Kukla M, Fukui S, Lysaker PH, Mueser KT. A randomized controlled trial of illness management and recovery with an active control group. Psychiatric Services 2014;65:1005-11.

Santiago-Arizona 1985 \{published data only\} Santiago JM, McCall-Perez F, Bachrach LJ. Integrated services for chronic mental patients: theoretical perspective and experimental results. General Hospital Psychiatry 1985;7:309-15.

\section{Sato 2012 \{published data only\}}

Sato S, Ikebuchi E, Anzai N, Inoue S. Effects of psychosocial program for preparing long-term hospitalized patients with schizophrenia for discharge from hospital: randomized controlled trial. Psychiatry and Clinical Neurosciences 2012;66:474-81.

\section{Schmidt 2005 \{published data only\}}

Schmidt LT. Comparison of Service Outcomes of Case Management Teams With and Without a Consumer Provider. New York: University of Medicine and Dentistry of New York, 2005.

\section{Segal 2010 \{published data only\}}

Segal SP, Silverman CJ, Temkin TL. Self-help and community mental health agency outcomes: a recoveryfocused randomized controlled trial. Psychiatric Services 2010;61(9):905-10.

\section{Segal 2011 \{published data only\}}

Segal SP, Silverman CJ, Temkin TL. Outcomes from consumeroperated and community mental health services: a randomized controlled trial. Psychiatric Services 2011;62:915-21.

\section{Sha 2010 \{published data only\}}

Sha R, Cai J, Zhu WM, Fu PX, Bo ZS, Song YH, et al 沙蓉, 蔡 军, 朱卫明, 付培金䊗, 柏忠生, 宋月红, et al. The effect of skill training on returning to community for chronic schizophrenia [《\#返社区技能训练程\#》对慢性精神分裂症康复效果的分 析]. Journal of Psychiatry [\#\#\#\#\#] 2010;23(05):340-2.

Shen WW 2013 \{published data only\}

Shen WW, Zhang ZQ, Chen J, Huang X, Gong S, Deng H 申文 武, 张倬秋, 陈娟, 黄霞, 龚姝, 邓红. The effect of HospitalCommunity-Family rehabilitation intervention on quality of life of schizophrenia patients [“医院-社区-家庭”一体化精神康 复模式对精神分裂症患者生存质量的影响]. Chinese Journal of Evidence-Based Medicine [中国循证医学杂志] 2013, issue 10:1176-9. 
Shern-USA2 \{published and unpublished data\}

Greenwood RM, Schaefer-McDaniel N, Winkel G, Tsemberis S. Decreasing psychiatric symptoms by increasing choice in services for adults with histories of homelessness. American Journal of Community Psychology 2005;36(3/4):223-38.

Gulcur L, Stefancic A, Shinn M, Tsemberis S, Fischer S. Housing, hospitalization, and cost outcomes for homeless individuals with psychiatric disabilities participating in continuum of care and Housing First programmes. Journal of Community and Applied Social Psychology 2003;13:171-86.

Gulcur L, Tsemberis S, Stefancic A, Greenwood RM. Community integration of adults with psychiatric disabilities and histories of homelessness. Community Mental Health Journal 2007;43(3):211-28

Hul L. Further information on pathways to housing [personal communication]. Email to: M Dieterich 10 June 2009.

Padgett DK, Gulcur L, Tsemberis S. Housing First services for people who are homeless with co-occurring serious mental illness and substance abuse. Research on Social Work Practice 2006;16(1):74-83.

Tsemberis S, Gulcur L, Nakae M. Housing First, consumer choice, and harm reduction for homeless individuals with a dual diagnosis. American Journal of Public Health 2004;94(4):651-6. [PUBMED: 15054020]

Tsemberis SJ, Moran L, Shinn M, Asmussen SM, Shern DL. Consumer preference programmes for individuals who are homeless and have psychiatric disabilities: a drop-in center and a supported housing programme. American Journal of Community Psychology 2003;32(3-4):305-17. [PUBMED: 14703266]

\section{Shern-USA3 \{published data only\}}

Stefancic A, Tsemberis S. Housing First for long term shelter dwellers with psychiatric disabilities in a suburban county: a four-year study of housing access and retention. Journal of Primary Prevention 2007;28:265-79.

\section{Shern-USA4 \{published data only\}}

Tsemberis S, Eisenberg RF. Pathways to housing: supported housing for street-dwelling homeless individuals with psychiatric disabilities. Psychiatric Services 2000;51(4):487-93.

\section{Solomon 2014 \{published data only\}}

Hanrahan NP, Solomon P, Hurford MO. A pilot randomized control trial testing a transitional care model for acute psychiatric conditions. Journal of the American Psychiatric Nurses Association 2014;20:315-27.

* Solomon P, Hanrahan NP, Hurford M, DeCesaris M, Josey L. Lessons learned from implementing a pilot RCT of transitional care model for individuals with serious mental illness. Archives of Psychiatric Nursing 2014;28(4):250-5. [DOI: 10.1016/ j.apnu.2014.03.005]

\section{Solomon-Pennsylvania1 \{published data only\}}

Solomon P, Draine J. Consumer case management and attitudes concerning family relations among persons with mental illness. Psychiatric Quarterly 1995;66(3):249-61. [PUBMED: 7568532]

Solomon P, Draine J. Family perceptions of consumers as case managers. Community Mental Health Journal 1994;30(2):165-76. [PUBMED: 8013213]

Solomon P, Draine J. One-year outcomes of a randomized trial of consumer case management. Evaluation and Programme Planning 1995;18:117-27.

Solomon P, Draine J. Perspectives concerning consumers as case managers. Community Mental Health Journal 1996;32(1):41-6. [PUBMED: 8635316]

Solomon P, Draine J. Satisfaction with mental health treatment in a randomized trial of consumer case management. Journal of Nervous and Mental Disease 1994;182(3):179-84.

Solomon P, Draine J. The efficacy of a consumer case management team: 2-year outcomes of a randomized trial. Journal of Mental Health Administration 1995;22(2):135-46. [PUBMED: 10142127]

\section{Somers 2013 \{published data only\}}

Somers JM, Patterson ML, Moniruzzaman A, Currie L, Rezansoff SN, Palepu A, et al. Vancouver At Home: Pragmatic randomized trials investigating Housing First for homeless and mentally ill adults. Trials 2013;14:365. [PUBMED: 24176253]

\section{Song 2013 \{published data only\}}

Song PJ, Pei SY, Sun SH 宋珀槿, 裴双义, 孙淑红. The effect of family psychological intervention combined with social skill training on rehabilitation in patients with schizophrenia [家庭 心理干预联合社会技能训练治疗康复期精神分裂症患者的疗 效] . Chinese Journal of Gerontology [Zhongguo lao nian xue za zhi; 中国老年学杂志] 2013, issue 20:5019-20.

\section{Stein 1974 \{published data only\}}

The 1974 APA achievement award winners. Gold award: a community treatment program. Mendota Mental Health Institute, Madison, Wisconsin. Hospital and Community Psychiatry 1974; Vol. 25, issue 10:669-72. [PUBMED: 4416614]

Stein-Wisconsin \{published data only\}

Stein LI, Test MA. Alternative to mental hospital treatment: I. Conceptual model, treatment programme, and clinical evaluation. Archives of General Psychiatry 1980;37(4):392-7. [PUBMED: 7362425]

Stein LI, Test MA, Marx AJ. Alternative to the hospital: a controlled study. American Journal of Psychiatry 1975;132:517-22. [PUBMED: 164129]

Test MA, Stein LI. Alternative to mental hospital treatment. III. Social cost. Archives of General Psychiatry 1980;37(4):409-12. [PUBMED: 7362426]

Test MA, Stein LI. Training in community living: a follow-up look at a Gold-Award programme. Hospital and Community Psychiatry 1976;27(3):193-4. [PUBMED: 819348] 
Test MA, Stein LI. Training in community living: research design and results. In: Stein LI, Test MA editor(s). Alternatives to Mental Hospital Treatment. New York: Plenum Publishing Corporation, 1978:57-74.

Weisbrod BA, Test MA, Stein LI. Alternative to mental hospital treatment: II. Economic benefit cost analysis. Archives of General Psychiatry 1980;37(4):400-5. [PUBMED: 6767462]

\section{Stultz 2014 \{published data only\}}

Stultz N. Home treatment for acute psychiatric care. clinicaltrials.gov/ct2/show/NCT02322437 (first received 11 December 2014).

\section{Sungur 2011 \{published data only\}}

Sungur M, Soygur H, Guner P, Ustun B, Cetin I, Falloon IR. Identifying an optimal treatment for schizophrenia: a 2-year randomized controlled trial comparing integrated care to a high-quality routine treatment. International Journal of Psychiatry in Clinical Practice 2011;15:118-27.

\section{Supereden 2012 \{published data only\}}

ISRCTN61621571. Sustaining positive engagement and recovery (SUPEREDEN) - the next step after early intervention for psychosis. Study 3: Improving social recovery in young people with emerging severe social disability: a proof of principle randomised controlled trial. www.isrctn.com/ISRCTN61621571 (first received 26 June 2012).

\section{Susser-New York 1997 \{published data only\}} Jones K, Colson PW, Holter MC, Lin S, Valencia E, Susser E, et al. Cost-effectiveness of critical time intervention to reduce homelessness among persons with mental illness. Psychiatric Services 2003;54(6):884-90. [PUBMED: 12773605]

Susser E, Valencia E, Conover S, Felix A, Tsai WY, Wyatt RJ. Preventing recurrent homelessness among mentally ill men: a "critical time" intervention after discharge from a shelter. American Journal of Public Health 1997;87(2):256-62.

\section{Tang 2013 \{published data only\}}

Tang J, Wu KM, Liu DA, Zhou XL, Li JJ, Zhou Q 唐兢, 吴克明, 刘德安, 周晓林, 李建军, 周卿. The effect of the communityfamily rehabilitation intervention for patients with chronic schizophrenia [社区-家庭康复 对慢性精神分裂症患者的干预 分析]. Shanghai Medical and Pharmaceutical Journal [上海医 药] 2013, issue 08:60-2.

\section{Tao-China 2004 \{published data only\}}

Tao YL, Yong YL, Tian CW, Wei YY, Yu HC, Yuan A, et al. Effect of early intervention on quality of life in patients with first episode schizophrenia. Chinese Journal of Clinical Rehabilitation 2004;8(18):3464-5.

Teague-New Hampshire 1995 \{published data only\} Teague GB, Drake RE, Ackerson TH. Evaluating the use of continuous treatment teams for persons with mental illness and substance abuse. Psychiatric Services 1995;46:689-95.
Thornicroft-Maryland 1991 \{published data only\}

Thornicroft G, Breakey WR. The COSTAR programme 1: Improving social networks of the long term mentally ill. British Journal of Psychiatry 1991;159:245-9.

Tomita 2011 \{published data only\}

Tomita A, Herman DB. The impact of critical time intervention in reducing psychiatric rehospitalization after hospital discharge. Psychiatric Services 2012;63(9):935-7. [DOI: 10.1176/ appi.ps.201100468]

Tomita MA. Examining the Impact and Theoretical Pathway of Critical Time Intervention on Psychiatric Re-Hospitalization Outcomes Among Formerly Homeless Individuals With Severe Mental Illness [Dissertation]. Columbia University, 2012.

Toro-New York 1997 \{published data only\}

Toro PA, Bellavia CW, Wall DD, Passero-Rabideau JM, Daeschler CV, Thomas DM. Evaluating an intervention for homeless persons: results of a field experiment. Journal of Consulting and Clinical Psychology 1997;65:476-84.

Tyrer-UK 1995 \{published and unpublished data\}

Tyrer P, Morgan J, Van Horn E, Jayakody M, Evans K, Brummell $\mathrm{R}$, et al. A randomised controlled study of close monitoring of vulnerable psychiatric patients. Lancet 1995;345(8952):756-9. [MEDLINE: 7891486]

van Meijel 2003 \{published data only\} van Meijel B. Relapse Prevention in Patients With Schizophrenia: a Nursing Intervention Study [Dissertation]. Utrecht: Universiteit Utrecht, 2003.

\section{Vesterager 2011 \{published data only\}}

Vesterager L, Christensen TT, Olsen BB, Krarup G, Forchhammer HB, Melau M, et al. Cognitive training plus a comprehensive psychosocial programme (opus) versus the comprehensive psychosocial programme alone for patients with first-episode schizophrenia (the NEUROCOM trial): a study protocol for a centrally randomised, observer-blinded multicentre clinical trial. Trials 2011;12:35.

Vincent-Ohio 1977 \{published data only\}

Vincent P, Price JR. Evaluation of a VNA Mental Health Project. Nursing Research 1977;26:361-7.

Wang 2008a \{published data only\}

Wang YH. Effect of community nursing on schizophrenic patients in convalescence [社区护理对康复期精神分裂症患者 的影响]. Journal of Clinical Nursing 2008;7(3):16-7.

\section{Wang F 2012 \{published data only\}}

Wang F, Li HL王飞, 李红丽. The short-term efficacy of family rehabilitation for patients with chronic schizophrenia [家庭式 康复治疗对慢性精神分裂症患者的近期疗效观察]. Chinese Magazine of Drug Abuse Prevention and Treatment [中国药物 滥用防治杂志] 2012, issue 05:269-72.

Wang FY 2013 \{published data only\}

Wang FY 王凤英. Family rehabilitation for discharged patients with schizophrenia [家庭康复在精神分裂症出院患者中的 
应用] . Journal of Qilu Nursing [齐鲁护理杂志] 2013, issue 13:99-100.

\section{Wang YL 2012 \{published data only\}}

Wang YL 王玉玲. The effect of family intervention on the rehabilitation of patients with schizophrenia [家庭护理干预 对精神分裂症患者康复的影响]. Psychiatric Nursing Risk Management Training Class and Academic Communication [ 精 神科护理风险管理培训班及学术交流会] 2012:1.

\section{Wang YQ 2010a \{published data only\}}

Wang YQ 王英群. Effects of psychological intervention on social functions of schizophrenics in rehabilitation period [心理干 预对康复期精神分裂症患者社会功能的影响]. Journal of Shandong Medical College [\#\#\#\#\#\#\#\#\#\#\#] 2010;32(07):545-7.

Wang Z 2012 \{published data only\}

Wang Z, Liu XW, Tan YY 王志, 刘晓伟, 谈幼玉. Family intervention effect on social dysfunction in rehabilitation period of schizophrenia [家庭干预在精神分裂症患者社会功能障碍 康复中的效果分析]. Journal of Military Surgeon in Southwest China [西南军医] 2012, issue 02:208-10.

\section{Wen 2010 \{published data only\}}

Wen L, Liu DJ 文丽, 刘杜鹃. Influence of PDCA home care intervention for patients with schizophrenia [家庭护理干预对 精神分裂症患者的影响]. Chinese Journal of Misdiagnostics [\#\# \#\#\#\#] 2010;10(11):2529-30.

\section{Wirshing 2006 \{published data only\}}

Wirshing DA, Guzik LH, Zorick TS, Pierre JM, Resnick SA, Goldstein $D$, et al. Community re-entry program training module for schizophrenic inpatients improves treatment outcomes. Schizophrenia Research 2006;1-3:338-9.

\section{Wood-New Zealand 1994 \{published data only\}}

Wood K, Anderson J. The effect on hospital admissions of psychiatric case management involving general practitioners: preliminary results. Australian and New Zealand Journal of Psychiatry 1994;28:223-9.

\section{Wu 2013 \{published data only\}}

Wu J, Zhang SB, Tang YL, Tan Y 吴菁, 章三斌, 唐义莲, 谭悦. The effects of group-psychotherapy and family therapy for the schizophrenics in rehabilitation period [团体心理治疗和家 庭治疗对康复期精神分裂症患者疗效的对照研究] . China Journal of Health Psychology [\#\#\#\#\#\#\#\#] 2013;21(12):1780-2.

\section{Wunderink 2015 \{published data only\}}

Wunderink L. A trial comparing the effectiveness of Assertive Community Treatment enhanced with evidence based interventions with standard Assertive Community Treatment. www.isrctn.com/ISRCTN37364372 (first received 15 March 2013). [DOI: 10.1186/ISRCTN37364372]

\section{Xing 2013 \{published data only\}}

Xing F, Lu QF 邢锋, 鲁青芳. Effects of community comprehensive rehabilitation training for patients with chronic schizophrenia [社区综合康复训练对慢性精神分裂症患者效 果观察] . China Medicine and Pharmacy [中国医药科学] 2013, issue 17:79-80,130.
Yang 2013 \{published data only\}

Yang X, Guo LR 杨新, 郭丽蓉. Effects of community rehabilitation for patients with schizophrenia [精神分裂症患者 社区康复训练的疗效观察]. China Journal of Pharmaceutical Economics [中国药物经济学] 2013, issue 04:412-3.

Yao 2013 \{published data only\}

Yao FJ, Wang ZM, Qin ZH 姚丰菊, 王志敏, 秦志华. The effect of case management on community rehabilitation of female patients with schizophrenia [个案管理对社区女性精神分裂症 患者康复的影响]. China Medicine and Pharmacy [中国医药科 学] 2013, issue 15:13-5.

\section{Yao 2014 \{published data only\}}

Yao ZZ, Xu Q, Wu LF, Fu WZ, Lu Y, Zhao AP. Effects of day nursing care in community on social function of patients with chronic schizophrenia. Journal of Shanghai Jiaotong University (Medical Science) 2014;34:830-5.

\section{Yu 2011 \{published data only\}}

Yu X, Jia JD, Guo P, Zhang YH 余学, 贾金鼎, 郭平, 张䎦华. Effects of community-based comprehensive intervention on medicine consumption and social function in patients with schizophrenia [社区综合干预对分裂症患者药物消耗量和社会 功能的影响]. Chinese Journal of Health Psychology [\#\#\#\#\#\#\#\#] 2011;19(07):772-4.

\section{Yuan 2013 \{published data only\}}

Yuan YF, Yu ZY, Shen JB 袁月芳, 俞智勇, 沈建斌. The impact of community case management on the rehabilitation quality for patients with schizophrenia [社区个案管理对精神分裂症 患者康复质量的影响]. Nursing Journal of Chinese People's Liberation Army [解放军护理杂志] 2013, issue 09:35-7.

Zheng H, Chen SL, Tang AD, Zhou LJ, Ji WD 郑宏, 陈思路, 汤爱 娣, 周路佳, 季卫东. The effects of comprehensive community service team for schizophrenia in community [综合服务团 队对社区精神分裂症患者综合干预的研究]. China Journal of Health Psychology [中国健康心理学杂志] 2012, issue 11:1615-7.

Zheng H, Chen XL, Niu X, Huang JJ, Zhou LJ, Tang AD 郑 宏, 陈思路, 牛昕, 黄嘉俊, 周路佳, 汤爱娣. Effects of comprehensive community interventions for families of schizophrenia patients [社区综合服务団队对精神分裂症患者 家庭的干预效果分析]. Chinese General Practice [中国全科医 学] 2013, issue 01:90-2.

\section{Zhang SY 2013 \{published data only\}}

Zhang SY, Zhang ZX, Dong CL 张淑云, 张仲霞, 董春玲. Effects of intensive family care for patients with chronic schizophrenia [对慢性精神分裂症患者加强家庭护理干预的效果分析]. Chinese Journal of Trauma and Disability Medicine [中国伤残 [医学] 2013, issue 02:170-1.

\section{Zhang YF 2012 \{published data only\}}

Zhang YF 张燕锋. The effect of active interview on the rehabilitation of schizopheria in community of Luoding City [罗定市社区主动访视对精神分裂症的康复研究]. Medical Innovation of China [中国医学创新] 2012, issue 12:138-9. 
Zhang YM 2013 \{published data only\}

Zhang YM, Zhang ZW, Jiang LP, Zhao GX 张玉敏, 张振文, 蒋令 朋, 赵桂霞. Effects of community comprehensive intervention on medication compliance and quality of life in patients with schizophrenia [社区综合干预对精神分裂症患者服药依从性和 生活质量的影响].China Medicine and Pharmacy [中国医药科 学] 2013, issue 18:63-64,84.

\section{Zhao HM 2013 \{published data only\}}

Zhao HM, Li WX, He R 赵红梅, 李文秀, 何锐. The effect of community-based rehabilitation for patients with schizophrenia [社区康复措施在康复期精神分裂症患者中的应用效果观察]. Hainan Medical Journal [海南医学] 2013, issue 06:836-8.

\section{Zhu 2009 \{published data only\}}

Zhu YX, Liu Y, Sun QX, Wang HS 朱玉星, 刘勇, 孙群星, 王宏 升. Effects of psychologcial nursing care on cognition function of patients with schizophrenia [心理护理对慢性精神分裂 症患者认知功能的影响]. China Practical Medical [\#\#\#\#\#\#] 2009;4(22):190-2.

\section{Zhu DP 2012 \{published data only\}}

Zhu DP 竹道平. Influence of family psychological interventions on the rehabilitation of schizophrenia patients [家庭心理干预 对精神分裂症患者社区康复的影响]. China Practical Medical [中国实用医药] 2012, issue 15:240-1.

\section{References to studies awaiting assessment}

\section{Bonsack-Switzerland \{published data only\}}

Bonsack C. Efficacy of Transitional Case Management Following Psychiatric Hospital Discharge. clinicaltrials.gov/ct2/show/ record/NCT02258737 (first received 29 September 2014).

\section{ChiCTR-TRC-13003407 \{published data only\}}

ChiCTR-TRC-13003407. Adaptation and assessment of the Family-Based Assertive Community Treatment model for the treatment of schizophrenia in China: a randomized controlled trial. apps.who.int/trialsearch/Trial2.aspx?TrialID=ChiCTRTRC-13003407 (first received 25 March 2013).

\section{Dick-UK 2000 \{published data only\}}

Dick P. Randomised controlled trial of a community rehab programme in the treatment of chronic functional psychosis. National Research Register 2000.

Dick P. Randomised controlled trials of a community rehabilitation programme in the treatment of chronic functional psychosis. National Research Register 2001; Vol. 1.

\section{Guo-China 2003 \{published data only\}}

Guo H, Deng J, Li Z. The influence of the effect of community intervention with schizophrenics' family background and relevant factors. Chinese Journal of Clinical Medicine Practice 2003;2(9):779-84.

\section{Kawanishi-Japan 2014 \{published data only\}}

Kawanishi C, Aruga T, Ishizuka N, Yonemoto N, Otsuka K, Kamijo $\mathrm{Y}$, et al. Assertive case management versus enhanced usual care for people with mental health problems who had attempted suicide and were admitted to hospital emergency departments in Japan (ACTION-J): a multicentre, randomised controlled trial. Lancet Psychiatry 2014;1:193-201.

Kossobudzka-Poland 2001 \{published data only\}

Slupczynska-Kossobudzka E, Boguszewska L, Wojtowitz S. Effectiveness of mobile community teams in four centers a two-year follow up study. Postępy Psychiatrii i Neurologii 2001;10:289-99.

\section{Li 2010 \{published data only\}}

Li SC, Lu ZS, Hu YW, Yang RX, Qin D, Ge XW, et al 李守春, 卢 振胜, 胡雅伟, 杨瑞香, 秦东, 葛欣伟, et al. Influence of community comprehensive intervention on the quality of life in patients with chronic schizophrenia [社区综合干预对慢性精神 分裂症患者生活质量的影响]. Journal of Clinical Psychiatry [\#\# \#\#\#\#\#\#] 2010;20(1):46-8.

\section{Linszen-Netherlands 2002 \{published data only\}}

Linszen D. Early and critical period intervention in first episode schizophrenia: relapse, chronicity, early stabilisation, predictors over 4 years and new research. Schizophrenia Research 2004;70(1):66.

Linszen D, Wouters L, Dingemans P, De Haan L, Nieman D. Early and 3-year sustained intervention in first episode schizophrenia: relapse, stabilization and its predictors. Schizophrenia Research 2004;67(1):18.

Linszen DH, De Haan L, Dingemans P, van Bruggen M, Hofstra N, van Engelsdorp $\mathrm{H}$, et al. Treatment reluctance in first episode schizophrenia: Lack of insight, non-compliance and cannabis abuse predict bad outcome after eighteen months intervention. Schizophrenia Research 2003;60(1):325.

Linszen DH, Dingemans PM. Sustained intervention in recent onset schizophrenia: three year results of a controlled clinical trial. Schizophrenia Research 2002;53(3 Suppl 1):14.

\section{Manuel 2009 \{published data only\}}

Manuel JI. A longitudinal analysis of psychiatric medication adherence and provider continuity among individuals with cooccurring disorders. Dissertation Abstracts International Section A: Humanities and Social Sciences 2009;69(10A):4126.

O'Donnell-Australia 1999 \{published data only\}

O'Donnell M, Parker G, Proberts M, Matthews R, Fisher D, Johnson $B$, et al. A study of client focused case management and consumer advocacy: the community and consumer service project. Australian and New Zealand Journal of Psychiatry 1999;33(5):684-93. [PUBMED: 10544992]

Rivera-New York 2007 \{published data only\}

Rivera JJ, Sullivan AM, Valenti SS. Adding consumer-providers to Intensive Case Management: does it improve outcome?. Psychiatric Services 2007;58(6):802-9. [PUBMED: 17535940]

Ruggeri-Italy \{published data only\}

Ruggeri M, Bonetto C, Lasalvia A, De Girolamo G, Fioritti A, Rucci $P$, et al. A multi-element psychosocial intervention for early psychosis (get up piano trial) conducted in a catchment area of 10 million inhabitants: study protocol for a pragmatic 
cluster randomized controlled trial. Trials 2012;13:73. [DOI: 10.1186/1745-6215-13-73; PUBMED: 22647399]

\section{Sells-Connecticut 2006 \{published data only\}}

Sells D, Black R, Davidson L, Rowe M. Beyond generic support: incidence and impact of invalidation in peer services for clients with severe mental illness. Psychiatric Services 2008;59(11):1322-7.

Sells D, Davidson L, Jewell C, Falzer P, Rowe M. The treatment relationship in peer-based and regular case management for clients with severe mental illness. Psychiatric Services 2006;57(8):1179-84. [PUBMED: 16870970]

\section{Sharifi-Iran 2009 \{published data only\}}

Sharifi V, Amini H, Tehranidoost M, Yunesian M, JalaliRoudsari M, Sobhebidari P, et al. A randomized trial of a home aftercare service for patients with severe mental disorders in Iran. World Psychiatry 2009;8(Suppl 1):P03.175.

\section{Su-China 2008 \{published data only\}}

Su M, Wang CQ, Lin LX [苏勉, 王彩琴, 凌礼雄]. The effect of community intervention on the quality of life in patients with schizophrenia [社区干预对精神分裂症患者生活质量的影响]. Journal of Tropical Medicine [\#\#\#\#\#] 2008;8(2):138, 147-9.

\section{Tan-China 2005 \{published data only\}}

Tan B, He YF, Xiang EP. The observation of cure effects and medical expenses of community rehabilitation on schizophrenia. Journal of Hubei Institute for Nationalities Medical Edition 2005;22(2):31-3.

Tan B, Xiang EP, He YF. Effect of community prevention and treatment in patients with schizophrenia and their family economic burden. Chinese Journal of Clinical Rehabilitation 2005;9(28):24-6.

\section{Verhaegh-Netherlands 2006 \{published data only\}}

Verhaegh MJ, Bongers IM, Kroon H, Garretsen HF. Assertive Community Treatment for patients with a first episode psychosis. Model fidelity and specific adaptations for particular target groups [Assertive Community Treatment bij patienten met een eerste psychose. Modelgetrouwheid en doelgroepspecifieke aanpassingen]. Tijdschrift voor Psychiatrie 2007;49(11):789-98. [PUBMED: 17994498]

Verhaegh MJ, Bongers IM, Kroon H, Garretsen HF. Model fidelity of assertive community treatment for clients with first-episode psychosis: a target group-specific application. Community Mental Health Journal 2009;45(1):12-8. [PUBMED: 18925435]

Verhaegh MJM. Researching the effect of Assertive Community Treatment (ACT) for first episode psychosis: the use of peerinterviewers in a quasi-experimental study. Schizophrenia Research 2006;86(Suppl 1):S170.

\section{Wang 2009 \{published data only\}}

Wang YH, Tang JS, Chai XS, Zheng CQ, Zhang JX, Weng Z 王延 祜, 唐济生, 柴新生, 郑崇泉, 张敬悬, 翁正. The effects of comprehensive intervention on social rehabilitation in patients with schizophrenia [社区综合干预对精神分裂症患者社会康复
效果的对照研究]. Shandong Archives of Psychiatry [山东精神 [医学] 2009; Vol. 22, issue 4:244-6.

\section{Zhang 2009 \{published data only\}}

Zhang HZ, Jin XX 张海杰, 靳新霞. Effects of community intervention on social function and quality of life in patients with schizophrenia [社区干预对精神分裂症患者社会功能及 生存质量的影响]. Shandong Archives of Psychiatry [\#\#\#\#\#] 2009;22(5):368-70.

\section{Zoeteman-Netherlands \{published data only\}}

Zoeteman JB. Assertive community treatment (ACT) versus case management in treating homeless patients with severe mental illness; a randomized controlled trial. apps.who.int/trialsearch/ Trial2.aspx?TrialID=NTR896 (first received 10 November 2006).

\section{References to ongoing studies}

Koike-Japan \{published data only\}

Nishida A, Koike S, Yamasaki S, Ando S, Nakamura T, Harima H, et al. Comprehensive early intervention for patients with first-episode psychosis in Japan (J-CAP): study protocol for a randomised controlled trial. apps.who.int/trialsearch/ Trial2.aspx?TrialID=JPRN-UMIN000005092 (first received 15 February 2011).

\section{Lutgens 2015 \{published data only\}}

Lutgens D, lyer S, Joober R, Brown TG, Norman R, Latimer E, et al. A five-year randomized parallel and blinded clinical trial of an extended specialized early intervention vs. regular care in the early phase of psychotic disorders: study protocol. BMC Psychiatry 2015 Feb 14;15:22.

\section{Malla-Canada \{published data only\}}

Malla A, Norman R, lyer S, Joober R, Brown T, Schmitz N, et al. Extending specialized early intervention service from 2 to 5 years: a randomized controlled trial. Eighth International Conference on Early Psychosis; 2012 Oct 11-13; San Francisco. 2012.

Rondeau MC, Rho A, lyer S, Joober R, Schmitz N, Latimer E, et al. A randomized controlled evaluation of 'extended specialized early intervention service' vs. 'regular care' for long-term management of early psychosis. Early Intervention in Psychiatry 2012;6:72.

\section{NCT01313052 \{published data only\}}

NCT01313052. Forensic assertive community treatment: an emerging model of service delivery. clinicaltrials.gov/ct2/show/ NCT01313052 (first received 8 March 2011).

RISE - Ethiopia \{published data only\}

NCT02160249. RISE (Rehabilitation Intervention for People With Schizophrenia in Ethiopia): a cluster-randomised trial. clinicaltrials.gov/ct2/show/NCT02160249 (first received 3 June 2014).

Walsh-Connecticut \{published data only\}

Walsh B, Srihari VH, Woods S. Randomized trial of usual care versus specialized, phase-specific care in the public 
sector for first episode psychosis. clinicaltrials.gov/ct2/show/ NCT00309452 (first received 29 March 2006).

\section{Additional references}

\section{Altman 1996}

Altman DG, Bland JM. Detecting skewness from summary information. BMJ 1996;313(7066):1200. [PUBMED: 8916759]

\section{Andreasen 1982}

Andreasen NC. Negative symptoms in schizophrenia. Archives of General Psychiatry 1982;39:784-8.

\section{Andreasen 1984}

Andreasen NC. Scale for the Assessment of Positive Symptoms (SAPS). lowa City: University of lowa, 1984.

\section{Andreasen 1989}

Andreasen N. Scale for the Assessment of Negative Symptoms (SANS). British Journal of Psychiatry 1989;7:53-8.

\section{APA 1987}

American Psychiatric Association. Diagnostic and Statistical Manual of Mental Disorders. 3rd Edition. Washington, DC: American Psychiatric Association, 1987.

\section{APA 1994}

American Psychiatric Association. Diagnostic and Statistical Manual of Mental Disorders. 4th Edition. Washington, DC: American Psychiatric Association, 1994.

\section{Asberg 1978}

Asberg M, Montgomery SA, Perris C, Schalling D, Sedvall G. A comprehensive psychopathological rating scale. Acta Psychiatrica Scandinavica 1978;271:5-27.

\section{Baker 1983}

Baker R, Hall J. REHAB: A new assessment instrument for chronic psychiatric patients. Schizophrenia Bulletin 1988;14(1):97-111.

\section{Beck 1979}

Beck A, Rush A, Shaw B, Emery G. Cognitive Therapy of Depression. New York: Guilford Press, 1979.

\section{Bland 1997}

Bland JM. Statistics notes. Trials randomised in clusters. BMJ 1997;315:600.

\section{Boissel 1999}

Boissel JP, Cucherat M, Li W, Chatellier G, Gueyffier F, Buyse $M$, et al. The problem of therapeutic efficacy indices. 3. Comparison of the indices and their use [Apercu sur la problematique des indices d'efficacite therapeutique, 3 : comparaison des indices et utilisation. Groupe d'Etude des Indices D'efficacite.]. Therapie 1999;54(4):405-11. [PUBMED: 10667106]

\section{Bond 2015}

Bond GR, Drake RE. The critical ingredients of assertive community treatment. World Psychiatry: Official Journal of the World Psychiatric Association (WPA) 2015;14(2):240-2. [PUBMED: 26043344]

\section{Brewer 2015}

Brewer WJ, Lambert TJ, Witt K, Dileo J, Duff C, Crlenjak C, et al. Intensive case management for high-risk patients with first-episode psychosis: service model and outcomes. Lancet Psychiatry 2015;2(1):29-37. [PUBMED: 26359610]

\section{Burns 2001}

Burns T, Knapp M, Catty J, Healey A, Henderson J, Watt H, et al. Home treatment for mental health problems: a systematic review. Health Technology Assessment 2001;5(15):1-139. [PUBMED: 11532236]

\section{Burns 2008}

Burns T. Case management or assertive community treatment. What is the difference?. Epidemiologia e Psichiatria Sociale 2008; Vol. 17, issue 2:99-105. [PUBMED: 18589623]

\section{Burns 2009}

Burns T. End of the road for treatment-as-usual studies?. British Journal of Psychiatry 2009;195(1):5-6. [PUBMED: 19567887]

\section{Burns 2010}

Burns T. The rise and fall of assertive community treatment? International Review of Psychiatry (Abingdon, England) 2010;22(2):130-7. [PUBMED: 20504053]

\section{Burns 2012}

Burns T. Newer is not automatically better. Psychiatrist 2012;36:477.

\section{Catty 2002}

Catty J, Burns T, Knapp M, Watt H, Wright C, Henderson J, et al. Home treatment for mental health problems: a systematic review. Psychological Medicine 2002;32(3):383-401. [PUBMED: 11989985]

\section{Coldwell 2007}

Coldwell CM, Bender WS. The effectiveness of assertive community treatment for homeless populations with severe mental illness: a meta-analysis. American Journal of Psychiatry 2007;164(3):393-9. [PUBMED: 17329462]

\section{Deeks 2000}

Deeks J. Issues in the selection for meta-analyses of binary data. Eighth International Cochrane Colloquium; 2000 Oct 25-28; Cape Town. Cape Town: The Cochrane Collaboration, 2000.

\section{Department of Health 1990}

Department of Health. Community Care in the Next Decade and Beyond. London: HMSO, 1990.

\section{Department of Health 1999}

Department of Health. National Service Framework for Mental Health. London: DH, 1999. 


\section{Derogatis 1974}

Derogatis LR, Lipman RS, Rickels K, Uhlenhuth EH, Covi L. The Hopkins Symptom Checklist (HSCL): a self-report symptom inventory. Behavioral Science 1974;19(1):1-15.

\section{Derogatis 1983}

Derogatis LR, Melisaratos N. The Brief Symptom Inventory: an introductory report. Psychological Medicine 1983;13:595-605.

\section{Divine 1992}

Divine GW, Brown JT, Frazier LM. The unit of analysis error in studies about physicians' patient care behavior. Journal of General Internal Medicine 1992;7(6):623-9.

\section{Donner 2002}

Donner A, Klar N. Issues in the meta-analysis of cluster randomized trials. Statistics in Medicine 2002;21:2971-80.

\section{Drake 1996}

Drake RE, Mueser KT, McHugo GJ. Clinician rating scales: Alcohol Use Scale (AUS), Drug Use Scale (DUS), and Substance Abuse Treatment Scale (SATS). In: Sederer LI, Dickey B editor(s). Outcomes Assessment in Clinical Practice. Baltimore: Williams and Wilkins, 1996.

\section{Drukker 2008}

Drukker M, Maarschalkerweerd M, Bak M, Driessen G, a Campo J, de Bie A, et al. A real-life observational study of the effectiveness of FACT in a Dutch mental health region. $B M C$ Psychiatry 2008;8:93. [PUBMED: 19055813]

\section{Egger 1997}

Egger M, Davey-Smith G, Schneider M, Minder CSO. Bias in meta-analysis detected by a simple, graphical test. BMJ 1997;13:629-34.

\section{Elbourne 2002}

Elbourne D, Altman DG, Higgins JPT, Curtina F, Worthingtond HV, Vaile A. Meta-analyses involving crossover trials: methodological issues. International Journal of Epidemiology 2002;31(1):140-9.

\section{Endicott 1976}

Endicott J, Spitzer RL, Fleiss JL, Cohen J. The global assessment scale. A procedure for measuring overall severity of psychiatric disturbance. Archives of General Psychiatry 1976;33(6):766-71. [PUBMED: 938196]

\section{Endicott 1978}

Endicott J, Spitzer RL. A diagnostic interview: the schedule for affective disorders and schizophrenia. Archives of General Psychiatry 1978;35(7):837-44. [PUBMED: 678037]

\section{Fekete 1998}

Fekete DM, Bond GR, McDonel EC, Salyers MP, Chen A, Miller L. Rural assertive community treatment: a field experiment. Psychiatric Rehabilitation Journal 1998;21(4):371.

\section{Finnerty 2015}

Finnerty MT, Manuel JI, Tochterman AZ, Stellato C, Fraser LH, Reber CA, et al. Clinicians' perceptions of challenges and strategies of transition from assertive community treatment to less intensive services. Community Mental Health Journal 2015;51(1):85-95. [PUBMED: 24526472]

\section{First 1997}

First MB, Spitzer RL, Gibbon M, Williams JBW. Structured Clinical Interview for DSM-IV Axis I Disorders (SCID). Washington, DC: American Psychiatric Press, 1997.

\section{Gerber 1999}

Gerber GJ, Prince PN. Measuring client satisfaction with assertive community treatment. Psychiatric Services 1999;50(4):546-50. [PUBMED: 10211738]

\section{Gilburt 2012}

Gilburt H, Burns T, Copello A, Coulton S, Crawford M, Day E, et al. Assertive Community Treatment for alcohol dependence (ACTAD): study protocol for a randomised controlled trial. Trials 2012;13:19. [PUBMED: 22348423]

\section{Glover 2006}

Glover G, Arts G, Babu KS. Crisis resolution/home treatment teams and psychiatric admission rates in England. British Journal of Psychiatry 2006;189:441-5. [PUBMED: 17077435]

\section{Green 1987}

Green RS, Gracely EJ. Selecting a rating scale for evaluating services to the chronically mentally ill. Community Mental Health Journal 1987;23(2):91-102. [PUBMED: 3652669]

\section{Gulliford 1999}

Gulliford MC. Components of variance and intraclass correlations for the design of community-based surveys and intervention studies: data from the Health Survey for England 1994. American Journal of Epidemiology 1999;149:876-83.

\section{Henderson 1980}

Henderson S, Duncan-Jones P, Byrne DG, Scott R. Measuring social relationships. The Interview Schedule for Social Interaction. Psychological Medicine 1980;10(4):723-34. [PUBMED: 7208730]

\section{Higgins 2003}

Higgins JP, Thompson SG, Deeks JJ, Altman DG. Measuring inconsistency in meta-analyses. BMJ 2003;327:557-60.

\section{Higgins 2011}

Higgins JP, Green S, editor(s). Cochrane Handbook for Systematic Reviews of Interventions Version 5.1.0 (updated March 2011). The Cochrane Collaboration, 2011. Available from handbook.cochrane.org.

\section{Holloway 1991}

Holloway F. Case management for the mentally ill: looking at the evidence. International Journal of Social Psychiatry 1991;37:2-13.

\section{Holloway 1995}

Holloway F. Home treatment as an alternative to hospital admission. In: Tyrer P, Creed F editor(s). Community Psychiatry in Action. Cambridge: Cambridge University Press, 1995. 


\section{Intagliata 1982}

Intagliata J. Improving the quality of community care for the chronically mentally disabled: the role of case management. Schizophrenia Bulletin 1982;8:655-74.

\section{Johnson 2008}

Johnson S. So what shall we do about assertive community treatment?. Epidemiologia e Psichiatria Sociale 2008; Vol. 17, issue 2:110-4. [PUBMED: 18589625]

\section{Kay 1986}

Kay SR, Opler LA, Fiszbein A. Positive and Negative Syndrome Scale (PANSS) Manual. North Tonawanda, NY: Multi-Health Systems, 1986.

\section{Killaspy 2008}

Killaspy H, Johnson S, King M, Bebbington P. Developing mental health services in response to research evidence. Epidemiologia e Psichiatria Sociale 2008;17(1):47-56. [PUBMED: 18444458]

\section{Killaspy 2012}

Killaspy $\mathrm{H}$. Importance of specialisation in psychiatric services (commentary on): How did we let it come to this?. Psychiatrist 2012;36:364-5.

\section{Kirk 2013}

Kirk TA, Di Leo P, Rehmer P, Moy S, Davidson L. A case and care management program to reduce use of acute care by clients with substance use disorders. Psychiatric Services (Washington, DC) 2013;64(5):491-3. [PUBMED: 23632578]

\section{Krawiecka 1977}

Krawiecka M, Goldberg D, Vaughan M. A standardized psychiatric assessment scale for rating chronic psychotic patients. Acta Psychiatrica Scandinavica 1977;55(4):299-308. [PUBMED: 855676]

\section{Larsen 1979}

Larsen DL, Attkisson CC, Hargreaves WA, Nguyen TD. Assessment of client/patient satisfaction: development of a general scale. Evaluation and Programme Planning 1979;2(3):197-207. [PUBMED: 10245370]

\section{Lehman 1983}

Lehman A. The well being of chronic mental patients: assessing their quality of life. Archives of General Psychiatry 1983;40:369-73.

\section{Lehman 1988}

Lehman AF. A quality of life interview for the chronically mentally ill. Evaluation and Programme Planning 1988;11:51-62.

\section{Lehman 1993}

Lehman AF, Postrado LT, Rachuba LT. Convergent validation of quality of life assessments for persons with severe mental illnesses. Quality of Life Research 1993;2(5):327-33. [PUBMED: 8136797]

\section{Leucht 2005a}

Leucht S, Kane JM, Kissling W, Hamann J, Etschel E, Engel RR. What does the PANSS mean?. Schizophrenia Research 2005;79(2-3):231-8. [PUBMED: 15982856]

\section{Leucht 2005b}

Leucht S, Kane JM, Kissling W, Hamann J, Etschel E, Engel R. Clinical implications of Brief Psychiatric Rating Scale scores. British Journal of Psychiatry 2005;187:366-71. [PUBMED: 16199797]

\section{Low 2013}

Low L, Tan YY, Lim BL, Poon WC, Lee C. Effectiveness of assertive community management in Singapore. Annals of the Academy of Medicine, Singapore 2013;42(3):125-32. [PUBMED: 23604501]

\section{Malone 2007}

Malone D, Newron-Howes G, Simmonds S, Marriot S, Tyrer P. Community mental health teams (CMHTs) for people with severe mental illnesses and disordered personality. Cochrane Database of Systematic Reviews 2007, Issue 3. [DOI: 10.1002/14651858.CD000270.pub2]

\section{Marshall 2000}

Marshall M, Lockwood A, Bradley C, Adams C, Joy C, Fenton M. Unpublished rating scales: a major source of bias in randomised controlled trials of treatments for schizophrenia. British Journal of Psychiatry 2000;176:249-52.

\section{Marshall 2008}

Marshall M. What have we learnt from 40 years of research on Intensive Case Management?. Epidemiologia e Psichiatria Sociale 2008; Vol. 17, issue 2:106-9. [PUBMED: 18589624]

\section{McGrew 1994}

McGrew JH, Bond GR, Dietzen L, Salyers M. Measuring the fidelity of implementation of a mental health programme model. Journal of Consulting and Clinical Psychology 1994;62(4):670-8. [PUBMED: 7962870]

\section{McGrew 1995}

McGrew JH, Bond GR. Critical ingredients of assertive community treatment: judgements of the experts. Journal of Mental Health Administration 1995;22:113-25.

\section{McGuffin 1991}

McGuffin P, Farmer A, Harvey I. A polydiagnostic application of operational criteria in studies of psychotic illness: development and reliability of the OPCRIT system. Archives General Psychiatry 1991;48:764-70.

\section{McHugo 1995}

McHugo GJ, Drake RE, Burton HL, Ackerson TH. A scale for assessing the stage of substance abuse treatment in persons with severe mental illness. Journal of Nervous and Mental Disease 1995;183(12):762-7. [PUBMED: 8522938]

\section{Mueser 1995}

Mueser KT, Drake RE, Clark RE, McHugo GJ, Mercer-McFadden C, Ackerson TH. Evaluating Substance Abuse in Persons with 
Severe Mental Illness. Cambridge: MA: Human Services Research Institute, 1995.

\section{Murray 1996}

Murray CL, Lopez AD. The Global Burden of Disease. Cambridge: Harvard University Press, 1996.

\section{National Institute of Mental Health 1987}

National Institute of Mental Health. Towards a Model for a Comprehensive, Community-Based Mental Health System. Washington, DC: NIMH, 1987.

\section{Nishio 2014}

Nishio M, Sono T, Ishiguro T, Horiuchi K, Ambo H. How many Assertive Community Treatment Teams are needed in Japan? Estimate from Need Survey in Sendai City. Clinical Practice and Epidemiology in Mental Health 2014;10:184-90.

\section{Olfson 1990}

Olfson M. Assertive community treatment: an evaluation of the experimental evidence. Hospital and Community Psychiatry 1990;41:634-41.

\section{Oliver 1996}

Oliver J, Huxley P, Bridges K, Mohammed H. Quality of Life and Mental Health Services. London: Routledge, 1996.

\section{Oliver 1997}

Oliver JP, Huxley PJ, Priebe S, Kaiser W. Measuring the quality of life of severely mentally ill people using the Lancashire quality of life profile. Social Psychiatry and Psychiatric Epidemiology 1997;32(2):76-83. [PUBMED: 9050348]

\section{Overall 1962}

Overall JE, Gorham DR. The brief psychiatric rating scale. Psychological Reports 1962;10:799-812.

\section{Parker 1992}

Parker G, Rosen A, Emdur N, Hadzi-Pavlovic D. The life skills profile: psychometric properties of a measure assessing function and disability in schizophrenia. Acta Psychiatrica Scandinavica 1992;83:145-52.

\section{Pettersen 2014}

Pettersen H, Ruud T, Ravndal E, Havnes I, Landheim A. Engagement in assertive community treatment as experienced by recovering clients with severe mental illness and concurrent substance use. International Journal of Mental Health Systems 2014;8(1):40. [PUBMED: 25389446]

\section{Phelan 1993}

Phelan M, Slade M, Dunn G, Holloway G, Strathdee G, Thornicroft G, et al. Camberwell Assessment of Need. London: London Institute of Psychiatry, 1993.

\section{Phelan 1995}

Phelan M, Slade M, Thornicroft G, Dunn G, Holloway F, Wykes T, et al. The Camberwell Assessment of Need: the validity and reliability of an instrument to assess the needs of people with severe mental illness. British Journal of Psychiatry 1995;167(5):589-95. [PUBMED: 8564313]

\section{Priebe 1999}

Priebe S, Huxley P, Knight S, Evans S. Application and results of the Manchester Short Assessment of Quality of Life (MANSA). International Journal of Social Psychiatry 1999;45(1):7-12. [PUBMED: 10443245]

\section{R 2008 [Computer program]}

R Development Core Team, R Foundation for Statistical Computing. R: A language and environment for statistical computing. Available from www.R-project.org. Vienna: R Development Core Team, R Foundation for Statistical Computing, 2008.

\section{Risser 1975}

Risser NL. Development of an instrument to measure patient satisfaction with nurses and nursing care in primary care settings. Nursing Research 1975;24(1):45-52. [PUBMED: 1038021]

\section{Rosen 1989}

Rosen A, Hadzi-Pavlovic D, Parker G. The life skills profile: a measure assessing function and disability in schizophrenia. Schizophrenia Bulletin 1989;15(2):325-37. [PUBMED: 2749191]

\section{Rosen 2013}

Rosen A, Stein L, McGorry P, Harvey C, Birchwood M, Diamond R. Specialist community teams backed by years of quality research. Psychiatrist 2013;37:38.

\section{Rosenberg 1998}

Rosenberg SD, Drake RE, Wolford GL, Mueser KT, Oxman TE, Vidaver RM, et al. Dartmouth Assessment of Lifestyle Instrument (DALI): a substance use disorder screen for people with severe mental illness. American Journal of Psychiatry 1998;155:232-8.

\section{Rubin 1992}

Rubin A. Is case management effective for people with serious mental illness? A research review. Health and Social Work 1992;17:138-50.

\section{Ruggeri 2000}

Ruggeri M, Leese M, Thornicroft G, Bisoffi G, Tansella M. Definition and prevalence of severe and persistent mental illness. British Journal of Psychiatry 2000;177:149-55. [PUBMED: 11026955]

\section{Ruggeri 2008}

Ruggeri M, Tansella M. Case management or assertive community treatment: are they really alternative approaches?. Epidemiologia e Psichiatria Sociale 2008; Vol. 17, issue 2:93-8. [PUBMED: 18589622]

\section{Schinnar 1990}

Schinnar AP, Rothbard AB, Kanter R, Jung YS. An empirical literature review of definitions of severe and persistent mental illness. American Journal of Psychiatry 1990;147(12):1602-8. [PUBMED: 2244636] 


\section{Schneider 1983}

Schneider, Leonard C, Struening EL. SLOF: a behavioral rating scale for assessing the mentally ill. Social Work Research and Abstracts 1983;19(3):9-21.

\section{Schooler 1979}

Schooler N, Weissman M, Hogarty G. Social adjustment scale for schizophrenics. In: Hargreaves W editor(s). Resource Material for Community Mental Health Program Evaluators. Rockville, MD: National Institute of Mental Health, 1979.

\section{Scott 1995}

Scott JE, Dixon LB. Assertive community treatment and case management for schizophrenia. Schizophrenia Bulletin 1995;21:657-67.

\section{Shern 1994}

Shern DL, Wilson NZ, Coen AS, Patrick DC, Foster M, Bartsch DA. Client outcomes II: longitudinal client data from the Colorado treatment outcome study. Milbank Quarterly 1994;72:123-48.

\section{Slade 1997}

Slade M, Powell R, Strathdee G. Current approaches to identifying the severely mentally ill. Social Psychiatry and Psychiatric Epidemiology 1997;32(4):177-84. [PUBMED: 9184462]

\section{Slade 1999}

Slade M, Thornicroft G, Loftus L, Phelan M, Wykes T. The Camberwell Assessment of Need (CAN). London: Gaskell, 1999.

\section{Sobell 1980}

Sobell MB, Maisto SA, Sobell LC, Cooper AM, Cooper T, Sanders B. Developing a prototype for evaluating alcohol treatment effectiveness. Evaluating Alcohol and Drug Abuse Treatment Effectiveness. New York: Pergamon, 1980:129-50.

\section{Solomon 1992}

Solomon P. The efficacy of case management services for severely mentally disabled clients. Community Mental Health Journal 1992;28:163-80.

\section{Spitzer 1978}

Spitzer RL, Endicott J, Robins E. Research diagnostic criteria: rationale and reliability. Archives of General Psychiatry 1978;35(6):773-82. [PUBMED: 655775]

\section{Stein 1980}

Stein LI, Test MA. Alternative to mental hospital treatment. I. Conceptual model, treatment programme, and clinical evaluation. Archives of General Psychiatry 1980;37(4):392-7. [PUBMED: 7362425]

\section{Stein 1999}

Stein GS. Usefulness of the health of the nation outcome scales. British Journal of Psychiatry 1999;174:375-7. [PUBMED: 10616599]

\section{Strauss 1972}

Strauss JS, Carpenter WT Jr. The prediction of outcome in schizophrenia. I. Characteristics of outcome. Archives of General Psychiatry 1972;27(6):739-46. [PUBMED: 4637891]

\section{Strauss 1974}

Strauss JS, Carpenter WT Jr. The prediction of outcome in schizophrenia. II. Relationships between predictor and outcome variables: a report from the WHO international pilot study of schizophrenia. Archives of General Psychiatry 1974;31(1):37-42. [PUBMED: 4835985]

\section{Thompson 1990}

Thompson KS, Griffity EEH, Leaf PJ. A historical review of the Madison Model of community care. Hospital and Community Psychiatry 1990;41:625-34.

\section{Tyrer 1979}

Tyrer PJ, Remington M. Controlled comparison of day-hospital and outpatient treatment for neurotic disorders. Lancet 1979;1(8124):1014-6. [PUBMED: 86727]

\section{Tyrer 1990}

Tyrer P. Personality disorder and social functioning. In: Peck DF, Shapiro CM editor(s). Measuring Human Problems: A Practical Guide. Chichester: John Wiley, 1990:119-42.

\section{Tyrer 2005}

Tyrer P, Nur U, Crawford M, Karlsen S, McLean C, Rao B, et al. The social functioning questionnaire: a rapid and robust measure of perceived functioning. International Journal of Social Psychiatry 2005;51(3):265-75. [PUBMED: 16252794]

\section{Ukoumunne 1999}

Ukoumunne OC, Gulliford MC, Chinn S, Sterne JAC, Burney PGJ. Methods for evaluating area-wide and organisation-based intervention in health and health care: a systematic review. Health Technology Assessment 1999;3(5):1-75.

\section{Unden 1989}

Unden AL, Orth-Gomer K. Development of a social support instrument for use in population surveys. Social Science \& Medicine 1989;29(12):1387-92. [PUBMED: 2629121]

\section{Velligan 2005}

Velligan D, Prihoda T, Dennehy E, Biggs M, Shores-Wilson K, Crismon L, et al. Brief psychiatric rating scale expanded version: how do new items affect factor structure?. Psychiatry Research 2005;135:217-28.

\section{Warner 1995}

Warner R, de Girolamo G. Epidemiology of Mental Disorders and Psychosocial Problems. Schizophrenia. Geneva: World Health Organization, 1995.

\section{Weiden 1994}

Weiden P, Rapkin B, Mott T, Zygmunt A, Goldman D, HorvitzLennon M, et al. Rating of medication influences (ROMI) scale in schizophrenia. Schizophrenia Bulletin 1994;20(2):297-310. [PUBMED: 7916162]

\section{Weissman 1971}

Weissman MM, Paykel ES, Siegel R, Klerman GL. The social role performance of depressed women: comparisons with a normal group. American Journal of Orthopsychiatry 1971;41:390-405. 


\section{Weissman 1974}

Weissman MM, Klerman GL, Paykel ES, Prusoff B, Hanson B. Treatment effects on the social adjustment of depressed patients. Archives of General Psychiatry 1974;30(6):771-8. [PUBMED: 4832185]

\section{WHO 1992}

World Health Organization. International Statistical Classification of Diseases and Related Health Problems (ICD-10). Geneva: WHO, 1992.

\section{WHO 1998}

World Health Organization, Division of Mental Health. Schedule for Clinical Assessment in Neuropsychiatry, Version 2.1. Present State Examination. Geneva: WHO, 1998.

\section{WHO 2001}

World Health Organization. Mental Health: New Understanding, New Hope. Geneva: WHO, 2001.

\section{WHO 2001a}

World Health Organization. Disability Assessment Schedule. Geneva: WHO, 2001.

\section{Wing 1974}

Wing J, Cooper JE, Sartorius N. The Description and Classification of Psychiatric Symptoms: An Instruction Manual for the PSE and Catego System. Cambridge: Cambridge University Press, 1974.

\section{Wing 1998}

Wing JK, Beevor AS, Curtis RH, Park SB, Hadden S, Burns A. Health of the Nation Outcome Scales (HoNOS). Research and development. British Journal of Psychiatry 1998;172:11-8. [PUBMED: 9534825]

\section{Witheridge 1982}

Witheridge TF, Dincin J, Appleby L. Working with the most frequent recidivists: a total team approach to assertive resource management. Psychosocial Rehabilitation Journal 1982;5:9-11.

\section{Witheridge 1991}

Witheridge TF. The "active ingredients" of assertive outreach. In: Cohen NL editor(s). Psychiatric Outreach to the Mentally III (New Directions for Mental Health Services, No. 52). San Francisco: Jossey-Bass, 1991:47-64.

\section{CHARACTERISTICS OF STUDIES}

\section{Characteristics of included studies [ordered by study ID]}

\section{Wykes 1986}

Wykes T, Sturt E. The measurement of social behaviour in psychiatric patients: an assessment of the reliability and validity of the SBS schedule. British Journal of Psychiatry 1986;148:1-11. [PUBMED: 3082403]

\section{Xia 2007}

Xia J, Adams CE, Bhagat N, Bhagat V, Bhoopathi P, El-Sayeh H, et al. The Leeds Outcomes Stakeholders Survey (LOSS) Study. 15th Cochrane Colloquium; 2007 Oct 23-27; Sao Paulo. The Cochrane Collaboration, 2007.

\section{Xia 2009}

Xia J, Adams CE, Bhagat N, Bhagat V, Bhoopathi P, El-Sayeh H, et al. Loss to outcomes stakeholder survey: the LOSS study. Psychiatric Bulletin 2009;33(7):254-7.

\section{Zigmond 1983}

Zigmond AS, Snaith RP. The hospital anxiety and depression scale. Acta Psychiatrica Scandinavica 1983;67(6):361-70. [PUBMED: 6880820]

\section{References to other published versions of this review} Burns 2007

Burns T, Catty J, Dash M, Roberts C, Lockwood A, Marshall M. Use of Intensive Case Management to reduce time in hospital in people with severe mental illness: systematic review and metaregression. BMJ 2007;335:336-40.

\section{Marshall 2000a}

Marshall M, Gray A, Lockwood A, Green R. Case management for people with severe mental disorders. Cochrane Database of Systematic Reviews 2000, Issue 2. [DOI: 10.1002/14651858.CD000050]

\section{Marshall 2000b}

Marshall M, Lockwood A. Assertive community treatment for people with severe mental disorders. Cochrane Database of Systematic Reviews 2000, Issue 2. [DOI: 10.1002/14651858.CD001089]

* Indicates the major publication for the study

\section{Aberg-Wistedt-Sweden 1995}

\begin{tabular}{ll} 
Methods & Allocation: randomised. \\
& Design: single centre. \\
& Duration: 24 months. \\
& Country: Stockholm, Sweden. \\
\hline Participants & Diagnosis: schizophrenic disorder (DSM-III-R). \\
& N $=40$. \\
& Setting: community setting.
\end{tabular}


Aberg-Wistedt-Sweden 1995 (Continued)

Age: 25 to 55 years, mean 38 years.

Sex: $65 \%$ M (26M, 14F).

Ethnicity: not reported.

History: recently admitted to ward or currently in outpatient department.

I. ICM: interdisciplinary-team based. Caseload: $1: 2.5 . \mathrm{N}=20$.
2. Standard care: multidisciplinary psychiatric outpatient team, specialised in people with schizophre-
nia. Caseload: $\sim 1: 10 / 15 . \mathrm{N}=20$.

nia. Caseload: 1:10/15. N = 20.

Global state: leaving the study early.
Unable to use -
Service use: average number of days in hospital per month and number of emergency visits (no mean,
no SD).
Quality of life: (no mean, no SD).
Social network size: (measure validated on children only, no mean, no SD).
Burden of care: (no mean, no SD).

Notes

\section{Risk of bias}

\begin{tabular}{|c|c|c|}
\hline Bias & Authors' judgement & Support for judgement \\
\hline $\begin{array}{l}\text { Random sequence genera- } \\
\text { tion (selection bias) }\end{array}$ & Unclear risk & $\begin{array}{l}\text { Randomised. } \\
\text { No further details. }\end{array}$ \\
\hline $\begin{array}{l}\text { Allocation concealment } \\
\text { (selection bias) }\end{array}$ & Unclear risk & No details \\
\hline $\begin{array}{l}\text { Blinding (performance } \\
\text { bias and detection bias) } \\
\text { All outcomes }\end{array}$ & Unclear risk & $\begin{array}{l}\text { Primary outcome: not available. } \\
\text { Secondary outcomes: clinician/participant mediated - rating - Unclear. }\end{array}$ \\
\hline $\begin{array}{l}\text { Blinding of participants } \\
\text { and personnel (perfor- } \\
\text { mance bias) } \\
\text { All outcomes }\end{array}$ & High risk & $\begin{array}{l}\text { Problematic to blind participants and those providing the intervention in stud- } \\
\text { ies comparing ICM intervention with standard care. }\end{array}$ \\
\hline $\begin{array}{l}\text { Blinding of outcome as- } \\
\text { sessment (detection bias) } \\
\text { All outcomes }\end{array}$ & Unclear risk & $\begin{array}{l}\text { Service utilisation (emergency and inpatient services): data collected from pa- } \\
\text { tient records. Blinding not reported. } \\
\text { Participant's quality of life, size of social networks, and their relatives' burden } \\
\text { of care self reported. }\end{array}$ \\
\hline $\begin{array}{l}\text { Incomplete outcome data } \\
\text { (attrition bias) } \\
\text { All outcomes }\end{array}$ & Unclear risk & The study did not address this outcome. \\
\hline $\begin{array}{l}\text { Selective reporting (re- } \\
\text { porting bias) }\end{array}$ & High risk & Most outcomes of interest are reported incompletely. \\
\hline Other bias & Low risk & No details. No evidence of the presence of other bias. \\
\hline
\end{tabular}


Audini-UK 1994

\begin{tabular}{|c|c|}
\hline Methods & $\begin{array}{l}\text { Allocation: randomised. } \\
\text { Design: single centre. } \\
\text { Duration: } 15 \text { months. } \\
\text { Country: London, UK. }\end{array}$ \\
\hline Participants & $\begin{array}{l}\text { Diagnosis: serious mental illness ( } 30 \% \text { schizophrenia according DSM-III-R). } \\
\mathrm{N}=66 . \\
\text { Setting: psychiatric community services. } \\
\text { Age: } 18 \text { to } 64 \text { years, median } 37 \text { years. } \\
\text { Sex: } 45 \% \mathrm{M}(30 \mathrm{M}, 36 \mathrm{~F}) . \\
\text { Ethnicity: } 26 \% \text { Afro-Caribbean. } \\
\text { History: mean } 0.2 \text { admissions in last year; completed at least } 18 \text { months in ACT programme*. } \\
\text { Excluded: primary addiction, primary organic brain damage. }\end{array}$ \\
\hline Interventions & $\begin{array}{l}\text { 1. ICM: ACT (Stein and Test model). } \\
\text { Caseload: } 1: 12 . \mathrm{N}=33^{* *} \text {. } \\
\text { 2. Standard care: routine care from psychiatric services, as outpatients or inpatients, or both as neces- } \\
\text { sary, with community support services. } \mathrm{N}=33 \text {. }\end{array}$ \\
\hline Outcomes & $\begin{array}{l}\text { Service use: average number of days in hospital per month }{ }^{\star \star \star} \text {. } \\
\text { Admitted to hospital. } \\
\text { Average number hospital admissions }{ }^{\star \star \star} \text {. } \\
\text { Death: suicide. } \\
\text { Global state: leaving the study early. } \\
\text { GAF. } \\
\text { Social functioning: SAS, employment status. } \\
\text { Mental state: BPRS } 24-i t e m, \text { PSE. } \\
\text { Participant satisfaction: CSQ. } \\
\text { Costs: costs of all care. } \\
\text { Unable to use - } \\
\text { Carer satisfaction: Relative's Satisfaction (scale not peer reviewed, attrition }>50 \% \text { ). }\end{array}$ \\
\hline Notes & $\begin{array}{l}\text { Loss to follow-up: } 12.1 \% \\
\text { *Participants in this study were recruited from the treatment arm of a trial on ACT } 20 \text { to } 30 \text { months } \\
\text { long. } \\
{ }^{\star \star A u t h o r s ~ r e p o r t ~ t h a t ~ " t h e ~ t e a m ~ b e c a m e ~ d e p l e t e d ~ a n d ~ d e m o r a l i s e d " ~ i n ~ t h e ~ c o u r s e ~ o f ~ t h i s ~ t r i a l . ~} \\
{ }^{\star \star \star} \text { Variance not reported - data from another study used. }\end{array}$ \\
\hline
\end{tabular}

\section{Risk of bias}

\begin{tabular}{lll}
\hline Bias & Authors' judgement & Support for judgement \\
\hline $\begin{array}{l}\text { Random sequence genera- } \\
\text { tion (selection bias) }\end{array}$ & Unclear risk & Randomised. \\
& & No further details. \\
\hline $\begin{array}{l}\text { Allocation concealment } \\
\text { (selection bias) }\end{array}$ & Unclear risk & No details \\
\hline $\begin{array}{l}\text { Blinding (performance } \\
\text { bias and detection bias) } \\
\text { All outcomes }\end{array}$ & Unclear risk & Primary outcome: clinician/participant mediated - rating - Unclear. \\
\hline
\end{tabular}


Audini-UK 1994 (Continued)

Blinding of participants High risk Problematic to blind participants and those providing the intervention in studand personnel (performance bias)

All outcomes ies comparing ICM intervention with standard care.

\section{Blinding of outcome as- Unclear risk sessment (detection bias)}

All outcomes

\section{Clinical (mental state) and social function assessed by independent raters. Blinding not tested.}

Participant's and relative's satisfaction self reported.

$\begin{array}{ll}\begin{array}{l}\text { Incomplete outcome data } \\ \text { (attrition bias) }\end{array} & \text { Low risk } \\ \text { All outcomes } & \begin{array}{l}\text { Number and reasons for attrition reported. Missing outcome data balanced } \\ \text { in numbers across intervention groups, with similar reasons for missing data } \\ \text { across groups. }\end{array}\end{array}$

All outcomes across groups.

\begin{tabular}{lll}
\hline $\begin{array}{l}\text { Selective reporting (re- } \\
\text { porting bias) }\end{array}$ & Low risk & All listed outcomes are reported completely. \\
\hline Other bias & Low risk & No details. No evidence of the presence of other bias. \\
\hline
\end{tabular}

\section{Bjorkman-Sweden 2002}

\begin{tabular}{ll}
\hline Methods & Allocation: randomised. \\
& Design: single centre. \\
& Duration: 36 months. \\
& Country: Lund, Sweden. \\
\hline Participants & Diagnosis*: serious mental illness, according to DSM-III-R. \\
$\mathrm{N}=77$. & Setting: community psychiatric service. \\
& Age*: 19 to 55 years, mean $\sim 37$ years. \\
& Sex: $36 \mathrm{M}, 41 \mathrm{~F}$. \\
& Ethnicity: not reported. \\
& History: i. serious mental illness for $>2$ yrs, ii. impairment due to illness (social-relationship, housing, \\
& or work situation) for more than 2 yrs, iii. no primary diagnosis of substance- or alcohol-related disor- \\
ders, iv. informed consent given.
\end{tabular}

Interventions

1. ICM: Case Management service based on the Strength Model. Caseload: 1:9. N=33.

2. Standard care: comprehensive psychiatric service with joint management for outpatient, inpatient, and day care facilities. $\mathrm{N}=44$.

Outcomes Service use: average number of days in hospital per month, not remaining in contact with psychiatric services, admitted to hospital.

Death: suicide.

Global state: leaving the study early, GAF.

Social functioning: Strauss-Carpenter Scale, social network (ISSI).

Mental state: general symptoms, SCL-90.

Quality of life: LQoLP.

Participant satisfaction: CAN.

Unable to use -

Client satisfaction: questionnaire by the Swedish Institute for Health Services Development (modified version, not peer reviewed).

$\begin{array}{ll} & { }^{*} 51.9 \% \text { schizophrenia-like disorder. } \\ & { }^{*} \text { ICM group significantly older than standard care group (5 yrs older on average). }\end{array}$


Bjorkman-Sweden 2002 (Continued)

Risk of bias

\begin{tabular}{|c|c|c|}
\hline Bias & Authors' judgement & Support for judgement \\
\hline $\begin{array}{l}\text { Random sequence genera- } \\
\text { tion (selection bias) }\end{array}$ & Low risk & Randomised: used a computer random number generator. \\
\hline $\begin{array}{l}\text { Allocation concealment } \\
\text { (selection bias) }\end{array}$ & Unclear risk & $\begin{array}{l}\text { Random selection performed by one of the trialist. } \\
\text { No further details. }\end{array}$ \\
\hline $\begin{array}{l}\text { Blinding (performance } \\
\text { bias and detection bias) } \\
\text { All outcomes }\end{array}$ & Unclear risk & $\begin{array}{l}\text { Primary outcomes: clinician/participant mediated - rating - Unclear. } \\
\text { Secondary outcomes: interviewer rated - rating - Unclear. } \\
\text { Interviewers formally blind to participant group allocation. Not tested. }\end{array}$ \\
\hline $\begin{array}{l}\text { Blinding of participants } \\
\text { and personnel (perfor- } \\
\text { mance bias) } \\
\text { All outcomes }\end{array}$ & High risk & $\begin{array}{l}\text { Problematic to blind participants and those providing the intervention in stud- } \\
\text { ies comparing ICM intervention with standard care. }\end{array}$ \\
\hline $\begin{array}{l}\text { Blinding of outcome as- } \\
\text { sessment (detection bias) } \\
\text { All outcomes }\end{array}$ & Unclear risk & $\begin{array}{l}\text { Clinical (mental state) and social function assessed by independent raters. } \\
\text { Blinding not tested. } \\
\text { Service utilisation. Blinding not reported. } \\
\text { Participant's and relative's satisfaction self reported, not blinded. }\end{array}$ \\
\hline $\begin{array}{l}\text { Incomplete outcome data } \\
\text { (attrition bias) } \\
\text { All outcomes }\end{array}$ & Unclear risk & $\begin{array}{l}\text { Analysis performed on an ITT basis, but "clients who were not available for or } \\
\text { refused contact at follow-up were excluded from the respective analysis on an } \\
\text { individual basis". }\end{array}$ \\
\hline $\begin{array}{l}\text { Selective reporting (re- } \\
\text { porting bias) }\end{array}$ & Low risk & All listed outcomes of interest reported. \\
\hline Other bias & Low risk & No details. No evidence of the presence of other bias. \\
\hline
\end{tabular}

Bond-Chicago1 1990

\begin{tabular}{|c|c|}
\hline Methods & $\begin{array}{l}\text { Allocation: randomised. } \\
\text { Design: single centre. } \\
\text { Duration: } 12 \text { months. } \\
\text { Country: Chicago, Illinois, USA. }\end{array}$ \\
\hline Participants & $\begin{array}{l}\text { Diagnosis*: serious mental illness (schizophrenia, schizoaffective disorder, affective disorder, personal- } \\
\text { ity disorder) according to RDC and SADS. } \\
\mathrm{N}=88 . \\
\text { Setting: private psychiatric rehabilitation agency. } \\
\text { Age: > } 18 \text { years, mean } 34 \text { years. } \\
\text { Sex: } 56.8 \% \mathrm{M}(50 \mathrm{M}, 38 \mathrm{~F}) \text {. } \\
\text { Ethnicity: non-white } 36 \% \text {. } \\
\text { History }{ }^{\star *}: \text { i. } 3 \text { admissions in the last } 2 \text { years or total of } 5 \text { admissions in life, ii. had no prior contact of } \\
\text { more than } 1 \text { month's duration with either study programme, iii. informed consent given. }\end{array}$ \\
\hline Interventions & $\begin{array}{l}\text { 1. ICM }{ }^{* \star \star} \text { : a large-city adaptation of ACT model according to Stein and Test. Caseload } 1: 10 . N=45 \text {. } \\
\text { 2. Standard care }{ }^{\star * \star} \text { : provided from drop-in centre (day treatment, central meeting place, no require- } \\
\text { ment for frequent contacts). Caseload: } 1: \geqq 20 . N=43 \text {. }\end{array}$ \\
\hline
\end{tabular}


Bond-Chicago1 1990 (Continued)

Outcomes

Service use: average number of days in hospital per month, not remaining in contact with psychiatric services, admitted to hospital, average number of admissions.

Death: all causes, suicide.

Global state: leaving the study early.

Social functioning: police contact, arrested, imprisoned, accomodation status.

Unable to use -

Global functioning: GAS (no SD, no sample size providing data).

Social functioning: Areas of Difficulty Checklist (ADC) (scale not peer reviewed, no SD).

Quality of life: Life Satisfaction Checklist (LSC) (scale not peer reviewed, no SD).

Satisfaction with care: Satisfaction with Services (SWS), author-modified version of CSQ (not peer re-

viewed, no SD).

Cost: no SD, no sample size.

Notes

${ }^{*}$ Schizophrenia 37\%; primary or secondary diagnosis of substance abuse $=26 \%$.

${ }^{* *}$ At the time of study admission, $62 \%$ in ICM group and 54\% in SC group were in a psychiatric hospital.

***Author reporting: "ACT had been in existence for $6 \mathrm{yrs}$, SC service for $2 \mathrm{yrs}$. In both programmes staff were enthusiastically committed to the respective programme philosophy".

\section{Risk of bias}

\begin{tabular}{lll}
\hline Bias & Authors' judgement & Support for judgement \\
\hline $\begin{array}{l}\text { Random sequence genera- } \\
\text { tion (selection bias) }\end{array}$ & Unclear risk & Randomised. No further details. \\
\hline $\begin{array}{l}\text { Allocation concealment } \\
\text { (selection bias) }\end{array}$ & Unclear risk & $\begin{array}{l}\text { Random assignment achieved by individual sealed envelopes (not specified if } \\
\text { opaque), the assignment was carried out by a person unconnected to research } \\
\text { programme. }\end{array}$
\end{tabular}

\begin{tabular}{|c|c|c|}
\hline $\begin{array}{l}\text { Blinding (performance } \\
\text { bias and detection bias) } \\
\text { All outcomes }\end{array}$ & Unclear risk & $\begin{array}{l}\text { Primary outcome: clinician/participant mediated - rating - Unclear. } \\
\text { Secondary outcomes: most are clinician/participant mediated - rating - Un- } \\
\text { clear. }\end{array}$ \\
\hline
\end{tabular}

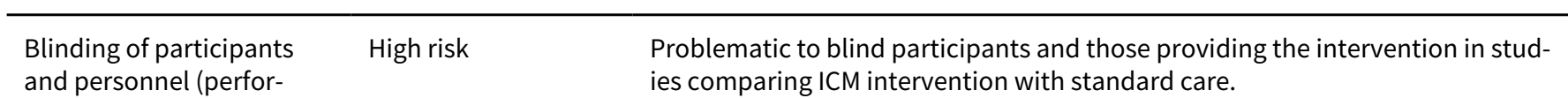
ies comparing ICM intervention with standard care.

mance bias)

All outcomes

\begin{tabular}{|c|c|c|}
\hline $\begin{array}{l}\text { Blinding of outcome as- } \\
\text { sessment (detection bias) } \\
\text { All outcomes }\end{array}$ & High risk & Interviewer was not blind to treatment condition. \\
\hline $\begin{array}{l}\text { Incomplete outcome data } \\
\text { (attrition bias) } \\
\text { All outcomes }\end{array}$ & High risk & $\begin{array}{l}\text { Missing outcome data not balanced in numbers across intervention groups, } \\
\text { but similar reasons for missing data across groups. }\end{array}$ \\
\hline $\begin{array}{l}\text { Selective reporting (re- } \\
\text { porting bias) }\end{array}$ & High risk & $\begin{array}{l}\text { Some outcomes of interest are reported incompletely and are not usable (due } \\
\text { to missing SD or sample size providing data). }\end{array}$ \\
\hline Other bias & Low risk & $\begin{array}{l}\text { Publicly funded (by the State Department of Mental Health). No further details. } \\
\text { No evidence of the presence of other bias. }\end{array}$ \\
\hline
\end{tabular}

Bond-Indiana1 (A)

Methods Methods: see above Bond-Indiana1 1988.


Bond-Indiana1 (A) (Continued)

Participants
\begin{tabular}{ll}
$\mathrm{N}=61$. \\
\hline Interventions
\end{tabular}
$\begin{aligned} & \text { Interventions: see above Bond-Indiana1 } 1988 . \\
& \text { 2. Standard care: } \mathrm{N}=32 .\end{aligned}$

\begin{tabular}{ll}
\hline Outcomes & Service use: average number of days in hospital per month*. \\
& Death: suicide. \\
& Social functioning: contact with the police. \\
& Unable to use - \\
Costs: total inpatient cost, total treatment cost (no SD).
\end{tabular}

Bond-Indiana1 (B)

\begin{tabular}{ll}
\hline Methods & Methods: see above Bond-Indiana1 1988. \\
\hline Participants & Participants: see above Bond-Indiana1 1988. \\
& $\mathrm{~N}=64$. \\
\hline Interventions & Interventions: see above Bond-Indiana1 1988. \\
& $\begin{array}{l}\text { 1. ICM: } \mathrm{N}=34 . \\
\text { 2. Standard care: } \mathrm{N}=30 .\end{array}$ \\
\hline Outcomes & Service use: average number of days in hospital per month*. \\
\hline Notes & *Variance not reported - data from another study used. \\
\hline
\end{tabular}

Bond-Indiana1 (C)

\begin{tabular}{ll}
\hline Methods & Methods: see above Bond-Indiana1 1988. \\
\hline Participants & Participants: see above Bond-Indiana1 1988. \\
& $\mathrm{~N}=42$. \\
\hline Interventions & Interventions: see above Bond-Indiana1 1988. \\
& $\begin{array}{l}\text { 1. ICM: } \mathrm{N}=21 . \\
\text { 2. Standard care: } \mathrm{N}=21 .\end{array}$ \\
\hline Outcomes & Service use: average number of days in hospital per month*. \\
\hline Notes & *Variance not reported - data from another study used. \\
\hline
\end{tabular}


Bond-Indiana1 1988

\begin{tabular}{|c|c|}
\hline Methods & $\begin{array}{l}\text { Allocation: randomised. } \\
\text { Design: multicentre, } 3 \text { centres }(3 \mathrm{CMHCS})^{\star} \text {. } \\
\text { Duration: } 6 \text { months. } \\
\text { Country: Indiana, USA. }\end{array}$ \\
\hline Participants & $\begin{array}{l}\text { Diagnosis }{ }^{\star \star} \text { : psychotic disorder (DSM-III). } \\
\mathrm{N}=67 . \\
\text { Setting: CMHCs. } \\
\text { Age: > } 17 \text { years, mean } 35 \text { years. } \\
\text { Sex: } 61.6 \% \mathrm{M}(103 \mathrm{M}, 64 \mathrm{~F}) \text {. } \\
\text { Ethnicity: black (understood to be African-American) } 34 \% \text {. } \\
\text { History }{ }^{\star \star \star}: \text { high risk of hospitalisation, described as: i. discharged within last year and either rehospi- } \\
\text { talised } \geqq 3 \text { times in previous } 2 \text { years or determined by mental health services staff to be at high risk for } \\
\text { readmission, ii. committed or awaiting commitment to hospitals, iii. presenting for admission at CMHC } \\
\text { inpatient unit and having } \geqq 4 \text { psychiatric hospitalisations in last } 2 \text { years. }\end{array}$ \\
\hline
\end{tabular}

Interventions 1. ICM: Assertive Case Management, according to the ACT model (Stein and Test), in addition to all other available mental health programmes. Caseload: $1: 8-12 . \mathrm{N}=84$.

2. Standard care: as provided at CMHCs (including case management services with large caseload). $\mathrm{N}=$ 83.

\begin{tabular}{|c|c|}
\hline \multirow[t]{2}{*}{ Outcomes } & $\begin{array}{l}\text { Service use: average number of days in hospital per month }{ }^{\star \star \star \star} \text {, (data available for single centre, see } \\
\text { below); admitted to hospital. } \\
\text { Death: all causes (data from centre A only). } \\
\text { Global state: leaving the study early. } \\
\text { Social functioning: contact with the police (data from centre A only). }\end{array}$ \\
\hline & $\begin{array}{l}\text { Unable to use - } \\
\text { Quality of life: self report instrument (no data, scale not peer reviewed, compiled by the therapist). } \\
\text { Global state: compliance with medication (data not reported). } \\
\text { Costs: available only for centre A (no SD). }\end{array}$ \\
\hline Notes & 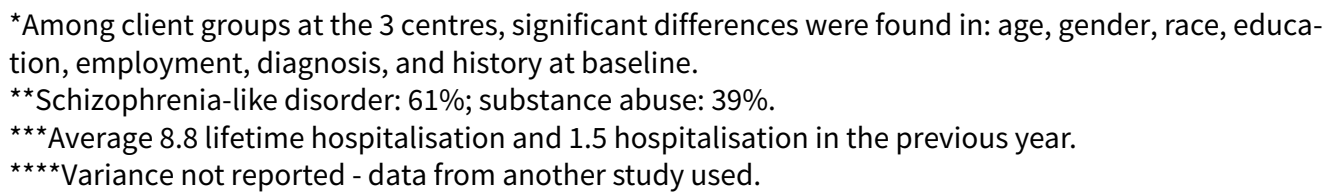 \\
\hline
\end{tabular}

\section{Risk of bias}

\begin{tabular}{lll}
\hline Bias & Authors' judgement & Support for judgement \\
\hline $\begin{array}{l}\text { Random sequence genera- } \\
\text { tion (selection bias) }\end{array}$ & Unclear risk & Randomised for single centre. No further details. \\
\hline $\begin{array}{l}\text { Allocation concealment } \\
\text { (selection bias) }\end{array}$ & Unclear risk & No details. \\
\hline $\begin{array}{l}\text { Blinding (performance } \\
\text { bias and detection bias) }\end{array}$ & Unclear risk & $\begin{array}{l}\text { Primary outcome: clinician/participant mediated - rating - Unclear. } \\
\text { All outcomes }\end{array}$ \\
\hline $\begin{array}{l}\text { Blinding of participants } \\
\text { and personnel (perfor- } \\
\text { mance bias) }\end{array}$ & Unclear risk & clear. \\
$\begin{array}{l}\text { All outcomes } \\
\text { Blinding of outcome as- } \\
\text { sessment (detection bias) }\end{array}$ & Unclear risk & No details \\
\hline
\end{tabular}


Bond-Indiana1 1988 (Continued)

All outcomes

\begin{tabular}{lll}
$\begin{array}{l}\text { Incomplete outcome data } \\
\text { (attrition bias) } \\
\text { All outcomes }\end{array}$ & Unclear risk & $\begin{array}{l}\text { Number of lost to follow-up reported, but no reasons for missing data provid- } \\
\text { ed. }\end{array}$ \\
\hline $\begin{array}{l}\text { Selective reporting (re- } \\
\text { porting bias) }\end{array}$ & High risk & $\begin{array}{l}\text { Prespecified outcomes not reported (medication compliance) or reported in- } \\
\text { completely (days in hospital: SD missing). }\end{array}$ \\
\hline Other bias & Low risk & No details. No evidence of the presence of other bias.
\end{tabular}

Bush-Georgia 1990

\begin{tabular}{|c|c|}
\hline Methods & $\begin{array}{l}\text { Allocation: randomised. } \\
\text { Design: single site. } \\
\text { Duration: } 12 \text { months. } \\
\text { Country: Atlanta, USA. }\end{array}$ \\
\hline Participants & $\begin{array}{l}\text { Diagnosis* : severe mental illness. } \\
\mathrm{N}=28 \text {. } \\
\text { Age: } 25 \text { to } 56 \text { years. } \\
\text { Sex: } 57 \% \mathrm{M}(16 \mathrm{M}, 12 \mathrm{~F}) \text {. } \\
\text { Ethnicity: } 50 \% \text { African-American. } \\
\text { History: high rates of hospital readmissions ( } 2 \text { to } 18 \text { previous admissions), difficulty in community liv- } \\
\text { ing. }\end{array}$ \\
\hline Interventions & $\begin{array}{l}\text { 1. ICM: clinical case management, providing intensive support and outreach (according to the Stein and } \\
\text { Test model } T C L \text { ). } N=14 \text {. } \\
\text { 2. non-ICM: standard care providing case management at a lower level of intensity and rehabilitation } \\
\text { services. } N=14 \text {. }\end{array}$ \\
\hline
\end{tabular}

Outcomes Service use: average number of days in hospital per month ${ }^{\star \star}$.

Death: all causes, suicide.

Unable to use -

Service use: number of hospital admissions (no individual group data); emergency room visits (no individual group data).

Global state: compliance: adherence to service and medication plan (incompletely reported data).

Social functioning: appropriate living status (incompletely reported data).

Costs: no individual group data.

Notes $\quad \begin{aligned} & { }^{*} 86 \% \text { schizophrenia. } \\ & { }^{*} \text { Variance not reported - data from another study used. }\end{aligned}$

\section{Risk of bias}

\begin{tabular}{lll}
\hline Bias & Authors' judgement & Support for judgement \\
\hline $\begin{array}{l}\text { Random sequence genera- } \\
\text { tion (selection bias) }\end{array}$ & Unclear risk & Randomised. No further details. \\
\hline $\begin{array}{l}\text { Allocation concealment } \\
\text { (selection bias) }\end{array}$ & Unclear risk & No details \\
\hline $\begin{array}{l}\text { Blinding (performance } \\
\text { bias and detection bias) }\end{array}$ & Unclear risk & Primary outcome: clinician/participant mediated - rating - Unclear. \\
\end{tabular}


Bush-Georgia 1990 (Continued)

All outcomes
Secondary outcome: leaving the study early - clinician/participant mediated rating - Unclear.

\begin{tabular}{|c|c|c|}
\hline $\begin{array}{l}\text { Blinding of participants } \\
\text { and personnel (perfor- } \\
\text { mance bias) } \\
\text { All outcomes }\end{array}$ & High risk & $\begin{array}{l}\text { Problematic to blind participants and those providing the intervention in stud- } \\
\text { ies comparing ICM intervention with standard care. }\end{array}$ \\
\hline $\begin{array}{l}\text { Blinding of outcome as- } \\
\text { sessment (detection bias) } \\
\text { All outcomes }\end{array}$ & Unclear risk & $\begin{array}{l}\text { Service use and appropriate living conditions collected from records. Blinding } \\
\text { not reported. }\end{array}$ \\
\hline $\begin{array}{l}\text { Incomplete outcome data } \\
\text { (attrition bias) } \\
\text { All outcomes }\end{array}$ & Low risk & No missing outcome data. \\
\hline $\begin{array}{l}\text { Selective reporting (re- } \\
\text { porting bias) }\end{array}$ & High risk & $\begin{array}{l}\text { Outcomes not pre-stated. Most of the reported outcomes are reported incom- } \\
\text { pletely (data not usable). }\end{array}$ \\
\hline Other bias & Low risk & No details. No evidence of the presence of other bias. \\
\hline
\end{tabular}

\section{Chan-Hong Kong 2000}

$\begin{array}{ll}\text { Methods } & \text { Allocation: randomised. } \\ & \text { Design: single site. } \\ & \text { Duration: } 11 \text { months (5-month intervention period, 6-month follow-up). } \\ \text { Country: Hong Kong. } & \\ \text { Diagnosis: chronic schizophrenia. } \\ \text { Participants } 62 . \\ \text { Setting: recruited from a community mental hospital. } \\ \text { Age: } 21 \text { to } 65 \text { years. } \\ \text { Sex: } 71 \% \text { female. } \\ \text { Ethnicity: not reported. } \\ \text { History: suffered from schizophrenia for } 2 \text { or more years; had } 3 \text { or more hospitalisations in the past } 24 \\ \text { months before admission; required supervision in living skills; unemployed for } 3 \text { or more months; and } \\ \text { unreliable in compliance to treatment. }\end{array}$

I. ICM: based on the developed case management model, with a community psychiatric nurse case
manager co-ordinating care. Caseload 1:3. N = 31.
2. Standard care: traditional community psychiatric nursing care. Caseload 1:3. $\mathrm{N}=31$.

Outcomes

All of the usable outcomes are provided at the 6 months' follow-up.

Service use: unplanned admission through the Accident and Emergency Department; day hospital care; outpatient visits; home visits.

Costs: direct healthcare costs.

Unable to use -

SD or equivalent not reported: BPRS; Specific Level of Functioning scale (SLOF); Patient Satisfaction Instrument (PSI).

Average number of days in hospital per month: it may have been possible to calculate this outcome (imputing SD), but we decided not to use it as the study reports data only on "unplanned admission", therefore the available data misses data on overall admission (planned and unplanned). 
Chan-Hong Kong 2000 (Continued)

Notes

\section{Risk of bias}

\begin{tabular}{lll}
\hline Bias & Authors' judgement & Support for judgement \\
\hline $\begin{array}{ll}\text { Random sequence genera- } \\
\text { tion (selection bias) }\end{array}$ & Unclear risk & $\begin{array}{l}\text { "Patients who met the inclusion criteria and provided consent were ran- } \\
\text { domised to case management or conventional care after recruitment"; no fur- } \\
\text { ther details were provided. }\end{array}$
\end{tabular}

\begin{tabular}{lll}
\hline $\begin{array}{l}\text { Allocation concealment } \\
\text { (selection bias) }\end{array}$ & Unclear risk & No details on allocation concealment were reported. \\
\hline $\begin{array}{l}\text { Blinding (performance } \\
\text { bias and detection bias) }\end{array}$ & Unclear risk & No details reported. \\
All outcomes & \\
\hline
\end{tabular}

Blinding of participants Unclear risk Details on blinding participants and personnel were not reported.

and personnel (perfor-

mance bias)

All outcomes

Blinding of outcome as- Unclear risk Details on blinding outcome assessors were not reported.

sessment (detection bias)

All outcomes

\begin{tabular}{lll}
\hline $\begin{array}{l}\text { Incomplete outcome data } \\
\text { (attrition bias) } \\
\text { All outcomes }\end{array}$ & Unclear risk & $\begin{array}{l}\text { Analyses were reported as ITT; it was not reported whether any participants } \\
\text { left early. }\end{array}$ \\
\hline $\begin{array}{l}\text { Selective reporting (re- } \\
\text { porting bias) }\end{array}$ & High risk & $\begin{array}{l}\text { For many of the outcome scales, only items that had a significant difference } \\
\text { between experimental and control groups were reported. }\end{array}$ \\
\hline Other bias & Low risk & $\begin{array}{l}\text { The study seems to be free of other bias. The study reports that baseline char- } \\
\text { acteristics were similar, although only age and gender were reported. }\end{array}$ \\
\hline
\end{tabular}

Chandler-California1 (A)

\begin{tabular}{ll}
\hline Methods & Site A: Long Beach. \\
& Methods: see above Chandler-California1 1991. \\
\hline Participants & Participants: see above Chandler-California1 1991. \\
& $\mathrm{~N}=256$. \\
\hline Interventions & Interventions: see above Chandler-California1 1991. \\
& ICM $=127$. \\
& SC $=129$. \\
\hline Outcomes & $\begin{array}{l}\text { Service use: average number of days per month in hospital, not remaining in contact with psychiatric } \\
\text { services, admitted to hospital. } \\
\text { Global state: leaving the study early. } \\
\text { Social functioning: employment status, arrested, imprisoned, accomodation status. } \\
\text { Costs: direct costs of psychiatric hospital care. }\end{array}$ \\
\hline
\end{tabular}


Chandler-California1 (A) (Continued)

Notes

Chandler-California1 (B)

\begin{tabular}{ll} 
Methods & Site B: Stanislaus County. \\
& Methods: see above Chandler-California1 1991. \\
\hline Participants & Participants: see above Chandler-California1 1991. \\
& $\mathrm{~N}=260$. \\
\hline Interventions & Interventions: see above Chandler-California1 1991. \\
& SCM $=125$. \\
\hline Outcomes & Service use: average number of days per month in hospital, not remaining in contact with psychiatric \\
services, admitted to hospital. \\
Global state: leaving the study early. \\
Social functioning: employment, arrested, imprisoned, accomodation status. \\
Costs: direct costs of psychiatric hospital care.
\end{tabular}

Notes

$\begin{array}{ll}\text { Methods } & \text { Allocation: randomised. } \\ \text { Design: multicentre, } 2 \text { sites } & \text { Duration: } 36 \text { months. } \\ \text { Country: California, USA. }\end{array}$
Participants Diagnosis: serious and persistent mental illness (DSM-III-R).* $\mathrm{N}=516$.
Setting: 1 urban, 1 rural but integrated service agencies.
Age: $30 \%>45$ years.
Sex: $52 \%$ M.
Ethnicity: 26\% non-white.
History ${ }^{\star}$ : i. functional impairment due to mental disorder, ii. eligibility for public assistance, iii. not a primary diagnosis of substance abuse, iv. informed consent given.
Interventions $\quad$ 1. ICM ${ }^{\star \star \star}$ : ACT provided by integrated service agencies, according to Training in Community Living Pro- gramme (Stein and Test). Caseload: 1:10. $\mathrm{N}=252$.
2. Standard care: usual mental health service (i.e. outpatients: day treatment, case management; inpa- tients: minimal rehabilitation services). $\mathrm{N}=264$.

\section{Outcomes}

\section{Service use: average number of days in hospital per month}

Following outcomes available for single centre.

Service use: not remaining in contact with psychiatric services, admitted to hospital.

Global state: leaving the study early.

Social functioning: employment, arrested, imprisoned, accomodation status.

Costs: cost of psychiatric hospital care.

Unable to use - 
Chandler-California1 1991 (Continued)

Mental state: general symptoms: Colorado Symptom Index (no data reported).

Self esteem: New York Self Esteem Scale (no data reported).

Quality of life: Lehman's Quality of Life Instrument (no data reported).

Social functioning: level of social activities (scale not peer reviewed).

Family burden: subscales adapted from Tessler's Family Burden Interview (not peer reviewed, attrition

$>50 \%)$.

Participant satisfaction: scale for overall satisfaction with mental health services (scale not peer re-

viewed).

Costs: direct costs of health care and of all care (no SD), all mental health care (not listed as review outcome of interest).

\begin{tabular}{ll}
\hline Notes & ${ }^{*} 61 \%$ schizophrenia \\
& ${ }^{* \star *} 28 \%$ admitted in previous year \\
& vices and social and therapeutic activities. Site B emphasises avoiding hospitalisation through use of \\
& crisis house.
\end{tabular}

\section{Risk of bias}

\begin{tabular}{|c|c|c|}
\hline Bias & Authors' judgement & Support for judgement \\
\hline $\begin{array}{l}\text { Random sequence genera- } \\
\text { tion (selection bias) }\end{array}$ & Unclear risk & Randomised for single centre. No further details. \\
\hline $\begin{array}{l}\text { Allocation concealment } \\
\text { (selection bias) }\end{array}$ & Unclear risk & No details \\
\hline $\begin{array}{l}\text { Blinding (performance } \\
\text { bias and detection bias) } \\
\text { All outcomes }\end{array}$ & Unclear risk & $\begin{array}{l}\text { Primary outcome: clinician/participant mediated - rating - Unclear. } \\
\text { Secondary outcomes: clinician/participant mediated - rating - Unclear. }\end{array}$ \\
\hline $\begin{array}{l}\text { Blinding of participants } \\
\text { and personnel (perfor- } \\
\text { mance bias) } \\
\text { All outcomes }\end{array}$ & High risk & $\begin{array}{l}\text { Problematic to blind participants and those providing the intervention in stud- } \\
\text { ies comparing ICM intervention with standard care. }\end{array}$ \\
\hline $\begin{array}{l}\text { Blinding of outcome as- } \\
\text { sessment (detection bias) } \\
\text { All outcomes }\end{array}$ & Unclear risk & $\begin{array}{l}\text { Employment, arrest, conviction, homelessness, and service use (hospitalisa- } \\
\text { tion) were compiled from state and local databases. Blinding not reported. } \\
\text { For mental state (symptomology), independent research staff conducted inter- } \\
\text { views. Blinding not reported. } \\
\text { Quality of life and personal safety self reported. Not blinded. }\end{array}$ \\
\hline $\begin{array}{l}\text { Incomplete outcome data } \\
\text { (attrition bias) } \\
\text { All outcomes }\end{array}$ & Unclear risk & $\begin{array}{l}\text { Number of lost to follow-up reported, but reasons for missing data not provid- } \\
\text { ed. LOCF for continuous data. }\end{array}$ \\
\hline $\begin{array}{l}\text { Selective reporting (re- } \\
\text { porting bias) }\end{array}$ & High risk & $\begin{array}{l}\text { Listed outcomes of interest not reported (continuous data from scales not re- } \\
\text { ported; days in hospital reported only for site A, no SD). }\end{array}$ \\
\hline Other bias & Low risk & $\begin{array}{l}\text { Publicly funded (California Department of Mental Health, NIMH). No details. } \\
\text { No evidence of the presence of other bias. }\end{array}$ \\
\hline
\end{tabular}

Curtis-New York 1992

Methods Allocation: randomised.


Curtis-New York 1992 (Continued)

Design: single centre.

Duration: 14 months.

Country: New York, USA.

Follow-up*: range of 18 to 52 months.

Participants
Detting: Harlem Hospital Center $(\mathrm{HHC})$.
Se: 292.
Sex: $59 \% \mathrm{~F}$.
Ethnicity: $91 \%$ black (understood to be African-American).
History ${ }^{\star \star \star}:$ i. about to be discharged from hospital, ii. local residents, iii. without a sole diagnosis of
substance abuse or organic mental disorder, iv. inpatients for $>7$ days, and not eligible for the "Com-
munity Support System" programme - that is no psychiatric admission of $>6$ months duration $/ 3$ admis-
sions of $>10$ days within the last 2 years, v. informed consent given.

Interventions 1. ICM: intensive outreach case management from a multidisciplinary team at HHC, which implemented a discharge treatment plan and monitored clinical and social problems. The team did not "assume direct responsibility for care but [...] help[ed] the patient enrol in a day hospital programme, adult mental health clinic, rehabilitation programme, or alcohol treatment programme". Caseload: 1:17. N = 147 .

2. Standard care: routine aftercare, within the discharge treatment plan prescribed for each patient by HHC; "most received at least initial treatment form various divisions of the departments of psychiatry within the Health and Hospitals Corporation". $\mathrm{N}=145$.

Outcomes Service use: average number of days in hospital per month, admitted to hospital.
Death: all causes and suicide.

Unable to use -

Use of ambulatory services: this outcome is not listed as an outcome of interest for the review. Quality of life: measuring instrument written by trialists for this particular trial and was not published in peer-reviewed journal (EAF - Evaluation Aftercare Form).

Notes $\quad \begin{aligned} & { }^{\star} \text { Follow-up period variable, depending on date of participant's entry into the study. } \\ & { }^{\star \star} \text { Schizophrenia } 38 \% \text {; alcohol or drug abuse or dependence } 39 \% . \\ & { }^{* \star} \text { Mean number of previous admissions }>1 .\end{aligned}$
Some more severely ill clients not included in this part of study as they were eligible for "Community
Support System" programme group.

\section{Risk of bias}

\begin{tabular}{lll}
\hline Bias & Authors' judgement & Support for judgement \\
\hline $\begin{array}{l}\text { Random sequence genera- } \\
\text { tion (selection bias) }\end{array}$ & Unclear risk & Randomised. No further details. \\
\hline $\begin{array}{l}\text { Allocation concealment } \\
\text { (selection bias) }\end{array}$ & Unclear risk & No details. \\
\hline $\begin{array}{l}\text { Blinding (performance } \\
\text { bias and detection bias) }\end{array}$ & Unclear risk & $\begin{array}{l}\text { Primary outcome: clinician/participant mediated - rating - Unclear. } \\
\text { All outcomes }\end{array}$ \\
\hline $\begin{array}{l}\text { Blinding of participants } \\
\text { and personnel (perfor- } \\
\text { mance bias) }\end{array}$ & High risk & $\begin{array}{l}\text { Problematic to blind participants and those providing the intervention in stud- } \\
\text { All outcomes }\end{array}$ \\
\hline
\end{tabular}


Curtis-New York 1992 (Continued)

$\begin{array}{lll}\begin{array}{l}\text { Blinding of outcome as- } \\ \text { sessment (detection bias) }\end{array} & \text { Unclear risk } & \begin{array}{l}\text { Service use (rehospitalisations, hospital-based ambulatory services) and mor- } \\ \text { tality derived from the shared medical billings systems. Blinding not reported. }\end{array}\end{array}$ All outcomes

\begin{tabular}{lll}
\hline $\begin{array}{l}\text { Incomplete outcome data } \\
\text { (attrition bias) } \\
\text { All outcomes }\end{array}$ & Unclear risk & Missing data are not addressed. \\
\hline $\begin{array}{l}\text { Selective reporting (re- } \\
\text { porting bias) }\end{array}$ & Low risk & Listed outcomes are reported completely. \\
\hline Other bias & Low risk & $\begin{array}{l}\text { Funded by public institution (New York City Health and Hospitals Corporation } \\
\text { and foundations). No details. No evidence of the presence of other bias. }\end{array}$ \\
\hline
\end{tabular}

\section{Cusack-North Carolina}

\begin{tabular}{ll}
\hline Methods & Allocation: randomised. \\
& Design: single site. \\
& Duration: 24 months. \\
Country: USA. \\
Duration: 2 years. \\
\hline Diagnosis: DSM-IV axis I major mental disorder. \\
N $=134$. \\
Setting: Harlem Hospital Center (HHC). \\
Age: 37 (SD 10) years. \\
Sex: $59 \%$ male. \\
Ethnicity: $63 \%$ Caucasian (understood to be white participants). \\
History: detained in county prison at enrolment and diagnosed with major mental disorder. Comor- \\
bid substance abuse (66\%) or additional mental disorder diagnosis were not excluded. Candidates ever \\
charged with a serious, violent offence, or "third strike" candidates were excluded.
\end{tabular}

Interventions ICM: Forensic Assertive Community Treatment $(\mathrm{FACT})^{\star}$, with team-based mental health and sub-
stance abuse services, as well as support for housing, employment assistance, benefits applications,
advocacy, and a full-time peer recovery specialist and a full-time probation officer. Caseload: $<20 . \mathrm{N}=$
72.
$\begin{aligned} & \text { 2. Standard care: treatment as usual (TAU): services routinely available in the county-operated public } \\ & \text { behavioural health system, such as psychiatric assessment, psychiatric medications in both outpatient } \\ & \text { and inpatient general hospital settings, outpatient mental health and substance abuse counselling, } \\ & \text { and case management. } \mathrm{N}=62 .\end{aligned}$

Outcomes Service use: mean number of outpatient visits.

Social functioning: contact with legal system (bookings, jail days, convictions).

Costs: direct costs of psychiatric hospital care; direct costs for outpatient care, jail.

Not used -

Service use: mean number of hospital days (could not be converted to average number of days per month), crisis contacts.

*With high fidelity to the ACT model, DACTS scores 4.5 and 4.6.

\section{Risk of bias}


Cusack-North Carolina (Continued)

\section{Bias Authors' judgement Support for judgement}

Random sequence genera- Low risk Randomisation using a random number table, assignment in blocks of 2. tion (selection bias)

Allocation concealment Unclear risk Allocation concealment details were not reported.

(selection bias)

Blinding (performance Unclear risk No details

bias and detection bias)

All outcomes

Blinding of participants Unclear risk No details on blinding participants and personnel were reported.

and personnel (perfor-

mance bias)

All outcomes

Blinding of outcome as- Low risk Results relied on external administrative data.

sessment (detection bias)

All outcomes

\begin{tabular}{lll}
\hline $\begin{array}{l}\text { Incomplete outcome data } \\
\text { (attrition bias) } \\
\text { All outcomes }\end{array}$ & Low risk & $\begin{array}{l}\text { All analyses were ITT; outcomes were observed regardless of active or contin- } \\
\text { ued participation. }\end{array}$ \\
\hline $\begin{array}{l}\text { Selective reporting (re- } \\
\text { porting bias) }\end{array}$ & Low risk & Listed outcomes are reported completely. \\
\hline Other bias & Low risk & $\begin{array}{l}\text { No indication of other bias; baseline characteristics similar between groups, } \\
\text { except, on average, participants in the FACT group were nearly 4.5 years old- } \\
\text { er than participants in the TAU condition. However, after adjusting for age, the } \\
\text { results were essentially the same. }\end{array}$ \\
\hline
\end{tabular}

Drake-NHamp (A)

\begin{tabular}{ll}
\hline Methods & Methods: see above Drake-NHamp 1998. \\
\hline Participants & $\begin{array}{l}\text { Participants: see above Drake-NHamp 1998. For this centre, sample providing data: } \\
\mathrm{N}=23 .\end{array}$ \\
\hline Interventions & $\begin{array}{l}\text { Interventions: see above Drake-NHamp 1998. For this centre, sample providing data: } \\
\text { 2. ICM: } \mathrm{N}=11 .\end{array}$ \\
\hline Outcomes & Service use: average number of days in hospital per month. \\
\hline Notes & \\
\hline
\end{tabular}

Drake-NHamp (B)

\begin{tabular}{ll}
\hline Methods & Methods: see above Drake-NHamp 1998. \\
\hline Participants & Participants: see above Drake-NHamp 1998. For this centre, sample providing data: \\
\hline
\end{tabular}


Drake-NHamp (B) (Continued)

$N=23$.

Interventions: see above Drake-NHamp 1998. For this centre, sample providing data:
I. ICM: $\mathrm{N}=10$.
2. Standard care: $\mathrm{N}=13$.

2. Standard care: $\mathrm{N}=13$.

Outcomes Service use: average number of days in hospital per month.

Notes

Drake-NHamp (C)

\begin{tabular}{ll}
\hline Methods & Methods: see above Drake-NHamp 1998. \\
\hline Participants & Participants: see above Drake-NHamp 1998. For this centre, sample providing data: \\
N $=25$.
\end{tabular}

\begin{tabular}{ll}
\hline Interventions & Interventions: see above Drake-NHamp 1998. For this centre, sample providing data: \\
1. ICM: $N=14$. & 2. Standard care: $N=11$.
\end{tabular}

Outcomes Service use: average number of days in hospital per month.

\section{Notes}

Drake-NHamp (D)

\begin{tabular}{ll}
\hline Methods & Methods: see above Drake-NHamp 1998. \\
\hline Participants & $\begin{array}{l}\text { Participants: see above Drake-NHamp 1998. For this centre, sample providing data: } \\
\mathrm{N}=60 .\end{array}$ \\
\hline Interventions & $\begin{array}{l}\text { Interventions: see above Drake-NHamp 1998. For this centre, sample providing data: } \\
\text { 1. ICM: } \mathrm{N}=30 .\end{array}$ \\
\hline Outcomes & Service use: average number of days in hospital per month. $\mathrm{N}=30$. \\
\hline Notes & \\
\hline
\end{tabular}

Drake-NHamp (E)

\begin{tabular}{ll}
\hline Methods & Methods: see above Drake-NHamp 1998. \\
\hline Participants & Participants: see above Drake-NHamp 1998. For this centre, sample providing data: \\
& $\mathrm{N}=32$. \\
\hline Interventions & Interventions: see above Drake-NHamp 1998. For this centre, sample providing data: \\
& 1. ICM: $\mathrm{N}=17$. \\
& 2. Standard care: $\mathrm{N}=15$. \\
\hline
\end{tabular}


Drake-NHamp (E) (Continued)

Outcomes Service use: average days per month in hospital.

\section{Notes}

\section{Drake-NHamp (F)}

\begin{tabular}{ll}
\hline Methods & Methods: see above Drake-NHamp 1998. \\
\hline Participants & $\begin{array}{l}\text { Participants: see above Drake-NHamp 1998. For this centre, sample providing data: } \\
\mathrm{N}=17 .\end{array}$ \\
\hline Interventions & $\begin{array}{l}\text { Interventions: see above Drake-NHamp 1998. For this centre, sample providing data: } \\
\text { 1. ICM: } \mathrm{N}=9 .\end{array}$ \\
\hline Outcomes & Standard care: $\mathrm{N}=8$. \\
\hline Notes & \\
\hline
\end{tabular}

Drake-NHamp (G)

\begin{tabular}{ll}
\hline Methods & Methods: see above Drake-NHamp 1998. \\
\hline Participants & Participants: see above Drake-NHamp 1998. For this centre, sample providing data: \\
& $\mathrm{N}=16$. \\
\hline Interventions & $\begin{array}{l}\text { Interventions: see above Drake-NHamp 1998. For this centre, sample providing data: } \\
\text { 2. Standard care: } \mathrm{N}=9 .\end{array}$ \\
\hline Outcomes & Service use: average number of days in hospital per month. \\
\hline Notes &
\end{tabular}

Methods Allocation: randomised.

Design: multicentre (7 sites(CHMCs), 2 in urban areaand 5 in rural area).

Duration: 36 months.

Country: New Hampshire, USA.
Participants Diagnosis: schizophrenia, schizoaffective or bipolar disorder (DSM-III-R) and active substance user dis- order within past 6 months (DSM-III-R) ${ }^{*}$.
$\mathrm{N}=225$.
Setting: CHMCs.
Age: 18 to 60 years, mean 34 years.
Sex: $74.4 \%$ M (167M, 58F).
Ethnicity: ethnic minority $3.4 \%$.
History: i. no mental retardation or medical conditions that would prevent participation, ii. written in- formed consent given.


Drake-NHamp 1998 (Continued)

Interventions $\quad$ 1. ICM: ACT teams with special training in substance abuse treatment. Caseload: $1: 12$. N $=109$.

2. Non-ICM: standard non-Intensive Case Management, incorporating most of the ACT principle, but providing less individual service for substance abuse. Caseload: 1:25. $\mathrm{N}=114$.

Outcomes Service use: average number of days in hospital per month, not remaining in contact with psychiatric services.

Death: all causes.

Global state: leaving the study early.

Mental state: BPRS-24 item.

Social functioning: average days in stable accomodation, Alcohol Use Scale (AUS), Substance Abuse Treatment Scale (SATS), alcohol use days (TLFB), remission from alcohol use disorder.

Quality of life: QOLI.

Unable to use -

Substance abuse: drug use scale (DUS), drug use days (TLFB) and not in remission: attrition $>50 \%$, Addiction Severity Index (ASI) (data not reported).

Service use: Service Utilization Interview (data not reported).

Costs: direct costs of psychiatric hospital care (no SD), average total study cost (not listed as outcome of interest in the review).

Notes

*53\% schizophrenia, $22 \%$ schizoaffective, $24 \%$ bipolar disorder, $72.6 \%$ alcohol abuse, $41.8 \%$ drug abuse.

\section{Risk of bias}

\begin{tabular}{|c|c|c|}
\hline Bias & Authors' judgement & Support for judgement \\
\hline $\begin{array}{l}\text { Random sequence genera- } \\
\text { tion (selection bias) }\end{array}$ & Unclear risk & Randomised. No further details. \\
\hline $\begin{array}{l}\text { Allocation concealment } \\
\text { (selection bias) }\end{array}$ & Unclear risk & No details \\
\hline $\begin{array}{l}\text { Blinding (performance } \\
\text { bias and detection bias) } \\
\text { All outcomes }\end{array}$ & Unclear risk & $\begin{array}{l}\text { Primary outcome: clinician/participant mediated - rating - Unclear. } \\
\text { Secondary outcomes: some are clinician/participant mediated - rating - Un- } \\
\text { clear, some are interviewer rated - rating - Unclear. Interviewer blind, not test- } \\
\text { ed. }\end{array}$ \\
\hline $\begin{array}{l}\text { Blinding of participants } \\
\text { and personnel (perfor- } \\
\text { mance bias) } \\
\text { All outcomes }\end{array}$ & Unclear risk & Blinding not reported. \\
\hline $\begin{array}{l}\text { Blinding of outcome as- } \\
\text { sessment (detection bias) } \\
\text { All outcomes }\end{array}$ & Unclear risk & $\begin{array}{l}\text { Independent raters, blind to study condition, separately rated alcohol and } \\
\text { drug use as well as substance abuse treatment. Service use was obtained from } \\
\text { outpatient records and hospital records, blinding not reported. Blinding not } \\
\text { reported for mental state, quality of life, or homelessness. }\end{array}$ \\
\hline $\begin{array}{l}\text { Incomplete outcome data } \\
\text { (attrition bias) } \\
\text { All outcomes }\end{array}$ & Unclear risk & $\begin{array}{l}\text { Number of lost to follow-up is reported, but reasons for missing data are not } \\
\text { reported for each randomised treatment group (reported overall, information } \\
\text { not usable). }\end{array}$ \\
\hline $\begin{array}{l}\text { Selective reporting (re- } \\
\text { porting bias) }\end{array}$ & Low risk & Prespecified outcomes of interest are reported. \\
\hline Other bias & Low risk & No information available. No evidence of the presence of other bias. \\
\hline
\end{tabular}




\begin{tabular}{|c|c|}
\hline Methods & $\begin{array}{l}\text { Allocation: randomised. } \\
\text { Design*: multicentre, } 3 \text { sites, all in city area (data for single centre not available). } \\
\text { Duration: } 18 \text { months. } \\
\text { Country: Connecticut, USA. }\end{array}$ \\
\hline Participants & $\begin{array}{l}\text { Diagnosis**: major depression, bipolar disorder, schizophrenia, schizoaffective disorder according to } \\
\text { DSM-III-R. } \\
N=262 \text {. } \\
\text { Age: mean } 41 \text { years. } \\
\text { Sex: } 64 \% \text { M. } \\
\text { History }{ }^{\star \star \star}: \text { high level of service use (defined as: } \mathrm{i} . \geqq 2 \text { psychiatric hospitalisations in last } 2 \text { years, ii. } 1 \\
\text { psychiatric hospitalisation longer than } 180 \text { days in last yr, or iii. } \geqq 2 \text { contacts with crisis services in last } 2 \\
\text { years) and significant difficulties meeting the demands of everyday life (defined as: } 1 \text {. homeless in past } \\
\text { year, or ii. requiring extensive supervision or assistance at least weekly to meet personal-care needs), } \\
\text { informed consent given. }\end{array}$ \\
\hline
\end{tabular}

I. ICM: Assertive Community Treatment teams (Stein and Test model), but having a richer staff and
achieving 24 -hour coverage. Caseload: $1: 5-7 . \mathrm{N}=130^{\star \star \star \star}$.
2. Non-ICM: generalist model, but providing mobile case managers. Caseload: $1: 25-30 . \mathrm{N}=132^{\star \star \star \star}$.

Outcomes

Service use: average number of days in hospital per month.

Death: all causes.

Global state: leaving the study early.

Unable to use -

Mental state: modified version of SCL-90 (no peer-reviewed scale, no SD).

Social functioning: accomodation status, number of days homeless (no SD), imprisoned (not reported).

Quality of life: modified version of QOLI (no peer-reviewed scale, no SD).

Carer satisfaction: Family Burden Interview (no SD).

Costs: data reported incompletely.

\begin{tabular}{|c|c|}
\hline Notes & $\begin{array}{l}{ }^{\star} \text { Authors state that the same treatments provided in different sites were not identical ("different styles } \\
\text { of providing services were used at different sites"), but single-centre data not available. } \\
{ }^{\star *} 67 \% \text { schizophrenia or schizoaffective disorder. } \\
{ }^{\star \star *} 62 \% \text { participants had lifetime history of substance abuse or dependence and } 25 \% \text { participants were } \\
\text { hospitalised at the study entry. }\end{array}$ \\
\hline
\end{tabular}

\section{Risk of bias}

\begin{tabular}{lll}
\hline Bias & Authors' judgement & Support for judgement \\
\hline $\begin{array}{l}\text { Random sequence genera- } \\
\text { tion (selection bias) }\end{array}$ & Unclear risk & $\begin{array}{l}\text { Randomised. Randomisation counterbalanced "so that within each site clients } \\
\text { had 50\% likelihood of being assigned to either ICM or non-ICM and counter- } \\
\text { balanced so that clients hospitalised at the time of assignment had 50\% likeli- } \\
\text { hood of being assigned to either ICM or non-ICM." }\end{array}$ \\
\hline $\begin{array}{ll}\text { Allocation concealment } \\
\text { (selection bias) }\end{array}$ & Unclear risk & No details \\
\hline $\begin{array}{l}\text { Blinding (performance } \\
\text { bias and detection bias) } \\
\text { All outcomes }\end{array}$ & Unclear risk & $\begin{array}{l}\text { Primary outcome: clinician/participant mediated - rating - Unclear. } \\
\text { Secondary outcomes: leaving the study early - clinician/participant mediated } \\
\text { - rating - Unclear. }\end{array}$ \\
\hline
\end{tabular}

Blinding of participants

Unclear risk Blinding not reported. and personnel (performance bias) 
Essock-Connecticut1 1995 (Continued)

All outcomes

$\begin{array}{lll}\begin{array}{l}\text { Blinding of outcome as- } \\ \text { sessment (detection bias) }\end{array} & \text { Unclear risk } & \begin{array}{l}\text { Service use (hospitalisation), housing (homelessness, temporary housing): } \\ \text { source of data and blinding not reported. }\end{array}\end{array}$

All outcomes

$\begin{array}{ll}\begin{array}{l}\text { Incomplete outcome data } \\ \text { (attrition bias) }\end{array} & \text { Low risk } \\ \text { All outcomes } & \begin{array}{l}\text { Number and reasons for missing data clearly reported. Missing outcome data } \\ \text { balanced across intervention groups. }\end{array}\end{array}$

All outcomes

Selective reporting (re- High risk Listed outcome of interest not reported or reported incompletely.
porting bias)

porting bias)

\begin{tabular}{|c|c|c|}
\hline Other bias & Low risk & $\begin{array}{l}\text { Funded by public health institutes and private foundation. No other details } \\
\text { provided. No evidence of the presence of other bias. }\end{array}$ \\
\hline
\end{tabular}

Essock-Connecticut2 2006

$\begin{array}{ll}\text { Methods } & \text { Allocation: randomised. } \\ \text { Design: multicentre, } 2 \text { urban sites (data for single centre not available). } & \text { Duration: } 36 \text { months. } \\ \text { Country: Bridgeport, Connecticut, USA. }\end{array}$

Participants

Diagnosis*: schizophrenia, schizoaffective disorder, depression with psychotic features, bipolar disorder according to DSM-III-R.

$N^{\star \star}=198$ (randomised to site $A: N=100$, to site $B: N=98$ ).

Setting: 2 state-operated outpatient CMHCs.

Age: mean 36.5 years (SD 7.8 years).

Sex: $72 \%$ M (143M, 55F).

Ethnicity: African-American 55\%; Hispanic 14\%.

History: i. active substance use disorder (abuse or dependence on alcohol or other drugs previous 6 months), ii. high service use previous 6 months ( $\geqq 2$ between: psychiatric hospitalisation, stays in a psychiatric crisis or respite programme, emergency department visit, or incarceration), iii. homeless or unstably housed, iv. poor independent living skills, v. no pending legal charge, vi. no medical condition or mental retardation, vii. written informed consent given.

$\begin{array}{ll}\text { Interventions } & \mathrm{ICM}^{\star \star \star}: \text { Assertive Case Management. Caseload: } 1: 10-15 . \mathrm{N}=99 . \\ \text { 2. Non- } \mathrm{ICM}^{\star \star \star}: \text { Clinical Case Management provided by clinicians. Caseload: } 1: 20-30 . \mathrm{N}=99 .\end{array}$

Outcomes Service use: average number of days in hospital per month.

Unable to use -

Global state: leaving the study early, GAS ( $\mathrm{N}$ for single intervention group not reported).

Social functioning: days in stable accomodation ( $\mathrm{N}$ for single intervention group not reported), impris-

oned (days in jail reported with days in hospital).

Social functioning: SATS, AUS, DUS (scales rated by clinicians), days of alcohol/drug use ( $\mathrm{N}$ for single in-

tervention group not reported).

Mental state: BPRS-24 item ( $\mathrm{N}$ for single intervention group not reported).

Client satisfaction: General Life Satisfaction ( $N$ for single intervention group not reported).

Costs: not reported.

\begin{tabular}{ll}
\hline Notes & ${ }^{\star} 76 \%$ schizophrenia or schizoaffective disorder; $17 \%$ mood disorder; $74 \%$ alcohol disorder; $81 \%$ sub- \\
& stance use disorder. \\
& \\
& port to which group they were assigned. \\
& ${ }^{\star \star \star}$ Both interventions addressed substance use disorders.
\end{tabular}


Essock-Connecticut2 2006 (Continued)

Risk of bias

\begin{tabular}{|c|c|c|}
\hline Bias & Authors' judgement & Support for judgement \\
\hline $\begin{array}{l}\text { Random sequence genera- } \\
\text { tion (selection bias) }\end{array}$ & Low risk & $\begin{array}{l}\text { Randomised using separate computer-generated randomisation stream for } \\
\text { each site. }\end{array}$ \\
\hline $\begin{array}{l}\text { Allocation concealment } \\
\text { (selection bias) }\end{array}$ & Unclear risk & No details \\
\hline $\begin{array}{l}\text { Blinding (performance } \\
\text { bias and detection bias) } \\
\text { All outcomes }\end{array}$ & Unclear risk & No details \\
\hline $\begin{array}{l}\text { Blinding of participants } \\
\text { and personnel (perfor- } \\
\text { mance bias) } \\
\text { All outcomes }\end{array}$ & Unclear risk & No details \\
\hline $\begin{array}{l}\text { Blinding of outcome as- } \\
\text { sessment (detection bias) } \\
\text { All outcomes }\end{array}$ & Unclear risk & No details \\
\hline $\begin{array}{l}\text { Incomplete outcome data } \\
\text { (attrition bias) } \\
\text { All outcomes }\end{array}$ & Unclear risk & $\begin{array}{l}\text { Insufficient reporting of missing data (number and reasons for missing data } \\
\text { reported for the total sample, not for each intervention group). } 7 \text { randomised } \\
\text { participants were lost due to administrative reason, but their intervention allo- } \\
\text { cation was not reported. }\end{array}$ \\
\hline $\begin{array}{l}\text { Selective reporting (re- } \\
\text { porting bias) }\end{array}$ & High risk & $\begin{array}{l}\text { Listed outcomes of interest are fully reported for each site, but authors did not } \\
\text { provide sample size. }\end{array}$ \\
\hline Other bias & Low risk & Publicly funded. No further details. No evidence of the presence of other bias. \\
\hline
\end{tabular}

\section{Ford-UK 1995}

$\begin{array}{ll}\text { Methods } & \text { Allocation: randomised. } \\ & \text { Design: single centre (multisite study, but randomisation is used in only one site; we have reported da- } \\ \text { ta from this site). } \\ \text { Duration: } 18 \text { months. } \\ \text { Country: North Southwark, London, UK. } \\ \text { Diagnosis*: psychotic illness, severely and persistently mentally ill (criteria not reported). } \\ \text { N }=77 . \\ \text { Setting: Centre for Mental Health. } \\ \text { Age: mean } 46 \text { years. } \\ \text { Sex: } 47 \% \text { M. } \\ \text { Ethnicity: } 30 \% \text { ethnic minority. } \\ \text { History: i. either: recent inpatient admission, or impairment in social functioning, or problems in com- } \\ \text { pliance with medication/treatment regimens, or problem in receiving help from multi-agency; ii. no pri- } \\ \text { mary diagnosis of organic psychosis, personality disorder, drug/alcohol abuse, learning difficulty. }\end{array}$

Interventions

1. ICM: multidisciplinary team, providing Assertive Community Care not following any specific model of case management. Caseload: $<15$ clients per case manager. ICM provided in addition to standard mental health service. $\mathrm{N}=39$.

2. Standard care: provided by psychiatric services. $\mathrm{N}=38$. 
Ford-UK 1995 (Continued)

Outcomes
Service use: average number of days in hospital per month, admitted to hospital.

Death $^{\star \star}$ : all causes and suicide.

Global state: leaving the study early, compliance with medication.

Social functioning: imprisoned.

Mental state: BPRS-18 item.

Quality of life: objective quality of life through QOLI.

Costs: direct costs of psychiatric hospital care, direct costs of all care.

Unable to use -

Social functioning: Life Skill Profile (LSP) (rated by the therapist, not independent rater).

Social support: data not reported due to "high level of missing data".

*Schizophrenia $81 \%$.

${ }^{\star \star}$ Difference of 1 death (all causes) between 2 reports ( 3 to 4 deaths reported in ICM group). (Number of suicide consistent between reports). Reported lowest number of death here.

\section{Risk of bias}

\begin{tabular}{|c|c|c|}
\hline Bias & Authors' judgement & Support for judgement \\
\hline $\begin{array}{l}\text { Random sequence genera- } \\
\text { tion (selection bias) }\end{array}$ & Low risk & Randomised. Sequence of random number generated by computer program. \\
\hline $\begin{array}{l}\text { Allocation concealment } \\
\text { (selection bias) }\end{array}$ & Unclear risk & No details \\
\hline $\begin{array}{l}\text { Blinding (performance } \\
\text { bias and detection bias) } \\
\text { All outcomes }\end{array}$ & Unclear risk & $\begin{array}{l}\text { Primary outcome: clinician/participant mediated - rating - Unclear. } \\
\text { Secondary outcomes: some are clinician/participant mediated - rating - Un- } \\
\text { clear, some are interviewer rated - rating - Unclear. No details. }\end{array}$ \\
\hline $\begin{array}{l}\text { Blinding of participants } \\
\text { and personnel (perfor- } \\
\text { mance bias) } \\
\text { All outcomes }\end{array}$ & High risk & $\begin{array}{l}\text { Problematic to blind participants and those providing the intervention in stud- } \\
\text { ies comparing ICM intervention with standard care. }\end{array}$ \\
\hline $\begin{array}{l}\text { Blinding of outcome as- } \\
\text { sessment (detection bias) } \\
\text { All outcomes }\end{array}$ & Unclear risk & $\begin{array}{l}\text { Service use, costs, convictions/imprisonment, and mortality were collected by } \\
\text { independent researchers. Blinding not reported. }\end{array}$ \\
\hline $\begin{array}{l}\text { Incomplete outcome data } \\
\text { (attrition bias) } \\
\text { All outcomes }\end{array}$ & High risk & $\begin{array}{l}\text { Number and reasons for missing dichotomous outcome data reported; im- } \\
\text { balance in numbers and reasons for missing data across intervention groups, } \\
\text { which is not addressed for continuous outcome data. }\end{array}$ \\
\hline $\begin{array}{l}\text { Selective reporting (re- } \\
\text { porting bias) }\end{array}$ & Low risk & $\begin{array}{l}\text { Listed outcome of interest reported, or if not reported, reason is provided (i.e. } \\
\text { social support data are not reported due to high level of missing data). }\end{array}$ \\
\hline Other bias & Low risk & No details. No evidence of the presence of other bias. \\
\hline
\end{tabular}

Hampton-Illinois (A)

\begin{tabular}{ll} 
Methods & Site A - Bridge West. \\
& Methods: see above Hampton-Illinois 1992. \\
\hline Participants & $\begin{array}{l}\text { Participants: see above Hampton-Illinois } 1992 . \\
\text { Site A provided the following information: }\end{array}$
\end{tabular}


Hampton-Illinois (A) (Continued)

Diagnosis: 78\% (74/95) primary diagnosis of major mental illness (schizophrenia, affective disorder, other psychosis), $13 \%$ (12/95) have primary diagnosis of alcohol or substance abuse.

$\mathrm{N}=95$.

Sex: $80 \%$ M (76M, 19F).

Ethnicity: non-white: $50.5 \%$ (48/95).

Age: average 38 years.

History: average age at first psychiatric contact 23.5 years, average duration of homeless before entering the study 44.6 months.

Interventions

Interventions: see also above Hampton-Illinois 1992.

Site A provided the following information:

1. ICM: $\mathrm{N}=48$.

2. Standard care: provided by psychiatric services, including traditional office-based outpatient care and case management. $\mathrm{N}=47$.

Outcomes

Service use: average number of days in hospital per month*, not remaining in contact with psychiatric services.

Death: all causes.

Global state: leaving the study early.

Social functioning: accomodation status.

Unable to use -

Service use: mean number of admissions (data not reported).

Social functioning: imprisoned (data not reported).

Notes *Variance not reported - data from another study used.

Hampton-Illinois (B)

Methods Site B - Bridge South.

Methods: see above Hampton-Illinois 1992.

Participants Participants: see above Hampton-Illinois 1992.

Site $B$ provided the following information:

Diagnosis: $54 \%$ (38/70) have primary diagnosis of schizophrenia, $27 \%(19 / 70)$ have primary diagnosis of affective disorder, $1 \%(1 / 70)$ have primary diagnosis of substance abuse.

$\mathrm{N}=70$.

Sex: $71 \%$ M (M59/70).

Age: average 35 years.

History: average age at first psychiatric contact 23 years, average homeless before entering the study

18 months.

Interventions Interventions: see above Hampton-Illinois 1992.

Site $B$ provided the following information:

1. ICM: $\mathrm{N}=34$.

2. Standard care: $\mathrm{N}=36$.

Outcomes

Service use: average number of days in hospital per month*.

Global state: leaving the study early.

Unable to use $e^{\star \star}$ -

Service use: not remaining in contact with psychiatric services.

Death: all causes.

Social functioning: accomodation status, imprisoned. 
Hampton-Illinois (B) (Continued)

Notes

*Variance not reported - data from another study used.

${ }^{\star \star}$ Data excluded due to an incomplete report (figures in project report do not add up; > than total $\mathrm{N}$ in study).

Hampton-Illinois 1992

$\begin{array}{ll}\text { Methods } & \text { Allocation: randomised. } \\ & \text { Design: multicentre (2 centres: Site A - Bridge West; Site B - Bridge South). } \\ & \text { Duration: } 12 \text { months. } \\ & \text { Country: Chicago, USA. } \\ & \text { Diagnosis*: serious mental illness. } \\ & \text { N }=165 . \\ & \text { Setting: community mental health service. } \\ & \text { Age: } \geqq 18 \text { years, mean } 37 \text { years. } \\ & \text { Sex: } 77 \% \text { M (127M, 38F). } \\ & \text { Ethnicity: } 55.7 \% \text { black (understood to be African-American). } \\ & \text { History: i. admitted to inpatient units of } 2 \text { state hospitals, ii. homeless on admission or at risk of home- } \\ & \text { lessness on discharge, iii. informed consent given (site A). }\end{array}$

$\begin{array}{ll}\text { Interventions } & \text { 1. ICM: Assertive Case Management (Stein and Test model). Caseload: } \sim 1: 10 . \mathrm{N}=82 . \\ \text { 2. Standard care: provided by psychiatric services. } \mathrm{N}=83 \text {. (Site A included traditional office-based out- } \\ \text { patient care and case management.) }\end{array}$

Outcomes

Outcomes from different centres are reported separately; we assumed a single-centre randomisation procedure. See below in Hampton-Illinois (A) and Hampton-Illinois (B).

\begin{tabular}{lll}
\hline Notes & *Schizophrenia 42\%. \\
\hline Risk of bias & Authors' judgement & Support for judgement \\
\hline Bias & Unclear risk & $\begin{array}{l}\text { Randomised. No further details. } \\
\text { Random sequence genera- } \\
\text { tion (selection bias) }\end{array}$ \\
$\begin{array}{l}\text { Although this was not explicit, we assumed an independent randomisation for } \\
\text { to reduce bias". We interpreted this as meaning that different days were used } \\
\text { for recruitment to the whole study, and not that group of allocation was deter- } \\
\text { mined by day. }\end{array}$
\end{tabular}

\begin{tabular}{ll}
\hline $\begin{array}{l}\text { Allocation concealment } \\
\text { (selection bias) }\end{array}$ & Unclear risk \\
& No further details.
\end{tabular}

\begin{tabular}{|c|c|c|}
\hline $\begin{array}{l}\text { Blinding (performance } \\
\text { bias and detection bias) } \\
\text { All outcomes }\end{array}$ & Unclear risk & $\begin{array}{l}\text { Primary outcome: clinician/participant mediated - rating - Unclear. } \\
\text { Secondary outcomes: some clinician/participant mediated - rating - Unclear. }\end{array}$ \\
\hline $\begin{array}{l}\text { Blinding of participants } \\
\text { and personnel (perfor- } \\
\text { mance bias) } \\
\text { All outcomes }\end{array}$ & Unclear risk & No details \\
\hline
\end{tabular}

\begin{tabular}{ll}
\hline Blinding of outcome as- & Unclear risk
\end{tabular}


Hampton-Illinois 1992 (Continued)

All outcomes

Incomplete outcome data Unclear risk Insufficient reporting of attrition/exclusions to permit judgement.
(attrition bias)

(attrition bias)

All outcomes

\section{Selective reporting (re- High risk porting bias)}

Other bias

Unclear risk
In both centres, Hampton-Illinois (A) and Hampton-Illinois (B), some listed outcomes of interest were not reported or were reported incompletely (i.e. days spent in hospital: no SD).

Publicly funded (National Institute of Mental Health). It is unclear whether the study was interrupted earlier in Hampton-Illinois (B). Authors reported: "The study sample for Site B was inadequate due to late start, implementation problems and high dropout rate"; for this reason, they reported data only by 6 months.

$\begin{array}{ll}\text { Methods } & \text { Allocation: randomised. } \\ & \text { Design: single centre. } \\ \text { Duration: } 24 \text { months. } \\ \text { Country: London, UK. }\end{array}$

Diagnosis*: "heavy psychiatric service users", no diagnostic criteria reported.
N $=193$.
Setting: community psychiatric services.
Age: 16 to 64 years, mean 39 years.
Sex: $53 \%$ M $(102 \mathrm{M}, 91 \mathrm{~F})$.
History: admitted within the last 3 years and had at least 2 admissions in the last 6.5 years.
Excluded: participants who were continuously hospitalised during 8 months' recruitment.

Interventions

1. ICM: enhanced community management on ACT principles (Stein model) provided by dedicated multiprofessional team. Caseload: 1:8-15. $\mathrm{N}=97$.

2. Non-ICM: locality-based community psychiatric services using the UK Care Programme Approach.

Caseload: $1 \geqq 20 . \mathrm{N}=96$.

Outcomes Service use: average number of days in hospital per month.

Death: all causes, suicide.

Global state: leaving the study early, compliance, Rating of Medication Influences (ROMI).

Social functioning: Social Functioning Questionnaire (SFQ).

Mental state: Krawiecka Scale (KS), Health of the Nation Outcome Scale (HoNOS), Hospital Anxiety and

Depression Scale (HADS).

Participant satisfaction: Camberwell Assessment of Need (CAN).

Costs: direct costs of psychiatric hospital care.

Unable to use -

Mental state: general symptoms, Well-being Questionnaire (W-BQ) (not peer-reviewed scale and modified from the original).

\begin{tabular}{|c|c|}
\hline Notes & $\begin{array}{l}{ }^{*} \text { Schizophrenia } 65 \% . \\
{ }^{\star *} \text { Median number of } 5 \text { admissions over } 6.5 \text { years. }\end{array}$ \\
\hline
\end{tabular}

\section{Risk of bias}

Bias Authors' judgement Support for judgement


Harrison-Read-UK 2000 (Continued)
Random sequence genera- Low risk
Random sequence generated by computer program. tion (selection bias)

\begin{tabular}{lll}
\hline $\begin{array}{l}\text { Allocation concealment } \\
\text { (selection bias) }\end{array}$ & Unclear risk details \\
\hline
\end{tabular}

\begin{tabular}{|c|c|c|}
\hline $\begin{array}{l}\text { Blinding (performance } \\
\text { bias and detection bias) } \\
\text { All outcomes }\end{array}$ & Unclear risk & $\begin{array}{l}\text { Primary outcome: clinician/participant mediated - rating - Unclear. } \\
\text { Secondary outcomes: some are interviewer rated - rating - No. Interviewers } \\
\text { are not blind to treatment assignment. }\end{array}$ \\
\hline
\end{tabular}

Blinding of participants $\quad$ Unclear risk $\quad$ Blinding not reported.
and personnel (perfor-
mance bias)
All outcomes

\begin{tabular}{lll}
\hline $\begin{array}{l}\text { Blinding of outcome as- } \\
\text { sessment (detection bias) } \\
\text { All outcomes }\end{array}$ & High risk & Interviewers were not blinded. \\
\hline $\begin{array}{l}\text { Incomplete outcome data } \\
\text { (attrition bias) }\end{array}$ & Unclear risk & $\begin{array}{l}\text { Number of lost to follow-up reported, but reasons for attrition not reported. } \\
\text { All outcomes }\end{array}$ \\
\hline
\end{tabular}

\begin{tabular}{lll}
\hline $\begin{array}{l}\text { Selective reporting (re- } \\
\text { porting bias) }\end{array}$ & Low risk & Listed outcomes of interest are fully reported. \\
\hline Other bias & Low risk & $\begin{array}{l}\text { Publicly funded (National Health Service). No further details. No evidence of } \\
\text { other bias. }\end{array}$ \\
\hline
\end{tabular}

Herinckx-Oregon 1996

$\begin{array}{ll}\text { Methods } & \text { Allocation: randomised. } \\ & \text { Design: single centre. } \\ & \text { Duration: } 29 \text { months. } \\ \text { Country: Portland, Oregon, USA. }\end{array}$
Participants Diagnosis: schizophrenia, major affective disorder, paranoid disorder, or another severe mental disor- der; diagnostic criteria not reported.
Interventions $\quad 1 . \mathrm{ICM}^{\star \star}$ : Assertive Community Treatment from combined teams staffed by consumers $(\mathrm{N}=58)$ and not staffed by consumers $(N=59)$. ACT following the Stein and Test model. Caseload: $1: 10 . N=117$. 2. Standard care ${ }^{\star \star *}$ : provided by 1 of 4 CMHCs and a number of smaller, more specialised agencies (none providing assertive outreach). Average caseload $\sim 1: 27 . \mathrm{N}=61$.

\section{Outcomes}

Social functioning: accomodation status, arrests. 
Herinckx-Oregon 1996 (Continued)

Unable to use -

Social functioning: employment status, illicit drug use (not reported).

Mental state: general symptoms (not reported).

Quality of life: measurement instrument not specified (data not reported).

Satisfaction with services: measurement instrument not specified (not reported).

Notes

${ }^{*} 60 \%$ psychotic disorders, $40 \%$ affective disorders, $33 \%$ severe alcohol or drug use comorbidity, $61 \% 2+$ admissions in last 6 months.

${ }^{\star *}$ Staff members were self identified mental health consumers DSM-III-R axis I diagnosis ( $50 \%$ of staff had a diagnosis of bipolar disorder).

$* \star \star$ In the standard care group: caseload 1:15 is provided to 33\% participants.

$\star \star \star \star$ Disengagement does not include who moved out, who refused to be re-interviewed, death.

\section{Risk of bias}

\begin{tabular}{|c|c|c|}
\hline Bias & Authors' judgement & Support for judgement \\
\hline $\begin{array}{l}\text { Random sequence genera- } \\
\text { tion (selection bias) }\end{array}$ & Unclear risk & $\begin{array}{l}\text { Randomised. Ratio randomisation between interventions: ICM:SC }=2: 1 \text {. No fur- } \\
\text { ther details. }\end{array}$ \\
\hline $\begin{array}{l}\text { Allocation concealment } \\
\text { (selection bias) }\end{array}$ & Unclear risk & No details \\
\hline $\begin{array}{l}\text { Blinding (performance } \\
\text { bias and detection bias) } \\
\text { All outcomes }\end{array}$ & Unclear risk & $\begin{array}{l}\text { Primary outcome: clinician/participant mediated - rating - Unclear. } \\
\text { Secondary outcomes: clinician/participant mediated - rating - Unclear. }\end{array}$ \\
\hline $\begin{array}{l}\text { Blinding of participants } \\
\text { and personnel (perfor- } \\
\text { mance bias) } \\
\text { All outcomes }\end{array}$ & High risk & $\begin{array}{l}\text { Problematic to blind participants and those providing the intervention in stud- } \\
\text { ies comparing ICM intervention with standard care. }\end{array}$ \\
\hline $\begin{array}{l}\text { Blinding of outcome as- } \\
\text { sessment (detection bias) } \\
\text { All outcomes }\end{array}$ & Unclear risk & Source of data not reported. \\
\hline $\begin{array}{l}\text { Incomplete outcome data } \\
\text { (attrition bias) } \\
\text { All outcomes }\end{array}$ & Unclear risk & Missing data are not addressed. \\
\hline $\begin{array}{l}\text { Selective reporting (re- } \\
\text { porting bias) }\end{array}$ & High risk & Not all listed outcomes of interest are reported. \\
\hline Other bias & Low risk & No details. No evidence of the presence of other bias. \\
\hline
\end{tabular}

Holloway-UK 1996

\begin{tabular}{ll} 
Methods & Allocation: randomised. \\
& Design: single centre. \\
& Duration: 18 months. \\
& Country: East Lambeth, London, UK. \\
\hline Participants & Diagnosis*: hospital diagnosis of functional psychosis (no diagnostic criteria stated). \\
& N $=70$. \\
& Setting: community psychiatric service. \\
& Age: 16 to 64 years, mean $~ 35$ years. \\
& Sex: $66 \% \mathrm{M}(46 \mathrm{M}, 24 \mathrm{~F})$.
\end{tabular}


Holloway-UK 1996 (Continued)

Ethnicity: not available.

History**: referred by teams as being "hard to treat" (previous non-compliance with treatment, frequent readmission or poor symptomatic response to conventional management), ii. live locally, iii. informed consent given.

Interventions

1. ICM: intensive team-based Case Management. "Continuing care team" providing Assertive Case Management (according to the Clinical Case Management Model. Caseload: 1:8. $\mathrm{N}=35$.

2. Standard care: provided by community psychiatric nursing service. $\mathrm{N}=35$.

Outcomes

Service use: average number of days in hospital per month, not remaining in contact with psychiatric services, admitted to hospital.

Death: all causes and suicide.

Global state: leaving the study early.

Social functioning: Disability Assessment Scale (DAS).

Mental state: Comprehensive Psychopathological Rating Scale (CPRS), Schedule for the Assessment of

Negative Symptoms (SANS), depression, Beck Depression inventory (BDI).

Quality of life: Lancashire Quality of Life Profile (LQoLP).

Behaviour: Social Behaviour Scale (SBS).

Unable to use -

Participant satisfaction: satisfaction interview (not peer reviewed, scale developed by the research team).

\begin{tabular}{l}
\hline Notes $\quad{ }^{*} 66 \%$ schizophrenia/schizoaffective disorder; $26 \%$ bipolar disorder. \\
${ }^{\star *}$ All had 1 or more psychiatric admission, years since onset of illness $\simeq$ mean 11 years.
\end{tabular}

\section{Risk of bias}

\begin{tabular}{|c|c|c|}
\hline Bias & Authors' judgement & Support for judgement \\
\hline $\begin{array}{l}\text { Random sequence genera- } \\
\text { tion (selection bias) }\end{array}$ & Unclear risk & Randomised. No further details. \\
\hline $\begin{array}{l}\text { Allocation concealment } \\
\text { (selection bias) }\end{array}$ & Unclear risk & Randomisation by sealed envelopes (not stated if opaque). \\
\hline $\begin{array}{l}\text { Blinding (performance } \\
\text { bias and detection bias) } \\
\text { All outcomes }\end{array}$ & Unclear risk & $\begin{array}{l}\text { Primary outcome: clinician/participant mediated - rating - Unclear. } \\
\text { Secondary outcomes: most are interviewer rated - rating - No. Interviewers are } \\
\text { not blind to treatment condition. }\end{array}$ \\
\hline $\begin{array}{l}\text { Blinding of participants } \\
\text { and personnel (perfor- } \\
\text { mance bias) } \\
\text { All outcomes }\end{array}$ & High risk & $\begin{array}{l}\text { Problematic to blind participants and those providing the intervention in stud- } \\
\text { ies comparing ICM intervention with standard care. }\end{array}$ \\
\hline $\begin{array}{l}\text { Blinding of outcome as- } \\
\text { sessment (detection bias) } \\
\text { All outcomes }\end{array}$ & Unclear risk & $\begin{array}{l}\text { Source of data and blinding not reported for mortality and service use. Raters } \\
\text { were not blinded for mental state and social functioning outcomes. }\end{array}$ \\
\hline $\begin{array}{l}\text { Incomplete outcome data } \\
\text { (attrition bias) } \\
\text { All outcomes }\end{array}$ & Low risk & $\begin{array}{l}\text { YES - Primary outcomes: average number of days in hospital per month; not } \\
\text { remaining in contact with psychiatric services. No missing data. } \\
\text { NO - Secondary outcomes: imbalance in numbers for missing data across in- } \\
\text { tervention groups. }\end{array}$ \\
\hline $\begin{array}{l}\text { Selective reporting (re- } \\
\text { porting bias) }\end{array}$ & Low risk & All listed outcomes of interest are fully reported. \\
\hline Other bias & Low risk & No details. No evidence of the presence of other bias. \\
\hline
\end{tabular}


Jerrell-SCarolina1 1991

\begin{tabular}{|c|c|}
\hline Methods & $\begin{array}{l}\text { Allocation: randomised. } \\
\text { Design: single-centre trial. } \\
\text { Duration: } 18 \text { months. } \\
\text { Country: South Carolina, USA. }\end{array}$ \\
\hline Participants & $\begin{array}{l}\text { Diagnosis*: psychotic or major affective disorders (DSM-III-R, according to the Computer based Diag- } \\
\text { nostic Interview Schedule Revised (C-DIS-R)). } \\
\mathrm{N}=122 . \\
\text { Age: } 18 \text { to } 59 \text { years, } 55 \%>34 \text { years. } \\
\text { Sex: } 68 \mathrm{M}, 54 \mathrm{~F} \text {. } \\
\text { Ethnicity: } 28 \% \text { non-white. } \\
\text { Setting: large urban mental health service. } \\
\text { History: i. participants were being discharged from the most recent of } 2+\text { inpatient admissions in last } \\
\text { year or subacute care episodes or lengthy residential treatment and repeated emergency psychiatric } \\
\text { visits; ii. } 2+\text { years of poor work history, eligible for public assistance, poor living skills, poor social sup- } \\
\text { port, history of inappropriate behaviour. }\end{array}$ \\
\hline
\end{tabular}

Interventions
2. ${ }^{\star \star}$ ICM (1): Programme Assertive Community Treatment (2): Intensive Broker Case Management Model. Caseload: $1: 15 / 18 . \mathrm{N}=42$.
3. Standard care: from 1 of 4 multidisciplinary psychiatric teams. Clinical approach according to a gen-
eralist model with supplemental case management provided to $\simeq 25 \%$ of the most unstable clients.
Caseload: $1: 35$ to $1: 45 . N=40$.

\begin{tabular}{ll}
\hline Outcomes & Service use: average number of days in hospital per month ${ }^{\star \star \star}$, average visits to emergency room ${ }^{\star \star \star}$. \\
& Social functioning: Social Adjustment Scale-II (SAS-II), Role Functioning Scale (RFS). \\
& Unable to use - \\
& Mental state: not reported with the psychometric measurement instrument. \\
& Social functioning: use of alcohol and drug, legal system involvement (not reported). \\
& Participant satisfaction: satisfaction with service (instrument not stated, data not reported). Satisfac- \\
& tion with Life Scale (SLS) (not reported). \\
& Costs: direct costs of psychiatric inpatient care (no SD). \\
& ${ }^{*}$ Schizophrenia 75\%. \\
& ${ }^{* \star B o t h ~ i n t e r v e n t i o n ~(1) ~ a n d ~ i n t e r v e n t i o n ~(2) ~ w o u l d ~ f i t ~ i n t o ~ t h e ~ d e f i n i t i o n ~ o f ~ I n t e n s i v e ~ C a s e ~ M a n a g e m e n t ~}$ \\
& as described in this review, hence they could be considered as a single intervention. But as they were \\
& reported separately in the original study, it was not possible to present data from these two samples \\
Notes & combined (it is not possible to sum up SD data). We decided to present only data from intervention (1) \\
& versus standard care. \\
& $* \star$ Variance not reported - data from another study used.
\end{tabular}

\section{Risk of bias}

\begin{tabular}{lll}
\hline Bias & Authors' judgement & Support for judgement \\
\hline $\begin{array}{l}\text { Random sequence genera- } \\
\text { tion (selection bias) }\end{array}$ & Unclear risk & Randomised. No further details. \\
\hline $\begin{array}{l}\text { Allocation concealment } \\
\text { (selection bias) }\end{array}$ & Unclear risk & No information \\
\hline $\begin{array}{l}\text { Blinding (performance } \\
\text { bias and detection bias) } \\
\text { All outcomes }\end{array}$ & Unclear risk & $\begin{array}{l}\text { Primary outcome: clinician/participant mediated - rating - Unclear. } \\
\text { Secondary outcomes: interviewer rated - rating - Unclear. No information. }\end{array}$ \\
\hline
\end{tabular}


Jerrell-SCarolina1 1991 (Continued)
Blinding of participants
Unclear risk
Blinding not reported. and personnel (perfor- mance bias)
All outcomes

\begin{tabular}{|c|c|c|}
\hline $\begin{array}{l}\text { Blinding of outcome as- } \\
\text { sessment (detection bias) } \\
\text { All outcomes }\end{array}$ & Unclear risk & $\begin{array}{l}\text { Cost-of-care data were based on both public and private mental health ser- } \\
\text { vices. Blinding not reported. Blinding for interviewers for social adjustment, } \\
\text { mental state not reported. }\end{array}$ \\
\hline
\end{tabular}

\begin{tabular}{lll}
\hline $\begin{array}{l}\text { Incomplete outcome data } \\
\text { (attrition bias) } \\
\text { All outcomes }\end{array}$ & Unclear risk & $\begin{array}{l}\text { Number of randomised participants is not stated; only number of randomised } \\
\text { participants completing the study period is reported. }\end{array}$ \\
\hline $\begin{array}{l}\text { Selective reporting (re- } \\
\text { porting bias) }\end{array}$ & High risk & $\begin{array}{l}\text { Listed outcome of interest not reported or reported incompletely (i.e. service } \\
\text { use: no SD). }\end{array}$ \\
\hline Other bias & Low risk & $\begin{array}{l}\text { Publicly funded (National Institute of Mental Health). No details. No evidence } \\
\text { of the presence of other bias. }\end{array}$ \\
\hline
\end{tabular}

Johnston-Australia 1998

$\begin{array}{ll}\text { Methods } & \text { Allocation: randomised. } \\ & \text { Design: single centre. } \\ & \text { Duration: } 12 \text { months. } \\ \text { Country: Sydney, Australia. } & \text { Setting: Eastern Suburb Mental Health Service (ESMHS). } \\ & \text { Diagnosis*: schizophrenia, bipolar disorder (diagnostic criteria not reported). } \\ \text { N }=73 . & \\ \text { Setting: Eastern Suburb Mental Health Service (ESMHS). } \\ \text { Age: } 16 \text { to } 70 \text { years, mean } ~ 42 \text { years. } \\ \text { Sex: } 56 \% \text { M (41M, 32F). } \\ \text { History: at least } 3 \text { of: i. high relapse rate over previous } 2 \text { years, ii. poor compliance, iii. disturbing behav- } \\ \text { iour, iv. frequent changes of accommodation, v. poor budgeting skills, vi. low quality of life, vii. difficul- } \\ \text { ty to manage in existing service. Resident of the ESMHS catchment area. No primary diagnosis of sub- } \\ \text { stance misuse, organic brain disorder, or intellectual disability. }\end{array}$

$\begin{array}{ll}\text { Interventions } & \text { 1. ICM }{ }^{\star *} \text { : Caseload: } 1: 8-10 . \mathrm{N}=37 . \\ \text { 2. Non-ICM: Caseload: } 1: 20-40 . \mathrm{N}=36 .\end{array}$

\section{Outcomes}
Service use: average number of days in hospital per month, not remaining in contact with psychiatric services, admission to hospital.
Death: all causes.
Global state: leaving the study early, compliance with medication.
Social functioning: accomodation status, number living in supported accomodation, employment, participants spending at least 1 day employed, participants on paid employment, number of partici- pants having contact with police or legal system.
Behaviour: number of participants having incident of self harm or harm to others.
Costs: direct costs of all care.
Unable to use -
Service use: number admitted to hospital (not reported), use of general practitioner (not listed as re- view outcome of interest).
Global state: clinically significant improvement (as Life Skill Profile (LSP) improvement $\geqq 18$ points/12 months) (scale assessment completed by the therapist, not reported what measurement used).
Social functioning: accomodation changes (not listed as review outcome of interest), LSP (assessment completed by the therapist).


Johnston-Australia 1998 (Continued)

Costs: direct costs of psychiatric hospital care (no SD).

Notes

${ }^{\star}$ Schizophrenia $89 \%$.

${ }^{\star \star}$ Main difference between teams was in ratio of staff to participant. Both are multidisciplinary, co-or-

dinate and provide a variety of services, have access to inpatient, rehabilitation, and 24-hour crisis service.

\section{Risk of bias}

\begin{tabular}{|c|c|c|}
\hline Bias & Authors' judgement & Support for judgement \\
\hline $\begin{array}{l}\text { Random sequence genera- } \\
\text { tion (selection bias) }\end{array}$ & Unclear risk & Randomised. No further details. \\
\hline $\begin{array}{l}\text { Allocation concealment } \\
\text { (selection bias) }\end{array}$ & Unclear risk & No details \\
\hline $\begin{array}{l}\text { Blinding (performance } \\
\text { bias and detection bias) } \\
\text { All outcomes }\end{array}$ & Unclear risk & $\begin{array}{l}\text { Primary outcome: clinician/participant mediated - rating - Unclear. } \\
\text { Secondary outcomes: most are clinician/participant mediated - rating - Un- } \\
\text { clear. }\end{array}$ \\
\hline $\begin{array}{l}\text { Blinding of participants } \\
\text { and personnel (perfor- } \\
\text { mance bias) } \\
\text { All outcomes }\end{array}$ & Unclear risk & Blinding not reported. \\
\hline $\begin{array}{l}\text { Blinding of outcome as- } \\
\text { sessment (detection bias) } \\
\text { All outcomes }\end{array}$ & Unclear risk & $\begin{array}{l}\text { Service use (hospitalisations), social functioning (accomodation status, em- } \\
\text { ployment, police and legal involvement), behaviour (self harm and harm to } \\
\text { others): collected and reported by the case manager. Blinding not reported. }\end{array}$ \\
\hline $\begin{array}{l}\text { Incomplete outcome data } \\
\text { (attrition bias) } \\
\text { All outcomes }\end{array}$ & Low risk & $\begin{array}{l}\text { YES - Primary outcomes: average number of days in hospital per month, not } \\
\text { remaining in contact with psychiatric services. Numbers and reasons for miss- } \\
\text { ing data clearly reported and balanced between groups. } \\
\text { NO - Secondary outcomes: imbalance in numbers for missing data across in- } \\
\text { tervention groups. }\end{array}$ \\
\hline
\end{tabular}

Selective reporting (re- Low risk All listed outcomes of interest are fully reported.

porting bias)

Other bias Low risk No details. No evidence of the presence of other bias.

Lehman-Maryland1 1994

$\begin{array}{ll}\text { Methods } & \text { Allocation: randomised. } \\ & \text { Design: single centre. } \\ & \text { Duration: } 12 \text { months. } \\ \text { Country: Baltimore, Maryland, USA. } & \\ & \text { Diagnosis*: severe mental disorder (schizophrenia, schizoaffective disorder, or any other diagnosis axis } \\ \text { I and extensive prior hospitalisation history, according to the DSM-III-R). } \\ \text { N }=152 . \\ \text { Setting: CMHCs. } \\ \text { Age: } 18 \text { to } 64 \text { years, mean } ~ 37 \text { years. } \\ \text { Sex: } 67 \% \mathrm{M}(102 \mathrm{M}, 50 \mathrm{~F}) . \\ \text { History }{ }^{\star \star}: \text { i. homeless }\end{array}$


Lehman-Maryland1 1994 (Continued)

Interventions $\quad$ 1. ICM: Programme of Assertive Community Treatment (Stein and Test model). Caseload: $1: 10-12 . \mathrm{N}=$ 77.

2. Standard care: care from community mental health centres and emergency facilities, though also a small amount of non-ICM. $\mathrm{N}=75$.

Outcomes Service use: average number of days in hospital per month, admitted to hospital, average number of admissions to emergency room (mean adjusted for race as covariate).

Global state: leaving the study early.

Social functioning: not living independently, days in stable accomodation.

Mental state: Colorado Symptom Index (CSI) (mean adjusted for race as covariate).

Quality of life: Lehman's Quality of Life Index (QOLI), satisfaction with general well-being (mean adjusted for race as covariate).

Costs: direct cost of psychiatric hospital, direct costs of all health care.

Unable to use -

Social functioning: days in prison (reported data are not complete).

Quality of life: specific items reported from Quality of Life Scale (QOLS) (not global assessments).

Social functioning: objective QOLS (no data), days homeless (split reporting of different types of homelessness, no SD).

General health: Medical Outcomes Study 36-Item Short Form Health Survey (SF-36) (mean adjusted for race as covariate) (not listed as outcome of interest for the review).

Notes

*Schizophrenia-like disorder: 58\%, bipolar disorder 20\%, major depression 8\%, comorbid for substance use disorders $71 \%$.

${ }^{\star \star} 74 \%$ homeless for at least 1 year in total; $34 \%$ homeless for $\geqq 4$ years.

${ }^{* \star *}$ Homeless defined as: on street or shelter for $\geqq 5$ days last 45 or $\geqq 14$ last 180 , or in temporary accommodation with $\geqq 2$ residential moves in last 6 months.

${ }^{\star * \star \star}$ Severe mental disorder: defined as diagnosis of schizophrenia or schizophrenia-like illness or receiving benefit because of mental disorder or had another axis I disorder and either: $>2$ hospitalisations of $>21$ days in past 3 years or a total of $>42$ days prior to current hospitalisation or $\geqq 90$ days in psychiatric hospital or nursing home in past 3 years or mental disability lasting $>1$ year during which unable to spend $>75 \%$ of time in some gainful activity.

Note complex inclusion criteria.

\section{Risk of bias}

\begin{tabular}{|c|c|c|}
\hline Bias & Authors' judgement & Support for judgement \\
\hline $\begin{array}{l}\text { Random sequence genera- } \\
\text { tion (selection bias) }\end{array}$ & Unclear risk & Randomised (stratified random assignment). No further details. \\
\hline $\begin{array}{l}\text { Allocation concealment } \\
\text { (selection bias) }\end{array}$ & Unclear risk & No details \\
\hline $\begin{array}{l}\text { Blinding (performance } \\
\text { bias and detection bias) } \\
\text { All outcomes }\end{array}$ & Unclear risk & $\begin{array}{l}\text { Primary outcome: clinician/participant mediated - rating - Unclear. } \\
\text { Secondary outcomes: those interviewer rated - rating - Unclear. No details. }\end{array}$ \\
\hline $\begin{array}{l}\text { Blinding of participants } \\
\text { and personnel (perfor- } \\
\text { mance bias) } \\
\text { All outcomes }\end{array}$ & High risk & $\begin{array}{l}\text { Problematic to blind participants and those providing the intervention in stud- } \\
\text { ies comparing ICM intervention with standard care. }\end{array}$ \\
\hline $\begin{array}{l}\text { Blinding of outcome as- } \\
\text { sessment (detection bias) } \\
\text { All outcomes }\end{array}$ & Unclear risk & $\begin{array}{l}\text { Source of data not reported for service use (hospitalisations, emergency room } \\
\text { visits, outpatient visits for general medical care), social functioning (homeless- } \\
\text { ness, incarceration). Blinding not reported for mental state. } \\
\text { Quality of life and health survey self reported. }\end{array}$ \\
\hline
\end{tabular}


Lehman-Maryland1 1994 (Continued)

\begin{tabular}{|c|c|c|}
\hline $\begin{array}{l}\text { Incomplete outcome data } \\
\text { (attrition bias) }\end{array}$ & Unclear risk & $\begin{array}{l}\text { Insufficient reporting of attrition/exclusion (no reasons for missing data pro- } \\
\text { vided). }\end{array}$ \\
\hline
\end{tabular}

All outcomes

Not all listed outcomes of interest are reported completely.

Selective reporting (reporting bias)

High risk

$\begin{array}{ll}\text { Other bias } \quad \text { Low risk } & \begin{array}{l}\text { Publicly funded (Center for Mental Health Services, Maryland). No details. No } \\ \text { evidence of the presence of other bias. }\end{array}\end{array}$

\section{Macias-Utah 1994}

$\begin{array}{ll}\text { Methods } & \text { Allocation: randomised. } \\ & \text { Design: single centre. } \\ & \text { Duration: } 18 \text { months. } \\ \text { Country: Utah, USA. }\end{array}$

\begin{tabular}{|c|c|}
\hline Participants & $\begin{array}{l}\text { Diagnosis*: serious and persistent mental disorder. } \\
N=41 . \\
\text { Setting: mental health centre. } \\
\text { Age: } \text { not reported. } \\
\text { Sex: } 56 \% \mathrm{M} \text {. } \\
\text { Ethnicity: } 100 \% \text { Caucasian (understood to be white). } \\
\text { History: unclear, no primary diagnosis of mental retardation or substance abuse. }\end{array}$ \\
\hline Interventions & $\begin{array}{l}\text { 1. ICM: psychosocial rehabilitation programme at } \mathrm{CMHC}+\text { Case Management (CM is modelled as } \\
\text { Strengths } \mathrm{CM} \text { ). Caseload: } 1: 20 . \mathrm{N}=20 \text {. } \\
\text { 2. Standard care: psychosocial rehabilitation programme }{ }^{\star \star} \text { at } \mathrm{CMHC} . \mathrm{N}=21 \text {. }\end{array}$ \\
\hline Outcomes & $\begin{array}{l}\text { Service use: admitted to hospital. } \\
\text { Global state: leaving the study early. } \\
\text { Unable to use - } \\
\text { Mental state: Brief Psychological Well-Being Index (BPWI) (unpublished scales, designed by authors es- } \\
\text { pecially for the population under study). } \\
\text { Global state: Self Report Inventory (SRI) (unpublished scales, designed by authors especially for the } \\
\text { population under study). } \\
\text { Carer satisfaction: Utah Family Burden Scale (unpublished, designed by authors especially for the pop- } \\
\text { ulation under study). } \\
\text { Social functioning: Utah Case Management Consumer Assessment Record (not independently rated, } \\
\text { no summary score, no SD). }\end{array}$ \\
\hline Notes & $\begin{array}{l}\text { *Schizophrenia } 46 \% \text {, major depression } 22 \% \text {. } \\
\text { ** Described as "a high quality rehabilitation program that informally provides many services typical of } \\
\text { case management". It is not a specific package of care and does not refer to a specific intervention. }\end{array}$ \\
\hline
\end{tabular}

\section{Risk of bias}

\begin{tabular}{lll}
\hline Bias & Authors' judgement & Support for judgement \\
\hline $\begin{array}{l}\text { Random sequence genera- } \\
\text { tion (selection bias) }\end{array}$ & Unclear risk & Randomised. No further details. \\
\hline $\begin{array}{l}\text { Allocation concealment } \\
\text { (selection bias) }\end{array}$ & Unclear risk & No details \\
\hline
\end{tabular}


Macias-Utah 1994 (Continued)

$\begin{array}{ll}\begin{array}{l}\text { Blinding (performance } \\ \text { bias and detection bias) }\end{array} \quad \text { Unclear risk } & \text { Primary outcome: clinician/participant mediated - rating - Unclear. } \\ \text { All } \text { Secondary outcomes: clinician/participant mediated - rating - Unclear. }\end{array}$

All outcomes

Blinding of participants $\quad$ Unclear risk No details
and personnel (perfor-
mance bias)
All outcomes

Blinding of outcome as- Unclear risk No details

sessment (detection bias)

All outcomes

\begin{tabular}{|c|c|c|}
\hline $\begin{array}{l}\text { Incomplete outcome data } \\
\text { (attrition bias) }\end{array}$ & Unclear risk & $\begin{array}{l}\text { Insufficient reporting of attrition/exclusion (no reasons for missing data pro- } \\
\text { vided). }\end{array}$ \\
\hline
\end{tabular}

\begin{tabular}{lll}
\hline $\begin{array}{l}\text { Selective reporting (re- } \\
\text { porting bias) }\end{array}$ & Unclear risk & $\begin{array}{l}\text { One listed outcome of interest is not reported completely (Utah Case Manage- } \\
\text { ment Consumer Assessment Record: no summary report, no SD). }\end{array}$ \\
\hline Other bias & Low risk & $\begin{array}{l}\text { Funded by public institution (NIMH). No details. No evidence of the presence } \\
\text { of other bias. }\end{array}$ \\
\hline
\end{tabular}

\section{Marshall-UK 1995}

\begin{tabular}{ll}
\hline Methods & Allocation: randomised. \\
& Design: single centre. \\
& Duration: 14 months. \\
Country: Oxford, UK. & \\
\hline Participants & Diagnosis*: severe persistent psychiatric disorder. \\
& N $=80$. \\
& Setting: Oxford Social Service. \\
& Age: 20 to over 60 years, mean $~ 48$ years. \\
& Sex: $85 \%$ M (68M, 12F). \\
& Ethnicity: not reported. \\
& History**: i. either homeless, at risk of homelessness, living in supported, temporary or poor-quality \\
& accommodation, experiencing social isolation, or causing disturbances, ii. not already receiving case \\
& management, iii. informed consent given.
\end{tabular}

Interventions

1. ICM: Case Management from team of social services case managers (case managers are free to choose how much time to offer each patient, but at minimum provided some intervention). Caseload: $1: 10 . \mathrm{N}=40$.

2. Standard care: provided by CMHTs. $\mathrm{N}=40$.

Outcomes

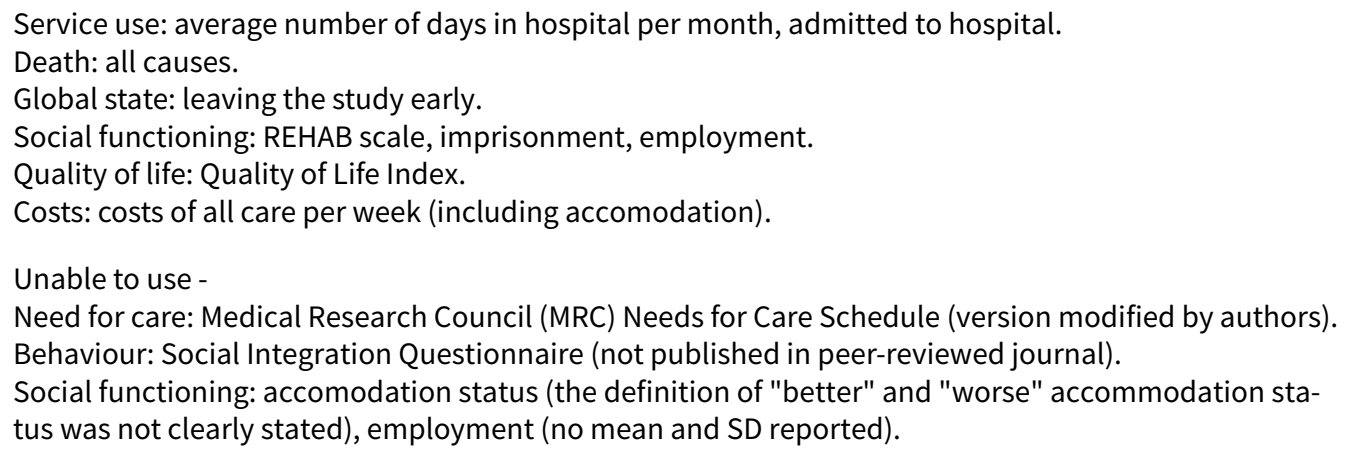


Marshall-UK 1995 (Continued)

Costs: direct cost of psychiatric hospital care and of health care (no SD).

Notes $\quad$ *Schizophrenia and related disorder $74 \%$.

${ }^{\star *} 40 \%$ illness $>1 \mathrm{yr}, 85 \%$ previous psychiatric admission.

\section{Risk of bias}

\begin{tabular}{|c|c|c|}
\hline Bias & Authors' judgement & Support for judgement \\
\hline $\begin{array}{l}\text { Random sequence genera- } \\
\text { tion (selection bias) }\end{array}$ & Low risk & Randomisation by permuted block. No further details. \\
\hline $\begin{array}{l}\text { Allocation concealment } \\
\text { (selection bias) }\end{array}$ & Unclear risk & Randomisation by sealed envelopes (not stated if opaque). \\
\hline $\begin{array}{l}\text { Blinding (performance } \\
\text { bias and detection bias) } \\
\text { All outcomes }\end{array}$ & Unclear risk & $\begin{array}{l}\text { Primary outcome: clinician/participant mediated - rating - Unclear. } \\
\text { Secondary outcomes: those interviewer rated - rating - Unclear. No details. }\end{array}$ \\
\hline $\begin{array}{l}\text { Blinding of participants } \\
\text { and personnel (perfor- } \\
\text { mance bias) } \\
\text { All outcomes }\end{array}$ & High risk & $\begin{array}{l}\text { Problematic to blind participants and those providing the intervention in stud- } \\
\text { ies comparing ICM intervention with standard care. }\end{array}$ \\
\hline $\begin{array}{l}\text { Blinding of outcome as- } \\
\text { sessment (detection bias) } \\
\text { All outcomes }\end{array}$ & Unclear risk & $\begin{array}{l}\text { Data for service use (hospitalisation), costs, social functioning (employment; } \\
\text { accomodation), non-psychiatric health care, psychiatric health care provided } \\
\text { by the respective healthcare providers. Blinding not reported. Mental state and } \\
\text { social functioning (social behaviour measured by REHAB) rated by a trained } \\
\text { observer. Blinding not reported. } \\
\text { Social functioning and quality of life self reported. }\end{array}$ \\
\hline $\begin{array}{l}\text { Incomplete outcome data } \\
\text { (attrition bias) } \\
\text { All outcomes }\end{array}$ & Unclear risk & $\begin{array}{l}\text { Insufficient reporting of attrition/exclusion (no reasons for missing data pro- } \\
\text { vided). Lost to follow-up reported at } 7 \text { months, not at } 14 \text { months. Reasons for } \\
\text { attrition reported only for the experimental sample. }\end{array}$ \\
\hline $\begin{array}{l}\text { Selective reporting (re- } \\
\text { porting bias) }\end{array}$ & Low risk & Listed outcomes reported completely. \\
\hline Other bias & Low risk & No details. No evidence of the presence of other bias. \\
\hline
\end{tabular}

McDonel-Indiana (A)

Methods $\quad$ McDonel-Indiana (A) refers to 4 centres grouped together, providing data on service use.

Methods: see above McDonel-Indiana 1997.

$\begin{array}{ll}\text { Participants } & \text { Participants: see above McDonel-Indiana } 1997 . \\ N=160 .\end{array}$

Interventions Interventions: see above McDonel-Indiana 1997.

1. ICM: $\mathrm{N}=80$.

2. Non-ICM: $\mathrm{N}=80$. 
McDonel-Indiana (A) (Continued)

Notes

McDonel-Indiana (B)

Methods McDonel-Indiana (B) refers to the 5 th centre alone, providing data on service use.

Methods: see above McDonel-Indiana 1997.

Participants Participants: see above McDonel-Indiana 1997.

$\mathrm{N}=40$.

Interventions Interventions: see above McDonel-Indiana 1997.

1. ICM: $\mathrm{N}=20$.

2. Control group: $\mathrm{N}=20$.

Outcomes Service use: average number of days in hospital per month.

Notes

McDonel-Indiana 1997

$\begin{array}{ll}\text { Methods } & \text { Allocation: randomised. } \\ & \text { Design: multicentre (5 rural sites). } \\ & \text { Duration: } 24 \text { months. } \\ \text { Country: Indiana, USA. }\end{array}$

Participants

Diagnosis*: severe mental illness (DSM-III-R coded between 295 and 301.99).

$\mathrm{N}=200$ (40 participants for each site).

Setting: 5 rural CMHCs.

Age: $>18$ years, mean $~ 38.1$ years (SD 11.1).

Sex: $43 \%$ M (86M, 114F).

Ethnicity: $98 \%$ Caucasian (understood to be white).

History $^{\star *}$ : i. poor utilisation of community mental health services and frequent use of psychiatric hospital or emergency room, ii. difficulties with the legal system or in maintaining stable housing, iii. more than 1 episode of intensive psychiatric care lasting $>2$ months, iv. impaired role functioning on a continuing or intermittent basis for at least 2 years.

I. ICM: Assertive Community Treatment (1 site had addition of Rhinelander model to ACT). Caseload:
1:10. $\mathrm{N}=100$.
2. Non-ICM: provided by the mental health services: office-based; subscribed to the tradition of individ-
ual Case Management (including day treatment, partial hospitalisation, outpatient therapy, residential
services). Caseload: $1: 30$ to $1: 60 . \mathrm{N}=100$.

Outcomes

Service use: average number of days in hospital per month (provided for 2 groups of centres, see below in McDonel-Indiana 1997 A and McDonel-Indiana 1997 B).

Global state: leaving the study early.

Unable to use -

Service use: admission (sample size not reported).

Global functioning: Global Assessment of Functioning (GAF) (rated by the therapist), compliance with medication on 11-item client-rated checklist (not a peer-reviewed scale).

Social functioning: Indiana Level of Functioning (Indiana LOF) (rated by the therapist), accomodation quality and employment scale (not a peer-reviewed scale). 
McDonel-Indiana 1997 (Continued)

Social functioning: days in jail, number of police contacts (sample size not reported).

Mental state: Brief Psychiatric Rating Scale (BPRS) (rated by the therapist).

Quality of life: scale modified by the trialist (not a peer-reviewed scale).

Satisfaction with services: satisfaction with service scale (not peer-reviewed scale).

$\begin{array}{ll}\text { Notes } & { }^{\star} \text { Schizophrenia } 48 \% \text {, affective disorder } 32 \% . \mathrm{N}=153 . \\ & { }^{\star \star} \text { Mean lifetime hospitalisation: } 8.4 \text { (SD 7.5), mean hospitalisation in the previous years: } 1.3 \text { (SD 1.1). N } \\ & =153 .\end{array}$

\section{Risk of bias}

\begin{tabular}{|c|c|c|}
\hline Bias & Authors' judgement & Support for judgement \\
\hline $\begin{array}{l}\text { Random sequence genera- } \\
\text { tion (selection bias) }\end{array}$ & Unclear risk & Randomised. No further details. \\
\hline $\begin{array}{l}\text { Allocation concealment } \\
\text { (selection bias) }\end{array}$ & Unclear risk & No details \\
\hline $\begin{array}{l}\text { Blinding (performance } \\
\text { bias and detection bias) } \\
\text { All outcomes }\end{array}$ & Unclear risk & $\begin{array}{l}\text { Primary outcome: clinician/participant mediated - rating - Unclear. } \\
\text { Secondary outcome: clinician/participant mediated - rating - Unclear. }\end{array}$ \\
\hline $\begin{array}{l}\text { Blinding of participants } \\
\text { and personnel (perfor- } \\
\text { mance bias) } \\
\text { All outcomes }\end{array}$ & Unclear risk & Blinding not reported. \\
\hline $\begin{array}{l}\text { Blinding of outcome as- } \\
\text { sessment (detection bias) } \\
\text { All outcomes }\end{array}$ & Unclear risk & $\begin{array}{l}\text { Service use (hospitalisation) was obtained from agency records and the state } \\
\text { Department of Mental Health database. Blinding not reported. } \\
\text { Accomodation, legal involvement, and education outcomes were self reported } \\
\text { and verified by case managers. Blinding not reported. }\end{array}$ \\
\hline $\begin{array}{l}\text { Incomplete outcome data } \\
\text { (attrition bias) } \\
\text { All outcomes }\end{array}$ & Unclear risk & $\begin{array}{l}\text { Insufficient reporting of attrition/exclusion (number of missing data is report- } \\
\text { ed, but no reasons provided). Number of participants randomised to each } \\
\text { group not clearly reported (some participants refused to participate and they } \\
\text { were not clearly accounted for in each group). }\end{array}$ \\
\hline $\begin{array}{l}\text { Selective reporting (re- } \\
\text { porting bias) }\end{array}$ & High risk & $\begin{array}{l}\text { Some listed outcomes of interest are reported incompletely (sample size not } \\
\text { reported for social functioning and admission). }\end{array}$ \\
\hline
\end{tabular}

Morse-Missouri1 1992

\begin{tabular}{ll} 
Methods & Allocation: randomised. \\
& Design: single site. \\
& Duration: 12 months. \\
& Country: Missouri, USA. \\
\hline Participants & Diagnosis*: psychiatric diagnosis (DSM-III-R). \\
& $\mathrm{N}=178^{\star \star}$ \\
& Setting: community (drop-in daytime centres for homeless in St Louis area, mental health clinic oper- \\
ated by Missouri Department of Mental Health). & Age: mean $~ 33.7$ years.
\end{tabular}


Morse-Missouri1 1992 (Continued)

Sex: 58\% M (103M, 75F).

Ethnicity: $52.5 \%$ non-white, mostly African-American.

History $^{\star \star \star}$ : i. serious psychiatric disorder defined as a) previous psychiatric hospitalisation or b) above 90th centile on Global Severity Index or c) above 90th centile on psychoticism, paranoid ideation, or depression subscales of the Brief Symptom Inventory severity index, ii. currently homeless, iii. plans to stay in the study area for its duration, iv. no serious violent behaviour.

\begin{tabular}{|c|c|}
\hline Interventions & $\begin{array}{l}\text { 1. ICM: clinical case management based on ACT principles (TCL model). Caseload: } 1: 10 . N=52 \text {. } \\
\text { 2. Standard care: traditional outpatient treatment provided at local mental health clinic (offered psy- } \\
\text { chotherapy, medication, and assistance in obtaining social services). } N=64 \text {. }\end{array}$ \\
\hline & $\begin{array}{l}\text { 3. Drop-in centres } \\
\text { respite when other emergency shelters closed. Provided food, clothing, showers, some recreational ac- } \\
\text { tivity. Social workers available for referrals to other social services, staff-client ratio } 1: 40 . \mathrm{N}=62 \text {. }\end{array}$ \\
\hline
\end{tabular}

Outcomes

Global state: leaving the study early.

Unable to use -

Social functioning: Personality and Social Network Adjustment Scale ( $\mathrm{N}$ for treatment groups not presented).

Social functioning: mean number of days spent homeless in past month ( $\mathrm{N}$ for treatment groups not presented), monthly quantity and frequency of alcohol consumption based on form developed by the National Institute on Alcohol Abuse and Alcoholism ( $\mathrm{N}$ for treatment groups not presented). Mental state: global severity index of Brief Symptom Inventory ( $\mathrm{N}$ for treatment groups not presented). Participant satisfaction: measuring instrument not reported ( $\mathrm{N}$ for treatment groups not presented). Self esteem: Rosenberg's Self Esteem Scale (not peer reviewed, $\mathrm{N}$ for treatment groups not presented). Costs: mean monthly income (not described as a review outcome of interest, $\mathrm{N}$ for treatment groups not presented).

Notes ${ }^{\star} 30.1 \%$ schizophrenia, $21 \%$ major depression, $8 \%$ bipolar, $5 \%$ other psychotic disorder, $12 \%$ alcohol
abuse, $4 \%$ other drug abuse, $15 \%$ other axis I disorder, $5 \%$ no diagnosis. Of those with major axis I dis-
order, $23 \%$ had dual diagnosis with substance abuse. $\mathrm{N}=155$ (as for remaining 23 participants, it was
not possible to assess diagnosis).
${ }^{\star \star}$ Initially 50 people assigned to each treatment group, but if they were lost to follow-up within the first
month after entering the study, they were replaced by random assignment.
${ }_{\star \star \star}$ Mean length of time since last stable address 16.6 months, $71.9 \%$ had previous psychiatric hospital-
isation.
${ }_{\star \star \star \star D}$ Data from the drop-in centre arm were not presented as the intervention provided did not fit the
inclusion criteria for any of the interventions addressed in this review.

\section{Risk of bias}

Bias Authors' judgement Support for judgement

Random sequence genera- Unclear risk tion (selection bias)
Randomised. No further details. Participants lost to follow-up within first month were replaced, replacement was performed through random allocation.

$\begin{array}{lll}\text { Allocation concealment } & \text { Unclear risk } & \text { Not described } \\ \text { (selection bias) }\end{array}$

Blinding (performance

bias and detection bias)

All outcomes
Unclear risk

Primary outcome: not available.

Secondary outcomes: clinician/participant mediated - rating - Unclear.

\begin{tabular}{|c|c|c|}
\hline $\begin{array}{l}\text { Blinding of participants } \\
\text { and personnel (perfor- } \\
\text { mance bias) } \\
\text { All outcomes }\end{array}$ & High risk & $\begin{array}{l}\text { Problematic to blind participants and those providing the intervention in stud- } \\
\text { ies comparing ICM intervention with standard care. }\end{array}$ \\
\hline
\end{tabular}


Morse-Missouri1 1992 (Continued)
Blinding of outcome as-
Unclear risk
Blinding not reported. sessment (detection bias)

All outcomes

\begin{tabular}{lll}
$\begin{array}{l}\text { Incomplete outcome data } \\
\text { (attrition bias) } \\
\text { All outcomes }\end{array}$ & Unclear risk & Number and reasons for lost to follow-up incompletely reported. \\
\hline $\begin{array}{l}\text { Selective reporting (re- } \\
\text { porting bias) }\end{array}$ & High risk & All data presented but not usable due to N for treatment groups missing
\end{tabular}

Other bias Low risk Publicly funded (grants from National Institute of Mental Health). No further
details. No evidence of the presence of other bias.

\section{Morse-Missouri3 2005}

\begin{tabular}{|c|c|}
\hline Methods & $\begin{array}{l}\text { Allocation: randomised. } \\
\text { Design: single site. } \\
\text { Duration: } 24 \text { months. } \\
\text { Country: Missouri, USA. }\end{array}$ \\
\hline Participants & $\begin{array}{l}\text { Diagnosis*: severe mental illness and substance abuse disorder (according to DSM-IV). } \\
N=196 . \\
\text { Age: } 18 \text { to } 66 \text { years, mean } 40 \text { years. } \\
\text { Sex: 80\% M. } \\
\text { Ethnic: } 73 \% \text { African-American, } 25 \% \text { Caucasian (understood to be white), } 2 \% \text { other minority. } \\
\text { History }{ }^{\star \star} \text { : currently homeless and severely mentally ill, not already enrolled in an Intensive Case Man- } \\
\text { agement programme. }\end{array}$ \\
\hline
\end{tabular}

I. ICM: clinical team trained to deliver psychiatric care package following ACT principles and practices.
Caseload: $1: 10$. Treatment of participant's substance abuse via referral to other community providers
for outpatient or individual substance abuse services, 12 -step group. $\mathrm{N}=46$.

2. Standard care: participants shown a list of community agencies that provided mental health and/or substance abuse treatment ${ }^{\star \star \star}$. Team also provided linkage assistance to help participants access these services. $\mathrm{N}=49$.

3. Integrated Community Treatment: combination of Integrated Treatment Services and Assertive Community Treatment (IACT), clinical team trained to deliver psychiatric care package following ACT principles and practices. Also trained to follow Integrated Treatment principles and practices. Substance abuse services provided directly via counselling and bi-weekly treatment groups. Substance abuse specialist part of IACT team. $\mathrm{N}=54^{\star \star \star \star}$.

\section{Outcomes}

Social functioning: number of days homeless per month, mean number of days used substances. Costs: inpatient psychiatric costs, healthcare costs, costs of all care.

Unable to use -

Service use: contacts with treatment programme - not clearly defined (not described as a review outcome of interest).

Global state: leaving the study early (overall loss given, no individual group data).

Mental state: 24 -item BPRS (data reported are not likely to be obtained through the stated measurement instrument, as rating scores reported are not consistent with this scale).

Satisfaction: client satisfaction (modified scale used; not peer reviewed).

Social functioning: substance abuse rating score (scale not described or referenced).

Costs: emergency shelter costs (not described as a review outcome of interest). 
Morse-Missouri3 2005 (Continued)

Notes
* $48 \%$ schizophrenia, 19\% schizoaffective disorder, $11 \%$ atypical psychotic disorder, $11 \%$ bipolar, $9 \%$ major depression-recurrent disorder, $2 \%$ delusional disorder.
${ }^{\star *}$ Mean 12.5 days homeless in previous month.
${ }^{* \star \star}$ Most agencies specialised in either mental health treatment or substance abuse treatment, but not both.
${ }^{\star \star \star \star}$ Data from this group not used in final analysis, as ACT plus another treatment.

\section{Risk of bias}

\begin{tabular}{|c|c|c|}
\hline Bias & Authors' judgement & Support for judgement \\
\hline $\begin{array}{l}\text { Random sequence genera- } \\
\text { tion (selection bias) }\end{array}$ & Unclear risk & Randomised, but no description given. \\
\hline $\begin{array}{l}\text { Allocation concealment } \\
\text { (selection bias) }\end{array}$ & Unclear risk & Not described \\
\hline $\begin{array}{l}\text { Blinding (performance } \\
\text { bias and detection bias) } \\
\text { All outcomes }\end{array}$ & Unclear risk & $\begin{array}{l}\text { Primary outcome: not available. } \\
\text { Secondary outcomes: interviewer rated - rating - Unclear. Not described. }\end{array}$ \\
\hline $\begin{array}{l}\text { Blinding of participants } \\
\text { and personnel (perfor- } \\
\text { mance bias) } \\
\text { All outcomes }\end{array}$ & High risk & $\begin{array}{l}\text { Problematic to blind participants and those providing the intervention in stud- } \\
\text { ies comparing ICM intervention with standard care. }\end{array}$ \\
\hline $\begin{array}{l}\text { Blinding of outcome as- } \\
\text { sessment (detection bias) } \\
\text { All outcomes }\end{array}$ & Unclear risk & $\begin{array}{l}\text { Service use data were obtained from service agencies, claim records, and par- } \\
\text { ticipant self report. Services provided. Blinding not reported. } \\
\text { Homelessness, income, alcohol consumption self reported. }\end{array}$ \\
\hline $\begin{array}{l}\text { Incomplete outcome data } \\
\text { (attrition bias) } \\
\text { All outcomes }\end{array}$ & Unclear risk & $\begin{array}{l}\text { Attrition described for the total sample and analysed for effect on outcome. } \\
\text { Number and reasons for loss to follow-up not reported for single arm. }\end{array}$ \\
\hline $\begin{array}{l}\text { Selective reporting (re- } \\
\text { porting bias) }\end{array}$ & Low risk & All outcomes reported. \\
\hline Other bias & Low risk & No details. No evidence of the presence of other bias. \\
\hline
\end{tabular}

Muijen-UK2 1994

\begin{tabular}{ll}
\hline Methods & Allocation: randomised. \\
& Design: single centre. \\
& Duration: 18 months. \\
Country: Greenwich Health District, London, UK.
\end{tabular}

Participants

Diagnosis ${ }^{\star}$ : severe mental illness: psychotic disorder (schizophrenia or affective psychosis) (diagnostic criteria not reported).

$\mathrm{N}=82$.

Setting: Health District.

Age: 18 to 64 years, mean 37 years.

Sex: $56 \%$ M (46M, 36F).

Race: $23.1 \%$ African/Afro-Caribbean.

History ${ }^{\star \star}$ : schizophrenia or affective psychosis lasting $>2$ years, $\geqq 2$ hospital admissions last 2 years, about to be discharged, no primary organic disorder. 
Muijen-UK2 1994 (Continued)

Interventions
1. ICM: Case Management approach provided by a Community Support Team (community psychiatric nurses and team leader). The team acts as advocate, practical assistance with welfare benefits and housing, no-discharge policy. Caseload: 1:8-11 for the first 15 months (until April 1990), then increased to caseload: 1:20-25 for the last 3 months, until the end of the trial. $N=41$.

2. Standard care: provided by community psychiatric nurses (CPNs) working independently and based in primary care. $\mathrm{N}=41$.

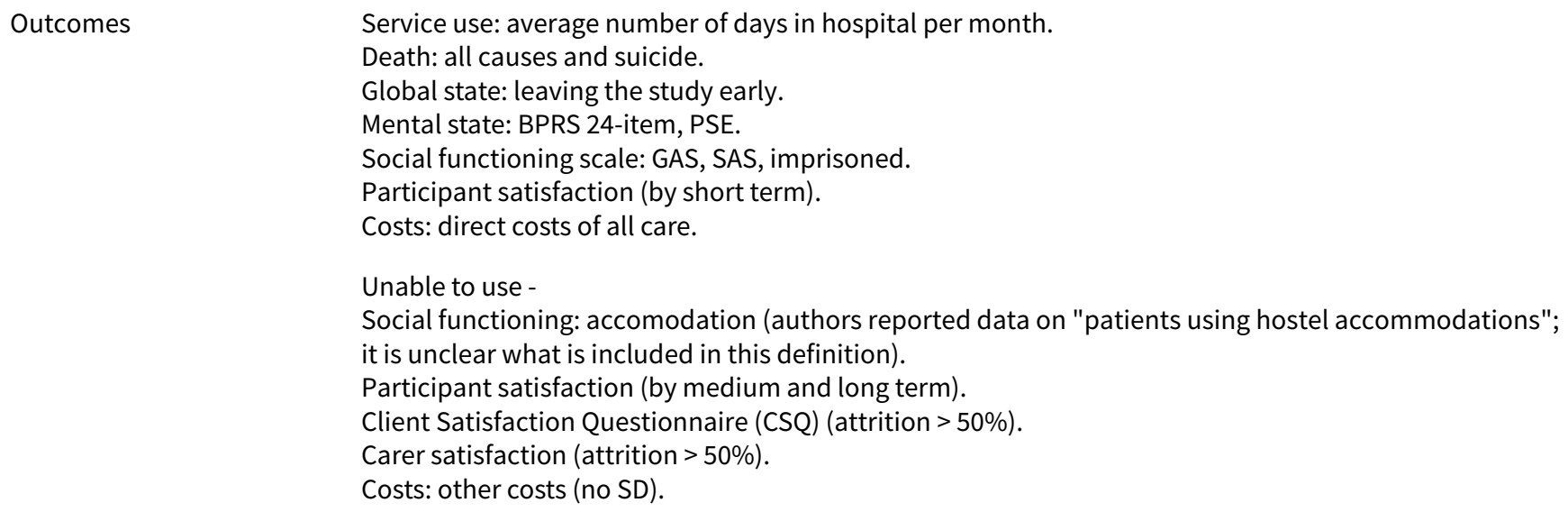

\section{Risk of bias}

\begin{tabular}{lll}
\hline Bias & Authors' judgement & Support for judgement \\
\hline $\begin{array}{l}\text { Random sequence genera- } \\
\text { tion (selection bias) }\end{array}$ & Unclear risk & Randomised. No details. \\
\hline $\begin{array}{l}\text { Allocation concealment } \\
\text { (selection bias) }\end{array}$ & Unclear risk & No details \\
\hline
\end{tabular}

Blinding (performance bias and detection bias) All outcomes
Primary outcome: clinician/participant mediated - rating - Unclear. Secondary outcomes: those interviewer rated - rating - Unclear. No details.

\begin{tabular}{lll}
\hline $\begin{array}{l}\text { Blinding of participants } \\
\text { and personnel (perfor- } \\
\text { mance bias) } \\
\text { All outcomes }\end{array}$ & High risk & $\begin{array}{l}\text { Problematic to blind participants and those providing the intervention in stud } \\
\text { ies comparing ICM intervention with standard care. }\end{array}$ \\
\hline $\begin{array}{l}\text { Blinding of outcome as- } \\
\text { sessment (detection bias) } \\
\text { All outcomes }\end{array}$ & Unclear risk & $\begin{array}{l}\text { Service use, income, and accomodation data were collected using the Client } \\
\text { Service Receipt Interview. Information was also taken from case records on } \\
\text { frequency and duration of input from CPNs. Blinding not reported. }\end{array}$
\end{tabular}

\begin{tabular}{|c|c|c|}
\hline $\begin{array}{l}\text { Incomplete outcome data } \\
\text { (attrition bias) } \\
\text { All outcomes }\end{array}$ & Unclear risk & $\begin{array}{l}\text { Number of lost to follow-up is stated, but reason for attrition is reported } \\
\text { generically, referred to the entire sample size and not the single intervention } \\
\text { sample. }\end{array}$ \\
\hline
\end{tabular}

\begin{tabular}{lll}
\hline $\begin{array}{l}\text { Selective reporting (re- } \\
\text { porting bias) }\end{array}$ & High risk & $\begin{array}{l}\text { All listed outcomes of interest are fully reported (but some economic out- } \\
\text { comes missing any variance measurement). }\end{array}$ \\
\hline Other bias & Low risk & No details. No evidence of the presence of other bias. \\
\hline
\end{tabular}




Methods
Allocation: randomised.
Design: multicentre (2 sites: Site A, New West; Site B, Surrey - individual centre data not reported).
Duration: 24 months.
Country: British Columbia, Canada.
Diagnosis ${ }^{\star}$ : majority of participants affected by schizophrenic disorder, others any DSM-III axis I or axis
II (including dual diagnosis).
N $=123$.
Setting: CMHC.
Ethnicity: data not available.
Age: 19 to 64 years (randomised sample age not reported).
Sex: $49.5 \%$ M (61M, 62F).
History: i. serious and persistent mental illness with impaired role functioning, ii. about to be dis-
charged from hospital, iii. high risk of rehospitalisation, iv. no primary diagnosis of substance abuse,
organic brain disease, or developmental disorder, v. no recent history of severe violence, vi. informed
consent to participate.

I. ICM: care from a CMHC plus additional Assertive Case Management according to Stein and Test mod-
el. Caseload: $\sim 1: 10 . \mathrm{N}=63$.
2. Standard care: care from a CMHC. $\mathrm{N}=60$.

\begin{tabular}{ll}
\hline Outcomes & Service use: average number of days in hospital per month ${ }^{\star \star}$, number of admissions ${ }^{\star \star}$. \\
& Death: all causes and suicide. \\
& Global state: leaving the study early. \\
& Unable to use - \\
& Quality of life: scale (not peer reviewed).
\end{tabular}

\begin{tabular}{ll}
\hline Notes & ${ }^{*}$ Schizophrenia-like disorder $(60.2 \%)$. \\
& ${ }^{*}$ Variance not reported - data from another study used. \\
\hline
\end{tabular}

\section{Risk of bias}

\begin{tabular}{|c|c|c|}
\hline Bias & Authors' judgement & Support for judgement \\
\hline $\begin{array}{l}\text { Random sequence genera- } \\
\text { tion (selection bias) }\end{array}$ & Unclear risk & Randomised. No further details. \\
\hline $\begin{array}{l}\text { Allocation concealment } \\
\text { (selection bias) }\end{array}$ & Unclear risk & No details \\
\hline $\begin{array}{l}\text { Blinding (performance } \\
\text { bias and detection bias) } \\
\text { All outcomes }\end{array}$ & Unclear risk & $\begin{array}{l}\text { Primary outcome: clinician/participant mediated - rating - Unclear. } \\
\text { Secondary outcome: those clinician/participant mediated - rating - Unclear. }\end{array}$ \\
\hline $\begin{array}{l}\text { Blinding of participants } \\
\text { and personnel (perfor- } \\
\text { mance bias) } \\
\text { All outcomes }\end{array}$ & High risk & $\begin{array}{l}\text { Problematic to blind participants and those providing the intervention in stud- } \\
\text { ies comparing ICM intervention with standard care. }\end{array}$ \\
\hline $\begin{array}{l}\text { Blinding of outcome as- } \\
\text { sessment (detection bias) } \\
\text { All outcomes }\end{array}$ & Unclear risk & $\begin{array}{l}\text { Hospitalisation based on hospital records. Blinding not reported. } \\
\text { Quality of life self reported. }\end{array}$ \\
\hline
\end{tabular}


Muller-Clemm-Canada 1996 (Continued)

Incomplete outcome data Unclear risk Number randomised not clearly reported, as authors declared that "Clients (attrition bias) who withdrew from the study within the first 6 months were replaced by other All outcomes clients".

$\begin{aligned} & \text { Selective reporting (re- } \\ & \text { porting bias) }\end{aligned}$ High risk Outcome length of hospitalisation reported incompletely (no SD).

Other bias Low risk No details. No evidence of the presence of other bias.

Okpaku-Tennessee 1997

\begin{tabular}{|c|c|}
\hline Methods & $\begin{array}{l}\text { Allocation: randomised. } \\
\text { Design: single centre. } \\
\text { Duration*: } 4 \text { months. } \\
\text { Country: Nashville, Tennessee, USA. }\end{array}$ \\
\hline Participants & $\begin{array}{l}\text { Diagnosis**: mental illness causing significant impairment. No further information provided. } \\
N=152 . \\
\text { Setting: urban mental health centres. } \\
\text { Age: } 18 \text { to } 55 \text { years, mean } 36.8 \text { years (SD 9.1). } \\
\text { Sex: } 59 \% \text { M. } \\
\text { Ethnicity: } 40 \% \text { non-white. } \\
\text { History: i. clients of mental health centres, ii. serious mental illness as judged by eligibility for disability } \\
\text { benefits. }\end{array}$ \\
\hline
\end{tabular}

Interventions

1. ICM ${ }^{\star \star *}$ : employment-oriented case management provided by psychiatric vocational rehabilitation specialists, supervised by the multidisciplinary team, vocational rehabilitation specialists. Caseload: 1:10. $N=73$

2. Non-ICM: standard case management services from CMHC. Caseload: 1:40 to $90 . \mathrm{N}=79$.

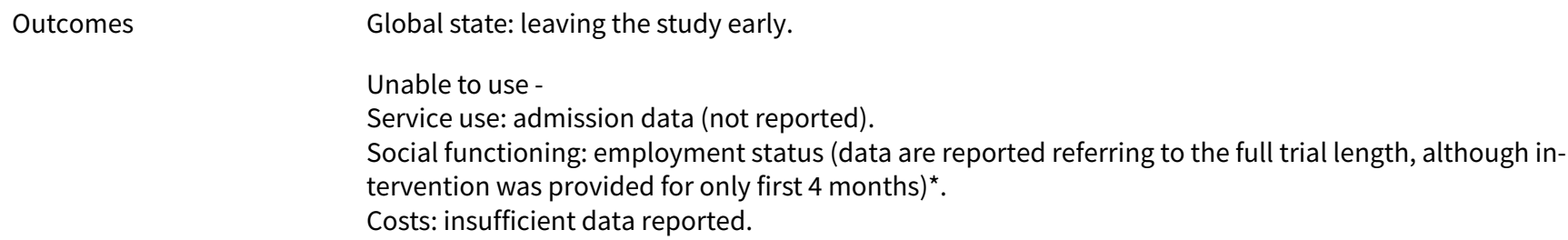

Notes

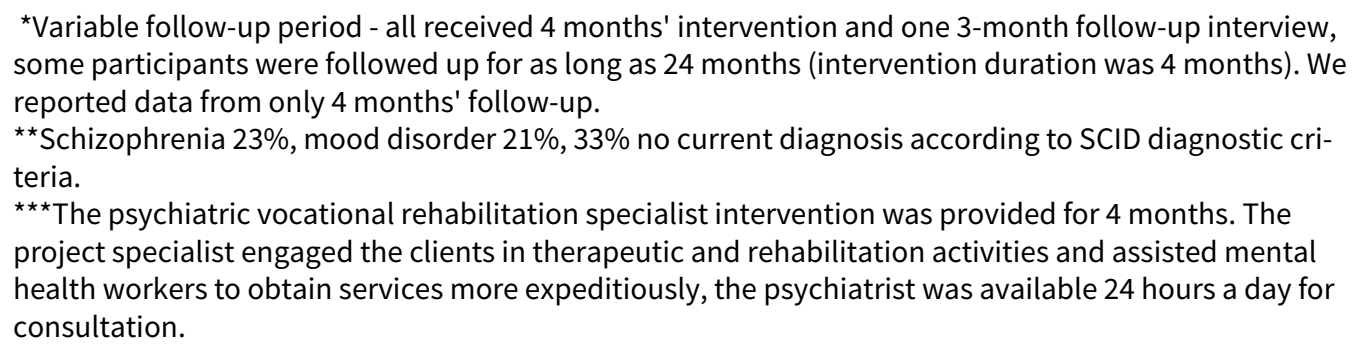

\section{Risk of bias}

\begin{tabular}{lll}
\hline Bias & Authors' judgement & Support for judgement \\
\hline $\begin{array}{l}\text { Random sequence genera- } \\
\text { tion (selection bias) }\end{array}$ & Unclear risk & Randomised. No further details. \\
\hline
\end{tabular}


Okpaku-Tennessee 1997 (Continued)

\begin{tabular}{lll}
$\begin{array}{l}\text { Allocation concealment } \\
\text { (selection bias) }\end{array}$ & Unclear risk & No details \\
\hline $\begin{array}{l}\text { Blinding (performance } \\
\text { bias and detection bias) } \\
\text { All outcomes }\end{array}$ & Unclear risk & $\begin{array}{l}\text { Primary outcome: not available. } \\
\text { Secondary outcome: clinician/participant mediated - rating - Unclear. }\end{array}$ \\
\hline
\end{tabular}

All outcomes

Blinding of participants $\quad$ Unclear risk $\quad$ Blinding not reported.
and personnel (perfor-
mance bias)
All outcomes

\begin{tabular}{lll}
\hline $\begin{array}{l}\text { Blinding of outcome as- } \\
\text { sessment (detection bias) } \\
\text { All outcomes }\end{array}$ & High risk & Hospitalisation, employment, income, illegal activity self reported. \\
\hline $\begin{array}{l}\text { Incomplete outcome data } \\
\text { (attrition bias) } \\
\text { All outcomes }\end{array}$ & Unclear risk & $\begin{array}{l}\text { Insufficient reporting of attrition/exclusion to permit judgement (number for } \\
\text { missing to follow-up is reported, but reason is not reported). }\end{array}$ \\
\hline $\begin{array}{l}\text { Selective reporting (re- } \\
\text { porting bias) }\end{array}$ & High risk & More listed outcomes of interest are reported incompletely. \\
\hline Other bias & Low risk & $\begin{array}{l}\text { Publicly funded (grant from the Social Security Administration). No further de- } \\
\text { tails. No evidence of the presence other bias. }\end{array}$ \\
\hline
\end{tabular}

\section{OPUS-Denmark 1999}

Allocation: randomised.
Design: multicentre ( 5 centres, in Copenhagen and Aarhus, data not available for single centres).
Duration: 24 months of active treatments after randomisation.
Follow-up: 5 and 10 years after randomisation (i.e. 3 and 8 years after the active intervention was pro-
vided). Starting from 2 years after randomisation, all participants received standard care (both those
randomised to the experimental intervention and those randomised to standard care).
Country: Copenhagen and Aahrus, Denmark.

Participants Diagnosis*: schizophrenia or schizophrenia-like psychosis (according to ICD-10) (main diagnosis and comorbidity based on SCAN 2.0 and SCAN 2.1).

$\mathrm{N}=547$.

Setting: public mental health service.

Age: 18 to 45 years, mean 26.6 years.

Sex: 59\% M (323M, 224F).

Ethnicity: not reported.

History $^{\star *}$ : i. prior treatment of mental disorder has not been adequate (i.e. $\leqq 12$ weeks of continuous antipsychotic medication in antipsychotic dosage), ii. absence of learning disability, organic mental disorder, and psychotic condition due to psychoactive substance use, iii. Danish speaker, iv. written informed consent, v. legal residence in the catchment area, vi. the use of psychoactive drug did not cause exclusion.

\begin{tabular}{ll} 
Interventions & $\begin{array}{l}\text { 1. ICM }{ }^{\star \star \star}: \text { modified Assertive Community Treatment (including individual case manager, recommenda- } \\
\text { tion of antipsychotic medications, psycho-educational family treatment }\end{array}$ \\
& $\begin{array}{l}\text { Caseload: } 1: 15 . \mathrm{N}=275 . \\
\text { 2. Standard care }\end{array}$ \\
\hline Outcomes & and social skill training).
\end{tabular}


OPUS-Denmark 1999 (Continued)

discharge to outpatient status)"; admitted to hospital (during previous 12 months); proportion not hospitalised; proportion without outpatient contacts; proportion without psychiatric emergency room contacts.

Death: all causes, suicide.

Global state: leaving the study early.

Social functioning: not working or in education, not living independently, alcohol and drug abuse diagnosed with SCAN, contact with the legal system - imprisonment (2-year treatment and 3-year follow-up), number of days in supported housing, number of days in a homeless shelter.

Mental state, specific symptoms: positive symptoms (Scale for Assessment of Positive Symptoms -

SAPS), negative symptoms (Scale for Assessment of Negative Symptoms - SANS), comorbidity with depression.

Participant satisfaction: Client Satisfaction Questionnaire (CSQ).

Behaviour: specific behaviour, self harm (measured by self reporting of suicide attempts).

Unable to use -

Global state: compliance with medications: defined "good compliance" as having taken the prescribed antipsychotic medications in the recommended doses regularly during previous 3 months (not reported), GAF (reported only subscale data, not global score).

Social functioning: Social Network Size (not published measure instrument), Social Network Schedule (SNS); various outcomes on accomodation status (as proportion spending at least 1 day in supported housing or in homeless shelter): not listed as a review outcome of interest.

Mental state, specific symptoms: disorganised dimension (not stated how it is measured) and suicidal ideation (not listed as a review outcome of interest).

Quality of life: not reported.

Participant satisfaction: Camberwell Assessment of Need (CAN) (not reported).

Relative satisfaction: Client Satisfaction Questionnaire 8-item, adapted version (not reported).

Behaviour: Social Behaviour Assessment Schedule (SBAS) (reported only score on subscale).

Use of coercive measure: not listed as a review outcome of interest.

Assessment of expressed emotion: not listed as a review outcome of interest.

Notes $\quad$ *Schizophrenia 66\%; schizotypal disorder 15\%; schizoaffective disorder $5 \%$.

${ }^{\star \star}$ Median duration of untreated psychosis $~ 50$ weeks.

${ }^{\star \star \star}$ In both interventions use of antipsychotics followed the guidelines from the Danish Psychiatry Society (recommending a low-dose strategy for patients with first-episode psychosis and the use of second-generation drugs as first choice).

${ }^{\star \star \star \star \star}$ Family treatment followed McFarlane's manual for psychoeducational treatment for multiple-family group (18 months' treatment, 1.5 hours twice a week, in a multiple-family group with 2 therapists and 4 to 6 patients with their families). Focus on problem solving and development of skills to cope with illness.

\section{Risk of bias}

Bias Authors' judgement Support for judgement

Random sequence genera- Low risk tion (selection bias)

\author{
Randomised
}

In Copenhagen, allocation sequence was computer generated. In Aahrus, a secretary drew a lot among 5 red and 5 white lots from a black box. Central randomisation. Randomisation was $1: 1$, in block of 6 , stratified for each of the 5 centres.

\begin{tabular}{ll}
\hline $\begin{array}{l}\text { Allocation concealment } \\
\text { (selection bias) }\end{array}$ & $\begin{array}{l}\text { In Cow risk } \\
\text { allocation. }\end{array}$ \\
& $\begin{array}{l}\text { In Aahrus, researchers were informed on the randomisation assignment after } \\
\text { they had finished the entry assessment. Block sizes were unknown to investi- } \\
\text { gator. }\end{array}$
\end{tabular}

Blinding (performance Unclear risk Primary outcome: clinician/participant mediated - rating - Unclear.


OPUS-Denmark 1999 (Continued)

All outcomes
Secondary outcome: those interviewer rated - rating - No. Reported: "Investigators were not blind to treatment allocation".

\begin{tabular}{|c|c|c|}
\hline $\begin{array}{l}\text { Blinding of participants } \\
\text { and personnel (perfor- } \\
\text { mance bias) } \\
\text { All outcomes }\end{array}$ & High risk & $\begin{array}{l}\text { Problematic to blind participants and those providing the intervention in stud- } \\
\text { ies comparing ICM intervention with standard care. }\end{array}$ \\
\hline $\begin{array}{l}\text { Blinding of outcome as- } \\
\text { sessment (detection bias) } \\
\text { All outcomes }\end{array}$ & Unclear risk & $\begin{array}{l}\text { Service use based on official registers, blinding not reported. Interviewers for } \\
\text { mental state unblinded. } \\
\text { Client satisfaction, suicide attempts, and suicidal ideation self reported. }\end{array}$ \\
\hline $\begin{array}{l}\text { Incomplete outcome data } \\
\text { (attrition bias) } \\
\text { All outcomes }\end{array}$ & Low risk & $\begin{array}{l}\text { Numbers and reasons for missing data are clearly reported. Analysis was con- } \\
\text { ducted on an ITT basis. }\end{array}$ \\
\hline $\begin{array}{l}\text { Selective reporting (re- } \\
\text { porting bias) }\end{array}$ & High risk & $\begin{array}{l}\text { Authors reported change of primary outcome stated in the protocol (from "re- } \\
\text { lapse and positive symptoms" to "psychotic and negative symptoms"), as high } \\
\text { attrition occurred in the former outcome measurements. Some listed out- } \\
\text { comes of interest are not reported. }\end{array}$ \\
\hline Other bias & Low risk & $\begin{array}{l}\text { Publicly funded. No declaration of interest. No further details. No evidence of } \\
\text { the presence of other bias. }\end{array}$ \\
\hline
\end{tabular}

Pique-California 1999

\begin{tabular}{ll}
\hline Methods & Allocation: randomised. \\
& Design: single centre. \\
& Duration: 24 months. \\
Country: San Francisco, USA. & \\
\hline Participants & Diagnosis: severe and persistent mental illness (psychiatric disorder as the primary source of disabili- \\
ty). & $\mathrm{N}=37$. \\
Setting: Department of Psychiatry, San Francisco General Hospital. & Age: $\geqq 18$ years. \\
Sex: not reported. & Ethnicity: European (understood to be white) or African-American. \\
History: high user of intensive treatment care who had experienced an unsatisfactory quality of life in \\
the community, i. recently hospitalised; ii. no primary diagnosis of (a) organic brain disease, (b) sub- \\
stance abuse disorder with no other psychiatric disorder, or (c) learning disability; iii. no history of vio- \\
lence not due to treatable psychiatric symptoms; iv. written informed consent.
\end{tabular}

I. ICM: standard care + Assertive Community Treatment according to Stein and Test model, culturally
focused on needs of Afro-American population. Caseload: $1: 10 . \mathrm{N}=22$.
2. Standard care: Caseload: $1: 30 . \mathrm{N}=15$.

2. Standard care: Caseload: 1:30. $\mathrm{N}=15$.

Global state: leaving the study early.
Unable to use -
Service use: average number of days in hospital per month (not reported).
Global functioning: GAF (attrition 57\%).
Costs: reported incompletely (no mean, no SD).

Notes 
Pique-California 1999 (Continued)

Risk of bias

\begin{tabular}{lll}
\hline Bias & Authors' judgement & Support for judgement \\
\hline $\begin{array}{ll}\text { Random sequence genera- } \\
\text { tion (selection bias) }\end{array}$ & Low risk & $\begin{array}{l}\text { Randomised. Stratified by sex, ethnicity, recruitment centre, assignment to in- } \\
\text { tervention 2:1. Randomisation sequence constructed by independent investi- } \\
\text { gator using a table of random permutation. }\end{array}$ \\
& &
\end{tabular}

\begin{tabular}{lll}
$\begin{array}{l}\text { Allocation concealment } \\
\text { (selection bias) }\end{array}$ & Unclear risk & $\begin{array}{l}\text { Randomisation sequence in sealed, opaque envelopes, unused assignment } \\
\text { envelopes kept in a locked container (available to the recruiter psychiatrists). }\end{array}$ \\
\hline $\begin{array}{l}\text { Blinding (performance } \\
\text { bias and detection bias) } \\
\text { All outcomes }\end{array}$ & Unclear risk & $\begin{array}{l}\text { Primary outcome: not available. } \\
\text { Secondary outcome: clinician/participant mediated - rating - Unclear. }\end{array}$ \\
\hline
\end{tabular}

\begin{tabular}{|c|c|c|}
\hline $\begin{array}{l}\text { Blinding of participants } \\
\text { and personnel (perfor- }\end{array}$ & High risk & $\begin{array}{l}\text { Problematic to blind participants and those providing the intervention in stud } \\
\text { ies comparing ICM intervention with standard care. }\end{array}$ \\
\hline
\end{tabular}

mance bias)

All outcomes

$\begin{array}{lll}\begin{array}{l}\text { Blinding of outcome as- } \\ \text { sessment (detection bias) }\end{array} & \text { Unclear risk } & \begin{array}{l}\text { Service use data were obtained from the county database. Costs were ob- } \\ \text { tained from billing records. Blinding not reported. }\end{array}\end{array}$

All outcomes

\begin{tabular}{|c|c|c|}
\hline $\begin{array}{l}\text { Incomplete outcome data } \\
\text { (attrition bias) }\end{array}$ & Unclear risk & $\begin{array}{l}\text { Author did not address this outcome. Reasons for missing outcome data not } \\
\text { reported. }\end{array}$ \\
\hline
\end{tabular}

All outcomes reported.

Selective reporting (re- High risk Listed outcome of interest reported incompletely (costs: no mean, no SD). porting bias)

Other bias Low risk No details. No evidence of the presence of other bias.

\begin{tabular}{ll} 
Methods & $\begin{array}{l}\text { Allocation: randomised. } \\
\text { Design: single centre. } \\
\text { Duration: } 24 \text { months. } \\
\text { Country: San Diego, California, USA. }\end{array}$ \\
\hline Participants & Diagnosis*: major disorder DSM-III-R axis I. \\
$\mathrm{N}=90$. & Setting: San Diego County Mental Health Service. \\
& Age: $>18$ years, 33\% $>40$ years, mean $~ 37$ years. \\
& Sex: $44 \% \mathrm{M}(40 \mathrm{M}, 50 \mathrm{~F})$. \\
& Ethnicity: $43 \%$ non-white (18\% African-American). \\
& History: $\geqq 3$ hospitalisations last 2.5 years.
\end{tabular}
Interventions
1. ICM: Assertive Community Treatment according to Stein and Test model. Caseload: 1:15. $N=30$.
2. Non-ICM: traditional CM programme, no team approach. Caseload: 1:40-60. $\mathrm{N}=30$.
3. Standard care: services offered by the public mental health system. $\mathrm{N}=30$.

Outcomes
Service use: average number of days in hospital per month. Costs: direct costs of psychiatric hospital care.

Unable to use - 
Quinlivan-California 1995 (Continued)

Service use: other service use than hospital (reported incompletely).

Global state: leaving the study early (not reported).

Costs: direct costs of other psychiatric care (outcomes relevant to this review not directly examined).

Notes $56 \%$ schizophrenia; $23 \%$ bipolar disorder.
This is a 3-arm study, data from the study are included in both comparisons addressed by the review:
ICM versus non-ICM and ICM versus standard care.

\section{Risk of bias}

\begin{tabular}{|c|c|c|}
\hline Bias & Authors' judgement & Support for judgement \\
\hline $\begin{array}{l}\text { Random sequence genera- } \\
\text { tion (selection bias) }\end{array}$ & Unclear risk & Randomised, no further details. \\
\hline $\begin{array}{l}\text { Allocation concealment } \\
\text { (selection bias) }\end{array}$ & Unclear risk & No information \\
\hline $\begin{array}{l}\text { Blinding (performance } \\
\text { bias and detection bias) } \\
\text { All outcomes }\end{array}$ & Unclear risk & $\begin{array}{l}\text { Primary outcome: clinician/participant mediated - rating - Unclear. } \\
\text { Secondary outcomes: - rating - Yes. No information provided, but available } \\
\text { outcomes are not likely to be influenced by lack of blinding. }\end{array}$ \\
\hline $\begin{array}{l}\text { Blinding of participants } \\
\text { and personnel (perfor- } \\
\text { mance bias) } \\
\text { All outcomes }\end{array}$ & Unclear risk & Blinding not reported. \\
\hline $\begin{array}{l}\text { Blinding of outcome as- } \\
\text { sessment (detection bias) } \\
\text { All outcomes }\end{array}$ & Low risk & $\begin{array}{l}\text { Hospitalisation data were obtained from the county mental health services di- } \\
\text { vision's management information system. Costs were based on budgeted unit } \\
\text { cost of each service multiplied by the total number of units as reported in the } \\
\text { management information system. }\end{array}$ \\
\hline
\end{tabular}

Incomplete outcome data Unclear risk The study did not address this outcome.

(attrition bias)

All outcomes

Selective reporting (re- Low risk Listed outcomes of interest are reported completely.

porting bias)

Other bias Low risk No data provided. No evidence of the presence of other bias.

\section{REACT-UK 2002}

\begin{tabular}{ll}
\hline Methods & Allocation: randomised. \\
Design: multicentre ( 2 centres, information for single centre not available). \\
Duration: 18 months. During this period, participants remained allocated in their trial arm. \\
Follow-up: 36 months and 10 years after randomisation (i.e. 18 months and 8.5 years after the active in- \\
tervention was provided). During the follow-up period, participants could remain in the originally allo- \\
cated intervention (ICM) or be transferred to the control one. \\
Country: London, UK. \\
Diagnosis*: SMI (schizophrenia, schizoaffective disorder, other chronic psychosis, bipolar affective dis- \\
order). \\
N $=251$. \\
Setting: community services in 2 inner London boroughs (Camden and Islington).
\end{tabular}


REACT-UK 2002 (Continued)

Age: mean 39 years (SD 11).

Sex: $58 \%$ M.

Ethnicity: African-Caribbean 36\%.

History: i. living independently or in low-supported accomodation, ii. under the care of CMHT $\geqq 12$ months and having difficulty engaging with standard community care, iii. recent high use of inpatient care (i.e. $\geqq 100$ consecutive inpatient days or $\geqq 5$ admissions during previous 2 years, or $\geqq 50$ consecutive inpatient days or $\geqq 3$ admissions previous 1 year), iv. substance misuse or personality disorder eligible if these were secondary diagnosis, v. no organic brain damage.

Interventions

1. ICM: Assertive Community Treatment (as described by McGrew 1995). Caseload: 1:12. N = 127 .

2. Non-ICM: services offered by CMHT (according to Care Programme Approach). Caseload: 1:35. $\mathrm{N}=$ 124.

Outcomes

Service use: average number of days in hospital per month, not remaining in contact with psychiatric services (defined as no face-to-face contacts between staff and client in previous 3 months), average admission, admitted to hospital.

Death: all causes and suicide.

Global state: leaving the study early, Health of the Nation Outcome Scale (HoNOS), Rating of Medication Influence scale (ROMI).

Social functioning: arrested, imprisoned, number homeless, living independently, living in supported accomodation, Life Skill Profile (LSP), substance abuse: assessed through various scales (Alcohol Use Scale - AUS, Drug Use Scale - DUS, Substance Abuse Treatment Scale - SATS), but reported as binary outcome.

Mental state: Brief Psychiatric Rating Scale (BPRS-24 item).

Behaviour: self harm, injury to others.

Quality of life: Manchester Short Assessment of Quality of Life (MANSA).

Participant satisfaction: Client Satisfaction Questionnaire modified version (CSQ-modified), Camberwell Assessment Need abbreviated form (CAN).

Unable to use -

Use of Mental Health Act (not listed as review outcome of interest).

Quality of engagement: adapted form of Homeless Engagement Acceptance Scale (HEAS) (not listed as review outcome of interest).

\section{Risk of bias}

\begin{tabular}{lll}
\hline Bias & Authors' judgement & Support for judgement \\
\hline $\begin{array}{l}\text { Random sequence genera- } \\
\text { tion (selection bias) }\end{array}$ & Low risk & $\begin{array}{l}\text { Randomised: permuted block randomisation with a block size of } 8 \text { ensuring } \\
\text { parity between CMHT in proportions randomised to ICM. }\end{array}$ \\
\hline $\begin{array}{l}\text { Allocation concealment } \\
\text { (selection bias) }\end{array}$ & Low risk & $\begin{array}{l}\text { The interviewer contacted an administrator at the trial centre who opened the } \\
\text { appropriate numbered envelope communicating the outcome of randomisa- } \\
\text { tion. Participants and referrers were informed of the treatment assignment by } \\
\text { letter. }\end{array}$
\end{tabular}

\begin{tabular}{|c|c|c|}
\hline $\begin{array}{l}\text { Blinding (performance } \\
\text { bias and detection bias) } \\
\text { All outcomes }\end{array}$ & Unclear risk & $\begin{array}{l}\text { Primary outcome: clinician/participant mediated - rating - Unclear. } \\
\text { Secondary outcomes: interviewer rated - rating - No. Interviewers were inde- } \\
\text { pendent of clinical care, but not blind. }\end{array}$ \\
\hline
\end{tabular}

Blinding of participants $\quad$ Unclear risk $\quad$ Blinding not reported.
and personnel (perfor-
mance bias)
All outcomes

\begin{tabular}{ll}
\hline Blinding of outcome as- & High risk
\end{tabular}
All outcomes 
Self harm, violence, contact with legal services, quality of life, compliance with medication, and mental state were obtained from interviews with clients. Other scales were completed by care co-ordinators. All additional data were collected from case notes. Assessors not blinded.

Incomplete outcome data Low risk
(attrition bias)

YES - Primary outcomes: average number of days in hospital per month. No missing data (except death, balanced in numbers across groups).

All outcomes

YES - Secondary outcomes: number and reasons for missing data are reported. Analysis carried out on an ITT basis.

\begin{tabular}{lll}
\hline $\begin{array}{l}\text { Selective reporting (re- } \\
\text { porting bias) }\end{array}$ & Low risk & All of the listed outcomes of interest are completely reported. \\
\hline Other bias & Low risk & $\begin{array}{l}\text { Publicly funded (Camden and Islington Health Authority; King's Fund; Depart- } \\
\text { ment of Health). Competing interesting declared: none. No further details. No } \\
\text { evidence of the presence of other bias. }\end{array}$ \\
\hline
\end{tabular}

Rosenheck-USA 1993

Methods
Allocation: randomised.
Design: multicentre. 10 sites: 6 General Medical and Surgical centres (GMS) and 4 Neuropsychiatric
(NP) centres. Data available both for single centre and for 2 pooled centre groups (GMS and NP). See
below.
Duration: 24 months.
Country: Northeastern United States
Participants Diagnosis*: primary psychiatric disorder.
$\mathrm{N}=873$.
Setting: community-based psychiatric care - Department of Veterans Affairs (VA).
Age: $48 \%>45$ years, mean 47.6 years.
Sex: $100 \%$ M.
Ethnicity: $20 \%$ non-white.
History: i. current inpatient in VA psychiatric unit, ii. no primary diagnosis of substance abuse or organ- ic brain disease, iii. recent high user of psychiatric care (definition varied between GMS and NP centres), iv. written consent.

\begin{abstract}
Interventions $\quad$ 1. ICM: Intensive Psychiatric Community Care programme, providing ACT intervention according to Stein and Test model. Caseload: average 1:7-15. $N=454$.

2. Standard care ${ }^{\star \star}$ : routine care from psychiatric services provided by Veterans Affairs, including inpatient and outpatient psychiatric treatment, psychopharmacological treatment, and rehabilitation service. $\mathrm{N}=419$.
\end{abstract}
Outcomes Service use: average number of days in hospital per month (both pooled data for GMS and NP centres and data for single centre available; see below) ${ }^{\star \star \star}$.
Global state: GAS (authors reported results pooled for GMS and NP centres; see below).
Mental state: BPRS-18 item, BSI (authors reported results pooled for GMS and NP centres; see below). Costs: total healthcare cost (authors reported results pooled for GMS and NP centres; see below).
Unable to use -
Global state: self reported measure (not published, not peer reviewed).
Participant satisfaction: satisfaction with service (measurement instrument not published, not peer re- viewed).
Social functioning: Addiction Severity Index (ASI) (reported only a subscale, not the overall score). Costs: non-healthcare costs (not listed as an outcome of interest in the review).

${ }^{\star *}$ Standard care provided by both types of centre did not differ programme wise. 
Rosenheck-USA 1993 (Continued)

***Pooled data for GMS and NP entered the meta-analysis; data for single centre entered in meta-regression.

\section{Risk of bias}

\begin{tabular}{lll}
\hline Bias & Authors' judgement & Support for judgement \\
\hline $\begin{array}{l}\text { Random sequence genera- } \\
\text { tion (selection bias) }\end{array}$ & Low risk & Randomised through coin tossing. \\
\hline $\begin{array}{l}\text { Allocation concealment } \\
\text { (selection bias) }\end{array}$ & Unclear risk & $\begin{array}{l}\text { No details (reported only that the assignment by coin tossing was performed } \\
\text { by an independent researcher). }\end{array}$ \\
\hline $\begin{array}{l}\text { Blinding (performance } \\
\text { bias and detection bias) } \\
\text { All outcomes }\end{array}$ & Unclear risk & $\begin{array}{l}\text { Primary outcome: clinician/participant mediated - rating - Unclear. } \\
\text { Secondary outcomes: interviewer rated - rating - Unclear. Authors report that } \\
\text { interviewers are independent, but it is not stated whether they are blind to } \\
\text { participant assignment. }\end{array}$ \\
\hline
\end{tabular}

Blinding of participants High risk Problematic to blind participants and those providing the intervention in stud-
and personnel (perfories comparing ICM intervention with standard care.

mance bias)

All outcomes

\begin{tabular}{lll}
\hline Blinding of outcome as- & Unclear risk & Service use (hospital and nursing home service use) was derived from the VA's \\
sessment (detection bias) & national computerised workload monitoring systems. Blinding not reported. \\
All outcomes & Costs were determined using VA's standardised Cost Distribution Report.
\end{tabular}

Incomplete outcome data Unclear risk

(attrition bias)

The study did not address this outcome.

All outcomes

\begin{tabular}{lll}
\hline $\begin{array}{l}\text { Selective reporting (re- } \\
\text { porting bias) }\end{array}$ & High risk & $\begin{array}{l}\text { Some listed outcomes of interest are not reported completely (i.e. substance } \\
\text { abuse, which is reported only as a subscale score and not general score). }\end{array}$ \\
\hline Other bias & Low risk & No details. No evidence of the presence of other bias. \\
\hline
\end{tabular}

Rosenheck-USA-GMS

\begin{tabular}{ll} 
Methods & Rosenheck-USA-GMS refers to 6 General Medical and Surgical centres (GMS) pooled together (centre A, \\
& B, D, F, I, J). \\
& Methods: see above Rosenheck-USA 1993. \\
\hline Participants & Participants: see above Rosenheck-USA 1993. \\
& Following data are specific for Rosenheck-USA-GMS: \\
N $=528$. & \\
Setting: Department of Veterans Affairs (VA) - 6 GMS centres enrolled in the Intensive Psychiatric Com- \\
munity Care programme. GMS centres are located in urban centres, provide shorter-term, crisis-orient- \\
ed, inpatient care. \\
History: i. current inpatient in VA psychiatric unit, ii. no primary diagnosis of substance abuse or organ- \\
ic brain disease, iii. recent high user of psychiatric care (defined as i. $\geqq 40$ days in hospital or ii. $\geqq 2$ ad- \\
missions in the previous year), iv. written consent.
\end{tabular}

\footnotetext{
Interventions

1. ICM ${ }^{*}$ : Intensive Psychiatric Community Care programme, providing ACT intervention according to Stein and Test model. Caseload: average 1:7-15. $N=271$.
} 
Rosenheck-USA-GMS (Continued)

2. Standard care: routine care from psychiatric services provided by Veterans Affairs, including inpatient and outpatient psychiatric treatment, psychopharmacological treatment, and rehabilitation service. $\mathrm{N}=257$.

\begin{tabular}{|c|c|}
\hline Outcomes & $\begin{array}{l}\text { Service use: average number of days in hospital per month }{ }^{\star \star} \text {. } \\
\text { Global state: GAS. } \\
\text { Mental state: BPRS-18 item, BSI. } \\
\text { Costs: costs of health care. }\end{array}$ \\
\hline Notes & $\begin{array}{l}{ }^{\star} \text { Centre } \mathrm{J} \text { : caseload } 1: 44 \text {. } \\
{ }^{\star \star} \text { Entered in the meta-analysis. }\end{array}$ \\
\hline
\end{tabular}

Rosenheck-USA-GMS (A)

\begin{tabular}{ll} 
Methods & Rosenheck-USA-GMS (A) is a GMS centre. \\
& Methods: see above Rosenheck-USA 1993 and Rosenheck-USA-GMS. \\
\hline Participants & Participants: see above Rosenheck-USA 1993 and Rosenheck-USA-GMS. \\
$\mathrm{N}=79$.
\end{tabular}

Interventions: see above Rosenheck-USA 1993 and Rosenheck-USA-GMS.
1. ICM: $\mathrm{N}=44$.
2. Standard care: $\mathrm{N}=35$.

Outcomes Service use: average number of days in hospital per month*.

Notes *Entered in the meta-regression.

Rosenheck-USA-GMS (B)

\begin{tabular}{ll}
\hline Methods & Rosenheck-USA-GMS (B) is a GMS centre. \\
& Methods: see above Rosenheck-USA 1993 and Rosenheck-USA-GMS. \\
\hline Participants & Participants: see above Rosenheck-USA 1993 and Rosenheck-USA-GMS. \\
N $=94$.
\end{tabular}

\begin{tabular}{ll}
\hline Interventions & Interventions: see above Rosenheck-USA 1993 and Rosenheck-USA-GMS. \\
& $\begin{array}{l}\text { 1. ICM: } \mathrm{N}=47 . \\
\text { 2. Standard care: } \mathrm{N}=47 .\end{array}$ \\
\hline Outcomes & Service use: average number of days in hospital per month ${ }^{\star}$. \\
\hline Notes & *Entered in the meta-regression. \\
\hline
\end{tabular}

Rosenheck-USA-GMS (D)

Methods Rosenheck-USA-GMS (D) is a GMS centre.


Rosenheck-USA-GMS (D) (Continued)

Methods: see above Rosenheck-USA 1993 and Rosenheck-USA-GMS.

\begin{tabular}{ll} 
Participants & Participants: see above Rosenheck-USA 1993 and Rosenheck-USA-GMS. \\
& $\mathrm{N}=102$. \\
\hline Interventions & Interventions: see above Rosenheck-USA 1993 and Rosenheck-USA-GMS. \\
& $\begin{array}{l}\text { 1. ICM: } \mathrm{N}=49 . \\
\text { 2. Standard care: } \mathrm{N}=53 .\end{array}$ \\
\hline Outcomes & Service use: average number of days in hospital per month*. \\
\hline Notes & *Entered in the meta-regression.
\end{tabular}

Rosenheck-USA-GMS (F)

Methods Rosenheck-USA-GMS (F) is a GMS centre.

Methods: see above Rosenheck-USA 1993 and Rosenheck-USA-GMS.

Participants $\begin{aligned} & \text { Participants: see above Rosenheck-USA } 1993 \text { and Rosenheck-USA-GMS. } \\ & N=78 .\end{aligned}$

Interventions: see above Rosenheck-USA 1993 and Rosenheck-USA-GMS.
1. ICM: $\mathrm{N}=43$.
2. Standard care: $\mathrm{N}=35$.

\begin{tabular}{ll}
\hline Outcomes & Service use: average number of days in hospital per month*. \\
\hline Notes & "Entered in the meta-regression. \\
\hline
\end{tabular}

Rosenheck-USA-GMS (I)

\begin{tabular}{ll}
\hline Methods & Rosenheck-USA-GMS (I) is a GMS centre. \\
& Methods: see above Rosenheck-USA 1993 and Rosenheck-USA-GMS. \\
\hline Participants & Participants: see above Rosenheck-USA 1993 and Rosenheck-USA-GMS. \\
& $\mathrm{N}=88$. \\
\hline Interventions & Interventions: see above Rosenheck-USA 1993 and Rosenheck-USA-GMS. \\
& $\begin{array}{l}\text { 1. ICM: } \mathrm{N}=44 . \\
\text { 2. Standard care: } \mathrm{N}=44 .\end{array}$ \\
\hline Outcomes & Service use: average number of days in hospital per month* \\
\hline Notes & ${ }^{\star}$ Entered in the meta-regression. \\
\hline
\end{tabular}


Rosenheck-USA-NP

\begin{tabular}{|c|c|}
\hline Methods & $\begin{array}{l}\text { Rosenheck-USA-NP refers to } 4 \text { Neuropsychiatric centres (NP) pooled together (centre C, E, G, H). } \\
\text { Methods: see above Rosenheck-USA } 1993 .\end{array}$ \\
\hline Participants & $\begin{array}{l}\text { Participants: see above Rosenheck-USA } 1993 \text {. } \\
\text { Following data are specific for Rosenheck-USA-NP: } \\
\mathrm{N}=345 \text {. } \\
\text { Setting: Department of Veterans Affairs (VA) - } 4 \text { NP centres enrolled in the Intensive Psychiatric Com- } \\
\text { munity Care programme. NP centres are large facilities providing long-term mental health care in sub- } \\
\text { urban or rural settings. } \\
\text { History*: i. current inpatient in VA psychiatric unit, ii. no primary diagnosis of substance abuse or or- } \\
\text { ganic brain disease, iii. recent high user of psychiatric care (defined as i. } \geqq 180 \text { days in hospital or ii. } \geqq 4 \\
\text { admissions in the previous year), iv. written consent. }\end{array}$ \\
\hline Outcomes & $\begin{array}{l}\text { Service use: average number of days in hospital per month }{ }^{\star \star} \text {. } \\
\text { Global state: GAS. } \\
\text { Mental state: BPRS-18 item, BSI. } \\
\text { Costs: direct costs of health care. }\end{array}$ \\
\hline Notes & $\begin{array}{l}{ }^{\star} \text { Characteristic at baseline differs between NP sites regarding inpatient days before programme en- } \\
\text { try. Difference was attributable to site C. To compensate for this imbalance, the authors made an ad- } \\
\text { justment. The adjusted value was used in all the calculations, but the results they yielded did not differ } \\
\text { substantially from those obtained with unadjusted value. } \\
{ }^{\star \star E n t e r e d ~ i n ~ t h e ~ m e t a-a n a l y s i s . ~}\end{array}$ \\
\hline
\end{tabular}

\section{Rosenheck-USA-NP (C)}

Methods Rosenheck-USA-NP (C) is a NP centre.

Methods: see above Rosenheck-USA 1993 and Rosenheck-USA-NP.

\begin{tabular}{ll}
\hline Participants & Participants: see above Rosenheck-USA 1993 and Rosenheck-USA-NP. \\
& $\mathrm{N}=93$. \\
\hline Interventions & Interventions: see above Rosenheck-USA 1993 and Rosenheck-USA-NP. \\
& $\begin{array}{l}\text { 1. ICM: } \mathrm{N}=50 . \\
\text { 2. Standard care: } \mathrm{N}=43 .\end{array}$ \\
\hline Outcomes & Service use: average number of days in hospital per month* \\
\hline Notes & ${ }^{*}$ Entered in the meta-regression. \\
\hline
\end{tabular}

Methods Rosenheck-USA-NP (E) is a NP centre.


Rosenheck-USA-NP (E) (Continued)

Methods: see above Rosenheck-USA 1993 and Rosenheck-USA-NP.

\begin{tabular}{ll} 
Participants & Participants: see above Rosenheck-USA 1993 and Rosenheck-USA-NP. \\
& $\mathrm{N}=67$. \\
\hline Interventions & Interventions: see above Rosenheck-USA 1993 and Rosenheck-USA-NP. \\
& $\begin{array}{l}\text { 1. ICM: } \mathrm{N}=34 . \\
\text { 2. Standard care: } \mathrm{N}=33 .\end{array}$ \\
\hline Outcomes & Service use: average number of days in hospital per month*. \\
\hline Notes & *Entered in the meta-regression.
\end{tabular}

Rosenheck-USA-NP (G)

Methods $\quad$ Rosenheck-USA-NP $(G)$ is a NP centre.

Methods: see above Rosenheck-USA 1993 and Rosenheck-USA-NP.

\begin{tabular}{ll}
\hline Participants & Participants: see above Rosenheck-USA 1993 and Rosenheck-USA-NP. \\
& $\mathrm{N}=71$ \\
\hline Interventions & Interventions: see above Rosenheck-USA 1993 and Rosenheck-USA-NP. \\
& $\begin{array}{l}\text { 1. ICM: } \mathrm{N}=40 . \\
\text { 2. Standard care: } \mathrm{N}=31 .\end{array}$
\end{tabular}

\begin{tabular}{ll}
\hline Outcomes & Service use: average number of days in hospital per month*. \\
\hline Notes & *Entered in the meta-regression. \\
\hline
\end{tabular}

Rosenheck-USA-NP (H)

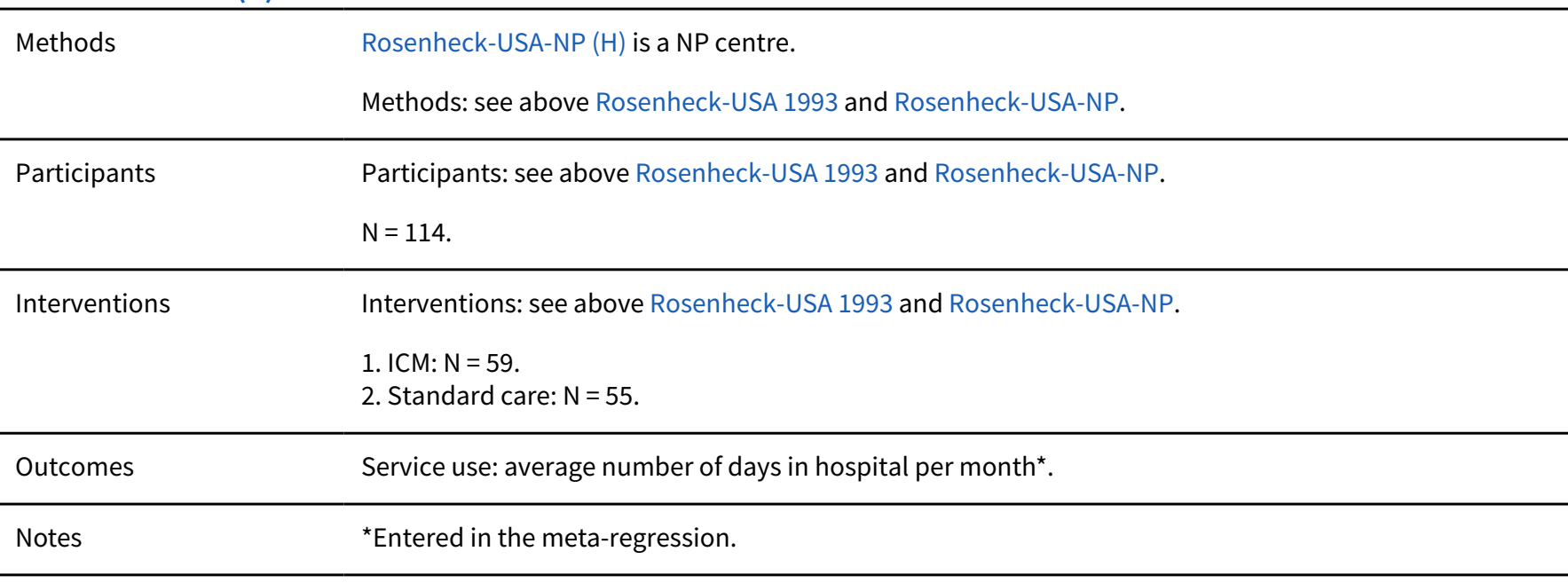


Salkever-SCarolina 1999

\begin{tabular}{|c|c|}
\hline Methods & $\begin{array}{l}\text { Allocation: randomised. } \\
\text { Design: single centre. } \\
\text { Duration: } 18 \text { months. } \\
\text { Country: Charleston, South Carolina, USA. }\end{array}$ \\
\hline Participants & $\begin{array}{l}\text { Diagnosis*: schizophrenia, schizoaffective disorder, bipolar disorder or other psychotic disorder (DSM- } \\
\text { III-R). } \\
\mathrm{N}=173 \text {. } \\
\text { Setting: Charleston and Darchester CMHCs. } \\
\text { Age: } 18 \text { to } 65 \text { years, mean } \simeq 35.5 \text { yrs. } \\
\text { Sex: } 54.8 \% \mathrm{M} \text {. }(\mathrm{N}=114) \text {. } \\
\text { Ethnicity: } 62.5 \% \text { non-white. ( } \mathrm{N}=114) \text {. } \\
\text { History: i. history or high risk for high services use patterns (i.e. long-term or multiple hospitalisation), } \\
\text { ii. difficulty with treatment compliance, independent living, or activities of daily living, iii. no primary } \\
\text { diagnosis of personality disorder or substance abuse or organic brain syndrome, iv. no need for } 24 \text {-hour } \\
\text { supervision, v. no assault behaviour in the previous year not associated with psychosis. }\end{array}$ \\
\hline
\end{tabular}

Interventions
(treatment based in $\mathrm{CMHC}$ and treatment run by nonprofit organisation located near $\mathrm{CMHC}$ ). Caseload:
ranging during the trial duration from 1:6.5 to 1:13. $\mathrm{N}=104$.
2. Non-ICM: standard care from $\mathrm{CMHC}$, providing primarily office-based case management programme.
Caseload: decreased during the trial duration from 1:68 to 1:34. $\mathrm{N}=69$.

Outcomes Service use: average number of days in hospital per month ${ }^{\star \star \star}$; admitted to hospital.
Global state: leaving the study early.

Unable to use -

Costs: direct costs of psychiatric hospital care (no SD).

\begin{tabular}{ll}
\hline Notes & ${ }^{*} \mathrm{~N}=114,81.5 \%$ schizophrenia or schizoaffective disorder, $50.8 \%$ secondary diagnosis of substance \\
& abuse disorder. \\
& ${ }^{* \star}$ PACT provided by the 2 sites did not differ. \\
& ${ }^{* \star *}$ Variance not reported - data from another study used. \\
\hline
\end{tabular}

\section{Risk of bias}

\begin{tabular}{|c|c|c|}
\hline Bias & Authors' judgement & Support for judgement \\
\hline $\begin{array}{l}\text { Random sequence genera- } \\
\text { tion (selection bias) }\end{array}$ & Unclear risk & Randomised. No further details. \\
\hline $\begin{array}{l}\text { Allocation concealment } \\
\text { (selection bias) }\end{array}$ & Unclear risk & No details \\
\hline $\begin{array}{l}\text { Blinding (performance } \\
\text { bias and detection bias) } \\
\text { All outcomes }\end{array}$ & Unclear risk & $\begin{array}{l}\text { Primary outcome: clinician/participant mediated - rating - Unclear. } \\
\text { Secondary outcomes: clinician/participant mediated - rating - Unclear. }\end{array}$ \\
\hline $\begin{array}{l}\text { Blinding of participants } \\
\text { and personnel (perfor- } \\
\text { mance bias) } \\
\text { All outcomes }\end{array}$ & Unclear risk & No details \\
\hline $\begin{array}{l}\text { Blinding of outcome as- } \\
\text { sessment (detection bias) } \\
\text { All outcomes }\end{array}$ & Unclear risk & No details \\
\hline
\end{tabular}


Salkever-SCarolina 1999 (Continued)
Incomplete outcome data Unclear risk
Insufficient reporting of missing data (number for missing data is provided, (attrition bias) but reason is provided for the whole sample, is not stated for single interven- All outcomes tion group).

\begin{tabular}{lll}
\hline $\begin{array}{l}\text { Selective reporting (re- } \\
\text { porting bias) }\end{array}$ & High risk & $\begin{array}{l}\text { Listed outcomes of interest are fully reported, but any variance measurements } \\
\text { are missing. }\end{array}$ \\
\hline Other bias & Low risk & $\begin{array}{l}\text { Publicly funded (by NIMH and grant from universities and university centres } \\
\text { for research). No further details. No evidence of the presence of other bias. }\end{array}$ \\
\hline
\end{tabular}

\section{Shern-USA1 2000}

$\begin{array}{ll}\text { Methods } & \text { Allocation: randomised. } \\ & \text { Design: single centre. } \\ & \text { Duration: } 24 \text { months. } \\ \text { Country: New York, USA. }\end{array}$
Participants Diagnosis*: severe mental illness causing severe disability (definition not clearly reported). $\mathrm{N}=168$. Setting: community services.
Age: $\geqq 18$ years, mean 40 years, range 21 to 66 years.
Sex: $76 \%$ M (128M, 40F).
Ethnicity: $61 \%$ black (understood to be African-American).
History: i. homeless for 7 of last 14 nights, ii. no primary diagnosis of substance abuse or learning dis- ability, iii. not considered dangerous to self or others, iv. informed consent given.

I. ICM: from the CHOICE programme, which aims to develop "housing readiness" (i.e. compliance with
psychiatric treatment and period of sobriety). The main features are: i. drop-in centre, ii. Case Manage-
ment intervention modelled according to main principles of the ACT model. Caseload: $\sim 1: 13 . \mathrm{N}=91$.
2. Standard care ${ }^{\star \star}:$ homelessness and specialty mental health services provided from a variety of
agencies (including drop-in centres, outreach services, mental health and health services, soup
kitchen, shelters). It could involve non-ICM. $\mathrm{N}=77$.

Social functioning: not living in stable accomodation.
Mental state: Colorado Symptom Index.
Quality of Life: Lehman's Quality of life Index (QOLI).
Unable to use -
Service use: hospital use (data partially reported).
Social functioning: police contact - imprisoned, arrested (data partially reported), change in propor-
tion of time spent in residential setting (incompletely reported).
Self esteem: Rosenberg's Self Esteem Scale (not peer reviewed).
Coping: Pearlin and Schooler's Mastery Scale (not listed as a review outcome of interest).
Participant satisfaction: unmet needs (measurement instrument not described).
Notes $\quad * \sim 9 \%$ no major mental illness.
${ }^{\star *}$ People randomised to standard care were just "provided informations by the research interviewers about local homelessness service programmes". No attempts were made to arrange the first contact to available local services.

\section{Risk of bias}

\begin{tabular}{lll}
\hline Bias & Authors' judgement & Support for judgement \\
\hline $\begin{array}{l}\text { Random sequence genera- } \\
\text { tion (selection bias) }\end{array}$ & Unclear risk & Randomised. No further details. \\
\hline
\end{tabular}


Shern-USA1 2000 (Continued)

\begin{tabular}{lll}
$\begin{array}{l}\text { Allocation concealment } \\
\text { (selection bias) }\end{array}$ & Unclear risk & No details \\
\hline $\begin{array}{l}\text { Blinding (performance } \\
\text { bias and detection bias) }\end{array}$ & High risk & $\begin{array}{l}\text { Primary outcome: not provided. } \\
\text { Secondary outcomes: interviewer mediated - rating - No. Not clearly stated, } \\
\text { but it is implicitly not blind. }\end{array}$ \\
\hline
\end{tabular}

Blinding of participants High risk Problematic to blind participants and those providing the intervention in stud-
and personnel (perfor- $\quad$ ies comparing ICM intervention with standard care.

mance bias)

All outcomes

\begin{tabular}{lll}
\hline $\begin{array}{l}\text { Blinding of outcome as- } \\
\text { sessment (detection bias) } \\
\text { All outcomes }\end{array}$ & High risk & $\begin{array}{l}\text { Service use and housing status were assessed in face-to face interviews, proto- } \\
\text { cols were used. Interviewers not blinded. }\end{array}$ \\
\hline $\begin{array}{l}\text { Incomplete outcome data } \\
\text { (attrition bias) }\end{array}$ & Unclear risk & $\begin{array}{l}\text { Authors declared "using alternative techniques for accommodate missing ob- } \\
\text { servations". Our main concern was regarding the high attrition rate the au- } \\
\text { thors declared, but was not clearly reported as presented data were already } \\
\text { transformed through statistician techniques accounting for missing observa- } \\
\text { tion. }\end{array}$
\end{tabular}

\begin{tabular}{lll}
\hline $\begin{array}{l}\text { Selective reporting (re- } \\
\text { porting bias) }\end{array}$ & High risk & $\begin{array}{l}\text { Some listed outcomes of interest are not usable due to incomplete reporting } \\
\text { (service use, social functioning, quality of life outcomes). }\end{array}$ \\
\hline Other bias & Low risk & $\begin{array}{l}\text { Publicly funded (NIMH). No further details. No evidence of the presence of oth- } \\
\text { er bias. }\end{array}$ \\
\hline
\end{tabular}

Solomon-Pennsylvania 1994

\begin{tabular}{|c|c|}
\hline Methods & $\begin{array}{l}\text { Allocation: randomised. } \\
\text { Design: single centre. } \\
\text { Duration: } 12 \text { months. } \\
\text { Country: Philadelphia, Pennsylvania, USA. } \\
\text { Not entering meta-regression. }\end{array}$ \\
\hline Participants & $\begin{array}{l}\text { Diagnosis*: seriously mentally ill (schizophrenia, affective or personality disorder according to DSM-III- } \\
\text { R). } \\
N=200 \text {. } \\
\text { Setting: CMHC. } \\
\text { Age: mean } 35.2 \text { years (SD 9.4). } \\
\text { Sex: } 86.5 \% \text { M. } \\
\text { Ethnicity: Afro-American } 81 \% \\
\text { History: i. about to be released from prison, ii. homeless (i.e. situational, episodically, or chronically } \\
\text { without a domicile or if jail detention resulted in the loss of a stable housing situation), iii. state hospi- } \\
\text { talisation } \geqq 60 \text { days in previous } 2 \text { years or a significant amount of outpatient treatment, iv. GAF } \leqq 40 \text { or } \\
\text { GAF } \leqq 60 \text { if age } \leqq 35 \text { years, v. written informed consent. }\end{array}$ \\
\hline Interventions & $\begin{array}{l}1^{\star \star} \text {. ICM: Assertive Community Treatment according to the Stein and Test model. Caseload: } 1: 8-12 . \mathrm{N}= \\
60 . \\
2^{\star \star} \text {. ICM: Intensive Case Management provided from an individual forensic case manager who worked } \\
\text { at CMHC, but as individual rather than as part of a treatment team. Caseload: not available. } \mathrm{N}=60 . \\
\text { 3. Standard care: from local CMHC ( } 2 \text { clients received ICM services at times during the year in the } \\
\text { study). } \mathrm{N}=80 \text {. }\end{array}$ \\
\hline
\end{tabular}


Solomon-Pennsylvania 1994 (Continued)

Social functioning: imprisonment ${ }^{\star \star \star}$.

Unable to use -

Service use: days in hospital, hospitalisation (data not available); drug and alcohol use Addiction

Severity Index (data not available); accomodation, employment, arrest (data not available), Pattison

Psychosocial Network Inventory (data not available).

Mental state: BPRS (data not available).

Quality of life: Lehman's Quality of Life Interview (objective and subjective components).

$\begin{array}{ll}\text { Notes } & \text { * Schizophrenia } 82.5 \% \text {, major affective disorder } 10 \% \text {, other (unspecified psychotic disorder and person- } \\ & \text { ality disorder) } 3 \%, \text { substance abuse disorder } 52 \% . \mathrm{N}=200 . \\ & { }^{* *} \text { We considered both arms as a single intervention. } \\ & { }^{* *} \text { Attrition in the control group }>50 \% .\end{array}$

\section{Risk of bias}

\begin{tabular}{lll} 
Bias & Authors' judgement & Support for judgement \\
\hline $\begin{array}{l}\text { Random sequence genera- } \\
\text { tion (selection bias) }\end{array}$ & Unclear risk & $\begin{array}{l}\text { Randomised. Authors reported that "slightly higher number of clients were } \\
\text { assigned to the control treatment", but it is not clear if it was a stratified ran- } \\
\text { domisation. No further details provided. }\end{array}$ \\
\hline
\end{tabular}

\begin{tabular}{ll}
\hline $\begin{array}{l}\text { Allocation concealment } \\
\text { (selection bias) }\end{array}$ & Unclear risk
\end{tabular}

\begin{tabular}{|c|c|c|}
\hline $\begin{array}{l}\text { Blinding (performance } \\
\text { bias and detection bias) }\end{array}$ & Unclear risk & $\begin{array}{l}\text { Primary outcome: not available. } \\
\text { Secondary outcome: clinician/participant mediated - rating - Unclear. }\end{array}$ \\
\hline
\end{tabular}

All outcomes

Blinding of participants $\quad$ High risk Participants and personnel not blinded.
and personnel (perfor-
mance bias)
All outcomes

\begin{tabular}{lll}
\hline $\begin{array}{l}\text { Blinding of outcome as- } \\
\text { sessment (detection bias) } \\
\text { All outcomes }\end{array}$ & Unclear risk & Blinding not reported. \\
\hline $\begin{array}{l}\text { Incomplete outcome data } \\
\begin{array}{l}\text { (attrition bias) } \\
\text { All outcomes }\end{array}\end{array}$ & Unclear risk & $\begin{array}{l}\text { Incomplete reporting of missing data (number of missing data is reported, but } \\
\text { reasons not provided). }\end{array}$ \\
\hline $\begin{array}{l}\text { Selective reporting (re- } \\
\text { porting bias) }\end{array}$ & High risk & $\begin{array}{l}\text { Some listed outcomes of interest are not reported (i.e. service use, quality of } \\
\text { life, etc.). }\end{array}$ \\
\hline Other bias & Low risk & No evidence of the presence of other bias. \\
\hline
\end{tabular}

Sytema-Netherlands 1999

\begin{tabular}{ll}
\hline Methods & Allocation: randomised. \\
& Design: single centre. \\
& Duration*: follow-up variable (minimum 12 months, maximum 24 months). \\
& Country: Netherlands. \\
\hline Participants & Diagnosis ${ }^{\star \star}:$ severe mental illness (diagnostic criteria not reported). \\
& $\mathrm{N}=118^{\star \star *}$. \\
& Setting: rural catchment area, community mental health service. \\
\hline
\end{tabular}


Sytema-Netherlands 1999 (Continued)

Age: mean 41.5 yrs (SD 11 years).

Sex: $68.6 \% \mathrm{M}$.

Ethnicity: not reported.

History: Health of the Nation Outcome Scale (HoNOS) $\geqq 15$.

$\begin{array}{ll}\text { Interventions } & \text { 1. ICM: ACT model according to a fidelity scale (DACTS). Caseload: } 1: 10 . \mathrm{N}=59 . \\ \text { 2. Standard care: provided by CMHT. Caseload: } 1: 40 . \mathrm{N}=59 .\end{array}$

Outcomes Service use: average number of days in hospital per month, not remaining in contact with psychiatric services (defined by authors as "not having any registered contact with the mental health services during the last 12 months of observation"), average number of admissions per month.

Death: all causes, suicide.

Social functioning: homeless at the end of the trial, average days per month in sheltered houses, Dartmouth Assessment of Lifestyle Interview (DALI).

Mental state: BPRS-24 item.

Participant satisfaction: Client Satisfaction Questionnaire (CSQ), Camberwell Assessment of Needs Short Appraisal Schedule (CANSAS).

Unable to use -

Social functioning: Social Functioning Scale (SFS) (data not clearly reported).

Notes *Participant inclusion in the study started April 2004 and was closed at 1 June 2005. All participants were followed up until August 2006.

${ }^{\star \star}$ Schizophrenia 51.7\%, major depression $13.5 \%$, bipolar 3\%.

$\star \star \star T h e ~ n u m b e r$ of participants randomised was 119 , but 1 participant was excluded from sample because he or she moved to another area directly after randomisation.

\section{Risk of bias}

\begin{tabular}{lll}
\hline Bias & Authors' judgement & Support for judgement \\
\hline $\begin{array}{l}\text { Random sequence genera- } \\
\text { tion (selection bias) }\end{array}$ & Low risk & Randomised, based on block design (block size of 5). \\
\hline $\begin{array}{l}\text { Allocation concealment } \\
\text { (selection bias) }\end{array}$ & Low risk & $\begin{array}{l}\text { Participant included in the study received ID number from service administra- } \\
\text { tion. The new ID number was emailed to a researcher who had a pre-arranged } \\
\text { list of ID numbers randomised. }\end{array}$ \\
\hline
\end{tabular}

\begin{tabular}{|c|c|c|}
\hline $\begin{array}{l}\text { Blinding (performance } \\
\text { bias and detection bias) }\end{array}$ & Unclear risk & $\begin{array}{l}\text { Primary outcome: clinician/participant mediated - rating - Unclear. } \\
\text { Secondary outcomes: interviewer rated - rating - No. Open-label. }\end{array}$ \\
\hline
\end{tabular}

All outcomes

\begin{tabular}{|c|c|c|}
\hline $\begin{array}{l}\text { Blinding of participants } \\
\text { and personnel (perfor- }\end{array}$ & High risk & $\begin{array}{l}\text { Problematic to blind participants and those providing the intervention in stud- } \\
\text { ies comparing ICM intervention with standard care. }\end{array}$ \\
\hline
\end{tabular}

mance bias)

All outcomes

\begin{tabular}{lll}
\hline $\begin{array}{l}\text { Blinding of outcome as- } \\
\text { sessment (detection bias) } \\
\text { All outcomes }\end{array}$ & Unclear risk & Blinding not reported. \\
\hline $\begin{array}{l}\text { Incomplete outcome data } \\
\text { (attrition bias) }\end{array}$ & Low risk & $\begin{array}{l}\text { YES - Primary outcomes: service use data. No missing data (but } 1 \text { participant } \\
\text { excluded from sample because he or she moved to another area directly after } \\
\text { randomisation). }\end{array}$ \\
& $\begin{array}{l}\text { UNCLEAR - Secondary outcomes: number of missing data is provided, but rea- } \\
\text { sons not stated. }\end{array}$
\end{tabular}


Sytema-Netherlands 1999 (Continued)
Selective reporting (re-
Low risk
All listed outcomes of interest are completely reported. porting bias)

Other bias Low risk

Publicly funded (grant from The Netherlands Organisation for Health Research and Development). No further details. No evidence of the presence of other bias.

\section{Test-Wisconsin 1985}

\begin{tabular}{ll}
\hline Methods & Allocation: randomised. \\
& Design: single centre. \\
& Duration: total duration of the trial 12 years, participants were followed at least 5 years, extended to 12 \\
years for those who first entered the study. Some data available at 4 years, some at 24 months. \\
Country: Dane County, Wisconsin, USA.
\end{tabular}

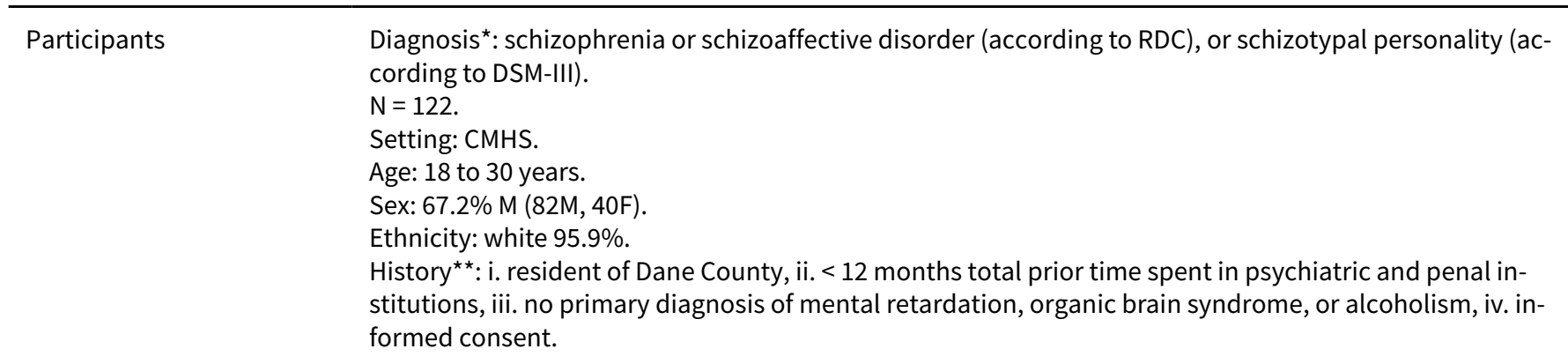

I. ICM: Assertive Community Treatment according to Stein and Test model. Caseload: $\sim 1: 9 . \mathrm{N}=75$.
2. Standard care: routine care from Dane County psychiatric services - included an unspecified degree
of case management. $\mathrm{N}=47$.

Outcomes Service use: average number of days in hospital per month, not remaining in contact with psychiatric services, admitted to hospital.

Death: all causes, suicide.

Global state: leaving the study early.

Social functioning: number homeless or living in sheltered accomodation (at least 1 day), not living independently, imprisoned.

Unable to use -

Mental state: BPRS, BSI (no data).

Social functioning: days homeless (no SD), days in jail (no SD), Community Adjustment Form (scale not peer reviewed, no data).

Quality of life: Satisfaction with Life Scale (scale not peer reviewed, no data).

$\begin{array}{ll}\text { Notes } & { }^{*} \text { Schizophrenia } 73.8 \% \text {; schizoaffective disorder } \sim 23 \% . \\ & { }^{* \star} \text { Average age first contact with mental health system: } 19.02 \text { yrs. }\end{array}$

\section{Risk of bias}

Bias Authors' judgement Support for judgement

Random sequence genera- Unclear risk Randomised (randomised assignment to experimental and control intervention (selection bias) tion on a ratio 6:4), no further details.

Allocation concealment $\quad$ Unclear risk No details
(selection bias)


Test-Wisconsin 1985 (Continued)
Blinding (performance Unclear risk
Primary outcome: clinician/participant mediated - rating - Unclear. bias and detection bias) Secondary outcomes: most are clinician/participant mediated - rating - Un- All outcomes clear.

\begin{tabular}{|c|c|c|}
\hline $\begin{array}{l}\text { Blinding of participants } \\
\text { and personnel (perfor- }\end{array}$ & High risk & $\begin{array}{l}\text { Problematic to blind participants and those providing the intervention in stud- } \\
\text { ies comparing ICM intervention with standard care. }\end{array}$ \\
\hline
\end{tabular}
mance bias)

All outcomes

\begin{tabular}{lll}
\hline $\begin{array}{l}\text { Blinding of outcome as- } \\
\text { sessment (detection bias) } \\
\text { All outcomes }\end{array}$ & Unclear risk & Blinding not reported. \\
\hline $\begin{array}{l}\text { Incomplete outcome data } \\
\text { (attrition bias) } \\
\text { All outcomes }\end{array}$ & Unclear risk & Number and reasons for missing data are not clearly reported. \\
\hline $\begin{array}{l}\text { Selective reporting (re- } \\
\text { porting bias) }\end{array}$ & High risk & Listed outcomes of interest not reported or reported incompletely, BPRS and \\
\hline Other bias & LSI (not reported), days in jail and days in hospital (no SD). \\
\hline
\end{tabular}

\section{UK700-UK (A)}

Methods Centre: St. George's - London.

Methods: see above UK700-UK 1999.

Participants $\quad$ Participants: see above UK700-UK 1999.
N $=196$.

Interventions Methods: see above UK700-UK 1999. Sample size providing data:

1. ICM: $\mathrm{N}=96$.

2. Non-ICM: $\mathrm{N}=99$.

Outcomes Service use: average number of days in hospital per month.

\section{Notes}

\section{UK700-UK (B)}

\begin{tabular}{ll} 
Methods & Centre: King's - London. \\
& Methods: see above UK700-UK 1999. \\
\hline Participants & Participants: see above UK700-UK 1999. \\
& N $=153$. \\
\hline Interventions & Methods: see above UK700-UK 1999. Sample size providing data: \\
& 1. ICM: N $=77$.
\end{tabular}


UK700-UK (B) (Continued)

2. Non-ICM: $\mathrm{N}=74$.

Outcomes Service use: average number of days in hospital per month.

Notes

UK700-UK (C)

\begin{tabular}{ll}
\hline Methods & Centre: Manchester. \\
& Methods: see above UK700-UK 1999. \\
\hline Participants & Participants: see above UK700-UK 1999. \\
& $N=158$.
\end{tabular}

$\mathrm{N}=158$.

Interventions Methods: see above UK700-UK 1999.

1. ICM: $\mathrm{N}=79$.

2. Non-ICM: $\mathrm{N}=79$.

Outcomes Service use: average number of days in hospital per month.

\section{Notes}

UK700-UK (D)

$\begin{array}{ll}\text { Methods } & \text { Centre: St. Mary's* - London. } \\ \text { Methods: see above UK700-UK } 1999 .\end{array}$

Participants $\quad$ Participants: see above UK700-UK 1999.
$\mathrm{~N}=201$.

$\mathrm{N}=201$.

Methods: see above UK700-UK 1999. Sample size providing data:
1. ICM: $N=99$.
2. Non-ICM: $N=101$.

\begin{tabular}{ll}
\hline Outcomes & Service use: average number of days in hospital per month. \\
\hline Notes & *In St. Mary's centre staff were randomly assigned to ICM or non-ICM position. \\
\hline
\end{tabular}

\section{UK700-UK 1999}

\begin{tabular}{ll}
\hline Methods & Allocation: randomised. \\
& Design: multicentre (4 sites, 3 in London and 1 in Manchester). \\
Duration: 24 months. & Country: UK.
\end{tabular}

$\begin{array}{ll}\text { Participants } & \text { Diagnosis*: psychotic illness (diagnosed through OPCRIT). } \\ & N=708 .\end{array}$


UK700-UK 1999 (Continued)

Setting: inner-city mental health services.

Age: 18 to 65 years, mean 37.3 years.

Sex: 57\% M (404M, 304F).

Ethnicity: Afro-Caribbean $28 \%$.

History: i. psychotic illness for at least 2 years, ii. $\geqq 2$ previous hospital admissions, 1 within past 2

years, iii. no organic brain disease or primary diagnosis of substance misuse, iv. written informed consent.

$\begin{array}{ll}\text { Interventions } & \text { 1CM**: Caseload: } 1: 10-15 . \mathrm{N}=353 . \\ \text { 2. Non-ICM: Caseload: } 1: 30-35 . \mathrm{N}=355 .\end{array}$

Outcomes

Service use: average number of days in hospital per month (available for single centre, see below), not remaining in contact with psychiatric services (reported as not remaining in contact with case manager), admitted to hospital, mean number of admissions.

Death: all causes and suicide.

Global state: leaving the study early.

Social functioning: time in independent living accomodation (reported as time spent in stable accomodation), imprisoned, Camberwell Assessment of Need (CAN).

Mental state: general symptoms CPRS, negative symptoms (SANS).

Behaviour: self harm, harm to others.

Quality of life: Lancashire Quality of Life Profile (LQoLP).

Participant satisfaction: Patients' satisfaction with health services questionnaire.

Costs: direct costs of all care.

Unable to use -

Social functioning: DAS (scale adapted by the trialist, not peer reviewed).

Mental state: subscale of CPRS for depression (Montgomery-Åsberg Depression Rating Scale) and for psychotic symptoms (no SD), for anxiety and for behavioural disturbance (no SD, not peer-reviewed scale).

Carer satisfaction: Experience of Caregiving Inventory, 12-Item General Health Questionnaire (attrition 83.6\%).

Costs: direct costs of health care and of psychiatric hospital care (no SD).

Adverse drug effects: Abnormal Involuntary Movement Scale (not listed as outcome of interest of the review).

Notes $\quad$ *Schizophrenia $38 \%$, schizoaffective $49 \%$, mania or bipolar $5 \%$.

**The team organisation varied slightly across centres.

\section{Risk of bias}

\begin{tabular}{lll}
\hline Bias & Authors' judgement & Support for judgement \\
\hline $\begin{array}{l}\text { Random sequence genera- } \\
\text { tion (selection bias) }\end{array}$ & Unclear risk & Randomised, randomisation stratified by centre. No further details. \\
\hline $\begin{array}{l}\text { Allocation concealment } \\
\text { (selection bias) }\end{array}$ & Low risk & $\begin{array}{l}\text { Randomised allocation assigned by telephone or fax by an independent statis- } \\
\text { tical centre. }\end{array}$ \\
\hline $\begin{array}{l}\text { Blinding (performance } \\
\text { bias and detection bias) } \\
\text { All outcomes }\end{array}$ & Unclear risk & $\begin{array}{l}\text { Primary outcome: clinician/participant mediated - rating - Unclear. } \\
\text { Secondary outcomes: interviewer rated - rating - No. (Researchers were not } \\
\text { masked to treatment allocation.) }\end{array}$ \\
\hline $\begin{array}{l}\text { Blinding of participants } \\
\text { and personnel (perfor- } \\
\text { mance bias) } \\
\text { All outcomes }\end{array}$ & Unclear risk & Blinding not reported. \\
\hline
\end{tabular}

$\begin{array}{lll}\text { Blinding of outcome as- } & \text { Unclear risk } \quad \text { Blinding not reported. }\end{array}$


UK700-UK 1999 (Continued)

All outcomes

Incomplete outcome data Low risk Number and reasons for attrition stated. Analysis performed on an ITT basis.
(attrition bias)

(attrition bias)

All outcomes

\begin{tabular}{lll}
\hline $\begin{array}{l}\text { Selective reporting (re- } \\
\text { porting bias) }\end{array}$ & Unclear risk & $\begin{array}{l}\text { Most of the listed outcomes are reported completely (the only exception is for } \\
\text { CPRS subscale: SD missing). }\end{array}$ \\
\hline Other bias & Low risk & $\begin{array}{l}\text { Publicly funded (grants from the UK Department of Health and NHS research } \\
\text { and development programme). No further details. No evidence of the pres- } \\
\text { ence of other bias. }\end{array}$ \\
\hline
\end{tabular}

ACT - Assertive Community Treatment

$\mathrm{CM}$ - Case Management

CMHC - Community Mental Health Center

CMHT - Community Mental Health Team

CPA - Care Programme Approach: the CPA is a combination of non-Intensive Case Management and care from a CMHT, introduced in England in the mid-1990s and becoming standard care thereafter

CPNS - Community Psychiatric Nursing Service

DSM-III-R - Diagnostic and Statistical Manual of Mental Disorders, 3rd Edition, Revised (APA 1987)

DSM IV - Diagnostic and Statistical Manual of Mental Disorders, 4th Edition (APA 1994)

F - female

ICD-10 - International Statistical Classification of Diseases and Related Health Problems 10th Revision (WHO 1992)

ICM - Intensive Case Management

ITT - intention to treat

LOCF - last observation carried forward

$M$ - male

$\mathrm{N}$ - number

NIMH - National Institute of Mental Health (USA)

non-ICM - non-Intensive Case Management

OPCRIT - Operational Criteria (McGuffin 1991)

OPD - outpatient department

PACT - Programme of Assertive Community Treatment

$\mathrm{P} / \mathrm{T}$ - part time

RDC - Research Diagnostic Criteria (Spitzer 1978)

SADS - Schedule for Affective Disorders and Schizophrenia interview (Endicott 1978)

SC - standard care

SCAN 2.0 - 2.1: Schedule for Clinical Assessment in Neuropsychiatry (SCAN 2.0 in 1998, SCAN 2.1 since 1999) (WHO 1998)

SCID - Structured Clinical Interview for DSM-IV (First 1997)

SD - standard deviation

SMI - severe mental illness

TCL - Training in Community Living

VA - Veterans administration

Yrs - years

Scales

$\mathrm{ACL}$ - Adjective Checklists

ASI - Addiction Severity Index

AUS - Alcohol Use Scale

BDI - Beck Depression Inventory

BPRS - Brief Psychiatric Rating Scale

BSI - Brief Symptom Inventory

CAN - Camberwell Assessment of Need Interview

CANSAS - Camberwell Assessment of Need Short Appraisal Schedule

CPRS - Comprehensive Psychopathological Rating Scale

C-DIS-R - Computer-based Diagnostic Interview Schedule Revised

CSI - Colorado Symptom Index

CSQ - Client Satisfaction Questionnaire

CSRI - Client Service Receipt Inventory 
DALI - Dartmouth Assessment of Lifestyle Interview

DACTS - Dartmouth Assertive Community Treatment Scale

DAS - Disability Assessment Scale

DUS - Drug Use Scale

GAF - Global Assessment of Functioning Scale

GAS - Global Assessment Scale

GSI - Global Severity Index

HADS - Hospital Anxiety and Depression Scale

HEAS - Homeless Engagement Acceptance Scale

HoNOS - Health of the Nation Outcome Scale

IMPS - Inpatient Multidimensional Psychiatric Scale

Indiana LOF - Indiana Level of Functioning

ISSI: Interview Schedule for Social Interaction

KS - Krawiecka Scale

LQoLP - Lancashire Quality of Life Profile

LSP - Life Skill Profile

MANSA - Manchester Short Assessment of Quality of Life

PANSS - Positive and Negative Syndrome Scale

PSE - Present State Examination

PSNAS - Personality and Social Network Adjustment Scale

QOLI - Lehman's Quality of Life Interview

REHAB - a scale of social functioning (REHAB GB: general behaviour); (REHAB DB: deviant behaviour)

ROMI - Rating of Medication Influences

RSES - Rosenberg Self Esteem Scale

SAI - Scale for the Assessment of Insight

SAI-E - Scale for the Assessment of Insight-expanded

SANS - Scale for the Assessment of Negative Symptoms

SAPS - Scale for the Assessment of Positive Symptoms

SAS - Social Adjustment Scale

SATS - Substance Abuse Treatment Scale

SBS - Social Behaviour Scale

SCL-90: Hopkins Symptoms Check List-90

SCRS - Short Clinical Rating Scale

SFQ - Social Functioning Questionnaire

SLS - Satisfaction with Life Scale

TLFB - Timeline Followback

W-BQ - Well-being Questionnaire

Characteristics of excluded studies [ordered by study ID]

\begin{tabular}{ll}
\hline Study & Reason for exclusion \\
\hline Agius-Croatia 2007 & Allocation: not randomised (cohort study). \\
\hline An 2013 & Allocation: randomised. \\
& Participants: schizophrenia. \\
& Interventions: 1 . Community-based follow-up interventions. \\
& 2. Care as usual. \\
& Not ICM. \\
\hline Allocation: randomised. \\
Participants: primary schizophrenia. \\
Interventions: 1. Family intervention. \\
2. Care as usual.
\end{tabular}




\begin{tabular}{|c|c|}
\hline Study & Reason for exclusion \\
\hline & Not ICM. \\
\hline \multirow[t]{2}{*}{ Bao 2012} & Allocation: unclear. \\
\hline & Not RCT. \\
\hline \multirow[t]{4}{*}{ Bao-China 2005} & Allocation: randomised. \\
\hline & Participants: people with schizophrenia. \\
\hline & Intervention: 1. Family intervention, community based. Not ICM. \\
\hline & 2. Standard care. \\
\hline \multirow[t]{3}{*}{ Barbic 2009} & Allocation: randomised. \\
\hline & $\begin{array}{l}\text { Participants: people who met DSM-IV diagnostic criteria for schizophrenia, schizoaffective disorder, } \\
\text { schizophreniform disorder, delusional disorder, or bipolar disorder. }\end{array}$ \\
\hline & Intervention: No relevant comparison: ACT vs ACT + Recovery Workbook. \\
\hline
\end{tabular}

Allocation: randomised.

Participants: age 18 to 65 years. DSM-IV diagnosis of bipolar I disorder (mania or mixed), schizophrenia, or schizoaffective disorder.

Intervention:

1. Psychoeducation program for families, explicity operationalised, provided within a package of care close to the ICM model (caseload 1:12).

2. Standard care: routine psychiatric treatments. Caseload not reported. $\mathrm{N}=62$.

\begin{tabular}{|c|c|}
\hline Bigelow-Oregon 1991 & Allocation: not randomised, quasi-experimental design. \\
\hline Bond-Chicago2 1989 & $\begin{array}{l}\text { Allocation: not randomised, matched-groups design. } \\
\text { Interventions: } 2 \text { types of crisis housing. }\end{array}$ \\
\hline Bond-Indiana2 1991 & $\begin{array}{l}\text { Allocation: not randomised, allocation to ACT and reference group was not random in } 1 \text { of the } 3 \\
\text { participating centres. The study could be included if separate data can be obtained from the } 2 \text { cen- } \\
\text { tres where randomisation took place. }\end{array}$ \\
\hline Borland-Washington 1989 & Allocation: not randomised. \\
\hline \multirow[t]{3}{*}{ Borras 2009} & Allocation: randomised. \\
\hline & $\begin{array}{l}\text { Participants: all meeting ICD-10 criteria for a diagnosis of schizophrenia or other non- affective psy- } \\
\text { choses. }\end{array}$ \\
\hline & $\begin{array}{l}\text { Interventions: no relevant comparison: traditional psychiatric outpatient care or case management } \\
\text { vs traditional psychiatric outpatient care or case management + self esteem module. }\end{array}$ \\
\hline \multirow[t]{5}{*}{ Botha 2014} & Allocation: randomised. \\
\hline & Participants: adults with severe mental illness. \\
\hline & Intervention: 1 . Modified assertive intervention. \\
\hline & 2. Standard care. \\
\hline & Caseload > 20 \\
\hline
\end{tabular}




\section{Study Reason for exclusion}

Participants: adult patients diagnosed with psychosis.

Interventions: 1. Community treatment orders (CTOS): program for patient who needs supervision after a period of involuntary hospital treatment and who, without it, is highly likely to relapse and be readmitted involuntarily. Not ICM.

2. Section 17 leave: a well-established rehabilitation practice, used for brief periods to assess the stability of a patient's recovery after or during a period of involuntary hospital treatment.

Burns-UK $1993 \quad$ Allocation: randomised.

Participants: people who were (a) aged between 18 and 74 years, (b) from the appropriate catchment area of Psychiatric Service, (c) not in treatment during the preceding 12 months, (d) able to be interviewed in English.

Intervention: multidisciplinary team home treatment, not ICM.

\section{Champney-Ohio $1992 \quad$ Allocation: randomised.}

Participants: adults with severe mental disabilities.

Intervention: all 4 comparison groups received some form of case management, no ACT. Neither standard care nor non-ICM in the comparison group.

Chandler 1999

Allocation: randomised.

Participants: volunteers randomly assigned to ACT.

Not exclusively community setting.

\section{Chandler 2000}

Allocation: randomised.

Participants: not clear, described as "high cost inpatient users".

Intervention: no relevant comparison: $\mathrm{ACT}+$ social skills training vs ACT.

\section{Chandler-California2 2006}

\section{Allocation: randomised.}

Participants: current serious mental illness and substance use disorder.

Intervention: 1. In-custody standard care + brief aftercare + Integrated Dual Disorders Treatment. Postcustody treatment includes ICM and specific intervention addressing substance abuse disorder. Not exclusively community setting; not only ICM.

2. Service as usual: in-custody standard care + usual postcustody services +60 days of postrelease case management and housing assistance. Not exclusively community setting.

Allocation: randomised.

Participants: 18 to 35 years with a diagnosis of schizophrenia, non-affective psychosis, affective disorders with psychotic features, or delusional disorder.

Intervention: 1. Community-based case management $(n=79)$.

2. Standard care: general psychiatric care with all auxiliary care options unchanged $(n=77)$.

All participants had been enrolled in the Early Assessment Service for Young People with Early Psychosis (EASY) for 2 years before starting this trial.

Not ICM, caseload 1:80. 


\section{Study Reason for exclusion}

Intervention: 1. Collaborative community-based care plus facility-based care.

2. Facility-based care alone. Not ICM.

Chen XZ 2012
Participants: schizophrenia.
Interventions:
1. Home visit.
2. Education.
Not ICM.

Chen-China $2007 \quad$ Allocation: randomised.

Participants: adults with schizophrenia (non-acute).

Intervention:

1. Antipsychotic + Digital Network + Community Support Network.

2. Antipsychotic + Community Support Network.

Digital Network involves providing online consultation to patients, emailing them periodically, providing online health education. Community Support Network involves providing physical, psychological, and rehabilitation treatment to non-acute patients. Not ICM.

COAST-UK $2004 \quad$ Allocation: randomised.

Participants: those with any functional psychosis.

Interventions: 1. ICM: Assertive Outreach Team model; caseload: 1:12. Also includes individual therapy for residual positive symptoms of psychosis (CBT) and family intervention, if appropriate. Not only ICM.

2. Non-ICM: usual services available to a local multidisciplinary team (according to Care Programme Approach); caseload: 1:35. Does not include any specialised psychological interventions.

\begin{tabular}{|c|c|}
\hline COMO-UK 2003 & $\begin{array}{l}\text { Allocation: cluster randomisation. } \\
\text { Participants: case manager providing care to patients with psychosis and comorbid substance } \\
\text { misuse ("dual diagnosis"). } \\
\text { Interventions: } 1 \text {. Training in dual-diagnosis intervention (i.e. i. treatment manual; ii. 5-day train- } \\
\text { ing course in assessment and management of dual diagnosis; iii. subsequent monthly supervision). } \\
\text { Not ICM. } \\
\text { 2. CMHT management as usual. }\end{array}$ \\
\hline
\end{tabular}
Allocation: randomised.

Participants: patients with serious mental illness as defined by federal Public Law 102-321 specifying diagnosis, duration, and level of disability (schizophrenia, schizoaffective, bipolar, depressive, other); age $\geq 18$ years.

Interventions: 1. Wellness Recovery Action Planning: an evidence-based practice that consisted of 9, 2.5-hour group sessions that were facilitated free of charge by 2 trained and certified instructors in recovery from mental illness, with backup instructors available as needed. Not ICM.

2. Choosing Wellness: Healthy Eating Curriculum: a nutrition education intervention holistically focused on wellness.

Participants: patients diagnosed with bipolar disorder, anxiety disorder, major depressive disorder, schizophrenia, personality disorder, panic disorder, age 16 to 64 years. 


\begin{tabular}{|c|c|}
\hline \multirow[t]{3}{*}{ Study } & Reason for exclusion \\
\hline & Intervention: 1. Co-design technique to optimise psychosocial recovery. Not ICM. \\
\hline & 2. Waiting list. \\
\hline \multirow[t]{5}{*}{ Cosden-California 2005} & Allocation: randomised. \\
\hline & Participants: offenders with serious mental illness. \\
\hline & Interventions: 1. ICM + non-adversarial court proceeding. \\
\hline & 2. Non-ICM + adversarial court proceeding. \\
\hline & 2 arms are provided with different intervention between groups in addition to ICM and non-ICM. \\
\hline \multirow[t]{3}{*}{ CRIMSON-UK 2008} & Allocation: randomised. \\
\hline & $\begin{array}{l}\text { Participants: diagnosis of psychotic illness; } \geqq 1 \text { admission to a psychiatric inpatient service during } \\
\text { previous } 2 \text { yrs. }\end{array}$ \\
\hline & $\begin{array}{l}\text { Interventions: } 1 \text {. Joint Crisis Plan: aims to empower the client and to facilitate early detection and } \\
\text { treatment of relapse. Plan contains client's treatment preferences for any future psychiatric emer- } \\
\text { gency, when the client may be too unwell to express clear views. Not ICM. 2. Standard treatment: as } \\
\text { provided by CMHT. }\end{array}$ \\
\hline
\end{tabular}

\begin{tabular}{|c|c|}
\hline Cui 2012 & $\begin{array}{l}\text { Allocation: randomised. } \\
\text { Participants: schizophrenia. } \\
\text { Interventions: } 1 \text {. Case management. } \\
\text { 2. Outpatient visits. } \\
\text { Not ICM. }\end{array}$ \\
\hline De Cangas-Canada 1994 & $\begin{array}{l}\text { Allocation: randomised. } \\
\text { Intervention: hospital-based care for those in control group, not community-based ICM. }\end{array}$ \\
\hline Dean-UK1 1990 & Allocation: not randomised. \\
\hline Dean-UK2 1993 & Allocation: not randomised, quasi-experimental study. \\
\hline Dekker-Netherlands 2002 & $\begin{array}{l}\text { Allocation: randomised, but randomisation is compromised because after initial randomisation } \\
\text { control group were combined with control group from another study that experienced problems. } \\
\text { Participants: chronic psychiatric patients with a history of several admissions. } \\
\text { Interventions: } 1 \text {. ACT; caseload: } 1: 30 \text {. Not ICM. } 2 \text {. Standard care. }\end{array}$ \\
\hline Deng-China 2006 & $\begin{array}{l}\text { Allocation: quasi-randomised (randomisation according to hospital admission number). } \\
\text { Participants: admitted to hospital for a first-onset schizophrenia. } \\
\text { Interventions: hospital based, not community based. }\end{array}$ \\
\hline Dharwadkar-Victoria 1994 & Allocation: not randomised, before-and-after design. \\
\hline Dinitz 1965 & $\begin{array}{l}\text { Allocation: randomised. } \\
\text { Participants: acutely mentally ill patients in a hospital setting. }\end{array}$ \\
\hline Er 2013 & $\begin{array}{l}\text { Allocation: randomised. } \\
\text { Participants: chronic schizophrenia. } \\
\text { Interventions: } 1 \text {. Antipsychotics + family education, and rehabilitation instruction } \\
\text { 2. Care as usual. } \\
\text { Not ICM. }\end{array}$ \\
\hline
\end{tabular}




\begin{tabular}{ll}
\hline Study & Reason for exclusion \\
\hline Fenton-Canada 1978 & $\begin{array}{l}\text { Allocation: randomised. } \\
\text { Participants: people with schizophrenia, acutely ill, requiring immediate hospital admission. } \\
\text { Intervention: home-care community-based treatment vs hospital care. Not ICM in the experimen- } \\
\text { tal group; comparison group not community based. }\end{array}$
\end{tabular}

Franklin-Texas $1987 \quad$ Allocation: randomised.

Participants: people who at least had 2 discharges from mental hospital. Intervention: Assertive Community Treatment caseload over 30. Not ICM.

Participants: schizophrenia.

Interventions: 1. Hospital-community-family integrated management. 2. Care as usual.

Caseload not reported.

\section{Standard care.}

\begin{tabular}{ll} 
Glick-New York 1986 & Allocation: randomised. \\
& Intervention: day hospital care vs outpatient group therapy, not ICM. \\
\hline Godley-Illinois 1994 & $\begin{array}{l}\text { Allocation: randomised. } \\
\text { Participants: major psychiatric and substance abuse diagnosis. } \\
\text { Intervention: } 1 . \text { ICM: specialised case management. Caseload 1:15. 2. Standard care. } \\
\text { Outcome: incomplete data reporting. Not providing usable outcomes. }\end{array}$
\end{tabular}

Goering-Canada $1988 \quad$ Allocation: not randomised, used historical controls.

Gold-SCarolina $2006 \quad$ Allocation: randomised.

Participants: adults with severe mental illness.

Intervention: ACT + Individual Placement and Support (integrated supported employment programme). Not only ICM.

2. Traditional programme: providing mental health and brokered case management services in parallel to vocational services. Not only standard-care services.

2 arms are provided with different interventions between groups in addition to ACT and standard care.

Allocation: randomised.

Participants: adults with severe mental illness.

Interventions: 1. Case management provided by village doctors.

2. Usual care. Not ICM. management caseload 1:10; cognitive-behavioural family treatment and home-based crisis management. Not only ICM. 


\section{Study Reason for exclusion}

2. Standard treatment: clinical-based case management caseload 1:10 and crisis inpatient treatment.

Gu-China 2010 Allocation: randomised (multicentre trial; 4 centres).

Participants: patients suffering from schizophrenia.

1. Integrated training: the intervention includes life skill training, occupational skill training, communication training, psycho-education, and family visiting. Delivered by nurses. Caseload is probably $1<20$.

\section{Standard care. Routine training, no more details.}

Not ICM in the intervention.

\begin{tabular}{|c|c|}
\hline Han SH 2012 & $\begin{array}{l}\text { Allocation: randomised. } \\
\text { Participants: primary schizophrenia. } \\
\text { Interventions: } 1 \text {. Antipsychotics, psycho-education, family visit, and instruction. } \\
\text { 2. Antipsychotics and psycho-education. } \\
\text { Not ICM. }\end{array}$ \\
\hline Hargreaves-California & Allocation: not randomised. \\
\hline Havassy-California 2000 & $\begin{array}{l}\text { Allocation: randomised. } \\
\text { Participants: seriously mentally ill adults with and without substance dependence. } \\
\text { Interventions: } 1 \text {. Intensive Clinical Case Management community-based. } \\
\text { 2. Expanded brokerage case management program (data on caseload not available; it could be ei- } \\
\text { ther ICM or non-ICM), hospital based, providing intensive support during the initial postdischarge } \\
\text { period, with a maximum of } 60 \text { days. } \\
\text { Outcome: poor reporting data results, not usable. } \\
2 \text { reasons for exclusion: intervention (not clear if the comparison intervention is ICM or non-ICM) } \\
\text { and not providing usable data (comparison is between the } 2 \text { subgroups "with or without substance } \\
\text { dependence", not between the } 2 \text { interventions). }\end{array}$ \\
\hline
\end{tabular}
dependence", not between the 2 interventions).

\begin{tabular}{ll}
\hline He-China 2004 & Allocation: quasi-randomised (randomised allocation according to register number). \\
\hline Hornstra-Kansas 1993 & Allocation: not randomised, historical controls. \\
\hline Hoult-Australia 1981 & $\begin{array}{l}\text { Allocation: randomised. } \\
\text { Participants: people with schizophrenia, acutely ill, requiring immediate hospital admission. } \\
\text { Interventions: ICM vs acute admission to a psychiatric hospital. }\end{array}$ \\
\hline Huang 2012 & Allocation: unclear. \\
& Not RCT. \\
\hline Huang 2013 & Pllocation: randomised. \\
Participants: schizophrenia. & Interventions: 1. Community nursing intervention and rehabilitation training. \\
2. Treatment as usual. & Not ICM.
\end{tabular}




\section{Study}

Hui 2014

\section{Reason for exclusion}

Allocation: randomised.

Participants: patients diagnosed with schizophrenia, schizophreniform disorder, schizoaffective disorder, delusional disorder, brief psychotic disorder, psychotic disorder not otherwise specified, or manic episodes with psychotic features (DSM-IV); age 22 to 55 years.

Interventions: 1. Early Intervention Program: specialised and individualised case management as well as community-based group programmes. Caseload $>20$.

2. Standard care.
Allocation: randomised.

Participants: severe mental illness.

Interventions:

1. Non-ICM: comprehensive case management; caseload 1:22. Not ICM.

2. Non-ICM: traditional case management; caseload 1:40. Not ICM.

3. Non-ICM: comprehensive case management; caseload 1:22 + high-level access to independent housing. Not ICM.

4. Non-ICM: traditional case management; caseload 1:40 + high-level access to independent housing. Not ICM.

\section{ISRCTN73683215}

\section{Allocation: randomised.}

Participants: adults with severe mental illness who received mental health care in clinical settings. Interventions: Not ICM. Boston Psychiatric Rehabilitation Approach versus treatment as usual.
Allocation: randomised. Randomisation is compromised because number of people excluded after randomisation is not reported (patients were excluded if they refused to participate after randomisation or if they had not been discharged from hospital within 6 months of entering the study). Participants: severe mental illness.

Intervention: 1. ICM, according to the ACT Stein and Test model. 2. Standard care: provided in the community setting.
Allocation: randomised.

Interventions: ACT vs 12 -step recovery programme and behavioural skills training + standard care. Not standard care only in the comparison group.
Allocation: randomised.

Participants: primary schizophrenia

Interventions: 1. Family intervention plus antipsychotics.

2. Antipsychotics alone.

Not ICM.

\begin{tabular}{ll}
\hline Jiang 2013 & Allocation: randomised. \\
& Participants: chronic schizophrenia. \\
& Interventions: 1 . Intergrated community-based intervention. \\
2. Care as usual. & \\
Not ICM.
\end{tabular}




\begin{tabular}{|c|c|}
\hline Study & Reason for exclusion \\
\hline & $\begin{array}{l}\text { Participants: patients meeting the criteria for schizophrenia according to ICD10 F.20.09 or schizoaf- } \\
\text { fective disorder according to ICD10 F.25.09; age } 18 \text { to } 70 \text { years. }\end{array}$ \\
\hline & $\begin{array}{l}\text { Interventions: } 1 \text {. Guided Self Determination: } 21 \text { worksheets designed to guide patient and men- } \\
\text { tal health professionals through autonomy-supportive problem solving. The worksheets are filled } \\
\text { in by the patient before and between conversations with their community nurse over } 10 \text { sessions, } \\
\text { each approximately } 1 \text { hour. Not ICM. }\end{array}$ \\
\hline & 2. Waiting list \\
\hline & *Trial status: ongoing (trial registration form only). \\
\hline
\end{tabular}

\begin{tabular}{ll}
\hline Kane-Virginia 2004 & Not randomised. \\
\hline Kilbourne 2014 & Allocation: randomised. \\
& Participants: Adult Veterans Administration (VA) Center patients diagnosed with an ICD-9 diagnosis \\
& of schizophrenia or related disorder or bipolar disorder who was lost to care. \\
& Interventions: 1: Re-Engage: a national VA outreach program consisting of risk assessment (i.e. \\
& identifying the patient's current status, including clinical care needs and current disposition) and \\
& outreach services (i.e. attempting to contact patients and invite them back to receive health ser- \\
vices). Not ICM.
\end{tabular}

\title{
2. Standard care.
}

\section{Klotz-California 2001}

$$
\text { Knight-California } 1990
$$

Kuldau-California 1977

Lafave-Canada 1996

Wrote to author for further information. Received email from D. Innes-Gomberg on 4 January 2016 stating that there is no data. Consequently, this study is excluded.

Allocation: not randomised, quasi-experimental design.

Allocation: randomised.

Interventions: rapid discharge vs hospital care, not ICM.

\section{Allocation: randomised.}

\begin{tabular}{ll}
\hline Langley 2009 & Allocation: randomised \\
& Participants: diagnosed with a DSM-IV axis 1 disorder. \\
& Interventions: ACT vs ICM. No relevant comparison: both interventions types of ICM. \\
\hline Langsley-Colorado 1968 & $\begin{array}{l}\text { Allocation: randomised. } \\
\text { Interventions: outpatient family crisis management vs hospital admission. Not ICM. }\end{array}$ \\
\hline Lehman-Maryland2 1993 & $\begin{array}{l}\text { Allocation: randomised. } \\
\text { Participants: dually diagnosed people. } \\
\text { Interventions: } 1 \text { ICM + experimental group treatment Being Sober Group (addressing the specific } \\
\text { problems of dually diagnosed adults). 2. Non-ICM. In the experimental group: not only ICM. }\end{array}$
\end{tabular}

Intervention: hospital-based care for control group, not community-based care. Neither standard care nor non-ICM in the comparison group.

\section{LEO-UK 1994}

\author{
Allocation: randomised. \\ Participants: people with non-affective psychosis. \\ Interventions: 1. ICM: Assertive Outreach model; caseload 1:7. Including also: i. CBT based on \\ manualised protocol; ii. family counselling and vocational strategies based on established proto- \\ cols. Not only ICM. \\ 2. Non-ICM: delivered by the sector community mental health teams (according to Care Pro- \\ gramme Approach). No CBT, no family counselling and vocational strategies.
}




\section{Study}

Li 2011

\section{Reason for exclusion}

Study design: randomised.

Participants: inpatients with schizophrenia (Chinese Classification of Mental Disorders (CCMD-3)). Interventions: hospital-based intervention, not community based.

Assessed by Sai Zhao.

Li 2012 Allocation: randomised.

Participants: primary schizophrenia.

Interventions: 1 . Community comprehensive intervention.

2. Care as usual.

Not ICM.

Allocation: randomised.

Participants: schizophrenia.

Interventions: 1. Care package involving family intervention, psycho-education, and drug therapy.

2. Care as usual.

Not ICM.

Allocation: randomised.

Participants: primary schizophrenia.

Interventions: 1 . Individulised rehabilitation training.

2. Care as usual.

Not ICM.

Allocation: randomised.

Participants: schizophrenia.

Interventions: 1. Family intervention plus usual care.

2. Care as usual.

Not ICM.

Li Ning 2013

Allocation: randomised.

Participants: schizophrenia.

Interventions: 1 . Community-based comprehensive intervention.

2. Standard community intervention.

Caseload not reported.

Li WX 2013

Allocation: randomised.

Participants: schizophrenia.

Interventions: 1 . Community-based interventions.

2. Long-term hospitalisation. 


\begin{tabular}{ll}
\hline Study & Reason for exclusion \\
\hline Not ICM. \\
\hline Li-China 2004 & $\begin{array}{l}\text { Not community setting: hospital setting. In Chinese. Assessment by Tao Yuan Li Jun and Chunbo } \\
\text { Lee. }\end{array}$
\end{tabular}

\begin{tabular}{|c|c|}
\hline Liang 2009 & $\begin{array}{l}\text { Allocation: not randomised. The author randomly selected } 188 \text { participants and divided them into } \\
2 \text { groups. } \\
\text { Participants: schizophrenia. } \\
\text { Interventions: talking therapy and instruction for medication. } \\
\text { Assessed by Sai Zhao. }\end{array}$ \\
\hline Liang 2012 & $\begin{array}{l}\text { Allocation: randomised. } \\
\text { Participants: schizophrenia. } \\
\text { Interventions: } 1 \text {. Family intervention and antipsychotic medication. } \\
\text { 2. Antipsychotics alone. } \\
\text { Not ICM. }\end{array}$ \\
\hline Liao 2010 & $\begin{array}{l}\text { Study design: RCT. } \\
\text { Participants: inpatients with schizophrenia. } \\
\text { Interventions: hospital-based intervention included psycho-education, social skills training, family } \\
\text { education, etc. } \\
\text { Assessed by Sai Zhao. }\end{array}$ \\
\hline Lichtenberg-Israel 2008 & $\begin{array}{l}\text { Allocation: randomised. } \\
\text { Participants: people with at least } 3 \text { psychiatric admissions during the previous } 2 \text { yrs. Diagnosis is } \\
\text { not stated. } \\
\text { Interventions: } 1 \text {. Non-ICM; caseload 1:30. Not ICM. } \\
\text { 2. Standard care: provided by the mental healthcare centres. }\end{array}$ \\
\hline
\end{tabular}

\begin{tabular}{|c|c|}
\hline Lin-China 1998 & Allocation: not randomised. \\
\hline Liu 2010 & $\begin{array}{l}\text { Study design: RCT. } \\
\text { Participants: inpatients with schizophrenia. } \\
\text { Interventions: hospital-based group psycho-education. } \\
\text { Assessed by Sai Zhao. }\end{array}$ \\
\hline Lloyd 2000 & Allocation: not randomised. \\
\hline Lu 2013 & $\begin{array}{l}\text { Allocation: randomised. } \\
\text { Participants: schizophrenia. } \\
\text { Interventions: } 1 \text {. Hospital-community integrated management model. } \\
\text { 2. Care as usual. } \\
\text { Caseload not reported. }\end{array}$ \\
\hline Malm-Sweden 2003 & $\begin{array}{l}\text { Allocation: randomised. } \\
\text { Participants: diagnosed according to DSM-IV schizophrenia, schizophreniform, schizoaffective, or } \\
\text { delusion disorders. } \\
\text { Intervention: } 1 . \text { "Integrated Care" vs } 2 \text {. "Rational Rehabilitation". } \\
\text { Both of the compared treatment programmes were delivered in the context of clinical case man- } \\
\text { agement by CMHTs. They actively incorporated some key features of ACT; caseloads } 1: 40 \text {. }\end{array}$ \\
\hline
\end{tabular}




\begin{tabular}{|c|c|}
\hline Study & Reason for exclusion \\
\hline & $\begin{array}{l}\text { The additional component in the Integrated Care programme was the continuous social network } \\
\text { resource group for each patient. Not ICM. }\end{array}$ \\
\hline Martin-Delaware 1993 & Allocation: not randomised. \\
\hline Marx-Wisconsin 1973 & $\begin{array}{l}\text { Allocation: randomised. } \\
\text { Interventions: hospital-based care for control group, not community-based care. Neither standard } \\
\text { care nor non-ICM in the comparison group. }\end{array}$ \\
\hline
\end{tabular}

Participants: patients meeting Mini International Neuropsychiatric Interview criteria for schizophrenia, schizoaffective disorder, bipolar I or II disorder, or recurrent major depressive disorder and methamphetamine, amphetamine, or cocaine dependence who had used stimulants during the past 30 days.

Intervention: 1. Contingency management for stimulant abstinence. Not ICM.

\section{Treatment as usual.}

\section{McFarlane-New York 1992}

Allocation: unclear if randomised. Interventions: ACT vs Family-Aided Assertive Community Treatment (FACT). Neither standard care nor non-ICM in the comparison group.

Allocation: unclear if randomised; control and treatment groups were "randomly selected" from a population already receiving ACT or standard care.

McGrew-Indiana 1994

Allocation: not randomised (study was a before-and-after design examining the effects of implementing ACT teams in 6 sites in Indiana).

McHugo-Washington DC 2004 Allocation: randomised.

Participants: adults with severe mental illness at high risk for homelessness.

Intervention: 1. Parallel housing services programme: including ICM - caseload 1:15, implemented by mobile ACT team, and housing by routine community-based landlords. Not only ICM.

2. Integrated housing services programme: including ICM - caseload 1:15; case management and housing services were provided by teams within a single agency and were closely co-ordinated. Neither standard care nor non-ICM in the comparison group. ment where every 2 months a key-worker assesses patient's subjective quality of life, treatment satisfaction, and wishes for additional/different support using a questionnaire (MECCA). It is expected that the results will directly feed into the therapeutic dialogue and be discussed by the patient and key-worker together. Not ICM.

2. Treatment as usual.

\section{Meneghelli-Italy 2000}

Merson-UK 1992
Allocation: not randomised.

\section{Allocation: randomised.}

Interventions: multidisciplinary team home treatment vs emergency assessment at hospital. Neither standard care nor non-ICM in the comparison group. 


\section{Study Reason for exclusion}

Interventions: strengths model of case management versus standard case management. As caseload is not reported for either group, it is unclear if experimental intervention could be considered ICM and comparison group could be considered standard care or non-ICM. The study could be included if more data can be obtained on caseload.

Morse-Missouri2 $1997 \quad$ Allocation: randomised.

Participants: homeless people with severe mental illness.

Interventions: 1. ICM: ACT, caseload 1:10; vs 2. Non-ICM: Brokered Case Management, caseload 1:85; vs 3. ACT plus community worker support, caseload 1:10.

Outcomes: unable to use data, numbers for treatment groups not presented.

Morthorst $2012 \quad$ Allocation: randomised.

Participants: patients admitted after a suicide attempt to acute emergency units, intensive care units, paediatric units, and psychiatric emergency rooms: age: $\geq 12$ (subgroup analysis according to age reported). Excluded patients who had been diagnosed with severe mental illness (i.e. schizophrenia spectrum disorders, severe depression, severe bipolar disorder).

\begin{tabular}{ll}
\hline Mosher-California 1975 & Allocation: not randomised, alternative assignment. \\
\hline Muijen-UK1 1992 & Allocation: randomised. \\
& Participants: those with severe mental illness requiring immediate emergency admission. \\
& Interventions: ICM vs acute admission to a psychiatric hospital.
\end{tabular}

Mulder-Missouri 1985 Allocation: randomised, but data from randomised and non-randomised patients not reported separately.

Participants: people with schizophrenia, acutely ill, presenting for psychiatric hospital admission. Interventions: 1. ICM: modelled according to the PACT model (as an alternative to current hospitalisation).

2. Standard care: usual hospital admission procedure, and at discharge the usual aftercare case management services.

NCT00781079 Allocation: randomised.

Participants: must have serious mental illness and must be working with Veterans Affairs ICM team.

Interventions: No relevant comparison: ICM + peer specialists vs ICM only.

Allocation: randomised.

Participants: aged 12 to 35 years, with prodromal schizophrenia, psychotic disorders, severe bipolar disorder with psychotic features, or severe major depression with psychotic features.

Interventions:

1. Family-Aided Assertive Community Treatment (FACT) (a combination of family psycho-education, ACT, supported education/employment, and psychotropic medication).

2. Enhanced standard treatment: the participants will receive the same psychotropic drugs, but will receive individual case management, family education, and crisis intervention.

Not ICM vs standard care or ICM vs non-ICM.

Nieves 2002

Allocation: not a randomised study: "quasi-experimental design with a matched-groups comparison of outcomes achieved by patients in two community mental health centers in the South Bronx area of New York City". 


\begin{tabular}{ll}
\hline Study & Reason for exclusion \\
\hline Pai-India 1982 & Allocation: not randomised, alternative assignment. \\
\hline Pioli 2006 & Allocation: randomised. \\
& Participants: aged between 18 and 65 years; affected by severe mental illness. \\
& Intervention: generic rehabilitation interventions, not ICM. \\
& Setting: conducted partially in residential rehabilitation centre.
\end{tabular}

Polak-Colorado $1976 \quad \begin{aligned} & \text { Allocation: randomised. } \\ & \text { Participants: not clearly specified. } \\ & \text { Interventions: admission to small "community-based therapeutic environments" vs standard hos- } \\ & \text { pital care. Not ICM. }\end{aligned}$

PRiSM-UK $1998 \quad$ Allocation: not randomised.

Ren-China $2004 \quad$ Allocation: not clearly stated if randomised.

Participants: people with chronic schizophrenia, admitted to rehabilitation hospital. Interventions: rehabilitation hospital based, not community based.

Allocation: randomised

Participants: patients diagnosed with schizophrenia with an evolution of 2 or more years since the moment of the diagnosis.

Interventions: 1 . Personalised in-home nursing care plan: periodic home visits, with maximum intervals of 21 days between visits. Not ICM.

\section{Standard care.}

\begin{tabular}{ll}
\hline Rossler-Germany1 1992 & Allocation: not randomised, case control study. \\
\hline Rossler-Germany2 1995 & Allocation: not randomised, case control study. \\
\hline Rutter-UK & $\begin{array}{l}\text { Allocation: randomised. } \\
\text { Participants. severe mental illness. } \\
\text { Interventions: } 1 \text {. ICM: case management provided by a case manager internal to the CMHT; case- } \\
\text { load 1:15. } \\
\text { 2. ICM: case management provided by a case manager outside the CMHT; caseload 1:15. The com- } \\
\text { parison group was neither standard care nor non-ICM. } \\
\text { Allocation: randomised. } \\
\text { Participants: adults with severe mental illness. } \\
\text { Interventions: Not ICM. Illness Management and Recovery (IMR) vs intensive problem solving. IMR: } \\
\text { psycho-education, cognitive behavioural approaches, relapse prevention, social skills training, and } \\
\text { coping skills training. }\end{array}$ \\
\hline
\end{tabular}

Participants: patients diagnosed with schizophrenia or schizoaffective disorder, currently receiving (or newly admitted to) mental health services at a Veterans Administration (VA) or community mental health centre; age 18 or older.

Interventions: 1. Group-based Illness Management and Recovery (IMR) program: developed to incorporate effective strategies including psycho-education, cognitive behavioural approaches to medication adherence, relapse prevention, social skills training, and coping skills training. 


\section{Study Reason for exclusion}

2. Equally intensive problem-solving (PS) control group.

Not ICM and irrelevant comparison.

Allocation: randomised.

Participants: those with serious mental illnesses, recently admitted to hospital and ready for discharge.

Intervention: 1. Treatment Network Team provided both in community- and hospital-based setting. Caseload not stated. Not ICM.

2. Standard care.

Unable to use all outcomes.

Allocation: randomised.

Participants: hospitalised patients.

\begin{tabular}{ll}
\hline Schmidt 2005 & Not randomised: retrospective cohort design using an approximation of random assignment. \\
\hline Segal 2010 & Allocation: randomised. \\
& Participants: adults with severe mental illness. \\
& Interventions: 1 . Community Mental Health Agencies (CMHA) treatment vs 2. CMHA treatment and \\
Self-Help Agencies. \\
Not ICM.
\end{tabular}

Segal $2011 \quad$ Allocation: randomised.

Participants: adults with serious mental illness (schizophrenia or schizoaffective disorder, major affective disorder).

Interventions: 1. CMHA outpatient treatment. Not ICM.

2. A combination of CMHA outpatient treatment and consumer-operated service programs (COSP).

\begin{tabular}{|c|c|}
\hline Sha 2010 & $\begin{array}{l}\text { Allocation: randomised. } \\
\text { Participants: inpatients with schizophrenia (Chinese Classification of Mental Disorders (CCMD-3)). } \\
\text { Interventions: hospital-based intervention, not community based. } \\
\text { Assessed by Sai Zhao. }\end{array}$ \\
\hline Shen WW 2013 & $\begin{array}{l}\text { Allocation: randomised. } \\
\text { Participants: schizophrenia. } \\
\text { Interventions: } 1 \text {. Hospital-community-family integrated management. } \\
\text { 2. Care as usual. } \\
\text { Caseload not reported. }\end{array}$ \\
\hline Shern-USA2 & $\begin{array}{l}\text { Allocation: randomised. } \\
\text { Participants: serious and persistent mental health disorder + homelessness. } \\
\text { Interventions: } 1 \text {. Pathways to Housing: supporting housing programme, providing permanent, in- } \\
\text { dependent housing + ACT (according to Stein and Test model); caseload 1:10. Not only ICM. } \\
\text { 2. Standard care: provided by social agencies (i.e. outreach teams, drop-in centres). If participant } \\
\text { has no current affiliation to service providers, information was given about where services could be } \\
\text { obtained. No active engagement. }\end{array}$ \\
\hline
\end{tabular}




\section{Study Reason for exclusion}

Participants: severe mental illness, co-occurring addiction and homelessness.

Interventions: 1. Housing First programme: providing permanent, independent housing without prerequisites for sobriety and treatment + ACT; vs 2. Standard care. Not only ACT.

Shern-USA4 Allocation: randomised.

Participants: homeless mentally ill population.

Interventions: 1. Pathways to Housing: supporting-housing programme, providing permanent, independent housing; vs 2. Standard residential treatment. Not ICM.

\section{Solomon 2014}

Intervention: Not ICM: Transitional Care Model is a time-limited (90 days), transitional intervention from release from acute hospitalisation to full engagement with community mental health care

1. Transitional Care with case management: nurse practitioner managing risk factors to prevent further cognitive or emotional decline, managing problem behaviours, assessing and managing physical symptoms, preventing functional decline; promoting adherence to therapies, assuring proper medical management and continuity of care, and helping case managers understand the integrated mental and physical care approach.

2. Usual case management: a case manager was assigned to the participant and a psychiatrist provided medication management.

Solomon-Pennsylvania1 Allocation: randomised. Intervention: 1 type of case management vs another. Neither standard care nor non-ICM in the comparison group.

Participants: homelessness or precarious housing participants with current mental disorder status meeting Mini-International Neuropsychiatric Interview criteria for major depressive episode, manic or hypomanic episode, post-traumatic stress disorder, mood disorder with psychotic features, and psychotic disorder.

Interventions: 1 . Housing First + ICM. Not ICM alone.

2. Usual care.

Allocation: randomised.

Participants: schizophrenia.

Interventions: 1. Family psychological care plus social skills training and antipsychotics.

2. Antipsychotics alone.

Not ICM.

Participants: adults with severe mental illness; age 18 to 62 years.

Interventions: 1 . Training in community living.

\section{Hospital care.}

Control group not in community. 


\section{Study Reason for exclusion}

Participants: patients in need for acute psychiatric inpatient care

Intervention: 1 . Community treatment (not specified).

2. Hospital admission and inpatient treatment. Control group not community treated.

*Trial status: ongoing (trial registration form only).

Sungur $2011 \quad$ Allocation: randomised.

Participants: between the ages of 18 and 45 years; a DSM-IV diagnosis of schizophrenia confirmed by Structured Clinical Interview for DSM-IV (SCID-IV) interviews; duration of illness of less than 10 years; living with a family member the year prior to admission; a resident of Ankara; completed written informed consent approved by the ethics committee (caregivers were also required to provide written informed consent.

Intervention: 1. Optimal case management. Caseload: 4:50.

2. Routine case management. Caseload: 4:50.

Participants: patients with nonaffective psychosis who had been with Early Intervention Service (EIS) between 1 and 2 years and who showed a low level of structured activity after at least 1 year of treatment (defined as 30 hours or less per week).

Interventions:1. Social recovery-oriented cognitive behavioural therapy + standard care. Not ICM.

\section{Standard care.}

Allocation: randomised.

Participants: severe mental illness.

Interventions: 1 . "Critical time intervention": a time-limited approach aimed at stabilising the patient's social support network, designed for transitions from various institutions to the community; the goal is to enhance continuity of care, strengthening the individual's long-term ties to services. Not ICM.

2. Standard care: community-based services.

Tang $2013 \quad$ Allocation: randomised.

Participants: schizophrenia.

Interventions: 1. Carers-provided home-based rehabilitation intervention.

2. Professional-provided home visit.

Not ICM.

Tao-China 2004

Allocation: randomised.

Participants: people with schizophrenia, admitted to hospital.

Intervention: provided pre-discharging. Hospital, not community, based.

\begin{tabular}{ll}
\hline Teague-New Hampshire 1995 & Allocation: not randomised. \\
\hline Thornicroft-Maryland 1991 & Allocation: not randomised. \\
\hline Tomita 2011 & Allocation: randomised. \\
& $\begin{array}{l}\text { Participants: } 150 \text { previously homeless individuals with severe and persistent mental illness after } \\
\text { discharge from inpatient treatment. }\end{array}$ \\
\hline
\end{tabular}




\begin{tabular}{ll}
\hline Study & Reason for exclusion \\
\hline & $\begin{array}{l}\text { Intervention: Critical Time Intervention (primarily designed to prevent homelessness) is not ICM; it } \\
\text { is transitional care from hospital to full engagement with community mental health services. }\end{array}$ \\
\hline Toro-New York 1997 & $\begin{array}{l}\text { Allocation: randomised. } \\
\text { Participants: a minority of the participants suffered from severe mental illness; around 80\% were } \\
\text { simply homeless. }\end{array}$ \\
\hline Tyrer-UK 1995 & $\begin{array}{l}\text { Allocation: randomised } \\
\text { Participants: psychotic illness. } \\
\text { Intervention: } 1 . \text { ICM. 2. Standard treatment: provided by social and psychiatric services. The case } \\
\text { managers for the treatment group were also the case managers for the control group (see Excluded } \\
\text { studies section). }\end{array}$
\end{tabular}

Allocation: randomised

Participants: adults with severe mental illness.

Interventions: 1. Relapse Prevention Plan: includes detecting the early signs of difficulty and actions that could be taken when psychotic relapse threatens (not ICM).

\section{Usual care.}

Allocation: randomised, parallel-group clinical trial.

Participants: outpatients, aged 18 to 35 years, diagnosed with first-episode psychosis or schizotypal disorder within F2 spectrum in ICD-10. Participants were in a postacute phase of illness, had sufficient comprehension of Danish (i.e. did not need an interpreter), and provided written informed consent.

Intervention: 1. OPUS + cognitive training vs 2. OPUS only.

OPUS consists of affiliation with a primary contact person, involvement of family, possibility of psycho-education and social skills training. Depending on individual needs, patients are can take part in group therapy, either social skills training or cognitive behavioural therapy.

No relevant comparison.

\begin{tabular}{ll}
\hline Vincent-Ohio 1977 & Allocation: not randomised, alternative assignment. \\
\hline Wang 2008a & Not randomised. \\
\hline Wang F 2012 & Allocation: randomised. \\
& Participants: chronic schizophrenia. \\
& Interventions: 1. Family rehabilitation intervention. \\
& 2. Care as usual. \\
& Not ICM. \\
\hline
\end{tabular}

Participants: schizophrenia.

Interventions: 1. Family rehabilitation intervention.

\section{Care as usual.}

Not ICM. 
Reason for exclusion

Participants: schizophrenia.

Interventions: 1. Family nursing intervention.

2. Care as usual.

Not ICM.

Wang YQ 2010a

Allocation: randomised.

Participants: inpatients with schizophrenia.

Interventions: hospital-based intervention.

Assessed by Sai Zhao.

Wang Z 2012

Allocation: randomised.

Participants: schizophrenia.

Interventions: 1. Family intervention plus antipsychotics.

2. Antipsychotics alone.

Not ICM.

\section{Wen 2010}

Allocation: randomised.

Participants: schizophrenia.

Interventions: family interventions; the nurses made an individualised treatment plan for each participant, then implemented intervention according to the plan. The effects of the intervention on outcomes were evaluated and treatments revised according to specific problems of the participant.

Assessed by Sai Zhao.

Allocation: randomised.

Participants: all with a diagnosis of DSM-IV schizophrenia or schizoaffective disorder.

Interventions: not ICM (Community Re-Entry Program), not outpatient/community setting (delivered during brief hospitalisations).

Allocation: not randomised, case control study.

\section{Wu 2013}

Allocation: randomised.

Participants: schizophrenia.

Interventions: 1 . Group psychotherapy combined with family intervention.

2. Care as usual.

Not ICM.

Allocation: randomised.

Participants: severely mentally ill patients.

Interventions: 1. Enhanced Assertive Community Treatment: ACT enhanced with evidence-based interventions.

\section{Standard care.}

*Trial status is ongoing (trial registration form only). 


\begin{tabular}{|c|c|}
\hline Study & Reason for exclusion \\
\hline Xing 2013 & $\begin{array}{l}\text { Allocation: randomised. } \\
\text { Participants: chronic schizophrenia. } \\
\text { Interventions: } 1 \text {. Rehabilitation training. } \\
\text { 2. Control group. } \\
\text { Not ICM. }\end{array}$ \\
\hline Yang 2013 & $\begin{array}{l}\text { Allocation: randomised. } \\
\text { Participants: schizophrenia. } \\
\text { Interventions: } 1 \text {. Rehabilitation training. } \\
\text { 2. Treatment as usual. } \\
\text { Not ICM. }\end{array}$ \\
\hline Yao 2013 & $\begin{array}{l}\text { Allocation: randomised. } \\
\text { Participants: schizophrenia. } \\
\text { Interventions: } 1 \text {. Case management model. } \\
\text { 2. No case management. } \\
\text { Not ICM. }\end{array}$ \\
\hline
\end{tabular}

\begin{tabular}{|c|c|}
\hline Yao 2014 & $\begin{array}{l}\text { Allocation: randomised. } \\
\text { Participants: chronic schizophrenia. } \\
\text { Interventions: 1. Day nursing care in community. } \\
\text { 2. Care as usual. } \\
\text { Not ICM. }\end{array}$ \\
\hline Yu 2011 & $\begin{array}{l}\text { Study design: not randomised. Allocation based on admission sequences. } \\
\text { Assessed by Sai Zhao. }\end{array}$ \\
\hline Yuan 2013 & $\begin{array}{l}\text { Allocation: randomised. } \\
\text { Participants: schizophrenia. } \\
\text { Interventions: } 1 \text {. Case management model. } \\
\text { 2. Conventional community management model. } \\
\text { Caseload not reported. }\end{array}$ \\
\hline Zhang SY 2013 & $\begin{array}{l}\text { Allocation: randomised. } \\
\text { Participants: schizophrenia. } \\
\text { Interventions: 1. Enhanced family nursing intervention. } \\
\text { 2. Conventional family intervention. } \\
\text { Not ICM. }\end{array}$ \\
\hline
\end{tabular}




\begin{tabular}{|c|c|}
\hline Study & Reason for exclusion \\
\hline Zhang YF 2012 & $\begin{array}{l}\text { Allocation: randomised. } \\
\text { Participants: schizophrenia. } \\
\text { Interventions: } 1 \text {. Community visit and antipsychotics. } \\
\text { 2. Antipsychotics alone. } \\
\text { Not ICM. }\end{array}$ \\
\hline Zhang YM 2013 & $\begin{array}{l}\text { Allocation: randomised. } \\
\text { Participants: schizophrenia. } \\
\text { Interventions: } 1 \text {. Comprehensive intervention (family visit, medicine compliance intervention, fam- } \\
\text { ily intervention, crisis intervention). } \\
\text { 2. Telephone follow-up. } \\
\text { Not ICM. }\end{array}$ \\
\hline Zhao HM 2013 & $\begin{array}{l}\text { Allocation: randomisation. } \\
\text { Participants: schizophrenia. } \\
\text { Intervention: } 1 \text {. Comprehensive community rehabilitation intervention. } \\
\text { 2. Standard drug therapy. } \\
\text { Caseload not reported. }\end{array}$ \\
\hline Zhu 2009 & $\begin{array}{l}\text { Allocation: not randomised. Allocation based on admission sequences. } \\
\text { Assessed by Sai Zhao. }\end{array}$ \\
\hline Zhu DP 2012 & $\begin{array}{l}\text { Allocation: randomised. } \\
\text { Participants: schizophrenia. } \\
\text { Interventions: } 1 \text {. Family intervention plus antipsychotics. } \\
\text { 2. Antipsychotics alone. } \\
\text { Not ICM. }\end{array}$ \\
\hline
\end{tabular}

ACT - Assertive Community Treatment

CBT - cognitive behavioural therapy

CMHA - Community Mental Health Agencies

CMHT: Community Mental Health Team

CPA - Care Programme Approach: the CPA is a combination of non-ICM and care from a CMHT, introduced in England in the mid-1990s and becoming standard care thereafter

DSM - Diagnostic and Statistical Manual of Mental Disorders

ICD-10 - 10th revision of the International Statistical Classification of Diseases and Related Health Problems

ICM - Intensive Case Management

non-ICM - non-Intensive Case Management

PACT - Programme of Assertive Community Treatment

$\mathrm{RCT}$ - randomised controlled trial

Characteristics of studies awaiting assessment [ordered by study ID] 
Bonsack-Switzerland

\begin{tabular}{ll}
\hline Methods & Allocation: randomised. \\
\hline Participants & Aged 18 to 65 years, following psychiatric hospitalisation. \\
\hline Interventions & 1. Transitional Case Management. \\
\hline 2. Standard care. \\
\hline \begin{tabular}{l} 
Adherence to outpatient care; working alliance; number of readmissions; degree of psychiatric \\
symptoms (SCL-90 R); etc. \\
\hline ClinicalTrials.gov (clinicaltrials.gov/ct2/show/NCT02258737): "This study has been completed."; no \\
results posted or publication announced.
\end{tabular} \\
\hline
\end{tabular}

\section{ChicTR-TRC-13003407}

\begin{tabular}{ll}
\hline Methods & Allocation: randomised. \\
\hline Participants & Adults with severe mental illness. \\
\hline Interventions & 1. Assertive Community Treatment. \\
& 2. Standard community service. \\
\hline
\end{tabular}

\section{Outcomes}

Notes Insufficient information in trial registry entry to determine inclusion/exclusion.

Written to investigator for clarification.

\section{Dick-UK 2000}

\begin{tabular}{ll}
\hline Methods & Allocation: randomised. \\
\hline Participants & People with chronic functional psychosis. \\
\hline Interventions & Community rehabilitation programme. \\
\hline Outcomes & \\
\hline Notes & Not available as full report, written to investigator for clarification, awaiting response. \\
\hline
\end{tabular}

\section{Guo-China 2003}

\begin{tabular}{ll}
\hline Methods & Allocation: randomised, no further details. \\
\hline Participants & Participants: people with schizophrenia (CCMD-3), living in the community. \\
\hline Interventions & Intervention: community-based. \\
\hline
\end{tabular}


1. Experimental intervention: provided by team composed by nurses, social worker; caseload 1:10. Providing: i. Expressed Emotion intervention; ii. psycho-education (how to prevent relapse, how to deal with adverse effects); iii. daily living activity.

2. Standard care. Awaiting further information on comparison treatment.

\begin{tabular}{ll}
\hline Outcomes & Outcomes: awaiting for data extraction. \\
\hline Notes & In Chinese. \\
& Written to investigator for clarification. \\
\hline
\end{tabular}

\section{Kawanishi-Japan 2014}

\begin{tabular}{ll}
\hline Methods & Allocation: randomised; multicentre. \\
\hline Participants & People with mental health problems who have attempted suicide. \\
\hline Interventions & 1. Assertive case management \\
& 2. Enhanced usual care. \\
\hline
\end{tabular}

\section{Outcomes}

Notes Caseload not reported.

Written to investigator for clarification.

\begin{tabular}{ll}
\hline Methods & Allocation: randomised. \\
\hline Participants & People with chronic psychoses, mostly schizophrenia; N = 107. \\
\hline Interventions & $\begin{array}{l}\text { 1. Mobile community teams. } \\
\text { 2. Traditional psychiatric care. }\end{array}$ \\
\hline
\end{tabular}

\section{Outcomes}

Notes To be assessed, in Polish.

\section{Li 2010}

\begin{tabular}{ll}
\hline Methods & Allocation: randomised. \\
\hline Participants & People with chronic schizophrenia. \\
\hline Interventions & $\begin{array}{l}\text { Interventions: community-based integrated intervention includes supportive psychosocial thera- } \\
\text { py, family therapy, rehabilitation training, and antipsychotics. } \\
\text { Caseload: the author did not state the caseload. }\end{array}$ \\
\hline
\end{tabular}


Li 2010 (Continued)

Outcomes

\begin{tabular}{ll}
\hline Notes & In Chinese. Assessed by Sai Zhao. (Li 2010). \\
& Insufficient information to determine inclusion or exclusion; written to investigator.
\end{tabular}

Linszen-Netherlands 2002

\begin{tabular}{ll}
\hline Methods & Allocation: randomised. \\
\hline Participants & Young patients with recent-onset psychosis. \\
\hline Interventions & \\
\hline Outcomes & $\begin{array}{l}\text { Not enough information in the published conference abstracts to determine intervention or age of } \\
\text { participants (described as "young"). } \\
\text { Notes }\end{array}$ \\
& Written to authors for more published reports. \\
\hline
\end{tabular}

Manuel 2009

\section{Methods}

\section{Participants}

\section{Interventions}

\section{Outcomes}

Notes To be assessed. PDF not yet retrieved.

\section{O'Donnell-Australia 1999}

\begin{tabular}{ll}
\hline Methods & Allocation: randomised. \\
\hline Participants & $\begin{array}{l}\text { Clients with schizophrenia and bipolar disorder, } 18 \text { to } 65 \text { years of age, referred for case manage- } \\
\text { ment by community health services. }\end{array}$ \\
\hline Interventions & $\begin{array}{l}\text { 1. Standard case management. } \\
\text { 2. Client-focused case management. } \\
\text { 3. Client-focused case management plus consumer advocacy. }\end{array}$ \\
\hline Outcomes & Written to authors asking for caseload. \\
\hline
\end{tabular}


Rivera-New York 2007

\begin{tabular}{ll}
\hline Methods & Allocation: randomised. \\
\hline Participants & 203 clients with severe and persistent mental illness. \\
\hline Interventions & ICM vs peer-enhanced ICM vs clinic-based ICM. \\
\hline
\end{tabular}

\section{Outcomes}

Notes Written to authors asking for caseload.

\section{Ruggeri-Italy}

\begin{tabular}{ll}
\hline Methods & Allocation: randomised. \\
\hline Participants & Adults with severe mental illness. \\
\hline Interventions & $\begin{array}{l}\text { 1. Multi-element psychosocial intervention. } \\
\text { 2. Standard care. }\end{array}$ \\
\hline
\end{tabular}

\section{Outcomes}

Notes Caseloads not reported. Unclear whether intervention is ICM.

Written to authors for clarification.

\section{Sells-Connecticut 2006}

\begin{tabular}{|c|c|}
\hline Methods & $\begin{array}{l}\text { Allocation: randomised. } \\
\text { Design: multicentre ( } 2 \text { cities in Connecticut, not stated which ones). } \\
\text { Duration: } 12 \text { months. } \\
\text { Country: Connecticut, USA. } \\
\text { Not entering meta-regression. }\end{array}$ \\
\hline Participants & $\begin{array}{l}\text { Diagnosis*: severe mental illness (schizophrenia spectrum disorder, major mood disorder, or both). } \\
\mathrm{N}=137 . \\
\text { Setting: public mental health centres, urban site. } \\
\text { Age: } 20 \text { to } 63 \text { years, mean } 41 \text { yrs (SD } 9 \text { years). } \\
\text { Sex: } 61 \% \text { M. } \\
\text { Ethnicity: African-American } 28.5 \% \text {. } \\
\text { History: i. treatment disengagement, ii. informed consent provided. }\end{array}$ \\
\hline Interventions & $\begin{array}{l}\text { 1. } \mathrm{ICM}^{\star \star} \text { : Case management services from peer providers partnered with } \mathrm{ACT} \text { teams. Peer case } \\
\text { manager. Caseload: } 1: 10-12 . \mathrm{N}=68 \text {. } \\
\text { 2. Non-ICM} \mathrm{CM}^{\star \star \star} \text { : regular case management from regular providers. Caseload: } 1: 20-24 . \mathrm{N}=69 \text {. }\end{array}$ \\
\hline Outcomes & $\begin{array}{l}\text { Unable to use - } \\
\text { Service use: } 26 \text {-item self reported measure of service use (not peer reviewed). } \\
\text { Level of engagement: rated using } 1 \text { item of Level of Care Utilization System (subscale not validat- } \\
\text { ed). } \\
\text { Social functioning: subscale from Addiction Severity Index (ASI) (subscale not peer reviewed, data } \\
\text { not reported). }\end{array}$ \\
\hline
\end{tabular}


Client-counsellor relationship: modified version of Barrett-Lennard Relationship Inventory (BLRI) (not peer reviewed, modified by authors).

\section{Notes}

*61\% psychiatric disorder; $63 \%$ major mood disorder; $72 \%$ substance abuse disorder; $70 \%$ co-occurring disorder (psychotic disorder, mood disorder, or both plus substance abuse disorder).

${ }^{\star \star}$ All peer staff had publicly disclosed histories of severe mental illness and some of co-occurring drug use disorder. They received broad-based training concerning the provision of case management service.

${ }^{\star \star \star}$ Regular providers worked alongside peer providers on the same treatment teams.

1 additional paper requested.

\section{Sharifi-Iran 2009}

\begin{tabular}{ll}
\hline Methods & Allocation: randomised. \\
\hline Participants & People with severe mental disorders. $\mathrm{N}=120$. \\
\hline Interventions & $\begin{array}{l}\text { 1. Home aftercare service: home visits by multidisciplinary teams, including general practitioners, } \\
\text { nurses, and social workers supervised by psychiatrists. }\end{array}$ \\
2. Treatment as usual.
\end{tabular}

\section{Outcomes}

Notes Conference abstract, not enough information to determine inclusion/exclusion.

Written to authors for clarification.

\section{Su-China 2008}

\begin{tabular}{ll}
\hline Methods & Allocation: randomised. \\
\hline Participants & People with schizophrenia. \\
\hline Interventions & $\begin{array}{l}\text { Interventions: community-based integrated intervention includes rehabilitation consultant, devel- } \\
\text { op individualised rehabilitation plan, telephone follow-up, and family psycho-education. } \\
\text { Caseload: the author did not state the caseload. }\end{array}$ \\
\hline
\end{tabular}

Outcomes

Notes In Chinese. To be assessed.

\section{Tan-China 2005}

\begin{tabular}{ll}
\hline Methods & Allocation: randomised, no further details. \\
\hline Participants & Diagnosis: schizophrenia. \\
\hline Interventions & $\begin{array}{l}\text { 1. Experimental intervention: provided by team, caseload not reported. Providing: i. antipsychotic } \\
\text { drug treatment; ii. family intervention; iii. occupational intervention; iv. rehabilitation intervention. }\end{array}$
\end{tabular}


Tan-China 2005 (Continued)

2. Control intervention: no details provided.

Outcomes Awaiting further information from author about experimental intervention caseload.

Notes In Chinese. To be assessed.

Verhaegh-Netherlands 2006

\begin{tabular}{ll}
\hline Methods & Allocation: unclear. \\
\hline Participants & People with first-episode psychosis, 18 to 35 years of age. \\
\hline Interventions & 1. ACT with different modalities. \\
2. Care as usual.
\end{tabular}

\section{Outcomes}

Notes Unclear if this is a randomised study (caseload is also unclear). Main paper in Dutch to be assessed. Written to authors for clarification.

\section{Wang 2009}

\begin{tabular}{ll}
\hline Methods & Allocation: randomised. \\
\hline Participants & People with schizophrenia. \\
\hline Interventions & $\begin{array}{l}\text { Interventions: community-based integrated intervention includes psycho-education, direction on } \\
\text { use of medication, social rehabilitation training, psychological intervention, and crisis intervention. } \\
\text { Caseload: the author did not state the caseload. }\end{array}$ \\
\hline
\end{tabular}

\section{Outcomes}

\begin{tabular}{ll}
\hline Notes & In Chinese. Asessed by Sai Zhao. (Wang 2009). \\
Insufficient information to determine inclusion or exclusion; written to investigator.
\end{tabular}

\section{Zhang 2009}

\begin{tabular}{ll}
\hline Methods & Allocation: randomised. \\
\hline Participants & People with schizophrenia. \\
\hline Interventions & $\begin{array}{l}\text { Interventions: community-based integrated intervention includes psycho-education, life skill train- } \\
\text { ing, and cognitive therapy. } \\
\text { Caseload: the author did not state the caseload. }\end{array}$ \\
\hline
\end{tabular}

\section{Outcomes}

\section{Notes}

In Chinese. Assessed by Sai Zhao. (Zhang 2009). 
Zoeteman-Netherlands

\begin{tabular}{ll}
\hline Methods & Allocation: randomised. \\
\hline Participants & Homeless patients with severe mental illness. \\
\hline Interventions & 1. ACT. \\
& 2. Case management. \\
\hline
\end{tabular}

\section{Outcomes}

Notes Trial completed, but no results or publication found. Caseload unclear.

Written to investigator for clarification.

ACT - Assertive Community Treatment

CCMD-3 - Chinese Classification of Mental Disorders

ICM - Intensive Case Management

SD - standard deviation

Characteristics of ongoing studies [ordered by study ID]

Koike-Japan

Trial name or title Comprehensive early intervention for patients with first-episode psychosis in Japan (J-CAP): study
protocol for a randomised controlled trial.

$\begin{array}{ll}\text { Methods } & \text { Allocation: randomised. } \\ \text { Blinding: Only assessors blinded. }\end{array}$

\begin{tabular}{ll}
\hline Participants & People who received a diagnosis of F2 or F3 (ICD-10), with psychotic symptoms. \\
\hline Interventions & 1. Comprehensive community-based care. \\
2. Standard care. & $\mathrm{N}=$ expected 150. \\
& Age: 15 to 35 years. \\
\hline Putcomes & Primary outcome: Global Assessment of Functioning (GAF-F) scores at the first endpoint. \\
& $\begin{array}{l}\text { Secondary outcomes: GAF-F at the second and last endpoints, symptom domain of Global Assess- } \\
\text { ment of Functioning (GAF-S), PANSS, the World Health Organization Quality of Life 26-item version } \\
\text { (WHOQOL-BREF), Brief Evaluation of Medication Influences and Beliefs (BEMIB), care satisfaction of } \\
\text { participants and their families, educational and vocational recovery rates, remission rate, readmis- } \\
\text { sion rate, lost to follow-up rate, self harm and suicide attempt rate, suicide rate, engagement be- } \\
\text { haviour, and direct and indirect costs at each endpoint. }\end{array}$ \\
\hline
\end{tabular}

Starting date March 2011.

Contact information skoike-tky@umin.ac.jp; nishida-at@igakuken.or.jp


Koike-Japan (Continued)

Notes
The University Hospital Medical Information Network Clinical Trials Registry (No. UMIN000005092).

Expected completion date: September 2017.

Lutgens 2015

Trial name or title A five-year randomized parallel and blinded clinical trial of an extended specialized early intervention vs. regular care in the early phase of psychotic disorders.

\begin{tabular}{ll}
\hline Methods & Allocation: randomised. \\
\hline Participants & People with first-episode psychosis. \\
\hline Interventions & $\begin{array}{l}\text { 1. Extended Specialized Early Intervention: modified assertive case management; psycho-educa- } \\
\text { tion for families; multiple-family intervention; cognitive behavioural therapy; and substance abuse } \\
\text { treatment and monitoring. } \\
\text { 2. Regular care. }\end{array}$ \\
\hline Outcomes & $\begin{array}{l}\text { Primary outcomes: proportion of participants in complete remission and mean length of remission } \\
\text { achieved. } \\
\text { Secondary outcomes: relapse, functioning, quality of life. }\end{array}$ \\
\hline Starting date & Danyael Lutgens: Danyael.Lutgens@douglas.mcgill.ca \\
\hline Notes & \\
\hline
\end{tabular}

\section{Malla-Canada}

\begin{tabular}{ll}
\hline Trial name or title & Extending specialized early intervention service from 2 to 5 years: a randomised controlled trial. \\
\hline Methods & Allocation: randomised. \\
\hline Participants & Adults with severe mental illness. \\
\hline Interventions & 1. Extended Specialized Early Intervention. \\
& 2. Usual care. \\
\hline Outcomes & Rates of sustained engagement, length of remission, health economic indices. \\
\hline Starting date & Ashok Malla: ashok.malla@mcgill.ca \\
\hline Contact information & Conference abstracts with brief interim results only. \\
\hline Notes &
\end{tabular}


NCT01313052

Trial name or title

Methods

Participants

Forensic Assertive Community Treatment: an emerging model of service delivery (FACT).

Allocation: randomised, open, and parallel.

Individuals diagnosed with any psychotic disorder such as schizophrenia, schizoaffective disorder, bipolar disorder with psychotic features, depressive disorder with psychotic features, and psychotic disorder, not otherwise specified, who currently face misdemeanor or violation charges and have not yet been sentenced; $\mathrm{N}=53$.

\begin{tabular}{ll}
\hline Interventions & $\begin{array}{l}\text { 1. Forensic Assertive Community Treatment (FACT): services of an Assertive Community Treatment } \\
\text { team and close supervision of a judge trained in the FACT model. }\end{array}$ \\
& $\begin{array}{l}\text { 2. Enhanced treatment as usual: expedited appointment at a clinic specialising in the treatment of } \\
\text { psychotic disorders; services of a therapist, psychiatrist, and case manager. }\end{array}$ \\
\hline Outcomes & Jail recidivism; mental health service utilisation. \\
\hline Starting date & May 2008. \\
\hline Contact information & Steven Lamberti, University of Rochester. \\
\hline Cotes & $\begin{array}{l}\text { Written to author for further information. Received email from S. Lamberti on } 30 \text { December } 2015 \\
\text { stating that they are unable to share the results at this time as they are being prepared for publica- } \\
\text { tion. Awaiting publication. }\end{array}$ \\
\hline
\end{tabular}

RISE - Ethiopia

\begin{tabular}{ll}
\hline Trial name or title & RISE (Rehabilitation Intervention for People With Schizophrenia in Ethiopia). \\
\hline Methods & Cluster randomised trial. \\
\hline Participants & $\begin{array}{l}\text { Adults with a diagnosis of schizophrenia spectrum disorder (schizophrenia, schizoaffective disor- } \\
\text { der, or schizophreniform disorder) using (DSM-IV) criteria. }\end{array}$ \\
\hline
\end{tabular}

Interventions
comprising psycho-education, adherence support, rehabilitation (including self care and social
skills), family support groups, and accessing existing community organisations. The intervention
also involves community awareness raising and education and mobilisation of community leaders;
antipsychotic medication prescribed by a nurse or clinical officer in a health centre; and basic psy-
cho-education.
2. Facility-based usual care: antipsychotic medication prescribed by a nurse or clinical officer in a
health centre and basic psycho-education.

Outcomes

Disability, symptom severity, Clinical Global Impression, relapse, functioning, economic activity of participant (employment, income, and household work), medication adherence, engagement with facility-based care, proportion with human rights problems, nutritional status (BMI), serious adverse events, etc.

\begin{tabular}{ll}
\hline Starting date & September 2015. \\
\hline Contact information & Mary De Silva, PhD MSc, London School of Hygiene and Tropical Medicine. \\
& Abebaw Fekadu, Addis Ababa University Department of Psychiatry. \\
\hline
\end{tabular}


RISE - Ethiopia (Continued)
Notes
Currently recruiting.

Walsh-Connecticut

\begin{tabular}{|c|c|}
\hline Trial name or title & Specialized Treatment Early in Psychosis (STEP). \\
\hline Methods & $\begin{array}{l}\text { Allocation: randomised. } \\
\text { Blinding: open label. }\end{array}$ \\
\hline Participants & $\begin{array}{l}\text { Diagnosis: schizophrenia spectrum psychosis or affective psychosis (DSM-IV, SCID). } \\
\mathrm{N}=\text { expected } 200 \text {. } \\
\text { Age: } 16 \text { to } 45 \text { years. } \\
\text { History: } \leqq 8 \text { weeks of received antipsychotic treatment lifetime, informed consent, no psychosis } \\
\text { believed due to substance use. }\end{array}$ \\
\hline Interventions & $\begin{array}{l}\text { 1. Specialised early treatment: including individual case management, antipsychotic prescription, } \\
\text { multifamily group therapy, group cognitive behavioural therapy, and cognitive remediation. } \\
\text { 2. Standard care: usual referral to CMHC. }\end{array}$ \\
\hline Outcomes & $\begin{array}{l}\text { Primary outcomes: service use: rehospitalisation (measured every } 6 \text { months for } 5 \text { yrs). } \\
\text { Secondary outcomes: relapse, overall functioning, quality of life, education and employment sta- } \\
\text { tus, treatment satisfaction, adherence, substance use, adverse effects (including self harm and vio- } \\
\text { lence), medication side effect, economic measures. }\end{array}$ \\
\hline Starting date & March 2006. \\
\hline Contact information & barbara.walsh@yale.edu; vinod.srihari@yale.edu \\
\hline Notes & $\begin{array}{l}\text { ClinicalTrials.gov identifier: NCT00309452 } \\
\text { Expected completion: September } 2011 .\end{array}$ \\
\hline & $\begin{array}{l}\text { NOTE: Results are available in Srihari VH, Tek C, Kucukgoncu S, Phutane VH, Breitborde NJ, Pollard } \\
\text { J, Ozkan B, Saksa J, Walsh BC, Woods SW. First-episode services for psychotic disorders in the U.S. } \\
\text { public sector: a pragmatic randomized controlled trial. Psychiatric Services } 2015 \text { Jul;66(7):705-12. }\end{array}$ \\
\hline
\end{tabular}

$\mathrm{BMI}$ - body mass index

CMHC - Community Mental Health Centre

DSM - Diagnostic and Statistical Manual of Mental Disorders

ICD-10 - International Statistical Classification of Diseases and Related Health Problems 10th Revision

PANSS - Positive and Negative Syndrome Scale

SCID - Structured Clinical Interview for DSM-IV (First 1997)

DATA AND ANALYSES

\section{Comparison 1. Intensive Case Management versus standard care}

\begin{tabular}{lllll}
\hline Outcome or subgroup title & No. of studies & $\begin{array}{l}\text { No. of partici- } \\
\text { pants }\end{array}$ & Statistical method & Effect size \\
\hline $\begin{array}{l}1 \text { Service use: 1. Average number of } \\
\text { days in hospital per month - by about } 24 \\
\text { months }\end{array}$ & 24 & 3595 & $\begin{array}{l}\text { Mean Difference (IV, Ran- } \\
\text { dom, 95\% Cl) }\end{array}$ & $-0.86[-1.37,-0.34]$ \\
\end{tabular}

Intensive case management for severe mental illness (Review) 


\begin{tabular}{|c|c|c|c|c|}
\hline Outcome or subgroup title & No. of studies & $\begin{array}{l}\text { No. of partici- } \\
\text { pants }\end{array}$ & Statistical method & Effect size \\
\hline 1.1 skewed data (sample size $\geqq 200$ ) & 5 & 1812 & $\begin{array}{l}\text { Mean Difference (IV, Ran- } \\
\text { dom, } 95 \% \mathrm{CI} \text { ) }\end{array}$ & $-0.46[-0.95,0.03]$ \\
\hline 1.2 skewed data (sample size $<200$ ) & 19 & 1783 & $\begin{array}{l}\text { Mean Difference (IV, Ran- } \\
\text { dom, } 95 \% \mathrm{CI} \text { ) }\end{array}$ & $-1.01[-1.74,-0.28]$ \\
\hline $\begin{array}{l}2 \text { Service use: } 1 \text {. Number of days in hos- } \\
\text { pital - by follow -up (skewed data, sample } \\
\text { size } \geqq 200 \text { ) }\end{array}$ & 1 & & $\begin{array}{l}\text { Mean Difference (IV, Ran- } \\
\text { dom, } 95 \% \mathrm{Cl} \text { ) }\end{array}$ & Subtotals only \\
\hline $\begin{array}{l}2.1 \text { by medium term FUP ( } 3 \text { years) (previ- } \\
\text { ous year) }\end{array}$ & 1 & 547 & $\begin{array}{l}\text { Mean Difference (IV, Ran- } \\
\text { dom, } 95 \% \mathrm{CI} \text { ) }\end{array}$ & $0.10[-10.26,10.46]$ \\
\hline $\begin{array}{l}2.2 \text { by long term FUP ( } 8 \text { years) (previous } \\
\text { year) }\end{array}$ & 1 & 547 & $\begin{array}{l}\text { Mean Difference (IV, Ran- } \\
\text { dom, } 95 \% \mathrm{CI} \text { ) }\end{array}$ & $4.30[-4.63,13.23]$ \\
\hline $\begin{array}{l}3 \text { Service use: } 2 \text {. Not remaining in contact } \\
\text { with psychiatric services }\end{array}$ & 9 & 1633 & $\begin{array}{l}\text { Risk Ratio (M-H, Random, } \\
95 \% \mathrm{Cl} \text { ) }\end{array}$ & $0.43[0.30,0.61]$ \\
\hline 3.1 by short term & 1 & 95 & $\begin{array}{l}\text { Risk Ratio (M-H, Random, } \\
95 \% \mathrm{Cl} \text { ) }\end{array}$ & $0.54[0.28,1.05]$ \\
\hline 3.2 by medium term & 3 & 1063 & $\begin{array}{l}\text { Risk Ratio (M-H, Random, } \\
95 \% \mathrm{Cl} \text { ) }\end{array}$ & $0.51[0.36,0.71]$ \\
\hline 3.3 by long term & 5 & 475 & $\begin{array}{l}\text { Risk Ratio (M-H, Random, } \\
95 \% \mathrm{Cl} \text { ) }\end{array}$ & $0.27[0.11,0.66]$ \\
\hline 4 Service use: 3a. Admitted to hospital & 16 & & $\begin{array}{l}\text { Risk Ratio (M-H, Random, } \\
95 \% \mathrm{Cl} \text { ) }\end{array}$ & Subtotals only \\
\hline 4.1 by short term & 2 & 244 & $\begin{array}{l}\text { Risk Ratio (M-H, Random, } \\
95 \% \mathrm{Cl})\end{array}$ & $0.61[0.22,1.69]$ \\
\hline 4.2 by medium term & 5 & 1303 & $\begin{array}{l}\text { Risk Ratio (M-H, Random, } \\
95 \% \mathrm{Cl} \text { ) }\end{array}$ & $0.85[0.77,0.93]$ \\
\hline 4.3 by long term & 11 & 1516 & $\begin{array}{l}\text { Risk Ratio (M-H, Random, } \\
95 \% \mathrm{Cl} \text { ) }\end{array}$ & $0.96[0.74,1.23]$ \\
\hline $\begin{array}{l}4.4 \text { by long term- during previous } 12 \\
\text { months }\end{array}$ & 1 & 547 & $\begin{array}{l}\text { Risk Ratio (M-H, Random, } \\
95 \% \mathrm{Cl})\end{array}$ & $0.67[0.52,0.86]$ \\
\hline $\begin{array}{l}4.5 \text { by short term FUP - unplanned admis- } \\
\text { sion through Emergency Department }\end{array}$ & 1 & 62 & $\begin{array}{l}\text { Risk Ratio (M-H, Random, } \\
95 \% \mathrm{Cl} \text { ) }\end{array}$ & $1.0[0.07,15.28]$ \\
\hline $\begin{array}{l}5 \text { Service use: } 3 b \text {. Average number of ad- } \\
\text { missions per month (skewed data) }\end{array}$ & & & Other data & No numeric data \\
\hline 5.1 by medium term & & & Other data & No numeric data \\
\hline 5.2 by long term & & & Other data & No numeric data \\
\hline $\begin{array}{l}6 \text { Service use: } 4 \text { a. Admitted to ER - by long } \\
\text { term }\end{array}$ & 1 & 178 & $\begin{array}{l}\text { Risk Ratio (M-H, Random, } \\
95 \% \mathrm{Cl} \text { ) }\end{array}$ & $1.13[0.72,1.76]$ \\
\hline
\end{tabular}




\section{Outcome or subgroup title} missions to ER (skewed data) - by medium term

\begin{tabular}{|c|c|c|c|c|}
\hline $\begin{array}{l}8 \text { Service use: } 5 \text { a. Received day hospital } \\
\text { care - by short term FUP }\end{array}$ & 1 & 62 & $\begin{array}{l}\text { Risk Ratio (M-H, Random, } \\
95 \% \mathrm{Cl} \text { ) }\end{array}$ & $2.0[0.19,20.93]$ \\
\hline $\begin{array}{l}9 \text { Service use: } 5 \text { b. Outpatient visits - by } \\
\text { short term FUP ( } 6 \text { months) }\end{array}$ & 1 & 62 & $\begin{array}{l}\text { Mean Difference (IV, Ran- } \\
\text { dom, } 95 \% \mathrm{CI} \text { ) }\end{array}$ & $0.29[-0.14,0.72]$ \\
\hline $\begin{array}{l}10 \text { Service use: } 5 c \text {. Outpatient visits - by } \\
\text { medium term (skewed data) }\end{array}$ & & & Other data & No numeric data \\
\hline $\begin{array}{l}11 \text { Service use: } 5 d \text {. Received home visits - } \\
\text { by short term FUP }\end{array}$ & 1 & 62 & $\begin{array}{l}\text { Mean Difference (IV, Ran- } \\
\text { dom, } 95 \% \mathrm{CI} \text { ) }\end{array}$ & $4.32[3.42,5.22]$ \\
\hline 12 Adverse event: $1 \mathrm{a}$. Death - any cause & 14 & & $\begin{array}{l}\text { Risk Ratio (M-H, Random, } \\
95 \% \mathrm{Cl} \text { ) }\end{array}$ & Subtotals only \\
\hline 12.1 by short term & 2 & 161 & $\begin{array}{l}\text { Risk Ratio (M-H, Random, } \\
95 \% \mathrm{Cl} \text { ) }\end{array}$ & $1.04[0.16,6.91]$ \\
\hline 12.2 by medium term & 6 & 901 & $\begin{array}{l}\text { Risk Ratio (M-H, Random, } \\
95 \% \mathrm{Cl} \text { ) }\end{array}$ & $0.78[0.23,2.62]$ \\
\hline 12.3 by long term & 9 & 1456 & $\begin{array}{l}\text { Risk Ratio (M-H, Random, } \\
95 \% \mathrm{Cl} \text { ) }\end{array}$ & $0.84[0.48,1.47]$ \\
\hline 12.4 by medium term FUP ( 3 years) & 1 & 547 & $\begin{array}{l}\text { Risk Ratio (M-H, Random, } \\
95 \% \mathrm{Cl} \text { ) }\end{array}$ & $0.59[0.22,1.61]$ \\
\hline 12.5 by long term FUP ( 8 years) & 1 & 547 & $\begin{array}{l}\text { Risk Ratio (M-H, Random, } \\
95 \% \mathrm{Cl} \text { ) }\end{array}$ & $0.92[0.45,1.88]$ \\
\hline 13 Adverse event: $1 \mathrm{~b}$. Death - suicide & 12 & & $\begin{array}{l}\text { Risk Ratio (M-H, Random, } \\
95 \% \mathrm{Cl} \text { ) }\end{array}$ & Subtotals only \\
\hline 13.1 by short term & 2 & 127 & $\begin{array}{l}\text { Risk Ratio (M-H, Random, } \\
95 \% \mathrm{Cl} \text { ) }\end{array}$ & $0.35[0.04,3.27]$ \\
\hline 13.2 by medium term & 4 & 819 & $\begin{array}{l}\text { Risk Ratio (M-H, Random, } \\
95 \% \mathrm{Cl} \text { ) }\end{array}$ & $0.98[0.17,5.60]$ \\
\hline 13.3 by long term & 9 & 1456 & $\begin{array}{l}\text { Risk Ratio (M-H, Random, } \\
95 \% \mathrm{Cl} \text { ) }\end{array}$ & $0.68[0.31,1.51]$ \\
\hline 13.4 by medium term FUP ( 3 years) & 1 & 547 & $\begin{array}{l}\text { Risk Ratio (M-H, Random, } \\
95 \% \mathrm{Cl} \text { ) }\end{array}$ & $0.74[0.17,3.28]$ \\
\hline 14 Global state: 1 . Leaving the study early & 21 & & $\begin{array}{l}\text { Risk Ratio (M-H, Random, } \\
95 \% \mathrm{Cl} \text { ) }\end{array}$ & Subtotals only \\
\hline 14.1 by short term & 5 & 598 & $\begin{array}{l}\text { Risk Ratio (M-H, Random, } \\
95 \% \mathrm{Cl} \text { ) }\end{array}$ & $0.79[0.44,1.41]$ \\
\hline
\end{tabular}




\begin{tabular}{|c|c|c|c|c|}
\hline Outcome or subgroup title & No. of studies & $\begin{array}{l}\text { No. of partici- } \\
\text { pants }\end{array}$ & Statistical method & Effect size \\
\hline 14.2 by medium term & 8 & 1699 & $\begin{array}{l}\text { Risk Ratio (M-H, Random, } \\
95 \% \mathrm{Cl} \text { ) }\end{array}$ & $0.60[0.51,0.70]$ \\
\hline 14.3 by long term & 13 & 1798 & $\begin{array}{l}\text { Risk Ratio (M-H, Random, } \\
95 \% \mathrm{Cl} \text { ) }\end{array}$ & $0.68[0.58,0.79]$ \\
\hline 14.4 by medium term FUP ( 3 years) & 1 & 547 & $\begin{array}{l}\text { Risk Ratio (M-H, Random, } \\
95 \% \mathrm{CI} \text { ) }\end{array}$ & $1.01[0.84,1.21]$ \\
\hline 14.5 by long term FUP ( 8 years) & 1 & 547 & $\begin{array}{l}\text { Risk Ratio (M-H, Random, } \\
95 \% \mathrm{CI})\end{array}$ & $0.88[0.70,1.09]$ \\
\hline $\begin{array}{l}15 \text { Global state: } 2 \text {. Average endpoint score } \\
\text { (GAF, high = good) }\end{array}$ & 5 & & $\begin{array}{l}\text { Mean Difference (IV, Ran- } \\
\text { dom, } 95 \% \mathrm{CI} \text { ) }\end{array}$ & Subtotals only \\
\hline 15.1 by short term & 4 & 797 & $\begin{array}{l}\text { Mean Difference (IV, Ran- } \\
\text { dom, } 95 \% \mathrm{CI} \text { ) }\end{array}$ & $2.07[0.28,3.86]$ \\
\hline 15.2 by medium term & 3 & 722 & $\begin{array}{l}\text { Mean Difference (IV, Ran- } \\
\text { dom, } 95 \% \mathrm{CI} \text { ) }\end{array}$ & $0.09[-3.11,3.28]$ \\
\hline 15.3 by long term & 5 & 818 & $\begin{array}{l}\text { Mean Difference (IV, Ran- } \\
\text { dom, } 95 \% \mathrm{CI} \text { ) }\end{array}$ & $3.41[1.66,5.16]$ \\
\hline $\begin{array}{l}16 \text { Global state: } 3 \text {. Not compliant with } \\
\text { medication - by long term }\end{array}$ & 1 & 71 & $\begin{array}{l}\text { Risk Ratio (M-H, Random, } \\
95 \% \mathrm{Cl} \text { ) }\end{array}$ & $0.35[0.15,0.81]$ \\
\hline $\begin{array}{l}17 \text { Social functioning: } 1 \text { a. Contact with le- } \\
\text { gal system (various measurements) }\end{array}$ & 11 & & $\begin{array}{l}\text { Risk Ratio (M-H, Random, } \\
95 \% \mathrm{Cl})\end{array}$ & Subtotals only \\
\hline $\begin{array}{l}17.1 \text { by short term - contact with the po- } \\
\text { lice }\end{array}$ & 1 & 61 & $\begin{array}{l}\text { Risk Ratio (M-H, Random, } \\
95 \% \mathrm{Cl})\end{array}$ & $2.57[0.73,9.04]$ \\
\hline 17.2 by medium term - arrested & 3 & 604 & $\begin{array}{l}\text { Risk Ratio (M-H, Random, } \\
95 \% \mathrm{Cl} \text { ) }\end{array}$ & $1.08[0.61,1.90]$ \\
\hline $\begin{array}{l}17.3 \text { by medium term - contact with the } \\
\text { police }\end{array}$ & 1 & 88 & $\begin{array}{l}\text { Risk Ratio (M-H, Random, } \\
95 \% \mathrm{Cl} \text { ) }\end{array}$ & $0.23[0.09,0.55]$ \\
\hline 17.4 by medium term - imprisoned & 4 & 804 & $\begin{array}{l}\text { Risk Ratio (M-H, Random, } \\
95 \% \mathrm{Cl} \text { ) }\end{array}$ & $0.80[0.39,1.64]$ \\
\hline 17.5 by long term - arrested & 1 & 178 & $\begin{array}{l}\text { Risk Ratio (M-H, Random, } \\
95 \% \mathrm{Cl})\end{array}$ & $0.66[0.32,1.37]$ \\
\hline 17.6 by long term - imprisoned & 5 & 908 & $\begin{array}{l}\text { Risk Ratio (M-H, Random, } \\
95 \% \mathrm{Cl} \text { ) }\end{array}$ & $0.86[0.45,1.65]$ \\
\hline $\begin{array}{l}18 \text { Social functioning: } 1 \text { b. Mean contacts } \\
\text { with legal system (skewed data) - by } \\
\text { medium term }\end{array}$ & & & Other data & No numeric data \\
\hline 18.1 Bookings & & & Other data & No numeric data \\
\hline 18.2 Jail days & & & Other data & No numeric data \\
\hline
\end{tabular}




\begin{tabular}{|c|c|c|c|c|}
\hline Outcome or subgroup title & No. of studies & $\begin{array}{l}\text { No. of partici- } \\
\text { pants }\end{array}$ & Statistical method & Effect size \\
\hline 18.3 Convictions & & & Other data & No numeric data \\
\hline $\begin{array}{l}19 \text { Social functioning: } 2 . \text { Employment sta- } \\
\text { tus (various measurements) }\end{array}$ & 6 & & $\begin{array}{l}\text { Risk Ratio (M-H, Random, } \\
95 \% \mathrm{Cl})\end{array}$ & Subtotals only \\
\hline $\begin{array}{l}19.1 \text { by medium term - not competitively } \\
\text { employed at the end of the trial }\end{array}$ & 1 & 88 & $\begin{array}{l}\text { Risk Ratio (M-H, Random, } \\
95 \% \mathrm{Cl})\end{array}$ & $1.00[0.91,1.10]$ \\
\hline $\begin{array}{l}19.2 \text { by medium term - not employed at } \\
\text { the end of the trial }\end{array}$ & 4 & 1136 & $\begin{array}{l}\text { Risk Ratio (M-H, Random, } \\
95 \% \mathrm{Cl} \text { ) }\end{array}$ & $0.89[0.79,1.00]$ \\
\hline $\begin{array}{l}19.3 \text { by long term - not employed at the } \\
\text { end of the trial }\end{array}$ & 4 & 1129 & $\begin{array}{l}\text { Risk Ratio (M-H, Random, } \\
95 \% \mathrm{Cl})\end{array}$ & $0.70[0.49,1.00]$ \\
\hline $\begin{array}{l}19.4 \text { by long term - not working/studying } \\
\text { in the previous year }\end{array}$ & 1 & 547 & $\begin{array}{l}\text { Risk Ratio (M-H, Random, } \\
95 \% \mathrm{CI})\end{array}$ & $0.86[0.74,0.99]$ \\
\hline $\begin{array}{l}19.5 \text { by medium term FUP ( } 3 \text { years) - not } \\
\text { working/studying in the previous year }\end{array}$ & 1 & 547 & $\begin{array}{l}\text { Risk Ratio (M-H, Random, } \\
95 \% \mathrm{Cl})\end{array}$ & $1.02[0.90,1.16]$ \\
\hline $\begin{array}{l}19.6 \text { by long term FUP ( } 8 \text { years) - not } \\
\text { working/studying in the previous year }\end{array}$ & 1 & 547 & $\begin{array}{l}\text { Risk Ratio (M-H, Random, } \\
95 \% \mathrm{Cl})\end{array}$ & $0.99[0.88,1.11]$ \\
\hline $\begin{array}{l}20 \text { Social functioning: } 3 \text { a. Accommoda- } \\
\text { tion status (various measurements) }\end{array}$ & 10 & & $\begin{array}{l}\text { Risk Ratio (M-H, Random, } \\
95 \% \mathrm{Cl} \text { ) }\end{array}$ & Subtotals only \\
\hline 20.1 by short term - homelessness & 1 & 95 & $\begin{array}{l}\text { Risk Ratio (M-H, Random, } \\
95 \% \mathrm{Cl})\end{array}$ & $0.04[0.00,0.70]$ \\
\hline 20.2 by medium term - homelessness & 1 & 88 & $\begin{array}{l}\text { Risk Ratio (M-H, Random, } \\
95 \% \mathrm{Cl})\end{array}$ & $0.32[0.03,2.95]$ \\
\hline $\begin{array}{l}20.3 \text { by medium term - not living indepen- } \\
\text { dently }\end{array}$ & 5 & 1303 & $\begin{array}{l}\text { Risk Ratio (M-H, Random, } \\
95 \% \mathrm{Cl})\end{array}$ & $0.80[0.66,0.97]$ \\
\hline 20.4 by long term - homelessness & 3 & 418 & $\begin{array}{l}\text { Risk Ratio (M-H, Random, } \\
95 \% \mathrm{CI})\end{array}$ & $0.78[0.34,1.82]$ \\
\hline $\begin{array}{l}20.5 \text { by long term - not living indepen- } \\
\text { dently }\end{array}$ & 4 & 1185 & $\begin{array}{l}\text { Risk Ratio (M-H, Random, } \\
95 \% \mathrm{Cl})\end{array}$ & $0.65[0.49,0.88]$ \\
\hline $\begin{array}{l}20.6 \text { by long term - not living in stable ac- } \\
\text { commodation }\end{array}$ & 1 & 168 & $\begin{array}{l}\text { Risk Ratio (M-H, Random, } \\
95 \% \mathrm{Cl})\end{array}$ & $0.80[0.65,0.98]$ \\
\hline $\begin{array}{l}21 \text { Social functioning: } 3 b \text {. Accomodation } \\
\text { status: mean number of days in support- } \\
\text { ed house (skewed data, sample size } \geqq \\
\text { 200) }\end{array}$ & 1 & & $\begin{array}{l}\text { Mean Difference (IV, Ran- } \\
\text { dom, } 95 \% \mathrm{CI} \text { ) }\end{array}$ & Subtotals only \\
\hline 21.1 by long term (previous year) & 1 & 547 & $\begin{array}{l}\text { Mean Difference (IV, Ran- } \\
\text { dom, } 95 \% \mathrm{CI} \text { ) }\end{array}$ & $0.30[-13.98,14.58]$ \\
\hline $\begin{array}{l}21.2 \text { by medium term FUP ( } 3 \text { years) (previ- } \\
\text { ous year) }\end{array}$ & 1 & 547 & $\begin{array}{l}\text { Mean Difference (IV, Ran- } \\
\text { dom, } 95 \% \mathrm{CI} \text { ) }\end{array}$ & $\begin{array}{l}-22.20[-38.47 \\
-5.93]\end{array}$ \\
\hline
\end{tabular}




\begin{tabular}{|c|c|c|c|c|}
\hline Outcome or subgroup title & No. of studies & $\begin{array}{l}\text { No. of partici- } \\
\text { pants }\end{array}$ & Statistical method & Effect size \\
\hline $\begin{array}{l}21.3 \text { by long term FUP ( } 8 \text { years) (previous } \\
\text { year) }\end{array}$ & 1 & 547 & $\begin{array}{l}\text { Mean Difference (IV, Ran- } \\
\text { dom, } 95 \% \mathrm{CI} \text { ) }\end{array}$ & $-6.70[-19.35,5.95]$ \\
\hline $\begin{array}{l}22 \text { Social functioning: } 3 c \text {. Accommo- } \\
\text { dation status (various measurements, } \\
\text { skewed data) }\end{array}$ & & & Other data & No numeric data \\
\hline $\begin{array}{l}22.1 \text { by medium term - average days per } \\
\text { month in stable accommodation }\end{array}$ & & & Other data & No numeric data \\
\hline $\begin{array}{l}22.2 \text { by long term - average days per } \\
\text { month in sheltered homes }\end{array}$ & & & Other data & No numeric data \\
\hline $\begin{array}{l}23 \text { Social functioning: } 4 \text { a. Substance } \\
\text { abuse }\end{array}$ & 1 & & $\begin{array}{l}\text { Risk Ratio (M-H, Random, } \\
95 \% \mathrm{Cl} \text { ) }\end{array}$ & Subtotals only \\
\hline 23.1 alcohol abuse - by long term & 1 & 547 & $\begin{array}{l}\text { Risk Ratio (M-H, Random, } \\
95 \% \mathrm{Cl} \text { ) }\end{array}$ & $0.55[0.26,1.17]$ \\
\hline 23.2 illicit drug abuse - by long term & 1 & 547 & $\begin{array}{l}\text { Risk Ratio (M-H, Random, } \\
95 \% \mathrm{Cl} \text { ) }\end{array}$ & $0.96[0.63,1.47]$ \\
\hline $\begin{array}{l}23.3 \text { substance abuse - by medium term } \\
\text { FUP ( } 3 \text { years) }\end{array}$ & 1 & 547 & $\begin{array}{l}\text { Risk Ratio (M-H, Random, } \\
95 \% \mathrm{Cl} \text { ) }\end{array}$ & $0.91[0.67,1.24]$ \\
\hline $\begin{array}{l}24 \text { Social functioning: } 4 b \text {. Substance } \\
\text { abuse (DALI, skewness not detectable) - } \\
\text { by medium term }\end{array}$ & & & Other data & No numeric data \\
\hline $\begin{array}{l}24.1 \text { alcohol abuse (DALI, }-4 \text { to }+6 \text {, high }= \\
\text { worse) }\end{array}$ & & & Other data & No numeric data \\
\hline $\begin{array}{l}24.2 \text { drug abuse (DALI, }-4 \text { to }+4 \text {, high }= \\
\text { worse) }\end{array}$ & & & Other data & No numeric data \\
\hline $\begin{array}{l}25 \text { Social functioning: } 4 c \text {. Substance } \\
\text { abuse - days used per month (skewed da- } \\
\text { ta) }\end{array}$ & & & Other data & No numeric data \\
\hline 25.1 by medium term & & & Other data & No numeric data \\
\hline 25.2 by long term & & & Other data & No numeric data \\
\hline $\begin{array}{l}26 \text { Social functioning: } 5 a \text {. Average end- } \\
\text { point score (various scales) }\end{array}$ & 3 & & $\begin{array}{l}\text { Mean Difference (IV, Ran- } \\
\text { dom, } 95 \% \mathrm{CI} \text { ) }\end{array}$ & Subtotals only \\
\hline 26.1 by short term (RFS, low = poor) & 1 & 80 & $\begin{array}{l}\text { Mean Difference (IV, Ran- } \\
\text { dom, } 95 \% \mathrm{CI} \text { ) }\end{array}$ & $-0.62[-2.23,0.99]$ \\
\hline $\begin{array}{l}26.2 \text { by short term (SAS-adapted version, } \\
\text { low }=\text { poor) }\end{array}$ & 1 & 80 & $\begin{array}{l}\text { Mean Difference (IV, Ran- } \\
\text { dom, } 95 \% \mathrm{CI} \text { ) }\end{array}$ & $-3.34[-7.55,0.87]$ \\
\hline $\begin{array}{l}26.3 \text { by medium term - social role perfor- } \\
\text { mance (DAS, high = poor) }\end{array}$ & 1 & 55 & $\begin{array}{l}\text { Mean Difference (IV, Ran- } \\
\text { dom, } 95 \% \mathrm{CI} \text { ) }\end{array}$ & $0.10[-0.40,0.60]$ \\
\hline
\end{tabular}




\begin{tabular}{|c|c|c|c|c|}
\hline Outcome or subgroup title & No. of studies & $\begin{array}{l}\text { No. of partici- } \\
\text { pants }\end{array}$ & Statistical method & Effect size \\
\hline 26.4 by medium term (RFS, low $=$ poor) & 1 & 80 & $\begin{array}{l}\text { Mean Difference (IV, Ran- } \\
\text { dom, } 95 \% \mathrm{CI} \text { ) }\end{array}$ & $-0.86[-2.72,1.00]$ \\
\hline $\begin{array}{l}26.5 \text { by medium term (SAS-adapted ver- } \\
\text { sion, low = poor) }\end{array}$ & 1 & 80 & $\begin{array}{l}\text { Mean Difference (IV, Ran- } \\
\text { dom, } 95 \% \mathrm{CI} \text { ) }\end{array}$ & $-3.30[-7.83,1.23]$ \\
\hline $\begin{array}{l}26.6 \text { by long term - social role perfor- } \\
\text { mance (DAS, high = poor) }\end{array}$ & 1 & 58 & $\begin{array}{l}\text { Mean Difference (IV, Ran- } \\
\text { dom, } 95 \% \mathrm{CI} \text { ) }\end{array}$ & $-0.20[-0.67,0.27]$ \\
\hline 26.7 by long term (ISSI, low = poor) & 1 & 62 & $\begin{array}{l}\text { Mean Difference (IV, Ran- } \\
\text { dom, } 95 \% \mathrm{CI} \text { ) }\end{array}$ & $3.20[0.11,6.29]$ \\
\hline 26.8 by long term (RFS, low = poor) & 1 & 80 & $\begin{array}{l}\text { Mean Difference (IV, Ran- } \\
\text { dom, } 95 \% \mathrm{CI} \text { ) }\end{array}$ & $-2.35[-4.05,-0.65]$ \\
\hline $\begin{array}{l}26.9 \text { by long term (SAS-adapted version, } \\
\text { low }=\text { poor) }\end{array}$ & 1 & 80 & $\begin{array}{l}\text { Mean Difference (IV, Ran- } \\
\text { dom, } 95 \% \mathrm{Cl} \text { ) }\end{array}$ & $-2.75[-7.13,1.63]$ \\
\hline $\begin{array}{l}26.10 \text { by long term (Strauss-Carpenter } \\
\text { Scale, low }=\text { poor) }\end{array}$ & 1 & 60 & $\begin{array}{l}\text { Mean Difference (IV, Ran- } \\
\text { dom, } 95 \% \mathrm{CI} \text { ) }\end{array}$ & $0.10[-1.17,1.37]$ \\
\hline $\begin{array}{l}27 \text { Social functioning: } 5 \mathrm{~b} \text {. Average end- } \\
\text { point score (various scales, skewed data) }\end{array}$ & & & Other data & No numeric data \\
\hline 27.1 by short term (SAS, high = poor) & & & Other data & No numeric data \\
\hline 27.2 by medium term (SAS, high = poor) & & & Other data & No numeric data \\
\hline 27.3 by long term (SAS, high = poor) & & & Other data & No numeric data \\
\hline 27.4 by long term $(\mathrm{REHAB}$, high $=$ poor $)$ & & & Other data & No numeric data \\
\hline $\begin{array}{l}28 \text { Mental state: } 1 \text { a. General symptoms - } \\
\text { average endpoint score (various scales) }\end{array}$ & 4 & & $\begin{array}{l}\text { Mean Difference (IV, Ran- } \\
\text { dom, } 95 \% \mathrm{CI} \text { ) }\end{array}$ & Subtotals only \\
\hline $\begin{array}{l}28.1 \text { by short term (BPRS- } 18 \text { items, high = } \\
\text { poor) }\end{array}$ & 2 & 668 & $\begin{array}{l}\text { Mean Difference (IV, Ran- } \\
\text { dom, } 95 \% \mathrm{CI} \text { ) }\end{array}$ & $-1.56[-6.85,3.73]$ \\
\hline 28.2 by short term (BSI, high = poor) & 2 & 668 & $\begin{array}{l}\text { Mean Difference (IV, Ran- } \\
\text { dom, } 95 \% \mathrm{CI} \text { ) }\end{array}$ & $-0.06[-0.19,0.06]$ \\
\hline 28.3 by short term (CSI, low = poor) & 1 & 125 & $\begin{array}{l}\text { Mean Difference (IV, Ran- } \\
\text { dom, } 95 \% \mathrm{CI} \text { ) }\end{array}$ & $-0.56[-0.84,-0.28]$ \\
\hline $\begin{array}{l}28.4 \text { by medium term (BPRS-18 items, } \\
\text { high = poor) }\end{array}$ & 2 & 662 & $\begin{array}{l}\text { Mean Difference (IV, Ran- } \\
\text { dom, } 95 \% \mathrm{CI} \text { ) }\end{array}$ & $-0.96[-2.42,0.51]$ \\
\hline 28.5 by medium term (BSI, high = poor) & 2 & 662 & $\begin{array}{l}\text { Mean Difference (IV, Ran- } \\
\text { dom, } 95 \% \mathrm{CI} \text { ) }\end{array}$ & $-0.02[-0.15,0.10]$ \\
\hline 28.6 medium term (CSI, low = poor) & 1 & 125 & $\begin{array}{l}\text { Mean Difference (IV, Ran- } \\
\text { dom, } 95 \% \mathrm{CI} \text { ) }\end{array}$ & $-0.35[-0.65,-0.05]$ \\
\hline $\begin{array}{l}28.7 \text { by long term (BPRS-18 items, high }= \\
\text { poor) }\end{array}$ & 3 & 777 & $\begin{array}{l}\text { Mean Difference (IV, Ran- } \\
\text { dom, } 95 \% \mathrm{CI} \text { ) }\end{array}$ & $-1.48[-3.69,0.74]$ \\
\hline
\end{tabular}




\begin{tabular}{|c|c|c|c|c|}
\hline Outcome or subgroup title & No. of studies & $\begin{array}{l}\text { No. of partici- } \\
\text { pants }\end{array}$ & Statistical method & Effect size \\
\hline 28.8 by long term (BSI, high = poor) & 2 & 647 & $\begin{array}{l}\text { Mean Difference (IV, Ran- } \\
\text { dom, } 95 \% \mathrm{CI} \text { ) }\end{array}$ & $-0.18[-0.31,-0.06]$ \\
\hline $\begin{array}{l}29 \text { Mental state: } 1 \text { b. General symptoms } \\
\text { - mean change from baseline (CSI, low = } \\
\text { poor ) - by long term }\end{array}$ & 1 & 168 & $\begin{array}{l}\text { Mean Difference (IV, Ran- } \\
\text { dom, } 95 \% \mathrm{CI} \text { ) }\end{array}$ & $-0.32[-0.53,-0.11]$ \\
\hline $\begin{array}{l}30 \text { Mental state: } 1 c . \text { General symptoms - } \\
\text { average endpoint score (various scales, } \\
\text { skewed data) }\end{array}$ & & & Other data & No numeric data \\
\hline $\begin{array}{l}30.1 \text { by short term (BPRS- } 24 \text { items, high }= \\
\text { poor) }\end{array}$ & & & Other data & No numeric data \\
\hline 30.2 by short term (PSE, high = poor) & & & Other data & No numeric data \\
\hline $\begin{array}{l}30.4 \text { by medium term (BPRS- } 24 \text { items, } \\
\text { high = poor) }\end{array}$ & & & Other data & No numeric data \\
\hline 30.5 by medium term (CPRS, high = poor) & & & Other data & No numeric data \\
\hline 30.6 by medium term (PSE, high = poor) & & & Other data & No numeric data \\
\hline $\begin{array}{l}30.8 \text { by long term (BPRS- } 18 \text { items, high }= \\
\text { poor) }\end{array}$ & & & Other data & No numeric data \\
\hline $\begin{array}{l}30.9 \text { by long term (BPRS- } 24 \text { items, high }= \\
\text { poor) }\end{array}$ & & & Other data & No numeric data \\
\hline 30.10 by long term (CPRS, high = poor) & & & Other data & No numeric data \\
\hline 30.11 by long term (PSE, high = poor) & & & Other data & No numeric data \\
\hline 30.12 by long term (SCL-90, high = poor) & & & Other data & No numeric data \\
\hline $\begin{array}{l}31 \text { Mental state: } 2 a \text {. Specific symptoms - } \\
\text { depression at follow up interview }\end{array}$ & 1 & & $\begin{array}{l}\text { Risk Ratio (M-H, Random, } \\
95 \% \mathrm{Cl} \text { ) }\end{array}$ & Subtotals only \\
\hline 31.1 by medium term & 1 & 547 & $\begin{array}{l}\text { Risk Ratio (M-H, Random, } \\
95 \% \mathrm{Cl} \text { ) }\end{array}$ & $0.77[0.56,1.04]$ \\
\hline 31.2 by long term & 1 & 547 & $\begin{array}{l}\text { Risk Ratio (M-H, Random, } \\
95 \% \mathrm{Cl} \text { ) }\end{array}$ & $0.83[0.57,1.21]$ \\
\hline 31.3 by medium term FUP ( 3 years) & 1 & 547 & $\begin{array}{l}\text { Risk Ratio (M-H, Random, } \\
95 \% \mathrm{Cl} \text { ) }\end{array}$ & $1.25[0.91,1.72]$ \\
\hline $\begin{array}{l}32 \text { Mental state: } 2 \text { b. Specific symptoms - } \\
\text { average endpoint score (various scales, } \\
\text { skewed data, sample size } \geqq 200 \text { ) }\end{array}$ & 1 & & $\begin{array}{l}\text { Mean Difference (IV, Ran- } \\
\text { dom, } 95 \% \mathrm{CI} \text { ) }\end{array}$ & Subtotals only \\
\hline $\begin{array}{l}32.1 \text { by long term - positive symptoms } \\
\text { (SAPS, high = poor) }\end{array}$ & 1 & 547 & $\begin{array}{l}\text { Mean Difference (IV, Ran- } \\
\text { dom, } 95 \% \mathrm{CI} \text { ) }\end{array}$ & $-0.22[-0.45,0.01]$ \\
\hline
\end{tabular}




\begin{tabular}{|c|c|c|c|c|}
\hline Outcome or subgroup title & No. of studies & $\begin{array}{l}\text { No. of partici- } \\
\text { pants }\end{array}$ & Statistical method & Effect size \\
\hline $\begin{array}{l}32.2 \text { by long term }- \text { negative symptoms } \\
\text { (SANS, high = poor) }\end{array}$ & 1 & 547 & $\begin{array}{l}\text { Mean Difference (IV, Ran- } \\
\text { dom, } 95 \% \mathrm{Cl} \text { ) }\end{array}$ & $-0.42[-0.62,-0.22]$ \\
\hline $\begin{array}{l}32.3 \text { by medium term FUP ( } 3 \text { years) - posi- } \\
\text { tive symptoms (SAPS, high = poor) }\end{array}$ & 1 & 547 & $\begin{array}{l}\text { Mean Difference (IV, Ran- } \\
\text { dom, } 95 \% \mathrm{Cl} \text { ) }\end{array}$ & $0.12[-0.15,0.39]$ \\
\hline $\begin{array}{l}32.4 \text { by medium term FUP ( } 3 \text { years) - neg- } \\
\text { ative symptoms (SANS, high = poor) }\end{array}$ & 1 & 547 & $\begin{array}{l}\text { Mean Difference (IV, Ran- } \\
\text { dom, } 95 \% \mathrm{Cl} \text { ) }\end{array}$ & $-0.10[-0.33,0.13]$ \\
\hline $\begin{array}{l}32.5 \text { by long term FUP ( } 8 \text { years) - positive } \\
\text { symptoms (SAPS, high = poor) }\end{array}$ & 1 & 547 & $\begin{array}{l}\text { Mean Difference (IV, Ran- } \\
\text { dom, } 95 \% \mathrm{Cl} \text { ) }\end{array}$ & $0.03[-0.21,0.27]$ \\
\hline $\begin{array}{l}32.6 \text { by long term FUP ( } 8 \text { years) - negative } \\
\text { symptoms (SANS, high = poor) }\end{array}$ & 1 & 547 & $\begin{array}{l}\text { Mean Difference (IV, Ran- } \\
\text { dom, } 95 \% \mathrm{CI} \text { ) }\end{array}$ & $0.06[-0.13,0.25]$ \\
\hline $\begin{array}{l}33 \text { Mental state: } 2 \text { c. Specific symptoms - } \\
\text { average endpoint score (various scales, } \\
\text { skewed data) }\end{array}$ & & & Other data & No numeric data \\
\hline $\begin{array}{l}33.3 \text { by medium term - depression symp- } \\
\text { toms ( } \mathrm{BDI} \text {, high = poor) }\end{array}$ & & & Other data & No numeric data \\
\hline $\begin{array}{l}33.4 \text { by medium term - negative symp- } \\
\text { toms (SANS, high = poor) }\end{array}$ & & & Other data & No numeric data \\
\hline $\begin{array}{l}33.7 \text { by long term - depression symptoms } \\
\text { (BDI, high = poor) }\end{array}$ & & & Other data & No numeric data \\
\hline $\begin{array}{l}33.11 \text { by long term - negative symptoms } \\
\text { (SANS, high = poor) }\end{array}$ & & & Other data & No numeric data \\
\hline $\begin{array}{l}34 \text { Behaviour: } 1 \text {. Specific behaviour - self- } \\
\text { harm }\end{array}$ & 3 & & $\begin{array}{l}\text { Risk Ratio (M-H, Random, } \\
95 \% \mathrm{Cl})\end{array}$ & Subtotals only \\
\hline 34.1 by medium term & 2 & 620 & $\begin{array}{l}\text { Risk Ratio (M-H, Random, } \\
95 \% \mathrm{Cl})\end{array}$ & $0.99[0.61,1.59]$ \\
\hline 34.2 by long term & 1 & 123 & $\begin{array}{l}\text { Risk Ratio (M-H, Random, } \\
95 \% \mathrm{Cl})\end{array}$ & $0.95[0.14,6.55]$ \\
\hline $\begin{array}{l}34.3 \text { attempted suicide - by long term } \\
\text { (during last } 12 \text { months) }\end{array}$ & 1 & 547 & $\begin{array}{l}\text { Risk Ratio (M-H, Random, } \\
95 \% \mathrm{Cl})\end{array}$ & $0.81[0.47,1.38]$ \\
\hline $\begin{array}{l}34.4 \text { attempted suicide - by medium term } \\
\text { FUP ( } 3 \text { years) (during last } 3 \text { years) }\end{array}$ & 1 & 547 & $\begin{array}{l}\text { Risk Ratio (M-H, Random, } \\
95 \% \mathrm{Cl})\end{array}$ & $0.95[0.56,1.62]$ \\
\hline $\begin{array}{l}35 \text { Behaviour: } 2 \text {. Social behaviour - aver- } \\
\text { age endpoint score (SBS, high = poor) }\end{array}$ & & & Other data & No numeric data \\
\hline 35.1 by medium term & & & Other data & No numeric data \\
\hline 35.2 by long term & & & Other data & No numeric data \\
\hline $\begin{array}{l}36 \text { Quality of life: 1a. Average endpoint } \\
\text { score (various scales) }\end{array}$ & 7 & & $\begin{array}{l}\text { Mean Difference (IV, Ran- } \\
\text { dom, } 95 \% \mathrm{Cl} \text { ) }\end{array}$ & Subtotals only \\
\hline
\end{tabular}




\begin{tabular}{|c|c|c|c|c|}
\hline Outcome or subgroup title & No. of studies & $\begin{array}{l}\text { No. of partici- } \\
\text { pants }\end{array}$ & Statistical method & Effect size \\
\hline $\begin{array}{l}36.1 \text { by short term - general well-being } \\
\text { (QOLI, high = better) }\end{array}$ & 1 & 125 & $\begin{array}{l}\text { Mean Difference (IV, Ran- } \\
\text { dom, } 95 \% \mathrm{CI} \text { ) }\end{array}$ & $0.53[0.09,0.97]$ \\
\hline $\begin{array}{l}36.2 \text { by medium term (LQoLP, high = bet- } \\
\text { ter) }\end{array}$ & 1 & 52 & $\begin{array}{l}\text { Mean Difference (IV, Ran- } \\
\text { dom, } 95 \% \mathrm{CI} \text { ) }\end{array}$ & $0.09[-0.60,0.78]$ \\
\hline $\begin{array}{l}36.3 \text { by medium term (MANSA - range } 1-7 \text {, } \\
\text { high = better) }\end{array}$ & 1 & 81 & $\begin{array}{l}\text { Mean Difference (IV, Ran- } \\
\text { dom, } 95 \% \mathrm{CI} \text { ) }\end{array}$ & $0.20[-0.29,0.69]$ \\
\hline 36.4 by long term (LQoLP, high = better) & 3 & 274 & $\begin{array}{l}\text { Mean Difference (IV, Ran- } \\
\text { dom, } 95 \% \mathrm{CI} \text { ) }\end{array}$ & $-0.13[-0.38,0.12]$ \\
\hline 36.5 by long term (QOLI, high = better) & 2 & 132 & $\begin{array}{l}\text { Mean Difference (IV, Ran- } \\
\text { dom, } 95 \% \mathrm{CI} \text { ) }\end{array}$ & $0.09[-0.24,0.42]$ \\
\hline $\begin{array}{l}37 \text { Quality of life: } 1 \text { b. Mean change from } \\
\text { baseline (QOLI, high = better, skewed da- } \\
\text { ta) - by long term }\end{array}$ & & & Other data & No numeric data \\
\hline $\begin{array}{l}38 \text { Participant satisfaction: 1a. Average } \\
\text { endpoint score (CSQ, high = better) }\end{array}$ & 3 & & $\begin{array}{l}\text { Mean Difference (IV, Ran- } \\
\text { dom, } 95 \% \mathrm{CI} \text { ) }\end{array}$ & Subtotals only \\
\hline 38.1 by short term & 1 & 61 & $\begin{array}{l}\text { Mean Difference (IV, Ran- } \\
\text { dom, } 95 \% \mathrm{Cl} \text { ) }\end{array}$ & $6.20[2.60,9.80]$ \\
\hline 38.2 by medium term & 2 & 500 & $\begin{array}{l}\text { Mean Difference (IV, Ran- } \\
\text { dom, } 95 \% \mathrm{CI} \text { ) }\end{array}$ & $1.93[0.86,3.01]$ \\
\hline 38.3 by long term & 2 & 423 & $\begin{array}{l}\text { Mean Difference (IV, Ran- } \\
\text { dom, } 95 \% \mathrm{CI} \text { ) }\end{array}$ & $3.23[2.31,4.14]$ \\
\hline $\begin{array}{l}39 \text { Participants satisfaction: } 1 \text { b. Aver- } \\
\text { age endpoint score (CSQ, high = better, } \\
\text { skewed data) - by short term }\end{array}$ & & & Other data & No numeric data \\
\hline $\begin{array}{l}40 \text { Participants need: } 1 \text {. Average endpoint } \\
\text { score (various scales, skewed data) }\end{array}$ & & & Other data & No numeric data \\
\hline $\begin{array}{l}40.1 \text { by medium term }- \text { met needs } \\
\text { (CANSAS, high = better) }\end{array}$ & & & Other data & No numeric data \\
\hline $\begin{array}{l}40.2 \text { by medium term }- \text { unmet needs } \\
\text { (CANSAS, high }=\text { poor) }\end{array}$ & & & Other data & No numeric data \\
\hline 40.4 by long term (CAN, high = poor) & & & Other data & No numeric data \\
\hline $\begin{array}{l}41 \text { Costs: 1a. Direct costs of psychiatric } \\
\text { hospital care - by medium term (Unit cost } \\
=\text { USD, fiscal year 1990) }\end{array}$ & 2 & 426 & $\begin{array}{l}\text { Mean Difference (IV, Ran- } \\
\text { dom, } 95 \% \mathrm{CI} \text { ) }\end{array}$ & $\begin{array}{l}-143.74[-272.40 \\
-15.08]\end{array}$ \\
\hline $\begin{array}{l}42 \text { Costs: } 1 \text { b. Direct costs of psychiatric } \\
\text { hospital care - skewed data }\end{array}$ & & & Other data & No numeric data \\
\hline 42.1 by medium term & & & Other data & No numeric data \\
\hline
\end{tabular}




\begin{tabular}{|c|c|c|c|c|}
\hline Outcome or subgroup title & No. of studies & $\begin{array}{l}\text { No. of partici- } \\
\text { pants }\end{array}$ & Statistical method & Effect size \\
\hline 42.2 by long term & & & Other data & No numeric data \\
\hline $\begin{array}{l}43 \text { Costs: } 2 \text { a. Direct healthcare costs - by } \\
\text { long term (Unit cost = USD, fiscal year } \\
\text { 1988) }\end{array}$ & 2 & 873 & $\begin{array}{l}\text { Mean Difference (IV, Ran- } \\
\text { dom, } 95 \% \mathrm{CI} \text { ) }\end{array}$ & $\begin{array}{l}-529.24[-2143.59 \\
1085.10]\end{array}$ \\
\hline $\begin{array}{l}44 \text { Costs: } 2 \text { b. Direct healthcare costs - } \\
\text { skewed data }\end{array}$ & & & Other data & No numeric data \\
\hline 44.1 by medium term & & & Other data & No numeric data \\
\hline 44.2 by short term FUP & & & Other data & No numeric data \\
\hline $\begin{array}{l}45 \text { Costs: } 3 \text {. Direct costs - other data - } \\
\text { skewed data }\end{array}$ & & & Other data & No numeric data \\
\hline 45.1 all care - by short term & & & Other data & No numeric data \\
\hline 45.2 all care - by medium term & & & Other data & No numeric data \\
\hline 45.3 all care - by long term & & & Other data & No numeric data \\
\hline $\begin{array}{l}45.4 \text { specific - outpatient care - by medi- } \\
\text { um term }\end{array}$ & & & Other data & No numeric data \\
\hline 45.5 specific - prison - by medium term & & & Other data & No numeric data \\
\hline
\end{tabular}

\section{Analysis 1.1. Comparison 1 Intensive Case Management versus standard care, Outcome 1 Service use: 1 . Average number of days in hospital per month - by about 24 months.}

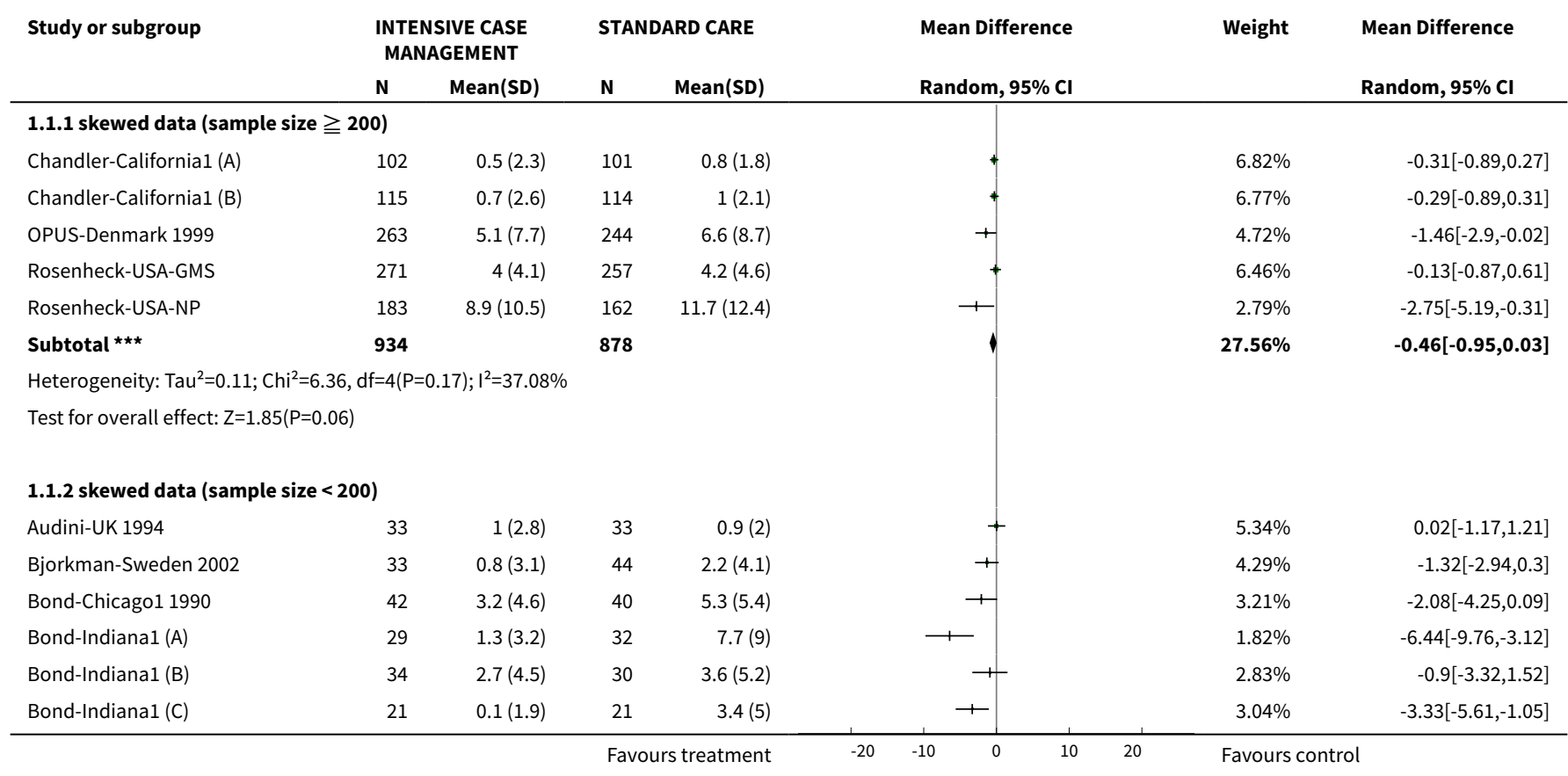




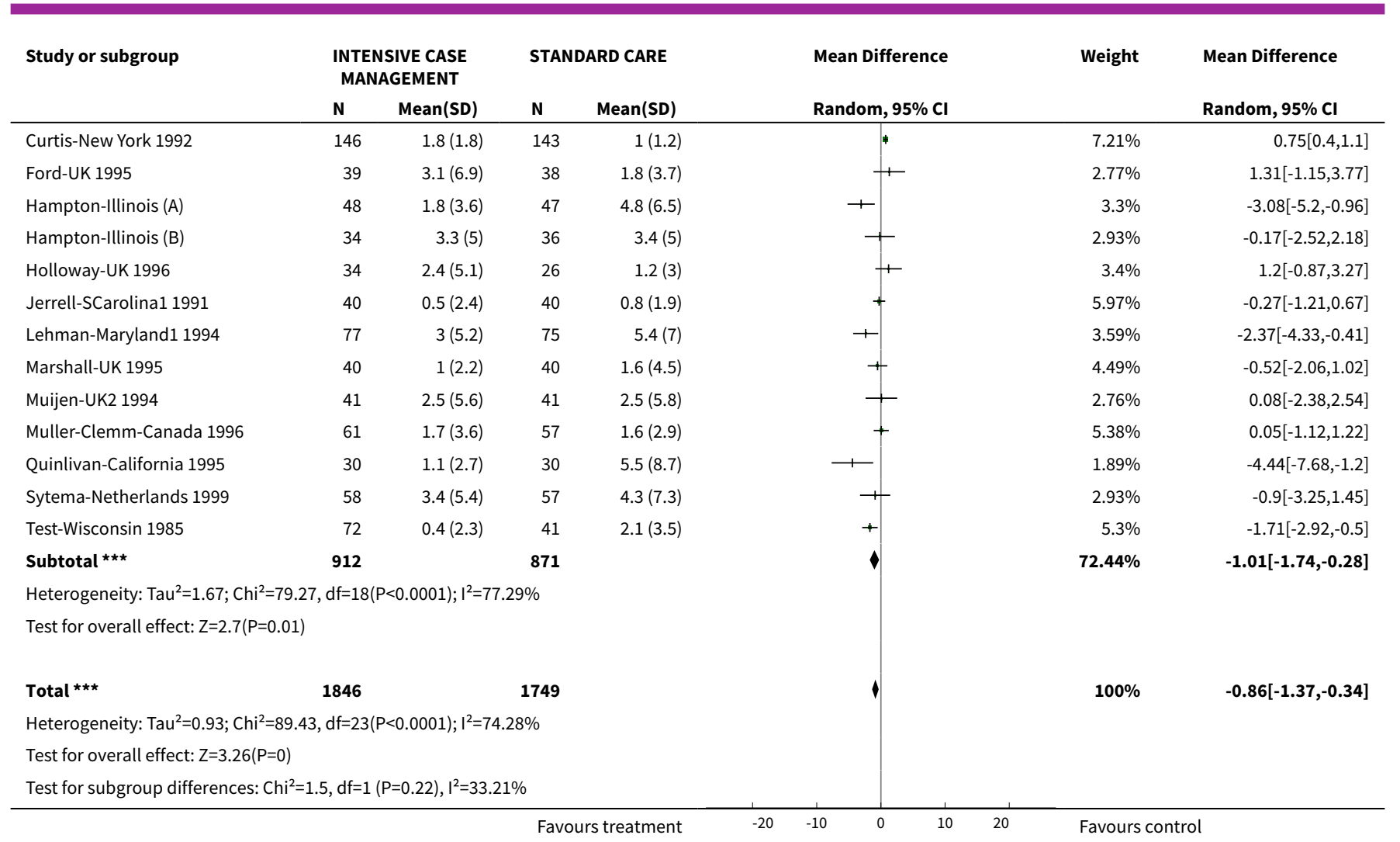

Analysis 1.2. Comparison 1 Intensive Case Management versus standard care, Outcome 2 Service use: 1a. Number of days in hospital - by follow -up (skewed data, sample size $\geqq 200$ ).

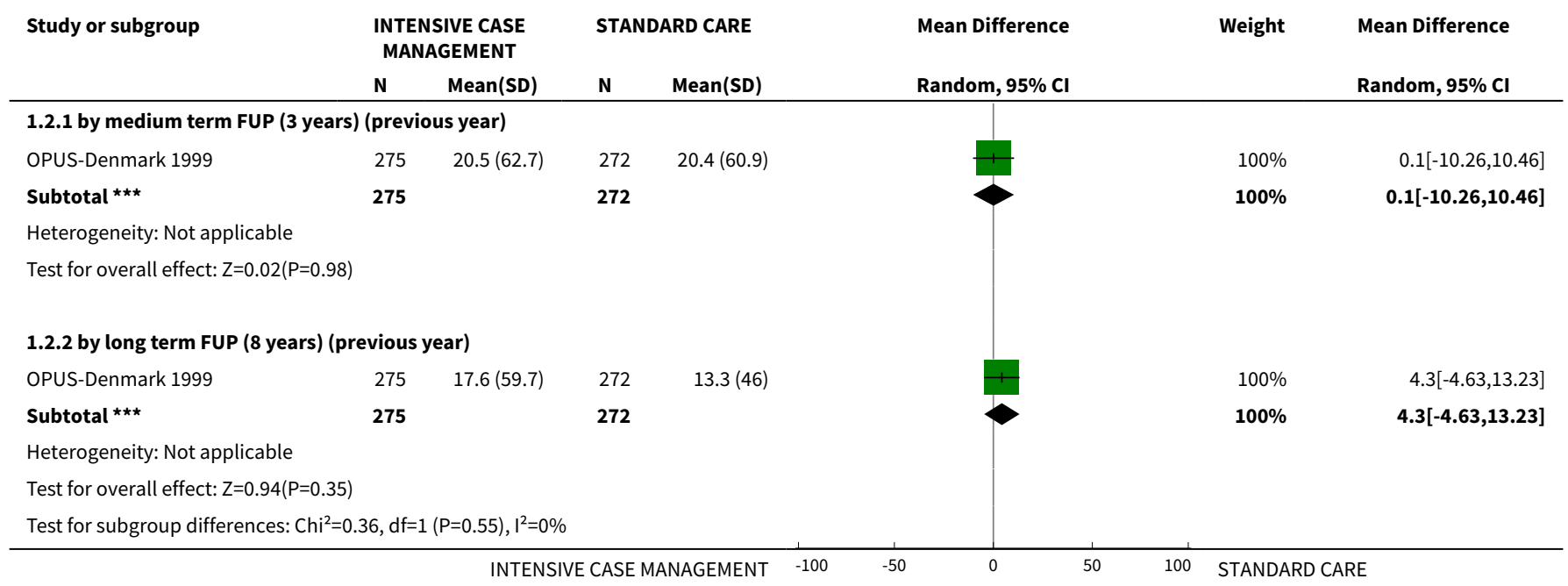


Analysis 1.3. Comparison 1 Intensive Case Management versus standard care, Outcome 3 Service use: 2 . Not remaining in contact with psychiatric services.

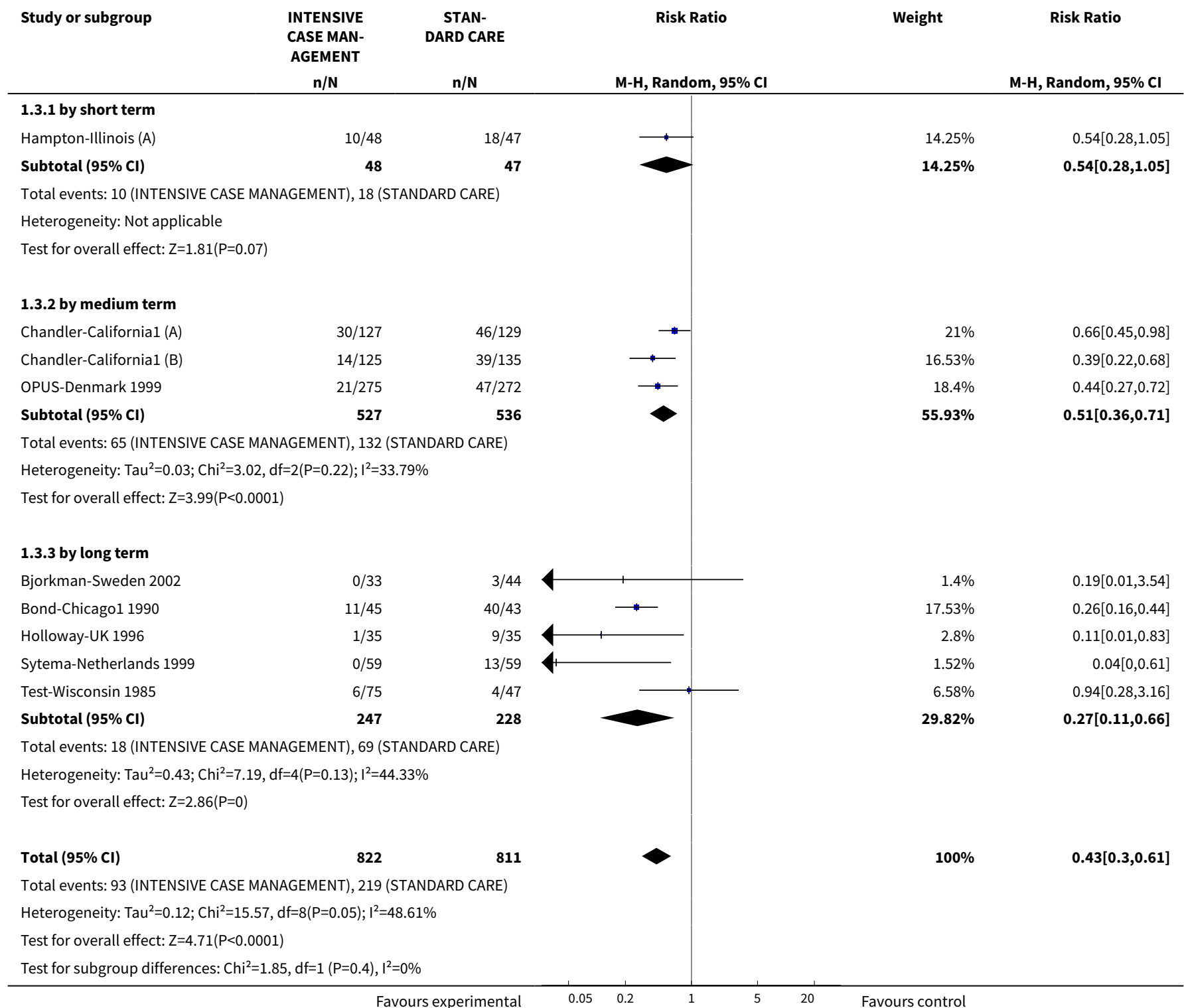

Analysis 1.4. Comparison 1 Intensive Case Management versus standard care, Outcome 4 Service use: 3a. Admitted to hospital.

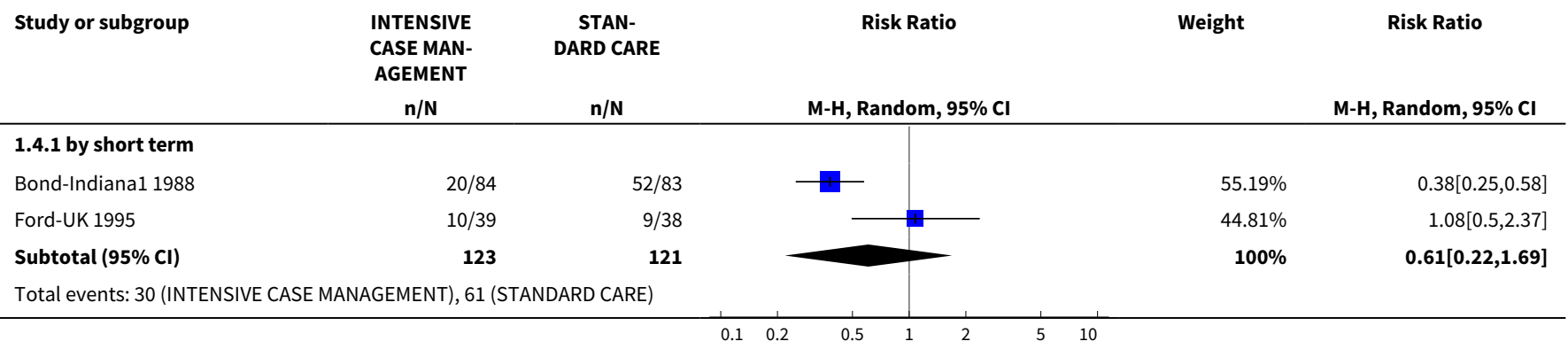




\begin{tabular}{|c|c|c|c|c|c|}
\hline Study or subgroup & $\begin{array}{l}\text { INTENSIVE } \\
\text { CASE MAN- } \\
\text { AGEMENT }\end{array}$ & $\begin{array}{l}\text { STAN- } \\
\text { DARD CARE }\end{array}$ & M-H, Random, $95 \% \mathrm{Cl}$ & Weight & M-H, Random, $95 \% \mathrm{Cl}$ \\
\hline \multicolumn{6}{|c|}{ Heterogeneity: $\mathrm{Tau}^{2}=0.45 ; \mathrm{Chi}^{2}=5.36, \mathrm{df}=1(\mathrm{P}=0.02) ; \mathrm{I}^{2}=81.36 \%$} \\
\hline \multicolumn{6}{|c|}{ Test for overall effect: $Z=0.96(P=0.34)$} \\
\hline \multicolumn{6}{|l|}{ 1.4.2 by medium term } \\
\hline Bond-Chicago1 1990 & $34 / 45$ & $37 / 43$ & $\rightarrow$ & $21.18 \%$ & $0.88[0.72,1.08]$ \\
\hline Chandler-California1 (A) & $27 / 127$ & $28 / 129$ & & $4.06 \%$ & $0.98[0.61,1.56]$ \\
\hline Chandler-California1 (B) & $29 / 125$ & $44 / 135$ & $\longrightarrow$ & $5.55 \%$ & $0.71[0.48,1.06]$ \\
\hline Lehman-Maryland1 1994 & $42 / 77$ & $45 / 75$ & $\rightarrow$ & $11.78 \%$ & $0.91[0.69,1.2]$ \\
\hline OPUS-Denmark 1999 & $162 / 275$ & $193 / 272$ & 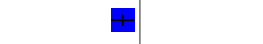 & $57.43 \%$ & $0.83[0.73,0.94]$ \\
\hline Subtotal $(95 \% \mathrm{Cl})$ & 649 & 654 & $\boldsymbol{\nabla}$ & $100 \%$ & $0.85[0.77,0.93]$ \\
\hline \multicolumn{6}{|c|}{ Heterogeneity: $\mathrm{Tau}^{2}=0 ; \mathrm{Chi}^{2}=1.58, \mathrm{df}=4(\mathrm{P}=0.81) ; \mathrm{I}^{2}=0 \%$} \\
\hline \multicolumn{6}{|c|}{ Test for overall effect: $Z=3.43(P=0)$} \\
\hline \multicolumn{6}{|l|}{ 1.4.3 by long term } \\
\hline Audini-UK 1994 & $9 / 33$ & $9 / 33$ & & $6.15 \%$ & $1[0.45,2.2]$ \\
\hline Bjorkman-Sweden 2002 & $15 / 33$ & $27 / 44$ & - & $10.3 \%$ & $0.74[0.48,1.15]$ \\
\hline Chandler-California1 (A) & $42 / 127$ & $39 / 129$ & + & $11.48 \%$ & $1.09[0.76,1.57]$ \\
\hline Chandler-California1 (B) & $50 / 125$ & $61 / 135$ & + & $12.58 \%$ & $0.89[0.67,1.18]$ \\
\hline Curtis-New York 1992 & $75 / 146$ & $51 / 143$ & $\rightarrow$ & $12.75 \%$ & $1.44[1.1,1.89]$ \\
\hline Ford-UK 1995 & $16 / 39$ & $14 / 38$ & & $8.65 \%$ & $1.11[0.64,1.95]$ \\
\hline Herinckx-Oregon 1996 & $54 / 117$ & $25 / 61$ & + & $11.5 \%$ & $1.13[0.79,1.61]$ \\
\hline Holloway-UK 1996 & $15 / 35$ & $15 / 35$ & 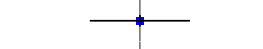 & $8.92 \%$ & $1[0.58,1.72]$ \\
\hline Macias-Utah 1994 & $0 / 20$ & $8 / 21$ & & $0.79 \%$ & $0.06[0,1]$ \\
\hline Test-Wisconsin 1985 & $15 / 75$ & $27 / 47$ & * & $9.26 \%$ & $0.35[0.21,0.58]$ \\
\hline Subtotal $(95 \% \mathrm{Cl})$ & 790 & 726 & & $100 \%$ & $0.96[0.74,1.23]$ \\
\hline \multicolumn{6}{|c|}{ Total events: 308 (INTENSIVE CASE MANAGEMENT), 286 (STANDARD CARE) } \\
\hline \multicolumn{6}{|c|}{ Heterogeneity: $\operatorname{Tau}^{2}=0.11 ; \mathrm{Chi}^{2}=32.88, \mathrm{df}=10(\mathrm{P}=0) ; \mathrm{I}^{2}=69.59 \%$} \\
\hline \multicolumn{6}{|c|}{ Test for overall effect: $Z=0.35(P=0.72)$} \\
\hline \multicolumn{6}{|c|}{ 1.4.4 by long term- during previous 12 months } \\
\hline OPUS-Denmark 1999 & $72 / 275$ & $106 / 272$ & & $100 \%$ & $0.67[0.52,0.86]$ \\
\hline Subtotal $(95 \% \mathrm{Cl})$ & 275 & 272 & & $100 \%$ & $0.67[0.52,0.86]$ \\
\hline \multicolumn{6}{|c|}{ Total events: 72 (INTENSIVE CASE MANAGEMENT), 106 (STANDARD CARE) } \\
\hline \multicolumn{6}{|c|}{ Heterogeneity: Not applicable } \\
\hline \multicolumn{6}{|c|}{ Test for overall effect: $Z=3.14(P=0)$} \\
\hline \multicolumn{6}{|c|}{$\begin{array}{l}\text { 1.4.5 by short term FUP - unplanned admission through Emergency } \\
\text { Department }\end{array}$} \\
\hline Chan-Hong Kong 2000 & $1 / 31$ & $1 / 31$ & & $100 \%$ & $1[0.07,15.28]$ \\
\hline Subtotal $(95 \% \mathrm{Cl})$ & 31 & 31 & & $100 \%$ & $1[0.07,15.28]$ \\
\hline \multicolumn{6}{|c|}{ Total events: 1 (INTENSIVE CASE MANAGEMENT), 1 (STANDARD CARE) } \\
\hline \multicolumn{6}{|c|}{ Heterogeneity: Not applicable } \\
\hline Test for overall effect: Not & & & & & \\
\hline
\end{tabular}


Analysis 1.5. Comparison 1 Intensive Case Management versus standard care, Outcome 5 Service use: 3b. Average number of admissions per month (skewed data).

\begin{tabular}{|c|c|c|c|c|c|c|}
\hline \multicolumn{7}{|c|}{ Service use: $3 \mathrm{~b}$. Average number of admissions per month (skewed data) } \\
\hline Study & Intervention & & Mean & SD & Total & Note \\
\hline \multicolumn{7}{|c|}{ by medium term } \\
\hline Bond-Chicago1 1990 & 1. ICM & 0.16 & & 0.15 & 42 & \\
\hline Bond-Chicago1 1990 & 2. Standard care & 0.26 & & 0.23 & 40 & \\
\hline \multicolumn{7}{|c|}{ by long term } \\
\hline Audini-UK 1994 & 1. ICM & 0.02 & & $0.05^{\star}$ & 33 & \\
\hline $\begin{array}{l}\text { Muller-Clemm-Canada } \\
1996\end{array}$ & 1. ICM & 0.09 & & $0.05^{*}$ & 61 & \\
\hline $\begin{array}{l}\text { Muller-Clemm-Canada } \\
1996\end{array}$ & 2. Standard care & 0.08 & & $0.06^{\star}$ & 57 & $\begin{array}{l}{ }^{\star} \text { Carried over from } \\
\text { Sytema-Netherlands. }\end{array}$ \\
\hline $\begin{array}{l}\text { Sytema-Netherlands } \\
1999\end{array}$ & 1. ICM & 0.05 & & 0.05 & 58 & \\
\hline $\begin{array}{l}\text { Sytema-Netherlands } \\
1999\end{array}$ & 2. Standard care & 0.05 & & 0.06 & 57 & \\
\hline
\end{tabular}

Analysis 1.6. Comparison 1 Intensive Case Management versus standard care, Outcome 6 Service use: 4a. Admitted to ER - by long term.

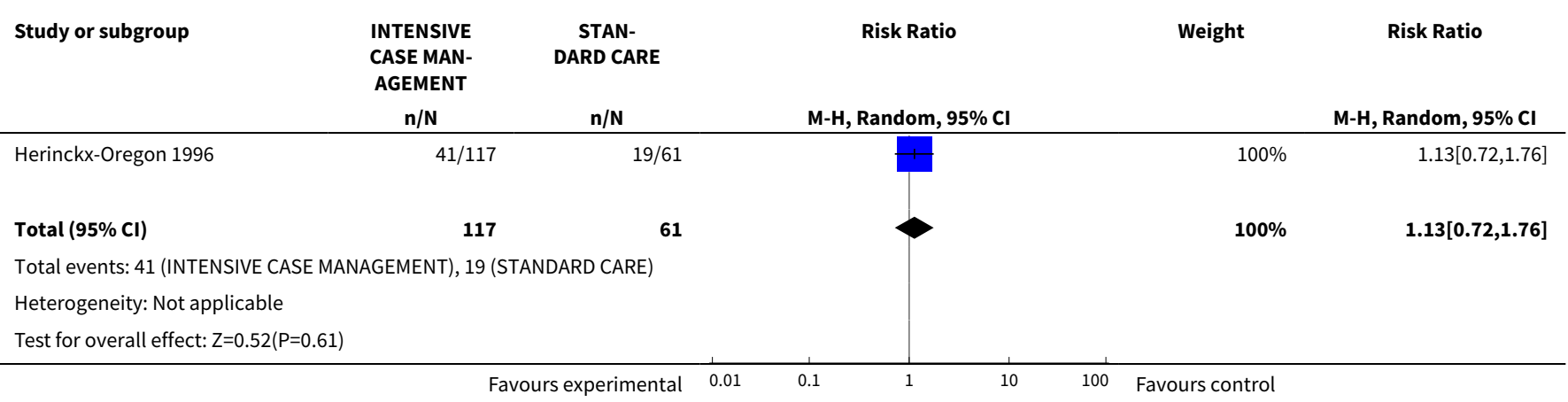

Analysis 1.7. Comparison 1 Intensive Case Management versus standard care, Outcome 7 Service use: 4b. Average number of admissions to ER (skewed data) - by medium term.

\begin{tabular}{|c|c|c|c|c|c|}
\hline \multicolumn{6}{|c|}{ Service use: $\mathbf{4 b .}$. Average number of admissions to ER (skewed data) - by medium term } \\
\hline Study & Intervention & Mean & SD & Total & Note \\
\hline Jerrell-SCarolina1 1991 & 1. ICM & 0.85 & $1.7^{\star}$ & 40 & \\
\hline Jerrell-SCarolina1 1991 & 2. Standard care & 0.73 & $3.3^{\star}$ & 40 & $\begin{array}{l}{ }^{*} \text { Carried over from } \\
\text { Lehman-Maryland1. }\end{array}$ \\
\hline Lehman-Maryland1 1994 & 1. ICM & 0.9 & 1.7 & 77 & \\
\hline Lehman-Maryland1 1994 & 2. Standard care & 2 & 3.3 & 75 & \\
\hline
\end{tabular}


Analysis 1.8. Comparison 1 Intensive Case Management versus standard care, Outcome 8 Service use: 5 a. Received day hospital care - by short term FUP.

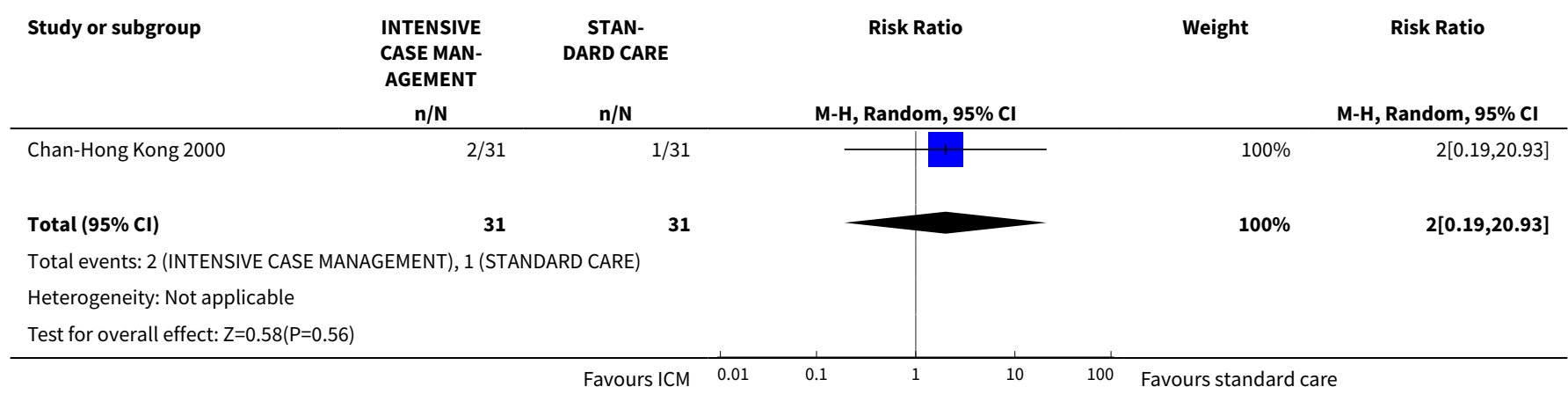

Analysis 1.9. Comparison 1 Intensive Case Management versus standard care, Outcome 9 Service use: 5b. Outpatient visits - by short term FUP (6 months).

\begin{tabular}{|c|c|c|c|c|c|c|c|}
\hline \multirow[t]{2}{*}{ Study or subgroup } & \multicolumn{2}{|c|}{ Experimental } & \multicolumn{2}{|c|}{ Control } & \multirow{2}{*}{$\begin{array}{l}\text { Mean Difference } \\
\text { Random, } 95 \% \mathrm{Cl}\end{array}$} & \multirow[t]{2}{*}{ Weight } & \multirow{2}{*}{$\begin{array}{l}\text { Mean Difference } \\
\text { Random, } 95 \% \mathrm{CI}\end{array}$} \\
\hline & $\mathbf{N}$ & Mean(SD) & $\mathbf{N}$ & Mean(SD) & & & \\
\hline Chan-Hong Kong 2000 & 31 & $4.5(0.8)$ & 31 & $4.2(0.9)$ & & $100 \%$ & $0.29[-0.14,0.72]$ \\
\hline Total *** & 31 & & 31 & & & $100 \%$ & $0.29[-0.14,0.72]$ \\
\hline \multicolumn{8}{|l|}{ Heterogeneity: Not applicable } \\
\hline Test for overall effect: $\mathrm{Z}=1.34(\mathrm{P}=0.18)$ & & & & & & & \\
\hline
\end{tabular}

Analysis 1.10. Comparison 1 Intensive Case Management versus standard care, Outcome 10 Service use: $5 c$. Outpatient visits - by medium term (skewed data).

Service use: $\mathbf{5 c}$. Outpatient visits - by medium term (skewed data)

\begin{tabular}{llllll}
\multicolumn{1}{c}{ Study } & Intervention & & Mean & SD & Note \\
\hline Cusack-North Carolina & ICM & 95.9 & 57.1 & & 72 \\
\hline Cusack-North Carolina & Standard care & 43.3 & 47.9 & & 62 \\
\hline
\end{tabular}

Analysis 1.11. Comparison 1 Intensive Case Management versus standard care, Outcome 11 Service use: 5d. Received home visits - by short term FUP.

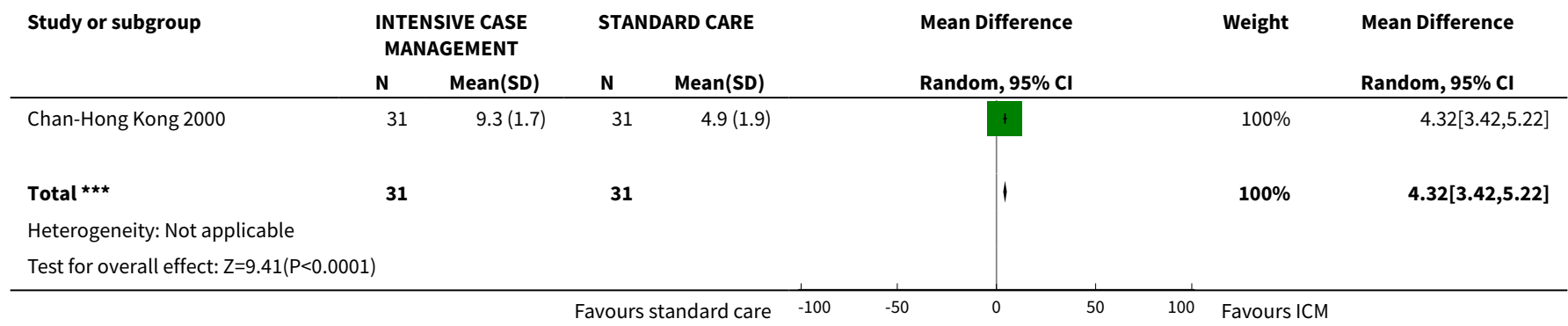


Analysis 1.12. Comparison 1 Intensive Case Management versus standard care, Outcome 12 Adverse event: 1a. Death - any cause.

\begin{tabular}{cccc} 
Study or subgroup & $\begin{array}{c}\text { INTENSIVE } \\
\text { CASE MAN- } \\
\text { AGEMENT }\end{array}$ & $\begin{array}{c}\text { STAN- } \\
\text { DARD CARE }\end{array}$ & Risk Ratio \\
& $\mathbf{n} / \mathrm{N}$ & $\mathrm{n} / \mathrm{N}$ & $\mathrm{M}-\mathrm{H}$, Random, $95 \% \mathrm{Cl}$ \\
\hline
\end{tabular}

Weight

Risk Ratio

M-H, Random, 95\% Cl

\subsection{1 by short term}

Audini-UK 1994

$0 / 33$

$2 / 48$

Hampton-Illinois (A)

Subtotal $(95 \% \mathrm{Cl})$

81

$1 / 33$

$1 / 47$

80

Total events: 2 (INTENSIVE CASE MANAGEMENT), 2 (STANDARD CARE)

Heterogeneity: $\mathrm{Tau}^{2}=0 ; \mathrm{Chi}^{2}=0.77, \mathrm{df}=1(\mathrm{P}=0.38) ; \mathrm{I}^{2}=0 \%$

Test for overall effect: $\mathrm{Z}=0.04(\mathrm{P}=0.97)$

1.12.2 by medium term

Audini-UK 1994

Bond-Chicago1 1990

Johnston-Australia 1998

Marshall-UK 1995

OPUS-Denmark 1999

Sytema-Netherlands 1999

Subtotal $(95 \% \mathrm{CI})$

$\begin{array}{rr}0 / 33 & 1 / 33 \\ 1 / 45 & 0 / 43 \\ 0 / 1 & 0 / 1 \\ 2 / 40 & 0 / 40 \\ 1 / 275 & 3 / 272 \\ 1 / 59 & 2 / 59 \\ \mathbf{4 5 3} & \mathbf{4 4 8}\end{array}$

Total events: 5 (INTENSIVE CASE MANAGEMENT), 6 (STANDARD CARE)

Heterogeneity: $\mathrm{Tau}^{2}=0 ; \mathrm{Chi}^{2}=3.11, \mathrm{df}=4(\mathrm{P}=0.54) ; \mathrm{I}^{2}=0 \%$

Test for overall effect: $Z=0.4(P=0.69)$

\subsection{3 by long term}

Audini-UK 1994

Bjorkman-Sweden 2002

Curtis-New York 1992

Ford-UK 1995

Holloway-UK 1996

Muijen-UK2 1994

Muller-Clemm-Canada 1996

OPUS-Denmark 1999

Test-Wisconsin 1985

Subtotal $(95 \% \mathrm{Cl})$

$\begin{array}{rr}0 / 33 & 1 / 33 \\ 0 / 33 & 1 / 44 \\ 11 / 147 & 10 / 145 \\ 3 / 39 & 0 / 38 \\ 1 / 35 & 2 / 35 \\ 0 / 41 & 0 / 41 \\ 2 / 63 & 3 / 60 \\ 1 / 275 & 6 / 272 \\ 6 / 75 & 4 / 47 \\ \mathbf{7 4 1} & \mathbf{7 1 5}\end{array}$

Total events: 24 (INTENSIVE CASE MANAGEMENT), 27 (STANDARD CARE)

Heterogeneity: $\mathrm{Tau}^{2}=0 ; \mathrm{Chi}^{2}=5.43, \mathrm{df}=7(\mathrm{P}=0.61) ; \mathrm{I}^{2}=0 \%$

Test for overall effect: $\mathrm{Z}=0.62(\mathrm{P}=0.53)$

\subsection{4 by medium term FUP ( 3 years)}

OPUS-Denmark 1999

$6 / 275$

$10 / 272$

Subtotal $(95 \% \mathrm{CI})$

275

272

Total events: 6 (INTENSIVE CASE MANAGEMENT), 10 (STANDARD CARE)

Heterogeneity: Not applicable

Test for overall effect: $\mathrm{Z}=1.02(\mathrm{P}=0.31)$

1.12.5 by long term FUP ( 8 years)

OPUS-Denmark 1999

$14 / 275 \quad 15 / 272$

Subtotal $(95 \% \mathrm{CI})$

275

272

Total events: 14 (INTENSIVE CASE MANAGEMENT), 15 (STANDARD CARE)

Heterogeneity: Not applicable

Test for overall effect: $Z=0.22(P=0.83)$

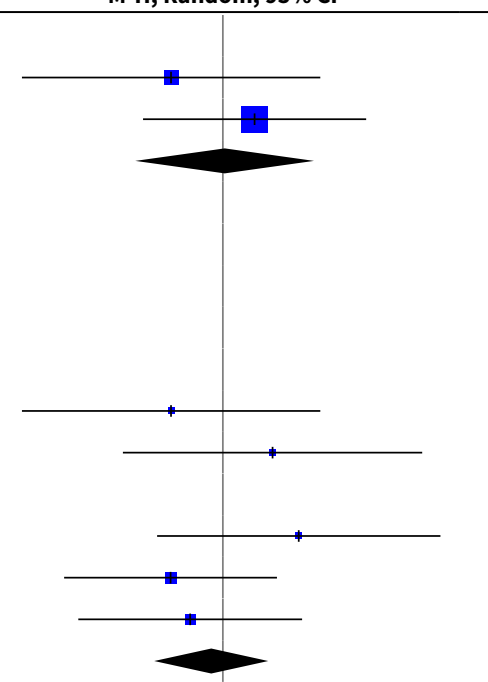

$35.86 \%$

$0.33[0.01,7.9]$

$64.14 \%$

$1.96[0.18,20.88]$

$1.04[0.16,6.91]$ $\begin{array}{rr}3.12 \% & 0.33[0.01,7.9] \\ 3.11 \% & 0.44[0.02,10.5] \\ 45.96 \% & 1.09[0.48,2.48] \\ 3.64 \% & 6.83[0.36,127.84] \\ 5.64 \% & 0.5[0.05,5.27] \\ & \text { Not estimable } \\ 10.17 \% & 0.63[0.11,3.67] \\ 7.03 \% & 0.16[0.02,1.36] \\ 21.32 \% & 0.94[0.28,3.16] \\ \mathbf{1 0 0 \%} & \mathbf{0 . 8 4}[\mathbf{0 . 4 8 , 1 . 4 7}]\end{array}$

$100 \%$

$0.59[0.22,1.61]$

$100 \%$

$0.59[0.22,1.61]$ 
Analysis 1.13. Comparison 1 Intensive Case Management versus standard care, Outcome 13 Adverse event: 1b. Death - suicide.

$\begin{array}{lcc}\text { Study or subgroup } & \text { STAN- } & \text { Risk Ratio } \\ & \text { CASE MAN- } & \text { DARD CARE } \\ \text { AGEMENT } & & \end{array}$

$n / N$

M-H, Random, 95\% Cl

M-H, Random, 95\% Cl

\subsection{1 by short term}

Audini-UK 1994

Bond-Indiana1 (A)

$0 / 33$

$0 / 29$

$1 / 33$

Bond-Indiana1 (A)

62

$1 / 32$

Total events: 0 (INTENSIVE CASE MANAGEMENT), 2 (STANDARD CARE)

Heterogeneity: $\mathrm{Tau}^{2}=0 ; \mathrm{Chi}^{2}=0, \mathrm{df}=1(\mathrm{P}=0.97) ; \mathrm{I}^{2}=0 \%$

Test for overall effect: $Z=0.92(P=0.36)$

\subsection{2 by medium term}

Audini-UK 1994

Bond-Chicago1 1990

$0 / 33$

$1 / 45$

OPUS-Denmark 1999

$1 / 275$

Sytema-Netherlands 1999

Subtotal $(95 \% \mathrm{Cl})$

$0 / 59$

412

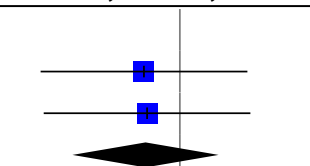

$1 / 33$

$0 / 43$

$1 / 272$

$0 / 59$

407

65

Total events: 2 (INT Heterogeneity: $\mathrm{Tau}^{2}=0 ; \mathrm{Chi}^{2}=0.89, \mathrm{df}=2(\mathrm{P}=0.64) ; \mathrm{I}^{2}=0 \%$

Test for overall effect: $\mathrm{Z}=0.02(\mathrm{P}=0.98)$

\subsection{3 by long term}

Audini-UK 1994

Bjorkman-Sweden 2002

$0 / 33 \quad 1 / 33$

Curtis-New York 1992

Ford-UK 1995

Holloway-UK 1996

Muijen-UK2 1994

Muller-Clemm-Canada 1996

OPUS-Denmark 1999

Test-Wisconsin 1985

Subtotal $(95 \% \mathrm{CI})$

$0 / 33$

$1 / 147$

$1 / 39$

$0 / 35$

$0 / 41$

$2 / 63$

$1 / 275$

$5 / 75$

741

Heterogeneity: $\mathrm{Tau}^{2}=0 ; \mathrm{Chi}^{2}=2.67, \mathrm{df}=7(\mathrm{P}=0.91) ; \mathrm{I}^{2}=0 \%$

Test for overall effect: $Z=0.94(P=0.35)$

1.13.4 by medium term FUP ( 3 years)

OPUS-Denmark 1999

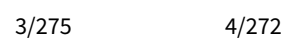

Subtotal $(95 \% \mathrm{Cl})$

275

272

Total events: 3 (INTENSIVE CASE MANAGEMENT), 4 (STANDARD CARE)

Heterogeneity: Not applicable

Test for overall effect: $\mathrm{Z}=0.39(\mathrm{P}=0.69)$

$1 / 33$

$1 / 44$

$2 / 145$

$1 / 35$

$0 / 41$

$2 / 60$

$4 / 272$

$3 / 47$

715

$49.95 \%$

$50.05 \%$

$0.33[0.01,7.9]$

$100 \%$

$0.37[0.02,8.66]$

$0.35[0.04,3.27]$

$30.27 \%$

$30.11 \%$

$39.62 \%$

$100 \%$

$6.34 \%$

$6.32 \%$

$11.13 \%$

$6.32 \%$

$6.33 \%$

$17.09 \%$

$13.31 \%$

$33.16 \%$

$100 \%$

$100 \%$

$100 \%$
$0.33[0.01,7.9]$

$2.87[0.12,68.58]$

$0.99[0.06,15.73]$

Not estimable

$0.98[0.17,5.6]$
$0.33[0.01,7.9]$

$0.44[0.02,10.5]$

$0.49[0.05,5.38]$

$2.93[0.12,69.64]$

$0.33[0.01,7.91]$

Not estimable

0.95[0.14,6.55]

$0.25[0.03,2.2]$

$1.04[0.26,4.17]$

$0.68[0.31,1.51]$

Favours experimental $\quad 0.001$ 


\section{Analysis 1.14. Comparison 1 Intensive Case Management versus standard care, Outcome 14 Global state: 1. Leaving the study early.}

\begin{tabular}{lrr} 
Study or subgroup & $\begin{array}{c}\text { INTENSIVE } \\
\text { CASE MAN- } \\
\text { AGEMENT } \\
\text { n/N }\end{array}$ & $\begin{array}{c}\text { STAN- } \\
\text { DARD CARE }\end{array}$ \\
\hline $\mathbf{1 . 1 4 . 1}$ by short term & & \\
Audini-UK 1994 & $2 / 33$ & $5 / 33$ \\
Bond-Indiana1 1988 & $18 / 84$ & $25 / 83$ \\
Hampton-Illinois (A) & $38 / 48$ & $29 / 47$ \\
Hampton-Illinois (B) & $34 / 34$ & $36 / 36$ \\
Solomon-Pennsylvania 1994 & $42 / 120$ & $52 / 80$ \\
Subtotal (95\% CI) & $\mathbf{3 1 9}$ & $\mathbf{2 7 9}$
\end{tabular}

Risk Ratio Weight

Risk Ratio

M-H, Random, 95\% Cl

Total events: 134 (INTENSIVE CASE MANAGEMENT), 147 (STANDARD CARE) Heterogeneity: $\mathrm{Tau}^{2}=0.35 ; \mathrm{Chi}^{2}=80.24, \mathrm{df}=4(\mathrm{P}<0.0001) ; \mathrm{I}^{2}=95.01 \%$

Test for overall effect: $\mathrm{Z}=0.8(\mathrm{P}=0.43)$

\subsection{2 by medium term}

Bond-Chicago1 1990

$\begin{array}{rr}11 / 45 & 19 / 43 \\ 25 / 127 & 28 / 129 \\ 10 / 125 & 27 / 135 \\ 10 / 77 & 17 / 75 \\ 6 / 40 & 5 / 40 \\ 15 / 52 & 29 / 64 \\ 48 / 275 & 80 / 272 \\ 48 / 120 & 58 / 80 \\ \mathbf{8 6 1} & \mathbf{8 3 8}\end{array}$

Chandler-California1 (A)

Chandler-California1 (B)

Lehman-Maryland1 1994

M-H, Random, $95 \% \mathrm{Cl}$

$$
\begin{array}{r}
0.4[0.08,1.92] \\
0.71[0.42,1.2] \\
1.28[0.98,1.68] \\
1[0.95,1.06] \\
0.54[0.4,0.72] \\
\mathbf{0 . 7 9}[0.44,1.41]
\end{array}
$$

Marshall-UK 1995

Morse-Missouri1 1992

OPUS-Denmark 1999

Solomon-Pennsylvania 1994

861

838

Total events: 173 (INTENSIVE CASE MANAGEMENT), 263 (STANDARD CARE)

Heterogeneity: $\mathrm{Tau}^{2}=0 ; \mathrm{Chi}^{2}=6.28, \mathrm{df}=7(\mathrm{P}=0.51) ; \mathrm{I}^{2}=0 \%$

Test for overall effect: $Z=6.39(P<0.0001)$

\subsection{3 by long term}

Aberg-Wistedt-Sweden 1995

Audini-UK 1994

Bjorkman-Sweden 2002

Chandler-California1 (A)

Chandler-California1 (B)

Ford-UK 1995

Holloway-UK 1996

Macias-Utah 1994

Muijen-UK2 1994

Muller-Clemm-Canada 1996

OPUS-Denmark 1999

Pique-California 1999

Test-Wisconsin 1985

Subtotal $(95 \% \mathrm{Cl})$

$\begin{array}{rr}0 / 20 & 0 / 20 \\ 3 / 33 & 5 / 33 \\ 5 / 33 & 8 / 44 \\ 44 / 127 & 60 / 129 \\ 33 / 125 & 63 / 135 \\ 4 / 39 & 9 / 38 \\ 1 / 35 & 9 / 35 \\ 2 / 20 & 5 / 21 \\ 10 / 41 & 14 / 41 \\ 2 / 63 & 3 / 60 \\ 70 / 275 & 108 / 272 \\ 14 / 22 & 7 / 15 \\ 6 / 75 & 4 / 47 \\ 908 & 890\end{array}$

Total events: 194 (INTENSIVE CASE MANAGEMENT), 295 (STANDARD CARE) Heterogeneity: $\mathrm{Tau}^{2}=0 ; \mathrm{Chi}^{2}=11.22, \mathrm{df}=11(\mathrm{P}=0.42) ; \mathrm{I}^{2}=1.97 \%$

Test for overall effect: $Z=4.94(P<0.0001)$

1.14.4 by medium term FUP ( 3 years)

OPUS-Denmark 1999

Subtotal $(95 \% \mathrm{Cl})$

$\begin{array}{rr}124 / 275 & 122 / 272 \\ \mathbf{2 7 5} & \mathbf{2 7 2}\end{array}$

272

$100 \%$

$1.01[0.84,1.21]$

Total events: 124 (INTENSIVE CASE MANAGEMENT), 122 (STANDARD CARE) 


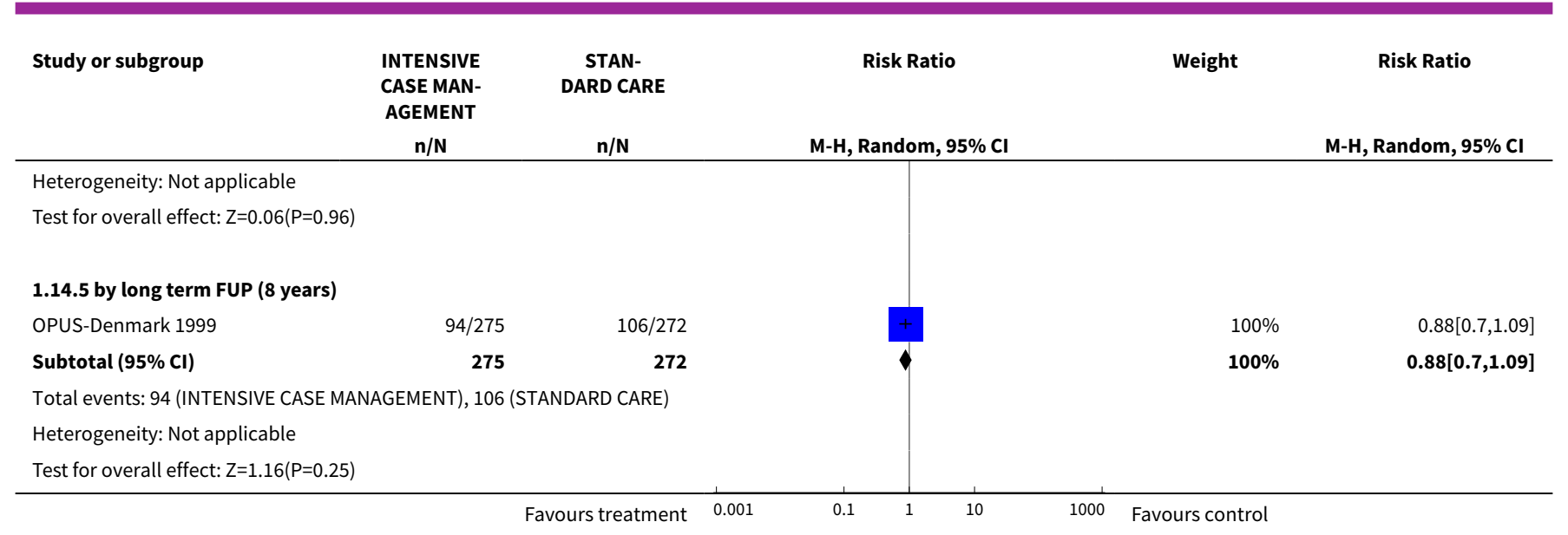

Analysis 1.15. Comparison 1 Intensive Case Management versus standard care, Outcome 15 Global state: 2. Average endpoint score (GAF, high = good).

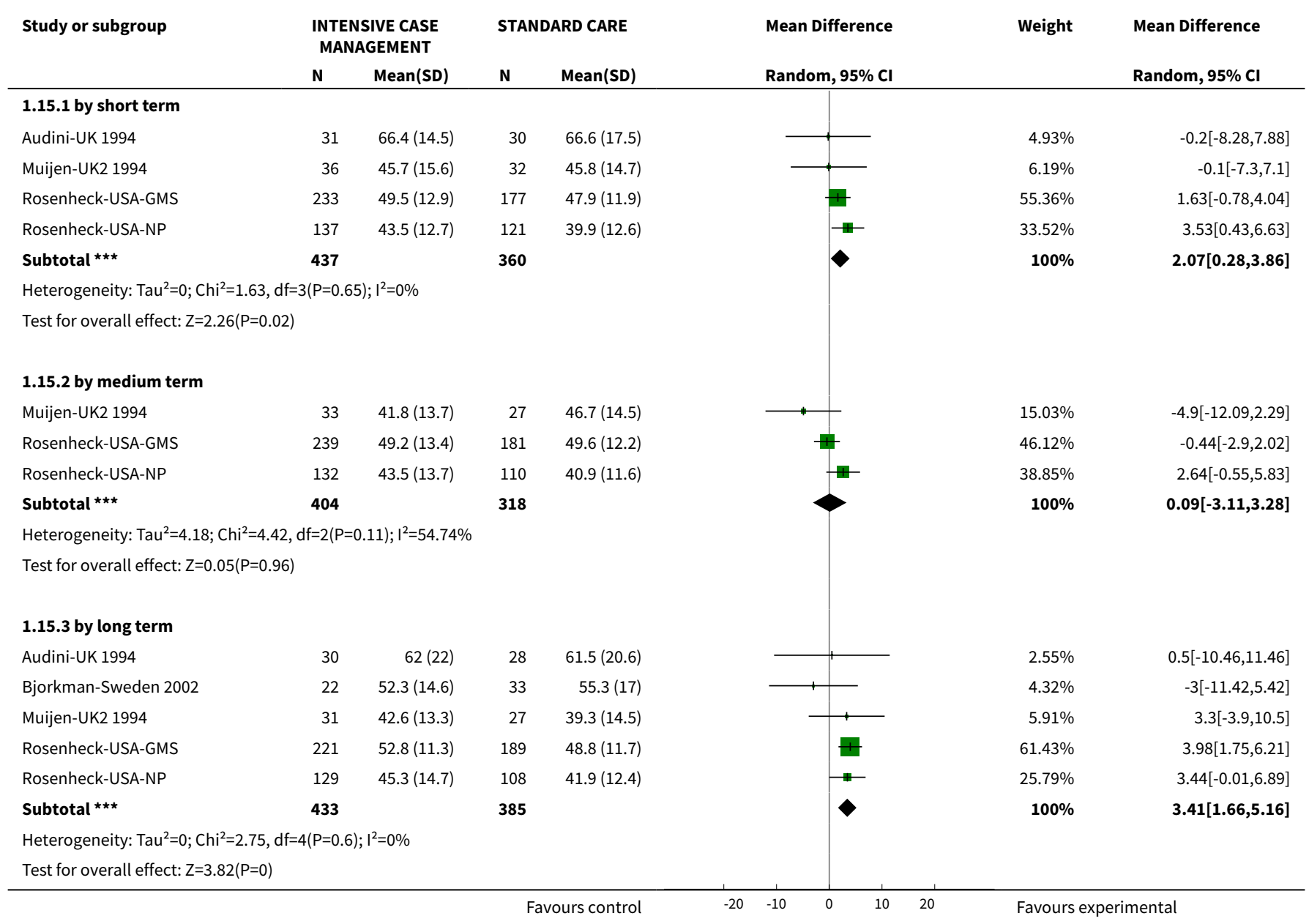


Analysis 1.16. Comparison 1 Intensive Case Management versus standard care, Outcome 16 Global state: 3 . Not compliant with medication - by long term.

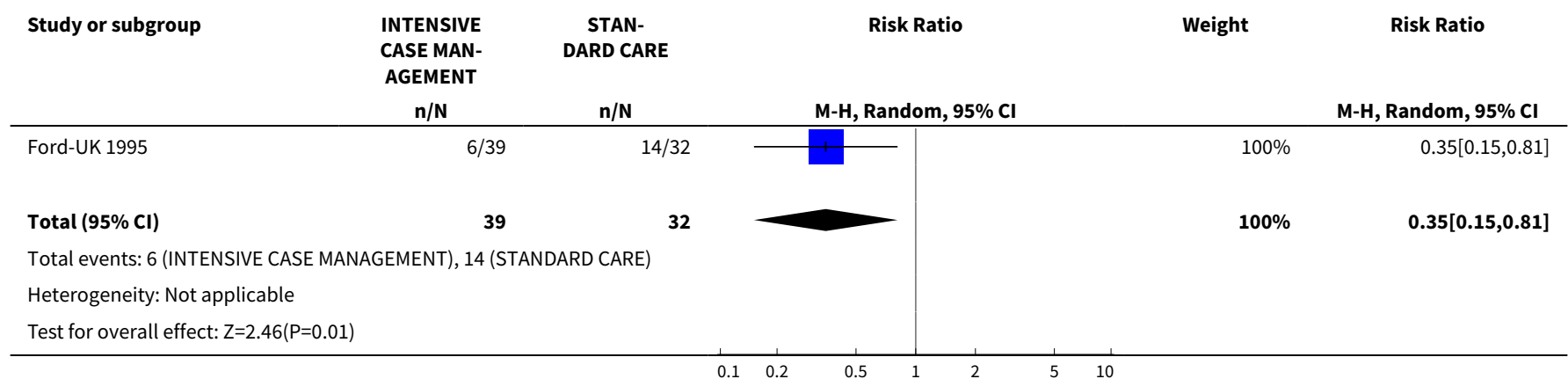

Analysis 1.17. Comparison 1 Intensive Case Management versus standard care, Outcome 17 Social functioning: 1a. Contact with legal system (various measurements).

\begin{tabular}{|c|c|c|c|c|c|}
\hline \multirow[t]{2}{*}{ Study or subgroup } & $\begin{array}{l}\text { INTENSIVE } \\
\text { CASE MAN- } \\
\text { AGEMENT }\end{array}$ & $\begin{array}{c}\text { STAN- } \\
\text { DARD CARE }\end{array}$ & \multirow[b]{2}{*}{ M-H, Random, 95\% Cl } & \multirow[t]{2}{*}{ Weight } & \multirow[b]{2}{*}{ M-H, Random, $95 \% \mathrm{Cl}$} \\
\hline & $n / N$ & $n / N$ & & & \\
\hline \multicolumn{3}{|c|}{ 1.17.1 by short term - contact with the police } & & & \\
\hline Bond-Indiana1 (A) & $7 / 29$ & $3 / 32$ & & $100 \%$ & $2.57[0.73,9.04]$ \\
\hline Subtotal $(95 \% \mathrm{CI})$ & 29 & 32 & & $100 \%$ & $2.57[0.73,9.04]$ \\
\hline \multicolumn{6}{|c|}{ Total events: 7 (INTENSIVE CASE MANAGEMENT), 3 (STANDARD CARE) } \\
\hline \multicolumn{6}{|l|}{ Heterogeneity: Not applicable } \\
\hline \multicolumn{6}{|c|}{ Test for overall effect: $Z=1.48(P=0.14)$} \\
\hline \multicolumn{6}{|c|}{ 1.17.2 by medium term - arrested } \\
\hline Bond-Chicagol 1990 & $4 / 45$ & $2 / 43$ & 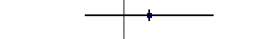 & $11.93 \%$ & $1.91[0.37,9.9]$ \\
\hline Chandler-California1 (A) & $10 / 127$ & $9 / 129$ & & $43.01 \%$ & $1.13[0.47,2.68]$ \\
\hline Subtotal $(95 \% \mathrm{Cl})$ & 297 & 307 & & $100 \%$ & $1.08[0.61,1.9]$ \\
\hline \multicolumn{6}{|c|}{ Total events: 23 (INTENSIVE CASE MANAGEMENT), 22 (STANDARD CARE) } \\
\hline \multicolumn{6}{|c|}{ Heterogeneity: $\operatorname{Tau}^{2}=0 ; \mathrm{Chi}^{2}=0.69, \mathrm{df}=2(\mathrm{P}=0.71) ; \mathrm{I}^{2}=0 \%$} \\
\hline \multicolumn{6}{|c|}{ Test for overall effect: $Z=0.25(P=0.8)$} \\
\hline \multicolumn{6}{|c|}{ 1.17.3 by medium term - contact with the police } \\
\hline Bond-Chicagol 1990 & $5 / 45$ & $21 / 43$ & & $100 \%$ & $0.23[0.09,0.55]$ \\
\hline Subtotal $(95 \% \mathrm{Cl})$ & 45 & 43 & & $100 \%$ & $0.23[0.09,0.55]$ \\
\hline \multicolumn{6}{|c|}{ Total events: 5 (INTENSIVE CASE MANAGEMENT), 21 (STANDARD CARE) } \\
\hline \multicolumn{6}{|l|}{ Heterogeneity: Not applicable } \\
\hline \multicolumn{6}{|c|}{ Test for overall effect: $Z=3.29(P=0)$} \\
\hline \multicolumn{6}{|c|}{ 1.17.4 by medium term - imprisoned } \\
\hline Chandler-California1 (B) & $4 / 125$ & $7 / 135$ & & $21.03 \%$ & $0.62[0.19,2.06]$ \\
\hline Solomon-Pennsylvania 1994 & $60 / 120$ & $29 / 80$ & & $45.68 \%$ & $1.38[0.98,1.94]$ \\
\hline Subtotal $(95 \% \mathrm{Cl})$ & 417 & 387 & & $100 \%$ & $0.8[0.39,1.64]$ \\
\hline \multicolumn{6}{|c|}{ Total events: 70 (INTENSIVE CASE MANAGEMENT), 49 (STANDARD CARE) } \\
\hline Heterogeneity: $\mathrm{Tau}^{2}=0.27$; Chi & $=3(P=0.1) ; I^{2}=51$. & & & & \\
\hline
\end{tabular}




\begin{tabular}{|c|c|c|c|c|c|}
\hline \multirow[t]{2}{*}{ Study or subgroup } & $\begin{array}{l}\text { INTENSIVE } \\
\text { CASE MAN- } \\
\text { AGEMENT }\end{array}$ & $\begin{array}{c}\text { STAN- } \\
\text { DARD CARE }\end{array}$ & Risk Ratio & \multirow[t]{2}{*}{ Weight } & Risk Ratio \\
\hline & $n / N$ & $n / N$ & M-H, Random, $95 \% \mathrm{Cl}$ & & M-H, Random, $95 \% \mathrm{Cl}$ \\
\hline \multicolumn{6}{|c|}{ Test for overall effect: $Z=0.61(P=0.54)$} \\
\hline \multicolumn{6}{|c|}{1.17 .5 by long term - arrested } \\
\hline Herinckx-Oregon 1996 & $14 / 117$ & $11 / 61$ & & $100 \%$ & $0.66[0.32,1.37]$ \\
\hline Subtotal $(95 \% \mathrm{Cl})$ & 117 & 61 & & $100 \%$ & $0.66[0.32,1.37]$ \\
\hline \multicolumn{6}{|c|}{ Total events: 14 (INTENSIVE CASE MANAGEMENT), 11 (STANDARD CARE) } \\
\hline \multicolumn{6}{|c|}{ Heterogeneity: Not applicable } \\
\hline \multicolumn{6}{|c|}{ Test for overall effect: $Z=1.11(P=0.27)$} \\
\hline \multicolumn{6}{|c|}{ 1.17.6 by long term - imprisoned } \\
\hline Ford-UK 1995 & $0 / 39$ & $0 / 38$ & & & Not estimable \\
\hline Marshall-UK 1995 & $0 / 40$ & $0 / 40$ & & & Not estimable \\
\hline Muijen-UK2 1994 & $1 / 41$ & $4 / 41$ & - & $8.87 \%$ & $0.25[0.03,2.14]$ \\
\hline OPUS-Denmark 1999 & $4 / 275$ & $2 / 272$ & $\longrightarrow$ & $13.97 \%$ & $1.98[0.37,10.71]$ \\
\hline Test-Wisconsin 1985 & $19 / 75$ & $14 / 47$ & & $77.16 \%$ & $0.85[0.47,1.53]$ \\
\hline Subtotal $(95 \% \mathrm{Cl})$ & 470 & 438 & 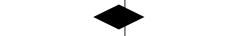 & $100 \%$ & $0.86[0.45,1.65]$ \\
\hline \multicolumn{6}{|c|}{ Total events: 24 (INTENSIVE CASE MANAGEMENT), 20 (STANDARD CARE) } \\
\hline \multicolumn{6}{|c|}{ Heterogeneity: $\operatorname{Tau}^{2}=0.05 ; \mathrm{Chi}^{2}=2.21, \mathrm{df}=2(\mathrm{P}=0.33) ; \mathrm{I}^{2}=9.48 \%$} \\
\hline \multicolumn{6}{|c|}{ Test for overall effect: $Z=0.46(P=0.65)$} \\
\hline
\end{tabular}

Analysis 1.18. Comparison 1 Intensive Case Management versus standard care, Outcome 18 Social functioning: 1 b. Mean contacts with legal system (skewed data) - by medium term.

\begin{tabular}{|c|c|c|c|c|c|}
\hline \multicolumn{6}{|c|}{ Social functioning: $1 \mathrm{~b}$. Mean contacts with legal system (skewed data) - by medium term } \\
\hline Study & Intervention & Mean & & SD & Total \\
\hline \multicolumn{6}{|c|}{ Bookings } \\
\hline Cusack-North Carolina & 1. ICM & 0.64 & 1.2 & & 72 \\
\hline Cusack-North Carolina & 2. Standard care & 1.42 & 1.86 & & 62 \\
\hline \multicolumn{6}{|c|}{ Jail days } \\
\hline Cusack-North Carolina & 1. ICM & 18.5 & 45.3 & & 72 \\
\hline Cusack-North Carolina & 2. Standard care & 35.3 & 56.9 & & 62 \\
\hline \multicolumn{6}{|c|}{ Convictions } \\
\hline Cusack-North Carolina & 1. ICM & 0.75 & 0.77 & & 72 \\
\hline Cusack-North Carolina & 2. Standard care & 0.85 & 1.03 & & 62 \\
\hline
\end{tabular}

Analysis 1.19. Comparison 1 Intensive Case Management versus standard care, Outcome 19 Social functioning: 2. Employment status (various measurements).

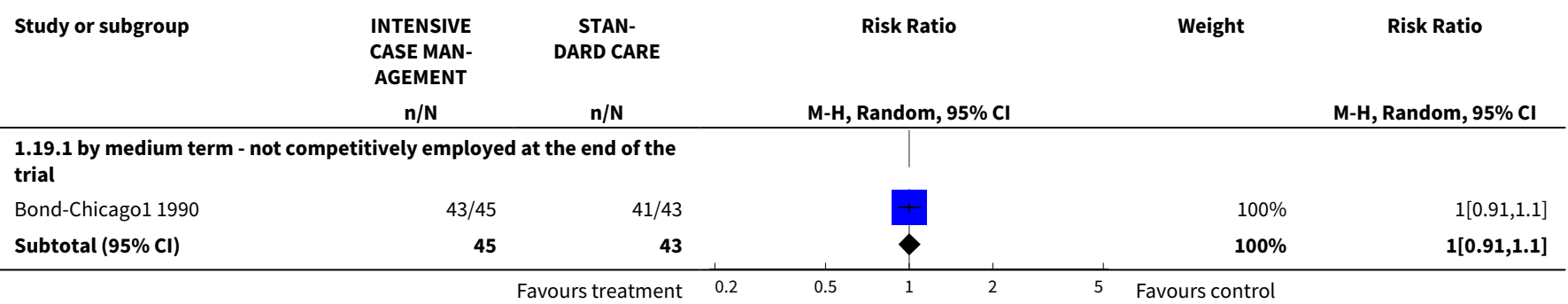




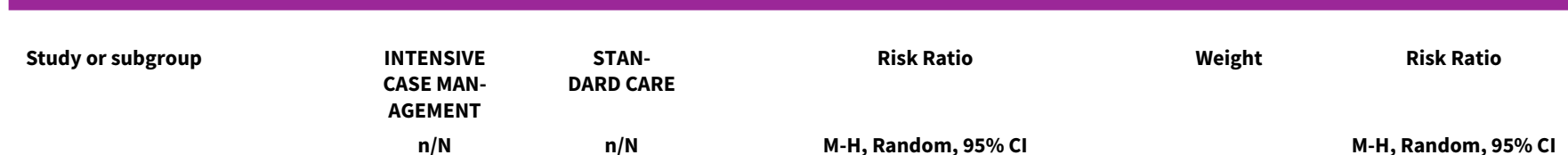

Total events: 43 (INTENSIVE CASE MANAGEMENT), 41 (STANDARD CARE)

Heterogeneity: Not applicable

Test for overall effect: $\mathrm{Z}=0.05(\mathrm{P}=0.96)$

1.19.2 by medium term - not employed at the end of the trial

Chandler-California1 (A)

$81 / 127 \quad 111 / 129$

Chandler-California1 (B)

$103 / 125 \quad 128 / 135$

Johnston-Australia 1998

$35 / 37$

$34 / 36$

OPUS-Denmark 1999

$179 / 275$

$188 / 272$

Subtotal $(95 \% \mathrm{Cl})$

564

572

Total events: 398 (INTENSIVE CASE MANAGEMENT), 461 (STANDARD CARE)

Heterogeneity: $\mathrm{Tau}^{2}=0.01 ; \mathrm{Chi}^{2}=11.86, \mathrm{df}=3(\mathrm{P}=0.01) ; \mathrm{I}^{2}=74.71 \%$

Test for overall effect: $\mathrm{Z}=2.04(\mathrm{P}=0.04)$

1.19.3 by long term - not employed at the end of the trial

Audini-UK 1994

$23 / 33$

Chandler-California1 (A)

$35 / 127$

$24 / 33$

Chandler-California1 (B)

$89 / 125$

$110 / 129$

OPUS-Denmark 1999

$168 / 275$

$120 / 135$

Subtotal $(95 \% \mathrm{Cl})$

560

$182 / 272$

Total events: 315 (INTENSIVE CASE MANAGEMENT), 436 (STANDARD CARE) Heterogeneity: $\mathrm{Tau}^{2}=0.12 ; \mathrm{Chi}^{2}=46.48, \mathrm{df}=3(\mathrm{P}<0.0001) ;\left.\right|^{2}=93.55 \%$

Test for overall effect: $\mathrm{Z}=1.95(\mathrm{P}=0.05)$

1.19.4 by long term - not working/studying in the previous year

OPUS-Denmark 1999

Subtotal $(95 \% \mathrm{Cl})$

275

272

M-H, Random, 95\% Cl

M-H, Random, 95\% Cl

Total events: 147 (INTENSIVE CASE MANAGEMENT), 170 (STANDARD CARE) Heterogeneity: $\mathrm{Tau}^{2}=0 ; \mathrm{Chi}^{2}=0, \mathrm{df}=0(\mathrm{P}<0.0001) ; \mathrm{I}^{2}=100 \%$

Test for overall effect: $\mathrm{Z}=2.13(\mathrm{P}=0.03)$

1.19.5 by medium term FUP ( 3 years) - not working/studying in the previous year

OPUS-Denmark 1999

$176 / 275 \quad 170 / 272$

Subtotal $(95 \% \mathrm{Cl})$

275

272

Total events: 176 (INTENSIVE CASE MANAGEMENT), 170 (STANDARD CARE)

Heterogeneity: Not applicable

Test for overall effect: $\mathrm{Z}=0.36(\mathrm{P}=0.72)$

1.19.6 by long term FUP ( 8 years) - not working/studying in the previous year

$\begin{array}{lll}\text { OPUS-Denmark } 1999 & 185 / 275 & 185 / 272\end{array}$

Subtotal $(95 \% \mathrm{CI}) \quad \mathbf{2 7 5} 272$

Total events: 185 (INTENSIVE CASE MANAGEMENT), 185 (STANDARD CARE)

Heterogeneity: Not applicable

Test for overall effect: $Z=0.19(P=0.85)$

Favours treatment

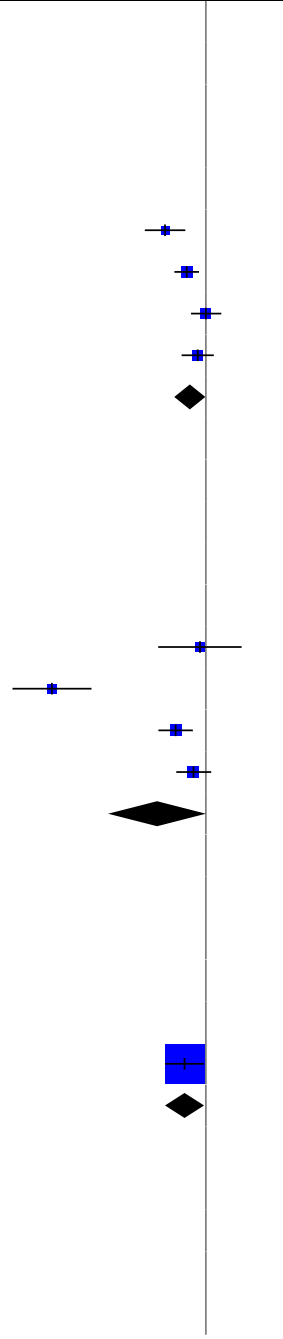

$21.49 \%$

$27.95 \%$

$25.68 \%$

$24.89 \%$

$100 \%$

$23.03 \%$

$23.44 \%$

$26.76 \%$

$26.77 \%$

$100 \%$

$100 \%$

$100 \%$

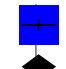

$100 \%$

$100 \%$

$1.02[0.9,1.16]$

$1.02[0.9,1.16]$
$0.96[0.7,1.3]$

$0.32[0.24,0.43]$

$0.8[0.71,0.91]$

$0.91[0.8,1.04]$

$0.7[0.49,1]$
$0.86[0.74,0.99]$

$\mathbf{0 . 8 6}[0.74,0.99]$

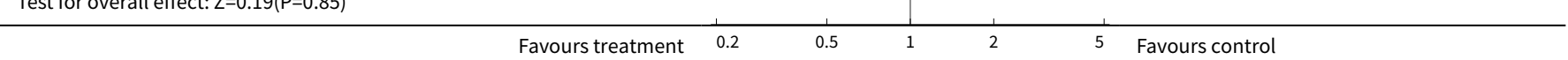


Analysis 1.20. Comparison 1 Intensive Case Management versus standard care, Outcome 20 Social functioning: 3a. Accommodation status (various measurements).

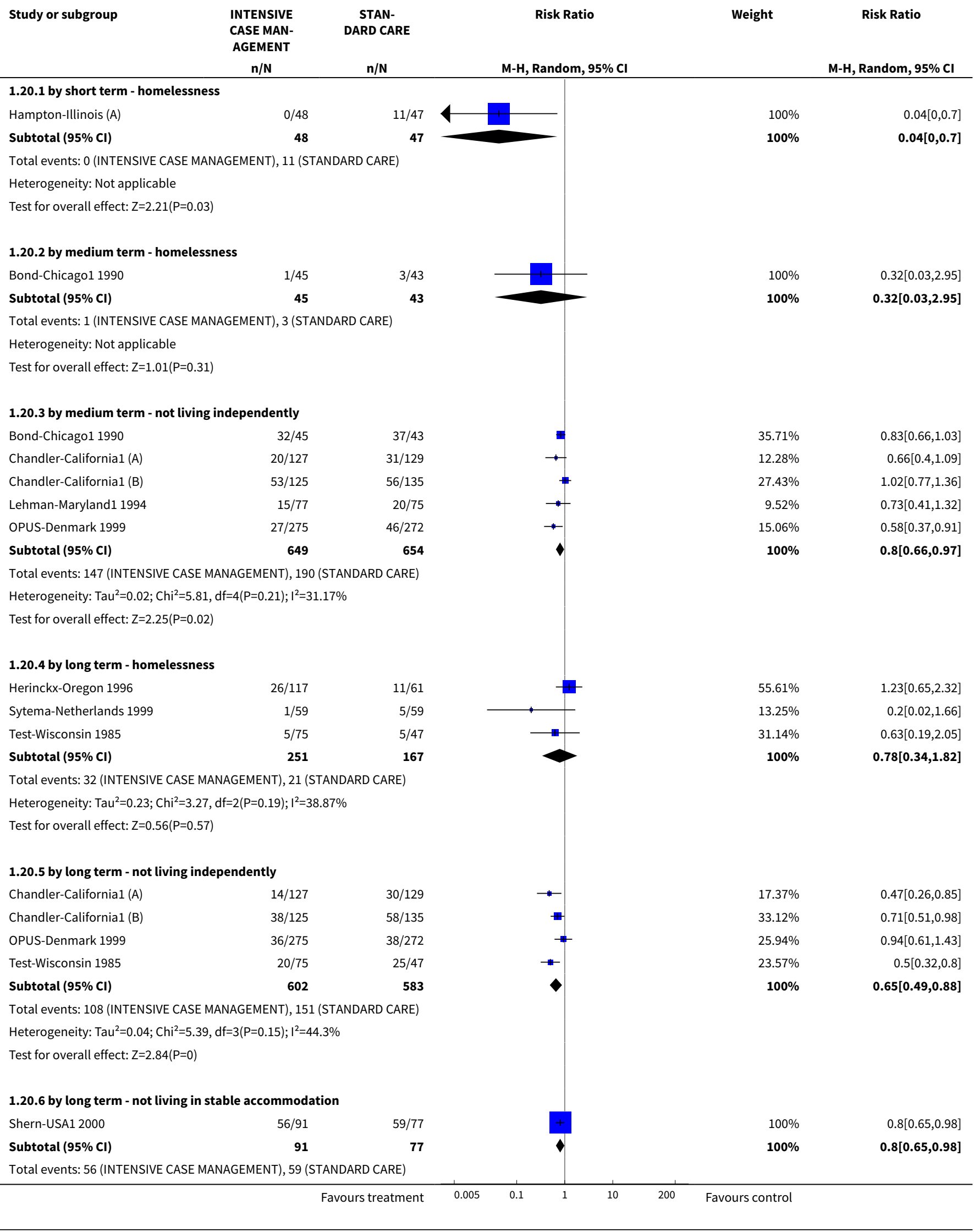




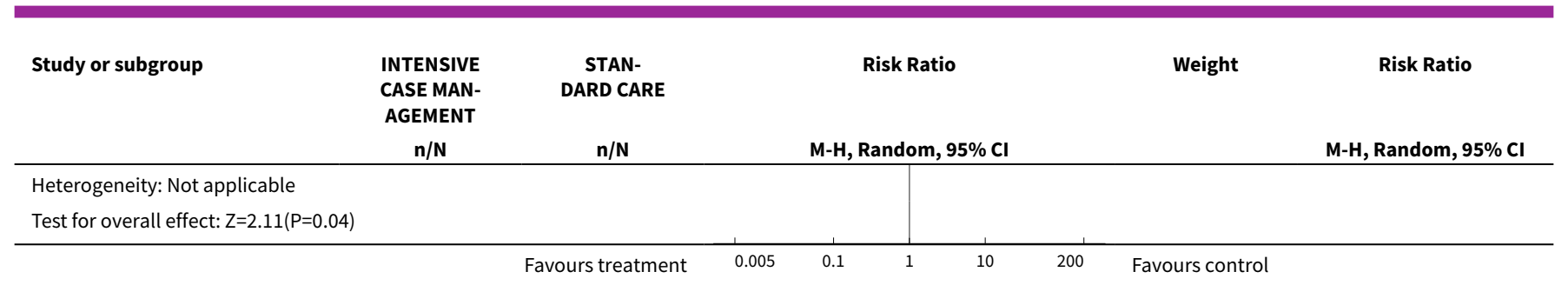

\section{Analysis 1.21. Comparison 1 Intensive Case Management versus standard care, Outcome 21 Social functioning:} 3b. Accomodation status: mean number of days in supported house (skewed data, sample size $\geqq 200$ ).

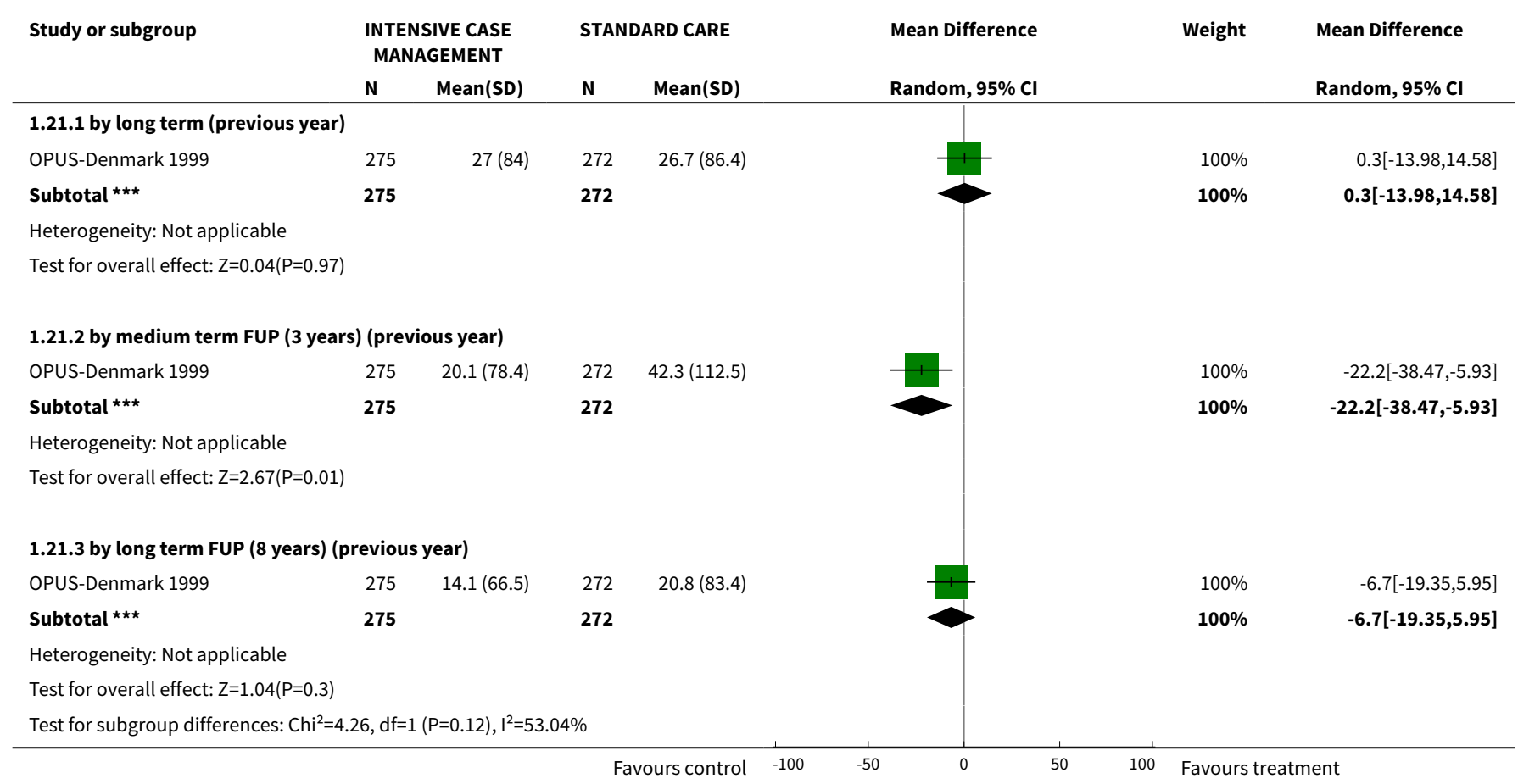

Analysis 1.22. Comparison 1 Intensive Case Management versus standard care, Outcome 22 Social functioning: 3c. Accommodation status (various measurements, skewed data).

\begin{tabular}{|c|c|c|c|c|}
\hline \multicolumn{5}{|c|}{ Social functioning: $3 c$. Accommodation status (various measurements, skewed data) } \\
\hline Study & Intervention & Mean & SD & Total \\
\hline \multicolumn{5}{|c|}{ by medium term - average days per month in stable accommodation } \\
\hline Lehman-Maryland1 1994 & 1. ICM & 17.5 & 9 & 77 \\
\hline Lehman-Maryland1 1994 & 2. Standard care & 13.34 & 9 & 75 \\
\hline Morse-Missouri3 2005 & 1. ICM & 5.77 & 7.42 & 54 \\
\hline Morse-Missouri3 2005 & 2. Standard care & 5.02 & 8.62 & 49 \\
\hline \multicolumn{5}{|c|}{ by long term - average days per month in sheltered homes } \\
\hline Morse-Missouri3 2005 & 1. ICM & 17.78 & 12.68 & 54 \\
\hline Morse-Missouri3 2005 & 2. Standard care & 12.59 & 13.27 & 49 \\
\hline Sytema-Netherlands 1999 & 1. ICM & 2.8 & 7.4 & 58 \\
\hline Sytema-Netherlands 1999 & 2. Standard care & 3.6 & 9.2 & 57 \\
\hline
\end{tabular}


Analysis 1.23. Comparison 1 Intensive Case Management versus standard care, Outcome 23 Social functioning: 4a. Substance abuse.

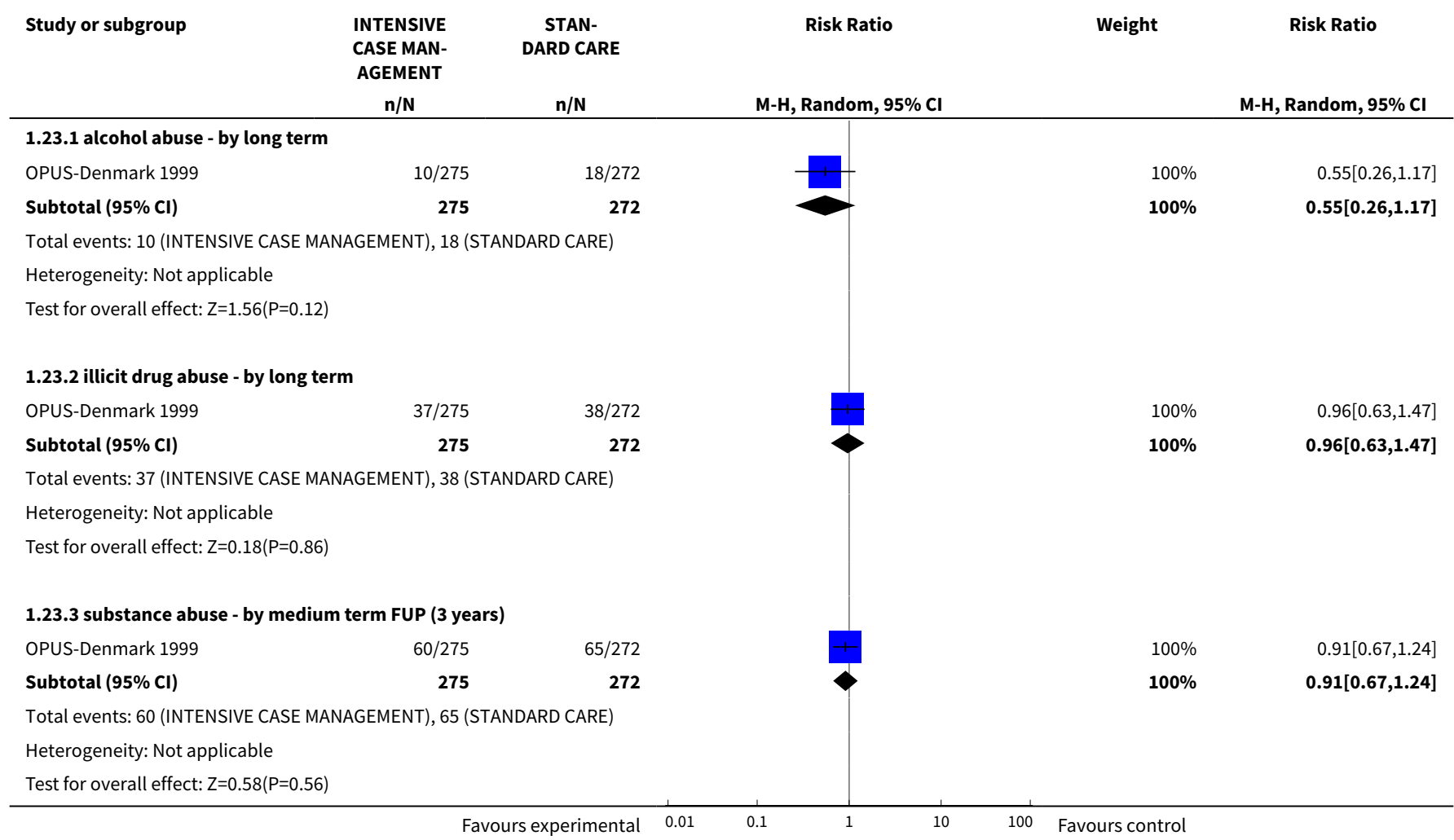

Analysis 1.24. Comparison 1 Intensive Case Management versus standard care, Outcome 24 Social functioning: 4b. Substance abuse (DALI, skewness not detectable) - by medium term.

\begin{tabular}{|c|c|c|c|c|c|}
\hline \multicolumn{6}{|c|}{ Social functioning: 4b. Substance abuse (DALI, skewness not detectable) - by medium term } \\
\hline Study & Intervention & Mean & & SD & Total \\
\hline \multicolumn{6}{|c|}{ alcohol abuse (DALI, -4 to +6 , high = worse) } \\
\hline Sytema-Netherlands 1999 & 1. ICM & -0.8 & 2.7 & & 45 \\
\hline Sytema-Netherlands 1999 & 2. Standard Care & -1.2 & 2.4 & & 36 \\
\hline \multicolumn{6}{|c|}{ drug abuse (DALI, -4 to +4 , high $=$ worse) } \\
\hline Sytema-Netherlands 1999 & 1. ICM & -1.4 & 1.3 & & 45 \\
\hline
\end{tabular}

Analysis 1.25. Comparison 1 Intensive Case Management versus standard care, Outcome 25 Social functioning: 4c. Substance abuse - days used per month (skewed data).

\begin{tabular}{|c|c|c|c|c|}
\hline \multicolumn{5}{|c|}{ Social functioning: 4c. Substance abuse - days used per month (skewed data) } \\
\hline Study & Intervention & Mean & SD & Total \\
\hline \multicolumn{5}{|c|}{ by medium term } \\
\hline Morse-Missouri3 2005 & 1. ICM & 6.25 & 7.84 & 54 \\
\hline Morse-Missouri3 2005 & 2. Standard care & 6.34 & 7.52 & 49 \\
\hline \multicolumn{5}{|c|}{ by long term } \\
\hline Morse-Missouri3 2005 & 1. ICM & 6.77 & 8.86 & 54 \\
\hline Morse-Missouri3 2005 & 2. Standard care & 6.42 & 7.84 & 49 \\
\hline
\end{tabular}




\section{Analysis 1.26. Comparison 1 Intensive Case Management versus standard care, Outcome 26 Social functioning: 5a. Average endpoint score (various scales).}

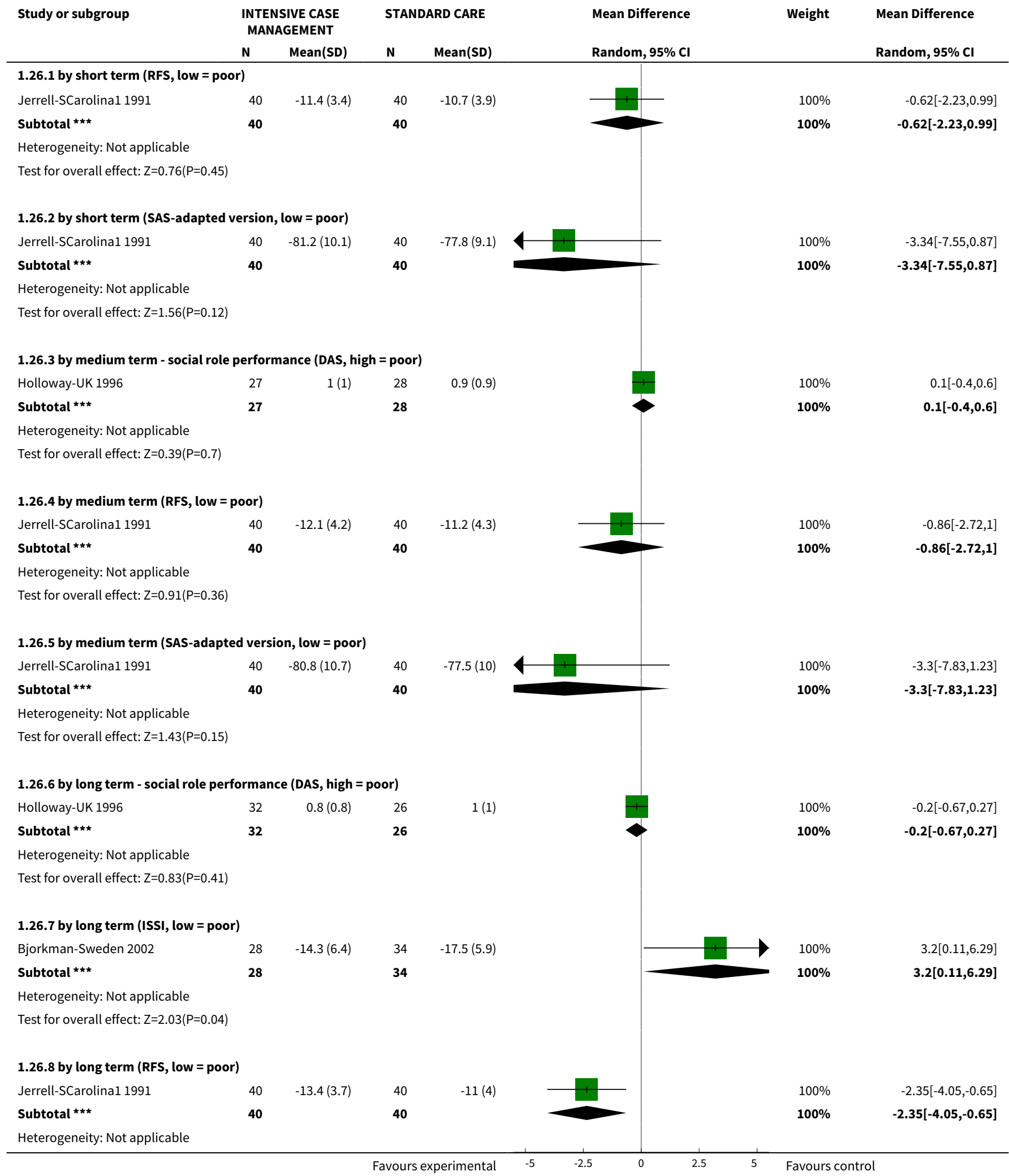




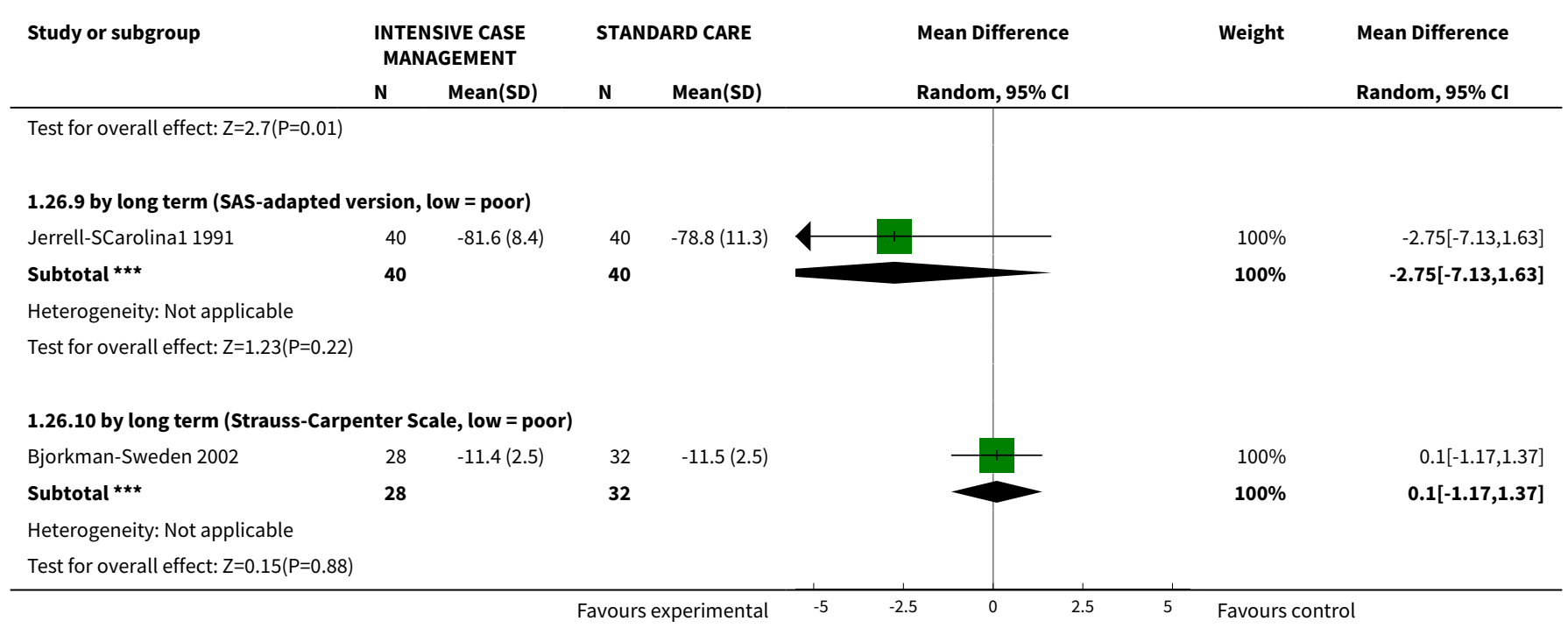

Analysis 1.27. Comparison 1 Intensive Case Management versus standard care, Outcome 27 Social functioning: 5 b. Average endpoint score (various scales, skewed data).

\begin{tabular}{|c|c|c|c|c|}
\hline \multicolumn{5}{|c|}{ Social functioning: $5 b$. Average endpoint score (various scales, skewed data) } \\
\hline Study & Intervention & Mean & SD & Total \\
\hline \multicolumn{5}{|c|}{ by short term (SAS, high = poor) } \\
\hline Muijen-UK2 1994 & 1. ICM & 3.9 & 1.1 & 35 \\
\hline Muijen-UK2 1994 & 2. Standard care & 3.9 & 1.6 & 29 \\
\hline \multicolumn{5}{|c|}{ by medium term (SAS, high = poor) } \\
\hline Muijen-UK2 1994 & 1. ICM & 4.3 & 1.6 & 29 \\
\hline Muijen-UK2 1994 & 2. Standard care & 3.7 & 1.5 & 25 \\
\hline \multicolumn{5}{|c|}{ by long term (SAS, high = poor) } \\
\hline Audini-UK 1994 & 1. ICM & 3.0 & 1.6 & 30 \\
\hline Audini-UK 1994 & 2. Standard care & 2.9 & 1.1 & 28 \\
\hline Muijen-UK2 1994 & 1. ICM & 3.6 & 1.4 & 24 \\
\hline Muijen-UK2 1994 & 2. Standard care & 4.2 & 1.4 & 22 \\
\hline \multicolumn{5}{|c|}{ by long term (REHAB, high = poor) } \\
\hline Marshall-UK 1995 & 1. ICM & 31.7 & 29.3 & 31 \\
\hline Marshall-UK 1995 & 2. Standard care & 40.83 & 19.65 & 30 \\
\hline
\end{tabular}

Analysis 1.28. Comparison 1 Intensive Case Management versus standard care, Outcome 28 Mental state: 1a. General symptoms - average endpoint score (various scales).

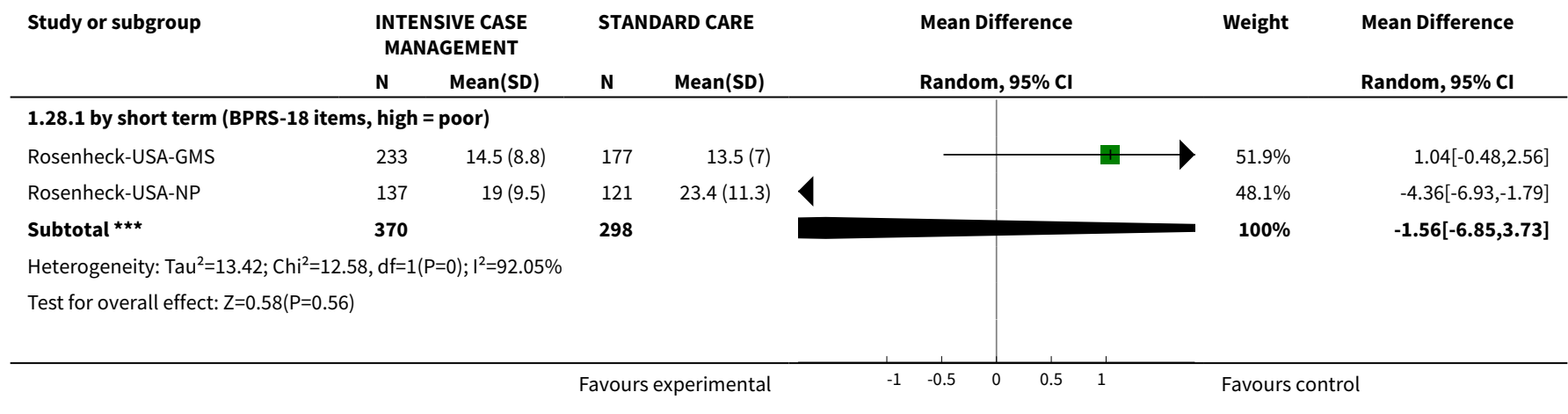




\begin{tabular}{|c|c|c|c|c|c|c|c|}
\hline \multirow[t]{2}{*}{ Study or subgroup } & \multicolumn{2}{|c|}{$\begin{array}{l}\text { INTENSIVE CASE } \\
\text { MANAGEMENT }\end{array}$} & \multicolumn{2}{|c|}{ STANDARD CARE } & \multirow{2}{*}{$\begin{array}{l}\text { Mean Difference } \\
\text { Random, } 95 \% \mathrm{Cl}\end{array}$} & \multirow[t]{2}{*}{ Weight } & \multirow{2}{*}{$\begin{array}{l}\text { Mean Difference } \\
\text { Random, } 95 \% \mathrm{Cl}\end{array}$} \\
\hline & $\mathbf{N}$ & $\operatorname{Mean}(S D)$ & $\mathbf{N}$ & Mean(SD) & & & \\
\hline \multicolumn{8}{|c|}{ 1.28.2 by short term (BSI, high = poor) } \\
\hline Rosenheck-USA-GMS & 233 & $1.2(0.9)$ & 177 & $1.3(0.9)$ & & $54.13 \%$ & $-0.07[-0.24,0.1]$ \\
\hline Rosenheck-USA-NP & 137 & $0.8(0.7)$ & 121 & $0.9(0.8)$ & & $45.87 \%$ & $-0.05[-0.24,0.14]$ \\
\hline \multicolumn{8}{|c|}{ Heterogeneity: $\mathrm{Tau}^{2}=0 ; \mathrm{Chi}^{2}=0.02, \mathrm{df}=1(\mathrm{P}=0.88) ; \mathrm{I}^{2}=0 \%$} \\
\hline \multicolumn{8}{|c|}{ Test for overall effect: $\mathrm{Z}=0.95(\mathrm{P}=0.34)$} \\
\hline \multicolumn{8}{|c|}{ 1.28.3 by short term (CSI, low = poor) } \\
\hline Lehman-Maryland1 1994 & 67 & $-4.2(0.8)$ & 58 & $-3.7(0.8)$ & & $100 \%$ & $-0.56[-0.84,-0.28]$ \\
\hline 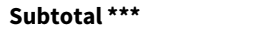 & 67 & & 58 & & & $100 \%$ & $-0.56[-0.84,-0.28]$ \\
\hline \multicolumn{8}{|c|}{ Test for overall effect: $Z=3.96(P<0.0001)$} \\
\hline \multicolumn{8}{|c|}{ 1.28.4 by medium term (BPRS-18 items, high = poor) } \\
\hline Rosenheck-USA-GMS & 239 & $14(9.3)$ & 181 & $14.4(9.3)$ & & $66.66 \%$ & $-0.46[-2.25,1.33]$ \\
\hline Rosenheck-USA-NP & 132 & $19(9.8)$ & 110 & $20.9(10.2)$ & & $33.34 \%$ & $-1.95[-4.49,0.59]$ \\
\hline 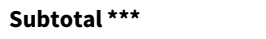 & 371 & & 291 & & & $100 \%$ & $-0.96[-2.42,0.51]$ \\
\hline \multicolumn{8}{|c|}{ Heterogeneity: $\operatorname{Tau}^{2}=0 ; \mathrm{Chi}^{2}=0.88, \mathrm{df}=1(\mathrm{P}=0.35) ; \mathrm{I}^{2}=0 \%$} \\
\hline \multicolumn{8}{|c|}{ Test for overall effect: $Z=1.28(P=0.2)$} \\
\hline \multicolumn{8}{|c|}{ 1.28.5 by medium term (BSI, high = poor) } \\
\hline Rosenheck-USA-GMS & 239 & $1.2(0.9)$ & 181 & $1.2(0.9)$ & & $55.19 \%$ & $-0.01[-0.18,0.16]$ \\
\hline Rosenheck-USA-NP & 132 & $0.7(0.7)$ & 110 & $0.8(0.8)$ & & $44.81 \%$ & $-0.04[-0.23,0.15]$ \\
\hline 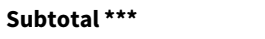 & 371 & & 291 & & & $100 \%$ & $-0.02[-0.15,0.1]$ \\
\hline \multicolumn{8}{|c|}{ Heterogeneity: $\mathrm{Tau}^{2}=0 ; \mathrm{Chi}^{2}=0.06, \mathrm{df}=1(\mathrm{P}=0.81) ; \mathrm{I}^{2}=0 \%$} \\
\hline \multicolumn{8}{|c|}{ 1.28.6 medium term (CSI, low = poor) } \\
\hline Lehman-Maryland1 1994 & 67 & $-4.1(0.9)$ & 58 & $-3.8(0.8)$ & & $100 \%$ & $-0.35[-0.65,-0.05]$ \\
\hline 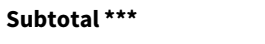 & 67 & & 58 & & & $100 \%$ & $-0.35[-0.65,-0.05]$ \\
\hline \multicolumn{8}{|c|}{ Heterogeneity: Not applicable } \\
\hline \multicolumn{8}{|c|}{ Test for overall effect: $Z=2.26(P=0.02)$} \\
\hline \multicolumn{8}{|c|}{ 1.28.7 by long term (BPRS-18 items, high = poor) } \\
\hline Morse-Missouri3 2005 & 65 & $2(0.5)$ & 65 & $2(0.6)$ & - & $41.06 \%$ & $0.02[-0.18,0.22]$ \\
\hline Rosenheck-USA-GMS & 221 & $11.4(8.3)$ & 189 & $14.3(9.6)$ & & $32.81 \%$ & $-2.96[-4.71,-1.21]$ \\
\hline Rosenheck-USA-NP & 129 & $15.6(9.2)$ & 108 & $17.6(11.1)$ & & $26.13 \%$ & $-1.96[-4.58,0.66]$ \\
\hline Subtotal $\star \star \star$ & 415 & & 362 & & & $100 \%$ & $-1.48[-3.69,0.74]$ \\
\hline \multicolumn{8}{|c|}{ Heterogeneity: $\mathrm{Tau}^{2}=3.11 ; \mathrm{Chi}^{2}=13.13, \mathrm{df}=2(\mathrm{P}=0) ; \mathrm{I}^{2}=84.77 \%$} \\
\hline \multicolumn{8}{|c|}{ Test for overall effect: $Z=1.3(P=0.19)$} \\
\hline 1.28.8 by long term (BSI, & & & & & & & \\
\hline Rosenheck-USA-GMS & 221 & $1.1(0.8)$ & 189 & $1.3(0.9)$ & + & $51.84 \%$ & $-0.23[-0.4,-0.06]$ \\
\hline Rosenheck-USA-NP & 129 & $0.6(0.6)$ & 108 & $0.7(0.8)$ & 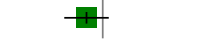 & $48.16 \%$ & $-0.13[-0.31,0.05]$ \\
\hline 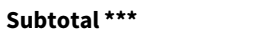 & 350 & & 297 & & $\gamma$ & $100 \%$ & $-0.18[-0.31,-0.06]$ \\
\hline Heterogeneity: $\operatorname{Tau}^{2}=0 ; \mathrm{Cr}$ & $1(P=0.4$ & $; 1^{2}=0 \%$ & & & & & \\
\hline Test for overall effect: $Z=2$ & & & & & & & \\
\hline
\end{tabular}


Analysis 1.29. Comparison 1 Intensive Case Management versus standard care, Outcome 29

Mental state: 1 b. General symptoms - mean change from baseline (CSI, low = poor ) - by long term.

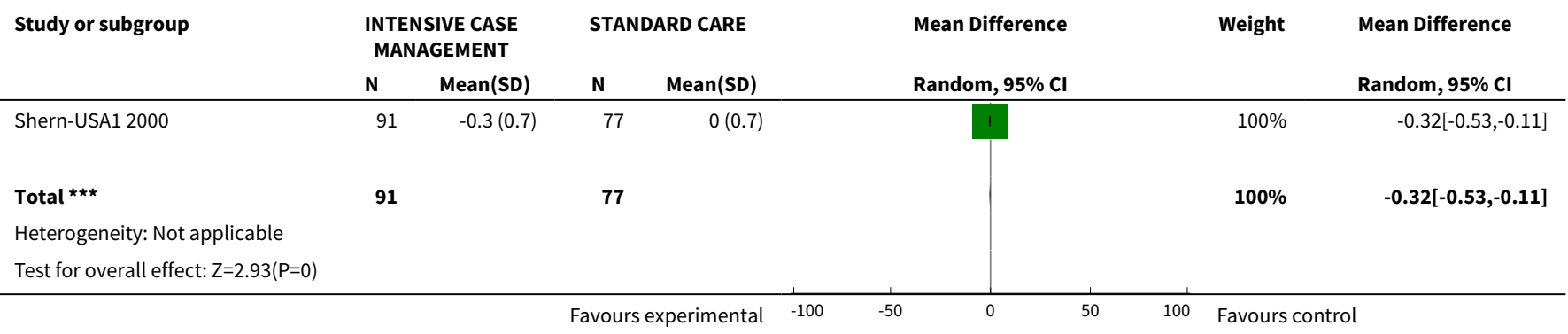

Analysis 1.30. Comparison 1 Intensive Case Management versus standard care, Outcome 30 Mental state: 1c. General symptoms - average endpoint score (various scales, skewed data).

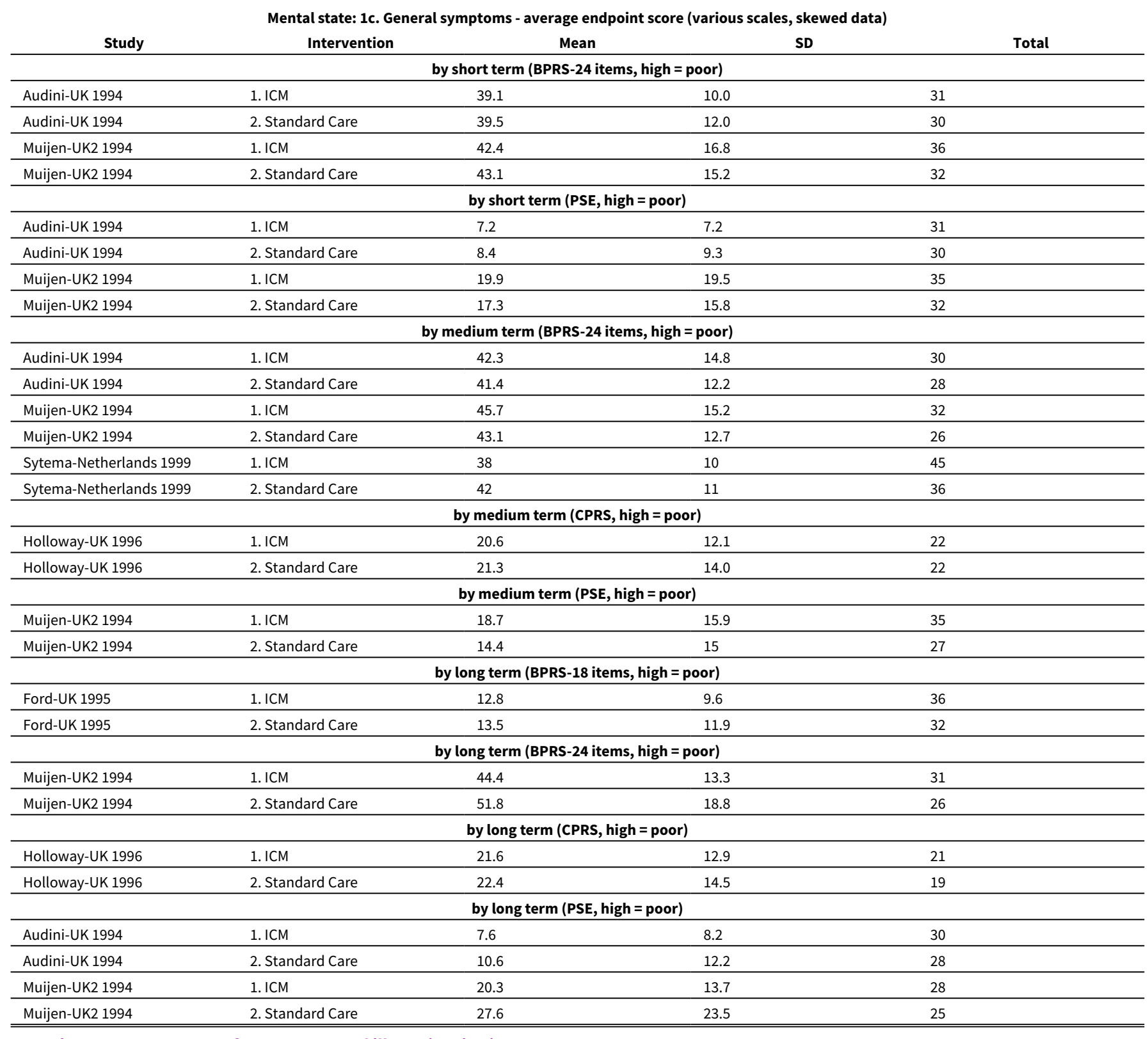


Mental state: 1c. General symptoms - average endpoint score (various scales, skewed data)

\begin{tabular}{llclc}
\multicolumn{1}{c}{ Study } & Intervention & Mean & Sotal & \\
\hline & & by long term (SCL-90, high = poor) & \\
\hline Bjorkman-Sweden 2002 & 1. ICM & 102 & 68.5 & \\
\hline Bjorkman-Sweden 2002 & 2. Standard Care & 81.4 & 55.1 & 27 \\
\hline
\end{tabular}

Analysis 1.31. Comparison 1 Intensive Case Management versus standard care, Outcome 31 Mental state: 2 a. Specific symptoms - depression at follow up interview.

\begin{tabular}{|c|c|c|c|c|c|}
\hline \multirow[t]{2}{*}{ Study or subgroup } & $\begin{array}{l}\text { INTENSIVE } \\
\text { CASE MAN- } \\
\text { AGEMENT }\end{array}$ & $\begin{array}{c}\text { STAN- } \\
\text { DARD CARE }\end{array}$ & \multirow[b]{2}{*}{ M-H, Random, $95 \% \mathrm{Cl}$} & \multirow[t]{2}{*}{ Weight } & \multirow[b]{2}{*}{ M-H, Random, $95 \% \mathrm{Cl}$} \\
\hline & $n / N$ & $\mathbf{n} / \mathbf{N}$ & & & \\
\hline OPUS-Denmark 1999 & $55 / 275$ & $71 / 272$ & & $100 \%$ & $0.77[0.56,1.04]$ \\
\hline Subtotal $(95 \% \mathrm{Cl})$ & 275 & 272 & & $100 \%$ & $0.77[0.56,1.04]$ \\
\hline \multicolumn{6}{|c|}{ Total events: 55 (INTENSIVE CASE MANAGEMENT), 71 (STANDARD CARE) } \\
\hline \multicolumn{6}{|c|}{ Heterogeneity: Not applicable } \\
\hline \multicolumn{6}{|c|}{ Test for overall effect: $Z=1.69(P=0.09)$} \\
\hline \multicolumn{6}{|l|}{ 1.31.2 by long term } \\
\hline OPUS-Denmark 1999 & $41 / 275$ & $49 / 272$ & & $100 \%$ & $0.83[0.57,1.21]$ \\
\hline Subtotal $(95 \% \mathrm{Cl})$ & 275 & 272 & & $100 \%$ & $0.83[0.57,1.21]$ \\
\hline \multicolumn{6}{|c|}{ Total events: 41 (INTENSIVE CASE MANAGEMENT), 49 (STANDARD CARE) } \\
\hline \multicolumn{6}{|c|}{ Test for overall effect: $Z=0.98(P=0.33)$} \\
\hline \multicolumn{6}{|c|}{1.31 .3 by medium term FUP ( 3 years) } \\
\hline OPUS-Denmark 1999 & $67 / 275$ & $53 / 272$ & & $100 \%$ & $1.25[0.91,1.72]$ \\
\hline Subtotal $(95 \% \mathrm{Cl})$ & 275 & 272 & & $100 \%$ & $1.25[0.91,1.72]$ \\
\hline \multicolumn{6}{|c|}{ Total events: 67 (INTENSIVE CASE MANAGEMENT), 53 (STANDARD CARE) } \\
\hline \multicolumn{6}{|c|}{ Heterogeneity: Not applicable } \\
\hline \multicolumn{6}{|c|}{ Test for overall effect: $\mathrm{Z}=1.37(\mathrm{P}=0.17)$} \\
\hline & & s experimental & 0.5 & urs control & \\
\hline
\end{tabular}

Analysis 1.32. Comparison 1 Intensive Case Management versus standard care, Outcome 32 Mental state: $\mathbf{2 b}$. Specific symptoms - average endpoint score (various scales, skewed data, sample size $\geqq 200$ ).

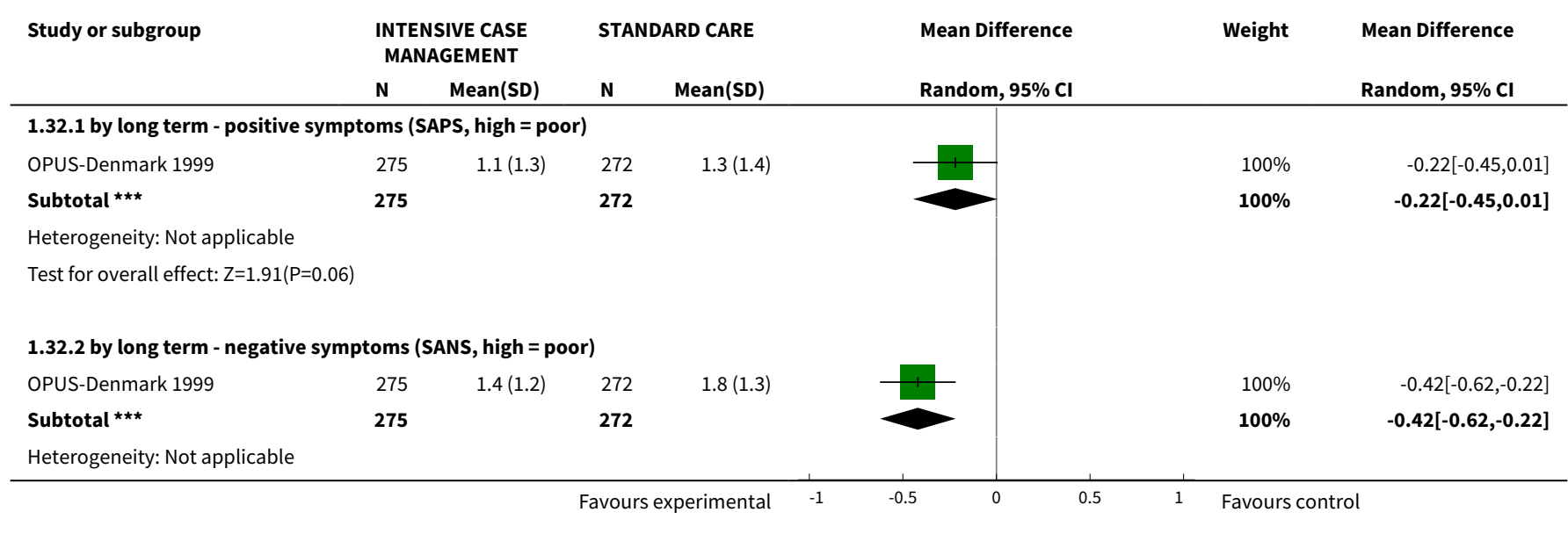


Study or subgroup

\section{INTENSIVE CASE STANDARD CARE}

Mean Difference

Weight

Mean Difference

N Mean(SD)

Random, $95 \% \mathrm{Cl}$

Random, 95\% Cl

Test for overall effect: $Z=4.07(P<0.0001)$

1.32.3 by medium term FUP ( 3 years) - positive symptoms (SAPS, high = poor)

$\begin{array}{lllll}\text { OPUS-Denmark } 1999 & 275 & 1.4(1.6) & 272 & 1.3(1.6)\end{array}$

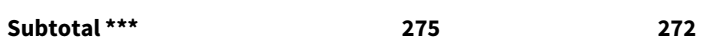

Heterogeneity: Not applicable

Test for overall effect: $\mathrm{Z}=0.87(\mathrm{P}=0.38)$

1.32.4 by medium term FUP ( 3 years) - negative symptoms (SANS, high = poor)

OPUS-Denmark $1999 \quad 275 \quad 1.7(1.3) \quad 272 \quad 1.8(1.5)$

Subtotal ***

275

Heterogeneity: Not applicable

Test for overall effect: $\mathrm{Z}=0.85(\mathrm{P}=0.4)$

1.32.5 by long term FUP ( 8 years) - positive symptoms (SAPS, high = poor)

OPUS-Denmark 1999

$275 \quad 1.2(1.4) \quad 272 \quad 1.2(1.4)$

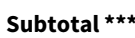

275

272

Heterogeneity: Not applicable

Test for overall effect: $\mathrm{Z}=0.25(\mathrm{P}=0.81)$

1.32.6 by long term FUP ( 8 years) - negative symptoms (SANS, high = poor)

OPUS-Denmark 1999

$100 \%$

$100 \%$

$0.12[-0.15,0.39]$

$0.12[-0.15,0.39]$

Heterogeneity: Not applicable

Test for overall effect: $\mathrm{Z}=0.63(\mathrm{P}=0.53)$

Test for subgroup differences: $\mathrm{Chi}^{2}=17.17, \mathrm{df}=1(\mathrm{P}=0), \mathrm{I}^{2}=70.88 \%$

Analysis 1.33. Comparison 1 Intensive Case Management versus standard care, Outcome 33 Mental state: $2 c$. Specific symptoms - average endpoint score (various scales, skewed data).

\begin{tabular}{|c|c|c|c|c|}
\hline \multicolumn{5}{|c|}{ Mental state: 2 c. Specific symptoms - average endpoint score (various scales, skewed data) } \\
\hline Study & Intervention & Mean & SD & Total \\
\hline \multicolumn{5}{|c|}{ by medium term - depression symptoms (BDI, high = poor) } \\
\hline Holloway-UK 1996 & 1. ICM & 11.5 & 8.9 & 23 \\
\hline Holloway-UK 1996 & 2. Standard care & 18.5 & 13.9 & 19 \\
\hline \multicolumn{5}{|c|}{ by medium term - negative symptoms (SANS, high = poor) } \\
\hline Holloway-UK 1996 & 1. ICM & 7.3 & 4 & 26 \\
\hline Holloway-UK 1996 & 2. Standard care & 6.3 & 4.4 & 22 \\
\hline \multicolumn{5}{|c|}{ by long term - depression symptoms (BDI, high = poor) } \\
\hline Holloway-UK 1996 & 1. ICM & 12.8 & 8.1 & 25 \\
\hline Holloway-UK 1996 & 2. Standard care & 14.8 & 11.5 & 17 \\
\hline \multicolumn{5}{|c|}{ by long term - negative symptoms (SANS, high = poor) } \\
\hline Holloway-UK 1996 & 1. ICM & 7.3 & 3.7 & 26 \\
\hline Holloway-UK 1996 & 2. Standard care & 7.1 & 4.1 & 20 \\
\hline
\end{tabular}


Analysis 1.34. Comparison 1 Intensive Case Management versus standard care, Outcome 34 Behaviour: 1. Specific behaviour - self-harm.

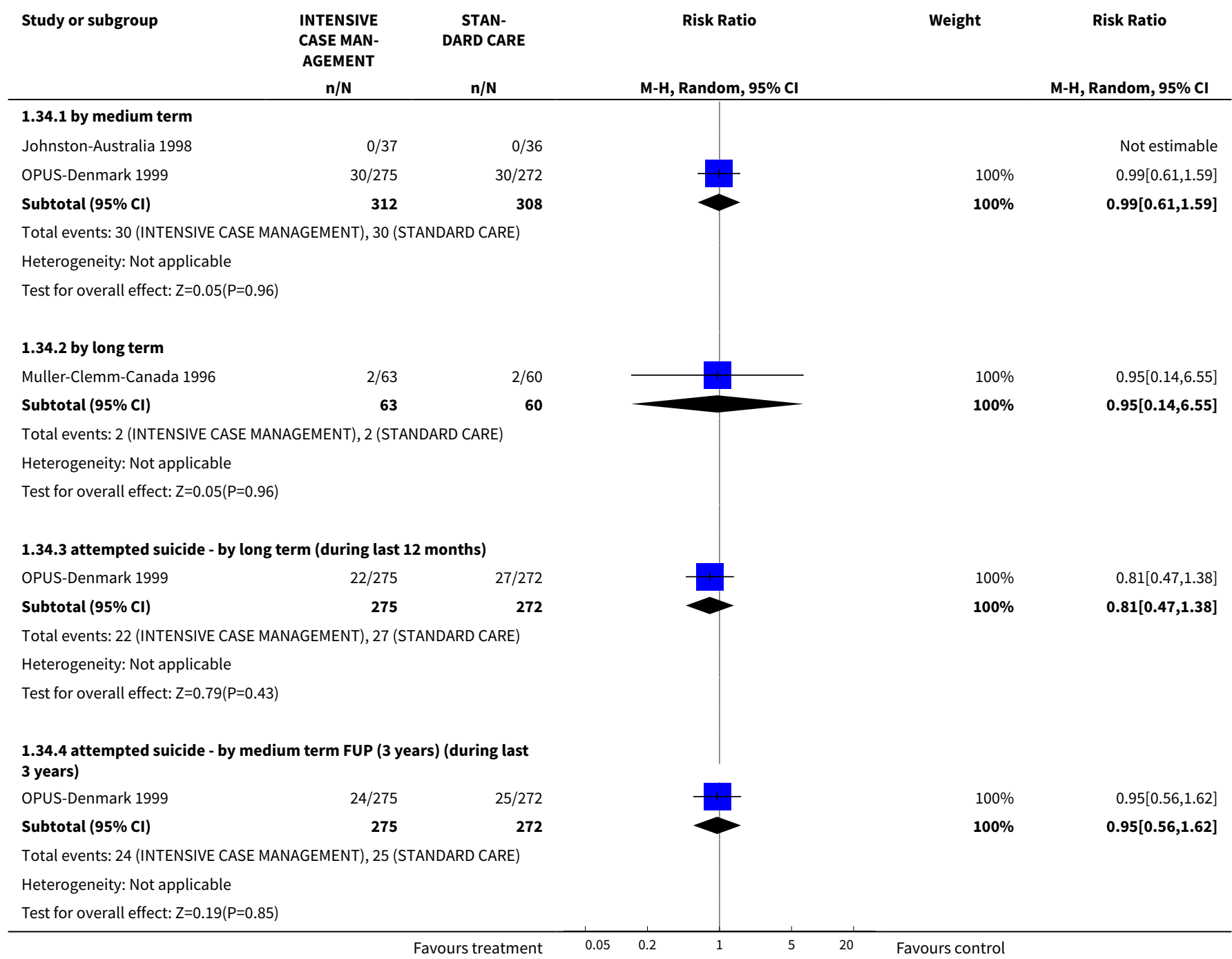

Analysis 1.35. Comparison 1 Intensive Case Management versus standard care, Outcome 35 Behaviour: 2. Social behaviour - average endpoint score (SBS, high = poor).

Behaviour: 2. Social behaviour - average endpoint score (SBS, high = poor)

\begin{tabular}{|c|c|c|c|c|}
\hline Study & Intervention & Mean & SD & Total \\
\hline \multicolumn{5}{|c|}{ by medium term } \\
\hline Holloway-UK 1996 & 1. ICM & 3.2 & 2.8 & 33 \\
\hline Holloway-UK 1996 & 2. Standard Care & 2.4 & 2.9 & 30 \\
\hline \multicolumn{5}{|c|}{ by long term } \\
\hline Holloway-UK 1996 & 1. ICM & 3.3 & 2.8 & 34 \\
\hline Holloway-UK 1996 & 2. Standard Care & 2.7 & 2.2 & 26 \\
\hline
\end{tabular}


Analysis 1.36. Comparison 1 Intensive Case Management versus standard care, Outcome 36 Quality of life: 1a. Average endpoint score (various scales).

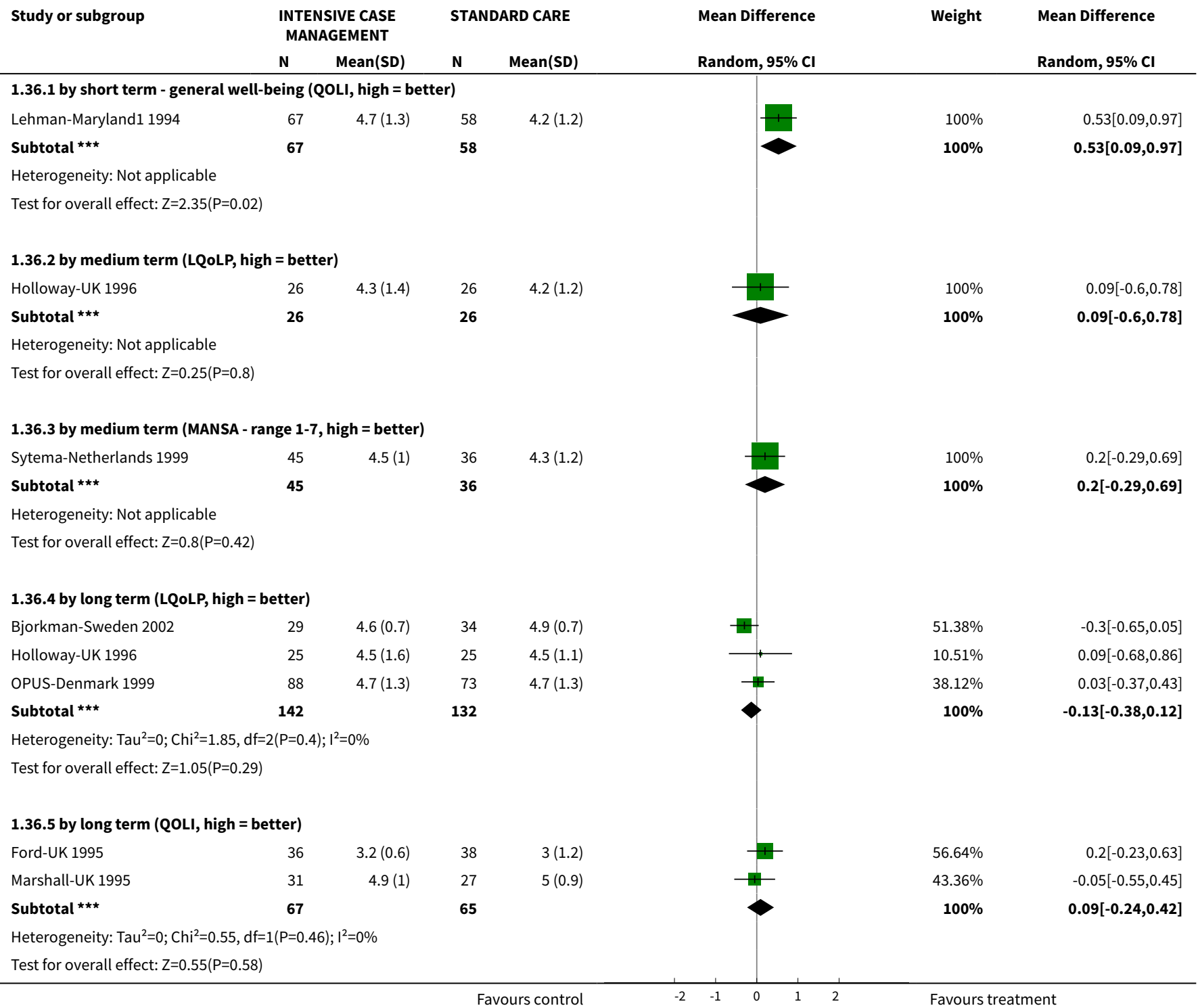

Analysis 1.37. Comparison 1 Intensive Case Management versus standard care, Outcome 37 Quality of life: 1b. Mean change from baseline (QOLI, high = better, skewed data) - by long term.

Quality of life: 1b. Mean change from baseline (QOLI, high = better, skewed data) - by long term

\begin{tabular}{lllll}
\multicolumn{1}{c}{ Study } & \multicolumn{1}{c}{ Intervention } & Mean & Total & \\
\hline Shern-USA1 2000 & 1. ICM & 1.19 & 1.99 & \\
\hline Shern-USA1 2000 & 2. Standard Care & -0.02 & 1.65 & \\
\hline
\end{tabular}


Analysis 1.38. Comparison 1 Intensive Case Management versus standard care,

Outcome 38 Participant satisfaction: 1a. Average endpoint score (CSQ, high = better).

\begin{tabular}{|c|c|c|c|c|c|c|c|}
\hline \multirow[t]{2}{*}{ Study or subgroup } & \multicolumn{2}{|c|}{$\begin{array}{l}\text { INTENSIVE CASE } \\
\text { MANAGEMENT }\end{array}$} & \multicolumn{2}{|c|}{ STANDARD CARE } & \multirow{2}{*}{$\begin{array}{l}\text { Mean Difference } \\
\text { Random, } 95 \% \mathrm{Cl}\end{array}$} & \multirow[t]{2}{*}{ Weight } & \multirow{2}{*}{$\begin{array}{l}\text { Mean Difference } \\
\text { Random, } 95 \% \mathrm{Cl}\end{array}$} \\
\hline & $\mathbf{N}$ & Mean(SD) & $\mathbf{N}$ & Mean(SD) & & & \\
\hline \multicolumn{8}{|l|}{ 1.38.1 by short term } \\
\hline Audini-UK 1994 & 31 & $27.6(3.9)$ & 30 & $21.4(9.3)$ & & $100 \%$ & $6.2[2.6,9.8]$ \\
\hline Subtotal $\star \star \star$ & 31 & & 30 & & & $100 \%$ & $6.2[2.6,9.8]$ \\
\hline \multicolumn{8}{|l|}{ Heterogeneity: Not applicable } \\
\hline \multicolumn{8}{|l|}{ 1.38.2 by medium term } \\
\hline OPUS-Denmark 1999 & 227 & $24.9(4.5)$ & 192 & $23(7.2)$ & t & $84.03 \%$ & $1.9[0.73,3.07]$ \\
\hline Sytema-Netherlands 1999 & 45 & $22.6(4.5)$ & 36 & $20.5(7.2)$ & & $15.97 \%$ & $2.1[-0.59,4.79]$ \\
\hline Subtotal $\star \star \star ~$ & 272 & & 228 & & & $100 \%$ & $1.93[0.86,3.01]$ \\
\hline \multicolumn{8}{|c|}{ Heterogeneity: $\mathrm{Tau}^{2}=0 ; \mathrm{Chi}^{2}=0.02, \mathrm{df}=1(\mathrm{P}=0.89) ; \mathrm{I}^{2}=0 \%$} \\
\hline \multicolumn{8}{|c|}{ Test for overall effect: $\mathrm{Z}=3.52(\mathrm{P}=0)$} \\
\hline \multicolumn{8}{|l|}{ 1.38.3 by long term } \\
\hline Audini-UK 1994 & 28 & $26.6(4.8)$ & 26 & $22.9(8.7)$ & & $5.84 \%$ & $3.7[-0.09,7.49]$ \\
\hline OPUS-Denmark 1999 & 205 & $26.1(3.7)$ & 164 & $22.9(5.2)$ & & $94.16 \%$ & $3.2[2.26,4.14]$ \\
\hline Subtotal ${ }^{\star \star \star}$ & 233 & & 190 & & & $100 \%$ & $3.23[2.31,4.14]$ \\
\hline \multicolumn{8}{|c|}{ Heterogeneity: $\mathrm{Tau}^{2}=0 ; \mathrm{Chi}^{2}=0.06, \mathrm{df}=1(\mathrm{P}=0.8) ; \mathrm{I}^{2}=0 \%$} \\
\hline Test for overall effect: $Z=6.9$ & & & & & & & \\
\hline
\end{tabular}

Analysis 1.39. Comparison 1 Intensive Case Management versus standard care, Outcome 39 Participants satisfaction: 1 b. Average endpoint score (CSQ, high = better, skewed data) - by short term.

Participants satisfaction: 1b. Average endpoint score (CSQ, high = better, skewed data) - by short term

\begin{tabular}{|c|c|c|c|c|c|}
\hline Study & Intervention & Mean & SD & Total & Note \\
\hline Muijen-UK2 1994 & 1. ICM & 26 & 5.4 & 30 & \\
\hline Muijen-UK2 1994 & 2. Standard Care* & 22 & 7.5 & 13 & ${ }^{\star}$ Attrition $>50 \%$. \\
\hline
\end{tabular}

Analysis 1.40. Comparison 1 Intensive Case Management versus standard care, Outcome 40 Participants need: 1. Average endpoint score (various scales, skewed data).

Participants need: 1. Average endpoint score (various scales, skewed data)

\begin{tabular}{|c|c|c|c|c|c|}
\hline Study & Intervention & Mean & SD & Total & Note \\
\hline \multicolumn{6}{|c|}{ by medium term - met needs (CANSAS, high = better) } \\
\hline $\begin{array}{l}\text { Sytema-Netherlands } \\
1999\end{array}$ & 1. ICM & 8.5 & 4.5 & 45 & \\
\hline $\begin{array}{l}\text { Sytema-Netherlands } \\
1999\end{array}$ & 2. Standard Care & 8.6 & 4.7 & 36 & \\
\hline \multicolumn{6}{|c|}{ by medium term - unmet needs (CANSAS, high = poor) } \\
\hline $\begin{array}{l}\text { Sytema-Netherlands } \\
1999\end{array}$ & 1. ICM & 1.4 & 1.9 & 45 & \\
\hline $\begin{array}{l}\text { Sytema-Netherlands } \\
1999\end{array}$ & 2. Standard Care & 1.6 & 1.7 & 36 & \\
\hline \multicolumn{6}{|c|}{ by long term (CAN, high = poor) } \\
\hline Bjorkman-Sweden 2002 & 1. ICM & 3.2 & 1.8 & 28 & \\
\hline Bjorkman-Sweden 2002 & 2. Standard Care & 4.6 & 3.8 & 36 & \\
\hline
\end{tabular}


Analysis 1.41. Comparison 1 Intensive Case Management versus standard care, Outcome 41 Costs: 1a. Direct costs of psychiatric hospital care - by medium term (Unit cost = USD, fiscal year 1990).

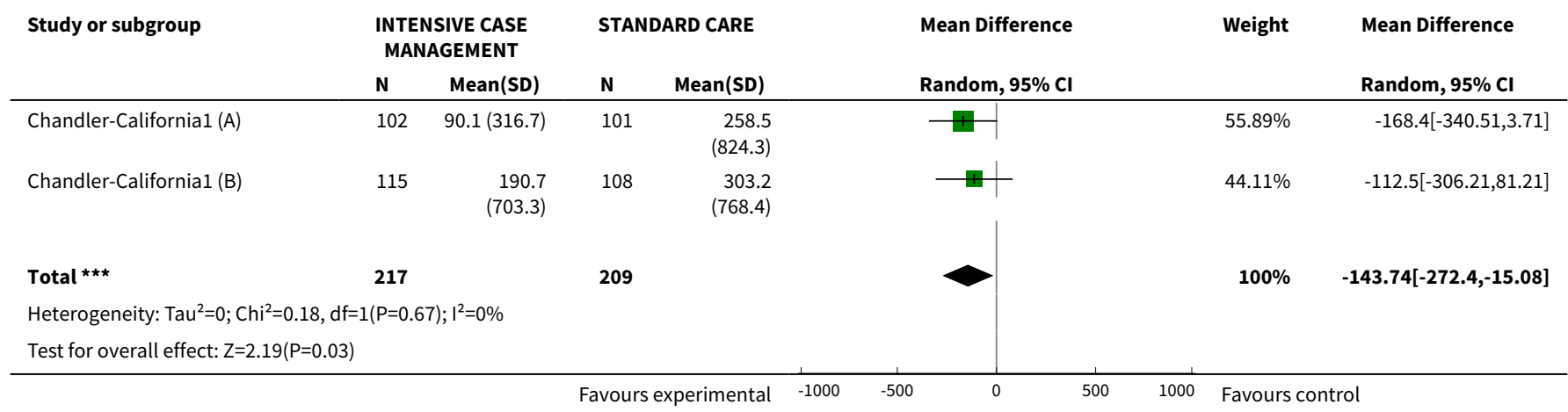

\section{Analysis 1.42. Comparison 1 Intensive Case Management versus standard care, Outcome 42 Costs: 1 b. Direct costs of psychiatric hospital care - skewed data.}

\begin{tabular}{|c|c|c|c|c|c|}
\hline \multicolumn{6}{|c|}{ Costs: 1b. Direct costs of psychiatric hospital care - skewed data } \\
\hline Study & Intervention & Mean & SD & Total & Note \\
\hline \multicolumn{6}{|c|}{ by medium term } \\
\hline Cusack-North Carolina & 1. $\mathrm{ICM}^{*}$ & 5,530 & 12,414 & 72 & $\begin{array}{l}{ }^{\star} \text { Unit cost US } \$ \text {, Inpa- } \\
\text { tient costs } \\
\text { Time period: } 12 \text { months. }\end{array}$ \\
\hline Cusack-North Carolina & 2. Standard care ${ }^{\star}$ & 8,827 & 19,289 & 62 & $\begin{array}{l}{ }^{*} \text { Unit cost US } \$ \text {, Inpa- } \\
\text { tient costs } \\
\text { Time period: } 12 \text { months. }\end{array}$ \\
\hline Lehman-Maryland1 1994 & 1. $\mathrm{ICM}^{\star}$ & 2,619 & 4,440 & 77 & \\
\hline Lehman-Maryland1 1994 & 2. Standard care ${ }^{\star}$ & 4,662 & 6,034 & 75 & $\begin{array}{l}{ }^{\star} \text { Unit cost US } \$ \text {, fiscal } \\
\text { year } 1994 . \\
t \text {-value }=2.34 \\
\text { Time period: } 12 \text { months. }\end{array}$ \\
\hline Morse-Missouri3 2005 & 1. $\mathrm{ICM}^{*}$ & 624 & 2,314 & 54 & \\
\hline Morse-Missouri3 2005 & 2. Standard care ${ }^{\star}$ & 439 & 1,596 & 49 & $\begin{array}{l}\text { * Unit cost US } \$ \text {, fiscal } \\
\text { year } 2001 . \\
\text { "No main effect of } \\
\text { of treatment condi- } \\
\text { tion for inpatient cost- } \\
\mathrm{s}, F(2,146)=0.10, \mathrm{p}=0.9 \text {, } \\
5^{2}=0.01 . " \\
\text { Time period: } 6 \text { months. }\end{array}$ \\
\hline
\end{tabular}

\begin{tabular}{|c|c|c|c|c|c|}
\hline \multicolumn{6}{|c|}{ by long term } \\
\hline Ford-UK 1995 & 1. $\mathrm{ICM}^{*}$ & 378 & 846 & 39 & \\
\hline Ford-UK 1995 & 2. Standard care* & 237 & 492 & 38 & $\begin{array}{l}{ }^{\star} \text { Unit cost } £ \text {, fiscal year } \\
\text { not reported, study base } \\
\text { year } 1990 . \\
{ }^{\star \star} \text { No statistical analysis } \\
\text { available from the paper. } \\
\text { Time period: } 18 \text { months. }\end{array}$ \\
\hline Morse-Missouri3 2005 & 1. $\mathrm{ICM}^{*}$ & 855 & 2,356 & 54 & \\
\hline Morse-Missouri3 2005 & 2. Standard care* & 455 & 1,065 & 49 & $\begin{array}{l}{ }^{\star} \text { Unit cost US } \$ \text {, fiscal } \\
\text { year } 2001 . \\
\star \star ~ " N o \text { main effect } \\
\text { of treatment condi- } \\
\text { tion for inpatient cost- } \\
\mathrm{s}, F(2,146)=0.10, p=0.9 \text {, } \\
5^{2}=0.01 . " \\
\text { Time period: } 6 \text { months. }\end{array}$ \\
\hline
\end{tabular}

\begin{tabular}{lllll}
\hline $\begin{array}{l}\text { Quinlivan-California } \\
1995\end{array}$ & 1. ICM $^{*}$ & 301 & 397 & 30
\end{tabular}


Costs: 1b. Direct costs of psychiatric hospital care - skewed data

\begin{tabular}{|c|c|c|c|c|c|}
\hline Study & Intervention & Mean & SD & Total & Note \\
\hline $\begin{array}{l}\text { Quinlivan-California } \\
1995\end{array}$ & 2. Standard care* & 1,636 & 2,593 & 30 & $\begin{array}{l}\text { * Unit cost US } \$ \text {, fiscal } \\
\text { year not reported, but } \\
\text { study was carried on } \\
\text { from April } 1990 \text { to March } \\
1992 . \\
\text { ** "Costs significant- } \\
\text { ly lower for the ICM } \\
\text { group ( } F=4.32, \mathrm{df}=2.87 \text {, } \\
\mathrm{p}=0.02 .) " \\
\text { Time period: } 24 \text { months. }\end{array}$ \\
\hline
\end{tabular}

\section{Analysis 1.43. Comparison 1 Intensive Case Management versus standard care, Outcome 43 Costs: 2 a. Direct healthcare costs - by long term (Unit cost = USD, fiscal year 1988).}

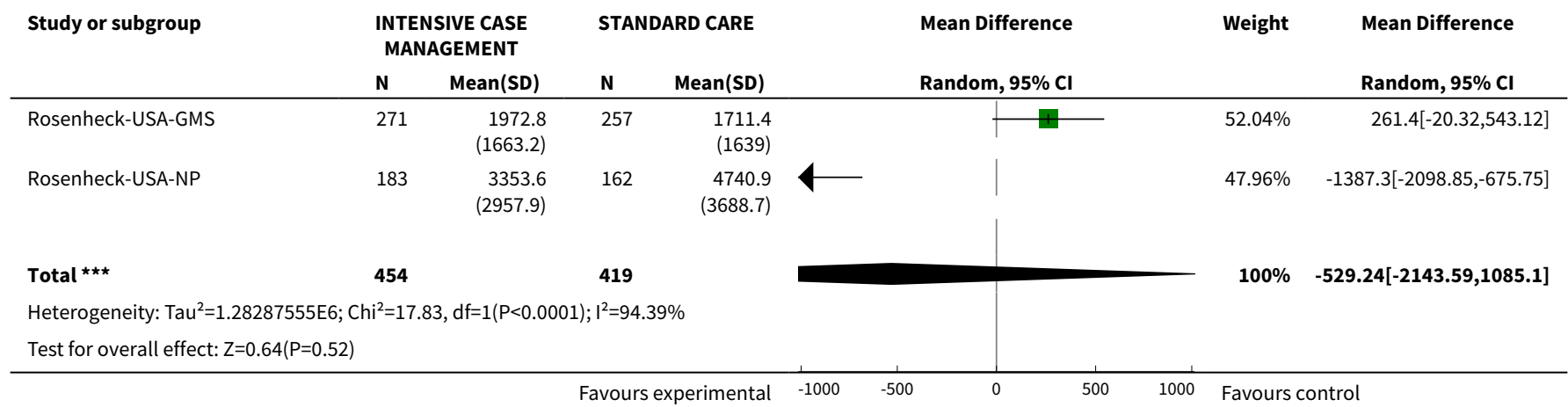

\section{Analysis 1.44. Comparison 1 Intensive Case Management versus standard care, Outcome $\mathbf{4 4}$ Costs: $\mathbf{2 b}$. Direct healthcare costs - skewed data.}

\begin{tabular}{|c|c|c|c|c|c|}
\hline \multicolumn{6}{|c|}{ Costs: 2 b. Direct healthcare costs - skewed data } \\
\hline Study & Intervention & Mean & SD & Total & Note \\
\hline \multicolumn{6}{|c|}{ by medium term } \\
\hline Lehman-Maryland1 1994 & 1. ICM ${ }^{*}$ & 4,229 & 5,058 & 77 & \\
\hline Lehman-Maryland1 1994 & 2. Standard care ${ }^{\star}$ & 5,540 & 6,368 & 75 & $\begin{array}{l}{ }^{\star} \text { Unit cost US } \$ \text {, fiscal } \\
\text { year } 1994 . \\
{ }_{\star \star} \text { 'Total per-case cost } \\
\text { did not reach statistical } \\
\text { significance }(p=0.07) \text {. } \\
\text { Transformation of to- } \\
\text { tal costs per case to ac- } \\
\text { count for non-normal- } \\
\text { ity (square root of to- } \\
\text { tal costs, } t \text {-test }=0.77, \\
\text { df }=1,134, N S \text { ) and non- } \\
\text { parametric analysis } \\
\text { (Wilcoxon test for ranks, } \\
Z=0.146, N S \text { ) also were } \\
\text { non-significant.' } \\
\text { Time period } 12 \text { months. }\end{array}$ \\
\hline \multicolumn{6}{|c|}{ by short term FUP } \\
\hline Chan-Hong Kong 2000 & 1. ICM & 14,833 & 1,539 & 31 & $\begin{array}{l}\text { HK } \$(H K \$ 8=U S \$ 1 \text {, at } \\
\text { time of study publica- } \\
\text { tion, 2000). } \\
\text { Statistically significant } \\
\text { difference }(P=0.017) \text {. }\end{array}$ \\
\hline Chan-Hong Kong 2000 & 2. Standard care & 11,230 & 7,979 & 31 & \\
\hline
\end{tabular}




\section{Analysis 1.45. Comparison 1 Intensive Case Management versus standard care, Outcome 45 Costs: 3. Direct costs - other data - skewed data.}

\begin{tabular}{|c|c|c|c|c|c|}
\hline \multicolumn{6}{|c|}{ Costs: 3. Direct costs - other data - skewed data } \\
\hline Study & Intervention & Mean & SD & Total & Note \\
\hline \multicolumn{6}{|c|}{ all care - by short term } \\
\hline Audini-UK 1994 & $1 . \mathrm{ICM}^{*}$ & 4,264 & 1,768 & 33 & \\
\hline Audini-UK 1994 & 2. Standard care ${ }^{\star}$ & 7,202 & 5,564 & 29 & $\begin{array}{l}{ }^{\star} \text { Unit cost } £ \text {, fiscal year } \\
1996 / 7 \text {. } \\
{ }^{\star \star} \text { 'Bivariate cost com- } \\
\text { parisons (after log trans- } \\
\text { formation) revealed sig- } \\
\text { nificant advantage for } \\
\text { ICM group ( } p=0.001) \text { '. } \\
{ }^{\star \star \star} \text { Time period: miss- } \\
\text { ing (it is not the monthly } \\
\text { cost per patient). }\end{array}$ \\
\hline
\end{tabular}

\begin{tabular}{llll}
\hline & & all care - by medium term \\
\hline Marshall-UK 1995 & 1. ICM & 1,044 & 425.3 \\
\hline Marshall-UK 1995 & 2. Standard care* & 1,108 & 530.4
\end{tabular}

\begin{tabular}{|c|c|c|c|c|c|}
\hline & & & & & $\begin{array}{l}1994 . \\
\star \star \text { 'No significant dif- } \\
\text { ferences between two } \\
\text { groups were found.' } \\
{ }^{\star \star \star} \text { Time period: mean } \\
\text { weekly cost. }\end{array}$ \\
\hline Morse-Missouri3 2005 & 1. $\mathrm{ICM}^{\star}$ & $2,946.8$ & $3,219.3$ & 54 & \\
\hline Morse-Missouri3 2005 & 2. Standard care ${ }^{\star}$ & $1,899.5$ & $3,629.6$ & 49 & 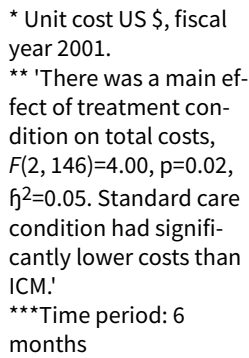 \\
\hline
\end{tabular}

\begin{tabular}{lllc}
\hline & & all care - by long term \\
\hline Audini-UK 1994 & 1. ICM $^{\star}$ & 10,192 & 3,900 \\
\hline Audini-UK 1994 & 2. Standard care & 15,288 & 17,160
\end{tabular}

\begin{tabular}{|c|c|c|c|c|c|}
\hline Audini-UK 1994 & 2. Standard carex & 15,288 & 17,160 & 28 & 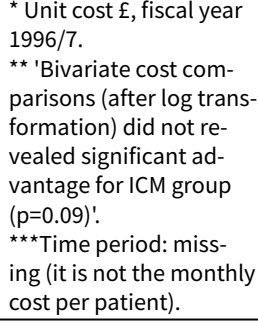 \\
\hline Ford-UK 1995 & 1. $\mathrm{ICM}^{*}$ & 1,813 & 1,347 & 39 & \\
\hline Ford-UK 1995 & 2. Standard care ${ }^{\star}$ & 717 & 768 & 38 & 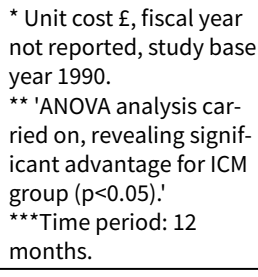 \\
\hline Marshall-UK 1995 & 1. $\mathrm{ICM}^{*}$ & 996 & 398 & 31 & \\
\hline Marshall-UK 1995 & 2. Standard care* & 1,088 & 562.4 & 30 & 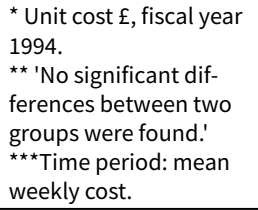 \\
\hline
\end{tabular}


Costs: 3. Direct costs - other data - skewed data

\begin{tabular}{|c|c|c|c|c|c|}
\hline Study & Intervention & Mean & SD & Total & Note \\
\hline Morse-Missouri3 2005 & 1. $\mathrm{ICM}^{*}$ & 3,190 & 3,441 & 54 & \\
\hline Morse-Missouri3 2005 & 2. Standard care ${ }^{\star}$ & 1,467 & 2,173 & 49 & 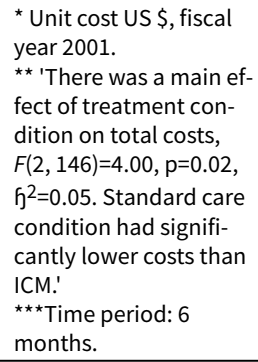 \\
\hline OPUS-Denmark 1999 & 1. $\mathrm{ICM}^{*}$ & 111,924 & 100,862 & 151 & \\
\hline OPUS-Denmark 1999 & 2. Standard Care* & 137,638 & 147,570 & 150 & 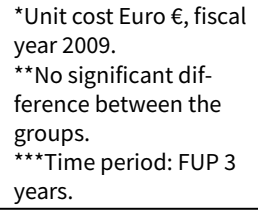 \\
\hline \multicolumn{6}{|c|}{ specific - outpatient care - by medium term } \\
\hline Cusack-North Carolina & 1. $\mathrm{ICM}^{*}$ & 13,481 & 9,547 & 72 & $\begin{array}{l}{ }^{\star} \text { Unit cost US } \$ . \\
{ }^{\star \star} \text { Time period: } 12 \\
\text { months. }\end{array}$ \\
\hline Cusack-North Carolina & 2. Standard care ${ }^{\star}$ & 5,118 & 6,184 & 62 & $\begin{array}{l}{ }^{\star} \text { Unit cost US } \$ . \\
{ }^{\star \star} \text { Time period: } 12 \\
\text { months. }\end{array}$ \\
\hline \multicolumn{6}{|c|}{ specific - prison - by medium term } \\
\hline Cusack-North Carolina & 1. $\mathrm{ICM}^{\star}$ & 1,848 & 4,533 & 72 & $\begin{array}{l}{ }^{\star} \text { Unit cost US \$. } \\
{ }^{\star \star} \text { Time period: } 12 \\
\text { months. }\end{array}$ \\
\hline Cusack-North Carolina & 2. Standard care ${ }^{\star}$ & 3,530 & 5,690 & 62 & $\begin{array}{l}{ }^{\star} \text { Unit cost US \$. } \\
{ }^{\star \star} \text { Time period: } 12 \\
\text { months. }\end{array}$ \\
\hline
\end{tabular}

\section{Comparison 2. Intensive Case Management versus non-Intensive Case Management}

\begin{tabular}{|c|c|c|c|c|}
\hline Outcome or subgroup title & No. of studies & $\begin{array}{l}\text { No. of partici- } \\
\text { pants }\end{array}$ & Statistical method & Effect size \\
\hline $\begin{array}{l}1 \text { Service use: } 1 \text {. Average number of days in } \\
\text { hospital per month - by about } 24 \text { months }\end{array}$ & 21 & 2220 & $\begin{array}{l}\text { Mean Difference (IV, Ran- } \\
\text { dom, } 95 \% \mathrm{CI} \text { ) }\end{array}$ & $-0.08[-0.37,0.21]$ \\
\hline 1.1 skewed data (sample size $\geqq 200$ ) & 3 & 694 & $\begin{array}{l}\text { Mean Difference (IV, Ran- } \\
\text { dom, } 95 \% \mathrm{Cl} \text { ) }\end{array}$ & $-0.58[-1.93,0.76]$ \\
\hline 1.2 skewed data (sample size $<200$ ) & 18 & 1526 & $\begin{array}{l}\text { Mean Difference (IV, Ran- } \\
\text { dom, } 95 \% \mathrm{Cl} \text { ) }\end{array}$ & $-0.03[-0.33,0.28]$ \\
\hline $\begin{array}{l}2 \text { Service use: } 1 \text { a. Average number of days } \\
\text { in hospital per month - by medium/long } \\
\text { term follow up (skewed data, sample size } \geqq \\
\text { 200) }\end{array}$ & 1 & & $\begin{array}{l}\text { Mean Difference (IV, Ran- } \\
\text { dom, } 95 \% \mathrm{CI} \text { ) }\end{array}$ & Subtotals only \\
\hline 2.1 by medium term FUP ( 18 months) & 1 & 237 & $\begin{array}{l}\text { Mean Difference (IV, Ran- } \\
\text { dom, } 95 \% \mathrm{CI} \text { ) }\end{array}$ & $0.60[-1.25,2.45]$ \\
\hline 2.2 by long term FUP ( 8.5 years) & 1 & 203 & $\begin{array}{l}\text { Mean Difference (IV, Ran- } \\
\text { dom, } 95 \% \mathrm{CI} \text { ) }\end{array}$ & $0.80[-1.47,3.07]$ \\
\hline
\end{tabular}




\begin{tabular}{|c|c|c|c|c|}
\hline Outcome or subgroup title & No. of studies & $\begin{array}{l}\text { No. of partici- } \\
\text { pants }\end{array}$ & Statistical method & Effect size \\
\hline $\begin{array}{l}3 \text { Service use: } 2 \text {. Not remaining in contact } \\
\text { with psychiatric services }\end{array}$ & 4 & & $\begin{array}{l}\text { Risk Ratio (M-H, Random, } \\
95 \% \mathrm{Cl} \text { ) }\end{array}$ & Subtotals only \\
\hline 3.1 by medium term & 1 & 73 & $\begin{array}{l}\text { Risk Ratio (M-H, Random, } \\
95 \% \mathrm{Cl} \text { ) }\end{array}$ & $0.27[0.08,0.87]$ \\
\hline 3.2 by long term & 3 & 1182 & $\begin{array}{l}\text { Risk Ratio (M-H, Random, } \\
95 \% \mathrm{Cl} \text { ) }\end{array}$ & $0.82[0.34,1.98]$ \\
\hline 3.3 by medium term FUP ( 18 months) & 1 & 251 & $\begin{array}{l}\text { Risk Ratio (M-H, Random, } \\
95 \% \mathrm{Cl} \text { ) }\end{array}$ & $0.42[0.17,1.05]$ \\
\hline $\begin{array}{l}4 \text { Service use: } 3 a \text {. Admitted to hospital - by } \\
\text { long term }\end{array}$ & 3 & 1132 & $\begin{array}{l}\text { Risk Ratio (M-H, Random, } \\
95 \% \mathrm{Cl} \text { ) }\end{array}$ & $0.91[0.75,1.12]$ \\
\hline $\begin{array}{l}5 \text { Service use: } 3 b \text {. Average number of ad- } \\
\text { missions (skewed data -sample size } \geqq 200 \text { ) }\end{array}$ & 2 & & $\begin{array}{l}\text { Mean Difference (IV, Ran- } \\
\text { dom, } 95 \% \mathrm{CI} \text { ) }\end{array}$ & Subtotals only \\
\hline 5.1 - by long term ( 24 months) & 1 & 678 & $\begin{array}{l}\text { Mean Difference (IV, Ran- } \\
\text { dom, } 95 \% \mathrm{CI} \text { ) }\end{array}$ & $-0.18[-0.41,0.05]$ \\
\hline 5.2 by medium term FUP ( 18 months) & 1 & 237 & $\begin{array}{l}\text { Mean Difference (IV, Ran- } \\
\text { dom, } 95 \% \mathrm{CI} \text { ) }\end{array}$ & $-0.10[-0.60,0.40]$ \\
\hline 5.3 by long term FUP ( 8.5 years) & 1 & 203 & $\begin{array}{l}\text { Mean Difference (IV, Ran- } \\
\text { dom, } 95 \% \mathrm{CI} \text { ) }\end{array}$ & $1.0[-0.25,2.25]$ \\
\hline $\begin{array}{l}6 \text { Service use: } 3 c \text {. Average number of ad- } \\
\text { missions (skewed data) - by medium term }\end{array}$ & & & Other data & No numeric data \\
\hline 7 Adverse event: 1 a. Death - any cause & 7 & & $\begin{array}{l}\text { Risk Ratio (M-H, Random, } \\
95 \% \mathrm{Cl} \text { ) }\end{array}$ & Subtotals only \\
\hline 7.1 by short term & 1 & 193 & $\begin{array}{l}\text { Risk Ratio (M-H, Random, } \\
95 \% \mathrm{Cl} \text { ) }\end{array}$ & $0.0[0.0,0.0]$ \\
\hline 7.2 by medium term & 3 & 294 & $\begin{array}{l}\text { Risk Ratio (M-H, Random, } \\
95 \% \mathrm{Cl} \text { ) }\end{array}$ & $2.92[0.12,69.43]$ \\
\hline 7.3 by long term & 5 & 1637 & $\begin{array}{l}\text { Risk Ratio (M-H, Random, } \\
95 \% \mathrm{Cl} \text { ) }\end{array}$ & $0.90[0.46,1.75]$ \\
\hline 7.4 by medium term FUP ( 18 months) & 1 & 251 & $\begin{array}{l}\text { Risk Ratio (M-H, Random, } \\
95 \% \mathrm{Cl} \text { ) }\end{array}$ & $0.98[0.32,2.95]$ \\
\hline 7.5 by long term FUP ( 8.5 years) & 1 & 251 & $\begin{array}{l}\text { Risk Ratio (M-H, Random, } \\
95 \% \mathrm{Cl} \text { ) }\end{array}$ & $1.15[0.63,2.09]$ \\
\hline 8 Adverse event: 1 b. Death - suicide & 8 & & $\begin{array}{l}\text { Risk Ratio (M-H, Random, } \\
95 \% \mathrm{Cl} \text { ) }\end{array}$ & Subtotals only \\
\hline 8.1 by short term & 1 & 193 & $\begin{array}{l}\text { Risk Ratio (M-H, Random, } \\
95 \% \mathrm{Cl} \text { ) }\end{array}$ & $0.0[0.0,0.0]$ \\
\hline
\end{tabular}




\begin{tabular}{|c|c|c|c|c|}
\hline Outcome or subgroup title & No. of studies & $\begin{array}{l}\text { No. of partici- } \\
\text { pants }\end{array}$ & Statistical method & Effect size \\
\hline 8.2 by medium term & 6 & 929 & $\begin{array}{l}\text { Risk Ratio (M-H, Random, } \\
95 \% \mathrm{Cl})\end{array}$ & $1.61[0.26,9.85]$ \\
\hline 8.3 by long term & 3 & 1152 & $\begin{array}{l}\text { Risk Ratio (M-H, Random, } \\
95 \% \mathrm{Cl} \text { ) }\end{array}$ & $0.88[0.27,2.84]$ \\
\hline 8.4 by medium term FUP ( 18 months) & 1 & 251 & $\begin{array}{l}\text { Risk Ratio (M-H, Random, } \\
95 \% \mathrm{Cl})\end{array}$ & $0.33[0.03,3.09]$ \\
\hline 9 Global state: 1 . Leaving the study early & 9 & 2195 & $\begin{array}{l}\text { Risk Ratio (M-H, Random, } \\
95 \% \mathrm{Cl})\end{array}$ & $0.72[0.52,0.99]$ \\
\hline 9.1 by medium term & 2 & 225 & $\begin{array}{l}\text { Risk Ratio (M-H, Random, } \\
95 \% \mathrm{Cl})\end{array}$ & $0.64[0.13,3.07]$ \\
\hline 9.2 by long term & 7 & 1970 & $\begin{array}{l}\text { Risk Ratio (M-H, Random, } \\
95 \% \mathrm{Cl})\end{array}$ & $0.70[0.52,0.95]$ \\
\hline $\begin{array}{l}10 \text { Global state: } 2 a . \text { Average endpoint score } \\
\text { (HoNOS, high = poor) - by long term }\end{array}$ & 1 & 239 & $\begin{array}{l}\text { Mean Difference (IV, Ran- } \\
\text { dom, } 95 \% \mathrm{CI} \text { ) }\end{array}$ & $-0.40[-1.77,0.97]$ \\
\hline $\begin{array}{l}11 \text { Global state: } 2 \text { b. Average endpoint score } \\
\text { (HoNOS, high = poor) - skewed data }\end{array}$ & & & Other data & No numeric data \\
\hline 11.1 medium term & & & Other data & No numeric data \\
\hline 11.2 long term & & & Other data & No numeric data \\
\hline $\begin{array}{l}12 \text { Global state: } 3 a \text {. Not compliant with } \\
\text { medication - by medium term }\end{array}$ & 1 & 73 & $\begin{array}{l}\text { Risk Ratio (M-H, Random, } \\
95 \% \mathrm{Cl})\end{array}$ & $1.14[0.42,3.05]$ \\
\hline $\begin{array}{l}13 \text { Global state: } 3 b \text {. Compliance with med- } \\
\text { ication - average endpoint sub-scale score } \\
\text { (ROMI) - by long term }\end{array}$ & 1 & & $\begin{array}{l}\text { Mean Difference (IV, Ran- } \\
\text { dom, } 95 \% \mathrm{CI} \text { ) }\end{array}$ & Subtotals only \\
\hline 13.1 compliance sub-scale (high = good) & 1 & 239 & $\begin{array}{l}\text { Mean Difference (IV, Ran- } \\
\text { dom, } 95 \% \mathrm{CI} \text { ) }\end{array}$ & $0.60[-0.05,1.25]$ \\
\hline $\begin{array}{l}13.2 \text { non-compliance sub-scale (high }= \\
\text { poor) }\end{array}$ & 1 & 239 & $\begin{array}{l}\text { Mean Difference (IV, Ran- } \\
\text { dom, } 95 \% \mathrm{CI} \text { ) }\end{array}$ & $-0.60[-1.63,0.43]$ \\
\hline $\begin{array}{l}14 \text { Global state: } 3 c \text {. Compliance with med- } \\
\text { ication - average endpoint sub-scale score } \\
\text { (ROMI, score } 1-3 \text {, skewed data) }\end{array}$ & & & Other data & No numeric data \\
\hline $\begin{array}{l}14.1 \text { medium term - compliance sub-scale } \\
\text { (high = good) }\end{array}$ & & & Other data & No numeric data \\
\hline $\begin{array}{l}14.2 \text { medium term - non-compliance sub- } \\
\text { scale (high = poor) }\end{array}$ & & & Other data & No numeric data \\
\hline $\begin{array}{l}14.3 \text { long term - compliance sub-scale } \\
\text { (high = good) }\end{array}$ & & & Other data & No numeric data \\
\hline
\end{tabular}




\begin{tabular}{|c|c|c|c|c|}
\hline Outcome or subgroup title & No. of studies & $\begin{array}{l}\text { No. of partici- } \\
\text { pants }\end{array}$ & Statistical method & Effect size \\
\hline $\begin{array}{l}14.4 \text { long term - non-compliance sub-scale } \\
\text { (high = poor) }\end{array}$ & & & Other data & No numeric data \\
\hline $\begin{array}{l}15 \text { Social functioning: } 1 \text {. Contact with legal } \\
\text { system (various measurements) }\end{array}$ & 3 & & $\begin{array}{l}\text { Risk Ratio (M-H, Random, } \\
95 \% \mathrm{Cl})\end{array}$ & Subtotals only \\
\hline $\begin{array}{l}15.1 \text { by medium term - contact with the po- } \\
\text { lice }\end{array}$ & 1 & 73 & $\begin{array}{l}\text { Risk Ratio (M-H, Random, } \\
95 \% \mathrm{Cl})\end{array}$ & $0.32[0.04,2.97]$ \\
\hline 15.2 by long term - imprisoned & 2 & 959 & $\begin{array}{l}\text { Risk Ratio (M-H, Random, } \\
95 \% \mathrm{Cl} \text { ) }\end{array}$ & $1.15[0.64,2.08]$ \\
\hline 15.3 by long term - arrested & 1 & 251 & $\begin{array}{l}\text { Risk Ratio (M-H, Random, } \\
95 \% \mathrm{Cl})\end{array}$ & $0.87[0.53,1.42]$ \\
\hline $\begin{array}{l}15.4 \text { by medium term FUP ( } 18 \text { months) - } \\
\text { imprisoned }\end{array}$ & 1 & 251 & $\begin{array}{l}\text { Risk Ratio (M-H, Random, } \\
95 \% \mathrm{Cl} \text { ) }\end{array}$ & $1.07[0.47,2.44]$ \\
\hline $\begin{array}{l}15.5 \text { by long term FUP ( } 8.5 \text { years) - impris- } \\
\text { oned }\end{array}$ & 1 & 214 & $\begin{array}{l}\text { Risk Ratio (M-H, Random, } \\
95 \% \mathrm{Cl} \text { ) }\end{array}$ & $0.7[0.43,1.14]$ \\
\hline $\begin{array}{l}16 \text { Social functioning } 2 . \text { Employment sta- } \\
\text { tus (various measurements) }\end{array}$ & 2 & & $\begin{array}{l}\text { Risk Ratio (M-H, Random, } \\
95 \% \mathrm{Cl})\end{array}$ & Subtotals only \\
\hline $\begin{array}{l}16.1 \text { spent }>1 \text { day employed - by medium } \\
\text { term }\end{array}$ & 1 & 73 & $\begin{array}{l}\text { Risk Ratio (M-H, Random, } \\
95 \% \mathrm{Cl})\end{array}$ & $1.46[0.45,4.74]$ \\
\hline $\begin{array}{l}16.2 \text { on paid employment - by medium } \\
\text { term }\end{array}$ & 1 & 73 & $\begin{array}{l}\text { Risk Ratio (M-H, Random, } \\
95 \% \mathrm{Cl} \text { ) }\end{array}$ & $0.97[0.14,6.54]$ \\
\hline $\begin{array}{l}16.3 \text { unemployed - by long term FUP ( } 8.5 \\
\text { years) }\end{array}$ & 1 & 214 & $\begin{array}{l}\text { Risk Ratio (M-H, Random, } \\
95 \% \mathrm{Cl} \text { ) }\end{array}$ & $1.10[0.91,1.34]$ \\
\hline $\begin{array}{l}17 \text { Social functioning: } 3 a \text {. Accommodation } \\
\text { status (various measurements) }\end{array}$ & 2 & & $\begin{array}{l}\text { Risk Ratio (M-H, Random, } \\
95 \% \mathrm{Cl} \text { ) }\end{array}$ & Subtotals only \\
\hline $\begin{array}{l}17.1 \text { by medium term - living in supported } \\
\text { accommodation }\end{array}$ & 1 & 73 & $\begin{array}{l}\text { Risk Ratio (M-H, Random, } \\
95 \% \mathrm{Cl} \text { ) }\end{array}$ & $2.59[0.75,9.01]$ \\
\hline 17.2 by long term - homelessness & 1 & 251 & $\begin{array}{l}\text { Risk Ratio (M-H, Random, } \\
95 \% \mathrm{Cl} \text { ) }\end{array}$ & $0.69[0.34,1.38]$ \\
\hline $\begin{array}{l}17.3 \text { by medium term FUP ( } 18 \text { months) - liv- } \\
\text { ing independently }\end{array}$ & 1 & 251 & $\begin{array}{l}\text { Risk Ratio (M-H, Random, } \\
95 \% \mathrm{Cl} \text { ) }\end{array}$ & $0.98[0.84,1.13]$ \\
\hline $\begin{array}{l}17.4 \text { by medium term FUP ( } 18 \text { months) - liv- } \\
\text { ing in supported accomodation }\end{array}$ & 1 & 251 & $\begin{array}{l}\text { Risk Ratio (M-H, Random, } \\
95 \% \mathrm{Cl})\end{array}$ & $0.83[0.38,1.77]$ \\
\hline $\begin{array}{l}17.5 \text { by medium term FUP ( } 18 \text { months) - } \\
\text { homelessness }\end{array}$ & 1 & 251 & $\begin{array}{l}\text { Risk Ratio (M-H, Random, } \\
95 \% \mathrm{Cl})\end{array}$ & $0.84[0.47,1.49]$ \\
\hline $\begin{array}{l}17.6 \text { by long term FUP ( } 8.5 \text { years) - living in } \\
\text { supported accomodation }\end{array}$ & 1 & 214 & $\begin{array}{l}\text { Risk Ratio (M-H, Random, } \\
95 \% \mathrm{Cl})\end{array}$ & $1.05[0.75,1.48]$ \\
\hline
\end{tabular}




\begin{tabular}{|c|c|c|c|c|}
\hline Outcome or subgroup title & No. of studies & $\begin{array}{l}\text { No. of partici- } \\
\text { pants }\end{array}$ & Statistical method & Effect size \\
\hline $\begin{array}{l}17.7 \text { by long term FUP ( } 8.5 \text { years) - home- } \\
\text { lessness }\end{array}$ & 1 & 214 & $\begin{array}{l}\text { Risk Ratio (M-H, Random, } \\
95 \% \mathrm{Cl})\end{array}$ & $0.92[0.55,1.53]$ \\
\hline $\begin{array}{l}18 \text { Social functioning: } 3 \mathrm{~b} \text {. Accommodation } \\
\text { status - average days per month in stable } \\
\text { accommodation }\end{array}$ & 2 & & $\begin{array}{l}\text { Mean Difference (IV, Ran- } \\
\text { dom, } 95 \% \mathrm{CI} \text { ) }\end{array}$ & Subtotals only \\
\hline 18.1 by short term & 1 & 203 & $\begin{array}{l}\text { Mean Difference (IV, Ran- } \\
\text { dom, } 95 \% \mathrm{CI} \text { ) }\end{array}$ & $-0.20[-2.48,2.08]$ \\
\hline 18.2 by medium term & 1 & 203 & $\begin{array}{l}\text { Mean Difference (IV, Ran- } \\
\text { dom, } 95 \% \mathrm{CI} \text { ) }\end{array}$ & $0.10[-2.15,2.35]$ \\
\hline 18.3 by long term & 2 & 901 & $\begin{array}{l}\text { Mean Difference (IV, Ran- } \\
\text { dom, } 95 \% \mathrm{CI} \text { ) }\end{array}$ & $-0.19[-1.37,1.00]$ \\
\hline $\begin{array}{l}19 \text { Social functioning: } 4 a \text {. Substance abuse } \\
\text { - by long term }\end{array}$ & 2 & & $\begin{array}{l}\text { Risk Ratio (M-H, Random, } \\
95 \% \mathrm{Cl})\end{array}$ & Subtotals only \\
\hline 19.1 alcohol abuse & 1 & 251 & $\begin{array}{l}\text { Risk Ratio (M-H, Random, } \\
95 \% \mathrm{Cl})\end{array}$ & $1.10[0.67,1.83]$ \\
\hline 19.2 illicit drug abuse & 1 & 251 & $\begin{array}{l}\text { Risk Ratio (M-H, Random, } \\
95 \% \mathrm{Cl})\end{array}$ & $1.08[0.69,1.71]$ \\
\hline $\begin{array}{l}\text { 19.3 alcohol - remission from alcohol use } \\
\text { disorder (AUS score < 3) }\end{array}$ & 1 & 223 & $\begin{array}{l}\text { Risk Ratio (M-H, Random, } \\
95 \% \mathrm{Cl} \text { ) }\end{array}$ & $0.86[0.65,1.14]$ \\
\hline $\begin{array}{l}20 \text { Social functioning: 4b. Substance abuse } \\
\text { - average endpoint score (SATS, low = poor) }\end{array}$ & 1 & & $\begin{array}{l}\text { Mean Difference (IV, Ran- } \\
\text { dom, } 95 \% \mathrm{CI} \text { ) }\end{array}$ & Subtotals only \\
\hline 20.1 by short term & 1 & 203 & $\begin{array}{l}\text { Mean Difference (IV, Ran- } \\
\text { dom, } 95 \% \mathrm{CI} \text { ) }\end{array}$ & $0.07[-0.28,0.42]$ \\
\hline 20.2 by medium term & 1 & 203 & $\begin{array}{l}\text { Mean Difference (IV, Ran- } \\
\text { dom, } 95 \% \mathrm{CI} \text { ) }\end{array}$ & $-0.11[-0.55,0.33]$ \\
\hline 20.3 by long term & 1 & 203 & $\begin{array}{l}\text { Mean Difference (IV, Ran- } \\
\text { dom, } 95 \% \mathrm{CI} \text { ) }\end{array}$ & $0.11[-0.41,0.63]$ \\
\hline $\begin{array}{l}21 \text { Social functioning: } 4 c . \text { Alcohol - abuse } \\
\text { (various measurements, skewed data) }\end{array}$ & & & Other data & No numeric data \\
\hline $\begin{array}{l}21.1 \text { short term - days using alcohol during } \\
\text { previous } 6 \text { months (TLFB) }\end{array}$ & & & Other data & No numeric data \\
\hline $\begin{array}{l}21.2 \text { short term - average endpoint score } \\
\text { (AUS, high = poor) }\end{array}$ & & & Other data & No numeric data \\
\hline $\begin{array}{l}21.3 \text { medium term - days using alcohol dur- } \\
\text { ing previous } 6 \text { months (TLFB) }\end{array}$ & & & Other data & No numeric data \\
\hline $\begin{array}{l}21.4 \text { medium term - average endpoint } \\
\text { score (AUS, high = poor) }\end{array}$ & & & Other data & No numeric data \\
\hline
\end{tabular}




\section{Outcome or subgroup title \\ 21.5 long term - days using alcohol during previous 6 months (TLFB)}

21.6 long term - average endpoint score (AUS, high = poor)

22 Social functioning: 5a. Average endpoint score (LSP, high = poor) - by long term

No. of studies

No. of partici-

pants

Statistical method

Other data

Other data

No numeric data
239
Mean Difference (IV, Ran- $\quad 4.0[-0.61,8.61]$ dom, $95 \% \mathrm{Cl}$ )

Other data

No numeric data

23 Social functioning: 5b. Average end-

point score (SFQ, high = poor) - skewed da-

ta

\begin{tabular}{|c|c|c|c|c|}
\hline 23.1 by medium term & & & Other data & No numeric data \\
\hline 23.2 by long term & & & Other data & No numeric data \\
\hline $\begin{array}{l}24 \text { Mental state: } 1 \text { a. General symptoms - } \\
\text { average endpoint score (various scales) }\end{array}$ & 2 & & $\begin{array}{l}\text { Mean Difference (IV, Ran- } \\
\text { dom, } 95 \% \mathrm{CI} \text { ) }\end{array}$ & Subtotals only \\
\hline $\begin{array}{l}24.1 \text { by short term (BPRS- } 24 \text { items, high = } \\
\text { poor) }\end{array}$ & 1 & 203 & $\begin{array}{l}\text { Mean Difference (IV, Ran- } \\
\text { dom, } 95 \% \mathrm{CI} \text { ) }\end{array}$ & $-0.65[-3.99,2.69]$ \\
\hline $\begin{array}{l}24.2 \text { by medium term (BPRS- } 24 \text { items, high } \\
=\text { poor) }\end{array}$ & 1 & 203 & $\begin{array}{l}\text { Mean Difference (IV, Ran- } \\
\text { dom, } 95 \% \mathrm{Cl} \text { ) }\end{array}$ & $-1.62[-4.76,1.52]$ \\
\hline $\begin{array}{l}24.3 \text { by long term (BPRS- } 24 \text { items, high }= \\
\text { poor) }\end{array}$ & 1 & 203 & $\begin{array}{l}\text { Mean Difference (IV, Ran- } \\
\text { dom, } 95 \% \mathrm{Cl} \text { ) }\end{array}$ & $-0.22[-3.32,2.88]$ \\
\hline 24.4 by long term (CPRS, high $=$ poor $)$ & 1 & 595 & $\begin{array}{l}\text { Mean Difference (IV, Ran- } \\
\text { dom, } 95 \% \mathrm{CI} \text { ) }\end{array}$ & $0.40[-1.83,2.63]$ \\
\hline $\begin{array}{l}25 \text { Mental state: } 1 \text { b. General symptoms - } \\
\text { average endpoint scores (various scales, } \\
\text { skewed data) }\end{array}$ & & & Other data & No numeric data \\
\hline $\begin{array}{l}25.1 \text { by medium term (Krawiecka Scale, } \\
\text { high = poor) }\end{array}$ & & & Other data & No numeric data \\
\hline $\begin{array}{l}25.2 \text { by long term (Krawiecka Scale, high = } \\
\text { poor) }\end{array}$ & & & Other data & No numeric data \\
\hline $\begin{array}{l}25.3 \text { by long term (BPRS 24-items, high = } \\
\text { good) }\end{array}$ & & & Other data & No numeric data \\
\hline $\begin{array}{l}26 \text { Mental state: } 2 \text { a. Specific symptoms: } \\
\text { negative symptoms - average endpoint } \\
\text { score (SANS, high = poor) - by long term }\end{array}$ & 1 & 593 & $\begin{array}{l}\text { Mean Difference (IV, Ran- } \\
\text { dom, } 95 \% \mathrm{CI} \text { ) }\end{array}$ & $0.20[-2.32,2.72]$ \\
\hline $\begin{array}{l}27 \text { Mental state: } 2 \text { b. Specific symptoms - } \\
\text { average endpoint scores (various scales, } \\
\text { skewed data) }\end{array}$ & & & Other data & No numeric data \\
\hline
\end{tabular}




\begin{tabular}{|c|c|c|c|c|}
\hline Outcome or subgroup title & No. of studies & $\begin{array}{l}\text { No. of partici- } \\
\text { pants }\end{array}$ & Statistical method & Effect size \\
\hline $\begin{array}{l}27.1 \text { medium term - anxiety (HADS, high = } \\
\text { poor) }\end{array}$ & & & Other data & No numeric data \\
\hline $\begin{array}{l}27.2 \text { medium term - depression (HADS, } \\
\text { high = poor) }\end{array}$ & & & Other data & No numeric data \\
\hline $\begin{array}{l}27.3 \text { long term - anxiety (HADS, high = } \\
\text { poor) }\end{array}$ & & & Other data & No numeric data \\
\hline $\begin{array}{l}27.5 \text { long term - depression (HADS, high = } \\
\text { poor) }\end{array}$ & & & Other data & No numeric data \\
\hline $\begin{array}{l}28 \text { Behaviour: } 1 \text {. Specific behaviour (vari- } \\
\text { ous measurements) }\end{array}$ & 3 & & $\begin{array}{l}\text { Risk Ratio (M-H, Random, } \\
95 \% \mathrm{Cl})\end{array}$ & Subtotals only \\
\hline $\begin{array}{l}28.1 \text { by medium term - harm to self or oth- } \\
\text { ers }\end{array}$ & 1 & 73 & $\begin{array}{l}\text { Risk Ratio (M-H, Random, } \\
95 \% \mathrm{Cl} \text { ) }\end{array}$ & $0.88[0.40,1.90]$ \\
\hline 28.2 by long term - self-harm & 2 & 959 & $\begin{array}{l}\text { Risk Ratio (M-H, Random, } \\
95 \% \mathrm{Cl})\end{array}$ & $1.00[0.69,1.46]$ \\
\hline 28.3 by long term - injury/assault to others & 2 & 959 & $\begin{array}{l}\text { Risk Ratio (M-H, Random, } \\
95 \% \mathrm{Cl})\end{array}$ & $1.09[0.85,1.40]$ \\
\hline $\begin{array}{l}28.4 \text { by medium term FUP ( } 18 \text { months) - } \\
\text { self harm }\end{array}$ & 1 & 251 & $\begin{array}{l}\text { Risk Ratio (M-H, Random, } \\
95 \% \mathrm{Cl})\end{array}$ & $0.85[0.44,1.67]$ \\
\hline $\begin{array}{l}28.5 \text { by medium term FUP ( } 18 \text { months) - in- } \\
\text { jury/assualt to others }\end{array}$ & 1 & 251 & $\begin{array}{l}\text { Risk Ratio (M-H, Random, } \\
95 \% \mathrm{Cl})\end{array}$ & $1.35[0.87,2.10]$ \\
\hline $\begin{array}{l}28.6 \text { by long term FUP ( } 8.5 \text { years) - self } \\
\text { harm }\end{array}$ & 1 & 214 & $\begin{array}{l}\text { Risk Ratio (M-H, Random, } \\
95 \% \mathrm{Cl} \text { ) }\end{array}$ & $0.81[0.51,1.27]$ \\
\hline $\begin{array}{l}28.7 \text { by long term FUP ( } 8.5 \text { years) - in- } \\
\text { jury/assault to others }\end{array}$ & 1 & 214 & $\begin{array}{l}\text { Risk Ratio (M-H, Random, } \\
95 \% \mathrm{Cl} \text { ) }\end{array}$ & $0.95[0.83,1.09]$ \\
\hline $\begin{array}{l}29 \text { Quality of life: } 1 . \text { Average endpoint score } \\
\text { (various scales) }\end{array}$ & 3 & & $\begin{array}{l}\text { Mean Difference (IV, Ran- } \\
\text { dom, } 95 \% \mathrm{CI} \text { ) }\end{array}$ & Subtotals only \\
\hline $\begin{array}{l}29.1 \text { by short term - overall life satisfaction } \\
\text { (QOLI, high = better) }\end{array}$ & 1 & 203 & $\begin{array}{l}\text { Mean Difference (IV, Ran- } \\
\text { dom, } 95 \% \mathrm{CI} \text { ) }\end{array}$ & $-0.02[-0.43,0.39]$ \\
\hline $\begin{array}{l}29.2 \text { by medium term - overall life satisfac- } \\
\text { tion (QOLI, high = better) }\end{array}$ & 1 & 203 & $\begin{array}{l}\text { Mean Difference (IV, Ran- } \\
\text { dom, } 95 \% \mathrm{CI} \text { ) }\end{array}$ & $-0.04[-0.43,0.35]$ \\
\hline 29.3 by long term (LQoLP, high = better) & 1 & 526 & $\begin{array}{l}\text { Mean Difference (IV, Ran- } \\
\text { dom, } 95 \% \mathrm{CI} \text { ) }\end{array}$ & $0.03[-0.10,0.16]$ \\
\hline $\begin{array}{l}29.4 \text { by long term (MANSA, range 1-7, high } \\
=\text { better) }\end{array}$ & 1 & 166 & $\begin{array}{l}\text { Mean Difference (IV, Ran- } \\
\text { dom, } 95 \% \mathrm{CI} \text { ) }\end{array}$ & $0.10[-0.19,0.39]$ \\
\hline $\begin{array}{l}29.5 \text { by long term - overall life satisfaction } \\
\text { (QOLI, high = better) }\end{array}$ & 1 & 203 & $\begin{array}{l}\text { Mean Difference (IV, Ran- } \\
\text { dom, } 95 \% \mathrm{CI} \text { ) }\end{array}$ & $0.10[-0.25,0.45]$ \\
\hline
\end{tabular}




\begin{tabular}{|c|c|c|c|c|}
\hline Outcome or subgroup title & No. of studies & $\begin{array}{l}\text { No. of partici- } \\
\text { pants }\end{array}$ & Statistical method & Effect size \\
\hline $\begin{array}{l}30 \text { Participant satisfaction/need: } 1 \text {. Aver- } \\
\text { age endpoint scores (various scale) - by } \\
\text { long term }\end{array}$ & 1 & & $\begin{array}{l}\text { Mean Difference (IV, Ran- } \\
\text { dom, } 95 \% \mathrm{CI} \text { ) }\end{array}$ & Subtotals only \\
\hline 30.1 Patient need: CAN (high = poor) & 1 & 585 & $\begin{array}{l}\text { Mean Difference (IV, Ran- } \\
\text { dom, } 95 \% \mathrm{CI} \text { ) }\end{array}$ & $-0.29[-0.69,0.11]$ \\
\hline $\begin{array}{l}30.2 \text { Patient Satisfaction with Health Ser- } \\
\text { vices (high }=\text { poor) }\end{array}$ & 1 & 490 & $\begin{array}{l}\text { Mean Difference (IV, Ran- } \\
\text { dom, } 95 \% \mathrm{CI} \text { ) }\end{array}$ & $-0.40[-1.25,0.45]$ \\
\hline $\begin{array}{l}31 \text { Participants need: } 1 \text {. Average endpoint } \\
\text { scores (various scales, skewed data) }\end{array}$ & & & Other data & No numeric data \\
\hline 31.1 by medium term (CAN, high $=$ poor) & & & Other data & No numeric data \\
\hline 31.2 by long term (CAN, high = poor) & & & Other data & No numeric data \\
\hline 31.3 by long term (CANSAS, high = poor) & & & Other data & No numeric data \\
\hline $\begin{array}{l}32 \text { Participant satisfaction: } 1 \text {. Average end- } \\
\text { point scores (CSQ-modified, high = better, } \\
\text { skewed data) - by long term }\end{array}$ & & & Other data & No numeric data \\
\hline $\begin{array}{l}33 \text { Costs: } 1 \text {. Direct costs of psychiatric hos- } \\
\text { pital care (skewed data) }\end{array}$ & & & Other data & No numeric data \\
\hline 33.3 by medium term & & & Other data & No numeric data \\
\hline 33.4 by long term & & & Other data & No numeric data \\
\hline $\begin{array}{l}34 \text { Costs: } 2 \text { a. Direct costs of all care - by } \\
\text { long term ( } 2 \text { years). Unit cost GBP, fiscal } \\
\text { year } 1997 / 98\end{array}$ & 1 & & $\begin{array}{l}\text { Mean Difference (IV, Ran- } \\
\text { dom, } 95 \% \mathrm{CI} \text { ) }\end{array}$ & Subtotals only \\
\hline $\begin{array}{l}35 \text { Costs: } 2 b \text {. Direct costs of all care - by } \\
\text { medium term (skewed data) }\end{array}$ & & & Other data & No numeric data \\
\hline $\begin{array}{l}36 \text { Costs: } 3 \text {. Total costs of care per patient - } \\
\text { Unit cost GBP }\end{array}$ & 2 & & $\begin{array}{l}\text { Mean Difference (IV, } \\
\text { Fixed, } 95 \% \mathrm{CI} \text { ) }\end{array}$ & Subtotals only \\
\hline 36.1 by 24 months, fiscal year $1997 / 98$ & 1 & 667 & $\begin{array}{l}\text { Mean Difference (IV, } \\
\text { Fixed, } 95 \% \mathrm{CI} \text { ) }\end{array}$ & $\begin{array}{l}1849.0[-1598.23 \\
5296.23]\end{array}$ \\
\hline $\begin{array}{l}36.2 \text { by } 18 \text { months, fiscal year 2003/2004 } \\
\text { ( GBP } 1 \text { = USD } 1.58 \text { ) }\end{array}$ & 1 & 243 & $\begin{array}{l}\text { Mean Difference (IV, } \\
\text { Fixed, } 95 \% \mathrm{CI} \text { ) }\end{array}$ & $\begin{array}{l}4031.00 \\
{[-2724.13} \\
10786.13\end{array}$ \\
\hline
\end{tabular}


Analysis 2.1. Comparison 2 Intensive Case Management versus non-Intensive Case Management, Outcome 1 Service use: 1. Average number of days in hospital per month - by about 24 months.

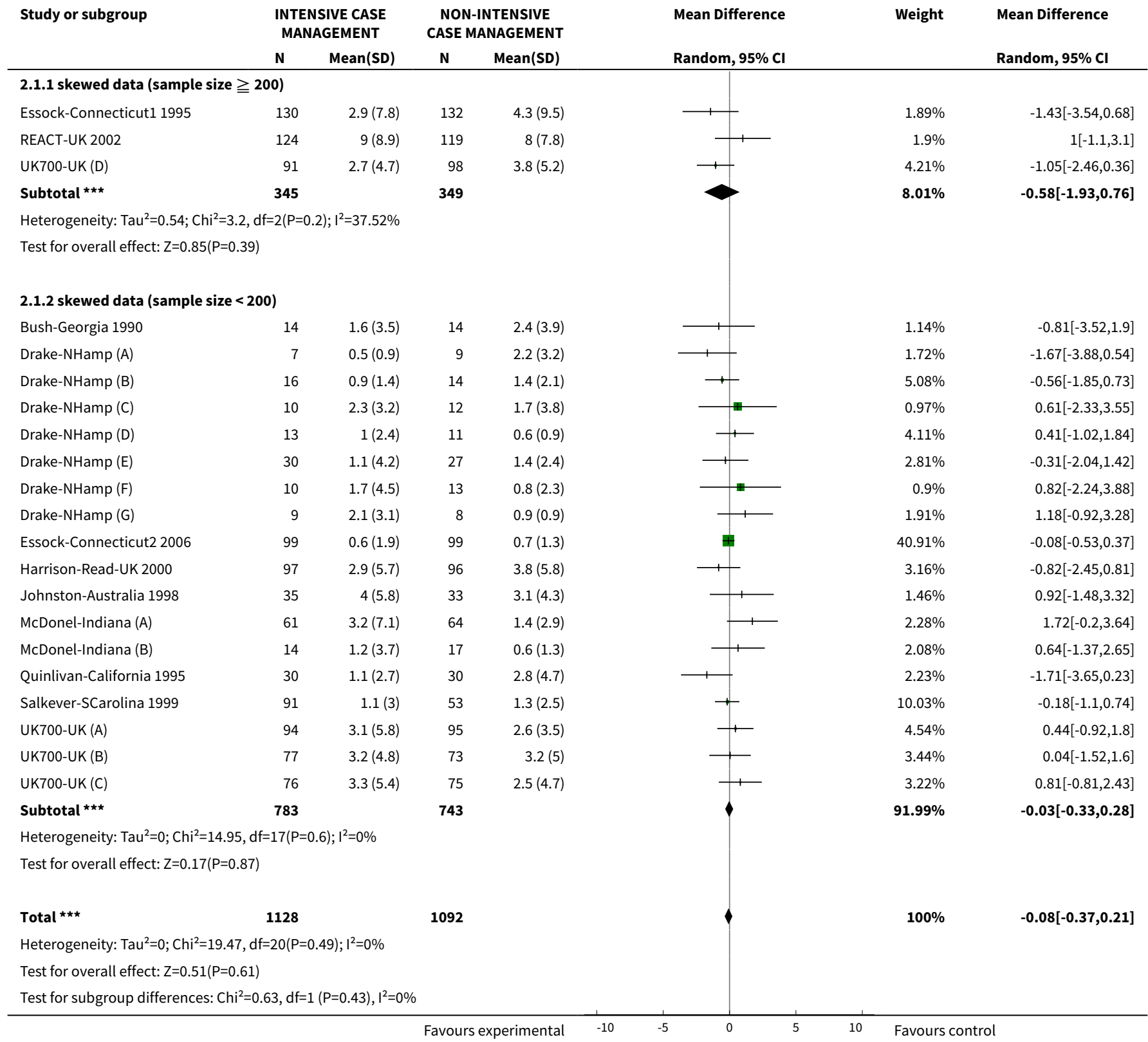

Analysis 2.2. Comparison 2 Intensive Case Management versus non-Intensive Case Management, Outcome 2 Service use: 1a. Average number of days in hospital per month - by medium/long term follow up (skewed data, sample size $\geqq 200$ ).

\begin{tabular}{|c|c|c|c|c|c|c|c|}
\hline \multirow[t]{2}{*}{ Study or subgroup } & \multicolumn{2}{|c|}{$\begin{array}{l}\text { INTENSIVE CASE } \\
\text { MANAGEMENT }\end{array}$} & \multicolumn{2}{|c|}{$\begin{array}{l}\text { NON-INTENSIVE } \\
\text { CASE MANAGEMENT }\end{array}$} & \multirow{2}{*}{$\begin{array}{l}\text { Mean Difference } \\
\text { Random, } 95 \% \mathrm{Cl}\end{array}$} & \multirow[t]{2}{*}{ Weight } & \multirow{2}{*}{$\begin{array}{l}\text { Mean Difference } \\
\text { Random, } 95 \% \mathrm{Cl}\end{array}$} \\
\hline & $\mathbf{N}$ & $\operatorname{Mean}(S D)$ & $\mathbf{N}$ & Mean(SD) & & & \\
\hline \multicolumn{8}{|c|}{ 2.2.1 by medium term FUP ( 18 months) } \\
\hline REACT-UK 2002 & 120 & $8(7.8)$ & 117 & $7.4(6.7)$ & & $100 \%$ & $0.6[-1.25,2.45]$ \\
\hline
\end{tabular}




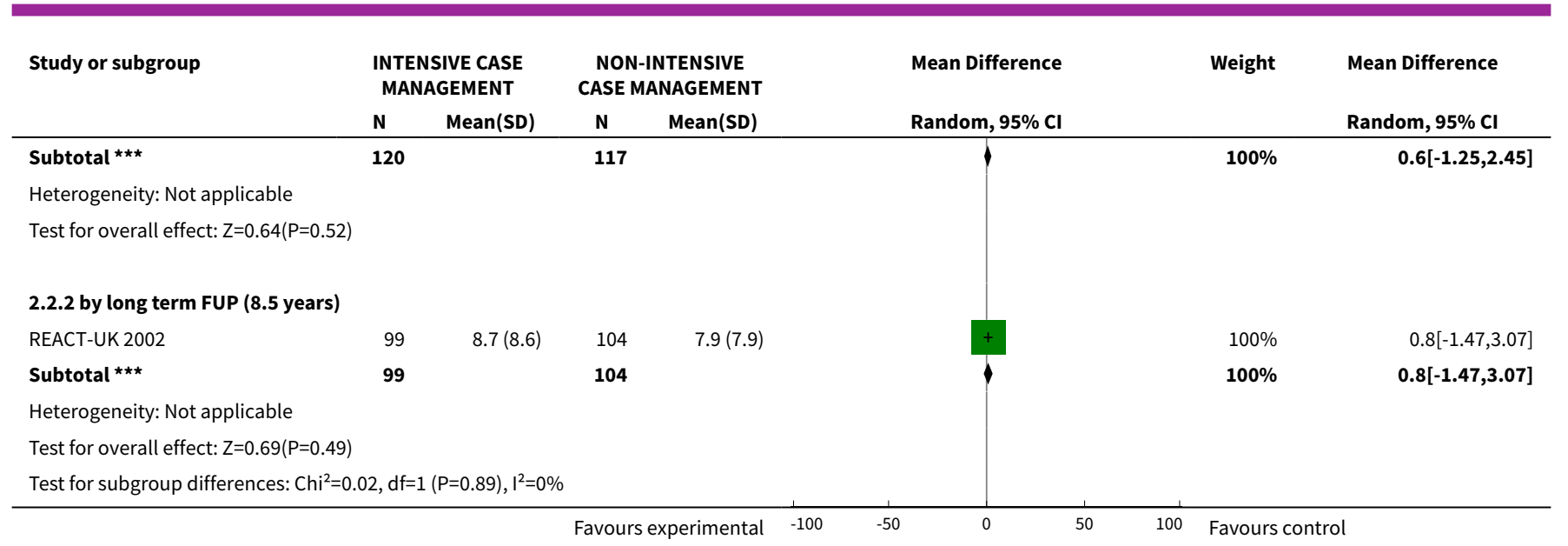

Analysis 2.3. Comparison 2 Intensive Case Management versus non-Intensive Case Management, Outcome 3 Service use: 2 . Not remaining in contact with psychiatric services.

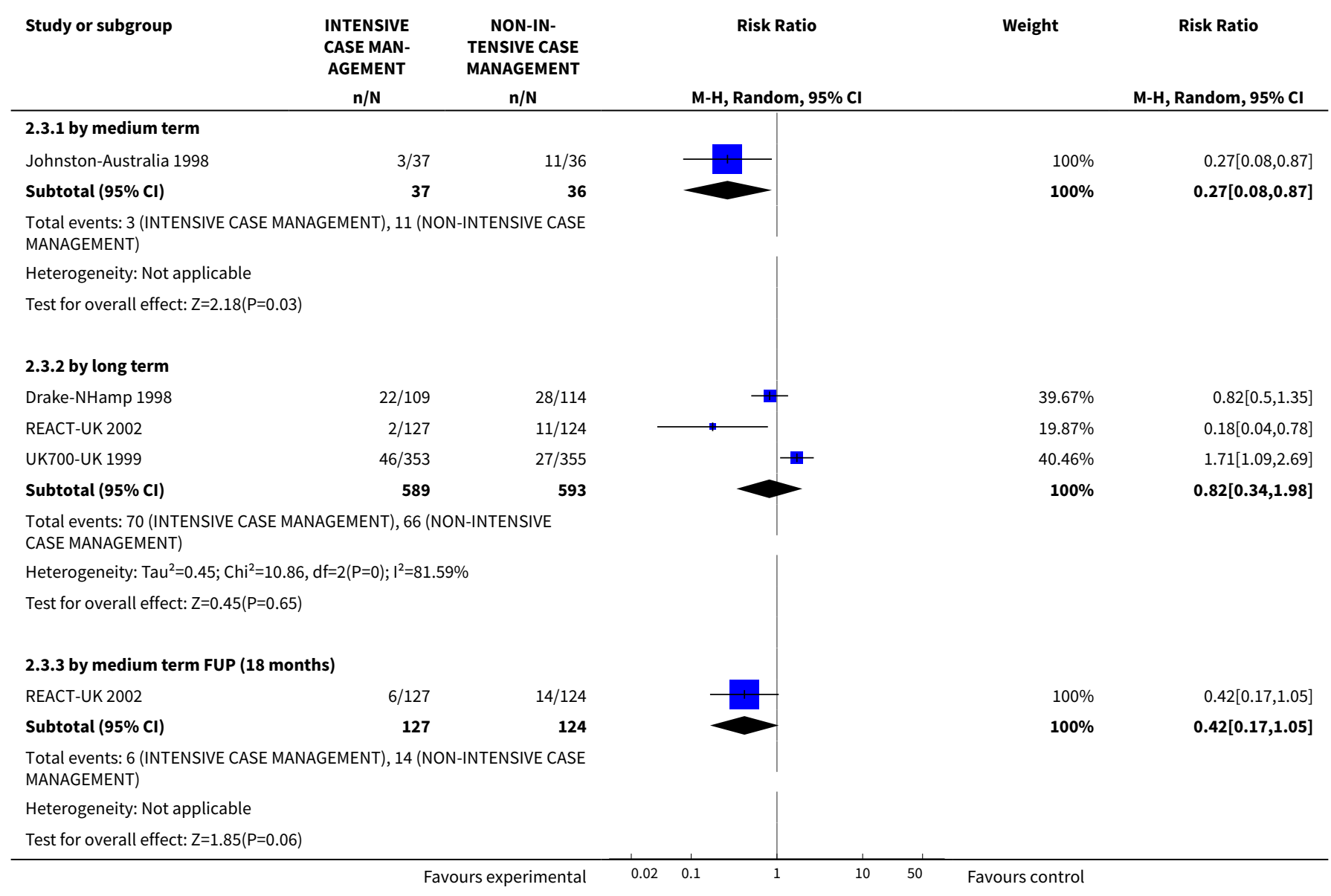


Analysis 2.4. Comparison 2 Intensive Case Management versus non-Intensive Case Management, Outcome 4 Service use: 3a. Admitted to hospital - by long term.

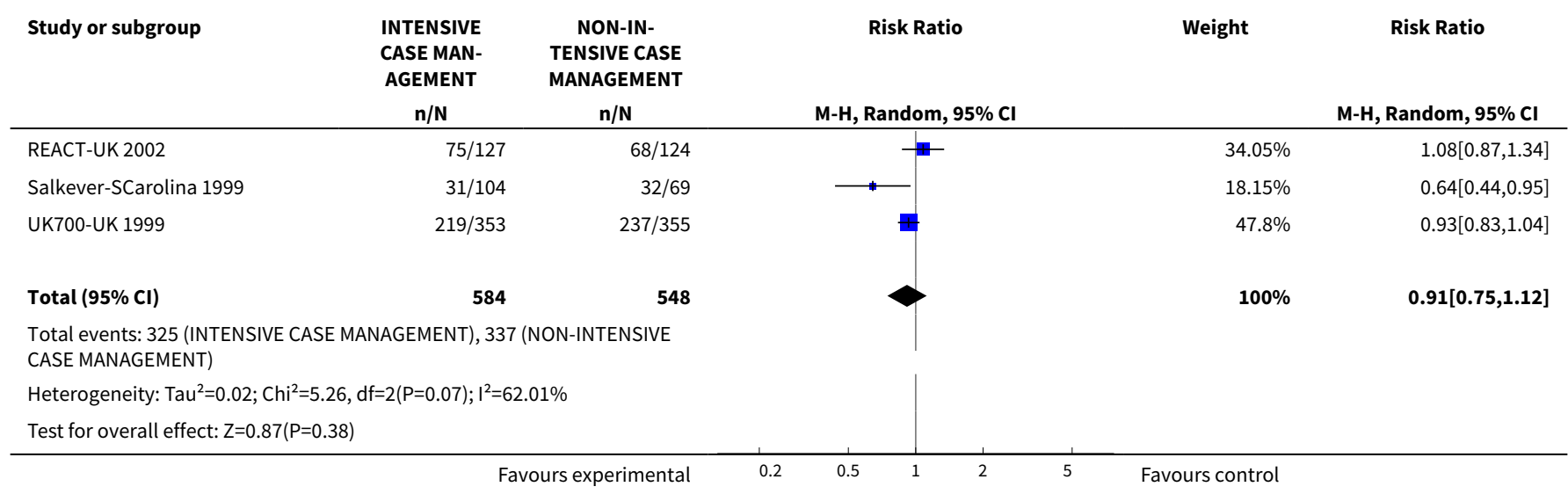

Analysis 2.5. Comparison 2 Intensive Case Management versus non-Intensive Case Management, Outcome 5 Service use: 3b. Average number of admissions (skewed data -sample size $\geqq 200$ ).

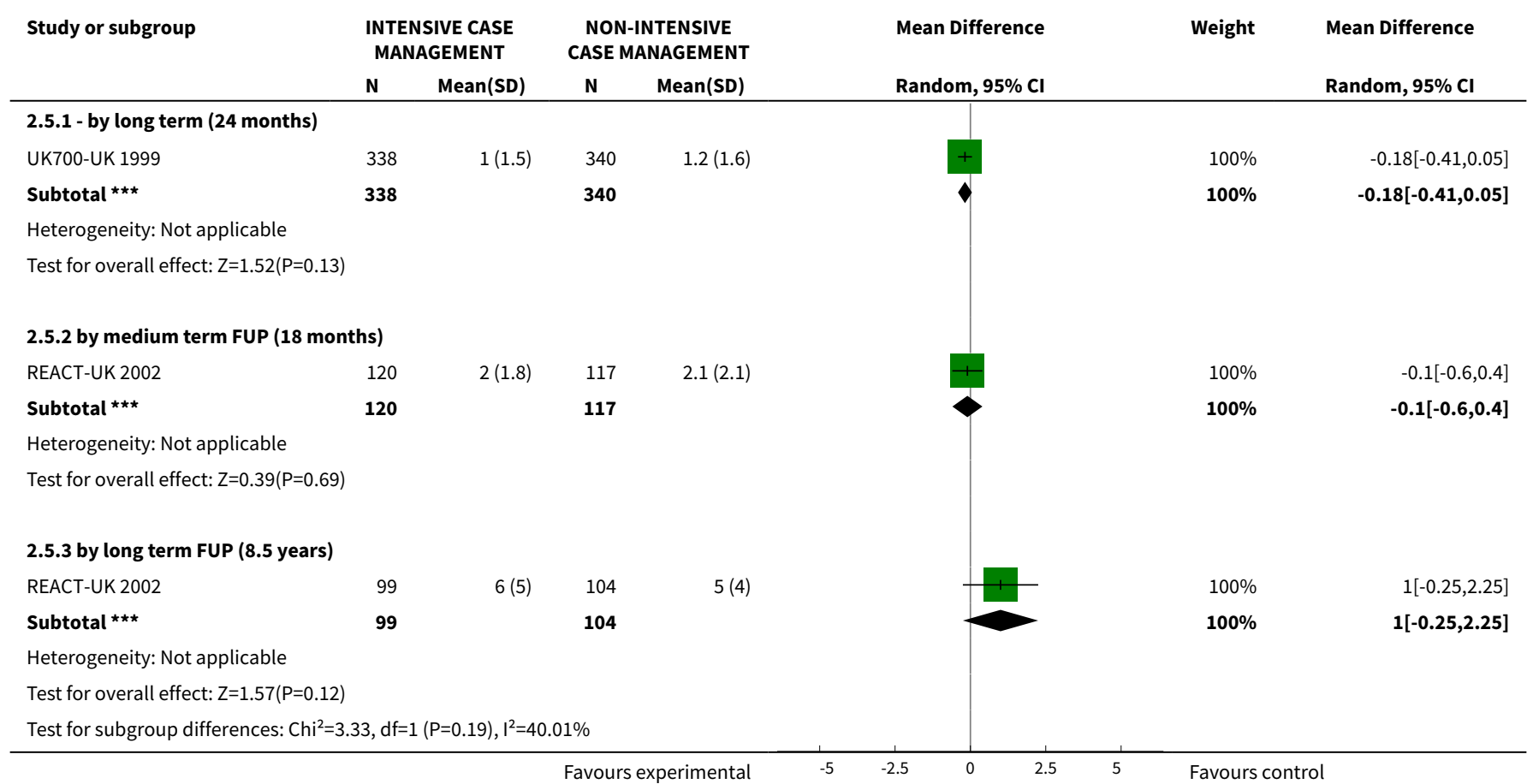

Analysis 2.6. Comparison 2 Intensive Case Management versus non-Intensive Case Management, Outcome 6 Service use: 3c. Average number of admissions (skewed data) - by medium term.

\begin{tabular}{cllll}
\multicolumn{1}{c}{ Study } & \multicolumn{2}{c}{$\begin{array}{c}\text { Service use: 3c. Average number of admissions (skewed data) - by medium term } \\
\text { Intervention }\end{array}$} & Mean & SD \\
\hline Johnston-Australia 1998 & 1. ICM & 1.6 & 2 & \\
\hline Johnston-Australia 1998 & 2. non-ICM & 1.9 & 2.4 & 35 \\
\hline
\end{tabular}


Analysis 2.7. Comparison 2 Intensive Case Management versus nonIntensive Case Management, Outcome 7 Adverse event: 1a. Death - any cause.

\begin{tabular}{lcccc} 
Study or subgroup & INTENSIVE & NON-IN- & Risk Ratio & Weight \\
CASE MAN- & TENSIVE CASE \\
AGEMENT & MANAGEMENT & & \\
& $\mathrm{n} / \mathrm{N}$ & $\mathrm{n} / \mathrm{N}$ & $\mathrm{M}-\mathrm{H}$, Random, $95 \% \mathrm{Cl}$ & $\mathrm{M}-\mathrm{H}, \mathrm{Random}, 95 \% \mathrm{Cl}$ \\
\hline
\end{tabular}

$\begin{array}{lrr}\text { 2.7.1 by short term } & & \\ \text { Harrison-Read-UK } 2000 & 0 / 97 & 0 / 96 \\ \text { Subtotal }(95 \% \mathrm{Cl}) & 97 & 96\end{array}$

Total events: 0 (INTENSIVE CASE MANAGEMENT), 0 (NON-INTENSIVE CASE MANAGEMENT)

Heterogeneity: Not applicable

Test for overall effect: Not applicable

\subsection{2 by medium term}

Bush-Georgia 1990

Harrison-Read-UK 2000

$\begin{array}{ll}0 / 14 & 0 / 14 \\ 0 / 97 & 0 / 96 \\ 1 / 37 & 0 / 36 \\ \mathbf{1 4 8} & \mathbf{1 4 6}\end{array}$

Subtotal $(95 \% \mathrm{CI})$

Total events: 1 (INTENSIVE CASE MANAGEMENT), 0 (NON-INTENSIVE CASE MANAGEMENT)

Heterogeneity: Not applicable

Test for overall effect: $\mathrm{Z}=0.66(\mathrm{P}=0.51)$

\subsection{3 by long term}

Drake-NHamp 1998

$3 / 109$

Essock-Connecticut1 $1995 \quad 2 / 130 \quad 2 / 132$

Harrison-Read-UK 2000

REACT-UK 2002

UK700-UK 1999

$0 / 97$

$0 / 96$

$3 / 127$

$8 / 353$

816

$4 / 124$

$8 / 355$

Subtotal $(95 \% \mathrm{Cl})$

821

Total events: 16 (INTENSIVE CASE MANAGEMENT), 18 (NON-INTENSIVE CASE MANAGEMENT)

Heterogeneity: $\mathrm{Tau}^{2}=0 ; \mathrm{Chi}^{2}=0.17, \mathrm{df}=3(\mathrm{P}=0.98) ; \mathrm{I}^{2}=0 \%$

Test for overall effect: $Z=0.32(P=0.75)$

2.7.4 by medium term FUP ( 18 months)

REACT-UK 2002

$6 / 127$

$6 / 124$

Subtotal $(95 \% \mathrm{CI})$

127

124

Total events: 6 (INTENSIVE CASE MANAGEMENT), 6 (NON-INTENSIVE CASE MANAGEMENT)

Heterogeneity: Not applicable

Test for overall effect: $\mathrm{Z}=0.04(\mathrm{P}=0.97)$

2.7.5 by long term FUP ( 8.5 years)

REACT-UK 2002

$20 / 127$

$17 / 124$

Subtotal $(95 \% \mathrm{Cl})$

127

124

Total events: 20 (INTENSIVE CASE MANAGEMENT), 17 (NON-INTENSIVE CASE MANAGEMENT)

Heterogeneity: Not applicable

Test for overall effect: $\mathrm{Z}=0.45(\mathrm{P}=0.65)$
Not estimable

Not estimable

96

$\mid$

Not estimable

Not estimable

$100 \%$ 2.92[0.12,69.43]

$100 \%$ $2.92[0.12,69.43]$
$100 \%$

$100 \%$

$20.47 \%$

$11.75 \%$

$20.4 \%$

$47.37 \%$

$100 \%$

$0.98[0.32,2.95]$

$0.98[0.32,2.95]$

Favours experimental $\quad 0.001$

0.1

Favours control 
Analysis 2.8. Comparison 2 Intensive Case Management versus nonIntensive Case Management, Outcome 8 Adverse event: 1b. Death - suicide.

\begin{tabular}{|c|c|c|c|c|c|}
\hline Study or subgroup & $\begin{array}{l}\text { INTENSIVE } \\
\text { CASE MAN- } \\
\text { AGEMENT }\end{array}$ & $\begin{array}{c}\text { NON-IN- } \\
\text { TENSIVE CASE } \\
\text { MANAGEMENT }\end{array}$ & Risk Ratio & Weight & Risk Ratio \\
\hline & $n / N$ & $n / N$ & M-H, Random, 95\% Cl & & M-H, Random, $95 \%$ C \\
\hline
\end{tabular}

$\begin{array}{lrr}\text { 2.8.1 by short term } & & \\ \text { Harrison-Read-UK } 2000 & 0 / 97 & 0 / 96 \\ \text { Subtotal }(95 \% \mathrm{Cl}) & 97 & 96\end{array}$

Total events: 0 (INTENSIVE CASE MANAGEMENT), 0 (NON-INTENSIVE CASE MANAGEMENT)

Heterogeneity: Not applicable

Test for overall effect: Not applicable

$\begin{array}{lrr}\text { 2.8.2 by medium term } & & \\ \text { Bush-Georgia 1990 } & 0 / 14 & 0 / 14 \\ \text { Harrison-Read-UK 2000 } & 0 / 97 & 0 / 96 \\ \text { UK700-UK (A) } & 0 / 97 & 2 / 99 \\ \text { UK700-UK (B) } & 0 / 77 & 0 / 76 \\ \text { UK700-UK (C) } & 1 / 79 & 0 / 79 \\ \text { UK700-UK (D) } & 4 / 100 & 1 / 101 \\ \text { Subtotal (95\% CI) } & \mathbf{4 6 4} & \mathbf{4 6 5}\end{array}$

Total events: 5 (INTENSIVE CASE MANAGEMENT), 3 (NON-INTENSIVE CASE MANAGEMENT)

Heterogeneity: $\mathrm{Tau}^{2}=0.64 ; \mathrm{Chi}^{2}=2.65, \mathrm{df}=2(\mathrm{P}=0.27) ; \mathrm{I}^{2}=24.43 \%$

Test for overall effect: $\mathrm{Z}=0.51(\mathrm{P}=0.61)$

\subsection{3 by long term}

Harrison-Read-UK 2000

REACT-UK 2002

$\begin{array}{rr}0 / 97 & 0 / 96 \\ 1 / 127 & 3 / 124 \\ 5 / 353 & 4 / 355 \\ \mathbf{5 7 7} & \mathbf{5 7 5}\end{array}$

Subtotal $(95 \% \mathrm{CI})$

577

96

|

Not estimable

Not estimable

Total events: 6 (INTENSIVE CASE MANAGEMENT), 7 (NON-INTENSIVE CASE MANAGEMENT)

Heterogeneity: $\mathrm{Tau}^{2}=0.04 ; \mathrm{Chi}^{2}=1.04, \mathrm{df}=1(\mathrm{P}=0.31) ; \mathrm{I}^{2}=4.09 \%$

Test for overall effect: $\mathrm{Z}=0.21(\mathrm{P}=0.83)$

2.8.4 by medium term FUP ( 18 months)

REACT-UK 2002

$1 / 127 \quad 3 / 124$

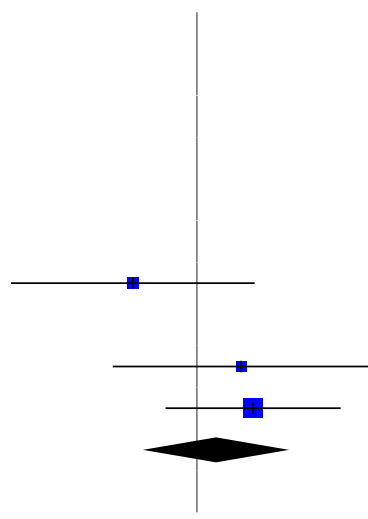

Not estimable

Not estimable

$0.2[0.01,4.2]$

Not estimable

$3[0.12,72.54]$

$4.04[0.46,35.52]$

$1.61[0.26,9.85]$

Subtotal $(95 \% \mathrm{Cl})$

127

124

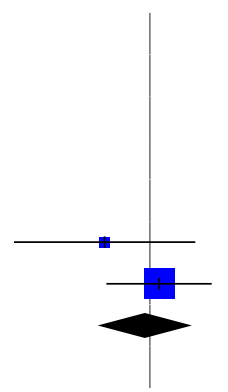

$26.04 \%$

$45.66 \%$

$100 \%$

Not estimable

$0.33[0.03,3.09]$

$1.26[0.34,4.64]$

$73.76 \%$

$\mathbf{0 . 8 8}[0.27,2.84]$

Total events: 1 (INTENSIVE CASE MANAGEMENT), 3 (NON-INTENSIVE CASE MANAGEMENT)

Heterogeneity: Not applicable

Test for overall effect: $Z=0.98(P=0.33)$

$\begin{array}{lllllll}\text { Favours experimental } & 0.005 & 0.1 & 1 & 10 & 200 & \text { Favours control }\end{array}$ 
Analysis 2.9. Comparison 2 Intensive Case Management versus non-Intensive Case Management, Outcome 9 Global state: 1. Leaving the study early.

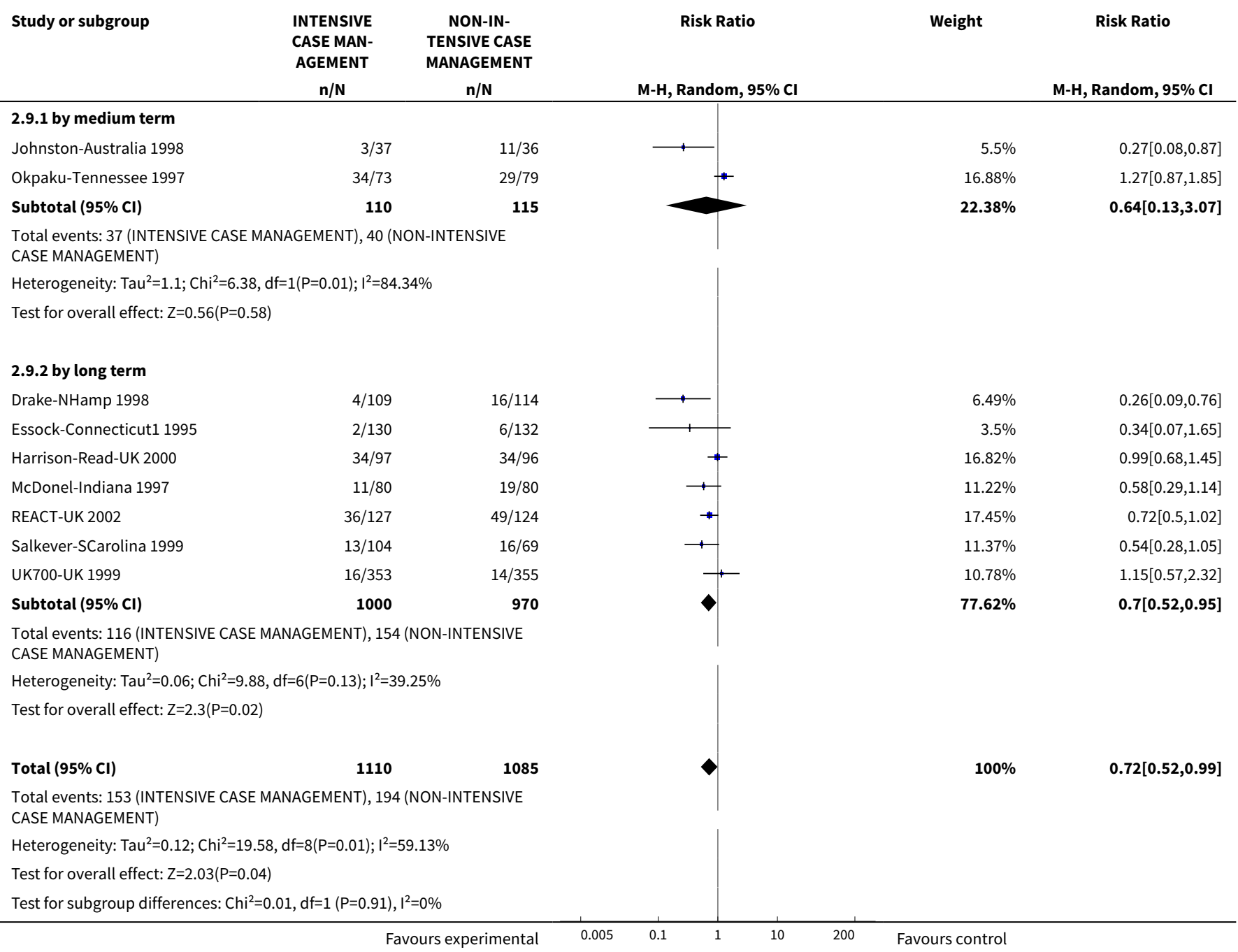

\section{Analysis 2.10. Comparison 2 Intensive Case Management versus non-Intensive Case Management, Outcome $10 \mathrm{Global}$ state: 2a. Average endpoint score (HoNOS, high = poor) - by long term.}

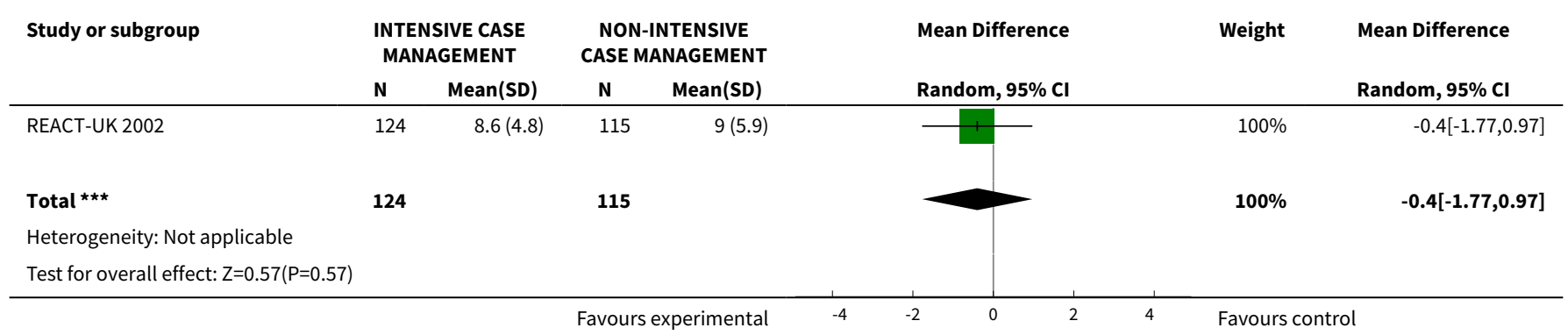


Analysis 2.11. Comparison 2 Intensive Case Management versus non-Intensive Case Management, Outcome 11 Global state: 2b. Average endpoint score (HoNOS, high = poor) - skewed data.

Global state: 2b. Average endpoint score (HoNOS, high = poor) - skewed data

\begin{tabular}{|c|c|c|c|c|}
\hline Study & Intervention & Mean & SD & Total \\
\hline \multicolumn{5}{|c|}{ medium term } \\
\hline Harrison-Read-UK 2000 & 1. ICM & 12 & 6.8 & 54 \\
\hline Harrison-Read-UK 2000 & 2. non-ICM & 11.4 & 6.4 & 64 \\
\hline \multicolumn{5}{|c|}{ long term } \\
\hline Harrison-Read-UK 2000 & 1. ICM & 11.9 & 5.9 & 60 \\
\hline Harrison-Read-UK 2000 & 2. non-ICM & 10.4 & 6.4 & 59 \\
\hline
\end{tabular}

Analysis 2.12. Comparison 2 Intensive Case Management versus non-Intensive Case Management, Outcome 12 Global state: 3a. Not compliant with medication - by medium term.

\begin{tabular}{|c|c|c|c|c|c|}
\hline \multirow[t]{2}{*}{ Study or subgroup } & $\begin{array}{l}\text { INTENSIVE } \\
\text { CASE MAN- } \\
\text { AGEMENT } \\
\end{array}$ & $\begin{array}{c}\text { NON-IN- } \\
\text { TENSIVE CASE } \\
\text { MANAGEMENT }\end{array}$ & Risk Ratio & Weight & Risk Ratio \\
\hline & $\mathbf{n} / \mathbf{N}$ & $\mathbf{n} / \mathbf{N}$ & M-H, Random, 95\% Cl & & M-H, Random, 95\% Cl \\
\hline Total $(95 \% \mathrm{Cl})$ & 37 & 36 & & $100 \%$ & $1.14[0.42,3.05]$ \\
\hline \multicolumn{6}{|c|}{$\begin{array}{l}\text { Total events: } 7 \text { (INTENSIVE CASE MANAGEMENT), } 6 \text { (NON-INTENSIVE CASE } \\
\text { MANAGEMENT) }\end{array}$} \\
\hline \multicolumn{6}{|c|}{ Heterogeneity: Not applicable } \\
\hline \multicolumn{6}{|c|}{ Test for overall effect: $\mathrm{Z}=0.25(\mathrm{P}=0.8)$} \\
\hline
\end{tabular}

Analysis 2.13. Comparison 2 Intensive Case Management versus non-Intensive Case Management, Outcome 13 Global state: 3b. Compliance with medication - average endpoint sub-scale score (ROMI) - by long term.

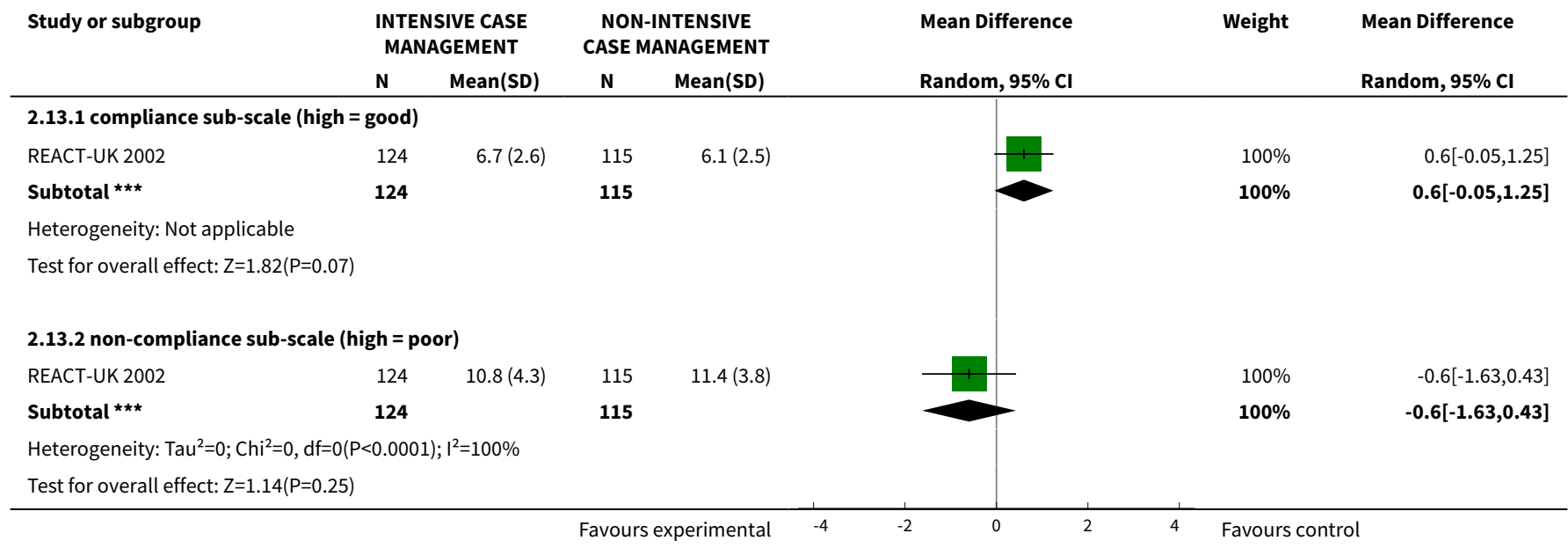


Analysis 2.14. Comparison 2 Intensive Case Management versus non-Intensive Case Management, Outcome 14 Global state: 3c. Compliance with medication - average endpoint sub-scale score (ROMI, score 1-3, skewed data).

Global state: 3c. Compliance with medication - average endpoint sub-scale score (ROMI, score 1-3, skewed data)

\begin{tabular}{|c|c|c|c|c|}
\hline Study & Intervention & Mean & & Total \\
\hline \multicolumn{5}{|c|}{ medium term - compliance sub-scale (high = good) } \\
\hline Harrison-Read-UK 2000 & 1. ICM & 1.8 & 0.4 & 49 \\
\hline Harrison-Read-UK 2000 & 2. non-ICM & 2.0 & 0.5 & 61 \\
\hline \multicolumn{5}{|c|}{ medium term - non-compliance sub-scale (high = poor) } \\
\hline Harrison-Read-UK 2000 & 1. ICM & 1.3 & 0.3 & 49 \\
\hline Harrison-Read-UK 2000 & 2. non-ICM & 1.2 & 0.3 & 61 \\
\hline Harrison-Read-UK 2000 & 1. ICM & 1.8 & 0.4 & 62 \\
\hline Harrison-Read-UK 2000 & 2. non-ICM & 1.9 & 0.5 & 60 \\
\hline \multicolumn{5}{|c|}{ long term - non-compliance sub-scale (high = poor) } \\
\hline Harrison-Read-UK 2000 & 1. ICM & 1.2 & 0.3 & 63 \\
\hline Harrison-Read-UK 2000 & 2. non-ICM & 1.2 & 0.3 & 61 \\
\hline
\end{tabular}

Analysis 2.15. Comparison 2 Intensive Case Management versus non-Intensive Case Management, Outcome 15 Social functioning: 1. Contact with legal system (various measurements).

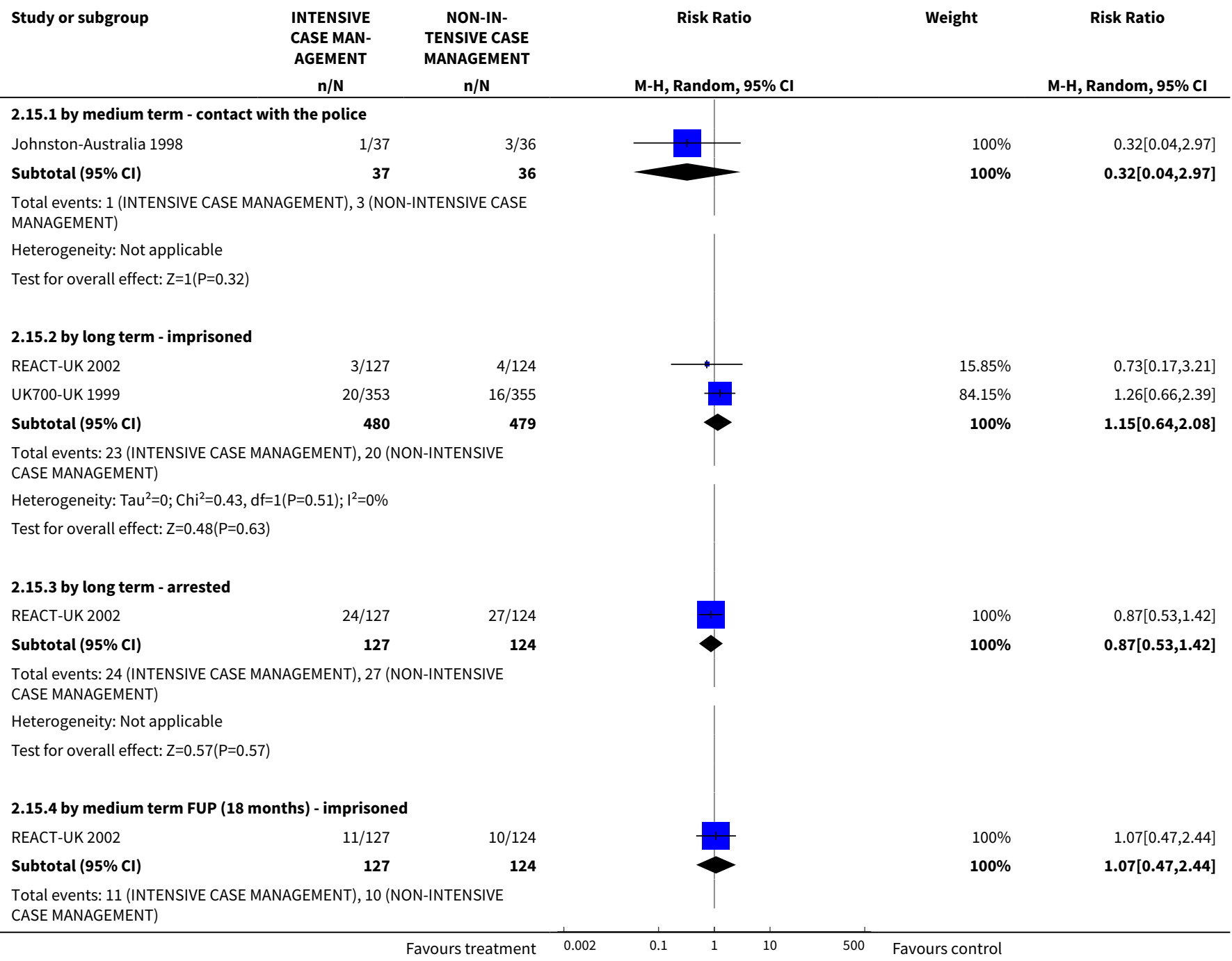




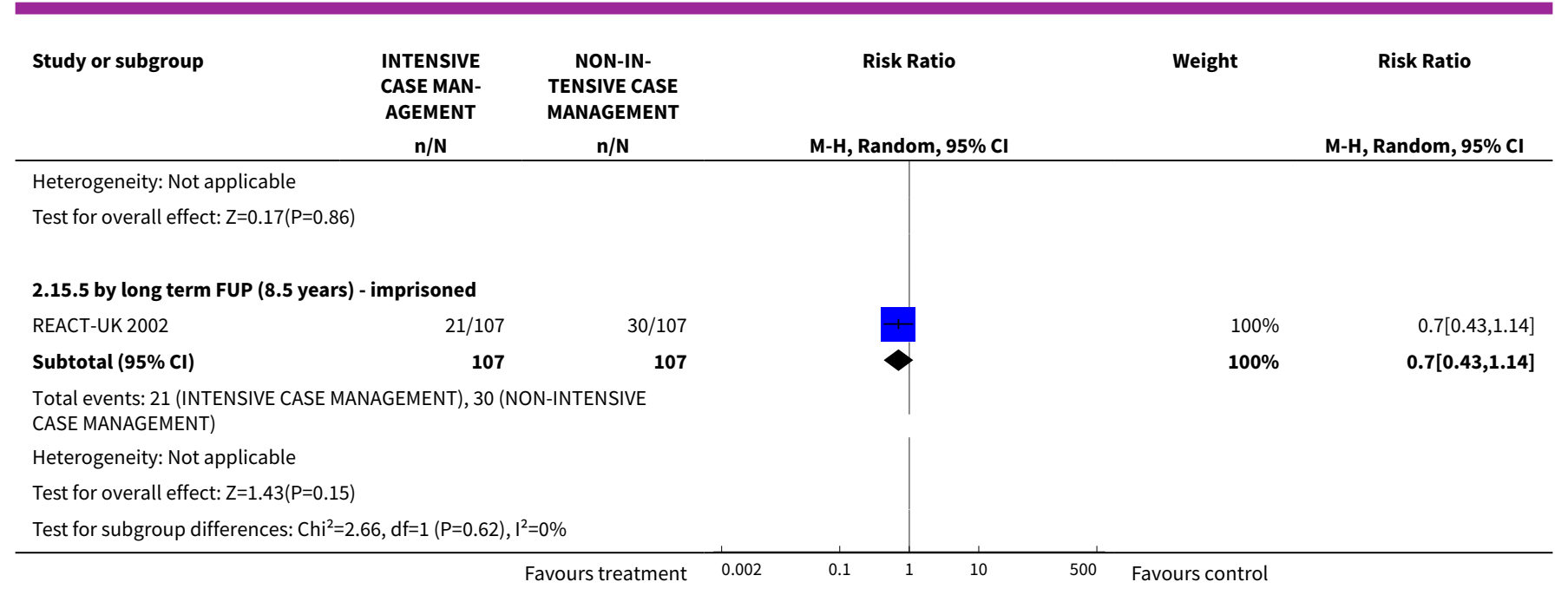

Analysis 2.16. Comparison 2 Intensive Case Management versus non-Intensive Case Management, Outcome 16 Social functioning 2. Employment status (various measurements).

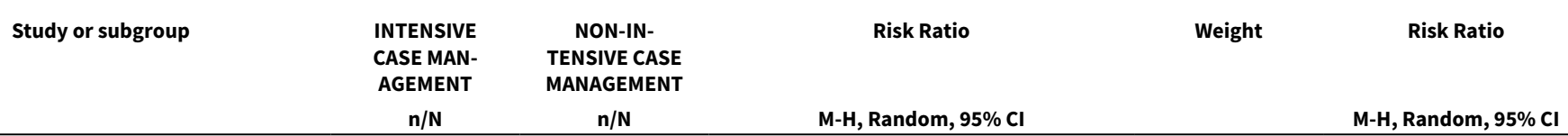

2.16.1 spent $>1$ day employed - by medium term

Johnston-Australia 1998

rm

Subtotal $(95 \% \mathrm{CI})$

$6 / 37 \quad 4 / 36$

37

36

M-H, Random, $95 \% \mathrm{Cl}$

Total events: 6 (INTENSIVE CASE MANAGEMENT), 4 (NON-INTENSIVE CASE MANAGEMENT)

Heterogeneity: Not applicable

Test for overall effect: $\mathrm{Z}=0.63(\mathrm{P}=0.53)$

2.16.2 on paid employment - by medium term

Johnston-Australia 1998

Total events: 2 (INTENSIVE CASE MANAGEMENT), 2 (NON-INTENSIVE CASE MANAGEMENT)

Heterogeneity: Not applicable

Test for overall effect: $\mathrm{Z}=0.03(\mathrm{P}=0.98)$

2.16.3 unemployed - by long term FUP (8.5 years)

$\begin{array}{lrr}\text { REACT-UK } 2002 & 74 / 107 & 67 / 107 \\ \text { Subtotal }(\mathbf{9 5} \% \mathbf{C I}) & \mathbf{1 0 7} & \mathbf{1 0 7}\end{array}$

Total events: 74 (INTENSIVE CASE MANAGEMENT), 67 (NON-INTENSIVE CASE MANAGEMENT)

Heterogeneity: Not applicable

Test for overall effect: $Z=1.01(P=0.31)$

$100 \%$

$100 \%$

$100 \%$

$1.1[0.91,1.34]$

$100 \%$

$1.1[0.91,1.34]$ 
Analysis 2.17. Comparison 2 Intensive Case Management versus non-Intensive Case Management, Outcome 17 Social functioning: 3a. Accommodation status (various measurements).

\begin{tabular}{lcc} 
Study or subgroup & $\begin{array}{c}\text { INTENSIVE } \\
\text { CASE MAN- } \\
\text { AGEMENT } \\
\text { n/N }\end{array}$ & $\begin{array}{c}\text { NON-IN- } \\
\text { TENSIVE CAS } \\
\text { MANAGEMENT }\end{array}$ \\
& $\mathbf{n} / \mathbf{N}$ \\
\hline 2.17.1 by medium term - living in supported accommodation \\
Johnston-Australia 1998 & $8 / 37$ & $3 / 36$ \\
Subtotal $(95 \% \mathrm{Cl})$ & $\mathbf{3 7}$
\end{tabular}

Total events: 8 (INTENSIVE CASE MANAGEMENT), 3 (NON-INTENSIVE CASE MANAGEMENT)

Heterogeneity: Not applicable

Test for overall effect: $Z=1.5(P=0.13)$

2.17.2 by long term - homelessness

REACT-UK $2002 \quad 12 / 127 \quad 17 / 124$

Subtotal $(95 \% \mathrm{CI})$

127

Total events: 12 (INTENSIVE CASE MANAGEMENT), 17 (NON-INTENSIVE CASE MANAGEMENT)

Heterogeneity: Not applicable

Test for overall effect: $Z=1.05(P=0.29)$

2.17.3 by medium term FUP (18 months) - living independently REACT-UK 2002

Total events: 93 (INTENSIVE CASE MANAGEMENT), 93 (NON-INTENSIVE CASE MANAGEMENT)

Heterogeneity: Not applicable

Test for overall effect: $\mathrm{Z}=0.32(\mathrm{P}=0.75)$

2.17.4 by medium term FUP ( 18 months) - living in supported accomodation

$\begin{array}{lrr}\text { REACT-UK } 2002 & 11 / 127 & 13 / 124 \\ \text { Subtotal }(95 \% \mathrm{Cl}) & \mathbf{1 2 7} & \mathbf{1 2 4}\end{array}$

Total events: 11 (INTENSIVE CASE MANAGEMENT), 13 (NON-INTENSIVE CASE MANAGEMENT)

Heterogeneity: $\mathrm{Tau}^{2}=0 ; \mathrm{Chi}^{2}=0, \mathrm{df}=0(\mathrm{P}<0.0001) ; \mathrm{I}^{2}=100 \%$

Test for overall effect: $Z=0.49(P=0.62)$

2.17.5 by medium term FUP (18 months) - homelessness

Total events: 18 (INTENSIVE CASE MANAGEMENT), 21 (NON-INTENSIVE CASE MANAGEMENT)

Heterogeneity: Tau $^{2}=0 ; \mathrm{Chi}^{2}=0, \mathrm{df}=0(\mathrm{P}<0.0001) ; \mathrm{I}^{2}=100 \%$

Test for overall effect: $Z=0.6(P=0.55)$

2.17.6 by long term FUP ( 8.5 years) - living in supported accomodation REACT-UK $2002 \quad 42 / 107 \quad 40 / 107$ Subtotal $(95 \% \mathrm{Cl})$

Total events: 42 (INTENSIVE CASE MANAGEMENT), 40 (NON-INTENSIVE CASE MANAGEMENT)

Heterogeneity: Not applicable

Test for overall effect: $\mathrm{Z}=0.28(\mathrm{P}=0.78)$

2.17.7 by long term FUP ( 8.5 years) - homelessness

$\begin{array}{lllllll}\text { Favours experimental } & 0.01 & 0.1 & 1 & 10 & 100 & \text { Favours control }\end{array}$

$100 \%$

$100 \%$ 


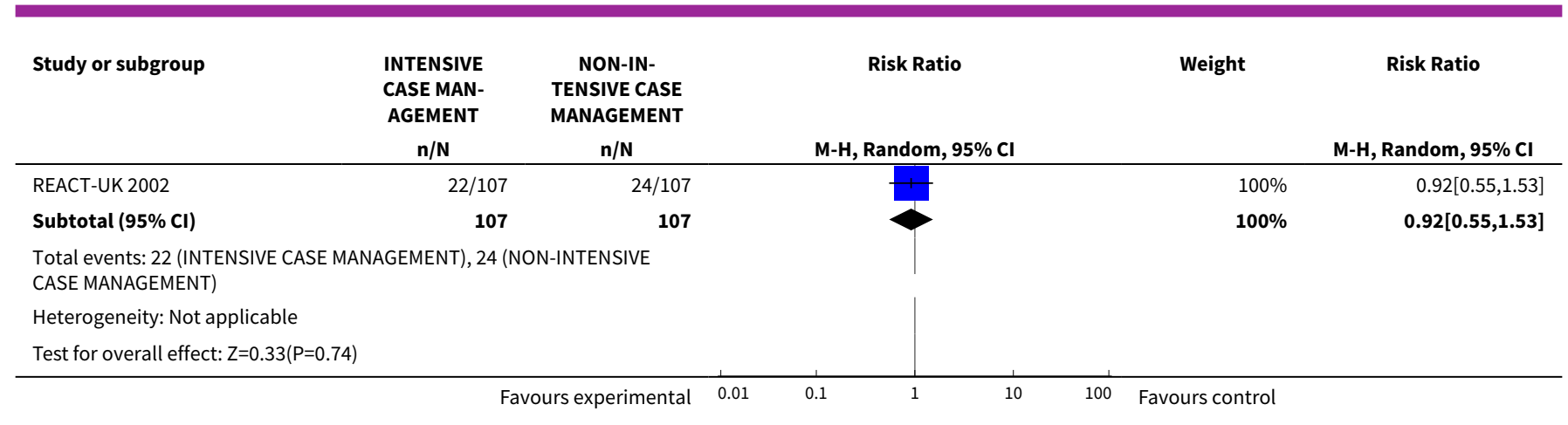

\section{Analysis 2.18. Comparison 2 Intensive Case Management versus non-Intensive Case Management, Outcome} 18 Social functioning: 3 b. Accommodation status - average days per month in stable accommodation.

\begin{tabular}{|c|c|c|c|c|c|c|c|}
\hline \multirow[t]{2}{*}{ Study or subgroup } & \multicolumn{2}{|c|}{$\begin{array}{l}\text { INTENSIVE CASE } \\
\text { MANAGEMENT }\end{array}$} & \multicolumn{2}{|c|}{$\begin{array}{l}\text { NON-INTENSIVE } \\
\text { CASE MANAGEMENT }\end{array}$} & \multirow{2}{*}{$\begin{array}{l}\text { Mean Difference } \\
\text { Random, } 95 \% \mathrm{Cl}\end{array}$} & \multirow[t]{2}{*}{ Weight } & \multirow{2}{*}{$\begin{array}{l}\text { Mean Difference } \\
\text { Random, } 95 \% \mathrm{Cl}\end{array}$} \\
\hline & $\mathbf{N}$ & Mean(SD) & $\mathbf{N}$ & $\operatorname{Mean}($ SD) & & & \\
\hline \multicolumn{8}{|l|}{2.18 .1 by short term } \\
\hline Drake-NHamp 1998 & 105 & $25.8(7.5)$ & 98 & $26(9)$ & + & $100 \%$ & $-0.2[-2.48,2.08]$ \\
\hline Subtotal $\star \star \star$ & 105 & & 98 & & 1 & $100 \%$ & $-0.2[-2.48,2.08]$ \\
\hline \multicolumn{8}{|c|}{ Heterogeneity: Not applicable } \\
\hline \multicolumn{8}{|c|}{ Test for overall effect: $\mathrm{Z}=0.17(\mathrm{P}=0.86)$} \\
\hline \multicolumn{8}{|c|}{2.18 .2 by medium term } \\
\hline Drake-NHamp 1998 & 105 & $25.3(7.2)$ & 98 & $25.2(9)$ & + & $100 \%$ & $0.1[-2 \cdot 15,2.35]$ \\
\hline Subtotal $\star \star \star ~$ & 105 & & 98 & & 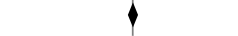 & $100 \%$ & $0.1[-2.15,2.35]$ \\
\hline \multicolumn{8}{|c|}{ Heterogeneity: $\operatorname{Tau}^{2}=0 ; \mathrm{Chi}^{2}=0, \mathrm{df}=0(\mathrm{P}<0.0001) ; \mathrm{I}^{2}=100 \%$} \\
\hline \multicolumn{8}{|c|}{ Test for overall effect: $\mathrm{Z}=0.09(\mathrm{P}=0.93)$} \\
\hline \multicolumn{8}{|l|}{ 2.18.3 by long term } \\
\hline Drake-NHamp 1998 & 105 & $28.6(8.8)$ & 98 & $28(9.7)$ & * & $21.48 \%$ & $0.6[-1.95,3.15]$ \\
\hline UK700-UK 1999 & 349 & $14.9(9.2)$ & 349 & $15.3(8.8)$ & + & $78.52 \%$ & $-0.4[-1.74,0.94]$ \\
\hline Subtotal $\star \star \star ~$ & 454 & & 447 & & 1 & $100 \%$ & $-0.19[-1.37,1]$ \\
\hline \multicolumn{8}{|c|}{ Heterogeneity: $\mathrm{Tau}^{2}=0 ; \mathrm{Chi}^{2}=0.46, \mathrm{df}=1(\mathrm{P}=0.5) ; \mathrm{I}^{2}=0 \%$} \\
\hline \multicolumn{8}{|c|}{ Test for overall effect: $Z=0.31(P=0.76)$} \\
\hline
\end{tabular}

\section{Analysis 2.19. Comparison 2 Intensive Case Management versus non-Intensive Case Management, Outcome 19 Social functioning: 4a. Substance abuse - by long term.}

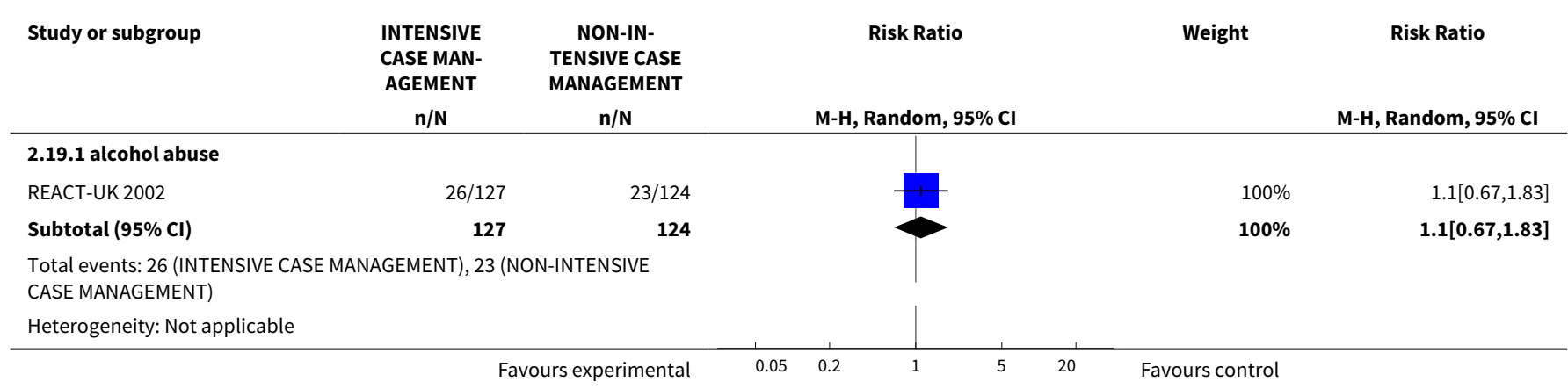




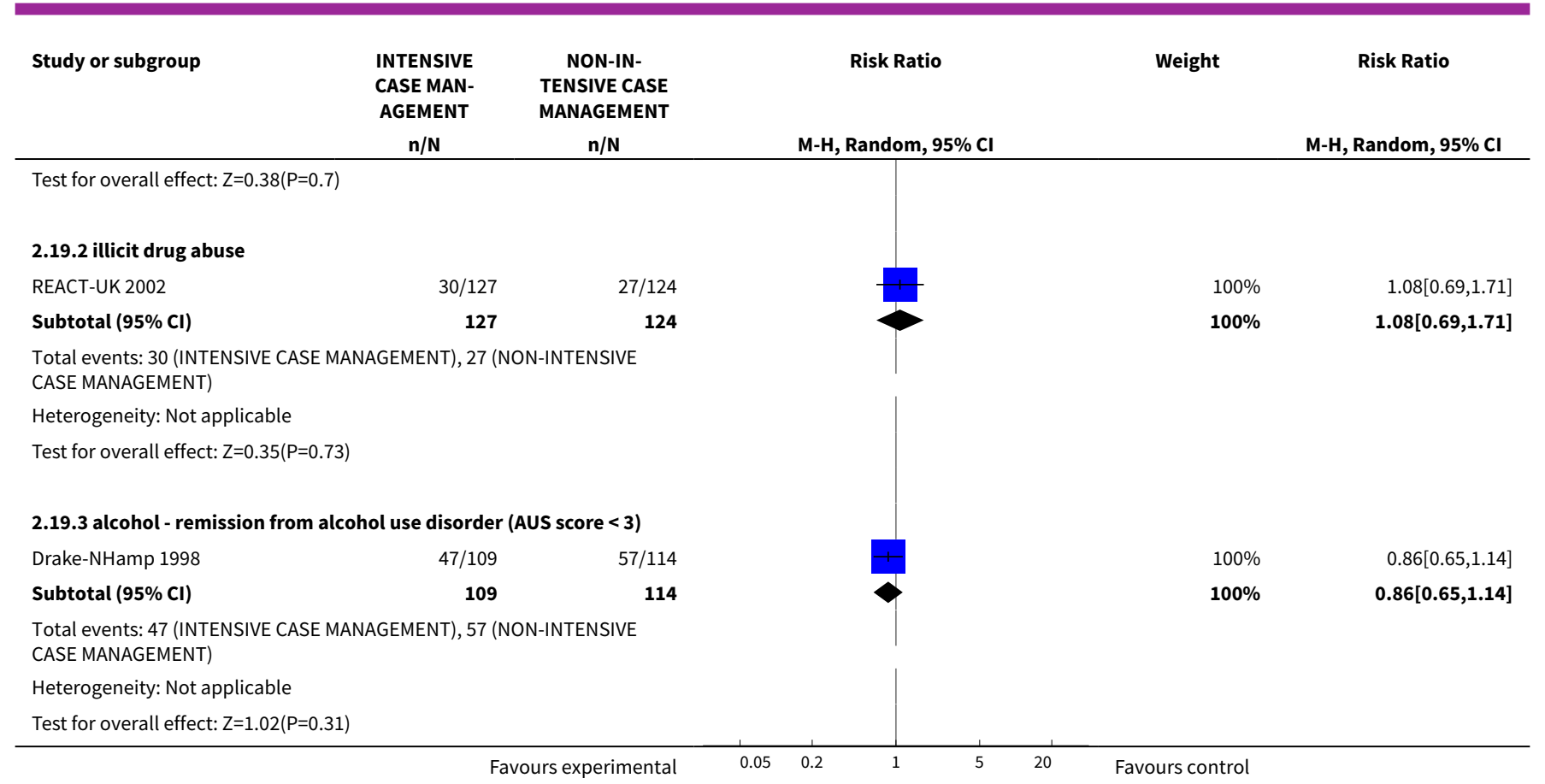

Analysis 2.20. Comparison 2 Intensive Case Management versus non-Intensive Case Management, Outcome 20 Social functioning: 4b. Substance abuse - average endpoint score (SATS, low = poor).

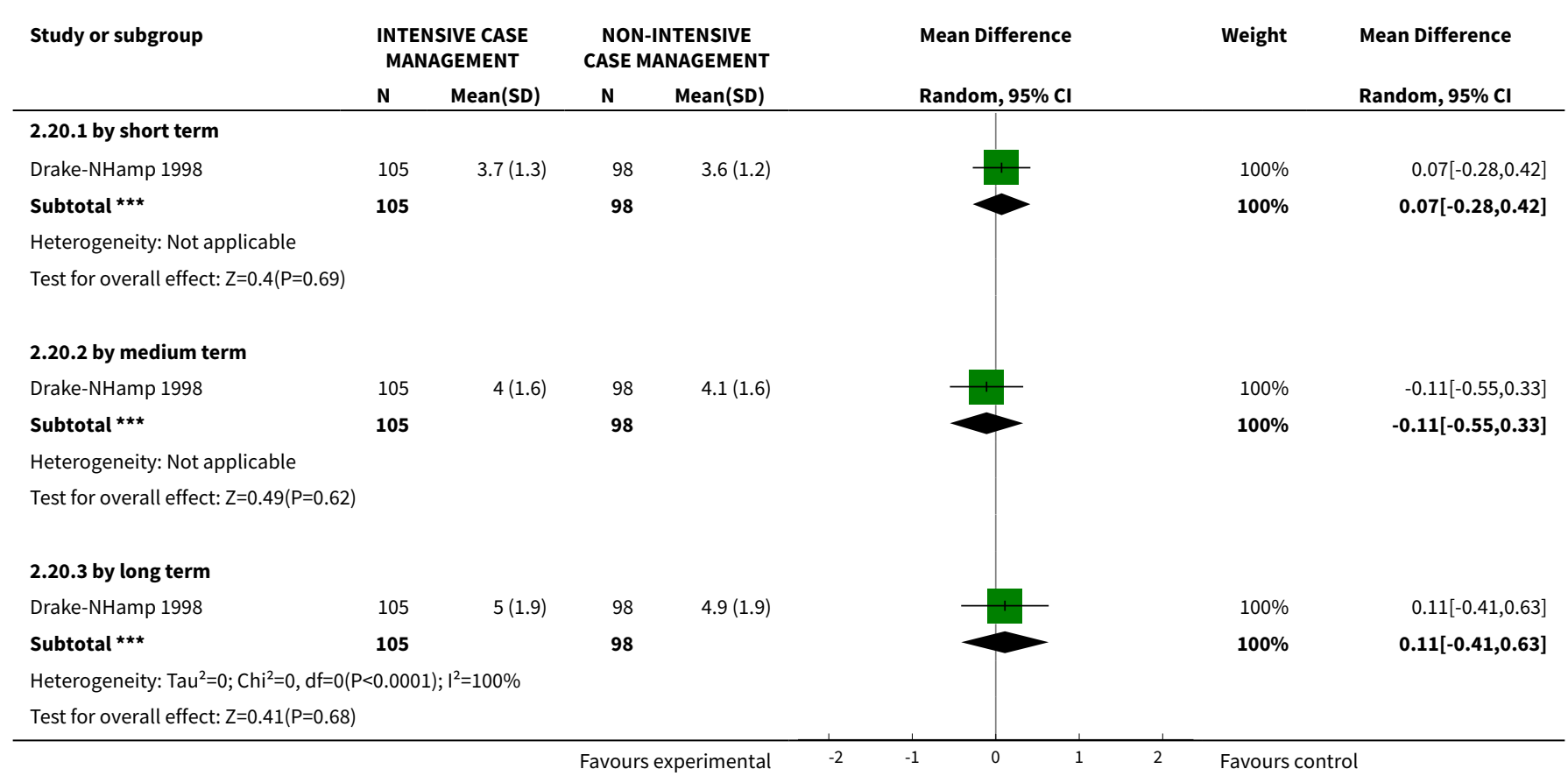


Analysis 2.21. Comparison 2 Intensive Case Management versus non-Intensive Case Management, Outcome 21 Social functioning: 4c. Alcohol - abuse (various measurements, skewed data).

\begin{tabular}{|c|c|c|c|c|}
\hline \multicolumn{5}{|c|}{ Social functioning: 4c. Alcohol - abuse (various measurements, skewed data) } \\
\hline Study & Intervention & Mean & SD & Total \\
\hline \multicolumn{5}{|c|}{ short term - days using alcohol during previous 6 months (TLFB) } \\
\hline Drake-NHamp 1998 & 1. ICM & 56.8 & 56.4 & 75 \\
\hline Drake-NHamp 1998 & 2. non-ICM & 47.5 & 58.4 & 68 \\
\hline \multicolumn{5}{|c|}{ short term - average endpoint score (AUS, high = poor) } \\
\hline Drake-NHamp 1998 & 1. ICM & 3.09 & 1.02 & 83 \\
\hline \multicolumn{5}{|c|}{ medium term - days using alcohol during previous 6 months (TLFB) } \\
\hline Drake-NHamp 1998 & 1. ICM & 59.1 & 53.3 & 75 \\
\hline Drake-NHamp 1998 & 2. non-ICM & 42.8 & 52.9 & 68 \\
\hline \multicolumn{5}{|c|}{ medium term - average endpoint score (AUS, high = poor) } \\
\hline Drake-NHamp 1998 & 1. ICM & 3.11 & 1.05 & 83 \\
\hline Drake-NHamp 1998 & 2. non-ICM & 2.8 & 1.13 & 73 \\
\hline Drake-NHamp 1998 & 1. ICM & 46.4 & 53.6 & 75 \\
\hline Drake-NHamp 1998 & 2. non-ICM & 43.6 & 57.3 & 68 \\
\hline \multicolumn{5}{|c|}{ long term - average endpoint score (AUS, high = poor) } \\
\hline Drake-NHamp 1998 & 1. ICM & 2.64 & 1.12 & 83 \\
\hline Drake-NHamp 1998 & 2. non-ICM & 2.77 & 1.18 & 73 \\
\hline
\end{tabular}

Analysis 2.22. Comparison 2 Intensive Case Management versus non-Intensive Case Management, Outcome 22 Social functioning: $5 \mathrm{a}$. Average endpoint score (LSP, high = poor) - by long term.

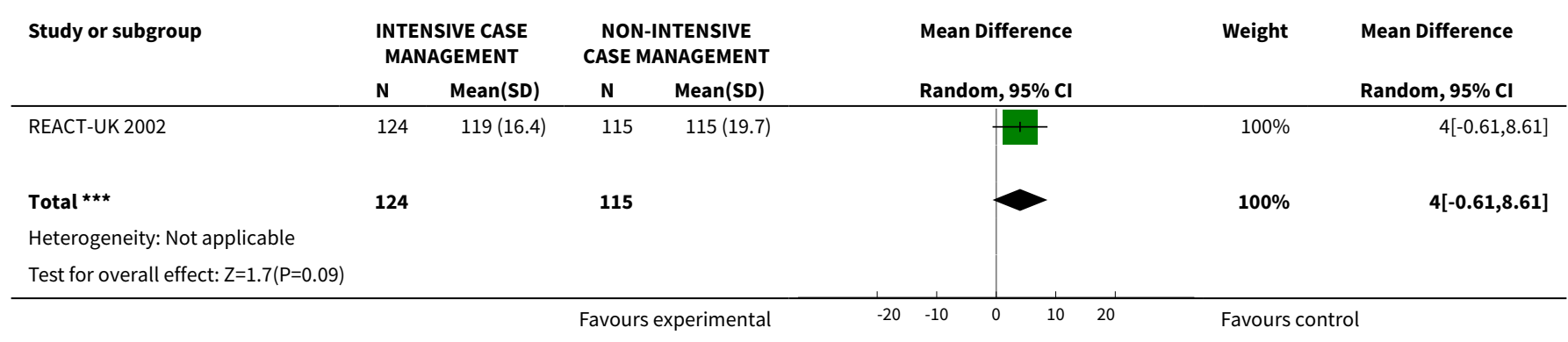

Analysis 2.23. Comparison 2 Intensive Case Management versus non-Intensive Case Management, Outcome 23 Social functioning: 5b. Average endpoint score (SFQ, high = poor) - skewed data.

Social functioning: 5 b. Average endpoint score (SFQ, high = poor) - skewed data

Study

Intervention

Mean

Tot

\begin{tabular}{lllll}
\hline & & by medium term & & \\
\hline Harrison-Read-UK 2000 & 1. ICM & 7.3 & 5.3 & \\
\hline Harrison-Read-UK 2000 & 2. non-ICM & 7.5 & 5.1 & \\
\hline & & & by long term & \\
\hline Harrison-Read-UK 2000 & 1. ICM & 8.9 & 49 & \\
\hline Harrison-Read-UK 2000 & 2. non-ICM & 7.9 & 5.9 & \\
\hline
\end{tabular}


Analysis 2.24. Comparison 2 Intensive Case Management versus non-Intensive Case Management, Outcome 24 Mental state: 1a. General symptoms - average endpoint score (various scales).

\begin{tabular}{|c|c|c|c|c|c|c|c|}
\hline \multirow[t]{2}{*}{ Study or subgroup } & \multicolumn{2}{|c|}{$\begin{array}{l}\text { INTENSIVE CASE } \\
\text { MANAGEMENT }\end{array}$} & \multicolumn{2}{|c|}{$\begin{array}{l}\text { NON-INTENSIVE } \\
\text { CASE MANAGEMENT }\end{array}$} & \multirow{2}{*}{$\begin{array}{l}\text { Mean Difference } \\
\text { Random, } 95 \% \mathrm{Cl}\end{array}$} & \multirow[t]{2}{*}{ Weight } & \multirow{2}{*}{$\begin{array}{l}\text { Mean Difference } \\
\text { Random, 95\% CI }\end{array}$} \\
\hline & $\mathbf{N}$ & Mean(SD) & $\mathbf{N}$ & Mean(SD) & & & \\
\hline \multicolumn{8}{|c|}{ 2.24.1 by short term (BPRS-24 items, high = poor) } \\
\hline Drake-NHamp 1998 & 105 & $42.2(12.2)$ & 98 & $42.9(12.1)$ & & $100 \%$ & $-0.65[-3.99,2.69]$ \\
\hline Subtotal $\star \star \star$ & 105 & & 98 & & & $100 \%$ & $-0.65[-3.99,2.69]$ \\
\hline \multicolumn{8}{|c|}{ Heterogeneity: Not applicable } \\
\hline \multicolumn{8}{|c|}{ 2.24.2 by medium term (BPRS-24 items, high = poor) } \\
\hline Drake-NHamp 1998 & 105 & $41.6(10.8)$ & 98 & $43.2(12)$ & & $100 \%$ & $-1.62[-4.76,1.52]$ \\
\hline Subtotal $\star \star \star$ & 105 & & 98 & & & $100 \%$ & $-1.62[-4.76,1.52]$ \\
\hline \multicolumn{8}{|c|}{ Heterogeneity: Not applicable } \\
\hline \multicolumn{8}{|c|}{ Test for overall effect: $Z=1.01(P=0.31)$} \\
\hline \multicolumn{8}{|c|}{ 2.24.3 by long term (BPRS-24 items, high = poor) } \\
\hline Drake-NHamp 1998 & 105 & $40.9(10.8)$ & 98 & $41.1(11.7)$ & & $100 \%$ & $-0.22[-3.32,2.88]$ \\
\hline 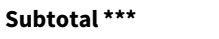 & 105 & & 98 & & & $100 \%$ & $-0.22[-3.32,2.88]$ \\
\hline \multicolumn{8}{|c|}{ Heterogeneity: Not applicable } \\
\hline \multicolumn{8}{|c|}{ Test for overall effect: $Z=0.14(P=0.89)$} \\
\hline \multicolumn{8}{|c|}{ 2.24.4 by long term (CPRS, high = poor) } \\
\hline UK700-UK 1999 & 311 & $18.5(13.8)$ & 284 & $18.1(13.9)$ & & $100 \%$ & $0.4[-1.83,2.63]$ \\
\hline Subtotal $\star \star \star$ & 311 & & 284 & & & $100 \%$ & $0.4[-1.83,2.63]$ \\
\hline \multicolumn{8}{|c|}{ Heterogeneity: Not applicable } \\
\hline Test for overall effect & & & & & & & \\
\hline
\end{tabular}

Analysis 2.25. Comparison 2 Intensive Case Management versus non-Intensive Case Management, Outcome 25 Mental state: 1b. General symptoms - average endpoint scores (various scales, skewed data).

\begin{tabular}{|c|c|c|c|c|}
\hline \multicolumn{5}{|c|}{ Mental state: 1b. General symptoms - average endpoint scores (various scales, skewed data) } \\
\hline Study & Intervention & Mean & SD & Total \\
\hline \multicolumn{5}{|c|}{ by medium term (Krawiecka Scale, high = poor) } \\
\hline Harrison-Read-UK 2000 & 1. ICM & 8.8 & 5.6 & 57 \\
\hline Harrison-Read-UK 2000 & 2. non-ICM & 8 & 4.5 & 65 \\
\hline \multicolumn{5}{|c|}{ by long term (Krawiecka Scale, high = poor) } \\
\hline Harrison-Read-UK 2000 & 1. ICM & 9.2 & 5.5 & 47 \\
\hline \multicolumn{5}{|c|}{ by long term (BPRS 24-items, high = good) } \\
\hline REACT-UK 2002 & 1. ICM & 32.9 & 9 & 91 \\
\hline REACT-UK 2002 & 2. non-ICM & 33.5 & 8.6 & 75 \\
\hline
\end{tabular}


Analysis 2.26. Comparison 2 Intensive Case Management versus nonIntensive Case Management, Outcome 26 Mental state: $2 a$. Specific symptoms: negative symptoms - average endpoint score (SANS, high = poor) - by long term.

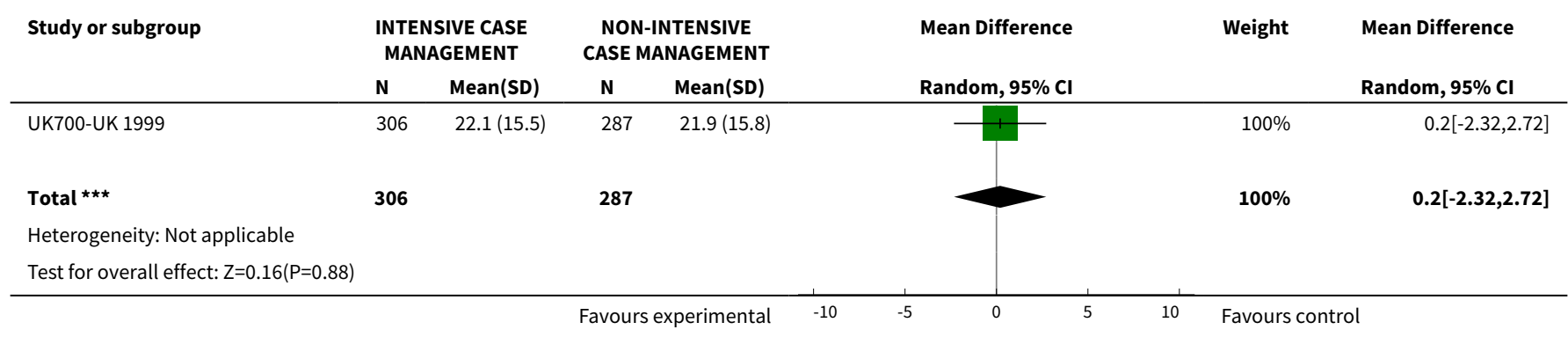

Analysis 2.27. Comparison 2 Intensive Case Management versus non-Intensive Case Management, Outcome 27 Mental state: 2 b. Specific symptoms - average endpoint scores (various scales, skewed data).

\begin{tabular}{|c|c|c|c|c|}
\hline \multicolumn{5}{|c|}{ Mental state: $\mathbf{2 b}$. Specific symptoms - average endpoint scores (various scales, skewed data) } \\
\hline Study & Intervention & Mean & SD & Total \\
\hline \multicolumn{5}{|c|}{ medium term - anxiety (HADS, high = poor) } \\
\hline Harrison-Read-UK 2000 & 1. ICM & 6.5 & 4.9 & 52 \\
\hline Harrison-Read-UK 2000 & 2. non-ICM & 6.7 & 4.6 & 61 \\
\hline \multicolumn{5}{|c|}{ medium term - depression (HADS, high = poor) } \\
\hline Harrison-Read-UK 2000 & 1. ICM & 6.4 & 5.4 & 52 \\
\hline \multicolumn{5}{|c|}{ long term - anxiety (HADS, high = poor) } \\
\hline Harrison-Read-UK 2000 & 1. ICM & 7.5 & 5.3 & 56 \\
\hline Harrison-Read-UK 2000 & 2. non-ICM & 6.4 & 4.6 & 58 \\
\hline \multicolumn{5}{|c|}{ long term - depression (HADS, high = poor) } \\
\hline Harrison-Read-UK 2000 & 1. ICM & 7.3 & 5.4 & 56 \\
\hline Harrison-Read-UK 2000 & 2. non-ICM & 6.8 & 5.6 & 58 \\
\hline
\end{tabular}

Analysis 2.28. Comparison 2 Intensive Case Management versus non-Intensive Case Management, Outcome 28 Behaviour: 1. Specific behaviour (various measurements).

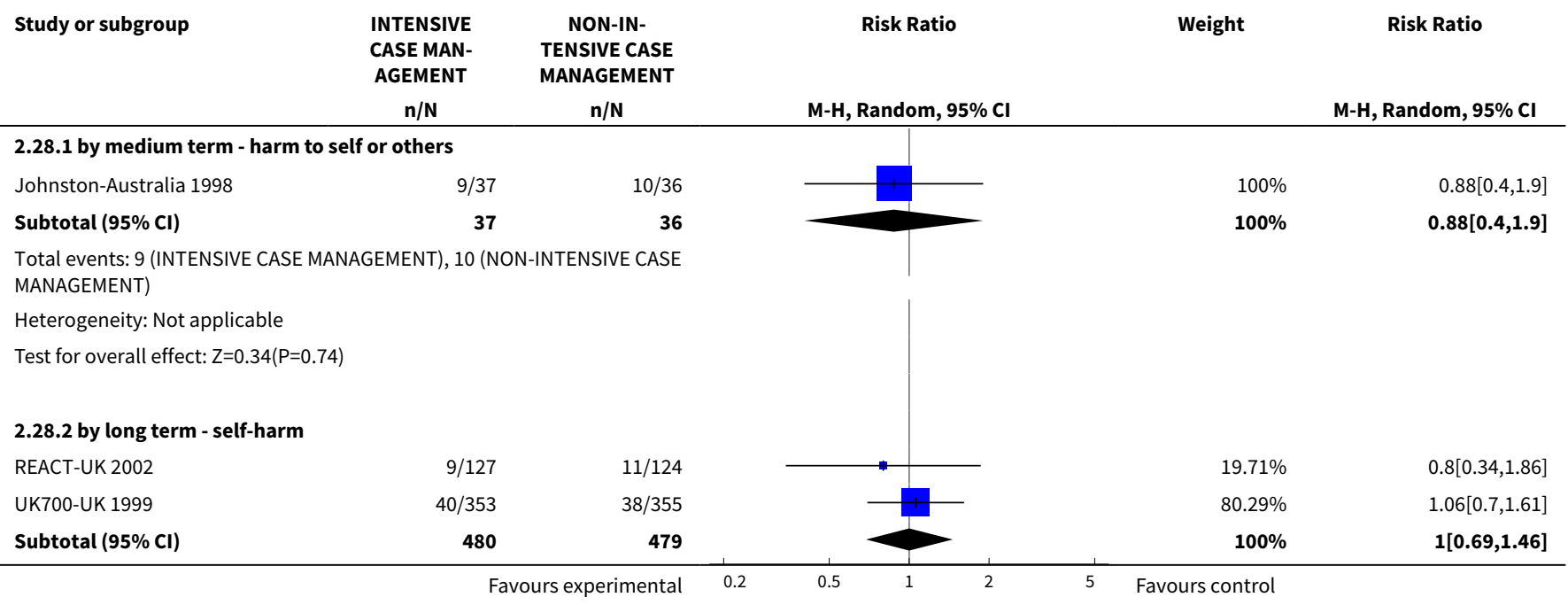




\begin{tabular}{|c|c|c|c|c|c|}
\hline Study or subgroup & $\begin{array}{c}\text { INTENSIVE } \\
\text { CASE MAN- } \\
\text { AGEMENT } \\
\mathbf{n} / \mathbf{N}\end{array}$ & $\begin{array}{c}\text { NON-IN- } \\
\text { TENSIVE CASE } \\
\text { MANAGEMENT } \\
n / \mathbf{N}\end{array}$ & M-H, Random, $95 \% \mathrm{Cl}$ & Weight & M-H, Random, $95 \% \mathrm{Cl}$ \\
\hline \multicolumn{6}{|c|}{$\begin{array}{l}\text { Total events: } 49 \text { (INTENSIVE CASE MANAGEMENT), } 49 \text { (NON-INTENSIVE } \\
\text { CASE MANAGEMENT) }\end{array}$} \\
\hline \multicolumn{6}{|c|}{ Heterogeneity: Tau $^{2}=0 ; \mathrm{Chi}^{2}=0.34, \mathrm{df}=1(\mathrm{P}=0.56) ; \mathrm{I}^{2}=0 \%$} \\
\hline \multicolumn{6}{|c|}{ 2.28.3 by long term - injury/assault to others } \\
\hline REACT-UK 2002 & $21 / 127$ & $15 / 124$ & 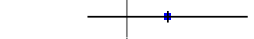 & $16.56 \%$ & $1.37[0.74,2.53]$ \\
\hline UK700-UK 1999 & $81 / 353$ & $78 / 355$ & - & $83.44 \%$ & $1.04[0.79,1.37]$ \\
\hline Subtotal $(95 \% \mathrm{Cl})$ & 480 & 479 & & $100 \%$ & $1.09[0.85,1.4]$ \\
\hline \multicolumn{6}{|c|}{$\begin{array}{l}\text { Total events: } 102 \text { (INTENSIVE CASE MANAGEMENT), } 93 \text { (NON-INTENSIVE } \\
\text { CASE MANAGEMENT) }\end{array}$} \\
\hline \multicolumn{6}{|c|}{ Heterogeneity: $\mathrm{Tau}^{2}=0 ; \mathrm{Chi}^{2}=0.62, \mathrm{df}=1(\mathrm{P}=0.43) ; \mathrm{I}^{2}=0 \%$} \\
\hline \multicolumn{6}{|l|}{ Test for overall effect: $Z=0.69(P=0.49)$} \\
\hline \multicolumn{6}{|c|}{ 2.28.4 by medium term FUP ( 18 months) - self harm } \\
\hline REACT-UK 2002 & $14 / 127$ & $16 / 124$ & & $100 \%$ & $0.85[0.44,1.67]$ \\
\hline Subtotal $(95 \% \mathrm{Cl})$ & 127 & 124 & & $100 \%$ & $0.85[0.44,1.67]$ \\
\hline \multicolumn{6}{|c|}{$\begin{array}{l}\text { Total events: } 14 \text { (INTENSIVE CASE MANAGEMENT), } 16 \text { (NON-INTENSIVE } \\
\text { CASE MANAGEMENT) }\end{array}$} \\
\hline \multicolumn{6}{|l|}{ Heterogeneity: Not applicable } \\
\hline \multicolumn{6}{|l|}{ Test for overall effect: $Z=0.46(P=0.65)$} \\
\hline \multicolumn{6}{|c|}{ 2.28.5 by medium term FUP ( 18 months) - injury/assualt to others } \\
\hline REACT-UK 2002 & $36 / 127$ & $26 / 124$ & - & $100 \%$ & $1.35[0.87,2.1]$ \\
\hline Subtotal $(95 \% \mathrm{Cl})$ & 127 & 124 & & $100 \%$ & $1.35[0.87,2.1]$ \\
\hline \multicolumn{6}{|c|}{ Heterogeneity: Not applicable } \\
\hline \multicolumn{6}{|l|}{ Test for overall effect: $Z=1.34(P=0.18)$} \\
\hline \multicolumn{6}{|c|}{ 2.28.6 by long term FUP ( 8.5 years) - self harm } \\
\hline REACT-UK 2002 & $25 / 107$ & $31 / 107$ & - & $100 \%$ & $0.81[0.51,1.27]$ \\
\hline Subtotal $(95 \% \mathrm{Cl})$ & 107 & 107 & & $100 \%$ & $0.81[0.51,1.27]$ \\
\hline \multicolumn{6}{|c|}{$\begin{array}{l}\text { Total events: } 25 \text { (INTENSIVE CASE MANAGEMENT), } 31 \text { (NON-INTENSIVE } \\
\text { CASE MANAGEMENT) }\end{array}$} \\
\hline \multicolumn{6}{|l|}{ Heterogeneity: Not applicable } \\
\hline \multicolumn{6}{|l|}{ Test for overall effect: $\mathrm{Z}=0.93(\mathrm{P}=0.35)$} \\
\hline \multicolumn{6}{|c|}{ 2.28.7 by long term FUP ( 8.5 years) - injury/assault to others } \\
\hline REACT-UK 2002 & $83 / 107$ & $87 / 107$ & & $100 \%$ & $0.95[0.83,1.09]$ \\
\hline Subtotal $(95 \% \mathrm{Cl})$ & 107 & 107 & & $100 \%$ & $0.95[0.83,1.09]$ \\
\hline \multicolumn{6}{|c|}{$\begin{array}{l}\text { Total events: } 83 \text { (INTENSIVE CASE MANAGEMENT), } 87 \text { (NON-INTENSIVE } \\
\text { CASE MANAGEMENT) }\end{array}$} \\
\hline \multicolumn{6}{|c|}{ Heterogeneity: Not applicable } \\
\hline Test for overall effect: $Z=0.68(P=0.5)$ & & & & & \\
\hline
\end{tabular}

Favours experimental 
Analysis 2.29. Comparison 2 Intensive Case Management versus non-Intensive Case Management, Outcome 29 Quality of life: 1. Average endpoint score (various scales).

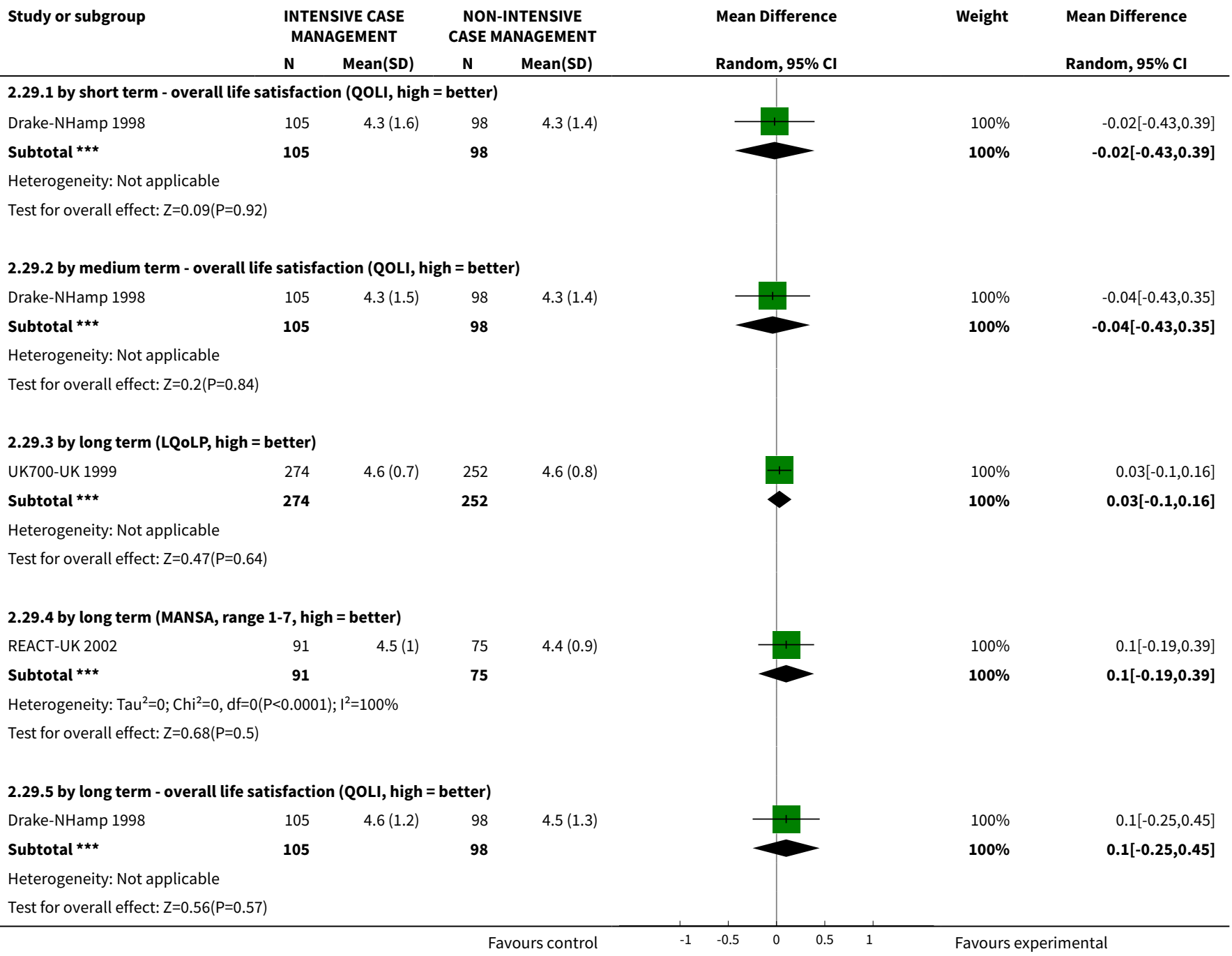

Analysis 2.30. Comparison 2 Intensive Case Management versus non-Intensive Case Management, Outcome 30 Participant satisfaction/need: 1. Average endpoint scores (various scale) - by long term.

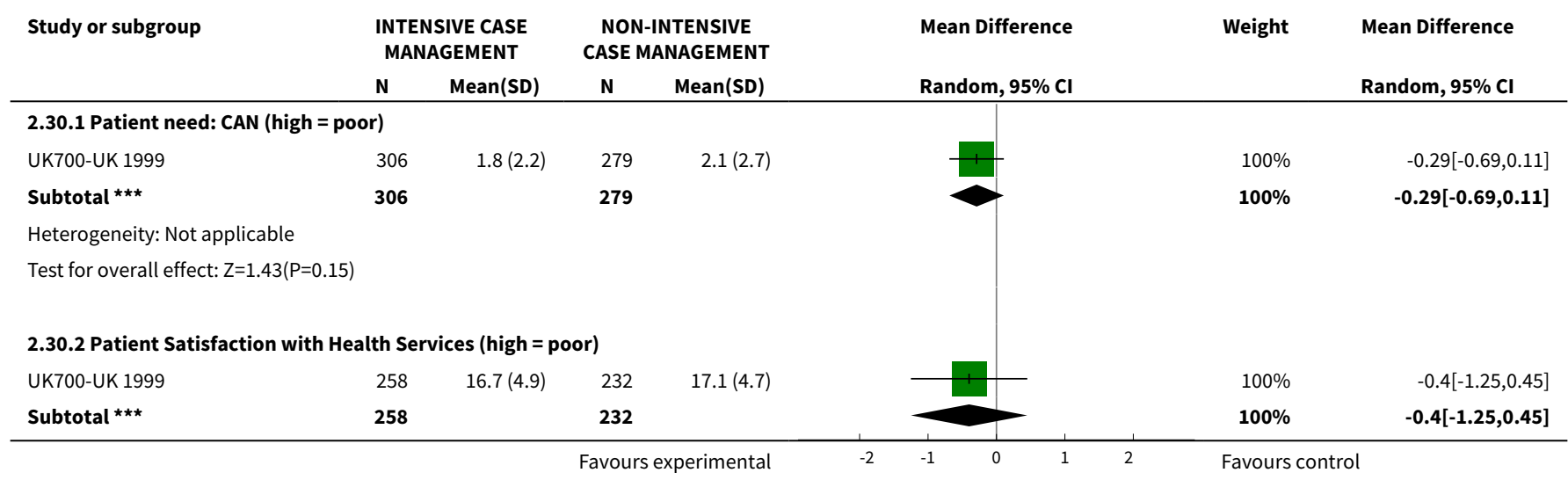




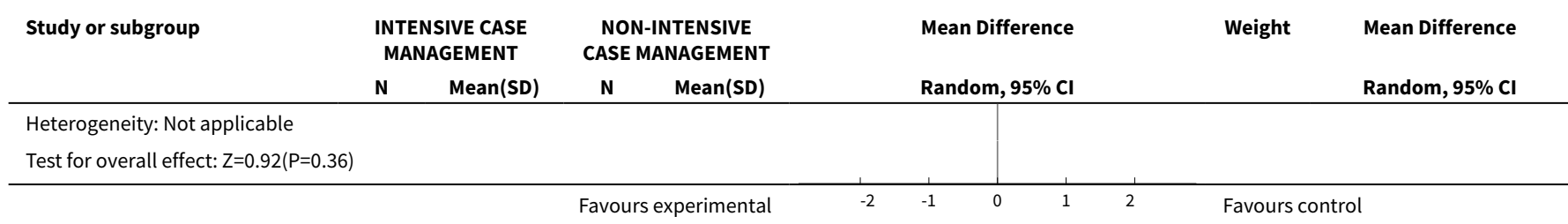

Analysis 2.31. Comparison 2 Intensive Case Management versus non-Intensive Case Management, Outcome 31 Participants need: 1. Average endpoint scores (various scales, skewed data).

Participants need: 1. Average endpoint scores (various scales, skewed data)

Study Intervention Mean Total

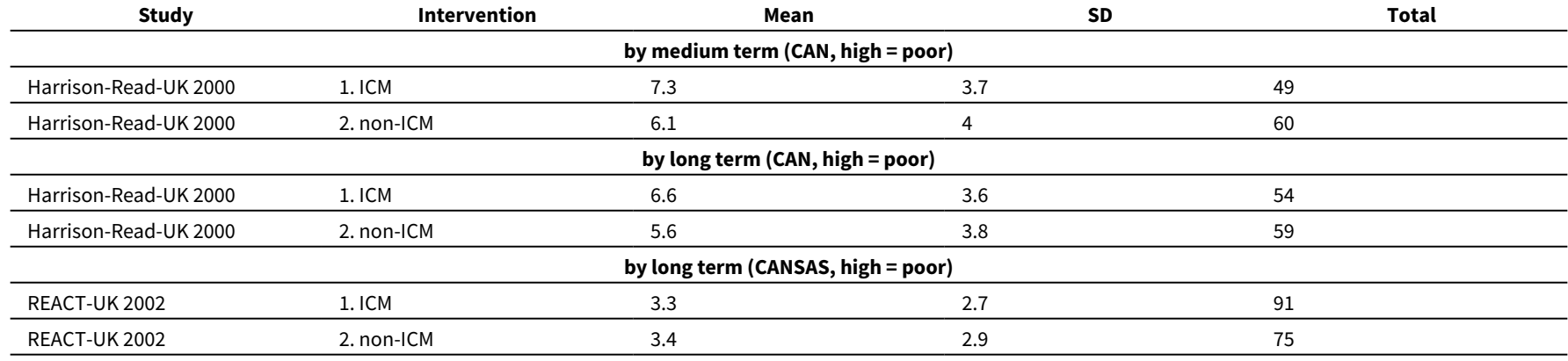

Analysis 2.32. Comparison 2 Intensive Case Management versus non-Intensive Case Management, Outcome 32 Participant satisfaction: 1. Average endpoint scores (CSQ-modified, high = better, skewed data) - by long term.

Participant satisfaction: 1. Average endpoint scores (CSQ-modified, high = better, skewed data) - by long term

\begin{tabular}{lllll}
\multicolumn{1}{c}{ Study } & \multicolumn{1}{c}{ Intervention } & Mean & SD & \multicolumn{1}{c}{ Total } \\
\hline REACT-UK 2002 & 1. ICM & 77.2 & 20 & 91 \\
\hline REACT-UK 2002 & 2. non-ICM & 70 & 20.6 & 75 \\
\hline
\end{tabular}

Analysis 2.33. Comparison 2 Intensive Case Management versus non-Intensive Case Management, Outcome 33 Costs: 1. Direct costs of psychiatric hospital care (skewed data).

Costs: 1 . Direct costs of psychiatric hospital care (skewed data)

\begin{tabular}{|c|c|c|c|c|c|}
\hline Study & Intervention & Mean & SD & Total & Note \\
\hline \multicolumn{6}{|c|}{ by medium term } \\
\hline Harrison-Read-UK 2000 & 1. $\mathrm{ICM}^{*}$ & 501 & 967.4 & 97 & \\
\hline Harrison-Read-UK 2000 & 2. Low ICM* & 527 & 753 & 96 & $\begin{array}{l}{ }^{\star} \text { Unit cost } £ \text {, fiscal year } \\
1995 / 6 \text {. } \\
\star \star ~ ' N o ~ s i g n i f i c a n t ~ d i f f e r- \\
\text { ence between groups. } \\
\text { Statistical analysis on } \\
\text { non-parametric data } \\
\text { were performed using } \\
\text { bootstrap methods'. } \\
{ }^{\star \star \star T i m e ~ p e r i o d: ~} 12 \\
\text { months. }\end{array}$ \\
\hline
\end{tabular}

\begin{tabular}{|c|c|c|c|c|c|}
\hline \multicolumn{6}{|c|}{ by long term } \\
\hline Harrison-Read-UK 2000 & 1. $\mathrm{ICM}^{\star}$ & 414 & 777.8 & 97 & \\
\hline Harrison-Read-UK 2000 & 2. Low ICM* & 478 & 890 & 96 & $\begin{array}{l}\text { * Unit cost } £ \text {, fiscal year } \\
1995 / 6 \text {. } \\
\text { ** 'No significant differ- } \\
\text { ence between groups. } \\
\text { Statistical analysis on } \\
\text { non-parametric data }\end{array}$ \\
\hline
\end{tabular}


Costs: 1. Direct costs of psychiatric hospital care (skewed data)

\begin{tabular}{|c|c|c|c|c|c|}
\hline Study & Intervention & Mean & SD & Total & Note \\
\hline & & & & & $\begin{array}{l}\text { were performed using } \\
\text { bootstrap methods'. } \\
\star \star \star \text { Time period: } 12 \\
\text { months. }\end{array}$ \\
\hline $\begin{array}{l}\text { Quinlivan-California } \\
1995\end{array}$ & 1. $\mathrm{ICM}^{\star}$ & 301 & 396.6 & 30 & \\
\hline $\begin{array}{l}\text { Quinlivan-California } \\
1995\end{array}$ & 2. Low ICM* & 959 & $1,572.7$ & 30 & 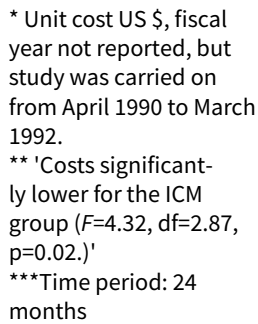 \\
\hline
\end{tabular}

Analysis 2.34. Comparison 2 Intensive Case Management versus non-Intensive Case Management, Outcome 34 Costs: 2a. Direct costs of all care - by long term (2 years). Unit cost GBP, fiscal year 1997/98.

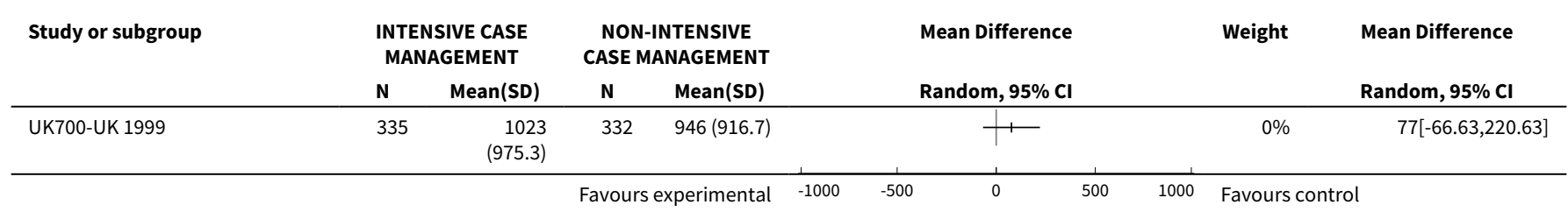

Analysis 2.35. Comparison 2 Intensive Case Management versus non-Intensive Case Management, Outcome 35 Costs: 2 b. Direct costs of all care - by medium term (skewed data).

Costs: 2 b. Direct costs of all care - by medium term (skewed data)

\begin{tabular}{|c|c|c|c|c|c|}
\hline Study & Intervention & Mean & SD & Total & Note \\
\hline Johnston-Australia 1998 & 1. $\mathrm{ICM}^{\star}$ & 2,408 & $2,581.4$ & 33 & \\
\hline Johnston-Australia 1998 & 2. Non-ICM ${ }^{\star}$ & 1,762 & 1,872 & 25 & 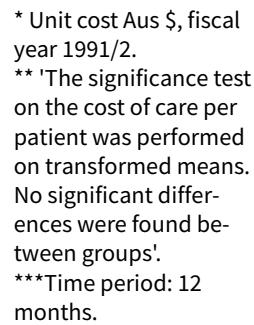 \\
\hline
\end{tabular}

Analysis 2.36. Comparison 2 Intensive Case Management versus non-Intensive Case Management, Outcome 36 Costs: 3 . Total costs of care per patient - Unit cost GBP.

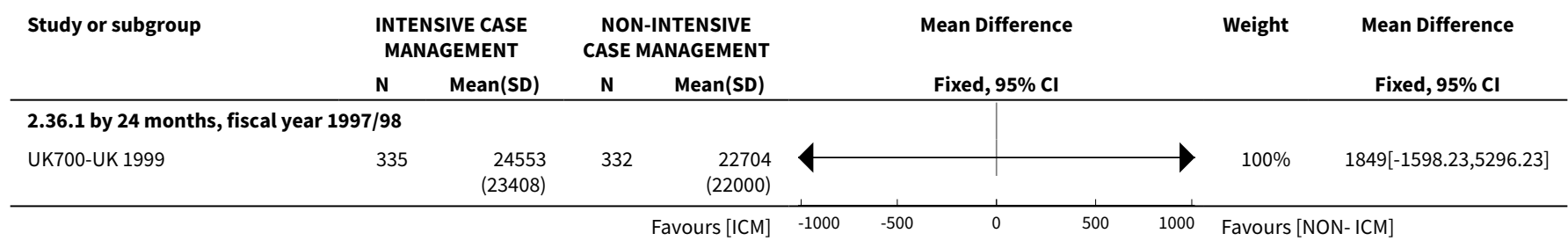




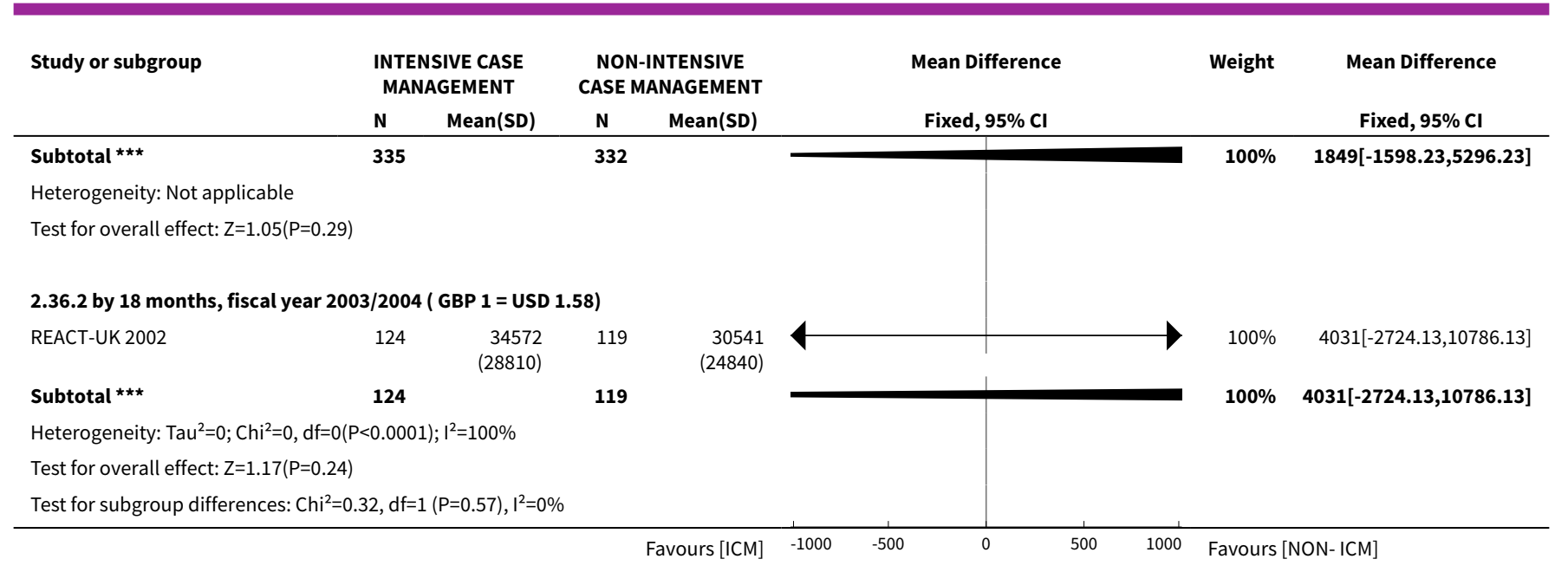

\section{ADDITIONAL TABLES}

\section{Table 1. Case Management and Assertive Community Treatment}

\section{Case Management (CM)}

The key principle of case management is that a single person - the 'case manager' - takes primary responsibility for a defined group of patients in the community. The case manager is responsible for (Holloway 1991):

- assessing the patient's needs;

- developing a care plan;

- arranging suitable care from community services;

- keeping contact with the patient.

Initially, in its simplest form (referred to as 'brokerage'), case managers were not mental health professionals, did not provide any direct care, and worked independently.

\section{Assertive Community Treatment (ACT)}

Assertive Community Treatment should be practiced according to a defined and validated model (Stein 1980), based on the consensus of an international panel of ACT experts (McGrew 1994; McGrew 1995). A key aspect of ACT is that it is a team-based approach, characteristically a multidisciplinary team including social workers, nurses, and psychiatrists, caring exclusively for a defined group of patients (McGrew 1995; Olfson 1990). Team members share responsibility for their clients, so it is common for several team members to work together in treating the same patient. Other characteristics of ACT are (Stein 1980):

- provide all necessary care themselves, rather than arranging for it to be provided by other services;

- provide care at home or in workplaces;

- carry low caseloads (usually 10 to 15 patients per member);

- practice 'assertive outreach', meaning that they persist in attempts to engage unco-operative clients;

- place particular emphasis on medication compliance;

- provide 24-hour emergency cover. 


\begin{tabular}{|c|c|c|c|c|c|c|c|}
\hline $\begin{array}{l}\text { Intensive Case Management versus standard } \\
\text { care }\end{array}$ & ICM & ICM & ICM & SC & SC & SC & Note \\
\hline Study ID & Mean & SD & Total & Mean & SD & Total & \\
\hline Audini-UK 1994 & 0.95 & $2.84^{\star}$ & 33 & 0.93 & $2.03^{\star}$ & 33 & ${ }^{\star}$ SD imputed \\
\hline Bjorkman-Sweden 2002 & 0.83 & 3.13 & 33 & 2.15 & 4.13 & 44 & \\
\hline Bond-Chicago1 1990 & 3.22 & 4.55 & 42 & 5.3 & 5.42 & 40 & \\
\hline Bond-Indiana1 (A) & 1.28 & $3.17^{\star}$ & 29 & 7.72 & $8.99^{\star}$ & 32 & ${ }^{\star}$ SD imputed \\
\hline Bond-Indiana1 (B) & 2.72 & $4.54^{\star}$ & 34 & 3.62 & $5.24^{\star}$ & 30 & *SD imputed \\
\hline Bond-Indiana1 (C) & 0.05 & $1.89^{\star}$ & 21 & 3.38 & $4.98^{\star}$ & 21 & *SD imputed \\
\hline Chandler-California1 (A) & 0.47 & $2.34^{*}$ & 102 & 0.78 & $1.84^{\star}$ & 101 & ${ }^{\star}$ SD imputed \\
\hline Chandler-California1 (B) & 0.67 & $2.55^{\star}$ & 115 & 0.96 & $2.07^{\star}$ & 114 & ${ }^{\star}$ SD imputed \\
\hline Curtis-New York 1992 & 1.77 & 1.79 & 146 & 1.02 & 1.18 & 143 & \\
\hline Ford-UK 1995 & 3.07 & 6.9 & 39 & 1.76 & 3.67 & 38 & \\
\hline Hampton-Illinois (A) & 1.75 & $3.63^{*}$ & 48 & 4.83 & $6.49^{\star}$ & 47 & ${ }^{\star}$ SD imputed \\
\hline Hampton-Illinois (B) & 3.25 & $5.01^{*}$ & 34 & 3.42 & $5.02^{\star}$ & 36 & *SD imputed \\
\hline Holloway-UK 1996 & 2.4 & 5.1 & 34 & 1.2 & 3 & 26 & \\
\hline Jerrell-SCarolina1 1991 & 0.53 & $2.40^{*}$ & 40 & 0.8 & $1.86^{\star}$ & 40 & ${ }^{\star}$ SD imputed \\
\hline Lehman-Maryland1 1994 & 3.04 & 5.15 & 77 & 5.41 & 7 & 75 & \\
\hline Marshall-UK 1995 & 1.04 & 2.18 & 40 & 1.56 & 4.45 & 40 & \\
\hline Muijen-UK2 1994 & 2.53 & 5.55 & 41 & 2.45 & 5.83 & 41 & \\
\hline Muller-Clemm-Canada 1996 & 1.68 & $3.56^{*}$ & 61 & 1.63 & $2.93^{*}$ & 57 & *SD imputed \\
\hline OPUS-Denmark 1999 & 5.11 & 7.7 & 263 & 6.57 & 8.73 & 244 & \\
\hline
\end{tabular}




\begin{tabular}{|c|c|c|c|c|c|c|c|}
\hline Quinlivan-California 1995 & 1.09 & 2.65 & 30 & 5.53 & 8.65 & 30 & \\
\hline Rosenheck-USA-GMS (A) & 3.63 & 3.89 & 44 & 3.71 & 2.76 & 35 & \\
\hline Rosenheck-USA-GMS (B) & 6.99 & 4.85 & 47 & 4.23 & 5.18 & 47 & \\
\hline Rosenheck-USA-NP (C) & 18.52 & 11.16 & 50 & 19.16 & 12.19 & 43 & \\
\hline Rosenheck-USA-GMS (D) & 2.8 & 3.31 & 49 & 3.26 & 3.98 & 53 & \\
\hline Rosenheck-USA-NP (E) & 4.13 & 5.24 & 34 & 3.05 & 4.61 & 33 & \\
\hline Rosenheck-USA-GMS (F) & 2.39 & 3.16 & 43 & 2.58 & 2.45 & 35 & \\
\hline Rosenheck-USA-NP (G) & 7.68 & 7.72 & 40 & 12.2 & 10.65 & 31 & \\
\hline Rosenheck-USA-NP (H) & 4.63 & 8.58 & 59 & 11.21 & 13.38 & 55 & \\
\hline Rosenheck-USA-GMS (I) & 5.62 & 4.67 & 44 & 7.8 & 6.63 & 44 & \\
\hline Sytema-Netherlands 1999 & 3.4 & 5.4 & 58 & 4.3 & 7.3 & 57 & \\
\hline Test-Wisconsin 1985 & 0.42 & $2.29^{\star}$ & 72 & 2.13 & $3.54^{\star}$ & 41 & ${ }^{\star}$ SD imputed \\
\hline $\begin{array}{l}\text { Intensive Case Management versus non-Inten- } \\
\text { sive Case Management }\end{array}$ & ICM & ICM & ICM & Non-ICM & Non-ICM & Non-ICM & Note \\
\hline Study ID & Mean & SD & Total & Mean & SD & Total & \\
\hline Bush-Georgia 1990 & 1.58 & $3.46^{\star}$ & 14 & 2.39 & $3.85^{\star}$ & 14 & ${ }^{*}$ SD imputed \\
\hline Drake-NHamp (A) & 0.5 & 0.94 & 7 & 2.17 & 3.21 & 9 & \\
\hline Drake-NHamp (B) & 0.85 & 1.43 & 16 & 1.41 & 2.06 & 14 & \\
\hline Drake-NHamp (C) & 2.28 & 3.2 & 10 & 1.67 & 3.84 & 12 & \\
\hline Drake-NHamp (D) & 1.04 & 2.44 & 13 & 0.63 & 0.91 & 11 & \\
\hline Drake-NHamp (E) & 1.08 & 4.15 & 30 & 1.39 & 2.36 & 27 & \\
\hline Drake-NHamp (F) & 1.66 & 4.49 & 10 & 0.84 & 2.33 & 13 & \\
\hline
\end{tabular}




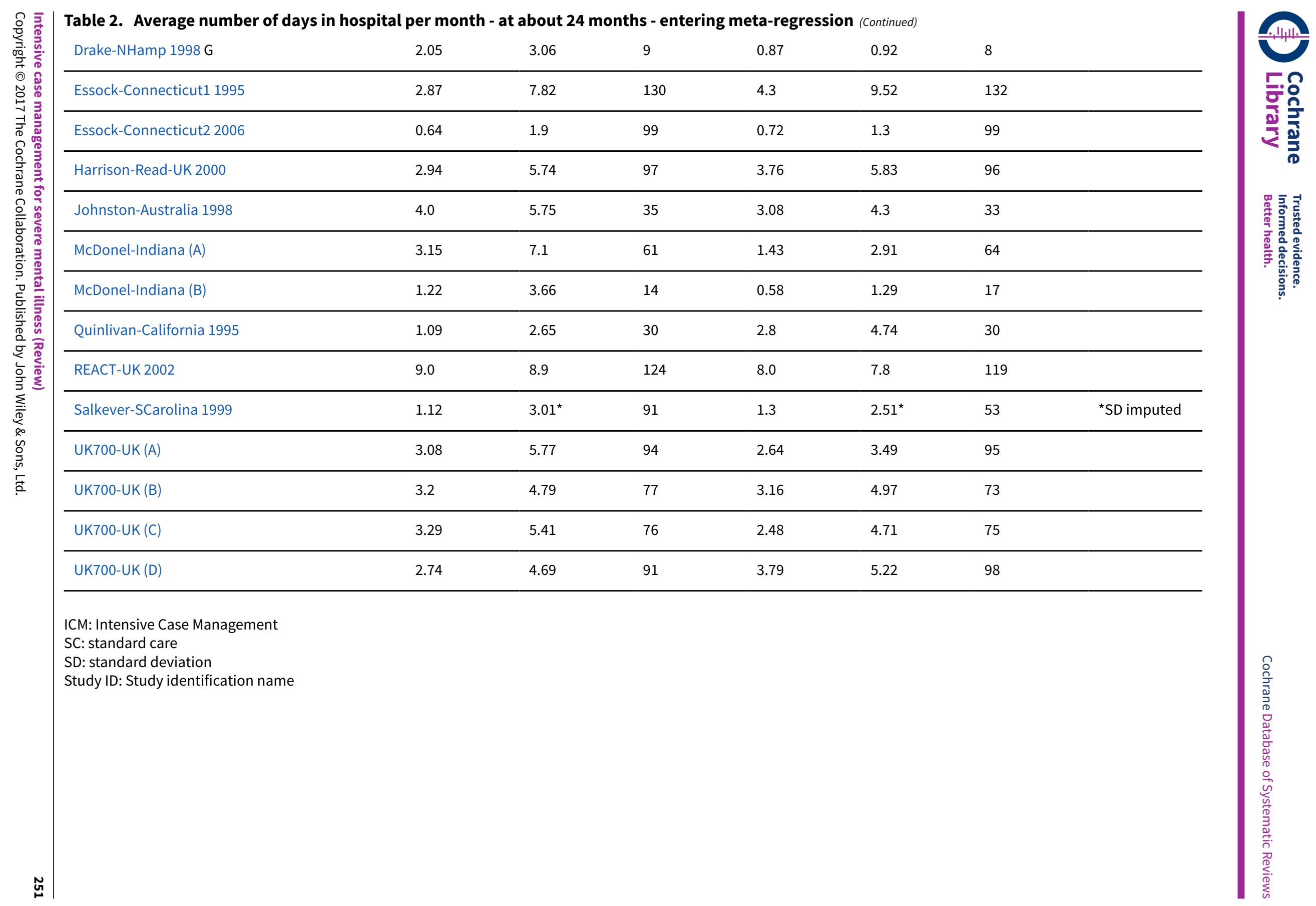


Table 3. Covariates entering meta-regression

\begin{tabular}{|c|c|c|c|c|c|c|}
\hline $\begin{array}{l}\text { Intensive Case Management versus stan- } \\
\text { dard care }\end{array}$ & $\begin{array}{l}\text { Baseline } \\
\text { hospital } \\
\text { use }\end{array}$ & $\begin{array}{l}\text { Baseline } \\
\text { hospital } \\
\text { use }\end{array}$ & IFACT & IFACT & IFACT & Note \\
\hline Study ID & Mean & Total & Total score & $\begin{array}{l}\text { Organisa- } \\
\text { tion sub- } \\
\text { scale score }\end{array}$ & $\begin{array}{l}\text { Staff sub- } \\
\text { scale score }\end{array}$ & \\
\hline Audini-UK 1994 & 1.08 & 66 & 6.7 & 3.5 & 3.2 & \\
\hline Bjorkman-Sweden 2002 & 5.63 & 77 & 7 & 4.5 & 2.5 & \\
\hline Bond-Chicago1 1990 & 7.83 & 88 & 6 & 4 & 2 & \\
\hline Bond-Indiana1 (A) & 14.17 & 61 & 9.2 & 7 & 2.2 & \\
\hline Bond-Indiana1 (B) & 4.95 & 64 & 2.2 & 1 & 1.2 & \\
\hline Bond-Indiana1 (C) & 10.86 & 42 & 7.4 & 5 & 2.4 & \\
\hline Chandler-California1 (A) & 0.5 & 203 & 8.5 & 5 & 3.5 & \\
\hline Chandler-California1 (B) & 1.14 & 229 & 6.6 & 5 & 1.6 & \\
\hline Curtis-New York 1992 & $0.95^{\star}$ & 289 & 5.8 & 3.5 & 2.3 & $\begin{array}{l}{ }^{*} \text { Mean im- } \\
\text { puted }\end{array}$ \\
\hline Ford-UK 1995 & 2.61 & 77 & 4.8 & 2 & 2.8 & \\
\hline Hampton-Illinois (A) & 5.6 & 95 & 6 & 4 & 2 & \\
\hline Hampton-Illinois (B) & 5.2 & 70 & 5 & 3 & 2 & \\
\hline Holloway-UK 1996 & 7.37 & 70 & 9.3 & 6 & 3.3 & \\
\hline Jerrell-SCarolina1 1991 & 2.85 & 80 & 8.8 & 5.5 & 3.3 & \\
\hline Lehman-Maryland1 1994 & $4.94^{\star}$ & 152 & 11 & 7 & 4 & $\begin{array}{l}{ }^{*} \text { Mean im- } \\
\text { puted }\end{array}$ \\
\hline Marshall-UK 1995 & $3.31^{*}$ & 80 & 4.9 & 4 & 0.9 & $\begin{array}{l}{ }^{\star} \text { Mean im- } \\
\text { puted }\end{array}$ \\
\hline Muijen-UK2 1994 & $8.43^{\star}$ & 82 & 5.4 & 3 & 2.4 & $\begin{array}{l}{ }^{\star} \text { Mean im- } \\
\text { puted }\end{array}$ \\
\hline Muller-Clemm-Canada 1996 & 4.07 & 123 & 6.2 & 4 & 2.2 & \\
\hline OPUS-Denmark 1999 & NA & 547 & 8 & 4 & 4 & $\begin{array}{l}\text { *Baseline } \\
\text { hospital } \\
\text { use: not } \\
\text { applica- } \\
\text { ble as first } \\
\text { episode }\end{array}$ \\
\hline
\end{tabular}


Table 3. Covariates entering meta-regression (Continued)

\begin{tabular}{|c|c|c|c|c|c|c|}
\hline Quinlivan-California 1995 & $4.50^{\star}$ & 60 & 6.4 & 4 & 2.4 & $\begin{array}{l}\text { *Mean im- } \\
\text { puted }\end{array}$ \\
\hline Rosenheck-USA-GMS (A) & 3.96 & 79 & 6 & 2 & 4 & \\
\hline Rosenheck-USA-GMS (B) & 5.83 & 94 & 3.8 & 2 & 1.8 & \\
\hline Rosenheck-USA-NP (C) & 19.8 & 93 & 7.7 & 5 & 2.7 & \\
\hline Rosenheck-USA-GMS (D) & 4.19 & 102 & 7 & 3 & 4 & \\
\hline Rosenheck-USA-NP (E) & 5.33 & 67 & 6.4 & 3.5 & 2.9 & \\
\hline Rosenheck-USA-GMS (F) & 3.22 & 78 & 6.6 & 3 & 3.6 & \\
\hline Rosenheck-USA-NP (G) & 11.42 & 71 & 8.4 & 5 & 3.4 & \\
\hline Rosenheck-USA-NP (H) & 11.4 & 114 & 6.4 & 4 & 2.4 & \\
\hline Rosenheck-USA-GMS (I) & 8.28 & 88 & 5.8 & 2 & 3.8 & \\
\hline Sytema-Netherlands 1999 & $12.17^{\star}$ & 118 & $7.6^{\star}$ & $5.1^{*}$ & $2.5^{\star}$ & $\begin{array}{l}\text { *Mean and } \\
\text { IFACT score } \\
\text { imputed }\end{array}$ \\
\hline Test-Wisconsin 1985 & 2.33 & 122 & 8.5 & 5.5 & 3 & \\
\hline $\begin{array}{l}\text { Intensive Case Management versus non- } \\
\text { Intensive Case Management }\end{array}$ & $\begin{array}{l}\text { Baseline } \\
\text { hospital } \\
\text { use }\end{array}$ & $\begin{array}{l}\text { Baseline } \\
\text { hospital } \\
\text { use }\end{array}$ & IFACT & IFACT & IFACT & Note \\
\hline Study ID & Mean & Total & Total score & $\begin{array}{l}\text { Organisa- } \\
\text { tion sub- } \\
\text { scale score }\end{array}$ & $\begin{array}{l}\text { Staff sub- } \\
\text { scale score }\end{array}$ & \\
\hline Bush-Georgia 1990 & 3.99 & 28 & 3.1 & 2 & 1.1 & \\
\hline Drake-NHamp (A) & 2.88 & 19 & 8 & 5 & 3 & \\
\hline Drake-NHamp (B) & 1.72 & 33 & 3.8 & 3 & 0.8 & \\
\hline Drake-NHamp (C) & 3.02 & 25 & 8.8 & 5.5 & 3.3 & \\
\hline Drake-NHamp (D) & 1.78 & 26 & 7.8 & 4.5 & 3.3 & \\
\hline Drake-NHamp (E) & 2.76 & 66 & 8.5 & 4.5 & 4 & \\
\hline Drake-NHamp (F) & 2.34 & 22 & 3.5 & 3 & 0.5 & \\
\hline Drake-NHamp 1998 (G) & 4.1 & 19 & 5 & 2 & 3 & \\
\hline Essock-Connecticut1 1995 & $2.81^{\star}$ & 262 & 8.5 & 4.5 & 4 & $\begin{array}{l}{ }^{\star} \text { Mean im- } \\
\text { puted }\end{array}$ \\
\hline Essock-Connecticut2 2006 & $1.08^{\star}$ & 198 & $10^{*}$ & $7^{\star}$ & $3^{\star}$ & $\begin{array}{l}\text { *Mean and } \\
\text { IFACT score } \\
\text { imputed }\end{array}$ \\
\hline
\end{tabular}


Table 3. Covariates entering meta-regression (Continued)

\begin{tabular}{|c|c|c|c|c|c|c|}
\hline Harrison-Read-UK 2000 & 4.11 & 193 & 7.6 & 4 & 3.6 & \\
\hline Johnston-Australia 1998 & 3.66 & 71 & 7.3 & 3.5 & 3.8 & \\
\hline McDonel-Indiana (A) & 4.2 & 152 & 4.2 & 3 & 1.2 & \\
\hline McDonel-Indiana (B) & 1.16 & 39 & 4.4 & 3 & 1.4 & \\
\hline Quinlivan-California 1995 & $2.96^{\star}$ & 60 & 6.4 & 4 & 2.4 & $\begin{array}{l}\text { *Mean im- } \\
\text { puted }\end{array}$ \\
\hline REACT-UK 2002 & 7.3 & 251 & 10.3 & 6.5 & 3.8 & \\
\hline Salkever-SCarolina 1999 & 3.06 & 144 & 7 & 5 & 2 & \\
\hline UK700-UK (A) & 4.55 & 196 & 8.8 & 5 & 3.8 & \\
\hline UK700-UK (B) & 4.66 & 153 & 4.5 & 3 & 1.5 & \\
\hline UK700-UK (C) & 4.33 & 158 & 4.2 & 2 & 2.2 & \\
\hline UK700-UK (D) & 4.59 & 200 & 8.5 & 5 & 3.5 & \\
\hline
\end{tabular}

Baseline hospital use: average number of days per month in hospital for all participants in the two years before the study began IFACT: Index of Fidelity to Assertive Community Treatment

NA: not applicable

Study ID: Study identification name

\section{Table 4. Interventions in Curtis-New York}

1. ICM: "Intensive outreach case management" from a multidisciplinary team at Harlem Hospital Center. This team implemented a discharge treatment plan and monitored clinical and social problems. The team did not "assume direct responsibility for care but [...] help[ed] the patient enrol in a day hospital programme, adult mental health clinic, rehabilitation programme, or alcohol treatment programme". Caseload: 1:17. $\mathrm{N}=147$.

2. Standard care: routine aftercare, within the discharge treatment plan prescribed for each patient from Harlem Hospital Center; "most received at least initial treatment from various divisions of the departments of psychiatry within the Health and Hospitals Corporation". $\mathrm{N}=145$.

\section{AP P E N D I CES}

\section{Appendix 1. Previous searches}

\section{Search method Assertive Community Treatment Review}

\subsection{Electronic searches}

\subsubsection{CINAHL (January 1982 to May 1997)}

It was searched using the CSG's terms for randomised controlled trials and the CSG's terms for schizophrenia combined with the phrase:

[and ((case or care) near management) or CPA or (Care near1 Programme near1 Approach) or (Assertive near1 Community near1 Treatment) or PACT or TCL or (Training near (community near1 living)) or (Madison near4 model)]

\subsubsection{The Cochrane Schizophrenia Group's Register (1997)}

It was searched using the phrase: 
[and ((case or care) and management) or CPA or (Care and Programme and Approach) or (Assertive and Community and Treatment) or PACT or TCL or (Training and (community and living)) or (Madison and model)]

\subsubsection{EMBASE (January 1980 to May 1997)}

It was searched using the CSG's terms for randomised controlled trials and the CSG's terms for schizophrenia combined with the phrase:

[and ((case or care) near management) or CPA or (Care near1 Programme near1 Approach) or (Assertive near1 Community near1 Treatment) or PACT or TCL or (Training near (community near1 living)) or (Madison near4 model)]

\subsubsection{MEDLINE (January 1966 to May 1997)}

It was searched using the CSG's terms for randomised controlled trials and the CSG's terms for schizophrenia combined with the phrase:

[and ((case or care) near management) or CPA or (Care near1 Programme near1 Approach) or (Assertive near1 Community near1 Treatment) or PACT or TCL or (Training near (community near1 living)) or (Madison near4 model)]

\subsubsection{PsycLIT (January 1974 to May 1997)}

It was searched using the CSG's terms for randomised controlled trials and the CSG's terms for schizophrenia combined with the phrase:

[and ((case or care) near management) or CPA or (Care near1 Programme near1 Approach) or (Assertive near1 Community near1 Treatment) or PACT or TCL or (Training near (community near1 living)) or (Madison near4 model)]

\subsection{Searching other resources}

\subsubsection{Reference searching}

Each of the randomised controlled trial identified was sought as a citation on the SCISEARCH database. Reports of articles that had cited these studies were inspected in order to identify further trials. Reference lists of all included trials and identified reviews were scanned for evidence of trials missed by the computerised search.

It should be noted that in electronic searches the phrase 'ACT' is not feasible as this common word generates a very large number of false positives.

\section{Search method Case Management Review}

\subsection{Electronic searches}

The Cochrane Schizophrenia Group's Register (1997), EMBASE (January 1980 to May 1995), MEDLINE (January 1966 to May 1995), PsycLIT (January 1974 to May 1995) and CINAHL were all searched for any text containing the following phrases:

[((case or care) and (management)) or CPA or (Care Programme Approach) or (Assertive Community Treatment) or (PACT) or (TCL) or (Training near2 community living) or (Madison near model)]

Each randomised controlled trial identified by the search was sought as a citation on the SCISEARCH database.

\subsection{Searching other resources}

\subsubsection{Hand searching}

Reports of articles that had cited these studies were inspected in order to identify further trials.

Citation lists of all included trials and identified reviews were scanned for evidence of trials missed by the computerised search.

\section{Search method 2009, 2012 versions ICM Review}

\subsection{Cochrane Schizophrenia Group Trials Register (February 2009)}

The register was searched using the phrase:

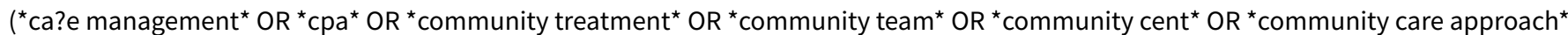

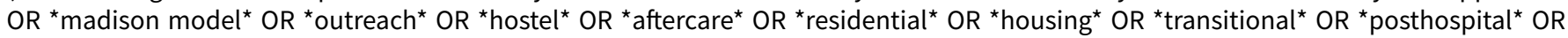

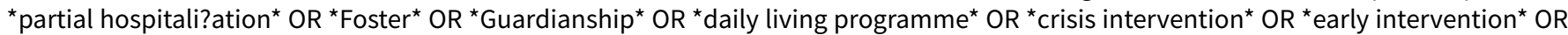

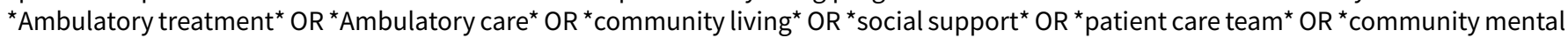
health* OR *patient participation* OR *assertive outreach* OR *drop-in hospital* OR *drop-in care* OR *drop-in treatment* OR *drop-

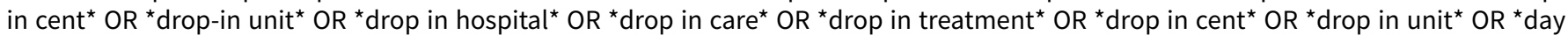

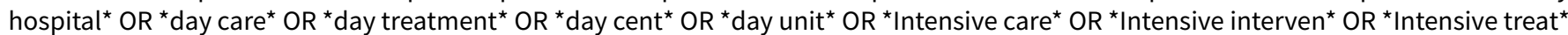

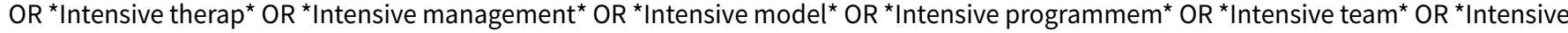

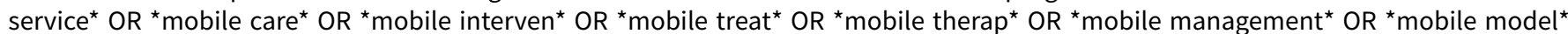
OR ${ }^{\star}$ mobile programmem ${ }^{\star}$ OR ${ }^{\star}$ mobile team ${ }^{\star}$ OR ${ }^{\star}$ mobile service ${ }^{\star}$ OR ${ }^{\star}$ outreach care ${ }^{\star}$ OR ${ }^{\star}$ outreach interven ${ }^{\star}$ OR ${ }^{\star}$ outreach treat ${ }^{\star}$ OR 
*outreach therap* OR *outreach management* OR *outreach model ${ }^{\star}$ OR *outreach programmem* OR *outreach team* OR *outreach

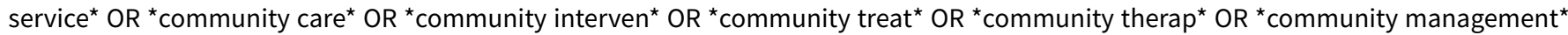

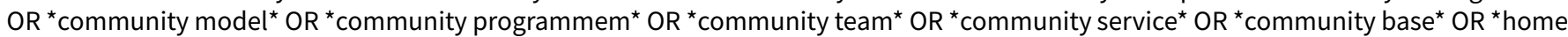

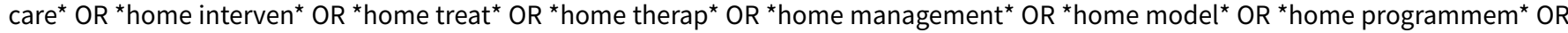

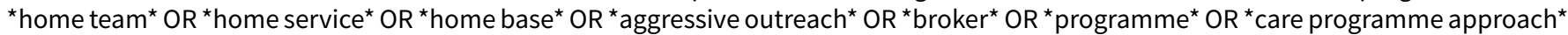
OR * care programme* in title, abstract, index terms of REFERENCE and in interventions of STUDY) OR ( ${ }^{\star} \mathrm{Pact}^{\star} \mathrm{OR}^{*} \mathrm{tcl}^{\star}$ In title) OR(Pact ${ }^{\star}$ OR $\mathrm{tcl}^{*}$ in abstract and index terms of REFERENCEand in interventions of STUDY)

This register is compiled by systematic searches of major databases, hand searches and conference proceedings (see Group's Module Specialised Register).

\subsection{Cochrane Schizophrenia Group Trials Register (August) 2012}

The Trials Search Co-ordinator searched the Cochrane Schizophrenia Group's Trials Register (August 2012)

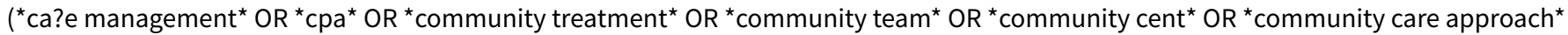
OR *madison model ${ }^{\star}$ OR * outreach* OR *hostel* OR *aftercare* OR *residential* OR *housing ${ }^{\star}$ OR *transitional* OR ${ }^{\star}$ posthospital ${ }^{\star}$ OR * partial hospitali?ation* $\mathrm{OR}^{\star}{ }^{\star}$ Foster ${ }^{\star} \mathrm{OR}{ }^{\star}$ Guardianship* $\mathrm{OR}{ }^{\star}$ daily living programme* OR * crisis intervention* OR *early intervention* OR

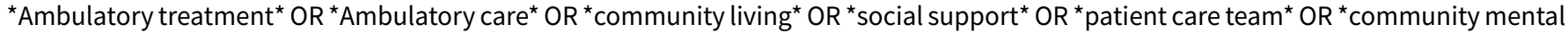
health* OR * patient participation* OR *assertive outreach* OR *drop-in hospital* OR *drop-in care* OR *drop-in treatment* OR *drop-

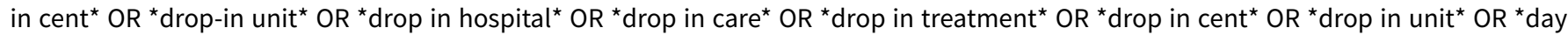

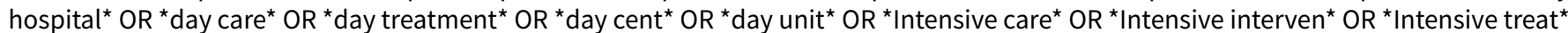

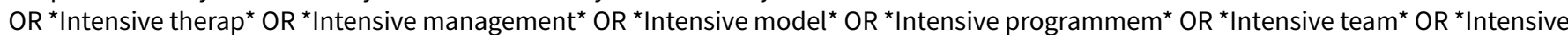

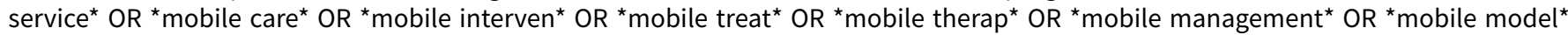

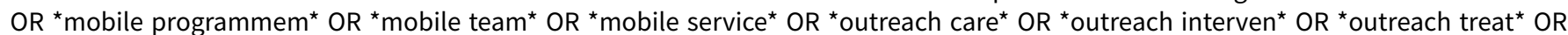
*outreach therap ${ }^{\star}$ OR *outreach management ${ }^{\star}$ OR *outreach model ${ }^{\star}$ OR *outreach programmem* OR *outreach team* OR *outreach

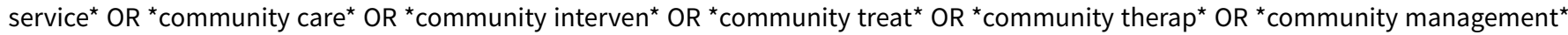

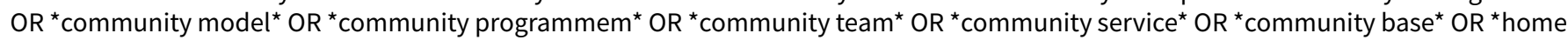
care* OR *home interven* OR *home treat* OR *home therap* OR *home management* OR *home model ${ }^{\star}$ OR *home programmem* OR

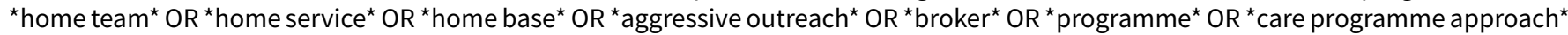
$\mathrm{OR}^{*}$ care programme* or ${ }^{*} \mathrm{Pact}^{\star} \mathrm{OR}{ }^{\star} \mathrm{tcl}^{\star}$ iin title, abstract, index terms of REFERENCE)

The Cochrane Schizophrenia Group's Trials Register is compiled by systematic searches of major databases, handsearches and conference proceedings (see Group's Module). Incoming trials are assigned to existing or new review titles.

\section{Appendix 2. Previous data collection and analyses methods}

\section{Selection of studies}

The principal reviewer (MD) inspected all abstracts of studies identified as above and identified potentially relevant reports. In addition, to ensure reliability, $\mathrm{CBI}$ inspected a random sample of these abstracts, comprising $10 \%$ of the total. Where disagreement occurred this was resolved by discussion, or where there was still doubt, the full article was acquired for further inspection. The full articles of relevant reports were acquired for reassessment and carefully inspected for a final decision on inclusion (see Criteria for considering studies for this review). Once the full articles were obtained, in turn MD and CBI inspected all full reports and independently decided whether they met inclusion criteria. MD and CBI were not blinded to the names of the authors, institutions or journal of publication. Where difficulties or disputes arose, we asked author MM for help and if it was impossible to decide, these studies were added to those awaiting assessment and the authors of the papers contacted for clarification.

\section{Data extraction and management}

\section{Extraction}

1.1 Data regarding criteria and outcomes

The principal reviewer (MD) extracted data from all included studies. In addition, to ensure reliability, $\mathrm{CBI}$ independently extracted data from a random sample of these studies, comprising $10 \%$ of the total. Again, any disagreement was discussed, decisions documented and, if necessary, authors of studies were contacted for clarification. With remaining problems MM helped clarify issues and those final decisions were documented. Data presented only in graphs and figures were extracted whenever possible, but were included only if two reviewers independently had the same result. Attempts were made to contact authors through an open-ended request in order to obtain missing information or for clarification whenever necessary. Where possible, we extracted data relevant to each component centre of multi-centre studies separately.

\subsection{Additional data}

\subsubsection{Fidelity}

This rating related to the Intensive Case Management intervention. This rated fidelity of the intervention to assertive community treatment on the 'team membership' and 'team structure and organisation' sub-scales of the Index of Fidelity to Assertive Community Treatment (IFACT) (McGrew 1994). 
This index was derived from a survey of 20 clinical experts in assertive community treatment and validated in a survey of 18 programmes.

a. The 'team membership' sub-scale comprises four items:

- ratio of patients to staff

- total size of team

- extent of psychiatric input

- extent of nursing input to the team

b. The 'structure and organisation' sub-scale comprises seven items, whether the team is:

- the primary source of care for its patients

- is situated away from the hospital

- meets daily

- shares responsibility for caseloads

- is available 24 hours a day

- has a team leader who is also a case manager

- offers unlimited time for its services

We chose IFACT because the sub-scales are brief and are possible to be completed from published or unpublished text. For each item on the index, a score of one indicates high fidelity to the model. Score ranges from 0 to 11, where the maximum score available on 'team membership' sub-scale is 4, and on 'structure and organisation' sub-scale is 7 , with higher scores indicating higher fidelity to the model.

We obtained fidelity data from published and unpublished trial reports, direct contact with trialists, and data previously obtained directly from trialists by previous reviews (Burns 2001, Catty 2002, Burns 2007). Two raters (MD and CBI) independently combined these data into a single fidelity score. Multicentre trials of Intensive Case Management often struggle to implement a uniform approach, with centres operating at different degrees of fidelity. Where possible, we rated each component centre separately.

1.2.2 Baseline hospital use

The average number of days per month in hospital for all participants in the two years before the study began.

We obtained this data from published and unpublished trial reports and from direct contact with trialists.

1.2.3 Service use: hospitalisation

We obtained the primary outcome mean number of days per month in hospital for the included studies from published and unpublished trial reports, direct contact with trialists and data previously obtained directly from trialists reported by a previous review (Burns 2007).

\section{Management}

2.1 Forms

Data were extracted onto standard, simple forms.

2.2 Data from multi-centre trials

Where possible MD and CBI verified independently calculated centre data against original trial reports.

\section{Scale-derived data}

We included continuous data from rating scales only if:

a. the psychometric properties of the measuring instrument had been described in a peer-reviewed journal (Marshall 2000); and

b. the measuring instrument was not written or modified by one of the trialists for that particular trial; and

c. the measuring instrument is either i. a self-report or ii. completed by an independent rater or relative (not the therapist).

4. Endpoint versus change data

We preferred to use scale endpoint data, which typically cannot have negative values and is easier to interpret from a clinical point of view. Change data are often not ordinal and are very problematic to interpret. If endpoint data were unavailable, we used change data.

\section{Skewed data}

5.1 General

Continuous data on clinical and social outcomes are often not normally distributed. To avoid the pitfall of applying parametric tests to nonparametric data, we aim to apply the following standards to all data before inclusion: a) standard deviations and means are reported in the paper or obtainable from the authors; b) when a scale starts from the finite number zero, the standard deviation, when multiplied by two, is less than the mean (as otherwise the mean is unlikely to be an appropriate measure of the centre of the distribution, (Altman 1996); c) if a scale started from a positive value (such as PANSS which can have values from 30 to 210) the calculation described above was modified to take the scale starting point into account. In these cases skew is present if $2 \mathrm{SD}>(\mathrm{S}-\mathrm{S} \mathrm{min}$ ), where $\mathrm{S}$ is the mean score and $\mathrm{S}$ min is the minimum score. Endpoint scores on scales often have a finite start and end point and these rules can be applied. When continuous data are presented on a scale which includes a possibility of negative values (such as change data), it is difficult to tell whether data are skewed 
or not. Skewed data from studies of less than 200 participants were entered in additional tables rather than into an analysis. Skewed data pose less of a problem when looking at means if the sample size is large and were entered into syntheses.

5.2 Specific - mean number of days in hospital

We implemented one exception to the above rule (5.1) in order to present more data, recognising that this is a 'post hoc' decision, but also that the rules as regards management of skewed data and how robust skewed data are within meta-analysis is unclear (Higgins 2011). Where mean number of days in hospital data were skewed, and they were provided by studies of less than 200 participants, we nevertheless entered those data into a sub-group of the overall analysis. We also presented the overall effect from all data pooled.

\section{Common measure}

To facilitate comparison between trials, we intended to convert variables that can be reported in different metrics, such as days in hospital (mean days per year, per week or per month) to a common metric (e.g. mean days per month).

\section{Conversion of continuous to binary}

Where possible, efforts were made to convert outcome measures to dichotomous data. This could be done by identifying cut-off points on rating scales and dividing participants accordingly into 'clinically improved' or 'not clinically improved'. It was generally assumed that if there had been a 50\% reduction in a scale-derived score such as the Brief Psychiatric Rating Scale (BPRS, Overall 1962) or the Positive and Negative Syndrome Scale (PANSS, Kay 1986), this could be considered as a clinically significant response ( Leucht 2005a, Leucht 2005b). If data based on these thresholds were not available, we used the primary cut-off point presented by the original authors.

8. Direction of graphs

Where possible, we entered data in such a way that the area to the left of the line of no effect indicates a favourable outcome for Intensive Case Management.

\section{Assessment of risk of bias in included studies}

Again working independently, the principal reviewer (MD) assessed risk of bias of all included studies and the second reviewer (CBI) assessed risk of bias from a random sample of these studies, comprising $10 \%$ of the total. MD and CBI assessed risk of bias using the tool described in the Cochrane Collaboration Handbook (Higgins 2011). This tool encourages consideration of how the sequence was generated, how allocation was concealed, the integrity of blinding at outcome, the completeness of outcome data, selective reporting and other biases. We would have excluded studies where allocation was clearly not concealed.

Trials with high risk of bias (defined as at least three out of five domains categorised as 'No') were not included in the meta-analysis. If the raters disagreed, the final rating was made by consensus with the involvement of another member of the review group. Where inadequate details of randomisation and other characteristics of trials are provided, authors of the studies were contacted in order to obtain further information. Non-concurrence in quality assessment was reported.

\section{Measures of treatment effect}

\section{Binary data}

For binary outcomes we calculated a standard estimation of the random-effects risk ratio (RR) and its $95 \%$ confidence interval (CI). It has been shown that RR is more intuitive (Boissel 1999) than odds ratios and that odds ratios tend to be interpreted as RR by clinicians (Deeks 2000). For statistically significant results we had planned to calculate the number needed to treat to provide benefit /to induce harm statistic (NNTB/H), and its 95\% confidence interval (CI) using Visual Rx (http://www.nntonline.net/) taking account of the event rate in the control group. This, however, was superseded by Summary of findings table 1 and the calculations therein.

\section{Continuous data}

2.1 Summary statistic

For continuous outcomes we estimated a random-effects mean difference (MD) between groups. We preferred not to calculate effect size measures (standardised mean difference SMD). However, in the case of where scales were of such similarity to allow presuming there was a small difference in measurement, we calculated it and, whenever possible, we transformed the effect back to the units of one or more of the specific instruments.

\section{Unit of analysis issues}

\section{Cluster trials}

Studies increasingly employ 'cluster randomisation' (such as randomisation by clinician or practice) but analysis and pooling of clustered data poses problems. Firstly, authors often fail to account for intra-class correlation in clustered studies, leading to a 'unit of analysis' error (Divine 1992) whereby $p$ values are spuriously low, confidence intervals unduly narrow and statistical significance overestimated. This causes type I errors (Bland 1997, Gulliford 1999).

Where clustering is not accounted for in primary studies, we presented data in a table, with a $\left(^{*}\right)$ symbol to indicate the presence of a probable unit of analysis error. In subsequent versions of this review we will seek to contact first authors of studies to obtain intra-class correlation coefficients for their clustered data and to adjust for this by using accepted methods (Gulliford 1999). Where clustering had 
been incorporated into the analysis of primary studies, we present these data as if from a non-cluster randomised study, but adjusted for the clustering effect.

We have sought statistical advice and have been advised that the binary data as presented in a report should be divided by a 'design effect'. This is calculated using the mean number of participants per cluster $(\mathrm{m})$ and the intra-class correlation coefficient (ICC) [Design effect $=$ $\left.1+(m-1)^{\star} I C C\right]$ (Donner 2002). If the ICC was not reported it was assumed to be 0.1 (Ukoumunne 1999).

If cluster studies has been appropriately analysed taking into account intra-class correlation coefficients and relevant data documented in the report, synthesis with other studies would have been possible using the generic inverse variance technique.

\section{Cross-over trials}

A major concern of cross-over trials is the carry-over effect. It occurs if an effect (e.g. pharmacological, physiological or psychological) of the treatment in the first phase is carried over to the second phase. As a consequence on entry to the second phase the participants can differ systematically from their initial state despite a wash-out phase. For the same reason cross-over trials are not appropriate if the condition of interest is unstable (Elbourne 2002). As both effects are very likely in severe mental illness, we only used data of the first phase of cross-over studies.

\section{Studies with multiple treatment groups}

Where a study involved more than two treatment arms, if relevant, the additional treatment arms were presented in comparisons. Where the additional treatment arms were not relevant, these data were not reproduced.

\section{Dealing with missing data}

1. Overall loss of credibility

At some degree of loss of follow-up, data must lose credibility (Xia 2007). For any particular outcome should more than $50 \%$ of data be unaccounted for, we did not reproduce these data or use them within analyses. If, however, more than $50 \%$ of those in one arm of a study were lost, but the total loss was less than $50 \%$, we marked such data with $\left(^{*}\right)$ to indicate that such a result may well be prone to bias.

\section{Binary}

In the case where attrition for a binary outcome is between 0 and $50 \%$ and where these data were not clearly described, data were presented on a 'once-randomised-always-analyse' basis (an intention to treat analysis). Those leaving the study early were all assumed to have the same rates of negative outcome as those who completed, with the exception of the outcome of death. A sensitivity analysis was undertaken testing how prone the primary outcomes were to change when data from only those who completed the study were compared with intention to treat data using the assumption outlined above.

In the case were number of death was more than $10 \%$ of the sample overall, the above statement was applied but attrition due to death was not imputed.

\section{Continuous}

\subsection{Attrition}

In the case where attrition for a continuous outcome is between 0 and $50 \%$ and data from only those who completed the study are reported, we have reproduced these.

\subsection{Standard deviations}

\subsubsection{General}

Where there are missing measures of variance for continuous data, but exact standard errors or confidence intervals for group means, or either ' $p$ ' or ' $t$ ' values for differences in means, we calculated standard deviation value according to method described in Section 7.7.3 of the Cochrane Handbook for Systematic Reviews of Interventions (Higgins 2011). If standard deviations were not reported and could not be calculated from available data, we asked authors to supply the data. In the absence of data from authors, we used the mean standard deviation from other studies.

\subsubsection{Standard deviation mean number of days per month in hospital}

For the primary outcome, mean number of days per month in hospital, if standard deviations were not reported and could not be calculated from available data, we asked authors for additional information. In the absence of data from authors, we imputed the missing standard deviations using a regression analysis of SD against mean from those trials that provided both. We documented in Table 2 in what studies we imputed SDs according to the above technique.

\subsection{Last observation carried forward}

We anticipated that in some studies the method of last observation carried forward (LOCF) would be employed within the study report. As with all methods of imputation to deal with missing data, LOCF introduces uncertainty about the reliability of the results. Therefore, where LOCF data has been used in the trial, if less than $50 \%$ of the data had been assumed, we reproduced these data and indicated that they are the product of LOCF assumptions.

\subsection{Incomplete data for meta-regression}


In some cases we anticipated that IFACT score variables would not all be available. If IFACT score could not be calculated from available data, we imputed it by multiple imputation using the mi library in R (R 2008). As explained above, we only made these assumptions if we were able to directly rate over $50 \%$ of the data. We documented in Table 3 in what studies we calculated IFACT score according to the above technique.

In some cases we anticipated that baseline hospital use data would not all be available. Missing data was imputed as for the IFACT scores. As explained above, we only make these assumptions if we were able to directly rate over $50 \%$ of the data. We documented for which studies we calculated baseline hospital use data according to the above technique (Table 3).

A sensitivity analysis was undertaken testing how prone the results from meta-regression were to change when data from only those who completed the studies were compared with the imputed data using the assumption outlined above.

\section{Assessment of heterogeneity}

1. Clinical heterogeneity

We considered all included studies initially, without seeing comparison data, to judge clinical heterogeneity. We simply inspected all studies for clearly outlying situations or people, which we had not predicted would arise. When such situations or participant groups arose, these were fully discussed.

In addition two potential sources of heterogeneity were specified a priori (fidelity and baseline level of hospital use (Data extraction and management). These data were extracted as described above.

\section{Methodological heterogeneity}

We considered all included studies initially, without seeing comparison data, to judge methodological heterogeneity. We simply inspected all studies for clearly outlying methods which we had not predicted would arise. Should such methodological outliers arise these will be fully discussed.

\section{Statistical heterogeneity}

3.1 Visual inspection

We visually inspected graphs to investigate the possibility of statistical heterogeneity.

\subsection{Employing the 12 statistic}

Heterogeneity between studies was investigated by considering the $\mathrm{I}^{2}$ method alongside the Chi ' $\mathrm{p}$ ' value. The $\mathrm{I}^{2}$ provides an estimate of the percentage of inconsistency thought to be due to chance (Higgins 2003). The importance of the observed value of $\mathrm{I}^{2} \mathrm{depends}$ on $\mathrm{i}$. magnitude and direction of effects and ii. strength of evidence for heterogeneity (e.g. ' $p$ ' value from Chi2 test, or a confidence interval for 12 ). 12 estimate greater than or equal to $50 \%$ accompanied by a statistically significant $\mathrm{Chi}^{2}$ statistic, was interpreted as evidence of substantial levels of heterogeneity (Section 9.5.2 - Higgins 2011). When substantial levels of heterogeneity were found in the primary outcome, we explored reasons for heterogeneity (Subgroup analysis and investigation of heterogeneity).

\section{Assessment of reporting biases}

Reporting biases arise when the dissemination of research findings is influenced by the nature and direction of results (Egger 1997). These are described in Section 10 of the Cochrane Handbook for Systematic Reviews of Interventions (Higgins 2011). We are aware that funnel plots may be useful in investigating reporting biases but are of limited power to detect small-study effects. We did not use funnel plots for outcomes where there were ten or fewer studies, or where all studies were of similar sizes. In other cases, where funnel plots were possible, we sought statistical advice in their interpretation.

\section{Data synthesis}

Where possible we employed a random-effects model for analyses. We understand that there is no closed argument for preference for use of fixed or random-effects models. The random-effects method incorporates an assumption that different studies are estimating different, yet related, intervention effects. According to our hypothesis of an existing variation across studies, to be explored further in the metaregression analysis despite being cautious that random-effects methods does put added weight onto the smaller of the studies - we favoured using random-effects model.

\section{Subgroup analysis and investigation of heterogeneity}

\section{Subgroup analyses}

We anticipate two sub-group analyses. For the first version of the protocol for this review we did not anticipate any sub-group analyses. On further consideration we now realise that analysis at separate time periods could be thought of as sub-groups. The second sub-group is within the primary outcome and relates to skewed and non-skewed data. This has been introduced late into this protocol and could be considered post hoc. However, we are also conscious that our original rule for management of these data could be considered overly cautious and result in some important data not being presented (Higgins 2011).

\section{Investigation of heterogeneity}


2.1 Anticipated heterogeneity - outcome of mean days per month in hospital

Investigation of heterogeneityformed part of the secondary objectives of the review. We hypothesised that the effect of Intensive Case Management on one of our primary outcomes (mean number of days per month in hospital) differs according to fidelity of intervention to the assertive community treatment model and the baseline level of hospital use.

The association of the IFACT score and the baseline number of days in hospital with the treatment effect was examined by performing random-effects meta-regression analysis in $\mathrm{R}$ ( $\mathrm{R}$ 2008). The script we used to perform meta-regression analyses is reported in Appendix 3. Meta-regression was also carried out using both variables within the same model. The relationship between the treatment effect and the two variables was also examined using a thin plate spline. If possible data from multi-centre studies were to be entered in the metaregression disaggregated into the component centre with outcome and fidelity data for each.

Meta-regression was performed:

a) only if at least ten studies per comparison are available (Higgins 2011)

b) all included studies were entered into the meta-regression. Comparison type was also tested as an additional regressor in the model.

2.2 Unanticipated heterogeneity - other outcomes

2.2.1 For outcomes other than the second primary outcome (not remaining in contact with psychiatric services)

If inconsistency was high this was reported and no exploration undertaken.

2.2.2 For outcome 'not remaining in contact with psychiatric services'

If inconsistency was high this was reported. First we investigated whether data had been entered correctly. Second, if data had been correct, the graph was visually inspected and studies outside of the company of the rest were successively removed to see if heterogeneity was restored. Should this occur with no more than $10 \%$ of the data being excluded, data were presented. If not, data were not pooled.

Should unanticipated clinical or methodological heterogeneity be obvious we simply stated hypotheses regarding these for future reviews or versions of this review. We did not anticipate undertaking analyses relating to these.

\section{Sensitivity analysis}

1. Implication of randomisation

We aimed to include trials in a sensitivity analysis if they are described in some way as to imply randomisation. For the primary outcomes we included these studies and if there was no substantive difference when the implied randomised studies were added to those with better description of randomisation, then all data were employed from these studies.

\section{Standard care caseload}

If data were available, a sensitivity analysis was undertaken testing how prone the primary outcomes were to change when trials comparing Intensive Case Management to standard community care caseload $\leqq 20$ were compared with trials comparing Intensive Case Management to standard community care caseload $>20$. If there was a substantial difference, we reported results and discussed them but continued to pool the data.

\section{Assumptions for lost binary data}

Where assumptions had to be made regarding people lost to follow-up (see Dealing with missing data) we compared the findings of the primary outcomes when we used our assumption compared with completer data only. If there was a substantial difference, we reported results and discussed them but continued to employ our assumption.

4. Assumptions for incomplete data for meta-regression

Where assumptions had to be made regarding missing SDs data in studies entering meta-regression (see Dealing with missing data), we compared the findings of the meta-regression on our primary outcome when we used our assumption compared with data taken from only those who completed the studies. A sensitivity analysis was undertaken testing how prone results from meta-regression were to change when data from those who completed were compared with imputed data using the assumption outlined above. If there was a substantial difference, we reported results and discussed them but continued to employ our assumption.

\section{Appendix 3. R script used to impute data and perform meta-regression analysis}

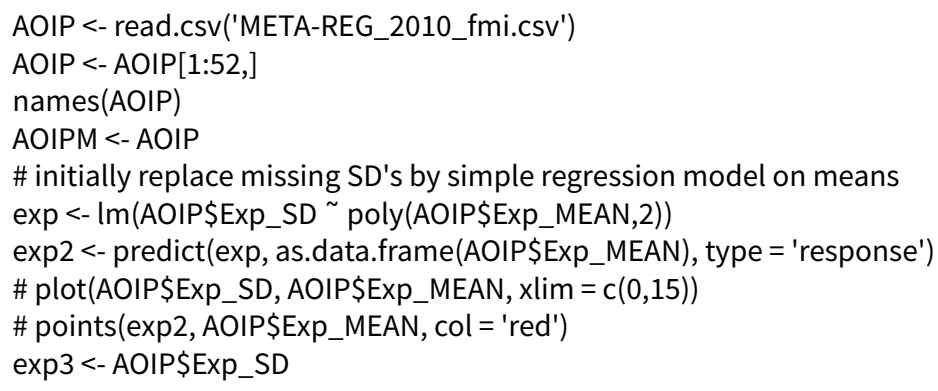


exp3[is.na(exp3)] <- exp2[is.na(exp3)]

AOIP\$Exp_SD $<-$ exp3

$\exp <-\operatorname{Im}($ AOIP\$Con_SD poly(AOIP\$Con_MEAN,2))

exp2 <- predict(exp, as.data.frame(AOIP\$Con_MEAN), type = 'response')

\# plot(AOIP\$Con_SD, AOIP\$Con_MEAN, $x \lim =\mathrm{c}(0,15))$

\# points(exp2, AOIP\$Con_MEAN, col = 'red')

exp3 $<$ - AOIP\$Con_SD

$\exp 3[$ is.na(exp3)] <- exp2[is.na(exp3)]

AOIP\$Con_SD <- exp3

library(mi)

AOIP2 <- cbind(AOIP[,18], AOIP[,5:11], AOIP[,13], AOIP[,16:17])

names(AOIP2) [1] <- names(AOIP) [18]

names(AOIP2) [9] <- names(AOIP) [13]

info.aoip $<-$ mi.info(AOIP2)

\# info.aoip <- update(info.aoip, "include",

\# list(trial_no="FALSE",cent.no="FALSE",trial_id="FALSE",centre_id="FALSE" ))

imp.aoip <- mi(AOIP2, info.aoip, n.iter = 30)

AOIP2 <- mi.data.frame(imp.aoip)

AOIP2 <- cbind(AOIP[,1:4],AOIP2)

\# recreate Fideltot

Fideltot $<$ - AOIP2\$Fid.Org + AOIP2\$Fidstaff

AOIP2 <- cbind(AOIP2, Fideltot)

\# set OPUS baseline mean to missing

is.na(AOIP2[,13]) <- 19

AOIP2.mi <- AOIP2

$A O I P<-$ AOIPM

\# write.csv(AOIP2, 'metaregdataset_imputed2010.csv')

\# meta analysis

library(meta)

AO1 <- metacont(AOIP\$Exp_N, AOIP\$Exp_MEAN, AOIP\$Exp_SD, AOIP\$Con_N, AOIP\$Con_MEAN, AOIP\$Con_SD, AOIP\$centre_id)

AO2 $<-$ metacont(AOIP2\$Exp_N, AOIP2\$Exp_MEAN, AOIP2\$Exp_SD, AOIP2\$Con_N, AOIP2\$Con_MEAN, AOIP2\$Con_SD, AOIP2\$centre_id)

AO3 <- metacont(AOIP2\$Exp_N, AOIP2\$Exp_MEAN, AOIP2\$Exp_SD, AOIP2\$Con_N, AOIP2\$Con_MEAN, AOIP2\$Con_SD, AOIP2\$centre_id, subset $=($ AOIP2\$SCVSLICM $==1))$

AO4 <- metacont(AOIP2\$Exp_N, AOIP2\$Exp_MEAN, AOIP2\$Exp_SD, AOIP2\$Con_N, AOIP2\$Con_MEAN, AOIP2\$Con_SD, AOIP2\$centre_id, subset $=($ AOIP2\$SCvsLICM ==2)

summary $(\mathrm{AO} 1)$

summary(AO2)

\# metaregression

\# Control type

AO2rct $<-\operatorname{Im}($ AO2\$TE AOIP2\$SCvsLICM, weights = AO2\$w.random $)$

summary(AO2rct)

\# Total fidelity score

AO2r1 <- $\operatorname{Im}(\mathrm{AO} 2 \$ T E ~ \sim ~ A O I P 2 \$ F i d e l t o t$, weights = AO2\$w.random $)$

summary $(\mathrm{AO} 2 \mathrm{r} 1)$

plot(AO2\$TE AOIP2\$Fideltot)

Intensive case management for severe mental illness (Review) 
abline $(\operatorname{lm}(\mathrm{AO} 2 \$ T E \sim$ AOIP2\$Fideltot, weights $=$ AO2\$w.random $))$

\# Interaction

AO2rint <- $\operatorname{Im}(\mathrm{AO} 2 \$ \mathrm{TE} \sim \mathrm{AOIP} 2 \$$ Fideltot * AOIP2\$SCvsLICM, weights $=\mathrm{AO} 2 \$$ w.random $)$

summary(AO2rint)

plot(AO2\$TE AOIP2\$Fideltot)

abline $(\operatorname{lm}(\mathrm{AO} 2 \$ T E \sim$ AOIP2\$Fideltot, weights $=$ AO2\$w.random $))$

\# Organizational fidelity score

$\mathrm{AO} 2 \mathrm{r} 2<-\operatorname{lm}(\mathrm{AO} 2 \$ \mathrm{TE} \sim \mathrm{AOIP} 2$ \$Fid.Org, weights $=\mathrm{AO} 2$ \$w.random $)$

summary $(\mathrm{AO} 2 \mathrm{r} 2)$

plot(AO2\$TE AOIP2\$Fid.Org)

abline $(\operatorname{lm}(A O 2 \$ T E \sim A O I P 2 \$ F i d . O r g$, weights $=A O 2 \$$ w.random $))$

\# more fancy plot

plot(AO1\$TE AOIP\$Fid.Org, pch = 21, bg = 'blue', cex = AO1\$w.random*6,

ylab = 'Treatment Effect (Days)', xlab = 'Organizational Fidelity Score')

abline $(\operatorname{Im}(\mathrm{AO} 2 \$ \mathrm{TE} \sim \mathrm{AOIP} 2 \$$ Fid.Org, weights $=\mathrm{AO} 2 \$$ w.random $))$

\# against baseline

AO2r4 <- $\operatorname{Im}($ AO2\$TE AOIP2\$Baseline_Mean, weights = AO2\$w.random)

summary $(\mathrm{AO} 2 \mathrm{r} 4)$

plot(AO2\$TE $\sim$ AOIP2\$Baseline_Mean)

abline $(\operatorname{lm}(A O 2 \$ T E \sim$ AOIP2\$Baseline_Mean, weights = AO2\$w.random $))$

\# fancy plot

plot(AO1\$TE AOIP\$Baseline_Mean, pch = 21, bg = 'blue', cex = A01\$w.random ${ }^{\star} 6$,

ylab = 'Treatment Effect (Days)', xlab = 'Baseline Days in Hospital')

abline $(\operatorname{lm}($ AO2\$TE AOIP2\$Baseline_Mean, weights = AO2\$w.random)

\# multivariate model

$\mathrm{AO} 2 \mathrm{r} 5<-\operatorname{Im}(\mathrm{AO} 2 \$ \mathrm{TE} \sim \mathrm{AOIP} 2 \$$ Baseline_Mean + AOIP2\$SCvsLICM + AOIP2\$Fid.Org, weights $=$ AO2\$w.random $)$

summary(AO2r5)

\# Thin plate spline

library(fields)

$x<-$ cbind(AOIP2\$Baseline_Mean[-19], AOIP2\$Fid.Org[-19])

fit $<-\operatorname{Tps}(x, A O 2 \$ T E[-19]$, weights = AO2\$w.random[-19], df = 10)

$\operatorname{par}(\mathrm{mfrow}=\mathrm{c}(2,1))$

surface(fit)

plot $\left(x, p c h=21, b g=\right.$ 'red', cex $=A 02 \$$ w.random $\left.[-19]^{*} 4\right)$

\section{Appendix 4. Results of searches from previous versions of this review}

1. Selection of studies (see Selection of studies).

When CBI inspected a random sample of study abstracts identified as above (see Search methods for identification of studies) comprising $10 \%$ of total abstracts the principal reviewer (MD) had inspected, disagreement did occur. Full articles were therefore acquired for further inspection. At this next stage MD and CBI had full agreement on the total sample of reports selected for further inspection.

When MD and $\mathrm{CBI}$ in turn inspected all full articles of relevant reports and independently decided whether they met inclusion criteria (see Criteria for considering studies for this review), they fully agreed and no difficulties or disputes arose on any report. It was not necessary contact third reviewer (MM) for clarifying issues.

2. Data extraction (see Data extraction and management)

When CBI independently extracted data from a random sample of included studies ( $10 \%$ of total) disagreements were discussed. MD and $\mathrm{CBI}$ reached full agreement on final decisions.

3. Original Cochrane reviews (Marshall 2000a, Marshall 2000b)

3.1 ACT (Marshall 2000b)

Fourteen trials had met inclusion for the ACT versus standard care comparison. The trials included were Aberg-Wistedt-Sweden 1995, Audini-UK 1994, Bond-Chicago1 1990, Bond-Indiana1 1988, Chandler-California1 1991, Hampton-Illinois 1992, Herinckx-Oregon 1996, Jerrell-SCarolina1 1991, Lehman-Maryland1 1994, Morse-Missouri1 1992, Quinlivan-California 1995, Rosenheck-USA 1993, SolomonPennsylvania 1994 and Test-Wisconsin 1985. All trials were included in the current review in the comparison ICM versus standard care. 
For the ACT versus hospital-based rehabilitation comparison, three trials had been eligible for inclusion (De Cangas-Canada 1994, LafaveCanada 1996, Marx-Wisconsin 1973). All three have been excluded from the current update as they did not met inclusion criteria.

Six trials were included in the ACT versus case management comparison (Bush-Georgia 1990, Essock-Connecticut1 1995, Jerrell-SCarolina1 1991, Morse-Missouri2 1997, Quinlivan-California 1995, Solomon-Pennsylvania 1994). Of the original six studies described above, two were included in the ICM versus standard care comparison as both are three arm studies comparing ICM (in two arms) versus standard care (Jerrell-SCarolina1 1991, Solomon-Pennsylvania 1994). Three trials were included in the ICM versus non-ICM comparison (BushGeorgia 1990, Essock-Connecticut1 1995, Quinlivan-California 1995). Quinlivan-California 1995 was included in both comparisons ICM versus standard care and ICM versus non-ICM, as it had three arms comparing three differentiated interventions (ICM, non-ICM and standard care). The sixth trial, Morse-Missouri2 1997, although previously included, was excluded from the current update because it contained no usable data, as number for treatment groups were not presented.

\subsection{Case Management (Marshall 2000a)}

Ten randomised controlled trials were included in the comparison of case management versus standard care (Curtis-New York 1992, FordUK 1995, Franklin-Texas 1987, Jerrell-SCarolina1 1991, Macias-Utah 1994, Marshall-UK 1995, Muijen-UK2 1994, Quinlivan-California 1995, Solomon-Pennsylvania 1994, Tyrer-UK 1995). Of these eight are now included (Curtis-New York 1992, Ford-UK 1995, Jerrell-SCarolina1 1991, Macias-Utah 1994, Marshall-UK 1995, Muijen-UK2 1994, Quinlivan-California 1995, Solomon-Pennsylvania 1994). Franklin-Texas 1987 and Tyrer-UK 1995 had to be excluded as they did not meet inclusion criteria on type of intervention.

\section{2009 update}

The February 2009 update search of Cochrane Schizophrenia Group's Register of trials yielded 2565 references. We selected 55 for further inspection. Of these 14 trials met the inclusion criteria and were included (Bjorkman-Sweden 2002, Drake-NHamp 1998, Essock-Connecticut2 2006, Harrison-Read-UK 2000, Johnston-Australia 1998, Morse-Missouri3 2005, Muller-Clemm-Canada 1996, OkpakuTennessee 1997, OPUS-Denmark 1999, Pique-California 1999, REACT-UK 2002, Sytema-Netherlands 1999, Salkever-SCarolina 1999, UK700UK 1999). Thirty one trials were excluded.

We added ten English language trials and five Chinese trials to those awaiting assessment and sought further information. Three trials which had been previously been awaiting assessment were able to be included as more reports had become available (Fekete 1998 now included as McDonel-Indiana 1997, Holloway-UK 1996, Shern-USA1 2000).

\section{Appendix 5. Dealing with missing data - standard deviation mean number of days per month in hospital (Intensive Case Management Protocol)}

3.2.2 Standard deviation mean number of days per month in hospital

For the primary outcome, mean number of days per month in hospital, if standard deviations were not reported and could not be calculated from available data, we asked authors for additional information. In the absence of data from authors, we calculated missing standard deviations using a regression analysis of standard deviation against mean, based on data from studies which did report these data. If the standard deviations calculated according to the above technique were available from a previous review (published and unpublished data) (Burns 2007), we used these data.

\section{Appendix 6. Dealing with missing data - incomplete data for meta-regression (Intensive Case Management Protocol)}

3.4 Incomplete data for meta-regression

In some cases we anticipate that IFACT score variables will not all be available. Where these missing data are from multi-centre studies from which we do have relevant data we assumed the missing variable score to be the mode of the available data from the other centres that we used as a reference. Where there was no clear reference centres, we tried to match the study to another we felt to be closest and used those scores. As explained above, we will only make these assumptions if we are able to directly rate over $50 \%$ of the data within each multi-centre study and overall.

In some cases we anticipate that baseline hospital use data will not all be available. Where these missing data are from multi-centre studies from which we do have relevant data we assumed the missing information to be the mean of the available data from the other centres that we used as a reference. Where there was no clear reference centres, we tried to match the study to another we felt to be closest and used those means. As explained above, we will only make these assumptions if we are able to directly rate over $50 \%$ of the data within each multi-centre study and overall.

\section{WHAT'S NEW}

\begin{tabular}{lll}
\hline Date & Event & Description \\
\hline 23 August 2016 & New search has been performed & $\begin{array}{l}\text { Results of } 2012 \text { and } 2015 \text { update searches added to the review. } \\
\text { Two new studies added to included studies. }\end{array}$ \\
\hline
\end{tabular}




\begin{tabular}{lll}
\hline Date & Event & Description \\
\hline 23 August 2016 & $\begin{array}{l}\text { New citation required but conclusions } \\
\text { have not changed }\end{array}$ & $\begin{array}{l}\text { Data from two new studies did not substantially alter results or } \\
\text { change overall conclusions. }\end{array}$ \\
\hline 10 April 2015 & Amended & $\begin{array}{l}\text { Search updated, and 299 possibly related references were added } \\
\text { to 'Classification pending references' of the review. }\end{array}$ \\
\hline
\end{tabular}

\section{HISTORY}

Protocol first published: Issue 3, 2009

Review first published: Issue 10, 2010

\begin{tabular}{lll}
\hline Date & Event & Description \\
\hline 12 August 2012 & Amended & $\begin{array}{l}\text { Update search of Cochrane Schizophrenia Group's Trial Regis- } \\
\text { ter (see Search methods for identification of studies), 81 studies } \\
\text { added to awaiting classification. }\end{array}$ \\
\hline 6 October 2010 & Amended & Contact details updated. \\
\hline
\end{tabular}

\section{CONTRIBUTIONSOF AUTHORS}

Marina Dieterich: developed and wrote protocol, participated in literature searches, selected studies and extracted data, wrote report for both versions.

Claire Irving: developed and wrote protocol, participated in studies selection and data extraction for original version.

Hanna Bergman: carried out trial selection and data extraction for 2015 update.

Mariam Khokhar: carried out trial selection and data extraction for 2015 update.

Bert Park: carried out meta-regression analysis, helped in writing the report for original version.

Max Marshall: developed and wrote protocol, helped in studies selection, final draft for original version.

\section{DECLARATIONSOF INTEREST}

Marina Dieterich: none known.

Claire Irving: none known.

Hanna Bergman: none known.

Mariam Khokhar: none known.

Bert Park: none known.

Max Marshall: was involved in one included study and has written extensively in this area.

\section{SOURCES OF SUPPORT}

\section{Internal sources}

- University of Verona, Italy.

Department of Medicine and Public Health, Section of Psychiatry and Clinical Psychology

\section{External sources}

- NIHR, UK.

Cochrane Incentive Award 15/81/30 - Intensive case management for severe mental illness

\section{DIFFERENCES BETWEEN PROTOCOL AND REVIEW}

The following section follows the numbering system of the methods of the review. 
1.) Types of outcome measures (see Types of outcome measures)

The time grouping of outcomes has been slightly amended in the review, introducing a different timing for follow-up assessed once the active intervention was stopped. We have reported the protocol version below.

Outcomes were grouped by time into short term (up to and including 6 months), medium term ( 7 months to up to and including 12 months) and long term (over 12 months). Where available, 24 months was the preferred follow-up point for calculating mean days per months in hospital. If more than one follow-up point within the same period were available, we reported the latest one.

2.) Some social functioning and costs outcomes have been slightly amended in the review (see Secondary outcomes). We have reported the protocol version below.

4. Social functioning

4.1 Imprisonment (i.e. police contacts \& arrests)

9. Economic

9.1 Costs of psychiatric hospital care

9.2 Costs of health care

9.3 Costs of all care

3.) Compliance with medication (see Secondary outcomes)

This secondary outcome was listed twice in the protocol due to inattention (both as global state and behaviour). We have amended it, listing 'compliance with medication' just under 'global state' outcome.

4.) Data regarding criteria and outcomes (see Data extraction and management)

We further clarified this section in the review. We have reported the protocol version below.

\section{Extraction}

1.1 Data regarding criteria and outcomes

Authors MD and CBI independently extracted data from included studies. Again, any disagreement was discussed, decisions documented and, if necessary, authors of studies were contacted for clarification. With remaining problems MM helped clarify issues and those final decisions were documented. Data presented only in graphs and figures were extracted whenever possible, but were included only if two reviewers independently had the same result. Attempts were made to contact authors through an open-ended request in order to obtain missing information or for clarification whenever necessary. Where possible, we extracted data relevant to each component centre of multicentre studies separately.

5.) Skewed data (see Data extraction and management)

We had not anticipated the following clarification in the protocol, but added it in the review.

We implemented one exception to the above rules in order to present more data, recognising that this is a post hoc decision, but also that the rules with regards to management of skewed data and how robust skewed data are within meta-analysis are unclear (Higgins 2011). Where mean number of days in hospital data were skewed, and they were provided by studies of fewer than 200 participants, we entered those data into a subgroup of the overall analysis. We also presented the overall effect from all data pooled.

6.) 'Summary of findings' table (see Data extraction and management)

The way the outcomes included in the 'Summary of findings' table were listed and presented were slightly rearranged, but not substantially modified. We have reported the protocol version below. Besides, we decided not to apply the low and high control risk calculation as stated in the protocol, and reported below.

Summary of findings table

We anticipate including the following long term main outcomes in a summary of findings table.

\section{Global state}

1.1 Hospitalisation: mean number of days per month in hospital

1.2 Relapse

1.3 Leaving the study early (lost to follow up)

2. Service use

2.1 Hospital admission across time

3. Adverse effect 
3.1 Death - suicide

4. Social functioning

4.1 Employment - unemployed at end of study

5. Mental state: general symptoms

5.1. Not improved to a clinically meaningful extent (as defined in trial)

Within the Summary of findings table we assumed for calculation of the low risk groups that the lowest control risk applied to all data. We did the same for the assumption of the highest risk groups.

7.) Assessment of risk of bias in included studies (see Assessment of risk of bias in included studies) We further clarified this section in the review. We have reported the protocol version below.

Again working independently, MD and CBI assessed risk of bias using the tool described in the Cochrane Collaboration Handbook (Higgins 2011). This tool encourages consideration of how the sequence was generated, how allocation was concealed, the integrity of blinding at outcome, the completeness of outcome data, selective reporting and other biases. We would have excluded studies where allocation was clearly not concealed.

8.) Binary (see Dealing with missing data, 2.)

We have further clarified this paragraph in the review. We have reported the protocol version below.

In the case where attrition for a binary outcome is between 0 and $50 \%$ and where these data were not clearly described, data were presented on a 'once-randomised-always-analyse' basis (an intention to treat analysis). Those leaving the study early were all assumed to have the same rates of negative outcome as those who completed, with the exception of the outcome of death. A sensitivity analysis was undertaken testing how prone the primary outcomes were to change when data from only those who completed the study were compared with intention to treat data using the assumption outlined above.

9.) Standard deviation mean number of days per month in hospital (see Dealing with missing data, 3.2.2)

Substantially amended. For original protocol version, please see Appendix 5.

10.) Incomplete data for meta-regression (see Dealing with missing data, 3.4)

Substantially amended. For original protocol version, please see Appendix 6.

11.) Statistical heterogeneity (see Assessment of heterogeneity, 3.2)

We further clarified the last paragraph in the review. We have reported the protocol version below.

3.2 Employing the 12 statistic

Heterogeneity between studies was investigated by considering the $\mathrm{I}^{2}$ method alongside the Chi ' $\mathrm{p}$ ' value. The $\mathrm{I}^{2}$ provides an estimate of the percentage of inconsistency thought to be due to chance (Higgins 2003). The importance of the observed value of $\mathrm{I}^{2}$ depends on $\mathrm{i}$. magnitude and direction of effects and ii. strength of evidence for heterogeneity (e.g. ' $p$ ' value from Chi 2 test, or a confidence interval for $\mathrm{I}^{2}$ ). $\mathrm{I}^{2}$ estimate greater than or equal to $50 \%$ accompanied by a statistically significant $\mathrm{Chi}^{2}$ statistic, was interpreted as evidence of substantial levels of heterogeneity (Section 9.5.2 - Higgins 2011) and reasons for heterogeneity were explored. If the inconsistency was high and the clear reasons were found, data were presented separately.

12.) Subgroup analyses (see Subgroup analysis and investigation of heterogeneity, 1.)

No subgroup analyses had been anticipated in the protocol.

13. Subgroup analyses (see Subgroup analysis and investigation of heterogeneity, 2.)

We further clarified this section in the review. We have reported the protocol version below.

2. Investigation of heterogeneity

2.1 Unanticipated heterogeneity

Should unanticipated clinical or methodological heterogeneity be obvious we will simply state hypotheses regarding these for future reviews or versions of this review. We do not anticipate undertaking analyses relating to these.

14.) Sensitivity analysis (see Sensitivity analysis, 4.)

Substantially amended. We have reported the protocol version below.

4. Assumptions for incomplete data for meta-regression

Where assumptions had to be made regarding missing trial data for meta-regression (see Dealing with missing data) we compared the findings of the meta-regression on primary outcome when we used our assumption compared with completer data only. A sensitivity 
analysis was undertaken testing how prone result from meta-regression were to change when 'completed' data only were compared to the imputed data using the above assumption. If there was a substantial difference, then only completed data were employed.

\section{INDEX TERMS}

\section{Medical Subject Headings (MeSH)}

${ }^{\star}$ Case Management; Community Mental Health Services [*methods]; Employment [statistics \& numerical data]; Hospitalization [statistics \& numerical data]; Mental Disorders [ ${ }^{*}$ therapy]; Outcome and Process Assessment, Health Care [ ${ }^{\star}$ methods]; Randomized Controlled Trials as Topic; Regression Analysis; Suicide [statistics \& numerical data]

\section{MeSH check words}

Humans 